Space and Iconicity in German Sign Language (DGS) 
(C) 2007, Pamela Perniss

Cover illustration: Pamela Perniss, inspired by El Lissitzky, Proun 99

Cover design: Ponsen \& Looijen bv, Wageningen

Printed and bound by Ponsen \& Looijen bv, Wageningen 


\title{
Space and Iconicity in German Sign Language (DGS)
}

\author{
een wetenschappelijke proeve \\ op het gebied van Letteren
}

\section{Proefschrift}

ter verkrijging van de graad van doctor

aan de Radboud Universiteit Nijmegen

op gezag van de rector magnificus prof. mr. S.C.J.J. Kortmann,

volgens besluit van het College van Decanen

in het openbaar te verdedigen op maandag 12 november 2007

om 13.30 uur precies

door

\section{Pamela M. Perniss}

geboren op 13 mei 1974 te Ludwigsburg (Duitsland) 
Promotores: $\quad$ Prof. Dr. Stephen C. Levinson

Prof. Dr. Ulrike Zeshan

Co-promotor: $\quad$ Dr. Asli Özyürek

Manuscriptcommissie:

Prof. Dr. Pieter C. Muysken

Prof. Dr. Karen Emmorey

Prof. Dr. Elisabeth Engberg-Pedersen

The research reported in this thesis was supported by a grant from the Max-PlanckGesellschaft zur Förderung der Wissenschaften, München, Germany. 


\section{Acknowledgments}

First and foremost I would like to thank my promotors Stephen Levinson and Ulrike Zeshan, and my co-promotor Asli Özyürek for their academic guidance, scientific expertise, and intellectual counsel throughout the maturation process of this thesis. I am thankful to have had the opportunity to write my thesis in such an outstanding academic institution as the MPI for Psycholinguistics represents. I would like to thank the members of the Language and Cognition group, in particular. Individuals have come and gone over the years, but at the core there remains an atmosphere in which ideas flourish and thrive, fostering an always stimulating and exciting intellectual environment, of which I am fortunate have been a part.

In addition to my (co-)promotors, many other people have taken the time to discuss with me the contents of the thesis and the ideas developed in it. I would like to thank especially David Birdsong, Niclas Burenhult, Onno Crasborn, Karen Emmorey, Marianne Gullberg, Theresa Hanske, Sotaro Kita, Paul Kockelman, Scott Liddell, John Lucy, Jill Morford, Victoria Nyst, Roland Pfau, Jennie Pyers, Waldemar Schwager, Gunter Senft, Ann Senghas, Dan Slobin, and Inge Zwitserlood. I am indebted to them for sharing their knowledge and expertise, and I have benefited greatly from it.

This thesis would not have been possible without the help and friendship of the many deaf people whom I have had the fortune of meeting since signing up for my first DGS course. I am especially grateful to Volker Maaßen and Peter Rapp from the Loor Ens school for DGS in Cologne, whose pedagogical and didactic skills paved my way into the world of signing, and who facilitated many of my contacts within the deaf community and much of the data collection for this thesis. I am immensely grateful to the deaf individuals who took the time to participate in this project, and give my heartfelt thanks also to Christian Ängenheister, Claudia Hingst, Murat Karabeke, Ulla Krause, Horst Sieprath, Anne Warnking, Isa Werth, Frank Winkens, and Uwe Zelle. The Loor Ens school and the Linguistics Departments at Cologne University and Aachen University kindly made available rooms in which I was able to make video recordings. In addition, I am indebted to Murat Karabeke for many hours of transcription and discussion of the DGS data.

The technical, financial, and administrative support offered by the MPI for Psycholinguistics is invaluable and incomparable. I am lucky to have had access to technical equipment of the highest quality and to premium annotation tools from the beginning. Thanks 
especially to Ric van Viersen and Ad Verbunt for answering to calls of technical need and distress.

My thanks goes also to my fellow PhD students, many of whom have become good friends over the years, and who together build and maintain a network of mutual support and understanding. Good friends here and afar have made the time vastly more enjoyable and have kept things bearable when they threatened not to be. I value and cherish the time spent with all of you - thank you dearly! And an extra big thanks to my paranimfen, Anita Wagner and Federico Rossano.

In more practical matters, I owe thanks to Arno Hazekamp, Jan Peter de Ruiter, and Inge Zwitserlood for help with the Dutch summary. Thanks also to Arno for so many other little and not-so-little things along the way. Special thanks and appreciation to Asli Özyürek, who got me through the final months of writing, and with whom I have the pleasure of continuing to work. Finally, my parents and family have encouraged and supported me always, and I thank them for standing by me. 
Acknowledgements

i

Table of contents

iii

Abbreviations and transcription conventions vii

Chapter 1. Introduction 1

1.1 Iconicity and sign language structure 3

1.2 The expression of location and motion in spoken and signed language 8

1.3 DGS background and data collection 11

1.3.1 Sociolinguistic and historical information 11

$\begin{array}{ll}\text { 1.3.2 Previous research on DGS } & 11\end{array}$

1.3.3 Contact with the deaf community and data collection $\quad 12$

1.3.4 Type of data 12

1.4 Structure of the dissertation 13

Chapter 2. Iconicity and sign language structure $\quad 17$

2.1 Meaning construction in signed language: Iconicity and beyond 17

2.1.1 Iconicity as a semiotic and linguistic notion 19

2.1.2 Iconicity in spoken and signed language 21

2.2 Sign language structure: Phonology 26

2.3 Sign language structure: The morphology of classifier predicates 32

2.3.1 Encoding size and shape information in spoken languages 34

2.3.2 Analyses of sign language classifier predicates as composed of discrete morphemes $\quad 35$

2.3.3 Analyses of sign language classifier predicates as composed of $\begin{array}{ll}\text { discrete and analogue elements } & 37\end{array}$

2.4 Sign language structure: Simultaneous constructions 39

2.4.1 Simultaneous constructions that reflect perceptual structure 40

2.4.2 Simultaneous constructions that reflect discourse structure 42

2.5 Summary 42

Chapter 3. The spatial domain in spoken and signed language 45

3.1 The spatial domain $\quad 45$

3.1.1 The encoding of location in language 46

3.1.1.1 Angular specification $\quad 47$

3.1.1.2 Non-angular specification $\quad 49$

3.1.2 The encoding of motion in language $\quad 50$

3.2 Frames of reference in spoken and signed language 52

3.2.1 Frame of reference research in spoken languages 55 
3.2.2 Frame of reference research in sign languages 58

$\begin{array}{lll}\text { 3.3 Signing perspective } & 63\end{array}$

3.4 Viewpoint in spatial descriptions in the visual-spatial modality 64

$\begin{array}{lll}3.4 .1 & \text { Signer vs. addressee viewpoint in spatial descriptions } & 64\end{array}$

3.4.2 Shared space in spatial descriptions $\quad 67$

3.5 Alignments between perspective, frames of reference, and classifier predicates 68

3.5.1 The relationship between frames of reference and signing perspective $\quad 70$

3.5.2 The relationship between classifier predicates and signing perspective $\quad 75$

$\begin{array}{lll}3.6 & \text { Summary } & 76\end{array}$

Chapter 4. Static scene spatial description in DGS

4.1 The structure of locative constructions in sign languages 77

4.2 Methodology $\quad 82$

4.2.1 Task description $\quad 82$

$\begin{array}{lll}\text { 4.2.2 Stimulus materials } & 83\end{array}$

4.2.3 Participants $\quad 84$

4.2.4 Data collection $\quad 84$

$\begin{array}{lll}4.2 .5 & \text { Data summary } & 85\end{array}$

4.2.6 Data coding 86

4.3 DGS locative descriptions $\quad 88$

4.3.1 Convention 1: Identification precedes spatialization 91

4.3.2 Convention 2: Classifiers encode all spatial information 102

4.3.3 Convention 3: Encode ground before figure 119

4.3.4 Convention 4: Use simultaneous classifier constructions 130

4.3.5 Convention 5: Sign from signer's (external) viewpoint 135

4.4 Summary and discussion $\quad 137$

Chapter 5. Perspective and frames of reference in DGS static scene $\begin{array}{lr}\text { spatial description } & 139\end{array}$

5.1 Data 140

5.2 Perspective in DGS static scene descriptions 141

5.3 Frames of reference in DGS static scene descriptions 143

5.4 Perspective and frame of reference alignment 147

5.5 Analysis of addressees' interpretations of static scene spatial descriptions 149

$\begin{array}{ll}\text { 5.5.1 Coding and stimulus categorization } & 151\end{array}$

5.5.2 Rotation and translation in interpretation 153

5.5.3 Rotation and translation interpretations in the data 154 $\begin{array}{lll}5.5 .3 .1 & \text { Simple scenes } & 155\end{array}$

5.5.3.2 Complex scenes $\quad 157$

5.5.4 When iconicity is not enough: miscommunications of spatial relationships 160

5.5.5 General discussion of interpretation results 163

$\begin{array}{lll}\text { 5.6 Summary and discussion } & 165\end{array}$ 
Chapter 6. Perspective and frames of reference in DGS event narratives

$\begin{array}{lll}\text { 6.1 Methodology } & 168\end{array}$

$\begin{array}{lll}6.1 .1 & \text { Stimulus materials and task description } & 168\end{array}$

$\begin{array}{ll}\text { 6.1.2 Data collection } & 169\end{array}$

$\begin{array}{ll}\text { 6.1.3 Data coding } & 169\end{array}$

6.2 The encoding of location in event narratives 175

$\begin{array}{ll}\text { 6.2.1 The use of signing perspective to encode location } & 175\end{array}$

$\begin{array}{ll}\text { 6.2.2 The use of frames of reference to encode location } & 179\end{array}$

6.3 The encoding of location and motion in event narratives 180

6.3.1 The use of signing perspective to encode location and motion $\quad 180$

6.3.2 The use of frames of reference to encode location and motion 182

6.4 Alignment between perspective and frames of reference in location and $\begin{array}{ll}\text { motion scenes } & 183\end{array}$

6.5 Encoding of viewpoint in the relative frame of reference in teller and $\begin{array}{ll}\text { reteller narratives } & 187\end{array}$

$\begin{array}{lll}\text { 6.6 Summary and discussion } & 190\end{array}$

\section{Chapter 7. The relationship between signing perspective and spatial/activity} predicates

7.1 The relationship between the use of perspective and different spatial/activity predicates in the sign language literature

7.2 Methodology 196

7.2.1 Stimulus materials and data collection 197

7.2.2 Coding of spatial/activity predicates and perspective 197

7.2.3 The relationship between perspective and classifier predicates 198

7.2.4 The relationship between signing perspective and lexical predicates $\quad 200$

7.2.5 The relationship between signing perspective and index signs 200

7.3 Combinations of perspective and spatial/activity predicates in the data:

$\begin{array}{ll}\text { Qualitative analysis } & 201\end{array}$

7.4 Distribution of perspective and spatial/activity predicates in the data:

$\begin{array}{ll}\text { Quantitative analysis } & 207\end{array}$

7.5 The relationship between semantic event components and classifier-

$\begin{array}{ll}\text { perspective constructions } & 210\end{array}$

7.5.1 The motivation for character perspective coding 210

7.5.2 The motivation for observer perspective coding 213

$\begin{array}{lll}\text { 7.6 Summary and discussion } & 216\end{array}$

Chapter 8. Form and function of simultaneity in DGS event narratives 219

8.1 Previous research on simultaneous constructions related to perspective 220

8.2 Discourse structure constraints of efficiency and informativeness 221

$\begin{array}{ll}\text { 8.3 Data } & 222\end{array}$

8.4 Non-aligned character perspective constructions: the use of multiple 
articulators to achieve full semantic specification

8.5 Double-perspective constructions: The use of multiple articulators

to achieve a mapping between different event space projections

8.5.1 Mapping between perspectives for spatial coherence:

efficiency of expression

8.5.2 Mapping between perspectives for spatial coherence:

informativeness of expression

8.6 Summary and discussion

Chapter 9. Conclusion

9.1 Summary of main findings

9.2 Methodological implications

9.3 Theoretical implications

244

9.3.1 Perspective and frames of reference analysis

9.3.2 Space, iconicity, and discourse

9.4 General conclusions

Appendix A: Stimulus pictures used in the spatial description task

References

Summary

Samenvatting

Zusammenfassung

Curriculum vitae 


\section{Abbreviations, of sign languages:}

AdaSL

ASL

Auslan

ÖGS

BSL

CSL

DSL

DGS

IPSL

ISL

ISL

Kata Kolok

KSL

LIS

LSF

NGT

NicaSL

NSL

LSQ

SSL

DSGS

TID

USL

VGT

general:

ABL

biman.

char

CL

cont

conv.

descr.

DIS

dom.

eleph

FoR

GCM

gen. man. activ.

ident.

intr.

lex. ident.
Adamorobe Sign Language (Ghana)

American Sign Language

Australian Sign Language

Österreichische Gebärdensprache, Austrian Sign Language

British Sign Language

Chinese Sign Language

Danish Sign Language

Deutsche Gebärdensprache, German Sign Language

Indo-Pakistani Sign Language

Irish Sign Language

Israeli Sign Language

Kata Kolok (Bali)

Korean Sign Language

Lingua Italiana dei Segni, Italian Sign Language

Langue des Signes Françaises, French Sign Language

Nederlandse Gebarentaal, Sign Language of the Netherlands

Nicaraguan Sign Language

Norwegian Sign Language

Langue des Signes Québécoises, Quebec Sign Language

Swedish Sign Language

Deutsch-Schweizerische Gebärdensprache, Swiss-German Sign

Language

Türk Işsaret Dili, Turkish Sign Language

Ugandan Sign Language

Vlaamse Gebarentaal, Flemish Sign Language

ablative case

bimanual

character

classifier

container

convention

descriptions

dispositional

dominant (hand)

elephant

frame of reference

general class marker

generic manual activity

identification

intrinsic

lexical identification 


\begin{tabular}{ll} 
LH & left hand \\
loc & location \\
masc & masculine \\
mot & motion \\
NA & not applicable \\
ndom. & non-dominant (hand) \\
non-feat. & non-featured \\
num. & numeral \\
obs & observer \\
ornt./orn. & orientation \\
persp. & perspective \\
PN & third person pronoun \\
post & posture \\
pred. & predicate \\
PREP & preposition \\
ref. & referent \\
rel. & relative \\
RH & right hand \\
rot. & rotation \\
SCM & specific class marker \\
sg & singular \\
simult. & simultaneous \\
spat. & spatialization \\
sth. & something \\
stim. pic. & stimulus pictures \\
trans. & translation \\
upr & upright entity \\
2-leg & 2-legged entity \\
\hline &
\end{tabular}

Transcription conventions:

BROWN
$\mathrm{CL}_{\mathrm{E}}$
$\mathrm{CL}_{\mathrm{H}}$
$\mathrm{CL}_{\mathrm{L}}$
$\mathrm{SASS}$
$\mathrm{CL}_{\mathrm{E}}$ (man)
$\mathrm{CL}_{\mathrm{H}}$ (ball)
$\mathrm{CL}_{\mathrm{H}}$ (ball): hold
$\mathrm{CL}_{\mathrm{E}}$ (man): locR,ornL
$\mathrm{CL}_{\mathrm{L}}$ (front limb)
$\mathrm{CL}_{\mathrm{L}}$ (front limb): stand
SASS(hat)
SASS(cone): locL
LEX
LOOK

lexical sign

entity classifier

handling classifier

limb classifier

size and shape specifier

the referent represented by the entity classifier is in brackets

the referent represented by the handling classifier is in brackets the type of handling is after the colon

the location and/or orientation of the referent represented with a classifier are after the colon

the type of limb represented by the limb classifier is in brackets the posture/manner of locomotion represented by the limb classifier is after the colon

the referent represented by the SASS is in brackets

the location of the referent represented with a SASS is after the colon

lexical predicate

directional predicate "to look at" 


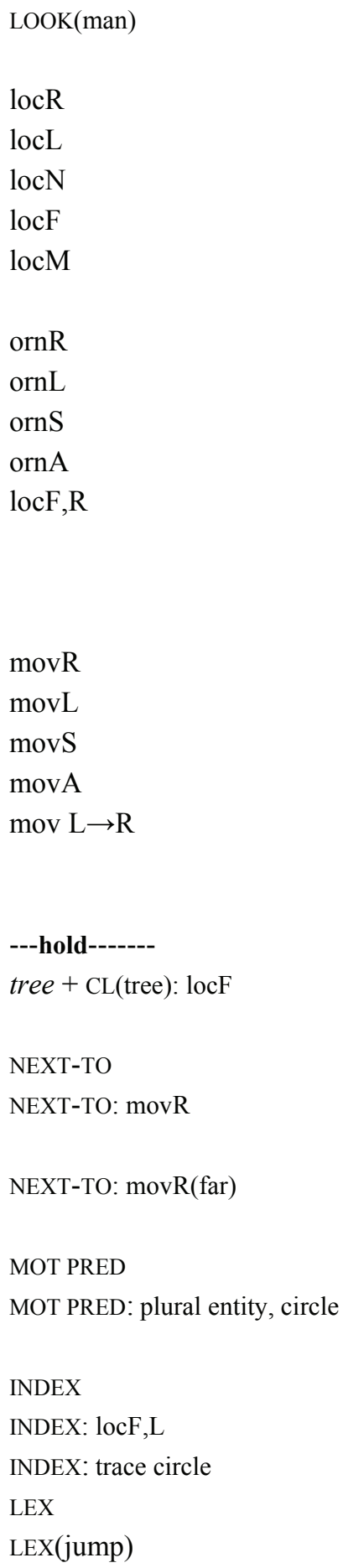

referent whose direction of looking is represented with the predicate is in brackets

location on the right side of sign space (lateral axis)

location on the left side of sign space (lateral axis)

location near to the signer's body (sagittal axis)

location far from the signer's body (sagittal axis)

location in the middle of sign space (for both lateral and sagittal axes)

orientation to the right

orientation to the left

orientation toward the signer

orientation away from the signer

location on the sagittal axis before the comma, location on the lateral axis after the comma (thus, in the example, the referent is located far from the signer's body on the right side of sign space)

movement of sign to the right

movement of sign to the left

movement of sign toward the signer

movement of sign away from the signer

movement of sign from place before the arrow to place after the arrow (thus, in the example, the sign moves from the left to the right)

a sign form holds for the duration of the dotted line

the italicized word is mouthed simultaneously with the

production of the classifier predicate

spatially modifiable prepositional sign

direction of spatial modification of the prepositional sign is after the colon

the length of the movement of the prepositional sign is in parentheses

general motion predicate

referent (type) referred to is before the comma, shape of the predicate in space is after the comma

pointing sign (index finger)

location of referent pointed to is after the colon

traced path of referents pointed to is after the colon

lexical spatial/activity predicate

meaning of lexical predicate is in parentheses 


\section{Introduction}

Chapter 1

Sign languages are the natural languages of the deaf. They are produced in the visual-spatial modality by using the hands, torso, eyes, face, and head as articulators, and are perceived visually. In this, they differ radically from spoken languages, which are produced by manipulations of the vocal tract, and auditorily perceived. This dissertation investigates the influence of the modality on the structure of German Sign Language (Deutsche Gebärdensprache, DGS) in the expression of spatial relationships. The discussion of the relevant theoretical domains is prefaced by a general introduction to sign languages as natural language systems and their use in deaf communities.

As Heßmann (2001) notes, most people would not include "hearing" on a list of attributes to describe themselves. For most people, the ability to hear, as one of our basic perceptual faculties, is taken for granted in the same way as is breathing. However, about $0.1 \%$ of the world's population cannot hear, either congenitally from birth or through trauma. A large portion of these people use a sign language as their primary and natural means of communication.

Only approximately $8 \%$ of deaf children are born to deaf parents; the large majority are born to hearing parents This makes the situation surrounding the acquisition and use of a signed language unique. Only this small percentage of sign language users acquires sign language "natively", that is, in what we consider to be the normal way, through immediate and constant exposure from birth. Thus, for the majority of deaf infants and children, one of the main consequences of being deaf is exclusion from the normal course of language exposure and acquisition. These children do not begin to learn sign language until they are brought into contact with other deaf children or adults - for example, at day care centers, at training centers, at schools, within their families, or at deaf clubs. It is here that they are in a situation that truly gives them access to a full language system. ${ }^{1}$

\footnotetext{
${ }^{1}$ Deaf children have partial access to the spoken language visually through lip-reading, and indeed, learning to lip-read plays a strong role in the education of deaf children in Europe and the United States.
} 
It is a common misconception that there is a universal sign language. ${ }^{2}$ Sign languages have developed in deaf communities all over the world, created over the course of decades or centuries through the contact and communication of deaf people with each other. The history of many of the sign languages that we speak of and research today, especially Western sign languages, can generally be dated back to the institutionalization of deaf education and/or the creation of national Federations of the Deaf. In some cases, the use of sign languages can be dated back much further (for example, in the Imperial Ottoman palace in the 16th -18 th centuries, cf. Miles 2000), but it is not clear to what extent there is continuity between the systems used in antiquity and those used today.

Research on sign languages as language systems (as opposed to the hodgepodge of mimes and gestures they were long taken to represent) began only about five decades ago. Linguistic inquiry into sign language structure was pioneered by descriptions of the Sign Language of the Netherlands (NGT) by Tervoort (1953) and American Sign Language (ASL) by Stokoe (1960). Since then, most sign language research has been done on ASL and European sign languages, but increasingly more sign languages around the world are coming under linguistic, sociolinguistic, and even psycholinguistic investigation.

Despite the difference in modality, sign and spoken languages have been found to share fundamental linguistic properties on all levels of linguistic structure, including phonology, morphology, and syntax (Klima and Bellugi 1979; Liddell 1980; Padden 1983; Stokoe 1960; Supalla 1982). ${ }^{3}$ Like spoken languages, sign languages have forms that belong to different lexical categories and that constitute a conventionalized lexicon (i.e. pairings of form and meaning that must be learned by users), and language-specific rules for their combination (Aarons 1996; Fischer 1975; Frishberg 1975; Supalla and Newport 1978). Furthermore, in deaf children with deaf parents, the process of acquisition of sign language has been shown to parallel the developmental course of spoken language acquisition (Newport and Meier 1985; Petitto and Marentette 1991), and neural substrates of spoken and sign language processing have been found to be similar (MacSweeney et al. 2002; Poizner et al. 1987). Over the last

\footnotetext{
${ }^{2}$ There is, however, a so-called "International Sign Language", which is a lingua franca used by signers of different national sign languages (see e.g. Supalla and Webb 1995). It is usually created ad hoc, though it has some "fixed" signs (often of ASL origin), and is used, for example, at international deaf and sign language research conferences (e.g. at WFD (World Federation of the Deaf) and TISLR (Theoretical Issues in Sign Language Research).

${ }^{3}$ The majority of this research has been on ASL, and indeed, the majority of studies cited in this dissertation refer to research on ASL. Where they do not, and it is relevant to the discussion, the specific sign language(s) are stated together with the reference. In addition, throughout the dissertation, I use the term "signed language" to refer to the fact of language in the visual-spatial modality, as opposed to the oral-aural modality of spoken languages, and "sign language(s)" to refer to specific languages in the visual-spatial modality, individually or as a group.
} 
decades, these findings have conclusively proven the status of sign languages as full human languages, on a par with spoken languages.

However, recent research has suggested that the visual-spatial modality influences phonological and morphosyntactic structure in such a way as to create modality effects in certain domains of linguistic expression. This is true especially for signed expressions that use space to express spatial and non-spatial relations, to mark the arguments of verbs, and to make pronominal reference (Emmorey 2002; Liddell 2003a; Meier 1990; Talmy 2003). Moreover, these domains have been found to exhibit more similarity across sign languages than across spoken languages (Aronoff et al. 2003; Aronoff et al. 2005; Woll 2003). This suggests that particular characteristics of the modality might constrain linguistic expression in a way that results in fundamental modality-driven differences between signed and spoken language (Meier 2002). One such characteristic is the potential of the visual-spatial modality for iconic representation. The three-dimensional visual space in which sign languages are produced, and the articulators that move within it, are remarkably well-suited for iconic representation of spatial information, in particular, of shape, location, motion, and action (Taub 2001; Wilcox 2004). This is evident at different levels of sign language structure. The role of iconicity in the lexicon and in certain morphosyntactic processes is discussed in the next section.

\subsection{Iconicity and sign language structure}

Iconicity within a language system refers to the existence of "structure-preserving correspondences" between a linguistic form and its meaning (see Taub 2001 and Wilcox 2006 for signed language; and see Peirce (1902) and de Saussure (1916) for language generally). In signed language, a direct relationship between form and meaning can be seen on the lexical level and in the use of morphologically complex predicates. For example, in DGS, as well as in ASL, the sign for TREE is made by holding the forearm vertically and slightly turning the hand (open, with fingers spread) at the wrist. The elbow rests on the back of the other hand, which forms the base, together with the horizontal forearm (see the first still in example (1) below). In the sign TREE, the "mapping" (i.e. the set of structure-preserving correspondences that defines the iconic relationship) between the linguistic form (the sign TREE) and the concept which it represents (the prototypical image of a tree) is evident in three main correspondences. The trunk is represented by the straight, vertical forearm, the branches are represented by the hand and the spread fingers, and the base/roots of the tree in the ground are represented by the placement of the other hand/forearm (Taub 2001: 29). 
The spatial medium of sign space also allows structure-preserving mappings of referent location and motion through the use of morphologically complex predicates known as classifier predicates, which are used in most sign languages studied to date (see chapter 2 , section 2.3). In these predicates, the handshape expresses information about the size and shape of the referent, and the position and movement of the hand(s) in sign space encode information about the location and motion of the referent(s) in the event space (Emmorey 2002; Engberg-Pedersen 1993; Schembri 2003; Schick 1990). In contrast to lexical signs like TREE, the handshape in these predicates is a morpheme that encodes information about a particular referent by "classifying" it according to certain semantic properties, like shape.

In discourse, the use of a classifier predicate to encode spatial information about a referent is typically preceded by a lexical sign that identifies the referent. Two main types of classifier predicates are relevant in this dissertation: entity classifiers (in which the handshape represents a whole entity) and handling classifiers (in which the handshape represents the manipulation of a referent). Example (1) shows the use of entity classifier predicates in DGS to describe the relationship between the man and the tree, as shown in the stimulus picture. (See the list of abbreviations and transcription conventions at the beginning of the dissertation.)

(1)
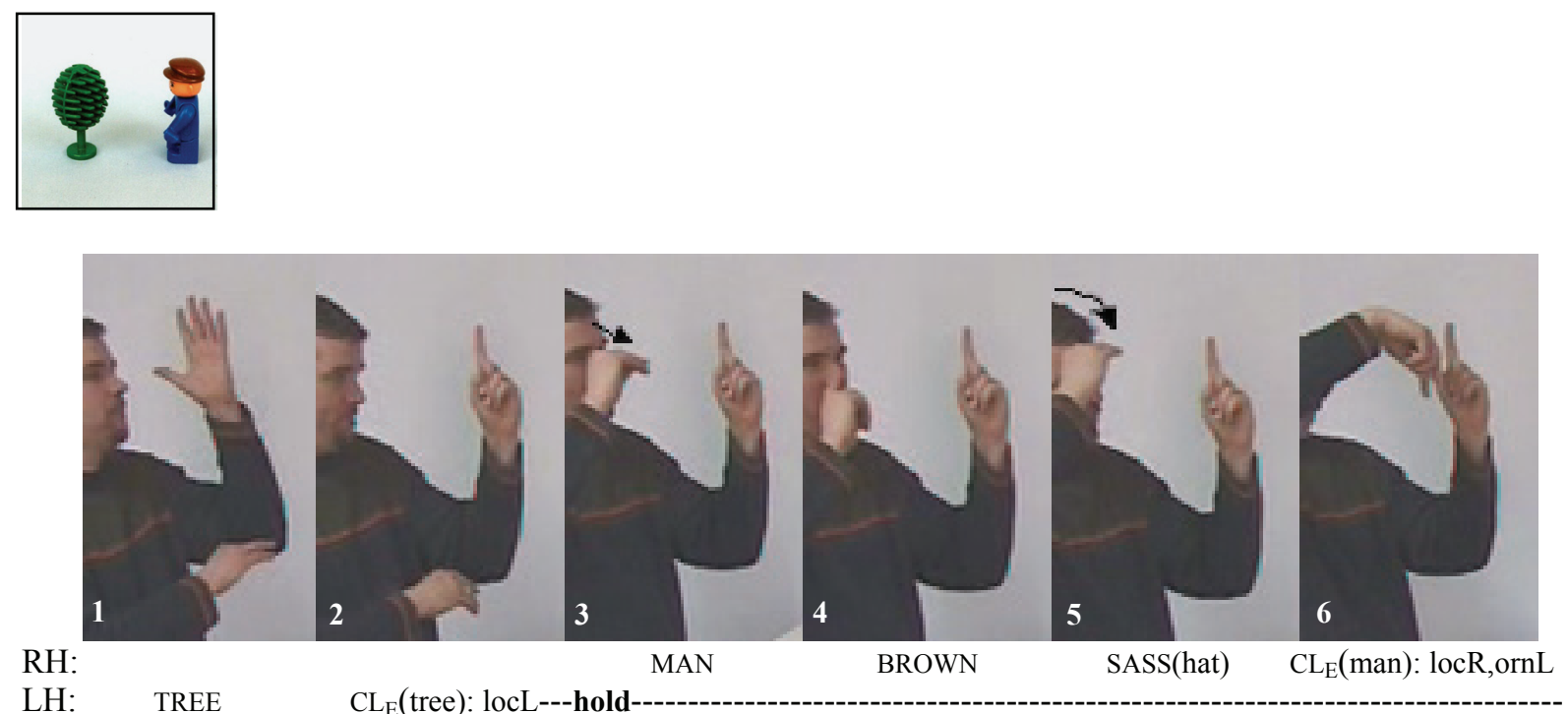

In this example, the signer first identifies the tree with the lexical sign TREE (in still 1) and then associates the tree with a particular location through the placement of the appropriate classifier form in sign space (in still 2). In DGS, the classifier form typically used to represent a tree (a long upright entity) is the so-called upright entity classifier, which is an upright index finger. Similarly, for the man, the signer first lexically identifies MAN (the right hand in still 
3 ), and then locates the man with respect to the tree. This is done in the example with the socalled 2-legged entity classifier, made with extended index and middle fingers, held apart and pointing downward (the right hand in still 6). Thus, through the appropriate placement of classifier forms in sign space, the signer creates an iconic representation of the spatial relationship between entities. The representation is possible because of the structurepreserving correspondences between the event space being described (here, the stimulus picture in example (1)) and the sign space. Just as in the stimulus, the classifier for the tree is placed on the signer's left side of sign space, and the classifier for the man is placed on the right side of sign space. Moreover, the structure-preserving correspondences between the entities being described (the man and the tree) and the linguistic forms used to represent them encode features of geometric form and orientation. Thus, the orientation of the 2-legged entity classifier form indicates that the man is facing the tree, as the back of the fingers is conventionally mapped to the front of the body (in still 6). In addition, as in a model, some metric properties, such as distance between the man and the tree, can be represented. Note also that the final representation is a "simultaneous classifier construction", in which two independent classifier predicates are simultaneously present in sign space (Engberg-Pedersen 1994; Leeson and Saeed 2002; Miller 1994). In such constructions, the affordance of the modality to create structure-preserving mappings is especially evident. The availability of two independent manual articulators makes possible the simultaneous representation of both entities, such that the spatial relationship between them is visually accessible. This is an aspect of signed language that is markedly different from the evanescent linear nature of spoken language. (See chapter 2, section 2.4 for a detailed discussion of simultaneous constructions.) Thus overall, this example shows how the modality affords the iconic mapping of relations which express spatial concepts of location, motion, form, and action. Indeed, sign languages are rich in such iconic forms and structures (Liddell 2003a; Taub 2001; Wilcox 2004). But, just how direct is this mapping? What, if any, are the limits to the iconicity which the modality affords?

At the lexical level, we see obvious constraints on the iconicity of signs. Every sign language has its own lexicon of conventionalized signs, and the meanings of iconic signs can often not be guessed by non-signers or users of a different sign language (Klima and Bellugi 1979; Pizzuto and Volterra 2000). The basis for an iconic sign is the prototypical image of the referent which it represents. However, this image is comprised of a multitude of elements. Depending on which elements are structurally preserved in individual sign forms, the iconic base of signs for the same referent can differ across sign languages. For example, in DGS, the 
sign EAGLE is an iconic sign because it depicts the hooked shape of an eagle's beak at the location of the nose. In another sign language, the sign EAGLE may look completely different, yet still be iconic. For example, in Ugandan Sign Language (USL), the iconic base of the sign is not the eagle's beak, but rather its claws. In addition, the phonological means by which the structure of elements is mapped from the form of the referent to the sign form differ across sign languages. For example, the Chinese Sign Language (CSL) sign for TREE represents only the shape of the tree's trunk, and does so by tracing its outline in space with an upward movement of both hands (see figure 2.1 in chapter 2). Which elements of the prototypical image of a referent become conventionalized in lexical signs, and by which phonological means, depends on language-specific form constraints as well as on culture-specific conceptualizations (Russo 2004; Taub 2001). ${ }^{4}$

In comparison, there is less known about the constraints on iconicity in the use of classifier predicates to represent location, motion, and action. As part of the language system, the status, composition, and analyzability of these complex forms remain the subject of debate in sign language linguistics. From its inception in the late seventies, the debate has ranged from a visual imagery analysis at one extreme (DeMatteo 1977; Mandel 1977) to a strict morphological analysis at the other extreme (Newport and Supalla 1980; Supalla 1978). In DeMatteo's (1977) view, the grammatical systems of sign and spoken languages differ fundamentally in the fact that signed visual representations are not discrete, but rather based on a set of "analogue rules" that map continuous aspects of a scene onto sign forms. The movement of the hands in space in the use of classifier predicates is taken to be an analogue representation of the visual image of the scene to be depicted. In contrast, Newport and Supalla (1980) argue that while the visual-spatial modality does indeed "offer the potential for an analogue, rather than a discrete, representational system" (p.191), the language itself (in this case, ASL) does not necessarily take advantage of this potential. Instead, classifier predicates of location and motion are analyzed as consisting of discrete morphemes that can combine in language-specific ways. A number of more recent accounts have settled in the middle, between the two extremes. In particular, Liddell $(1995,1998)$ has raised the issue of "listability", which points out that a strict morphological analysis cannot account for the potentially infinite possible locations in which spatially modifiable signs can be produced. At the same time, the meanings encoded by classifier predicates cannot be inferred from visual imagery alone. Liddell proposes that signs that depend on the use of meaningful locations in

\footnotetext{
${ }^{4}$ This is equally true for onomatopoetic words in spoken languages. They are language-specific and conventionalized imitations of sounds (cf. section 2.1.2 in chapter 2).
} 
sign space (including the system of classifier predicates, but also directional verbs, and pronouns) combine both linguistic and gestural elements. Handshape, some features of orientation, and some features of movement are analyzed as linguistic, while location and the directionality of movement are argued to be gestural features (see also Schembri 2002; Schembri et al. 2005). Such gestural features are taken to reflect imagistic representations, like the iconic and metaphoric manual gestures that co-occur with speech in spoken languages (McNeill 1992).

However, we still know little about how classifier predicates that represent location and motion are employed in actual discourse, especially in different types of discourse. In particular, we know little about the strength of influence of iconicity in shaping these usage patterns. One way to investigate this is through cross-linguistic comparison of sign languages. Some research on the use of classifier predicates has found striking similarities in the representation of spatial information across different sign languages (e.g. Aronoff et al. 2003 and Aronoff et al. 2005 for ASL and Israeli Sign Language (ISL), and Wilkinson et al. 2006 for ASL, French Sign Language (LSF), and Italian Sign Language (LIS)). ${ }^{5}$ The assumption supported by these findings is that the expression of spatial relationships is shaped by the exploitation of structure-preserving mappings, or iconicity, between conceptual space and sign space alone. The corollary assumption is that there should be no differences between sign languages in the representation of location and motion (see also Cuxac 1996; Sallandre and Cuxac 2002; Talmy 2003). However, other recent comparative research has shown that substantial differences exist between sign languages in the use of space for the expression of spatial information (e.g. Nyst and Perniss 2004 for Adamorobe Sign Language (Ghana) (AdaSL) and DGS, and Perniss and Özyürek 2004a, 2004b, in press for Turkish Sign Language (TID) and DGS). These results suggest that language-specific constraints are involved in shaping the influence of iconicity.

Overall, these findings suggest that more research is needed to reach a full understanding of the form and function of iconic mapping in the visual-spatial modality. This includes research within the spatial domain of location and motion, on different types of discourse, and across different sign languages. In addition, detailed research on individual sign languages is needed to discover the actual usage patterns and nuances of spatial language in different types of discourse across signers. This dissertation investigates the expression of spatial relationships in a specific sign language, DGS. The analysis focuses on the expression

\footnotetext{
${ }^{5}$ The acronym ISL is used for two different sign languages, Israeli Sign Language and Irish Sign Language. To avoid confusion the country name is spelled out in subsequent mentions of these two sign languages.
} 
of location and motion, the two major subdomains of the spatial domain, and for two types of discourse: static scene description (location) and event narratives (location and motion). In particular, it is concerned with the question of how frames of reference and perspective are used to express location and motion, and with characterizing the interplay between frames of reference, perspective, and the use of different kinds of classifier predicates. These domains are briefly introduced below.

\subsection{The expression of location and motion in spoken and signed language}

The subdomains of location and motion reflect the major division in our conceptual notion of space and have been the subject of extensive linguistic investigation and theorizing (e.g. Gennari et al. 2002; Jackendoff 1990; Levinson and Wilkins 2006b; Papafragou et al. 2002; Slobin 1996; Talmy 1985). As human beings, our lives depend to a large extent on the notion of space. We navigate through space and structure space to fulfill our needs (e.g. by erecting buildings, constructing streets, organizing our possessions). Moreover, we are proprioceptively aware of the extension in space possessed by our own body and those around us, both animate and inanimate. Since Kant (1768), the primacy of our experience with space, as well as the strength of our shared experience of space, has been taken as evidence for the universality of spatial cognition. Much research on the linguistic expression of location and motion has underscored the assumption of cognitive spatial universals (e.g. Jackendoff 1990; Landau and Jackendoff 1993; Li and Gleitman 2002). However, careful cross-linguistic comparisons have shown that far from being universal, there exist fundamentally different linguistic systems to describe space, displaying a wide range of semantic and morphosyntactic variation (Bowerman 1996; Levinson and Meira 2003; Levinson and Wilkins 2006b; Pederson et al. 1998). Companion studies have shown this variation to be reflected in conceptual structure and cognitive reasoning about space (Brown and Levinson 1993; Kita and Özyürek 2003; Levinson 2003; Levinson et al. 2002; Majid et al. 2004).

A key component in the expression of spatial information is the choice of frame of reference. Frame of reference systems are employed to express the spatial relationship between two objects (a figure and a ground object) that are located at some remove from one another in space (Levelt 1984, 1996; Levinson 1996a). In particular, the use of a frame of reference involves the specification of the direction in which the figure object is located with respect to the ground object. The direction can be specified in terms of a relative, intrinsic, or absolute frame of reference. The relative frame of reference involves the projection of the 
speaker's body coordinates onto the ground object, as in The man is left of the tree, and is dependent on the speaker's location with respect to the scene being described. The intrinsic frame of reference establishes a scene-internal relationship between the figure and the ground, based on the ground object's own intrinsic features, as in The cat is at the front of the car. Finally, the absolute frame of reference involves the use of fixed bearings, such as cardinal directions or landscape features, as in The man is East of the tree. (See chapter 3, section 3.2 for a more detailed exposition of frames of reference.)

Emmorey (1996) has discussed the use of these three frames of reference for location specification in ASL, giving examples of all three frames. The use of the relative frame of reference depends on the representation in sign space of the relationship between a figure and a ground from a particular viewpoint, e.g. the signer's own viewpoint as she views the scene. The use of the intrinsic frame of reference requires the use of inherently featured classifier predicates (recall e.g. the features of the 2-legged entity classifier that appears in example (1) above). The location of the figure is specified with respect to the intrinsic features of the classifier predicate that depicts the ground object. Finally, the use of signs for cardinal directions, such as NORTH or SOUTH, is associated with the use of an absolute frame of reference. Research on the distribution and instantiation of frames of reference in sign languages is discussed further in chapter 3.

A modality-specific feature of sign languages which is related to the use of frames of reference is the choice of signing perspective. Perspective refers to the way in which an event space is projected onto sign space. It determines how sign space is structured for spatial representation, and thus, plays a significant role in the depiction of spatial relationships. There are two ways in which this projection can take place, depending on the signer's conceptual location in relation to the event space. In one case, the signer is construed as external to the event space. From this observer perspective, the whole event space is projected onto the area of sign space in front of the body. In the other case, the signer is internal to the event space, in the role of a character within the event. This gives the signer a character perspective on the event space, which encompasses and surrounds the signer. ${ }^{6}$ The depiction of the location or motion of a figure object with respect to a ground object will differ depending on the perspective from which the event space being described is projected

\footnotetext{
${ }^{6}$ The terms "observer" and "character" are taken from McNeill (1992), who coined the terms "observer viewpoint" and "character viewpoint" to describe similar representations in co-speech gestures. I use the term "perspective" to differentiate between the two types of event space projection, and save the term "viewpoint" to refer to the signer's vs. addressee's view of an event space or sign space (see chapter 3 for more on perspective and viewpoint).
} 
onto sign space. (See chapter 3, section 3.3 for an overview of other definitions of these two types of spatial representation in the literature.) As an important spatial device in sign language, perspective interacts with frames of reference and the use of classifier predicates to represent referents.

In addition to the exploration of frames of reference and perspective in signed language (see also Emmorey 2001; Emmorey and Falgier 1999; Morgan 1999), research has addressed the relevance of viewpoint (i.e. the signer's vs. the addressee's view of a scene or of sign space itself) and the use of shared space (i.e. when the signer and addressee jointly view an environment) in spatial descriptions (Emmorey 1996, 2002) (see chapter 3, section 3.4). Finally, the addressee's comprehension of spatial information in sign space is an important factor in relation to frame of reference use that has received little attention in the literature (but see Emmorey et al. 1998 on mental rotation of sign locations by the addressee).

Most of the research that has been cited here has been carried out for ASL. There is a lack of research of a similar scope in the spatial domain in other sign languages. In addition, only little is known, in general, about spatial language in signed discourse. In comparison to spoken languages, much less is known about how different sign languages encode spatial relationships, and thus about the possible modality effects in this domain. Further research on coding within the spatial domain in sign languages is important not only because it is one of the most perspicuous examples of iconicity in the visual-spatial modality, but also because it is a linguistically and conceptually important domain. Moreover, understanding how space is mapped onto space in sign languages will further our knowledge of the relationship between language and cognition.

In summary, this dissertation looks at the structure of locative constructions in DGS and at the use of frames of reference and perspective in the expression of location and motion events in two types of discourse, namely in static scene descriptions and event narratives. In addition, as important evidence for the conventionalization of frame of reference use, the addressee's comprehension of the signed spatial information is analyzed for both types of discourse. Furthermore, it examines the use of classifier predicates in spatial expressions, focusing on their co-occurrence with different perspectives and frames of reference. In more general terms, the dissertation provides a usage-based account of iconic mapping in the visual-spatial modality. Through a systematic investigation of spatial expressions in DGS, it seeks to go beyond previous research in the spatial domain in sign languages, and in DGS, in particular, by investigating the impact of different constraints - e.g. linguistic, discourse, and articulatory — on iconicity in sign languages. 


\subsection{DGS background and data collection}

The next section presents some general information on DGS, including demographic, sociolinguistic, and historic information. It gives an overview of previous research on DGS, including linguistic, sociolinguistic, applied linguistic, and neurolinguistic studies. Finally, it provides details on the data collection for this dissertation, specifying the type of data, and how and where it was collected.

\subsubsection{Sociolinguistic and historical information}

Deutsche Gebärdensprache (DGS) is the sign language used by the estimated 80,000 deaf people living in Germany. There are dialectal differences centering around the bigger cities like Hamburg, Munich, Cologne, and Berlin, as well as between the former East and West Germany, but DGS is considered to be one sign language used throughout the country.

The first school for the deaf in Germany was established in 1778 in Leipzig by a man named Samuel Heinecke. Thus, the continuity of the language can be traced back for more than 200 years. The school in Leipzig was founded 18 years after the establishment of the school for the deaf in 1760 in Paris by Abbé de l'Epée - the first school of its kind in the world. Though Heinecke was a proponent of the oral method of teaching deaf children (that is, through lip-reading, without the use of signs), the popular method of the time in Europe was the bilingual method (a combination of signs and spoken language), spearheaded by de l'Epée in France. The two directors disputed the matter hotly for some time, but in the end, the bilingual method won out (Vogel 1999). The oral method did not become popular - and was not reinstated again in education - until the second half of the $19^{\text {th }}$ century. In schools for the deaf in Germany today, the oral method is still the dominant method of education. However, there is an increasing awareness of the importance of using sign language in deaf education among teachers at deaf schools.

\subsubsection{Previous research on $D G S$}

There has been a considerable amount of research on DGS, including linguistic research, but also many educational materials and dictionary projects, as well as many publications on sociolinguistic and pedagogical issues. The range of linguistic topics is wide, covering classifiers, negation, reciprocals, plural marking, morphosyntax, compounding, the role of mouthing, perspective, and discourse analysis (Ebbinghaus and Heßmann 1990, 1996; Erlenkamp 2002; Fehrmann 2001; Glück 2001; Glück and Pfau 1997, 1998; Heßmann 2001; Keller 1998; Perniss 2001, 2007a, 2007b; Pfau and Steinbach 2003, 2004; Prillwitz 1985; 
Steinbach and Pfau 2007). In addition, psycholinguistic studies have looked at disfluencies (Hohenberger et al. 2002, Leuninger et al. 2000), iconicity in the mental lexicon (Grote and Willmes 2003; Grote and Linz 2003), number processing (Iversen et al. 2004), and neurolinguistic processing (Klann and Huber 2005). In applied linguistics, sign language assessment tests have been created (e.g. the Aachen Test for Basic German Sign Language Competence (Aachener Test für Gebärdensprache (ATG)) (Fehrmann et al. 1991; Huber et al. 2001; see also Haug and Mann 2005) and the development of sign avatars, notation systems, and lexicon resources is ongoing (Hanke 2002; Hanke et al. 2001).

\subsubsection{Contact with the deaf community and data collection}

My own experience with DGS started with an introductory course in early 2000 at the Loor Ens school for DGS in Cologne, while studying at the University of Cologne. I continued taking courses at Loor Ens until the spring of 2003. During this time, in addition to following the courses, I actively sought contact with the deaf community in Cologne, beyond, but including, the teachers at the DGS school. For example, I frequently visited the Deaf Club in Cologne, joined regular pub meetings with a group of deaf people (including people from the DGS school), and took up a drawing course for the deaf and hard of hearing offered through the Deaf Club. In this way, I had ample opportunity to practice DGS in real discourse settings, and made friends and contacts within the wider deaf community in the Cologne area.

I started collecting data for my dissertation project in March 2002. I used two primary elicitation tasks that required pairs of signers, the analysis of which forms the substance of this dissertation. One task was a static-scene description task (see chapters 4-5), the other task involved telling event narratives (see chapters 6-8). In addition, I collected route descriptions, living space descriptions, and caught spontaneous conversation on camera, but this data was not ultimately included in this dissertation project. Between the months of March and August 2002, I conducted multiple data elicitation sessions, using rooms provided by the Loor Ens school and by the Linguistics Department at the University of Cologne. In May and June 2003, I also collected data at the University of Aachen.

\subsubsection{Type of data}

As mentioned above, though the expression of spatial information in the visual-spatial modality has received attention as an area of research, especially for ASL, our knowledge about the spatial domain in other sign languages is not very extensive. What are taken to be the facts of spatial description in DGS - in particular, the structure of basic locative 
constructions - has been hitherto based primarily on grammatical sketches and course materials, and is not founded on extensive empirical investigation. One reason for this is the lack of available sign language corpora from which information about actual usage in certain domains can be extracted. The collection of a data corpus on spatial descriptions was thus an important and necessary first objective of this study. Of course, any empirical study is at its strongest when it is based on naturalistic data, that is, on language produced in natural settings under natural conditions. However, a drawback to using naturalistic data (especially in the absence of other recorded language databases) is that there is no guarantee regarding mention of the content that needs to be investigated. Thus, the use of tasks designed specifically to elicit language of the particular type one is interested in is a valuable tool. Moreover, the use of elicitation tasks contributes to the creation of corpora that can help researchers gain knowledge about actual language usage. ${ }^{7}$

\subsection{Structure of the dissertation}

Chapter 2 introduces the notion of iconicity as a subject of semiotic and linguistic theory, and discusses the occurrence of iconicity in the language systems of both the signed and spoken modalities. It introduces the reader to the general structure of signed language, including the phonological components of signs and important morphosyntactic structures that will be relevant in the discussion of iconicity, especially the system of classifier predicates and the use of simultaneous constructions.

Chapter 3 gives an overview of the spatial domain, in particular, the subdomains of location and motion. The use of frames of reference in spatial description is defined, and an overview of research on frames of reference is given first for spoken languages and then for sign languages. The issue of signer's vs. addressee's viewpoint, as well as the use of shared space by signer and addressee is discussed in connection with frame of reference use. In addition, signing perspective is discussed as an important spatial device that is a prerequisite for spatial representation in the visual-spatial modality. The chapter ends with a definition of the relationships between frames of reference, perspective, and classifier predicates that I hold to be valid, and motivates alignments between them based on similarities in the properties that define their use.

\footnotetext{
${ }^{7}$ Large-scale sign language corpus projects currently underway that are not task-based are "Corpus Nederlandse Gebarentaal" by Inge Zwitserlood, Onno Crasborn, and Johan Ros at the Radboud University, Nijmegen and the "Auslan Archive Project" (Australian Sign Language (Auslan)) by Trevor Johnston, Adam Schembri, Louise de Beuzeville, and Della Goswell at Macquarie University, Sydney.
} 
The empirical investigation of the spatial domain begins in chapter 4 . The chapter investigates the structure of locative constructions in spatial descriptions of static scenes that contain two or more objects (both animate and inanimate), and presents the first detailed descriptive study of its kind for an individual sign language. The data analysis tests an hypothesis of the "canonical structure" of locative constructions. This "canonical structure" is defined by five conventions, motivated in part by principles of iconicity and economy of expression, that have been extracted from the literature on simple locative constructions in different sign languages (including DGS). The results of the analysis show that there is structural variation in DGS locative descriptions that cannot be explained by iconicity and economy alone. The variation is discussed in terms of grammatical and articulatory constraints on the use of locative constructions.

Chapter 5 addresses the use of perspective and frames of reference in DGS static scene descriptions. The analysis shows that DGS signers consistently map location and orientation information onto sign space in an observer perspective event space projection. Moreover, spatial information is represented in a relative frame of reference or in both relative and intrinsic frames simultaneously, as a special property of the modality. These results support an alignment between observer perspective and the relative frame, but also show that perspective and frames of reference are independent systems. A comprehension analysis shows that addressees (sitting across from the signers) predominantly demonstrate a mental rotation in interpreting the location and orientation information they see in the signer's sign space. The addressee's responses provide further evidence that the use of the relative frame of reference for static scene spatial descriptions is conventionalized in DGS.

In chapter 6, the use of perspective and frames of reference to encode location is examined in a different discourse type, namely in DGS event narratives of short cartoon stimulus films featuring a personified mouse and elephant. In addition, the use of perspective and frames of reference in the expression of motion events, which are prevalent in this type of narrative discourse, is investigated. The main questions to be answered are: Does the type of discourse (i.e. static description vs. narrative) influence the choice of perspective/frame of reference? Does perspective/frame of reference choice in narrative discourse in DGS differ between location vs. motion event expression? The results of the analysis show that, in event narratives, both location and motion events are predominantly expressed in a character perspective event space projection and in an intrinsic frame of reference. These results contrast with those of chapter 5 and indicate that discourse type strongly influences the coding of spatial information in DGS. The chapter also discusses further occurrences of the 
simultaneous use of the relative and intrinsic frames in the encoding of both location and motion. The results thereby provide additional evidence for the independence of perspective and frames of reference, but also for the alignments between them. Finally, the use of the relative frame of reference to encode location and motion in the event narratives is compared between the "teller" (the signer who has seen the stimulus) and "reteller" (the signer who has seen the signed narrative). As in the static scene descriptions, addressees preserved the spatial information they saw produced in the signer's space, which again provides evidence for the conventionalization of the relative frame of reference in DGS.

In chapter 7, the focus of analysis is on the relationship between signing perspective and different types of spatial/activity predicates (classifier predicates, lexical predicates, and index signs). The chapter presents a qualitative analysis, showing examples of different construction types that occur in the data, as well as a quantitative analysis, showing the distribution of the different construction types in the data. In addition, certain alignments between classifier predicates and perspective are shown to be motivated, based on iconicity principles of the scale of representation and on the type of information felicitously encoded by different classifier forms. The chapter ends with an analysis of the relationship between semantic event components and classifier-perspective alignments. In particular, the use of "aligned" vs. "non-aligned" constructions is discussed in relation to event simultaneity and iconicity of expression.

Chapter 8 looks at the role of another important modality-specific feature in sign language discourse, namely simultaneity in the expression of locative information. The focus is on the simultaneous use of manual articulators (i.e. the hands) and non-manual articulators (i.e. the torso, head, face, eyes) to represent aspects of an event associated with different perspectives. In particular, the use of a previously undescribed construction, which I call a "double-perspective construction", is discussed. The occurrence and function of constructions that exhibit the simultaneous use of perspectives in the DGS event narratives is motivated in terms of maintaining spatial coherence and in terms of discourse constraints of efficiency and informativeness. The constructions are discussed in comparison to similar constructions that have been attested in other sign languages. Their specific nature in DGS event narratives again suggests the existence of language-specific constraints on sign language structure in a domain that exhibits a high potential for iconic mapping.

The results and impact of the research presented in this dissertation are summarized in chapter 9, the concluding chapter. The methodological and theoretical implications are recapitulated, focusing particularly on perspective, frames of reference, iconicity, and 
simultaneity of expression. Overall, the findings have implications for our understanding of visual vs. linguistic/discourse constraints on the representation of imagistic content in the visual-spatial modality. Finally, it emphasizes the fact of differences and similarities found in investigations of the spatial domain in different sign languages, promoting our understanding of the variation within this domain.

In summary, this chapter has raised the theoretical questions behind this dissertation and has situated it within linguistic discussions regarding the iconic principles that drive sign language structure. It has established the spatial domain as an object of inquiry in linguistics and the cognitive sciences. Moreover, it has introduced the reader to the primary devices in signed language that are necessary for spatial description (i.e. classifier predicates, frames of reference, and perspective). Finally, it has emphasized the importance and necessity of careful, systematic investigation of sign language use, in different types of discourse, within the spatial domain. Only with such systematic investigation, to which this dissertation contributes, can we gain a true understanding of the possible variation in this domain and of the interaction between principles of iconicity and other constraints in sign language expressions. 


\section{Iconicity and sign language structure}

Chapter 2

The previous chapter introduced the notion of iconicity and its importance in sign language structures involving the representation of spatial concepts. Defined as structure-preserving mappings between form and meaning (Taub 2001), iconicity is apparent in the lexicon of sign languages, as well as in devices dedicated to the representation of entities, including their size, shape, number, movement, and location. This chapter begins with a discussion of meaning construction in signed language, focusing on the role of iconicity, from both a semiotic and a linguistic perspective (section 2.1). Then, to aid readers unfamiliar with the structure of signed language, I introduce the fundamental form components that are important to understanding the structure of signs (section 2.2). The morphologically complex system of classifier predicates, where iconicity plays a large role and which is thus especially relevant to this thesis, is discussed from different theoretical perspectives in section 2.3. Finally, section 2.4 defines simultaneous constructions and reviews the different functions which have been ascribed to them in the literature.

\subsection{Meaning construction in signed language: Iconicity and beyond}

A basic tenet of cognitive linguistics is that meaning is constructed not only from lexical semantics and syntactic structure, but from a combination of linguistic and non-linguistic knowledge (Coulson 2001; Fauconnier 1994, 1997; Langacker 1987, 1999). That is, the interpretation of utterances requires knowledge of grammatical structure, but also, and crucially, involves conceptualization and contextual understanding. In particular, grammar serves to symbolize and structure conceptual content. Through the combination of lexical and grammatical units, speakers frame things in certain ways in order to convey certain conceptualizations pertaining to the entities being talked about (Fillmore 1982).

Numerous researchers have applied a cognitive linguistics framework to the study of sign languages (Dudis 2004a; Janzen 2004; Liddell 2003a; Taub 2001; Wilcox 2004). For example, Liddell (2003a) states that "in addition to grammatical structure, understanding ASL involves conceptual mappings between semantic representations and numerous types of both spatial and non-spatial conceptualizations" (p. 65). Of primary interest in this 
dissertation are the spatial conceptualizations and representations related to form, location, and motion, and the role of iconicity (i.e. the presence of structure-preserving correspondences between form and meaning) in the construction of meaning in signed language.

We have seen an example of iconicity on the lexical level in chapter 1 . The DGS sign for TREE exhibits three form elements that directly correspond to the form of a prototypical tree: the trunk, the branches, and the base/roots. As figure 2.1 below shows, the signs for tree in other sign languages may also be iconic, but look quite different (as claimed e.g. for CSL and Danish Sign Language (DSL) by Klima and Bellugi 1979: 21). This is because every sign language will choose different characteristics of an image or concept to reflect in the phonological sign form itself (as also noted in chapter 1). Someone not familiar with the lexicon of a particular sign language will not be able to produce the sign for tree, although she may recognize the iconic elements of the sign and even guess the sign's meaning based on its iconic elements upon seeing the sign. There is no logical opposition here between iconicity and conventionality - the signs in figure 2.1 are iconic and completely conventionalized.

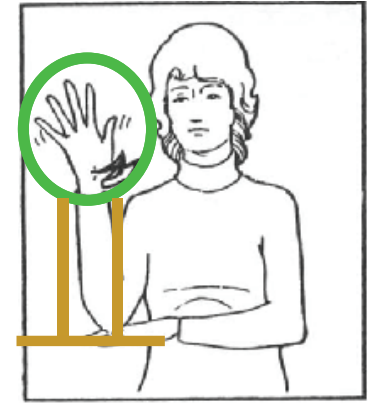

German Sign Language

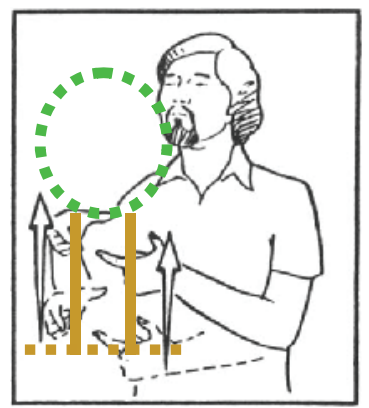

Chinese Sign Language

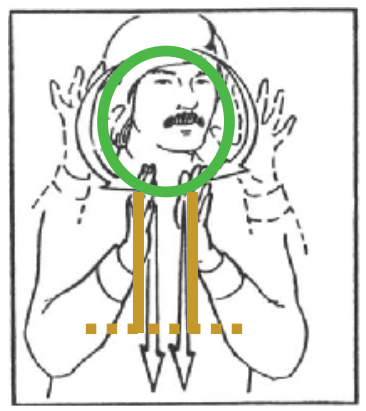

Danish Sign Language

Figure 2.1. The sign for tree in three different sign languages. In each language, the sign is different, yet each is clearly iconic of the referent. The DGS sign is a static sign (with internal movement of the hand only) that depicts the crown (the dominant hand), the trunk (the dominant forearm), and the base/roots (the non-dominant hand/forearm). The CSL sign exhibits an upward movement that bimanually traces the shape of only the trunk of the tree. The DSL sign also exhibits a bimanual movement outlining the shape of the tree, but traces both the crown and the trunk of the tree, moving downward. $^{8}$ Original figure reprinted by permission of the publisher from THE SIGNS OF LANGUAGE by Edward Klima and Ursula Bellugi, p. 21, Cambridge, Mass.: Harvard University Press, Copyright (C) 1979 by the President and Fellows of Harvard College.

\footnotetext{
${ }^{8}$ The line drawings used in figure 2.1 are from Klima and Bellugi (1979: 21). Note that in their example, the comparison is between ASL, CSL, and DSL. However, because the signs for tree in ASL and DGS are the same, and this dissertation is about DGS, I have made the comparison between DGS, CSL, and DSL. Moreover, as pointed out by Elisabeth Engberg-Pedersen (personal communication), the more common sign in DSL for tree looks very similar to the ASL and DGS signs for tree, except that instead of a rotating movement of the wrist, the fingers wiggle.
} 
Chapter 1 also introduced classifier predicates as forms that can iconically represent features of physical form, as well as of the location and motion of referents. In particular, classifier predicates exhibit structure-preserving, or iconic, correspondences between (1) the handshape and the referent's form, (2) the location of the hand in sign space and the referent's location in the event space being described, and (3) the movement of the hand in sign space and the referent's motion in the event space. The visual-spatial modality affords this type of iconicity because its main articulators (i.e. the hands) are visible entities in a spatial medium (i.e. sign space).

The role that iconicity plays in the system of classifier predicates (as well as in other systems, e.g. in pronouns, directional verbs, adverbs) has been treated in different ways from being emphasized to being severely downplayed - since the inception of sign language linguistics (see section 2.3 for a review; also Taub 2001). Regardless of the significance it is accorded, iconicity in sign language structure, and its contribution to meaning construction, has always been an important issue. With whatever sentiment, the notion of iconicity pervades the sign linguistics literature (e.g. Brennan 1990; Cuxac 1999; Emmorey 1996, 2002; Engberg-Pedersen 1993; Frishberg 1975; Klima and Bellugi 1979; Orlansky and Bonvillian 1984; Supalla 1986; Taub 2001; Wilcox 2004; Wilcox and Wilcox 1995).

As might be expected given its omnipresence, the terminology used to denote the role and manifestations of iconicity in sign languages is diverse. An assorted mix of terms, all somehow related to the notion of iconicity and all used more or less synonymously, abounds in the literature. They all describe or allude to the fact that the visual-spatial modality has a greater potential than the corresponding vocal modality for creating forms that resemble their referents. In addition to iconicity itself, we find the terms isomorphic, analogue, gradient, visually motivated, schematic, imagistic, and diagrammatic to describe the observed correspondences between form and meaning. The next sections define iconicity as a semiotic and linguistic notion, drawing on Peirce and de Saussure, in order to clarify the applicability and interchangeability of this multitude of terms.

\subsubsection{Iconicity as a semiotic and linguistic notion}

Our contemporary ideas about the relationship between a referent and the sign which stands for it have been greatly influenced by two primary figures: Charles Saunders Peirce (18391914) and Ferdinand de Saussure (1857-1913). ${ }^{9}$ In the jungle of terminology that revolves

\footnotetext{
${ }^{9}$ The philosophical debate has a much longer history, however. Plato tackled the question of the sign's relationship to its referent in his Kratylos.
} 
around the notion of iconicity in the sign language literature - for example, iconic, motivated, imagistic, diagrammatic, analogue, gradient, isomorphic, as listed above - one encounters both Peircean and Saussurean terms. This section aims to provide some clarity about the origins of the terms, their use, and applicability. Questions addressed here include: What is the relationship between arbitrariness and iconicity? What is the relationship between motivation and iconicity? What is the relationship between analogue/gradient representations and iconicity? What is imagistic and diagrammatic iconicity? In addition to illuminating the ways in which iconicity has been interpreted in sign language structures, I also address the role of iconicity in spoken languages.

For Peirce, an iconic relationship defines one of three primary modes, or types of relationship, through which a sign can represent an object or referent. Iconic signs stand in a relationship of similarity to their objects, such that the physical make-up of the sign is similar to the object itself. In contrast, indexic signs refer to their objects through a necessary or causal relationship, e.g. smoke is indexical of fire and the linguistic indexical today refers to the day on which it is uttered. The third type of sign, the symbolic sign, stands for its object by convention, and thus, the relationship between a symbol's form and its meaning is arbitrary.

The class of iconic signs is further subdivided into three categories: images, diagrams, and metaphors. Imagistic iconicity is the simplest form of iconicity, and may reflect the most common association with iconicity. The perceptual features of images resemble the perceptual features of the referents they stand for. Thus, there is a formal similarity between the sign and what it refers to. In the case of diagrammatic iconicity, or diagrams, it is the relationships between the components of the diagram, and not the individual components themselves, that resemble what they stand for. The relations between the parts of a diagram are analogous to the relations between the parts of the object that it represents. Metaphors, finally, rely on the representation of a parallelism between a source and a target domain. The notions of imagistic and diagrammatic iconicity will be returned to later in the discussion, in section 2.1.2.

We now turn to de Saussure, who in contrast to Peirce, focused his attention on the linguistic sign. He is most famously associated with the doctrine of the "arbitrariness of the sign". This expresses the idea that the relationship between a linguistic sign (i.e. the signifier) and the object or concept to which it refers (i.e. the signified) is based on convention alone. For example, there is nothing inherently in the sequence of phonemes $/ \mathrm{kæt} /$, other than conventions of the English language, which allows the sounds to refer to a domestic feline. 
The influence of the Saussurean dogma of the arbitrariness of the sign has been major, and indeed, it has been assumed to be one of the fundamental design features of human language, distinguishing it from other communication systems (Hockett 1960).

The counterpart of arbitrariness in the linguistic sign is generally taken to be iconicity, denoting a resemblance between form and meaning. However, de Saussure himself never actually used the term "iconicity". Instead, he opposed the terms arbitrary vs. motivated. De Saussure conceived of motivation in linguistic systems on two levels. The simpler level is that of individual linguistic signs: an arbitrary sign (like cat) in contrast to a motivated sign (like meow). Arbitrary signs dominate in the lexicon of any language, but there are small classes of motivated signs, involving onomatopoeia or sound symbolism (cf. Berlin 1994; Hinton et al. 1994a). On a more complex level, the concept of motivation constrains the arbitrariness of an entire linguistic system with respect to paradigmatic contrasts and syntagmatic combinability. ${ }^{10}$ De Saussure gives dix-neuf (nineteen in French) as an example: Though the French numeral words dix (ten) and neuf (nine) are themselves arbitrary signs, the word dix-neuf (nineteen) is motivated. Its meaning can be derived from its component parts and it stands in paradigmatic contrast with other signs which consist of or contain its parts, e.g. vingt-neuf (twenty-nine), dix (ten), soixante-dix (seventy) (de Saussure 1916).

The interpretation of the relationship between form and meaning in its application to both signed and spoken language structures is discussed in the next section.

\subsubsection{Iconicity in spoken and signed language}

With this background, we can examine the different terminology used to refer to iconic representations in sign languages. In addition, and as will be our first concern, we can identify different types of iconicity. We start with the notions imagistic iconicity (Peirce) and the motivation of individual signifiers (de Saussure), which overlap considerably when applied to linguistic signs (i.e. words). For a linguistic sign to be motivated or an icon to be imagistic, there must be a formal resemblance between the sign and its referent. That is, perceptual features of the sign must evoke perceptual features of the objects, whether these perceptual features are auditory or visual. In spoken languages, sound symbolic words or onomatopoeia are imagistic/motivated. The (auditory) phonological features of cock-a-

${ }^{10}$ See Haiman (1980) on isomorphism and motivation as two types of iconicity, which correspond to de Saussure's motivation on the level of the individual linguistic sign and the language system, respectively. Haiman notes that the degree of motivation in a language varies inversely with the size of its basic vocabulary (p. 535). That is, the more restricted the lexicon, the more perspicuous the grammar, since linguistic contrasts 
doodle-doo reflect those of the rooster's natural call. Nevertheless, these forms are conventionalized and conform to the phonotactic properties of the languages in which they occur. ${ }^{11}$ Individual spoken languages differ in their inventory size of onomatopoetic or sound symbolic forms, and in the degree of morphological productivity in creating such forms (cf. Hinton et al. 1994b).

Apart from iconicity on the lexical level, iconicity in spoken languages has been discussed in relation to phonetic representations, morphosyntactic complexity, and word order (Berlin 1992, 1994; Croft 2003; Givón 1985; Haiman 1980, 1985). This is the realm not of imagistic, but of diagrammatic iconicity. As Croft (2003: 102) puts it, thereby identifying the characteristics of diagrammatic iconicity, "the intuition behind iconicity is that the structure of language reflects in some way the structure of experience". Examples of such iconicity include that of sequence, contiguity, repetition, quantity, complexity, and cohesion (Croft 1990; Givón 1985, 1991; Greenberg 1963; Haiman 1980; Levinson 2000; Newmeyer 1992). For example, the principle of "iconicity of sequence" (or "sequential order") holds that the sequence of forms conforms to the sequence of experience, as in the famous collocation veni, vidi, vici. The principle of "iconicity of contiguity" (or "linguistic proximity") assumes that forms that belong together semantically will occur closer together morphosyntactically than forms that are semantically unrelated (cf. Bybee 1985).

Turning now to sign languages, we have already seen examples of imagistic iconicity/motivation on the lexical level. Signs like TREE or EAGLE in DGS visually reflect perceptual features of their referents. In addition to "imagistic iconicity" (Russo 2004; Taub 2001), the term "visual motivation" has been used in the sign literature to refer to imagistic iconicity on the lexical level (Cormier 2007; Sutton-Spence and Woll 1999; Vigliocco et al. 2005).

"Visual motivation" has also been used in a broader sense to encompass not only lexical iconicity, but also the topographic use of space (Cormier 2007; Perniss and Özyürek, in press). The topographic use of space refers to the meaningful (i.e. iconic) use of locations in sign space to represent referent locations, for example, with the use of classifier predicates. The idea that the use of classifier predicates is "visually motivated" relies on an assumption of "isomorphism" between the location/motion of the hands in sign space and the

must be grammaticalized from the small stock of lexical roots, making the relationships between different forms motivated.

${ }^{11}$ As de Saussure, for example, pointed out, different languages use a different series of phonemes to represent the rooster's call, e.g. German kikeriki or French cocorico. (Compare the examples of different signs for tree in figure 2.1.) 
location/motion of entities in real space. What is the relationship between this purported isomorphism and imagistic iconicity? An analysis of iconic forms based on analogue or gradient features - in contrast to the categorical or discrete features typically associated with linguistic forms - corresponds to this notion of iconicity. Indeed, previous analyses have proceeded from the assumption that signed representations involving classifiers are based on visual imagery, and that the continuous aspects of a real-world scene can be expressed in an equally continuous language code (DeMatteo 1977; Mandel 1977) (see section 2.3.3). The set of "analogue mapping rules" that underlie these analyses imply that the representations that can be achieved with classifier predicates are instances of imagistic iconicity.

There are many problems with analyses based purely on visual imagery (and thus on imagistic iconicity) (see Liddell 2003a, Taub 2001), but one must be careful not to throw the baby out with the bath water. Support for the idea of gradient and analogue representation is currently growing for the analysis of certain types of information in sign languages, including directionality, location, and size (Emmorey 2002; Emmorey and Herzig 2003; Liddell 2003a; Schembri et al. 2005). Liddell, in particular, takes the gradient aspects of representation to be "gestural", in the sense of "non-linguistic" and driven by the imagistic content of the language system (cf. McNeill 1992). In these analyses, then, the topographic use of space (i.e. the system of classifier predicates, but also directional verbs and pronouns) has an imagistic/analogue component, but cannot be fully explained by a reliance on isomorphism. To explain iconicity in the topographic use of space, we also need Peirce's notion of diagrammatic iconicity.

As Emmorey et al. (1995: 43) put it: "The spatial relations among signs correspond in a topographic manner to actual relations among objects described." They note further that it is possible "to specify the position of objects in a highly geometric and nonarbitrary fashion by situating certain sign forms (e.g., classifiers) in space such that they maintain the topographic relations of the world-space being described" (p.44). This characterization of the topographic use of space reveals the defining characteristic of diagrams. That is, it is not the signs themselves that must necessarily resemble their referents, but rather the relations between the signs in one system (e.g. the language code) must resemble the relations between referents in another system (e.g. the real world). Thus, as defined here, the system of classifier predicates as it is used to encode spatial relationships in sign languages relies principally on diagrammatic iconicity (while the choice of each individual location and the classifier forms themselves, i.e. the handshapes, have imagistic properties). 
Some researchers make explicit mention of both imagistic and diagrammatic iconicity in sign, but there is no true consensus on how signs and sign language structures are subsumed under the different types of iconicity (e.g. Cuxac 1999; Russo 2004; Sallandre and Cuxac 2002; Taub 2001). For example, Taub (2001) holds that ASL provides ample examples of both imagistic and diagrammatic iconicity (similar to the distinctions that I have made here), while Russo (2004) and Sallandre and Cuxac (2002) maintain that almost all examples of iconicity in signed language are imagistic. Still others may consider sign languages to exhibit primarily diagrammatic iconicity (cf. Cogill-Koez 2000, who stresses the schematic and templatic nature of classifier predicates). My own standpoint on this issue is that the correct analysis of classifier predicates combines both imagistic and diagrammatic iconic components, and that the visual-spatial modality affords signers the possibility of gradient modification (of location, orientation, and form, in particular).

A related issue, but which is not focused on in this dissertation, is how the notion of visual motivation applies to indexical signs, which are signs that point to their referents and include pronouns and directional verbs (Cormier 2007). ${ }^{12}$ In the case of indexicals in sign languages, I likewise maintain that they combine different types of Peircean iconic signs. When discourse referents are physically present (e.g. my interlocutor or other people in the room), pronouns and directional verbs literally point to their referents. Thus, to refer to my interlocutor (you), I point to her. Similarly, to ask my interlocutor whether she needs help with something, I direct the DGS sign HELP toward her. These signs are indexic signs in Peircean terms, because they refer to their referent through a necessary connection with that referent. The direction of the pronoun and verb is fully determined by the location of my addressee, and any other direction would not pick out the intended referent. Moreover, the interpretation of the reference depends on the context of use. If I were to use the same pronominal sign (produced in the same way) the next day while talking to someone else, the sign would refer to my new interlocutor (just as with pronouns, demonstratives, and time adverbials in spoken languages). If discourse referents are not physically present in the context of interlocution (e.g. a former work colleague or the author of a book), the indexical signs used to refer to them are not "connected" to their referent in the same way. Rather, the direction of the sign may be determined by the location of the referent in my conceptual representation, and by the association made between conceptual space and sign space. In the same way as for classifier predicates, the location itself is an analogical component of the

\footnotetext{
${ }^{12}$ See footnote 26 (in section 2.3.3) for a definition of directional verbs (also called agreement verbs (Padden 1983) and indicating verbs (Liddell 2003a)).
} 
sign's structure, determined by imagistic content. However, the signs remain indexical in the Peircean sense in that they necessarily pick out a referent that is characterized by being "not me" and "not you". ${ }^{13}$ Thus, I maintain that reference with pronouns and directional verbs to non-present entities combines Peircean imagistic and diagrammatic iconicity, as well as indexicality.

One theory that has been put forward in order to describe the manifestation of iconic representations in the visual-spatial modality is conceptual blending theory. Conceptual blending is assumed to be a general cognitive process that operates over "mental spaces", which are conceptual structures containing all the elements relevant for meaning construction and successful communication (Coulson and Fauconnier 1999; Fauconnier and Turner 1996). For signed representations, conceptual blending is advanced as a mechanism to explain the appearance of iconic mappings in structures that use space topographically (Dudis 2004a, 2004b, 2006; Liddell 1995, 2003a). For example, the construction in example (1) below can be described as a blend between the conceptual representation of the tree and the left hand, as well as a blend between the conceptual representation of the man and the right hand. In addition, the conceptual locations of the man and the tree are blended with corresponding locations in the space in front of the signer. The blending operation, and thus the possibility of iconic representation in sign space, is made possible by the structural correspondences between elements in the signer's conceptual representation of the event space and elements in "real space" (which includes the signer).

(1)

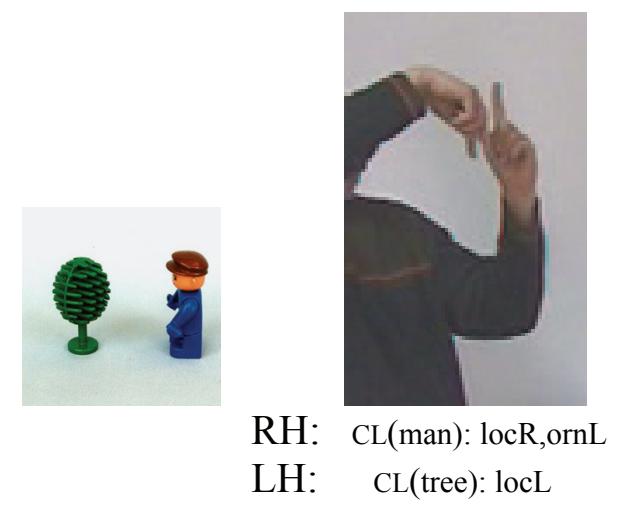

The idea of conceptual blending in sign language, as it is argued for by Liddell (e.g. in 2003a) and Dudis (e.g. in 2004b), allows an explanation of how entities are depicted in sign space and how the presence of iconicity in these structures is possible. However, as a theory for

\footnotetext{
${ }^{13}$ Thanks to Paul Kockelman for pointing this out to me. Note also the similarity to Meier's (1990) analysis of ASL pronouns as a two-person system, exhibiting first-person and non-first-person categories.
} 
explaining iconic representation, conceptual blending remains largely at the descriptive level. For example, it is not possible through the assumption of conceptual blending alone to predict under what conditions certain forms or constructions will be used. In addition, it is not possible to predict the appearance of certain forms over others, based on different types of constraints (e.g. articulatory, linguistic, or discourse constraints) (but see Dudis 2004a on the combination of different "partitionable zones" of the body). These issues are addressed for DGS narratives in chapters 7 and 8 of this dissertation.

We now have an understanding of the types of iconicity that are present in sign language structures that depend on the use of locations in sign space. Later in the dissertation, we will see the influence of other iconicity principles on the structure of spatial representations, including scale iconicity, temporal iconicity, and iconicity of perception. Of interest is how the potential of different types of iconic mappings in signed language is exploited in actual usage, when signers are faced with the task of representing location and motion events in different discourse types. In addition, a central question concerns what other constraints, besides iconicity, shape or affect spatial representation in sign space in DGS.

The rest of this chapter is dedicated to familiarizing the reader with the structure of sign language forms, focusing on sign phonology and the morphological structure of classifier predicates and simultaneous constructions. Classifier predicates are the iconic structure that is most relevant to this dissertation, as they are predominantly involved in spatial representations of location, motion, and action.

\subsection{Sign language structure: Phonology}

The analysis of linguistic structure in signed language has a relatively short history, dating back only five decades. Since then, linguists have elevated the status of sign languages in the minds of the general public from a system of mimes and gestures to a full-fledged linguistic system, on an equal footing as natural human languages with the more familiar spoken languages. In particular, sign languages exhibit phonological, morphological, and syntactic structure (Battison 1978; van der Kooij 2001; Meir 2002; Pfau 2002; Sandler 1989; Stokoe 1960). Nevertheless, the visual-spatial modality influences the nature of the building blocks of these systems and the nature of the constraints that govern their combination.

The phonology of a sign language is not, of course, a phono-logy in the strict sense (phōnē means "voice, sound" in Ancient Greek), but signs do exhibit Hockett's (1960) "double articulation" - they consist of meaningless elements that combine in certain ways to 
produce meaningful signs. This section discusses the phonological structure of signed language, providing examples from DGS.

Like words in spoken languages, lexical signs in sign languages are made up of meaningless components. There are four main phonological parameters that distinguish minimally between signs: handshape, place of articulation, movement, and hand orientation. ${ }^{14}$ Each of these contrastive categories is explained below. Their phonological status in DGS is illustrated with examples of minimal pairs in figures 2.3-2.7.

Handshape: The hand can be in various configurations, depending on finger extension and form (e.g. straight, bent, hooked, or curved). The inventories of handshapes vary from sign language to sign language, though there is a group of unmarked handshapes that occur with a high frequency across sign languages (Battison 1978; Sandler 1996) (shown in figure 2.2). Typically, handshapes are referred to by the letter of the finger alphabet or the numeral of the number system which they (also) denote. ${ }^{15}$ In what follows, I will use the accepted code letters to refer to the handshapes used in particular signs (e.g. B-hand). Figure 2.3 shows two DGS signs, a minimal pair, that contrast in handshape.
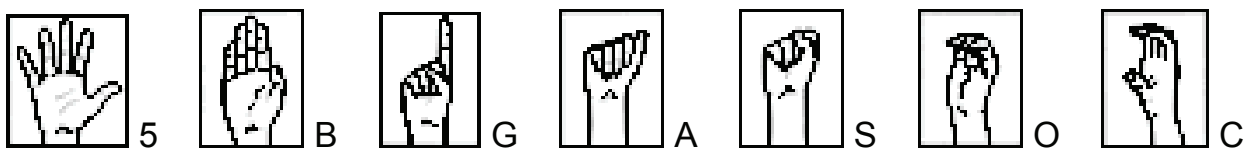

Figure 2.2. Examples of frequently occurring handshapes, and their letter identification.
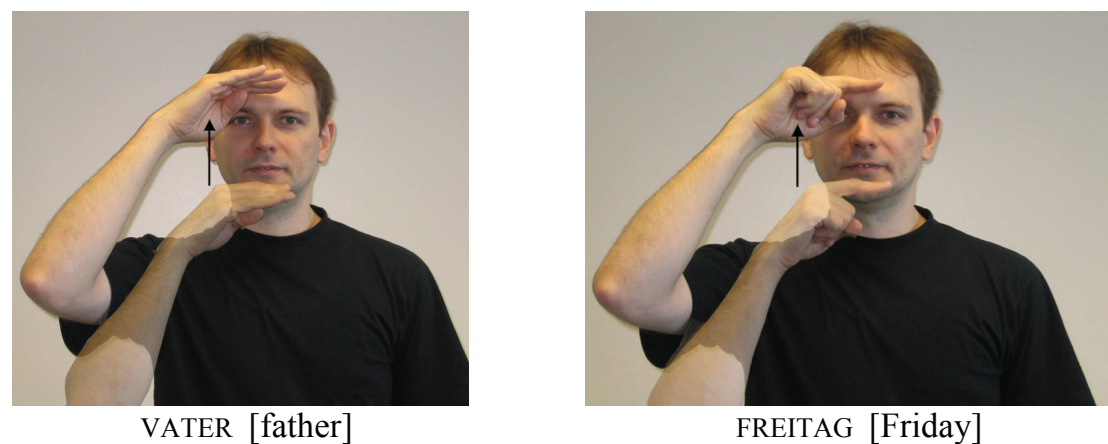

Figure 2.3. Example of a DGS minimal pair that contrasts in handshape. The sign VATER uses a Bhandshape, while the sign FREITAG uses a G-handshape.

\footnotetext{
${ }^{14}$ In some analyses of the phonological structure of signs, only three parameters are taken to be basic: handshape, place of articulation, and movement (e.g. Klima and Bellugi 1979; Sandler 1989; Stokoe 1960). Hand orientation appears as a feature of handshape (or hand configuration) in these models (e.g. Sandler 1989 for ASL).

${ }^{15}$ The handshapes are customarily named after the signs for letters of the alphabet or the number system in ASL.
} 
Place of articulation: Signs can have fixed points of articulation on the face or body, or can be executed in neutral space (i.e. in the area of space in front of the body). The chest, the shoulders, the arm, the wrist, the neck, and different parts of the head and face, including the ear, the mouth, the eye, the nose, the forehead, the side of the head, and the top of the head are all possible places of sign articulation. Figure 2.4 shows two DGS signs that contrast in place of articulation.
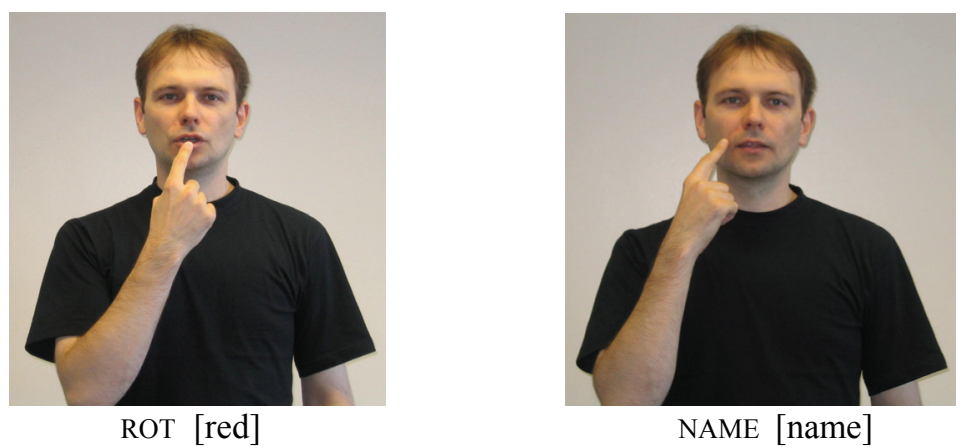

Figure 2.4. Example of a DGS minimal pair that contrasts in place of articulation. The sign ROT is made in the middle of the bottom lip, while the sign NAME is made at the same height, but next to the mouth on the cheek.

Movement: Many signs involve movement of the hand and/or fingers. The hand(s) can move in a straight or arc-shaped path which can be executed in different directions (e.g. sideways, forwards, or contralaterally across the body). In addition, the fingers can move "locally" by wiggling, bending, opening, or closing. Two DGS signs that contrast in their direction of movement are shown in figure 2.5.
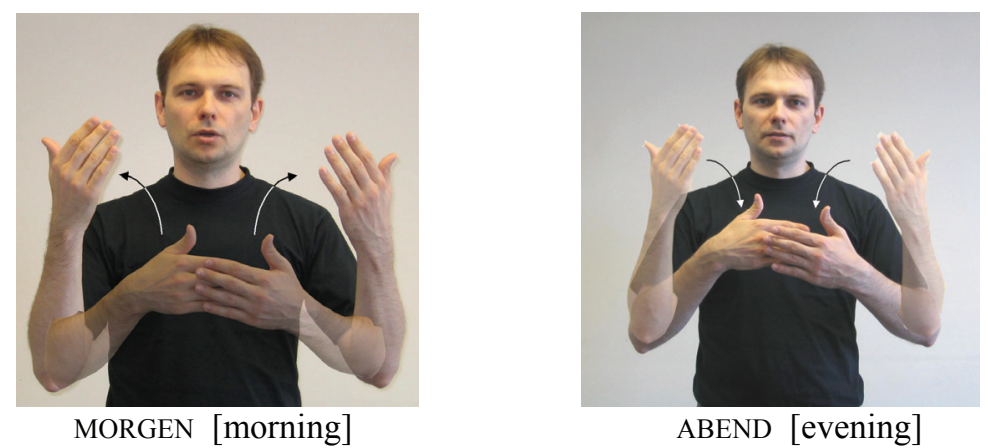

Figure 2.5. Example of a DGS minimal pair that contrasts in direction of movement. In the sign MORGEN, the hands move up and outward in an arcing movement. In ABEND, the hands have the same arcing movement, but move down and inward instead.

Hand orientation: Hand orientation can be characterized in terms of the orientation of the palm. The palm can be oriented upward, downward, toward the body, or away from the body. 
This is valid for all handshapes, irrespective of the degree to which the palm of the hand is covered by fingers. Figure 2.6 shows signs that contrast in hand orientation.

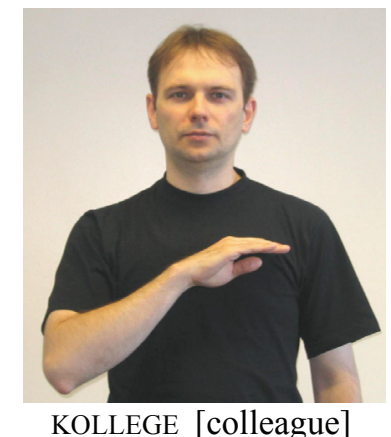

Figure 2.6. Example of a DGS minimal pair that contrasts in hand orientation. In KOLLEGE, the palm faces downward; in ARM, it faces upward.

Lexical signs can be one-handed (as in figures 2.3, 2.4, and 2.6) or two-handed (as in figure 2.5). Two-handed lexical signs (i.e. not simultaneous classifier constructions) are subject to two phonological well-formedness constraints - the symmetry condition and the dominance condition - formulated by Battison (1978) for ASL, but which are valid for other sign languages as well (see Pfau 1997 for DGS, van der Kooij 2001 for NGT, and Sutton-Spence and Woll 1999 for British Sign Language (BSL)). ${ }^{16}$

(a)

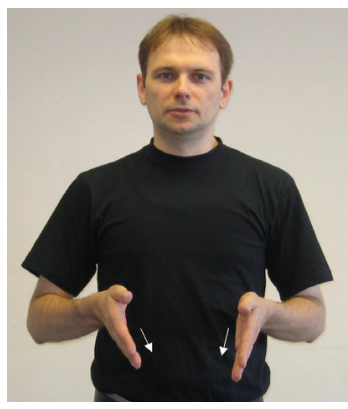

STRASSE [street]

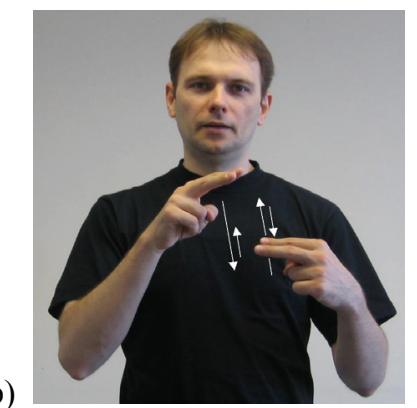

KENNENLERNEN [get to know]

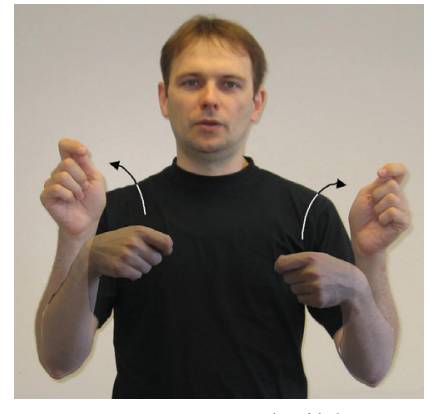

BAUEN [to build]

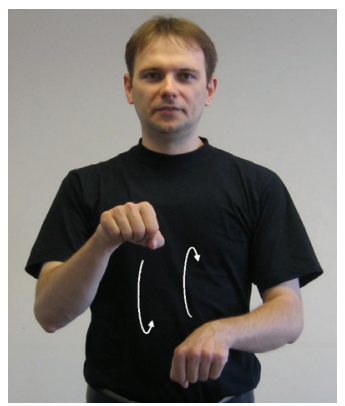

FAHRRAD [bike]

\footnotetext{
${ }^{16}$ These constraints may not hold to the same extent in some Asian sign languages (e.g. CSL or Korean Sign Language (KSL)), especially for signs that incorporate handshapes derived from the written character symbols (Byun 2004; Yang 2004).
} 
(c)

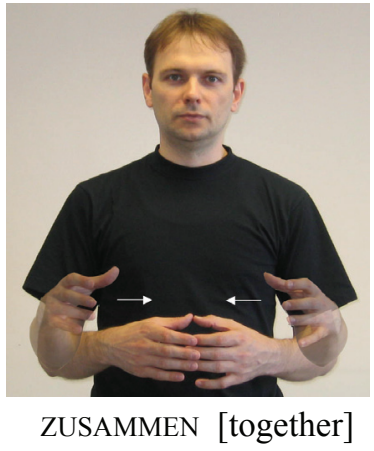

(d)

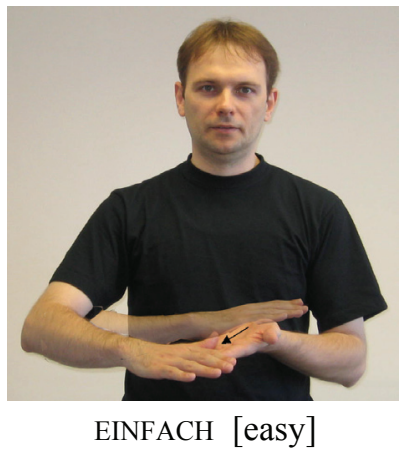

(e)

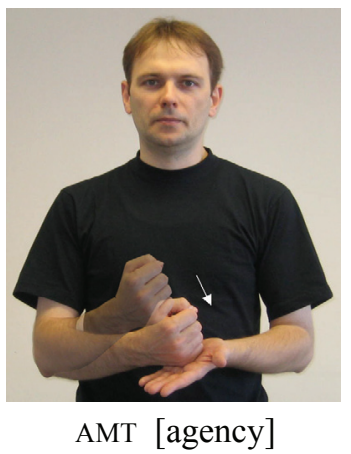

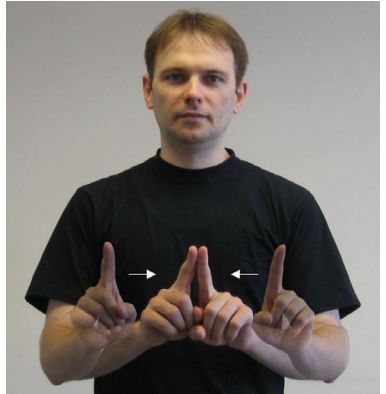

TREFFEN [meet]

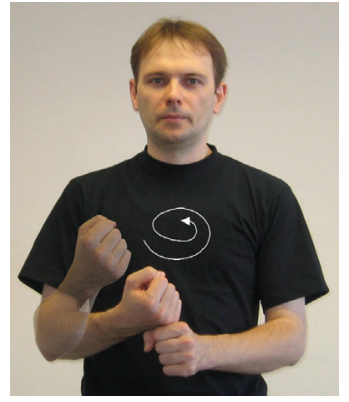

ARBEIT [work]

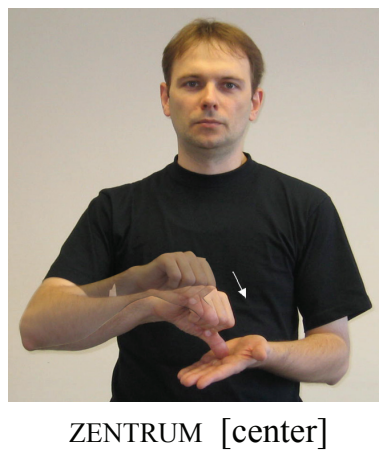

Figure 2.7. Examples of different types of two-handed signs. The signs in (a)-(c) display the symmetry condition. The movement of both hands is: (a) parallel, (b) alternating, or (c) symmetrical. The dominance condition is displayed in the signs in (d) and (e), where the hands have the same or different handshapes, respectively.

The symmetry condition applies to so-called "balanced" two-handed signs, in which both hands are active or moving. In such signs, both hands have the same specification for handshape and exhibit a parallel (figure 2.7a), alternating (figure 2.7b), or symmetrical (figure 2.7c) movement pattern. The dominance condition holds for two-handed signs in which only the dominant hand moves, while the other hand (the non-dominant hand) is stationary. In these "unbalanced" signs, the handshape of the non-dominant hand is either the same as (figure 2.7d) or different from (figure 2.7e) that of the dominant hand, and is generally restricted to the set of articulatorily simple, unmarked handshapes shown above (figure 2.2). 
Furthermore, most models of sign language phonology recognize the sequential structure inherent in the production of individual signs and propose phonological categories that differentiate between their static and dynamic segments. Prominent examples are Liddell and Johnson's (1989) analysis based on Movements (M) and Holds (H), Sandler's (1989) Hand Tier model, Brentari's (1998) Prosodic Model, and Uyechi's (1993) model of Visual Phonology (see Brentari 1995 for an overview).

In addition to these manual phonological components, signs can also include nonmanual phonological features. These fall into two main categories: mouth gestures (Mundgestik in German) and mouthings (Mundbilder in German) (see Boyes-Braem and Sutton-Spence 2001 for a discussion of the terminology and the relevant phenomena in numerous European sign languages). Both mouthings and mouth gestures occur simultaneously with the manual component of the sign. The former are derived from spoken words, while the latter display a phenomenon which has been called "echo phonology" (Woll 2001). ${ }^{17}$ Local movements of the hand (e.g. opening and closing) are paralleled by synchronous movements of the mouth (i.e. opening and closing). ${ }^{18}$ (For the use of mouthings in DGS, and their relation to spoken German, see Ebbinghaus 1998, Ebbinghaus and Heßmann 1996, and Keller 1999.) As we will see in the following chapters, other non-manual articulators, like the shoulders, torso, eyes, face, and head, can also be involved in the construction of meaning in signed representations.

This section has given a sketch of the phonological parameters that determine the form of lexical signs in DGS, and other sign languages. However, not all manual signs are subject to the phonological constraints outlined here. The system of classifier predicates in sign languages comprise morphologically complex signs whose production in space may violate the well-formedness principles that apply to lexical signs. The morphology of classifier predicates is discussed in the next section.

\footnotetext{
${ }^{17}$ Neuroimaging results on the processing of echo phonology by deaf and hearing (non-signing) subjects suggest that the phenomenon may reflect a mechanism associated with language evolution (Woll et al. 2006). Indeed, as Woll points out, a simple test shows that echo phonology is not specific to signed language. Opening your mouth in synchrony with opening your hand from a fist feels more natural than opening your mouth while closing your hand into a fist.

${ }^{18}$ In addition to phonological mouth gestures, there are mouth gestures that have a morphemic status in that they contribute a particular adjectival or adverbial meaning to a sign. The adjectival meanings include "small", "big", "soft", "hard", "thin", and "fat". In DGS, for example, rounded, slightly parted lips, and a slightly protruding tongue convey the meaning "small", while to indicate that something is "big", the lips start together, then part into a "baring teeth" gesture. Possible adverbial meanings include doing something "in a concentrated manner, intensely", "with effort", "with aversion", or "in a steady manner".
} 


\subsection{Sign language structure: The morphology of classifier predicates}

The linguistic structure of signed and spoken languages differs also in the dominant type of morphological process that is used to create complex forms. In contrast to spoken languages, the visual-spatial modality of sign languages relies to a great extent on non-concatenative morphology, where multiple morphemes are simultaneously layered upon or nested in a sign form (Klima and Bellugi 1979). ${ }^{19}$ Such simultaneous morphology is found especially in the expression of temporal concepts (e.g. aspectual marking) and spatial concepts (e.g. location and motion). An important instance of simultaneous morphology in sign languages - and highly relevant to the discussion of iconicity - is the system of classifier predicates. In addition, the availability of multiple, independently functioning articulators, such as the hands, face, and torso, makes possible even more complex morphosyntactic structures exhibiting simultaneity of expression. The special status of simultaneous constructions in signed language is the subject of section 2.4.

Classifier predicates are a special class of morphologically complex signs that have been found to exhibit similar properties across different sign languages and to exist in the majority of sign languages studied to date (see Schembri 2003 for an overview). Where they are used, classifier predicates are an important linguistic device for encoding spatial information. Through the (simultaneous) combination of handshape morphemes with certain movement patterns, classifier predicates express information about the size and shape, location, motion, and manipulation of referents.

In these predicates, the handshape refers to a specific entity by reflecting certain of its salient visual-geometric properties. That is, the handshape "classifies" the entity with respect to inherent properties of size and shape or, in some cases, semantic class. ${ }^{20}$ Example (1) above contains two classifier predicates: a G-hand for the tree (index finger extended upward to represent the long, upright form of the tree) and the so-called 2-legged entity classifier for the man (index and middle finger extend downward to represent two legs). Another example is the B-hand (held horizontally, palm down), which can be used to represent objects with

\footnotetext{
${ }^{19}$ This is not to say that sequential morphology is completely absent in sign languages. It exists, for example, in the suffixation of negation markers or agentive markers (Aronoff et al. 2003 for ASL and ISL; Pfau 2002 for DGS). Conversely, this is also not to say that simultaneous marking is completely absent in spoken languages (cf. the use of tone to mark tense in many African languages).

${ }^{20}$ The term classifier is widely used in the sign linguistics literature - and is used throughout this dissertation although its appropriateness is a matter of contention among sign researchers (see section 2.3.1 for classifiers in spoken languages; see also Schembri 2003 and Emmorey 2002 for discussions). Other names used in the literature for signs which include these forms are polymorphemic verbs (Engberg-Pedersen 1993), polycomponential signs (Slobin et al. 2001, 2003), productive signs (Brennan 1992; Heßmann 2001), or property markers (referring specifically to the handshape) (Slobin et al. 2003).
} 
broad, horizontal surfaces (like tables) (see figure 2.8a). In DGS, the same horizontal B-hand form is also used for the semantic class of four-wheeled vehicles (i.e. cars, buses, and trucks). The class of two-wheeled vehicles (i.e. bicycles and motorcycles), in contrast, is represented with a vertically-held B-hand (thumb on top) (see figure 2.8b). In ASL, no distinction is made between different types of land vehicles. Instead, the entire semantic class of vehicles is represented with a single handshape, in which the hand is held vertically and the thumb, index, and middle fingers are extended (thumb on top) (see figure 2.8c). In Kata Kolok, a village sign language used in Bali, the horizontally-held B-hand (used for cars in DGS) is used to represent airplanes, and not land vehicles. ${ }^{21}$ These different examples show that salient visual properties of the denoted referents are recognizable in classifier handshapes. However, it is also clear that the iconic, i.e. structure-preserving, correspondences they exhibit are highly schematized (cf. Cogill-Koez 2000) and conventionalized in different sign languages in language-specific ways.

(a)

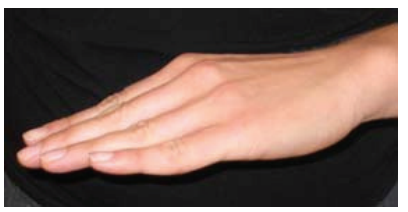

(b)

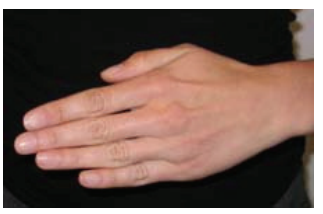

(c)

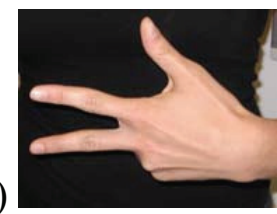

Figure 2.8. Handshapes used for land vehicles in DGS (4-wheeled in (a) and 2-wheeled in (b)) and ASL (in (c)).

The numerous classifications that have been proposed by different researchers (see section 2.3.2) minimally include three main types of classifier: (1) entity classifiers, (2) handling classifiers, and (3) size and shape specifiers (SASS) (cf. Schembri 2003). In entity classifiers, as in the examples above, the handshape represents a referent as a whole (e.g. a car or a table). The category of entity classifiers includes what have been called "static SASSes", "semantic", "whole entity", and "object" classifiers. In addition, it may include limb or body part classifiers, in which the hand represents individual body parts, e.g. front or back limbs, of an animate referent (cf. Engberg-Pedersen 1993; Supalla 1986; Zwitserlood 2003). In handling classifiers, the classifier handshape represents the way a referent is handled or manipulated by an agent. For example, an F-hand (contact between the index finger and thumb) can be used to represent holding a single flower or picking up a pencil (i.e. the handling of long and thin objects). This category also includes what have been called instrument classifiers, where the handshape depicts the whole entity (e.g. extended index and

\footnotetext{
${ }^{21}$ It is worth pointing out that these types of handshapes (i.e. entity classifiers, where the hand represents a whole referent in sign space; see below) are not used in AdaSL, a village sign language in Ghana (Nyst 2007).
} 
middle fingers to represent the form of a screwdriver), but the meaning refers to its manipulation by an agent. Finally, SASSes, or size and shape specifiers, denote handshapes in which the movement of the hands traces the outline of the referent. These forms have also been called "non-static SASSes", "extension", "perimeter", and "tracing" classifiers.

Classifier handshapes occur within predicates that depict the location and motion of referents through the position and movement of the hands in sign space. As discussed in chapter 1, the linguistic status of these predicates is controversial. Before I go into the different types of analyses of classifier predicates in signed language, I discuss the similarities between sign language classifier handshapes and spoken language devices, including classifiers, for encoding information about the size and shape of referents (section 2.3.1). The exposition of analyses of classifier predicates as purely morphological, i.e. linguistic, systems is given in section 2.3.2, while the analyses that incorporate both linguistic and iconic/gestural components are detailed in section 2.3.3.

\subsubsection{Encoding size and shape information in spoken languages}

In relation to signed language, the term "classifier" was originally used by Frishberg (1975: 715) in recognition of the use of certain handshapes in ASL to represent "certain semantic features of noun arguments." Subsequently, comparisons were made to the classificatory verb stems found in Athapaskan languages like Navajo and nominal classification systems with verbal classifiers in spoken languages, in general (McDonald 1982; Schick 1990; Supalla 1978, 1982; and more recently Benedicto and Brentari 2004; Sandler and Lillo-Martin 2003; Zwitserlood 2003). Numerous researchers have argued against the applicability of the term and have discussed problems with the comparison (Cogill-Koez 2000; Engberg-Pedersen 1993; Schembri 2003; Slobin et al. 2003; see also Aikhenvald 2003, Grinevald 2003).

However, there are other types of shape-based classificatory systems that occur in spoken languages that also bear a similarity to the system of classifier predicates in sign languages, for example, the noun class markers in Miraña (a North West Amazonian language) (Seifart 2005) or the set of dispositional verbs in Mayan languages (e.g. Tzeltal (Highland) and Yukatek (Lowland)) (Bohnemeyer and Brown, in press). In Miraña, referents introduced with specific nominal expressions can be tracked in discourse by pronominal expressions that contain class markers which encode information about the shape of the referent (e.g. oblong-shape, tube-shape, container-shape). The shape-based referent tracking mechanism in Miraña is very similar to the use of handshape classifiers in sign languages, which are used to identify referents based on properties of shape after they have been 
lexically identified (cf. the use of the term "proform" for sign language classifiers, BoyesBraem 1995; Edmondson 1990; Johnston 1996). ${ }^{22}$ Sign language classifier predicates may also be compared to the classes of (dis-)positional verb roots characteristic of Mayan languages. These verbs depict the disposition of figure objects relative to a ground by expressing salient spatial properties of the figure referents. The lexicalizations include features of shape, orientation, distribution, and posture (Bohnemeyer and Brown, in press). ${ }^{23}$

\subsubsection{Analyses of sign language classifier predicates as composed of discrete morphemes}

Supalla (1978), and subsequent publications, pioneered a strict morphological analysis of classifier predicates, in which all handshape, location, and movement units are taken to be discrete components (i.e. roots and affixes) that are specified in the grammar. Different researchers have proposed different analyses of the exact morphological composition of classifier predicates, but they are all based on the assumption of a particular inventory of handshape and movement morphemes, which can combine with each other in certain ways. Some analyses take the movement morphemes to be the verb root to which the handshape morpheme is affixed (Schick 1990; Supalla 1978). Others take the handshape to be the core of the classifier predicate, arguing that the handshape plays a greater role in determining its meaning (Engberg-Pedersen 1993; McDonald 1982). Emmorey (2002) sees no justification for treating either component as primary, and states simply that "classifier handshapes and movement [are] morphological units that combine to create classifier predicates" (p. 91).

Though different researchers define and classify the movement and handshape units in different ways, there is a general overlap in the analysis of form and function. The inventories of movement morphemes include those that specify location and/or existence and movement (e.g. Engberg-Pedersen 1993; Schick 1990; Supalla 1986; Zwitserlood 2003), as well as separate morphemes expressing action (e.g. Schick 1990), manner of motion, and extension (e.g. Engberg-Pedersen 1993; Zwitserlood 2003). The handshape unit encodes information

\footnotetext{
${ }^{22}$ For example, in Miraña, a basket can be introduced as a discourse referent (using a nominal expression + specific class marker for "container shape"), as in (a), and can be later unambiguously referred to through the use of a prounoun + class marker for "container shape", as in (b) (Seifart 2005: 284).
(a) $t s a-: b a j \quad u \beta i-: b a j$
one-SCM.cont basket-SCM.cont 'one basket'

(b) té:-báj $\quad$ pajné-tuщ PN-SCM.cont inside-ABL take-GCM.masc.sg 'from inside it (container, i.e. basket) he took'

${ }^{23}$ This example shows the use of the dispositional verb "stand vertically" in Tzeltal: wax-al ta lum p'in stand.vertically-DIS PREP ground pot '(A) pot is vertically standing on the ground.'
} 
about the size and shape of the referent, thereby fulfilling the putative classifying function, and is typically referred to as the "classifier morpheme" or simply the "classifier". Different researchers posit different categories of handshape morphemes, depending on the way in which the relationship to the referent is expressed (see table 2.1 for an overview). ${ }^{24}$

Table 2.1. Different classifications of movement and handshape morphemes in analyses of classifier predicates.

\begin{tabular}{|l|l|l|}
\hline Researcher & Movement morphemes & Handshape morphemes \\
\hline Supalla (1986) (ASL) & $\begin{array}{l}\text { stative root (existence) } \\
\text { contact root (location) } \\
\text { active root (motion) }\end{array}$ & $\begin{array}{l}\text { static size and shape specifier (SASS) } \\
\text { tracing size and shape specifier (SASS) } \\
\text { semantic classifier } \\
\text { body part classifier } \\
\text { body classifier } \\
\text { instrument classifier }\end{array}$ \\
\hline Schick (1990) (ASL) & $\begin{array}{l}\text { IMIT (activity) } \\
\text { MOV (motion) } \\
\text { DOT (location) }\end{array}$ & $\begin{array}{l}\text { CLASS classifier } \\
\text { HANDLE classifier } \\
\text { size and shape specifier (SASS) }\end{array}$ \\
\hline $\begin{array}{l}\text { Engberg-Pedersen (1993) } \\
\text { (DSL) }\end{array}$ & $\begin{array}{l}\text { position } \\
\text { motion } \\
\text { manner } \\
\text { extension }\end{array}$ & $\begin{array}{l}\text { whole entity classifier } \\
\text { handling/instrument classifier } \\
\text { limb classifier } \\
\text { extension/surface classifier }\end{array}$ \\
\hline
\end{tabular}

In addition to the classifications above, Zwitserlood (2003), in a slightly different analysis, distinguishes three subtypes of classifier predicates based on morphosyntactic criteria in her analysis of the classifier system in NGT. Predicates that express motion, location, and existence are collectively called VELMs (i.e. verbs of existence, location, and motion), and appear with hand configuration (i.e. the classifier representing a particular referent) as a bound morpheme. Tracing size and shape specifiers (SASSes) outline or trace the shape of the referent, with the handshape contributing additional shape information. The third subtype of classifier predicates are verbs of manner of motion, which indicate the manner in which a referent moves by depicting the movements of the referents' hands/arms or feet/legs (cf. body part classifiers).

The literature addressing the classifier predicate system in DGS (Glück 2001; Glück and Pfau 1997; Keller 1998) likewise focuses attention on the syntactic and morphosyntactic behavior of the predicates. A three-way division between classifier types, based on Schick (1990), distinguishes CLASS, HANDLE, and SASS classifiers. CLASS and HANDLE classifiers are grouped together as verb classifiers that appear as bound morphemes with verbs. In CLASS classifiers, also called subject classifiers, the handshape represents the

\footnotetext{
${ }^{24}$ Because I am not ultimately concerned with the morphological analysis of classifier predicates, I do not give an exhaustive summary of the different analyses proposed in the literature. For a more comprehensive overview, see Schembri (2003).
} 
subject of non-agentive, intransitive verbs. In the case of HANDLE, or object, classifiers, the handshape represents the (manipulation of the) object of agentive, transitive verbs. In contrast, SASSes are free morphemes that have adjectival character and appear as part of a complex noun phrase.

\subsubsection{Analyses of sign language classifier predicates as composed of discrete and analogue} elements

As introduced in chapter 1, Supalla's (1978) analysis of classifier predicates into discrete morphological components has a counterpart in DeMatteo (1977) and Mandel (1977). DeMatteo (1977) argues for an incorporation of visual imagery (in the form of "visual deep structures") into the linguistic analysis of signs. He suggests that signers use visual imagery to give the appropriate form to signs (in particular, to classifier predicates). Through a set of "analogue rules", signers map the continuous features of the world onto the continuous elements of the sign language code. In this way, signers achieve an iconic mapping between a spatial scene and its signed representation. Similarly, Mandel (1977) explores the "iconic devices" with which signers are able to "make pictures" of referents in signed language. The "gestures" used to make these pictures range on an iconicity scale from conventionalized iconic forms to fully mimetic forms. Though DeMatteo's model of analogue rules and visual deep structure was not subsequently developed, many researchers since then have incorporated "analogue movement" morphemes or analogue subsystems into their analysis of classifier predicates (for example, Schick's 1990 "IMIT" movement morpheme or EngbergPedersen's 1993 "analogue-(type)" motion morpheme.)

Specifically, the term analogue is used to define the relationship between the movements/locations of the hands in classifier predicates and the motion/locations of referents in an event space. Through the structural correspondences between the space being described and sign space, the hands can create a "visual analogue" of a motion event or of a configuration of objects. Such an analogue representation depends on the assumption of gradient, continuous properties in the language system (see Liddell 2003a for a discussion of the semantic problems of a fully visual analysis). Similarly, Engberg-Pedersen (1993) provides an in-depth discussion of "movement morphemes" vs. "movement analogues". As an example of a problem that a visual-analogues analysis of classifier predicates faces, she discusses the fact that movement within a classifier predicate does not always denote motion within an event (cf. fictive motion, Talmy 1996). 
Liddell $(1995,1996,2000,2003 a)$ is perhaps most articulate and thorough in his recognition that the analysis of classifier predicates - as well as the use of space in pronouns and so-called agreement (Padden 1983) or indicating (Liddell 2003a) verbs ${ }^{25}$ — cannot be based on visual imagery or the notion of continuous, analogue representation alone. Yet, Liddell argues that a fully grammatical analysis also fails to capture the way space is used for meaning construction in signed language. In his analysis, Liddell takes handshape and certain movement and orientation features to be lexically specified (and thus linguistic), but rejects a grammatical analysis of location specification (where locations in sign space are treated as "referential loci", as e.g. by Lillo-Martin and Klima 1990). Instead, he proposes that locations are imbued with meaning through their association with elements of mental representations. The locations to which signs are directed (e.g. classifier predicates, pronouns, and verbs) depend on the locations of conceptual entities projected onto sign space. Thus, the choice of location is argued to be not morphemically determined but rather to reflect gestural, i.e. nonlinguistic, components of the signs. Liddell argues that a morphemic analysis of the association of locations in sign space with referents confronts a major problem, namely the issue of "listability". The morphemes of a language, as components of the linguistic system, must be of a finite set, which can be listed along with their individual meanings in the grammar of the language.

Liddell's analysis is supported by the results of Emmorey and Herzig (2003), who used both production and comprehension experiments with signers and non-signers to test the categorical vs. gradient properties of classifier constructions in ASL. The results of their experiments reveal that, in production, signers modulate handshape in a gradient manner to express size and shape (e.g. the diameter of a medallion), while, in comprehension, handshape configurations were interpreted morphemically, according to different categories of size. In contrast, spatial location is treated gradiently in both production and comprehension. Moreover, signers and non-signers represent location in the same way, according to non-linguistic spatial categories. These results argue against the existence of a "base grid" of location morphemes as proposed by Supalla (1978).

\footnotetext{
${ }^{25}$ In general, the predicate system of sign languages is taken to have a tripartite structure (as proposed originally by Padden 1983): (1) plain verbs, (2) agreement verbs, and (3) spatial verbs. The form of plain verbs is invariant, and the movement is determined by their phonological form alone. In contrast, the movement of agreement verbs is determined by the verb's arguments. The verb begins at the "referential locus" (Lillo-Martin and Klima 1990) associated with the subject argument (or, more precisely, the agent (cf. Meir 1998)) and ends at the "referential locus" associated with the object (or the patient). Finally, the movement of spatial verbs (which often incorporate classifier handshapes, as in "place the cup at location X") is determined by the spatial location of referents.
} 
Both Liddell (2003a, 2003b) and Emmorey and Herzig (2003) suggest, then, that classifier predicates can be analyzed as containing parts that correspond to fixed, linguisticlike elements as well as variable, gesture-like elements (see also Emmorey 2002). Schembri (2002) has made similar claims, and has introduced the distinction between the view of sign language systems as homogenous or heterogenous systems. While homogenous systems contain only linguistic elements, heterogenous systems are characterized by containing both linguistic and non-linguistic (i.e. gestural elements). In a similar vein, Cogill-Koez (2000) presents an account of signed language as having two structured systems of representation, one linguistic and the other visual. In her view, the system of classifier predicates makes up the visual system and involves the use of schematic forms that contain both discrete and analogue-like elements. As already indicated in section 2.1.2 above in relation to iconicity, my own position on the composition of classifier predicates conforms to the idea that they are made up of both discrete (i.e. lexically-specified) and gradient/analogue (i.e. determined by imagistic content) elements.

However, an account of how mappings between conceptual space and sign space are accomplished does not yet reveal much about the choices that signers make in actual usage, and in different types of discourse. This general question, then, concerns the way iconic mappings are expressed in sign space. The next section addresses a part of this question by discussing the use of simultaneous constructions, that is, the form and function of two classifier predicates used simultaneously in sign space. A full understanding of the way the visual-spatial modality affords iconic mappings must take into account the interaction between classifier predicates and other systems that are relevant to spatial representation (see chapter 3), as well as the influence of linguistic and discourse constraints.

\subsection{Sign language structure: Simultaneous constructions}

As a general phenomenon, simultaneous constructions are defined as representations that are produced in more than one articulatory channel, whereby each channel bears distinct and independent meaning units which stand in some relationship to each other (Engberg-Pedersen 1994; Leeson and Saeed 2002; Miller 1994; Vermeerbergen 2001). Although this definition makes no commitment to the involvement of specific articulators, examples of simultaneous constructions within existing typologies in the literature typically involve the two manual articulators.

Researchers of different sign languages have identified numerous types of bimanual simultaneous constructions based on formal and functional properties (Engberg-Pedersen 
1993, 1994 for DSL; Leeson and Saeed 2002 for Irish Sign Language (ISL); Miller 1994 for Quebec Sign Language (LSQ); Vermeerbergen 2001 for Flemish Sign Language (VGT); Liddell 2003a for ASL; cf. also the review of literature on ASL presented in Miller 1994, which includes Friedman 1975; Gee and Kegl 1983; Klima and Bellugi 1979). Formally, two independent signs can be produced truly simultaneously (i.e. two one-handed signs are produced at the same time) or, more commonly, sequentially but in overlap (i.e. one sign is produced first and holds during the production of one or more other signs) (see figure 4.3 in section 4.3.4 in chapter 4). The functions that have been described for simultaneous constructions are listed below. They can be divided into two main groups depending on their structural organization and function. On the one hand, simultaneous constructions reflect spatial/temporal organization (see 1-3 in the list below); on the other hand, they reflect discourse organization (see 4-6 in the list below). These functions are discussed in more detail in sections 2.4.1 (perceptual structure) and 2.4.2 (discourse structure) below.

Functions of simultaneous constructions:

1. referent representation on both hands to express spatial information (in the depiction of the spatial relationship between two referents)

2. referent representation on both hands to express the temporal and spatial simultaneity of events (in the depiction of action or interaction between referents)

3. the expression of temporal simultaneity of events or states (aspectual information)

4. the hold of a topic on one hand while the other hand signs related information (topic comment structure)

5. the hold of an enumeration morpheme on one hand while the other hand signs one or more related signs

6. the hold of an index sign on one hand while the other hand signs one or more related signs

\subsubsection{Simultaneous constructions that reflect perceptual structure}

Previous research on simultaneous constructions has paid considerable attention to the use of the two manual articulators to express information about the spatial and temporal organization of an event. Thus, each hand represents a referent, and the simultaneous presence of the hands/referents in sign space reflects their overlap in the dimensions of space and time (cf. the depiction of the spatial relationship between the man and tree in example (1)). Though it is possible for both hands to appear in sign space simultaneously, in most cases the hands appear sequentially, such that a one-handed sign or one hand of a two-handed 
sign holds or perseveres during the production of one or more other signs. Sequentiallyproduced simultaneous constructions contain what Engberg-Pedersen $(1993,1994)$ analyzes as a hold-morpheme. In simultaneous constructions that express location information, the hold-morpheme typically represents the ground referent, and is typically associated with the non-dominant hand. After the ground object is identified and located in sign space, the dominant hand identifies and locates the figure object in relation to the (visually accessible) ground object. (See section 4.3 .3 in chapter 4 for the distribution of use of the dominant and non-dominant hand in DGS static scene spatial descriptions.)

Furthermore, the hold-morpheme is analyzed as neutral with respect to the semantic distinction between location and motion. This means that when the interaction between two moving referents is depicted in sign space, only the foregrounded referent is associated with a movement morpheme. For example, to depict the interaction of two basketball players, where both players are running and one is overtaken by the other, the hand depicting the overtaken player remains stationary, while the hand depicting the player doing the overtaking moves in space (Engberg-Pedersen 1993). The backgrounded player's motion is not actually represented by movement of the hand in space, but rather must be inferred from the context. In general, the maintenance of visually accessible background information contributes to the overall spatial coherence in event narratives (see chapter 8 on the role of simultaneous constructions in DGS narratives in contributing to spatial coherence).

In expressing the temporal simultaneity or temporal overlap of two events, the holdmorpheme backgrounds one event while the co-occurring event is depicted on the dominant hand. For example, to express drinking coffee while reading a newspaper, a signer can sign a sequence in which she first depicts reading the newspaper (i.e. holding a newspaper open in front of her with both hands), and then maintains the newspaper on her non-dominant hand with a hold-morpheme while depicting drinking coffee with the dominant hand (cf. Mathur 2002, for ASL).

Information related to the spatial and temporal structure of an event does not necessarily have to be encoded on the two manual articulators. Simultaneously occurring aspects of an event may also be expressed by other articulators, for example, the face or torso. This often entails the use of elements associated with different perspectives (cf. Aarons and Morgan 2003; Dudis 2004a, 20004b; Liddell 1998, 2000). 


\subsubsection{Simultaneous constructions that reflect discourse structure}

Simultaneous constructions that express discourse functions are analyzed in a similar way with respect to the relationship between backgrounded vs. foregrounded information and the use of the non-dominant vs. the dominant hand. In constructions expressing topic-comment structures, the topic is held on the non-dominant hand, while information related to it is expressed on the dominant hand. Other discourse-structural relationships that are expressed with simultaneous constructions include the simultaneous appearance of an enumeration morpheme or index sign on one hand together with one or a series of other signs on the other hand. For these constructions, in contrast to those that express primarily locative and/or temporal relationships, it is not uncommon for the signs on the two hands to get produced simultaneously (Vermeerbergen 2001 for VGT; Vogt-Svendsen and Bergman 2007 for Norwegian Sign Language (NSL) and Swedish Sign Language (SSL)).

Although he does not use the term simultaneous construction, Liddell (2003a) discusses similar constructions containing signs he calls buoys. These signs are produced on the nondominant hand and are held in place while the dominant hand continues signing. Semantically, their presence in sign space helps guide the procession of discourse. Liddell identifies four different types of buoys, two of which have counterparts in existing typologies of simultaneous constructions. List and pointer buoys essentially correspond to simultaneous constructions with index and enumeration signs, respectively. A theme buoy takes the form of a raised, vertical index finger on the non-dominant hand and signifies the discussion of an important discourse theme. Finally, a fragment buoy is an articulatory trace of a two-handed sign during a subsequent one-handed sign. Similar to what Mathur (2002) calls RESIDUE, it does not serve a semantic or syntactic function. Thus, they are not true simultaneous constructions as Miller (1994) defines them, where the use of the term construction is emphasized because the elements that are simultaneously expressed must stand in some relationship to each other, be it syntactic, discursive, or iconic.

Of particular interest to this dissertation are the simultaneous constructions that depict spatial information. In the prototypical simultaneous locative construction, two classifier predicates are simultaneously present in sign space, and each hand represents a different referent (see chapter 4). However, as mentioned, articulators other than the hands can be involved in the simultaneous contribution of meaning. Chapters 7 and 8 describe the simultaneous use of different articulators (including the hands, torso, shoulders, and eyes) in expressing information associated with different signing perspectives. 


\subsection{Summary}

The primary aims of this chapter have been to clarify how the notion of iconicity has been invoked to explain different sign language representations, especially those expressing spatial relationships, and to provide the reader with the building blocks to understand the nature of the relevant structures, e.g. the phonological structure of language in the visual-spatial modality (in particular, DGS). I have reviewed different analyses of the morphological structure of the system of classifier predicates, the spatial device most relevant to the expression of location and motion, and most important for this dissertation. Finally, the use of simultaneous constructions, a special affordance of the visual-spatial modality to express locative or discourse relations, was described, as it forms an important theme in the dissertation.

The next chapter provides additional theoretical background. I introduce the spatial domain, and the main subdomains of location and motion, as an object of linguistic inquiry in spoken and signed language. In addition, I introduce the systems relevant to spatial description in language, including frames of reference, perspective, and viewpoint. 


\section{The spatial domain in spoken and signed language}

Chapter 3

As the discussions in chapters 1 and 2 have shown, iconicity plays an important role in the expression of spatial information in signed language. As an object of investigation in both linguistics and the cognitive sciences, the spatial domain, and how it is encoded in language, merits a close theoretical review. This chapter focuses on the subdomains of location and motion (section 3.1), the use of frames of reference in spoken and signed languages (section 3.2), and the use of perspective in signed language (section 3.3). In relation to frames of reference, section 3.4 discusses the issue of viewpoint in signed descriptions and the use of shared space by signer and addressee (Emmorey 1996). Finally, section 3.5 discusses the relationship between frames of reference, perspective, and classifiers in signed language, and motivates alignments between the systems based on similarities in the properties that define their use.

\subsection{The spatial domain}

The study of the use of space is of central interest in the sign language literature. Space and time are pervasive in any event (e.g. giving, taking), and linguistic analyses of sign language structure are generally concerned with how a spatial medium is used to encode spatial aspects of an event. At times, space itself is the subject matter of expression, and we explicitly talk about and refer to space. This is the "spatial domain", which is our focus here.

As a semantic domain, the notion of "space" typically refers to the location and motion of referents with respect to other referents. Linguistically, the domain can be defined as comprising explicit or implicit "answers to where-questions". Studies on the spatial domain predominantly focus on the linguistic means of encoding location and motion, though the expression of shape as a semantic category is also important (see e.g. Bohnemeyer and Brown, in press; Brown 2006; Levinson 1994, 1996b; Seifart 2005).

For Talmy (1985: 60), the subdomains of location and motion both fall under the notion of a "motion event", which is defined as "a situation containing movement or the maintenance of an entity at a stationary location". A motion event can be described in terms of its major components, including the fact of motion itself (or lack of it in the case of static 
location), the moving (or located) figure entity, the ground object with respect to which the figure moves (or is located), as well as the path and manner of the figure's motion. Some languages, like English, use the same linguistic resources to express location and motion (as in the parallel of the prepositional phrase in the cup is on the table vs. he put the cup on the table). For English, Talmy's uniform treatment of motion and location (where location is simply a special case of a motion event) seems sufficient. However, the conceptual distinction between stasis vs. kinesis is not always neutral on the linguistic level. Many languages (e.g. Tzeltal or Tiryo) use very different systems for these subdomains, and they must be independently studied (see Levinson and Wilkins 2006b). In the following sections, I first discuss the semantic subfields of static location specification (section 3.1.1) and then move on to the encoding of kinetic motion events (section 3.1.2).

\subsubsection{The encoding of location in language}

In the spatial domain of location specification, a major divide can be drawn between situations where the figure and ground objects are coincident (i.e. in the same location) in space, on the one hand, and those where the objects are non-coincident (i.e. at a remove from each other in space), on the other hand. The latter case requires the use of coordinate systems, or frames of reference. Figure 3.1, adapted from Levinson (2003: 66), shows this delineation and the corresponding semantic subdomains available in languages. ${ }^{26}$ They are discussed in turn below.

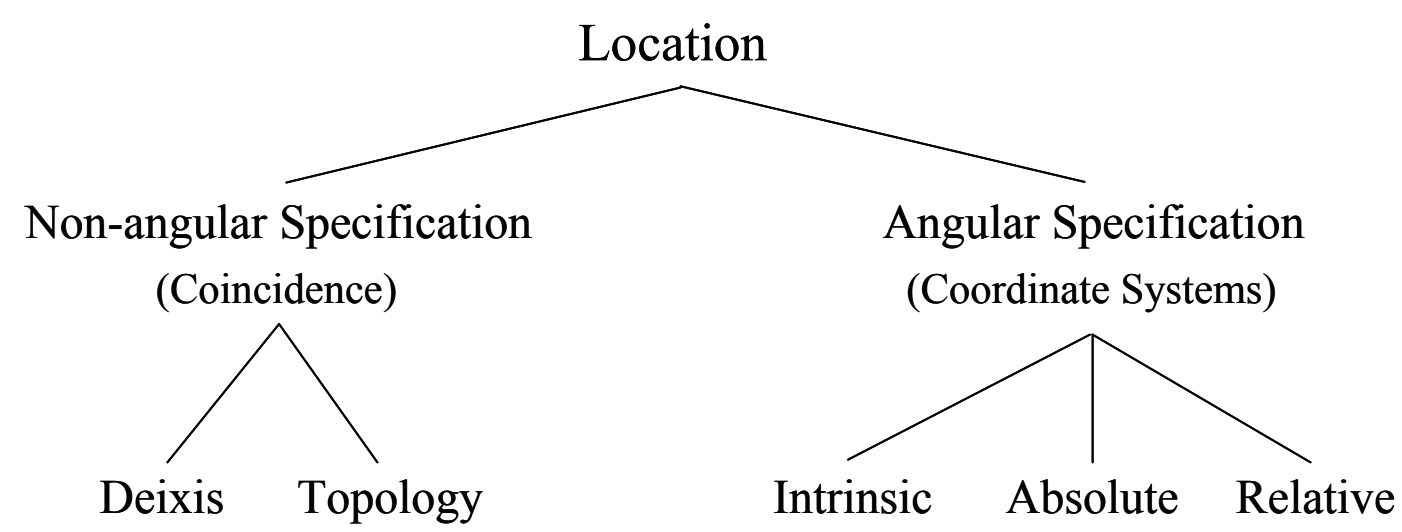

Figure 3.1. Semantic subfields in the domain of spatial location specification.

Coincidence in space comprises topological relations (e.g. contiguity, support, containment) and deixis, while non-coincidence defines relationships whose expression must contain the specification of a direction, and must thus utilize a frame of reference (intrinsic, absolute, 
relative). Another way of labeling this main divide, and the key to understanding it, is with the terms non-angular vs. angular specification. What it means to specify an angle, and thus the concept of angular vs. non-angular specification, is detailed in the following sections.

\subsubsection{Angular specification}

The concept of angular specification refers to the use of a system of polar coordinates. As stated in Levinson and Wilkins (2006a: 19):

$[\mathrm{O}]$ nce a figure object is removed in space from a relevant ground object or landmark, it becomes pertinent to specify a direction, or angle, relative to the landmark in which the figure may be found. Such angular or directional specifications of location require some form of coordinate system. Natural languages seem to employ only polar coordinates, specifying a direction by rotation around a ground object.

In a polar coordinate system, location is identified by specifying a distance and a direction. The location of a point $\mathrm{P}$ is indicated by the coordinate pair $(\mathrm{r}, \theta)$. The radial coordinate, $r$, is the distance of the point, $\mathrm{P}$, from the origin (i.e. a fixed point on a fixed line). The angular coordinate, $\theta$, specifies direction and is the angle made by the fixed line and the line made by r, which connects $P$ to the fixed point. This is illustrated in figure 3.2 below.

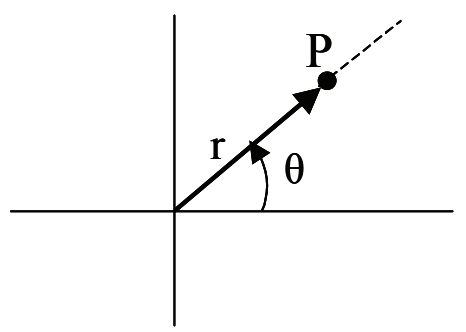

Figure 3.2. Specification of a point $\mathrm{P}$ in a polar coordinate system.

Applying this to the linguistic task of location specification, relating the location of the figure to the location of the ground requires designating an area of space that is not coincident with the ground object. This area of space is at a remove from the ground object and is arrived at by specifying a direction, projected from the ground object, which leads to the figure object if followed. In Levinson's terms, this area of space is the search domain in which to locate the figure, and it must be established by specifying a direction - which is to say, an angle with respect to the ground. The specification of an angle (i.e. angular specification, as it is labeled in figure 3.1) is tantamount to the use of a frame of reference, with which languages

\footnotetext{
${ }^{26}$ The figure does not include toponymy, i.e. the use of place names, to specify location.
} 
specify object location with respect to a spatially removed landmark or ground (Levinson 1996a, 2003; Levinson and Wilkins 2006a).

There are three major types of frame of reference that have been identified in natural languages: intrinsic, absolute, and relative (see section 3.2 for a description of each frame). All of them refer to instantiations of coordinate systems for object localization and thus share the following properties:

- As frames of reference, they are systems of (polar) coordinates.

- They are imposed on a spatial scene to specify the location of a figure object with respect to a non-contiguous ground object.

- They specify angular information about the horizontal direction into which a search domain must be projected from a ground object in order to be able to locate a figure object within it.

The use of a frame of reference to specify the location between a figure and a ground object is illustrated with a concrete example in figures 3.3 and 3.4 below. In the figures, the ground object is the plate, and the orange is the figure object which is to be located with respect to the plate. The diagram in figure 3.3 shows the superimposition of a coordinate system over the spatial scene containing the two objects. Importantly, the origin of the coordinate system is on the ground object, as it is in relation to the ground object that the figure must be located. The distance between the two objects is given by $r$. The direction in which the figure object is to be located with respect to the ground object is specified by the angle $\theta$. Note that for the purposes of a linguistic description involving angular specification, the direction of $r$ is not uniquely determined.
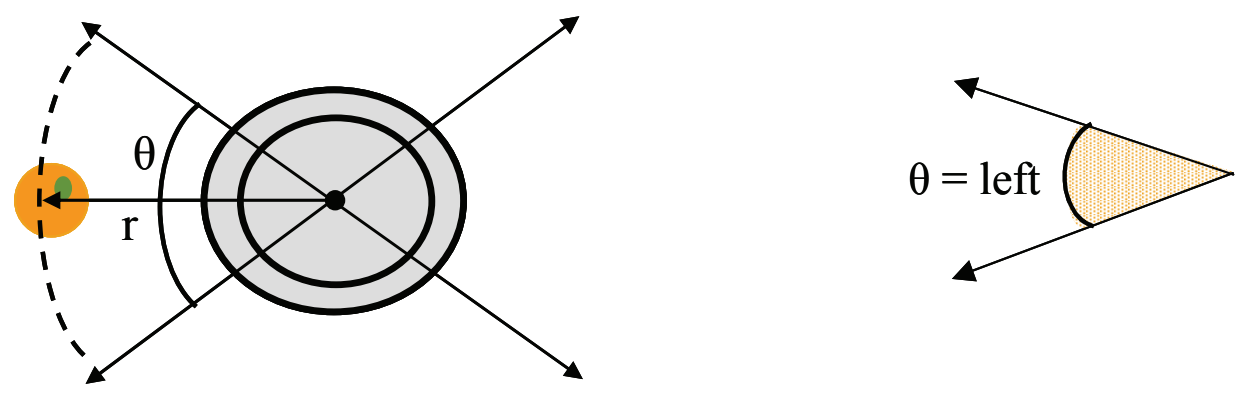

Figure 3.3. The superimposition of a coordinate system over a spatial array containing a ground object (i.e. the plate) and a figure object (i.e. the orange) shows how an angle can be determined to specify the location of the orange with respect to the plate. 

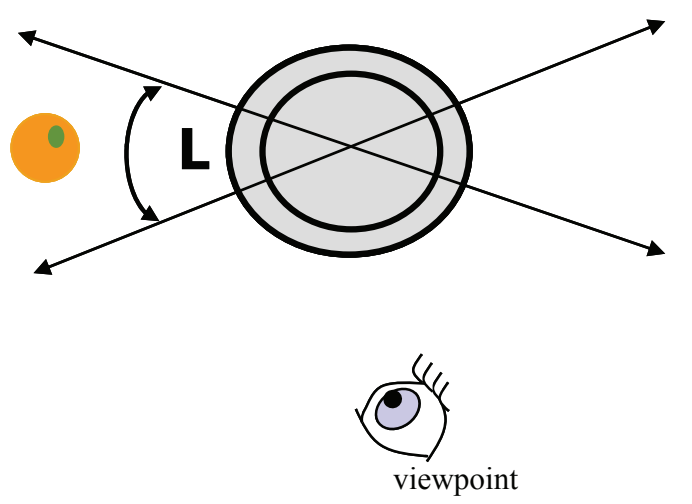

Figure 3.4. Using a relative frame of reference, the location of the figure object (i.e. the orange) can be specified with respect to the ground object (i.e. the plate), relative to a viewpoint, as The orange is to the left of the plate. By specifying an angle, the expression identifies the region of space to the left of the plate as the appropriate search domain in which to locate the orange.

The angle $\theta$ does not denote a single degree (i.e. one degree of a possible $360^{\circ}$ ), but rather encompasses a whole quadrant, as defined in figures 3.3 and 3.4 by the viewpoint-dependent relative spatial term left (in contrast to the terms right, front, or back). The actual location of the figure could be anywhere within the specified quadrant of direction. Thus, the direction of $r$ is only generally specified, but for the purposes of linguistic description, it sufficiently locates the figure object, i.e. the orange, with respect to the location of the ground object, i.e. the plate, relative to a viewpoint.

\subsubsection{Non-angular specification}

When figure and ground are coincident, it is not necessary to specify an area of space different from the ground object in which the figure can be located. The other major type of location specification thus relies on non-angular specification. Figure 3.1 above indicates two main ways in which the coincidence of figure and ground can be expressed: either as a deictic relationship or as a topological relationship.

The topological subdomain comprises relations of containment, contact, support, or contiguity between two objects (as expressed in English with prepositions such as in, on, at). In English, for example, we can express a contact or support relationship between the figure object (i.e. the orange) and the ground object (i.e. the plate) as The orange is on the plate. In deictic expressions like The orange is here, we have not two individual objects, but rather the orange as the figure object and the region of space denoted by the deictic term here as the ground. 
This dissertation (chapters 5 and 6, specifically) is concerned with the subdomain of angular specification, that is, with the use of frame of reference systems to encode spatial information. In addition to encoding location, frames of reference also appear in descriptions of motion. As we have seen, the use of a frame of reference entails the specification of a direction projected off the ground object. By "following" the specified direction, it is possible to find the figure. In the example in figure 3.4 above (The orange is to the left of the plate), the relative term left specifies the direction of the orange's location from the ground as seen from the reader's point of view. In the same way, left can specify the direction of a figure's motion with respect to a ground, as in The orange rolled to the left of the plate. The next section discusses the encoding of motion in spoken and signed language.

\subsubsection{The encoding of motion in language}

Languages differ typologically with respect to which components of a motion event are typically expressed and the linguistic means by which they are expressed. In the study of motion, many studies have focused on how manner and path are lexicalized across languages, including spoken and sign languages (Allen et al. 2006; Berman and Slobin 1994; Papafragou et al. 2002; Slobin and Hoiting 1994; Talmy 1985, 1991; Taub and Galvan 2001). According to Talmy $(1985,1991)$, languages can be grouped into two main typological categories depending on their dominant lexicalization patterns. Of particular importance is the encoding of what Talmy takes to be the core conceptual element of motion events, namely the path component. Verb-framed languages (e.g. Romance and Semitic languages) conflate motion and path in the main verb and express manner in a separate element. Satellite-framed languages (e.g. Germanic and Slavic languages), on the other hand, conflate manner and motion in the main verb, and express path in a separate satellite element. The sentences below illustrate the difference between verb-framed (example 1) and satellite-framed (example 2) languages. ${ }^{27}$

$\begin{array}{lllll}\text { (1) Jean } & \begin{array}{l}\text { a traversé } \\ {[\text { Path }]} \\ \text { crossed }\end{array} & \text { le pont } & \begin{array}{l}\text { à pied. } \\ \text { [Manner] } \\ \text { on foot. }\end{array} & \text { [French] } \\ \text { (2) John } & \begin{array}{l}\text { walked } \\ {[\text { Manner }]}\end{array} & \begin{array}{l}\text { across } \\ {[\text { Path }]}\end{array} & \text { the bridge. } & \text { [English] }\end{array}$

\footnotetext{
${ }^{27}$ Talmy (1985) distinguishes a third group of languages, represented by members of the Hokan (e.g. Atsugewi) and Athabaskan (e.g. Navajo) language families, in which verb roots encode motion together with information about the figure (e.g. -lup- 'for a small shiny spherical object to move/be located'). Path is encoded in a verb
} 
The lexicalization patterns exhibited by sign languages with respect to motion events have been described in different ways. Talmy (2003: 186) suggests that "spatial representation in signed classifier expressions is iconic with scene parsing in visual perception". This pertains to the representation of object size, form, and number, as well as to the representation of path contour, path length, manner and rate of motion, the repetition of a path, and the temporal progression of a path. Compared to spoken languages, Talmy (2003) argues that the spatial schemas of sign languages are less prepackaged and less discrete, and that they contain more independently variable elements as well as more spatial categories overall.

Slobin and Hoiting (1994) categorize sign languages (referring, in particular, to NGT and ASL) as "complex verb-framed languages". In terms of the crucial criterion of path representation, they argue that sign languages are verb-framed, since the representation of motion in a spatial medium (i.e. sign space) entails the representation of a path. They are "complex" because the spatial medium additionally affords the simultaneous representation of other components, in particular, the figure and the manner of motion.

Supalla (1990) discusses the grammatical restrictions in ASL on the combinability of morphemes within different types of verbs of motion. In essence, this identifies constraints on the simultaneous expression of different (simultaneous) aspects of an event. In particular, Supalla identifies restrictions on locomotion verbs (e.g. limping, walking, swimming) and path verbs (e.g. straight path, circular path). Supalla demonstrates that ASL does not allow the simultaneous representation of the figure's manner of locomotion together with the figure's path (including manner of motion along a path and direction of path). Instead, these components must be represented sequentially in two separate verbs, that is, in a serial verb construction. In these constructions, Supalla takes the manner of locomotion to appear in the verb stem and path to be represented in a dependent verb. ${ }^{28}$ Supalla attributes these restrictions to arbitrary linguistic rules, imposed on the distribution of morphologically distinct features.

In their analysis of ASL complex motion event descriptions, Taub and Galvan (2001) also found evidence that ASL signers presented different aspects of an event sequentially, consistently separating different pieces of information into different forms. For example, they found that signers represented a single falling event in at least three different ways, representing the motion/figure/path plus an additional element (e.g. the ground at the source

suffix relating to the ground (e.g. -lup-ict' 'for a small shiny spherical object to move into liquid'), and thus these languages fall under satellite-framed languages in the binary typology. 
or goal of the path motion). Thus, Taub and Galvan agree with Supalla that signers do not represent all features of an event simultaneously in a single form, but rather separate the features into pieces that can be represented sequentially. However, they disagree that this separation is due solely to linguistic constraints. Instead, they suggest that "many of the separation patterns in ASL stem from limitations on cognitive processing" (2001: 191). With respect to the typological classification of ASL, Taub and Galvan (2001) stress the high degree of simultaneity in ASL and suggest that Talmy's typology may need to be expanded to include a type in which path, manner, and figure is consistently conflated in a single verb form. Compared to motion event coding by English speakers, ASL signers consistently incorporate more conceptual elements (in particular, more motion, path, manner, affect, and spatial relationship information) (Taub and Galvan 2001). The analyses presented in chapters 7-8 of this dissertation deal with the conceptual elements (such as manner and path) that are expressed in different predicate constructions in the course of complex event narratives in DGS.

In addition to path, manner, and figure, the notion of a ground is an important component in the expression of motion events in language. The ground is the landmark or reference point with respect to which the figure motion of the object is specified. Often in motion descriptions, the ground is construed as the source or the goal of motion. Thus, the motion of the figure is expressed in terms of a directed "motion toward" or "motion from" a place. It is possible that both the source and the goal are specified in encoding the figure's motion (as "motion from A to B"). This creates a vector that does not explicitly encode (angular) direction. The source or goal may also be implied within a deictic verb of motion (e.g. in come (here)/go (there), where the goal/source of motion, respectively, is the place in which the utterance is made). In general, however, the specification of a direction indicates the use of a frame of reference. Chapters 5 and 6 of this dissertation concern location and motion descriptions in DGS that involve the use of a frame of reference. The following section describes the three linguistic frames of reference in detail and reviews the research on frame of reference use in spoken (section 3.2.1) and sign (section 3.2.2) languages.

\subsection{Frames of reference in spoken and signed language}

As we have seen in section 3.1, frame of reference systems are employed to express the spatial relationship between two objects (a figure and a ground object) that are at some

\footnotetext{
${ }^{28}$ In terms of Talmy's typology, then, Supalla's analysis should classify ASL as a satellite-framed language,
} 
distance from one another in space (Levinson 1996a, 2003; Levinson and Wilkins 2006a; Pederson et al. 1998). In this section, the three types of frames of reference - intrinsic, relative, absolute - are defined in more detail, and the research on frame of reference use in spoken and signed languages is discussed. ${ }^{29}$ Different languages manifest different variants and combinations of these three types of systems, but it is assumed that angular information in spatial description is exhaustively captured by these three types. The systematic investigation of frame of reference use for spatial reference has relied on the use of controlled elicitation tasks in a variety of typologically and genetically diverse languages. A concise description of the methodology of frame of reference elicitation in spoken languages is given in section 3.2.1. Section 3.2.2 addresses the research that has been conducted on the use of frames of reference in sign languages. First, the three frames of reference are defined here in more detail.

The intrinsic frame of reference makes use of conventionalized names given to sides or facets of objects based on certain language-specific criteria. Often, as in English, these criteria are functional, based on canonical orientation or direction of motion, but they can also be derived from the internal geometry of the object, as in Tzeltal (Brown 2006; Levinson 1994). The names given to sides or facets of an object are typically borrowed from body-part or landmark terms. In English, the front of a car, for example, is determined by direction of forward motion. The front of a television set is the screen, that is, the side canonically oriented toward the viewer (Miller and Johnson-Laird 1976). In expressions utilizing the intrinsic frame, the named facet defines a scene-internal binary relationship between figure and ground. By naming a facet of the ground object, a search domain in which to locate the figure object is projected off the named facet of the ground onto the surrounding horizontal plane. Thus, to say The tree is in front of the car projects a search domain off the side of the car designated as the front, such that the figure object, i.e. the tree, can be located in that region. This particular spatial relationship is shown in figure 3.5 below.

\footnotetext{
since the path element does not appear in the main verb.

${ }^{29}$ Frames of reference have been studied in many areas of cognitive science (e.g. developmental psychology, visual perception, brain sciences, psycholinguistics, linguistics), and different distinctions, based on the focus of interest, have been made (e.g. egocentric vs. allocentric, viewer-centered vs. object-centered, deictic vs. intrinsic) (Burgess et al. 1999; Clark 1973; Levelt 1996; Marr 1982; Miller and Johnson-Laird 1976). Linguistically, there is a tradition of distinguishing between deictic vs. intrinsic only. However, as Levinson (1996a) argues, this distinction cannot capture the difference between non-deictic relative uses and deictic intrinsic uses. The discussion of frames of reference in this dissertation follows Levinson (1996a and subsequent work).
} 


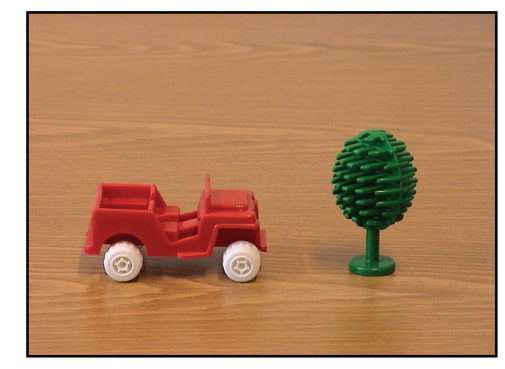

Figure 3.5. Example of a spatial relationship that can be expressed with an intrinsic frame of reference, i.e. The tree is in front of the car.

Expressions using the relative frame of reference encode the spatial relationship between a figure and a ground object from a viewpoint, or origo, distinct from either of the objects. Thus, a ternary, and not a binary relationship is coded. The origo from which the relationship between figure and ground is defined is typically the speaker, but can also be the addressee or any other third participant. An example of an expression utilizing the relative frame is The man is to the left of the tree (see figure 3.6). The location of the man with respect to the (nonfeatured) ground object (i.e. the tree) is indicated from the perspective of the viewer. The area of space that corresponds to the left of the tree is determined through a mapping of the viewer's own body axes onto the ground object. In English, the mapping is determined by a (mirror) reflection of the body axes or coordinates onto the non-featured ground object. Thus, the front of the tree, in English, refers the partitioned area around the tree that is directly opposite and visible to the viewer facing the tree. The tree does not have its own left, right, front, and back axes, and thus features must be secondarily derived through a particular mapping of the body axes onto the tree. ${ }^{30}$

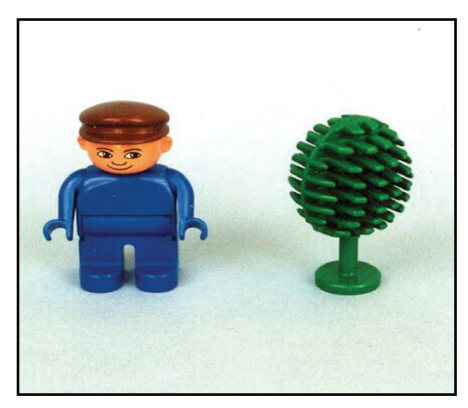

Figure 3.6. Example of a spatial relationship that can be expressed with a relative frame of reference, i.e. The man is left of the tree.

Similarly to the intrinsic frame, the absolute frame of reference encodes a binary relationship. The encoding, however, is based not on intrinsic features of a ground object, but

\footnotetext{
${ }^{30}$ There is linguistic variation within the relative frame of reference in how the mapping of the body coordinates onto a ground object occurs. In addition to reflection, the mapping can occur via translation (as in Hausa) or rotation (as in Tamil) (see Levinson 1996a, 2003).
} 
rather on conventional absolute bearings, like cardinal directions or landmark systems. With the use of fixed bearings, the coding of a spatial relationship does not depend on the position of the viewer relative to the objects, as in the relative frame. An example of an expression utilizing an absolute frame of reference is The man is West of the tree. This expression would correctly describe the picture in figure 3.6 above if West happened to be to your left (as the reader of this text). Moreover, the expression would be valid regardless of your location, or, in general, of the location of the speaker uttering an absolute expression. The predominant use of the absolute frame of reference relies on the community-wide availability of an abstract, fixed system of bearings. Effectively, speakers that use an absolute frame have absolute knowledge of their surroundings, which makes them capable of "dead reckoning" (Levinson 1996a). They can determine the location of a figure object with respect to a ground object in terms of fixed bearings at all times and from all locations, even in the absence of visual input of their surroundings.

\subsubsection{Frame of reference research in spoken languages}

The identification and determination of the use of frame of reference systems in natural spoken languages results from careful, controlled, and systematic data collection within a circumscribed semantic domain. The conceptual and linguistic variation within the frame of reference domain has been probed with stimuli that cover all possible oppositions within the domain (based on the construction of a so-called etic grid) (Levinson and Wilkins 2006a; Pederson et al. 1998). Their use for data elicitation presents a view of what distinctions are made in particular languages and how the possible discriminations are grouped conceptually and linguistically - yielding the emic division of the domain for each particular language. ${ }^{31}$

A particular set of stimuli used to elicit frame of reference use, the so-called Man and Tree task, is described here in detail. The task used in this dissertation to elicit static scene spatial descriptions in DGS (see chapter 4) was based loosely on the Man and Tree task. The Man and Tree task is a director-matcher photo matching task, whereby two speakers - one acting as the director and one acting as the matcher - sit side-by-side, facing in the same direction, but screened off from each other's view. Both speakers have, laid out in front of them, an identical set of photographs, each of which depicts a certain spatial configuration of a man and a tree. As a whole, the pictures manifest a structured set of oppositions contrasting

\footnotetext{
${ }^{31}$ The use of etic and emic in this context is derived from Pike's (1943) notion of "phonetics" to describe the production and perception of the sounds of speech, in general, as opposed to the "phonemic" inventory of individual languages (Levinson and Wilkins 2006a; Pederson et al. 1998).
} 
the relative locations of the two referents (the standing information) and the orientation of the man with respect to the tree (the facing information). Figure 3.7 below shows pairs of pictures that contrast in relative location only (pictures (a) and (b)) and in orientation of the man only (pictures (c) and (d)).

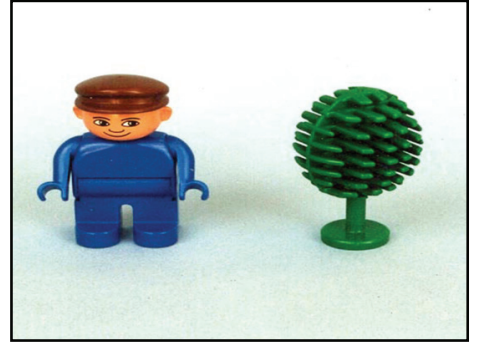

(a) Man left, tree right

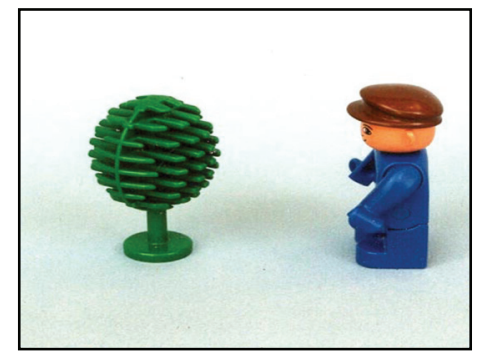

(c) Tree left, man facing tree

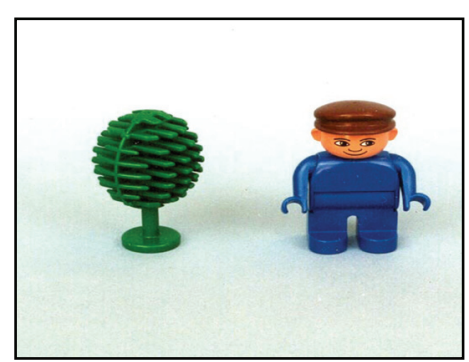

(b) Man right, tree left

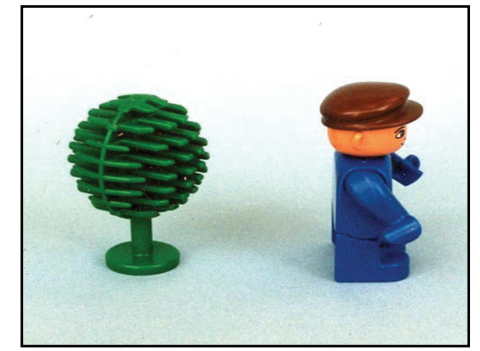

(d) Tree left, man facing away from tree

Figure 3.7. Sample pictures from the Man and Tree task with contrastive oppositions in location (pictures (a) and (b)) and orientation (pictures (c) and (d)).

The matcher selects pictures based on verbal descriptions by the director. Ensuing discussion between the director and the matcher ensures that the matcher has selected the same picture that the director has described. In the course of the interaction, distinguishing propositions allow the matcher to exclude a certain subset of pictures from his/her consideration. That is, they allow the matcher to identify a functional contrast and to subsequently include in his/her focal set only photographs for which the proposition is true. For example, for pictures (a) (d) in figure 3.7 above, saying the tree is on the left excludes only picture (a) from the set of possible targets. This proposition including the English relative term left distinguishes between the pictures based on a contrast between location on the transverse, or lateral, axis. In order for the speakers to be able to converge on a single picture (out of the subset of (b), (c), and (d)), the director must provide a further distinguishing proposition that specifies orientation information for the man. For example, the proposition the man is facing to the front together with the tree is on the left allows the matcher to select a single photograph. 
Note that the converse distinguishing proposition the tree is on the right is by itself sufficient to select a single photograph, namely photograph (a), from the set of four pictures in the figure.

By compiling the distinguishing propositions from all individual languages studied, it is possible to determine the functional equivalence of utterances across languages. The different terms found in functionally equivalent utterances provide cues to the way in which search domains for object location are specified. In this way, it is possible to determine which frames of reference are in use in a particular language and across multiple languages. Furthermore, the terms used in frame of reference locative descriptions in different languages appear in a variety of grammatical constructions and belong to different word classes. Thus, this research approach also sheds light on the semantic and morphosyntactic variation in the use of frames of reference across languages.

Overall, the research on frame of reference use in spoken languages has shown that there is considerable linguistic diversity in this domain (see Levinson 2003; Pederson et al. 1998). Firstly, there is variation between languages in the specific frames of reference used. Some languages use only the absolute frame, others use only the intrinsic frame, others use the relative and the intrinsic frame, while still others use some version of all three frames. In addition, there are variants within each of the three frames. For example, within the intrinsic frame, languages vary in the way inherent features are assigned to referents (e.g. by canonical orientation or function, or by internal geometry). Within the relative frame, languages vary with respect to the way the coordinates are mapped from the body onto the ground entity (cf. footnote 31 above), while the use of an absolute system may be based on abstract cardinal directions or on landscape features. Some generalizations can be made about the distribution of use of frames of reference across spoken languages. The use of the relative frame implies the intrinsic frame, with evidence that the former is derived from the latter (e.g. by the fact that many languages, like English, use the same terms in both the relative and intrinsic frames) (Levinson 1996a, 2003). As a rough generalization, the systematic use of true absolute frame of reference systems tends to be exclusive of the systematic use of the relative frame, and vice versa, while use of the intrinsic frame co-occurs with both the absolute and the relative frames. Moreover, in contrast to the relative frame, absolute systems tend to be found in non-urban communities.

Thus, we know quite a lot about frame of reference use in spoken languages, but what about in sign languages? Do we expect the same kind of variation in sign languages that we find in spoken languages, and along the same parameters? There has been comparatively little 
research done on frame of reference use in sign languages. The next section provides an overview.

\subsubsection{Frame of reference research in sign languages}

Compared to the research on spoken languages, the question of frame of reference use in sign languages has received little attention. To date, the use of the relative, intrinsic, and absolute frames of reference in the encoding of spatial relationships in sign languages has been addressed only by Emmorey $(1996,2002)$ for ASL and Arik (2006) for Turkish Sign Language (TID). Emmorey stresses that the use of a frame of reference in signed language is independent of the way in which sign space is structured for spatial representation by perspective. She gives examples of the usage of each of the three frame of reference systems in ASL in both types of perspective (or spatial format, in her terminology) (2002: 96-99). These examples of how the different frames of reference could be instantiated in ASL are described below.

One reason for the lack of research on frames of reference in sign languages may be that the iconic properties of spatial representation in the visual-spatial modality make the task seem straightforward. That is, classifier predicates are used to map spatial relationships between entities in sign space in a way that corresponds iconically to observed relationships between entities. ${ }^{32}$ Indeed, Emmorey et al. (1998: 241) notes that:

For the signer, the location of the classifier sign in signing space maps onto his mental image of the [entity's] location. Signers appear to obey a spatial mapping principle which states that locations within signing space map to isomorphic locations in either a physically observed space or an imagined space.

A close investigation of the kind outlined in section 3.2.1 above for spoken languages may seem superfluous for sign languages in the sense of contributing only intuitive and selfevident facts. However, there are some reasons to question the assumption that the visualspatial modality may exhibit a single, "natural" solution to spatial representation.

First, spatial descriptions elicited using the Man and Tree task from signers of Nicaraguan Sign Language (NicaSL) ${ }^{33}$, a young sign language which has emerged over the past 30 years, show no established convention for the viewpoint from which signers map

\footnotetext{
${ }^{32}$ However, there seem to be language-specific constraints on the way in which classifier handshapes can combine to express spatial relationships. In ASL, for example, it is not possible for a classifier handshape (e.g. representing a cat) to be placed on top of the vehicle classifier (Valli and Lucas 1995, cited in Emmorey 2001).

${ }^{33}$ In the literature, Nicaraguan Sign Language is generally referred to as NSL (e.g. Pyers and Senghas 2007; Senghas et al. 2004), however, I refer to it here as NicaSL in order to distinguish it from Norwegian Sign Language, which is also abbreviated as NSL.
} 
spatial relations onto sign space. With director (i.e. signer) and matcher (i.e. addressee) seated next to each other (but turned toward each other for the purposes of signing) signers gave descriptions both from their own and their addressee's viewpoint (Ann Senghas, unpublished data, personal communication). ${ }^{34}$ Another example comes from Kata Kolok, a village sign language in Bali. Kata Kolok uses an absolute frame of reference for indicating referent locations, which means that signers locate referents at their real-world locations, regardless of their own location and orientation. While spatial description has yet to be systematically studied in Kata Kolok, the use of an absolute frame of reference shows that there are possibilities besides the use of the relative frame of reference (with the signer as the deictic origo) in signed language. (See Marsaja (2003) and Perniss and Zeshan (in preparation) on the use of absolute locations in Kata Kolok. ${ }^{35}$ The use of the absolute frame of reference in signed language is discussed again later in this section, as well as in section 3.5. Finally, the interaction between frames of reference with the two primary perspectives from which an event space can be projected onto sign space imparts an additional complexity that must be carefully considered. As with all domains of linguistic inquiry, systematic investigations into specific language use must be made before we can fully understand frames of reference in the visual-spatial modality.

In the following, Emmorey's examples of frame of reference use in different perspectives in ASL are described in turn. Emmorey uses the terms "diagrammatic spatial format" and "viewer spatial format" for what I call observer perspective and character perspective event space projections, respectively. In the descriptions that follow, I use the terminology that I propose. It is essential to the analyses of perspective and frame of reference use in DGS presented in later chapters of this dissertation to achieve clarity about the possible instantiations of these systems. My own account of the interaction between frames of reference and perspective is given in section 3.5.

As we have seen, the intrinsic frame of reference uses designated facets of objects to specify a binary relationship between a figure and a ground. A search domain in which to locate the figure object is projected off a faceted side of the ground object by naming this side. As a binary relationship, the intrinsic frame of reference is "orientation-free", that is, it expresses a scene-internal orientation relationship that remains the same regardless of the

\footnotetext{
${ }^{34}$ It must be noted that these descriptions were elicited several years ago, in 1999/2000 and 2001/2002. It is possible that Nicaraguan signers, especially the younger signers, have established a convention by now. Indeed, at the time the task was performed, the younger signers exhibited a greater degree of systematicity in their descriptions than did the older signers.
} 
viewpoint from which the scene is viewed. For ASL, Emmorey shows how an intrinsic relationship can be represented in both observer and character perspective event space projections. In observer perspective, the signer may place a classifier form representing a human entity in front of a classifier form representing a vehicle (the front of which is represented by the fingertips; cf. figure 2.8(c) in chapter 2) (as in figure 3.8(a)). ${ }^{36}$ Thus, by placing another classifier form at the fingertips side of the vehicle classifier, the signer can express an intrinsic orientation relationship between the two objects. In character perspective, on the other hand, the binary relationship is between the signer's body and a classifier form. In Emmorey's example, the fingertips of the ASL vehicle classifier handshape point toward the body, thereby expressing the same binary intrinsic relationship as before (i.e. "the person is in front of the car"). Here, however, the person is represented not by an entity classifier, but rather by the signer's body (see figure 3.8(b)).

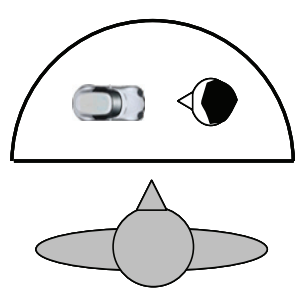

(a) Observer perspective

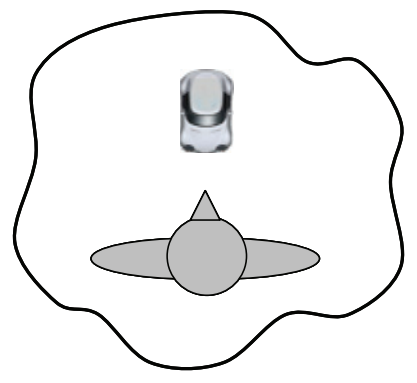

(b) Character perspective

Figure 3.8. Schematic illustrations of Emmorey's (2002) examples for the use of the intrinsic frame of reference in ASL, used in (a) observer perspective and in (b) character perspective. ${ }^{37}$

In contrast to the intrinsic frame, the relative frame of reference is "orientation-bound". That is, its use depends on the viewpoint from which a scene is viewed. Emmorey's examples for the use of the relative frame in ASL make explicit the ternary relationship between the figure, ground, and a particular origo or viewpoint. In the example using observer perspective, all three entities are located in the space in front of the signer's body (in which a diagram of the event space is created). A man is standing on a hill looking down into the valley, and sees a lake and a house. From the man's point of view (as the deictic origo), the house (the figure) is

\footnotetext{
${ }^{35}$ In addition, a PhD project by Connie de Vos is currently underway at the MPI for Psycholinguistics, Nijmegen about the use of pointing in Kata Kolok.

${ }^{36}$ Though there has been no systematic investigation of the conventional intrinsic properties of different DGS (or ASL) classifiers, my guess is that they are more similar to facet assignment in German than, for example, in Tzeltal.

${ }^{37}$ See Fridman-Mintz and Liddell (1998) for the use of a semi-circle area in front of the signer to indicate the use of observer perspective (i.e. "token space" in their terminology) and the use of a wavy-line area surrounding the signer to indicate the use of character perspective (i.e. "surrogate space" in their terminology). I use icons to represent the classifier forms in order to make the entities in the examples maximally identifiable.
} 
behind the lake (the ground) (as shown in figure 3.9(a)). On the other hand, in the use of character perspective to express a relative frame of reference relationship, the signer's view of the event space (from a place within it) corresponds to the deictic origo. In Emmorey's example, the signer maps a window to the left and a portrait to the right at eye-level, as though standing in a room and looking at the two objects ("the portrait is to the left of the window") (as in figure 3.9(b)).

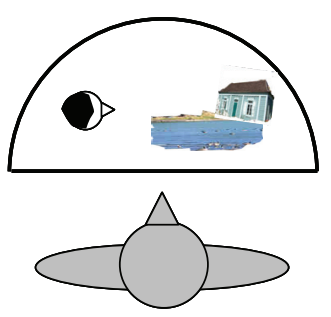

(a) Observer perspective

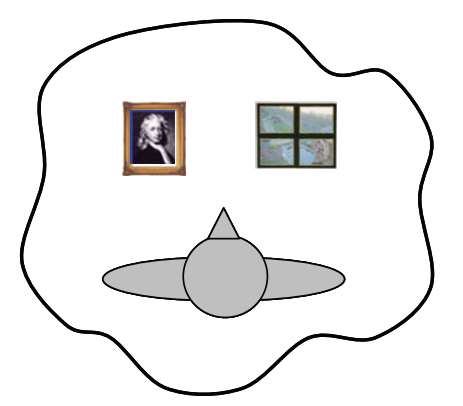

(b) Character perspective

Figure 3.9. Schematic illustrations of Emmorey's (2002) examples for the use of the relative frame of reference in ASL, used in (a) observer perspective and in (b) character perspective.

Finally, Emmorey takes the use of cardinal direction terms in ASL to correspond to the use of an absolute frame of reference. In Emmorey's examples, a signer conveys that she was "driving north". In citation form, NORTH is made by moving an N-hand vertically upwards (corresponding to the canonical orientation of standard maps). However, its direction in space can be modified to fit a particular spatial representation.

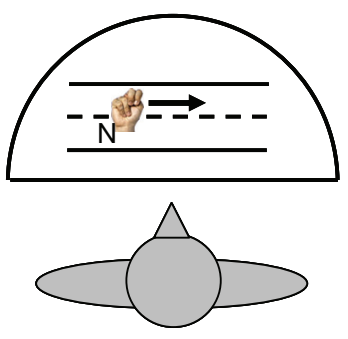

(a) Observer perspective

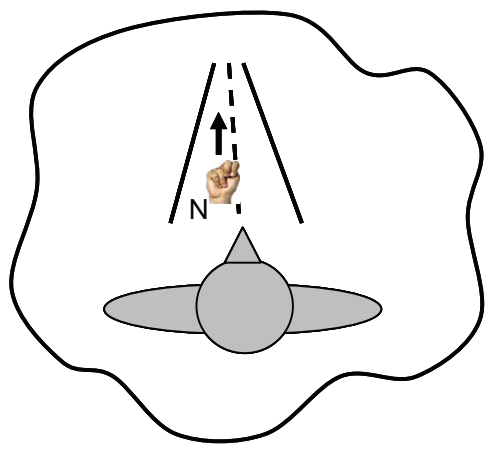

(b) Character perspective

Figure 3.10. Schematic illustrations of Emmorey's (2002) examples for the use of the absolute frame of reference in ASL, used in (a) observer perspective in (b) character perspective.

In observer perspective, the signer can map a street onto sign space laterally in front of the body. To indicate that the direction of driving along that street is to the north, the signer can sign NORTH along the street in sign space (e.g. to the right) (see figure 3.10(a)). In character 
perspective, on the other hand, the street is mapped onto sign space from the perspective of the signer as if she herself were moving along the street. The street thus extends outward from the body, and the use of the cardinal direction term NORTH to indicate "driving north" along the street is modified to move away from the body on the sagittal axis (see figure 3.10(b)). These examples for the use of an absolute frame of reference in ASL do not, arguably, exhibit a true use of an abstract system of absolute bearings (see section 3.5.1 for further discussion of this issue).

In a study exploring frame of reference use in ASL, signers who were asked to describe simple scenes containing two entities did so predominantly from their own point of view, in a relative frame of reference and in observer perspective (Emmorey 1996). In addition, Emmorey notes that signers encoded the scene-internal orientation of entities with intrinsically-featured classifiers, such that the relative and intrinsic frames were simultaneously present. ${ }^{38}$ However, some signers (up to three out of eight) described the scenes from the point of view of an addressee opposite them (even in the absence of an addressee). ${ }^{39}$ The deictic origo in these descriptions was thus the addressee and not the signer. Point of view was not explicitly indicated in any of the descriptions, though individual signers were consistent in their choice of point of view, suggesting that the location of the origo in the use of the relative frame of reference may not be fully conventionalized in ASL. On the other hand, the variation in point of view (or in the location of the origo in the relative frame), may not be related to conventionalization, but rather to the ambiguous nature of spatial descriptions of this type in general (see Levelt 1984; Schober 1993).

The issue of perspective has already received considerable attention in this chapter due to its co-occurrence with frames of reference. The distinction between different ways of structuring space for spatial representation (i.e. what I call signing perspective) is important in certain types of sign language discourse, especially event narratives. In the next section, I restate the definition of signing perspective (cf. chapter 1, section 1.2), based on the features that I consider to be most relevant. In addition, I give an overview of the different ways in which perspective has been talked about in the literature.

\footnotetext{
${ }^{38}$ Note that this corresponds to the schematic representation in figure 3.8 (a) if the signer is assumed to be the deictic origo. See section 3.5 for more on the simultaneous encoding of the relative and intrinsic frames in sign language.

39 Interestingly, one signer gave a "non-topographic" description of a picture showing a bowl and a banana. Instead of a simultaneous classifier construction depicting the spatial relationship between the two entities, the signer "produced the neutral phrase ON S-I-D-E" (Emmorey 1996: 179). Thus, the signer does not, in fact, use a
} 


\subsection{Signing perspective}

Signing perspective refers to the way in which signers project the event space being described onto sign space. Signers can create representations of an event space in sign space by associating entities with particular locations in the sign space. These representations take two major forms that can be characterized in terms of perspective. On the one hand, signers can have an external vantage point. The signer's role is thus that of an observer who can oversee the whole event space, which is mapped in a reduced size onto the space in front of the body. This corresponds to an observer perspective event space projection. On the other hand, the signer's vantage point can be within the event. Such an event-internal perspective is achieved by assuming the role of a character within the event. In this way, the character's location coincides with that of the signer, and entities in the event space are mapped onto sign space as they are seen by that character. This corresponds to a character perspective event space projection.

Perspective in the visual-spatial modality is an important spatial device, on which other spatial devices and systems are dependent. In fact, I argue that the choice of perspective is a prerequisite for frame of reference use. That is, a frame of reference expression must occur within an event space projection in which referent location and motion can be specified. (As we will see in chapter 6 , however, the use of perspective itself is not a prerequisite for the expression of information related to space.) The relationships that I take to hold between perspective and other spatial devices (i.e. frames of reference and classifier predicates) are outlined in section 3.5 below.

There is an abundance of terminology used in the literature to characterize the two main ways of structuring sign space for spatial representation. The use of perspective in different types of discourse involving spatial representation has been widely described in the literature, for different sign languages, and different researchers use their own terminology to discuss the phenomenon. Table 3.1 presents an overview of the different terminology used. ${ }^{40}$ There is substantial overlap between the criteria adduced to distinguish between the types of representation in each case. The overlap is large enough to assume that these are different names for the same phenomena. In the table, I list the different terminology under the headings "observer perspective" and "character perspective". Some characteristics that are

frame of reference to express the spatial relationship. Rather, the signer describes the picture in terms of a topological relation of proximity (as in English near).

${ }^{40}$ Though they are not included in the table, similar distinctions have been made for spatial structuring in spoken descriptions/narratives, as well as in the gestures that accompany speech (Clark and Gerrig 1990; Linde and Labov 1975; McNeill 1992; Taylor and Tversky 1992, 1996). 
ascribed to these representation types by different researchers are discussed in chapter 7 , where the distribution of use of perspectives in DGS event narratives is addressed.

Table 3.1. Different terminology used for "observer perspective" and "character perspective" in previous research on environment/event space descriptions in different sign languages.

\begin{tabular}{|l|l|l|l|}
\hline Reference & Sign language & Observer perspective & Character perspective \\
\hline Liddell (1995, 2000) & ASL & Token Space & Surrogate Space \\
\hline Liddell (2003a) & ASL & Depicting Space & Surrogate Space \\
\hline Morgan (1999, 2002) & BSL & $\begin{array}{l}\text { Fixed referential } \\
\text { framework/space }\end{array}$ & $\begin{array}{l}\text { Shifted referential } \\
\text { framework/space }\end{array}$ \\
\hline Poizner et al. (1987) & ASL & Fixed referential framework & Shifted referential framework \\
\hline Dudis (2004a) & ASL & Global viewpoint & Participant viewpoint \\
\hline Slobin et al. (2003) & ASL & Narrator perspective & Protagonist perspective \\
\hline Schick (1990) & ASL & Model space & Real-world space \\
\hline $\begin{array}{l}\text { Emmorey \& Falgier } \\
(1999)\end{array}$ & ASL & Diagrammatic spatial format & Viewer spatial format \\
\hline $\begin{array}{l}\text { Pyers \& Senghas } \\
(2007)\end{array}$ & ASL \& NicaSL & Diagrammatic space & Viewer space \\
\hline $\begin{array}{l}\text { Perniss \& Özyürek (in } \\
\text { press) }\end{array}$ & DGS \& TíD & Observer perspective & Character perspective \\
\hline
\end{tabular}

\subsection{Viewpoint in spatial descriptions in the visual-spatial modality}

In addition to perspective, viewpoint is an omnipresent feature of the visual-spatial modality. The fact that representations are spatially produced, and thus visually accessible, means that the signer and addressee automatically view the representation from a particular viewpoint. Viewpoint is crucial in the relative frame of reference, which requires an origo that is different from the figure and the ground. As the ASL spatial descriptions discussed in section 3.2.2 showed, signers can choose either the signer's or the addressee's viewpoint in relative frame of reference expressions. This is, of course, not particular to the visual-spatial modality. Speakers may also specify spatial relationships from their own or some other point of view. However, the nature of the visual-spatial modality has implications for the interpretation of viewpoint in spatial descriptions that are not present in the same way in the spoken modality.

\subsubsection{Signer vs. addressee viewpoint in spatial descriptions}

Canonically, signer and addressee face each other, as is schematized in figure 3.11 below. As we have seen, the iconic properties of the visual-spatial modality afford the possibility of mapping a scene viewed by the signer onto sign space as seen via the placement, orientation,

${ }^{41}$ Morgan (1999) uses the term "framework" where Morgan (2002) uses "space". 
and handshape of the hands. This corresponds to an observer perspective representation of the scene and to the use of a relative frame of reference, whereby the origo or viewpoint is the signer. Schober (1993) has shown that English speakers show a preference for taking the addressee's point of view in describing spatial arrays to - or rather for - the addressee (saying e.g. From your point of view, the man is to the left of the tree). Signers can also describe a spatial scene from the addressee's point of view. This corresponds to the use of a relative frame with the addressee as origo.

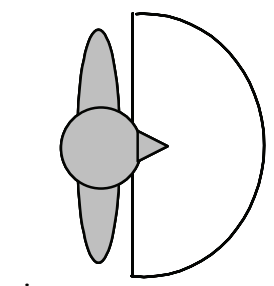

signer

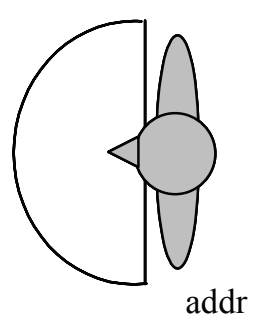

addr

Figure 3.11. Canonical positions of signer and addressee in signing interlocution.

However, spatial representation in a spatial medium puts a twist on the issue of viewpoint. The schematic illustrations in figures 3.12 and 3.13 illustrate this point. If the signer describes the scene depicting the man and the tree from her own point of view, as in figure 3.12, she places a classifier predicate depicting the tree at a location on the right in sign space and a classifier predicate representing the man on the left in sign space (in (b)).

(a) stimulus

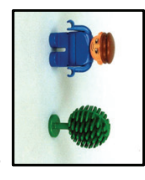

(b) description

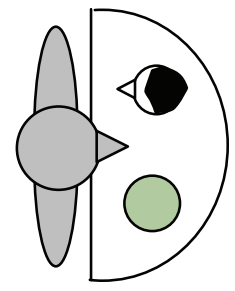

signer

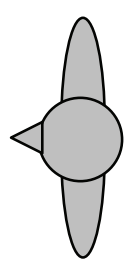

addr

Figure 3.12. Spatial description from the signer's viewpoint (in (b)). The signer represents the tree and the man in sign space from her own viewpoint as the viewer of the scene in the stimulus picture (in (a)). For the addressee, opposite the signer, the sign space representation as seen does not match the scene in the stimulus picture.

From the signer's point of view, this simultaneous classifier construction looks just like the stimulus picture (in (a)) with respect to both the standing (location) and facing (orientation) information of the two objects. What the addressee opposite the signer sees, however, does 
not look like the configuration in the stimulus picture. What the addressee sees in the signer's sign space, from her own point of view opposite the signer, is a classifier predicate depicting the tree on the left and a classifier predicate depicting the man on the right, and facing away from the addressee. In order to interpret this signed description as representing the spatial scene in the picture, the addressee must perform what effectively amounts to a mental rotation by $180^{\circ}$ of the scene that is visually presented in sign space.

Conversely, the signer can give the spatial description from the addressee's viewpoint, as schematized in figure 3.13. In this case, she would place the classifier depicting the tree on her left and the classifier for the man on her right, oriented away from her body. Here, what the addressee directly perceives in sign space matches the configuration in the picture. When a signer chooses to assume the addressee's viewpoint in giving a spatial description, it is the signer who must perform the rotation of the scene, not the addressee. ${ }^{42}$

(a) stimulus

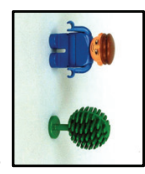

(b) description

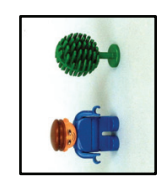

(c) stimulus viewed from addressee's point of view

Figure 3.13. Spatial description from the addressee's viewpoint. The signer represents the tree and the man in sign space (in (b)) not as she has viewed the scene in the stimulus (in (a)), but rather in a way that corresponds to the addressee's view of the scene (in (c)). For the addressee, opposite the signer, the sign space representation as she sees it in sign space, from her vantage point, matches the scene.

Emmorey et al. (1998) has shown that ASL signers prefer to give descriptions from their own point of view. Moreover, they found that addressees are better at interpreting relative locations in a signed spatial description when the description is given from the signer's viewpoint. In fact, both deaf and hearing signers are faster than non-signers at performing mental rotation in non-linguistic tasks, and show no rotation effect at all in linguistic tasks (Emmorey et al. 1993; Emmorey et al. 1998). These results suggest that ASL users are accustomed to performing a mental rotation of the spatial information they see in sign space. However, as discussed in section 3.2.2, Emmorey (1996) found that some signers consistently

\footnotetext{
${ }^{42}$ If the signer and addressee are not facing each other, but rather side by side (i.e. facing the same direction), then this question of different viewpoints does not arise.
} 
chose to represent scenes from the addressee's viewpoint in ASL descriptions of simple spatial scenes. The question of viewpoint conventionalization will be discussed further in chapter 5.

\subsubsection{Shared space in spatial descriptions}

In everyday discourse about spatial arrays, it is often the case that the spatial array in question is part of the interlocutors' physical environment. In the visual-spatial modality, when signer and addressee jointly view an environment, they use a "shared space" representation of the scene in sign space (Emmorey and Tversky 2002). In figure 3.14 below, the signer and addressee are both looking at the relevant spatial scene, that is, signer and addressee perceptually "share" the space containing the man and the tree.
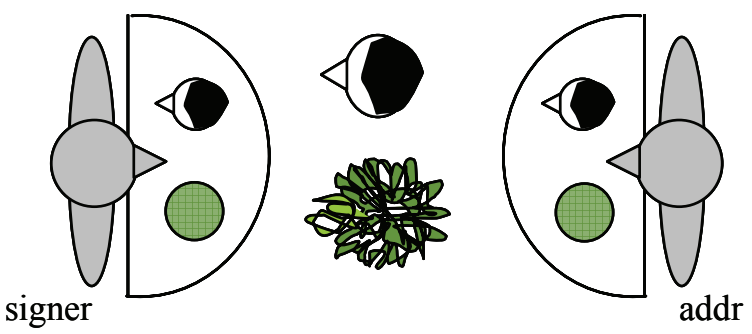

Figure 3.14. Illustration of shared space. When signers are jointly viewing an environment (facing each other), their different vantage points on the scene map to the same shared physical space. The description of the scene in sign space by the signer or the addressee matches the real-world locations of the objects.

As the figure shows, the difference between mapping spatial relationships onto sign space from the signer's or the addressee's viewpoint ceases to exist when an environment is jointly viewed by the signer and addressee. Both the signer's and the addressee's inscription of sign space maps to the jointly-viewed physical environment. Moreover, this holds both for the signer and addressee viewing their own sign space as well as each other's sign space.

For spoken language, it is precisely the opposite situation. In spoken languages that use the relative frame of reference (e.g. English), viewpoint becomes relevant in the case of a jointly-viewed environment, because interlocutors must take the other's viewpoint into consideration in specifying object location (see Emmorey 2002). This is especially clear in the case of objects of the same type (e.g. two trees), as figure 3.15 below shows. As mentioned, speakers tend to adopt their addressee's point of view in such cases, saying The one on your left more often than The one on the (my) right (Schober 1993). As these examples show, modality affects the role of viewpoint in spatial description. 


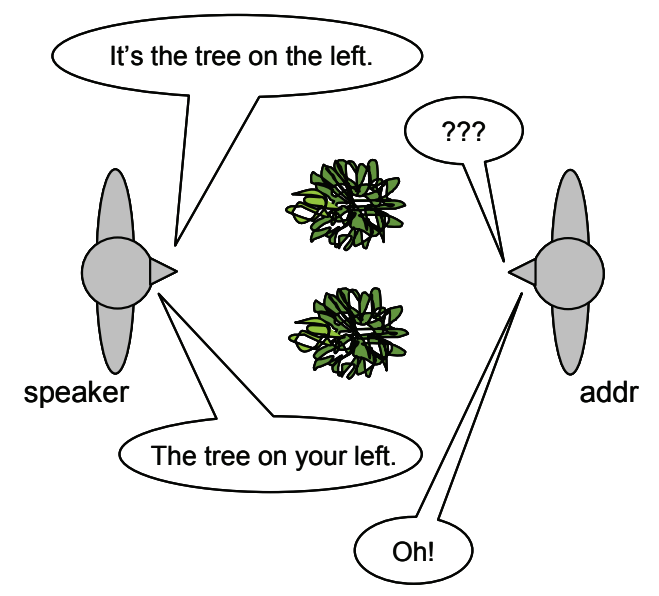

Figure 3.15. When speakers are jointly viewing an environment (facing each other), their different vantage points on the scene map to the same shared physical space. The description of the scene using relative frame of reference terms is ambiguous with respect to the deictic origo of the utterance.

In the previous sections, I have discussed the systems of representation that are relevant to understanding how spatial information is expressed in sign space (together with the system of classifier predicates, discussed in chapter 2, section 2.3). In the next section, I define the relationships between frames of reference, perspective, and classifier predicates as I see them. In particular, based on shared properties and iconic correspondences, I motivate an alignment between the relative frame of reference, observer perspective, and the use of entity classifiers, on the one hand, and between the intrinsic frame of reference, character perspective, and handling classifiers, on the other hand. The definitions that I present in section 3.5 are used throughout the remainder of the dissertation to guide the analyses of DGS spatial descriptions.

\subsection{Alignments between perspective, frames of reference, and classifier predicates}

As shown in section 3.2.1, the research on frame of reference use in spoken languages has proceeded according to a rigorous program of establishing how speakers specify the location of a figure object with respect to a ground object. The research has focused on the lexical content and linguistic structure of descriptions of spatial scenes containing two objects (a figure and ground), and has striven to identify how different languages express location and orientation information. In comparison, research on frame of reference use in signed language has been less rigorous, not concentrating, for example, on the structure of single locative expressions that specify the location of a figure with respect to a ground (but see Arik 2006 and Arik and Nadolske 2006 on TID). I take seriously the notion of the linguistic construal of a figure-ground relationship, whereby the origin of a system of coordinates is mapped onto 
the ground object in order to project a search domain in which the spatially-removed figure may be located.

As Emmorey (2002) recognizes, the use of a frame of reference system is independent of the perspective from which signers project an event space onto sign space. Thus, as shown in section 3.2.2, signers can potentially represent a spatial relationship in relative, intrinsic, or absolute terms in either signing perspective. However, as I will show below, there are shared semantic properties between the systems that motivate certain prototypical co-occurrences, which I refer to as alignments. Importantly, these alignments depend on a clear identification of the ground object, and on the number of entities involved in the relationship. In addition, I address the relationship between classifier predicates and signing perspective. Based on the correspondence between the size of the event space projection and the size of referent projection, I will similarly argue for certain alignments between classifier predicates and perspective. My use of the term alignment is based on parallels in the form and structure of representation. (This is different, for example, from Pickering and Garrod's (2004) use of the term to talk about the alignment of lexical, syntactic, and conceptual representations, and even non-linguistic "grooming" behaviors, between interlocutors in conversation.)

Because this will be central to what follows, it may be useful to recapitulate some of the terminology. I use the terms observer and character perspective to refer to the vantage point from which an event space is projected onto sign space. I distinguish between entity and handling classifiers based on the way in which referents are mapped onto the signer's hands. Finally, the three frames of reference with which it is possible to specify the location of a figure object with respect to a non-coincident ground object are the relative, intrinsic, and absolute frames. I proceed from the assumption that the use of a particular perspective is a prerequisite for the representation of spatial relationships in sign space. Table 3.2 shows the alignments that are detailed in sections 3.5.1 (frames of reference and perspective) and 3.5.2 (classifiers and perspective) below. ${ }^{43}$ In addition, section 3.5.1 discusses the use of the absolute frame in signed language.

Table 3.2. Alignments between signing perspective, frames of reference, and classifiers.

\begin{tabular}{|l|c|c|}
\hline & \multicolumn{2}{|c|}{ Alignments } \\
\hline Perspective & Observer & Character \\
\hline Frames of reference & Relative & Intrinsic \\
\hline Classifiers & Entity & Handling \\
\hline
\end{tabular}

\footnotetext{
${ }^{43}$ Remember that perspective is not to be equated with signing from a particular viewpoint or origo, which is relevant within the relative frame of reference. The discussion in 3.5.1 sheds light on this distinction.
} 


\subsubsection{The relationship between frames of reference and signing perspective}

To make the alignments clear, it is important to emphasize the underlying assumptions about frame of reference expressions and the spatial scenes to which they apply. Frames of reference apply to the linguistic description of spatial arrays, in which the location of one object (the figure) is specified with respect to another non-coincident object (the ground). In the case of the relative frame of reference, the figure's location is specified from a third location, namely that of a viewpoint from which the scene is viewed. The relation is thus ternary, and involves the figure, the ground, and a scene-external viewpoint. In the intrinsic frame of reference, the relationship is binary, involving only the scene-internal relationship between the figure and the ground.

As mentioned above, the study of frame of reference use in sign languages must be as precise in distinguishing individual figure-ground constructions as has been the frame of reference research on spoken languages. That is, a single frame of reference expression in a signed language must be characterized by the specification of a ground object, followed by that of a figure object in relation to the ground. ${ }^{44}$ In Emmorey's (2002) frame of reference examples, while the identification of a particular frame applies to the construction as a whole, based on the presence of the essential elements (e.g. two vs. three entities, featured vs. nonfeatured entities), the linguistic structure of the utterances used to produce the descriptions is not clear.

A frame of reference expression, as described in section 3.2, is characterized by the location of a figure object by the projection of a search domain off the ground object in which to locate the figure. In the relative frame of reference, the coordinate system which provides the basis for the angular specification is derived from the viewer's own body coordinates (front/back and left/right). Figure 3.16(a) shows how the specification of the figure's location depends on the viewer's location/orientation relative to the scene. A different viewpoint would require that a different angular, i.e. frame of reference, term be used in the linguistic description. The comparison between figure 3.16(a) and (b) shows the similarity between the use of observer perspective and the relative frame of reference to encode the spatial relationship between two objects. In observer perspective, the signer is external to the event, and the depiction of the scene in sign space (e.g. with entity classifiers to represent the figure

\footnotetext{
${ }^{44}$ The sign languages that have been studied to date have been found to exhibit a ground-figure ordering, in which the ground object is identified and located in sign space prior to the figure object. This order, also observed in drawing, may be due to a modality constraint (Emmorey 1996; Emmorey et al. 2002).
} 
and ground objects) depends on the signer's view of the scene. ${ }^{45}$ To express the relationship between the tree and the man, assume that the signer would first identify and locate the tree, and then identify and locate the man, while holding the tree in place (see chapter 4 for the structure of locative descriptions in DGS). Thus, the ground object is encoded first, and the figure object is located with respect to the ground. (The use of (small-scale) entity classifiers and a low horizontal signing plane are additional cues to the use of observer perspective (see Emmorey et al. 2000); see also chapter 7). Thus, the relative frame of reference and observer perspective are both characterized by expressing a ternary relationship between the figure, the ground, and the signer's/speaker's scene-external viewpoint.

(a)
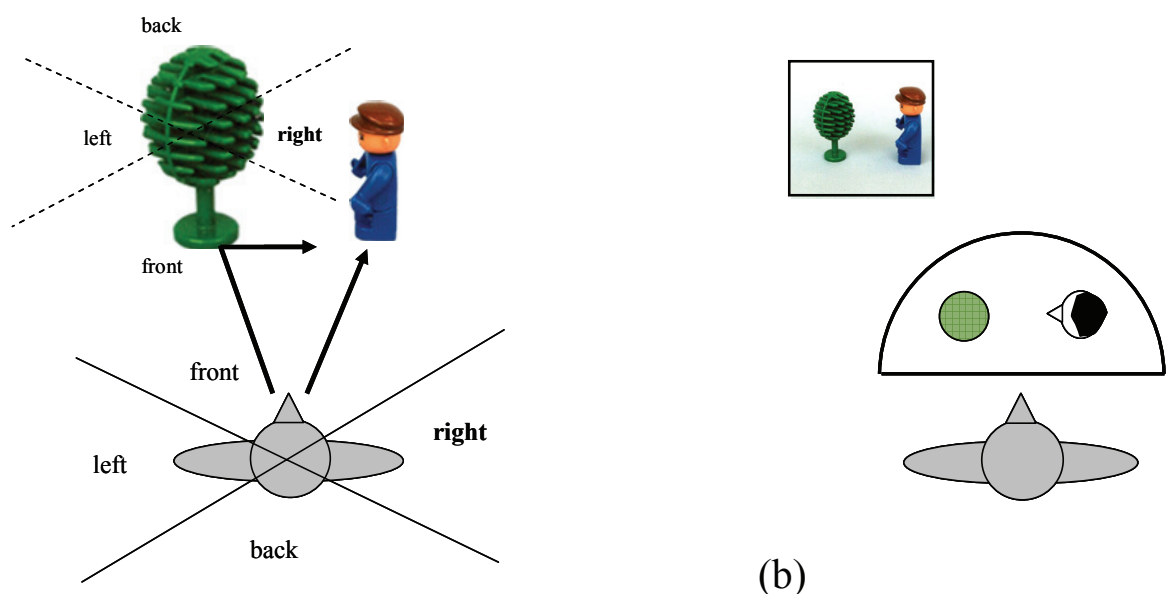

(b)

Figure 3.16. Commonality between encoding in the relative frame of reference (in (a), as The man is to the right of the tree) and the use of observer perspective in signing (in (b), where classifier predicates indicate the spatial relationship between the man and the tree).

Note that the relative frame of reference is necessary in spoken languages that do not use an absolute frame of reference at all scales to specify the location of a figure in relation to a nonfeatured ground object (like the tree in our examples). Thus, in English, the man's location with respect to the tree must be specified in relative terms, i.e. The man is to the right of the tree. In the case of featured ground objects, it is additionally possible to specify the figure's location in intrinsic terms. For example, in the scene in figure 3.16 above, the man could be construed as the featured ground object to express the intrinsic relationship The tree is in front of the man. This specifies orientation information, but not location information, since the intrinsic relationship is valid from any given viewpoint.

\footnotetext{
${ }^{45}$ For the purposes of explication, it is assumed that the signer's depiction of a scene in sign space corresponds to her own view of the event space, as opposed, for example, to that of an addressee. In fact, this will be tested for DGS in chapter 4.
} 
In the visual-spatial modality, however, location and orientation information can be simultaneously expressed in an observer perspective event space projection. In figure 3.16(b) above, the intrinsic relationship between the man and the tree is encoded through the use of the featured 2-legged entity classifier to represent the man. As Emmorey (1996) notes, the possibility of expressing the relative and intrinsic frames simultaneously may be unique to the visual-spatial modality. In spoken language, the encoding of "standing" and "facing" information using the relative and intrinsic frames, respectively, must be sequential (e.g. in two separate clauses, as in "The man is to the right of the tree, facing the tree") (cf. Pederson et al. 1998).

We now turn to the intrinsic frame of reference and the similarity it bears to the use of character perspective in signing. The intrinsic frame of reference expresses the relationship between a figure and a ground object based on the intrinsic (i.e. conventionally assigned) features of the ground object. In figure 3.17(a) below, the (featured) speaker is the ground (i.e. the origin of the coordinate system), and the tree is located in front of the speaker's body (see figure 3.17(a)). When signers use character perspective, event space is represented from the character's (scene-internal) perspective, and referents in sign space are located with respect to the signer's body. This makes the similarity between the intrinsic frame of reference and character perspective apparent. The signer's body (as the character) is the ground object with respect to which a figure object is located. This is shown in figure $3.17(\mathrm{~b})$.

(a)

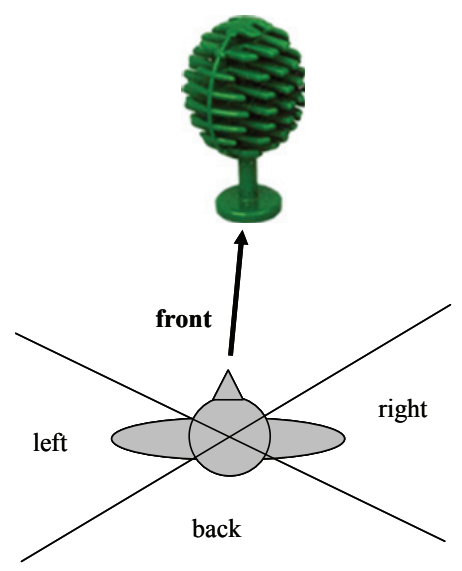

(b)

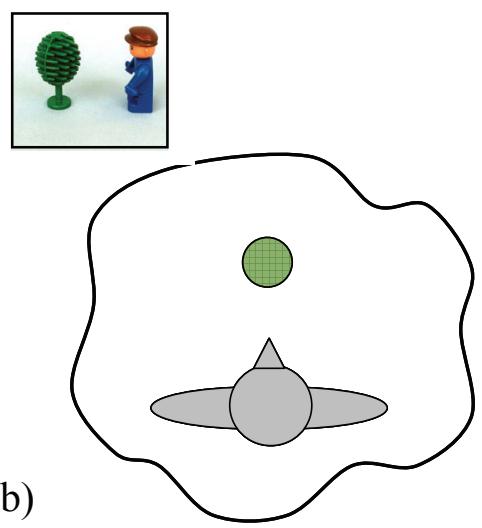

Figure 3.17. Commonality between encoding in the intrinsic frame of reference (in (a), as The tree is in front of the tree) and the use of character perspective in signing (in (b), where a classifier predicate indicates the spatial relationship between the man (as the signer) and the tree).

Note the similarity between figure 3.17(b) and Emmorey's (2002) example of the intrinsic frame in character perspective (shown below in figure 3.18(a); repeated from figure 3.8(b)). 
In both examples, the signer is construed as the ground, and the figure is located with respect to the ground (i.e. The tree is in front of me in figure 3.17(b) and The car is in front of me in figure 3.18(a)). How does this relate to Emmorey's (2002) example of a relative frame of reference expressed in character perspective (shown in figure 3.18(b); repeated from 3.9(b))? The portrait and the window are located in sign space from the signer's (i.e. character's) vantage point within the event space (as evidenced, for example, by the high placement of the signs in space (see Emmorey and Falgier 1999; Emmorey et al. 2000)). In my analysis, what we see in figure $3.18(\mathrm{~b})$ is the simultaneous expression of the relative and intrinsic frames of reference in a character perspective event space projection. The representation of both entities individually (as two separate figure objects) uses an intrinsic frame of reference, as both entities are located with respect to the signer's body (as the ground). However, the special spatial mapping possibilities of the modality, for example, that two entities can be simultaneously represented in space, afford the simultaneous expression of a relative relationship (i.e. The portrait is to the left of the window (from my point of view) in figure 3.18b). Thus, in the visual-spatial modality, it is possible to simultaneously express the relative and intrinsic frames of reference in both perspectives. This has not been previously highlighted in the literature. (Emmorey (2002) notes the simultaneous use of relative and intrinsic in observer perspective, but not in character perspective, in ASL). Chapters 5 and 6 discuss the occurrence of simultaneous relative and intrinsic expressions in both perspectives in DGS static scene descriptions and event narratives, respectively.

(a)

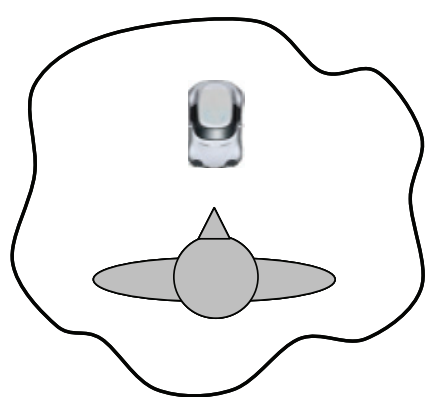

(b)

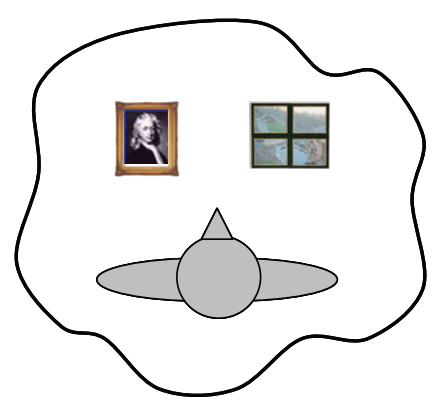

Figure 3.18. Comparison between Emmorey's (2002) examples of the use of (a) an intrinsic frame of reference in character perspective and (b) a relative frame of reference in character perspective.

The possibilities of simultaneous representation notwithstanding, I take the use of observer perspective and the relative frame, on the one hand, and the use of character perspective and the intrinsic frame, on the other hand, to represent the prototypical co-occurrences or alignments between the two systems, based on the formal similarities outlined above (and 
listed below in table 3.3). These alignments are relevant to the analyses presented in chapters 5 and 6.

Table 3.3. Formal similarities that motivate the alignments between the relative frame of reference and observer perspective, on the one hand, and the intrinsic frame of reference and character perspective, on the other hand.

\begin{tabular}{||l|l||l|l||}
\hline $\begin{array}{l}\text { Relative frame of } \\
\text { reference }\end{array}$ & Observer perspective & $\begin{array}{l}\text { Intrinsic frame of } \\
\text { reference }\end{array}$ & Character perspective \\
\hline $\begin{array}{l}\text { Viewpoint determines } \\
\text { relationship between } \\
\text { figure and ground }\end{array}$ & $\begin{array}{l}\text { Relationship between } \\
\text { referents encoded from } \\
\text { external perspective }\end{array}$ & $\begin{array}{l}\text { Intrinsic features of } \\
\text { ground determine } \\
\text { relationship between } \\
\text { figure and ground }\end{array}$ & $\begin{array}{l}\text { Signer's intrinsic features } \\
\text { determine referent } \\
\text { location }\end{array}$ \\
\hline $\begin{array}{l}\text { Viewpoint is different } \\
\text { from figure and ground } \\
\text { (scene-external) }\end{array}$ & $\begin{array}{l}\text { Signer is external to } \\
\text { represented scene }\end{array}$ & $\begin{array}{l}\text { Scene-internal } \\
\text { relationship between } \\
\text { figure and ground }\end{array}$ & $\begin{array}{l}\text { Signer is internal to } \\
\text { represented scene }\end{array}$ \\
\hline Ternary relationship & Ternary relationship & Binary relationship & Binary relationship \\
\hline
\end{tabular}

Finally, in this section, I discuss the use of the absolute frame of reference in signed language. In section 3.2.2, I noted that the examples that Emmorey (2002) gives for the use of an absolute frame of reference in ASL may arguably not exhibit a true use of absolute bearings (i.e. fixed, external bearings based on cardinal directions or landmarks). The signer states that she was driving North, using a modified form of the cardinal direction term NORTH, but does not truly direct the sign to the North. To exhibit true absolute frame of reference use in character perspective, the signer represented in Emmorey's (2002) example in figure 3.10(b) above, would have to literally turn her body to the North to indicate driving to the North. This is what we would expect from signers of Kata Kolok, the village sign language in Bali (mentioned in section 3.2.2), where signers point to the real-world locations of referents to refer to them, and thus do exhibit the use of an absolute system. ${ }^{46}$ Knowing which direction to turn (or point) is a telling sign of the use of a true absolute system. Users of such a system are oriented with respect to the absolute bearings regardless of where they are (even in a room without windows) (Levinson 1996; Levinson et al. 2002).

In contrast, speakers of a language like English predominantly use a relative system, but have available to them absolute terms (i.e. the cardinal direction terms) that they can use as an ancillary system. In general, speakers of English do not keep track of the cardinal directions (that is, they are generally not able to dead-reckon), and base their use of cardinal terms on abstract knowledge (gleaned, e.g., from maps). Thus, a speaker may say The city center is north of here without being independently able to point to the North. This is what

${ }^{46}$ This is true when Kata Kolok signers refer to referents within their village. Further research is needed, however, to determine how referent locations are referred to in tabletop space (cf. Pederson et al. 1998). 
we see in Emmorey's examples of an absolute frame used in ASL. This difference makes apparent an incompatibility of the visual-spatial modality, where space is used to talk about space, with the use of an ancillary (i.e. non-dead-reckoned) system of absolute terms (as found e.g. in the use of cardinal direction terms in English or ASL). ${ }^{47}$

\subsubsection{The relationship between classifier predicates and signing perspective}

As table 3.2 shows, I assume an alignment between the use of entity classifiers and observer perspective, on the one hand, and between handling classifiers and character perspective, on the other hand. These alignments can be motivated by a principle of scale iconicity, by which different parts of a representation should have the same size. The size of event space projection and the size of referent projection should correspond or align in size. The reduced size of the event space in observer perspective (where the whole event space is scaled down to fit into the area of space in front of the body) aligns in scale with the representation of whole entities on the hand, as with entity classifiers. The life-sized event space in character perspective aligns in scale with the representation of entities on the body and to the representation of the real-life interaction and manipulation of entities, as is possible with handling classifiers. ${ }^{48}$

In the literature, the relationship between the use of classifiers and the use of space for referent mapping is indirectly addressed in terms of argument structure or verb semantics (Benedicto and Brentari 2004; Engberg-Pedersen 1993; McDonald 1982; Zwitserlood, 2003). In these analyses, there is a systematic correspondence between entity classifiers and intransitive verbs, on the one hand, and handling classifiers and transitive verbs, on the other hand. In addition, McNeill (1992:119) makes a similar claim with respect to the distribution of occurrences of observer and character viewpoint gestures, namely that character viewpoint gestures appear more with transitive verbs, while observer viewpoint gestures appear more with intransitive verbs. Here, the expression of motion and location corresponds to the use of entity classifiers, while the expression of object manipulation and transfer corresponds to the use of handling classifiers. Although most argument structure analyses do not explicitly discuss signing perspective, they imply an alignment between the use of character

Balinese, the surrounding spoken language, also uses an absolute frame of reference. This is reflected in the directions of co-speech gestures (see e.g. Wassmann and Dasen 1998).

${ }^{47}$ Thanks to Martin Atherton for a discussion of this.

48 As we will see, signers do not always uphold scale iconicity. The mixing of scales will be discussed in chapters 7 and 8 as non-alignment of classifiers predicates and perspective. 
perspective/handling classifiers and observer perspective/entity classifiers. As stated, I emphasize that these alignments are based on the principle of scale iconicity.

\subsection{Summary}

In summary, this chapter has been concerned with the essential distinctions in the domain of spatial language, in general, and sign languages, in particular. An important outcome is the idea that perspective, frame of reference and viewpoint, and classifiers need to be distinguished in how sign languages refer to space, but are nevertheless closely connected in what I have called alignments. This will be crucial to what follows.

The next chapter analyzes the structure of DGS locative constructions used in static scene description, and discusses their structural variation in terms of iconicity, linguistic, discourse, and articulatory constraints. 


\section{Static scene spatial description in DGS}

Chapter 4

The goal of this chapter is to identify the DGS conventions for the expression of locative information in static scene descriptions. The structure of DGS locative constructions is discussed in relation to iconicity principles and different types of constraints, linguistic, discourse, and articulatory.

The analysis presented here is based on a task designed to elicit static spatial descriptions in the frame of reference domain (cf. Pederson et al. 1998). As detailed in chapter 3, frames of reference are needed to describe the location of a figure with respect to a non-coincident ground. For example, if somebody asks you Where did you leave the newspaper?, you might answer (The newspaper is) To the left of the TV. The use of the relative term left projects a search domain off the ground object (the television) in which the figure object (the newspaper) can be located. This is a different strategy from the non-angular expression (The newspaper is) On the kitchen table, which expresses a topological relationship, where the figure and the ground are coincident in location. From the data elicited with the task, it is possible to extract the major patterns observable in signers' spatial descriptions and to take initial steps toward a typology of locative constructions in DGS.

I begin with the structure of locative constructions, as they are described in the sign language literature (section 4.1). Section 4.2 is dedicated to detailing the methodology, including a description of the stimulus materials, the data collection, and the data coding. An analysis of the data is presented in section 4.3. Section 4.4 gives a summary and discussion of the results.

\subsection{The structure of locative constructions in sign languages}

Example (1) below is a DGS description of the spatial relationship between the man and the tree, as shown in the stimulus picture above the example. The structure of this description reflects what I hypothesize to be the "canonical structure" of DGS locative descriptions. In fact, the structural properties of the description reflect general assumptions in the literature addressing the structure of locative constructions across sign languages, including ASL, 
DGS, BSL, DSL, Swiss-German Sign Language (DSGS), and Austrian Sign Language (ÖGS).

(1)

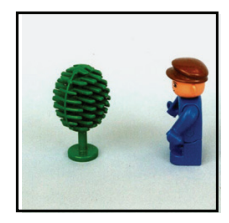

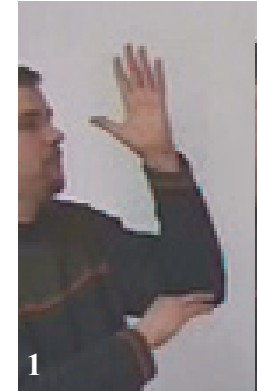

RH:

LH:

TREE

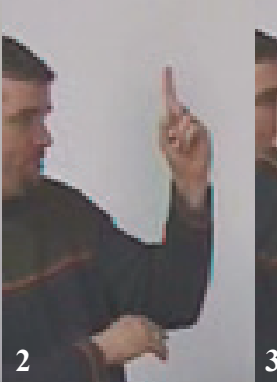

$\mathrm{CL}_{\mathrm{E}}($ tree): locL---hold

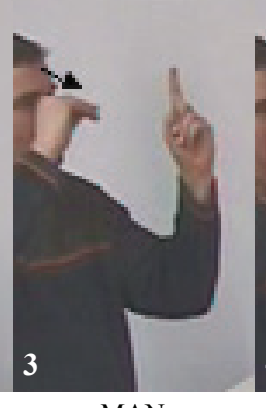

MAN

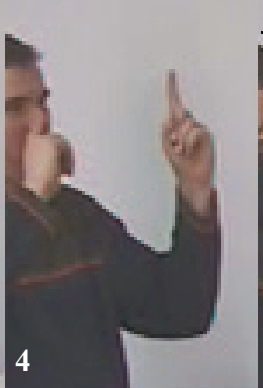

BROWN

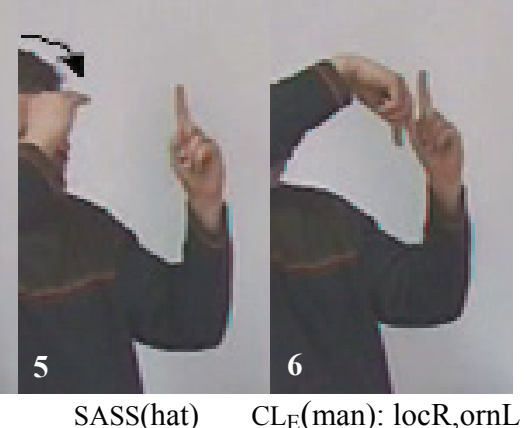

SASS(hat) $\quad \mathrm{CL}_{\mathrm{E}}$ (man): locR,ornL

There are five main structural properties that define the locative construction in example (1). They reflect, and are extracted from, what is found in the sign linguistics literature (reviewed below) about the syntax of locative constructions, the forms used to encode spatial information, the way in which the event space is mapped onto sign space, as well as general discourse-structure principles. These structural properties are: (1) referents are identified by a full NP before information about them is predicated; (2) classifier predicates are used to encode spatial information about the introduced referents; (3) the ground object (here, the tree) is encoded before the figure object (here, the man); (4) the spatial relationship between the two referents is depicted in sign space by means of a simultaneous classifier construction; and (5) the spatial scene is depicted from the signer's viewpoint as the viewer of the scene. These properties are expressed more elaborately in section 4.3 below as an hypothesis about the "canonical structure" of DGS locative constructions against which the data will be tested. It must be noted that these properties are typical of Western sign languages, in the first instance. What we know about village sign languages, in particular, AdaSL (Nyst 2007) and Kata Kolok (Marsaja 2003), as well as evidence from TID (Arik 2006) suggests that spatial descriptions in the visual modality might look very different from that in example (1). In addition, more research is needed on locative constructions in urban sign languages from other parts of the world, e.g. Asian and African sign languages, for comparison with those in Western sign languages. 
Though all of the five structural properties listed above are mentioned in the literature (primarily on Western sign languages), the literature overall is lacking in careful analyses of locative constructions, both across individual sign languages, and across different discourse types. In part, this may be a result of underlying assumptions about iconic mapping principles that guide spatial expression in the visual-spatial modality (see section 4.3). These assumptions lead to claims about widespread similarity across sign languages in the spatial domain due to modality effects (Aronoff et al. 2003, 2005; Meier 2002; Newport and Supalla 2000; Talmy 2003). Careful analyses of spatial descriptions in individual sign languages are necessary to uncover the language-specific linguistic and discourse constraints that may hold in this domain. Such an analysis is presented in this chapter for DGS static scene spatial description. The findings on DGS spatial description have important implications for claims that spatial description across sign languages can be explained on the basis of iconicity and modality effects alone.

The literature that explicitly addresses the structure of locative constructions is quite sparse, and most of it is not based on analyses of a corpus of data, reflecting actual usage. One reason for this may be, as mentioned above, the assumption of iconic mapping principles guiding spatial description. That is, the structure of locative constructions is assumed to be straightforward, derivable from the use and properties of classifier predicates (i.e. to represent referent form, location, and orientation) and from the affordances of the visual-spatial modality, in general (i.e. the potential for iconic mapping, the use of simultaneous classifier constructions). In the following, I move one by one through the five structural properties listed above that define the locative construction in example (1). For each one, I provide a review of the relevant literature.

The first property is, in fact, a general discourse property that holds for both spoken and signed language. Typically, referents are identified before information about them is predicated. In sign languages, the use of classifier predicates to express spatial information follows the lexical sign that identifies the referent depicted by the classifier form. This is explicitly noted for classifier predicate constructions that encode spatial information by Liddell (1980) and Emmorey (1996), as well as Emmorey et al. (2005) for ASL, EngbergPedersen (1993) for DSL, Johnston et al (2007) for Auslan, VGT, and Irish SL, Boyes-Braem (1995) for DSGS, and by Glück (2001), Glück and Pfau (1998), Keller (1998), Papaspyrou (1990), and Prillwitz (1985) for DGS.

In regard to the second property, most researchers who study classifier predicates in sign languages refer to their potential for iconically encoding spatial information related to 
referent location and orientation (e.g. Aronoff et al. 2003; Emmorey 1996, 2002; Liddell 2003a, 2003b; Talmy 2003 for ASL, Engberg-Pedersen 1993 for DSL, Brennan 1990 for BSL, Zwitserlood 2003 for NGT, and Zeshan 2003 for Indo-Pakistani Sign Language (IPSL)). However, analyses differ in regard to how this potential is realized. For example, Talmy (2003) assumes a high degree of iconicity, and emphasizes the ability to make very fine and nuanced spatial distinctions in the visual-spatial modality, as compared to the more restricted spatial schemas available to spoken languages. Liddell (2003b), on the other hand, argues that classifier predicates are "fixed lexical verbs" whose analyzability and compositionality varies depending on lexicalization. Zeshan (2003) also addresses the issues of lexicalization and grammaticalization in sign language, and discusses, in particular, the paradigmatic distribution of entity classifier predicates in IPSL. Moreover, Liddell (1980) notes that hand orientation in a classifier predicate can be "neutral" in the sense of indicating only the nature of the spatial relationship (e.g. The cat is on the fence), without specifying the cat's particular orientation in relation to the fence. These seem to be language-specific constraints on the ways in which potentially possible spatial categories (cf. Talmy 2003: 191) are represented within classifier predicates. In general, such constraints may influence the spatial information that may be encoded in a particular form.

Encoding the ground object before the figure object, another property that characterizes the description in example (1), is stated widely in the literature that mentions locative constructions (e.g. Emmorey 1996; Liddell 1980; Supalla 1990 for ASL; Engberg-Pedersen 1993, 1994 for DSL; Miller 1994 for LSQ; Leeson and Saeed 2002 for Irish SL; and Ebbinghaus and Heßmann 1990; Glück 2001; Keller 1998, 1999; Leuninger and Happ 1997 for DGS). As noted in chapter 3, Emmorey (1996) and Emmorey et al. (2002) suggest that the ground-figure order may be a modality constraint. In addition, the literature mentions the role of the non-dominant hand in encoding the ground object (e.g. Engberg-Pedersen 1993 for DSL; Hilzensauer and Skant 2001 for ÖGS). In the DGS literature, the order of mention of referents is also stated as an object-based principle, namely that in signed descriptions, fixed objects are encoded before movable objects, and big objects are encoded before small objects (Happ and Leuninger 1998; Leuninger and Happ 1997) (cf. Goldin-Meadow et al. 1996 for a similar finding for gestural representations in the absence of speech).

Many of these researchers also note that the classifier form encoding the ground is held in place during the production of subsequent signs, e.g. for the figure object (EngbergPedersen 1993, 1994; Leeson and Saeed 2002; Liddell 1980; Miller 1994). The end result is a simultaneous classifier construction, in which the spatial relationship between the two entities 
is represented by the relationship of the hands to one another in sign space. Thus, the modality affords the direct visual depiction of the spatial relationship itself. Finally, as discussed in chapter 2, and as will be argued again in section 4.3, the use of classifier predicates and simultaneous constructions can be subsumed under iconicity principles guiding spatial representation in the visual-spatial modality. That is, on the basis of iconicity, signers can create schematic correspondences between the hands in sign space and the realworld locations of entities being described (e.g. Aronoff et al. 2003; Emmorey 1996; Emmorey et al. 2002). The DGS literature also specifically addresses the affordance of the modality to allow iconic mappings of entity locations from the event space being described onto sign space (e.g. Ebbinghaus and Heßmann 1990, who speak of the "scenic principle"; Keller 1998).

In general, it should be noted that much of the literature on the expression of locative relationships in sign languages does not address these issues in much detail. References to the order of figure and ground, or to the use of the non-dominant hand to encode the ground, are sometimes made only in passing. For example, embedded in a discussion of the functions of different types of classifier predicates, Hilzensauer and Skant (2001) note that it is typically the dominant hand that expresses the location or motion of a referent, whereby "the other [non-dominant] hand may represent the 'ground' (in 'figure-ground' constructions, e.g. a cat sits on a fence) or a second object" (p. 97). ${ }^{49}$

With the exception of these references, there has been little investigation overall of the expression of spatial relationships in DGS, and mention of locative constructions is quite sporadic and unsystematic. As a case in point, an article devoted to an explication of the use of space in DGS syntax in different types of sentences (Sauer et al. 1997) discusses a wide range of construction types, including agreement verbs, spatial verbs, pronouns, topicalization, declaratives, questions, negation, and adverbials of time and place, but does not explicitly address locative constructions. What are taken to be the facts of spatial description in DGS is thus not founded on the investigation of actual use in discourse situations. One reason for this is the lack of available sign language corpora from which information about actual usage in certain domains can be extracted (see chapter 1, section 1.3.4). This situation is not unique to DGS, but is true for sign languages in general. This dissertation offers a data-based study of DGS spatial descriptions. The next section details the

\footnotetext{
49 The German text is: "die andere Hand kann den 'Grund' (in 'Figur-Grund'-Konstruktionen, z.B. eine Katze sitzt auf einem Zaun) oder ein zweites Object darstellen."
} 
methodology of data collection and coding of the static scene descriptions that form the basis for the analysis in this chapter.

\subsection{Methodology}

\subsubsection{Task description}

The director-matcher task that was used to collect DGS static scene descriptions required a director to describe a particular scene (i.e. an object configuration) and a matcher to reconstruct the scene based on the description. Scenes were presented to participants on photographs and consisted of plastic figurines set up in certain configurations.

To perform the task, two signers sat across from each other at a table. A divider was placed between them to shield the tabletop space directly in front of each of them from the other's view. ${ }^{50}$ Both signers had at their disposal an identical set of plastic figurines, comprising the objects that appeared in the photographs. The picture stimuli were given to the signer acting as director, who was asked to describe the object configuration shown on the pictures to the matcher. The signed description for each picture was to be produced by the director not from the photograph itself, however, but from a model of the pictured scene which he or she constructed on the tabletop using the available objects. The matcher was likewise asked to construct the scene on the tabletop, using the other set of plastic figures, according to his/her interpretation of the signed description.

Participants were told that both tabletop object configurations - the director's and the matcher's - should match the scene depicted in the picture given to the director. In each case, the experimenter (myself) assessed the match from a position directly beside or behind the camera (which was set up in line with the divider between the signers (see figure 4.2 in section 4.2.4 below)), from where the tabletop space in front of both signers was in clear view. If the matcher's object set-up did not match the scene depicted in the picture, I signaled this to the director, whereupon he/she repeated the description. Negotiation between the director and matcher about their respective object set-ups continued until both set-ups matched the scene depicted in the stimulus picture.

\footnotetext{
${ }^{50}$ In the signed examples given in this and the following chapter, the reader will notice that the signers' hands are level with the face, and thus quite high. This high horizontal plane is a result of the divider between the two
} 


\subsubsection{Stimulus materials}

The stimulus materials used for this task consisted of a total of 43 photographs of object configurations. The pictures included both animate and inanimate objects, and differed in complexity with respect to the number of objects and the nature of the configuration.

The stimuli set consisted of 31 simple and 12 complex scene stimulus pictures. The simple scenes featured two objects aligned either on the lateral (left/right) or the sagittal (near/far) axis, and were of three types: (1) two animates, i.e. two men (12 stimulus pictures) (see figure 4.1a), (2) one animate and one inanimate, i.e. one man and one tree (13 stimulus pictures) (see figure 4.1b), and (3) two inanimates, i.e. two cones (6 stimulus pictures) (see figure 4.1c). The complex scenes depicted scenes containing between three and nine distinct entities, with objects along both main axes and in more elaborate geometric arrangements (see figure 4.1d). All pictures manipulated referent location (i.e. standing information) and/or orientation (i.e. facing information). The full set of pictures used in the task is shown in appendix A. See table 4.2 below for an overview of the number of stimulus pictures and descriptions by type of picture.
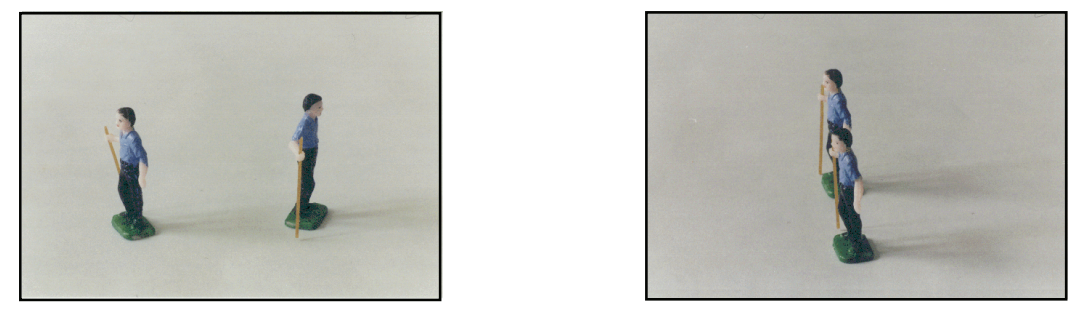

(a) Examples of two animates (man/man) stimulus pictures.
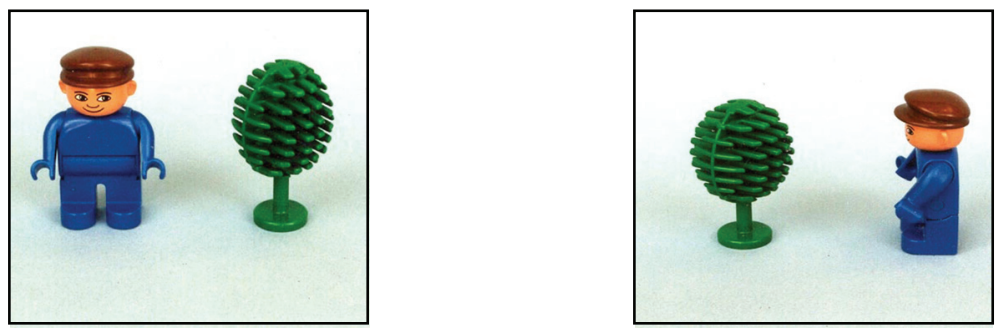

(b) Examples of one animate and one inanimate (man/tree) stimulus pictures. 

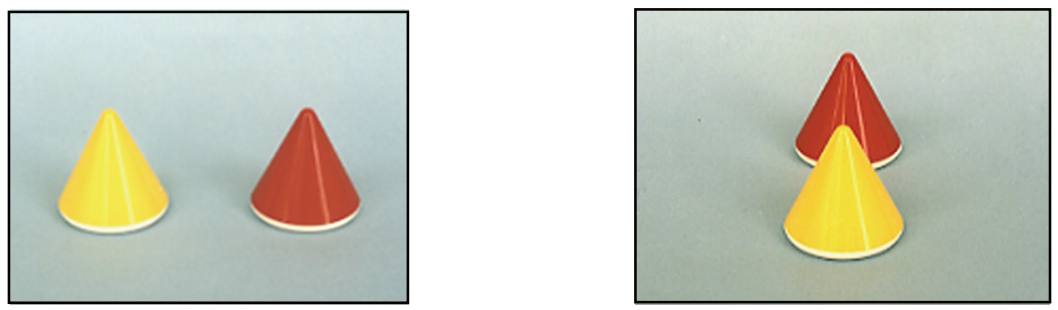

(c) Examples of two inanimates (cone/cone) stimulus pictures.
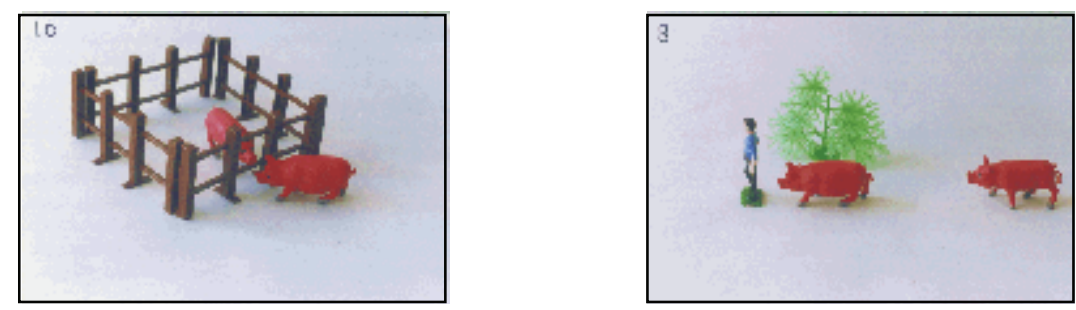

(d) Examples of complex scene stimulus pictures.

Figure 4.1. Examples of simple and complex scene stimulus pictures used in the task.

\subsubsection{Participants}

A total of eight signers participated in the task. Two signers were native signers (i.e. with deaf parents) (GL and FB); five signers were early signers (i.e. sign language acquisition by the age of six) (LL, KO, SR, JP, HT); one signer was a late signer (i.e. sign language acquisition after the age of six) (SB). All signers knew each other before performing the task.

\subsubsection{Data collection}

The stimulus pictures were divided into four groups, each of which included pictures of all four types of pictures (i.e. the three categories of simple scenes and the complex scenes, as shown in figure 4.1(a-d) above). As noted above, there were a total of 43 stimulus pictures used in the analysis, divided as follows: 10 stimulus pictures in group 1, 12 pictures in group 2, 11 pictures in group 3, and 10 pictures in group $4 .^{51}$ (Appendix A shows the stimulus pictures in each group in the order in which they were presented to the signers.) Data was collected from three pairs of signers for each of the four groups of pictures. The distribution of pairs of signers across the groups of stimulus pictures is given in table 4.1. As the table shows, individual signers were both directors and matchers, but the same signer was never in both roles for the same group of pictures.

\footnotetext{
${ }^{51}$ The numbers of pictures in each group differs slightly because some pictures that were originally included in the task were later excluded, e.g., because objects could not be recognized by the signers.
} 
Table 4.1. Distribution of director-matcher pairs across the four groups of stimulus pictures.

\begin{tabular}{|l|l|l|l|l|l||l|l||}
\hline \multicolumn{2}{|c|}{ Stim pic group 1 } & \multicolumn{2}{|c|}{ Stim pic group 2 } & \multicolumn{2}{l||}{ Stim pic group 3 } & \multicolumn{2}{l|}{ Stim pic group 4 } \\
\hline Director & Matcher & Director & Matcher & Director & Matcher & Director & Matcher \\
\hline GL & LL & LL & GL & LL & JP & JP & LL \\
\hline FB & KO & FB & SR & SR & FB & KO & FB \\
\hline SB & HT & HT & SB & SB & HT & HT & SB \\
\hline
\end{tabular}

Figure 4.2 shows the data collection set-up used for the task. The camera was aligned with the divider on the table. The camera angle captured the full tabletop space and both signers. The total amount of recorded material for this task was 2 hours 45 minutes. The average amount of time spent on a group of pictures was 13 minutes.

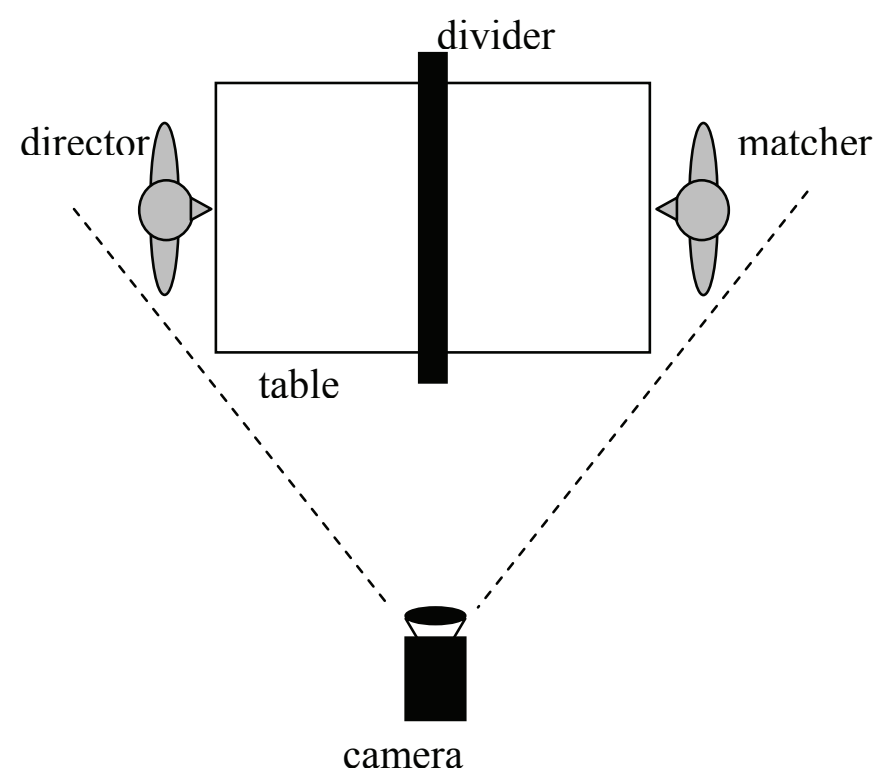

Figure 4.2. Data collection set-up for the static scene spatial description task.

\subsubsection{Data summary}

Altogether, a total of 122 descriptions were collected, comprised of 34 complex scene and 88 simple scene descriptions. ${ }^{52}$ The number of descriptions for each type of stimulus picture is given in table 4.2. The sections below present a detailed analysis of the simple scenes, whereby relevant issues with respect to the complex scene descriptions are given where they

\footnotetext{
52 As table 4.1 shows, the task was performed by three director-matcher pairs for each group of stimulus pictures. For the total of 43 stimulus pictures, this should correspond to 129 descriptions. However, because seven descriptions were not or not properly recorded on video, this left 122 descriptions for analysis.
} 
aid in generalizing about the observations made. The analysis is based on the director's first description of each scene only, before any response from the matcher.

Table 4.2. Number of stimulus pictures and descriptions by type, divided into pictures that depicted entities on the left/right lateral (lat.) or front/back sagittal (sag.) axis.

\begin{tabular}{|c|c|c|c|c|c|c|c|c|}
\hline \multirow{3}{*}{ stim. pic. } & \multicolumn{6}{|c|}{ simple scenes } & \multirow{3}{*}{$\begin{array}{c}\text { complex scenes } \\
12 \\
\end{array}$} & \multirow{3}{*}{\begin{tabular}{|c|} 
total \\
43 \\
\end{tabular}} \\
\hline & \multicolumn{2}{|c|}{$\operatorname{man} / \mathrm{man}$} & \multicolumn{2}{|c|}{$\mathrm{man} /$ tree } & \multicolumn{2}{|c|}{ cone/cone } & & \\
\hline & & $\begin{array}{l}\text { lat: } 10 \\
\text { sag: } 2\end{array}$ & 13 & $\begin{array}{l}\text { lat: } 7 \\
\text { sag: } 6\end{array}$ & 6 & $\begin{array}{l}\text { lat: } 3 \\
\text { sag: } 3\end{array}$ & & \\
\hline descr. & & $\begin{array}{l}\text { lat: } 28 \\
\text { sag: } 6\end{array}$ & 37 & $\begin{array}{l}\text { lat: } 20 \\
\text { sag: } 17\end{array}$ & 17 & $\begin{array}{l}\text { lat: } 9 \\
\text { sag: } 8\end{array}$ & 34 & 122 \\
\hline
\end{tabular}

\subsubsection{Data coding}

For each stimulus picture, the director's production (i.e. the signed description of the object configuration) was coded sign by sign for the following elements (see also table 4.3 below):

(1) Type of sign (lexical (e.g. MOUSE); numeral (e.g. TWO); classifier predicate (e.g. 2legged entity classifier); size and shape specifier (e.g. SASS(hat)); index sign (i.e. point to location); other predicate (e.g. directional predicate (e.g. LOOK)); meta-narrative element (e.g. AND-THEN))

(2) Mouthing (identifies the mouthing element that temporally coincides with the production of certain signs (e.g. Baum (tree), Mann (man)) ${ }^{53}$

(3) Hand that produces sign (dominant hand; non-dominant hand; bimanual)

(4) Type of classifier (e.g. 2-legged entity, upright entity)

(5) Hold of previous classifier (identifies the hand that holds and the entity represented with the hold)

(6) Simultaneous classifier construction (identifies the entities represented simultaneously)

(7) Location of signs in space (coded with respect to the signer's body as left (locL), middle (locM), right (locR) on the lateral axis, and as near (locN), middle (locM), far (locF) on the sagittal axis)

(8) Orientation of sign or direction of movement of sign (coded with respect to the signer's body as toward left (movL), toward right (movR), toward body (movS), away from body (movA))

(9) Figure / Ground object (identifies which object is the figure and which is the ground)

\footnotetext{
${ }^{53}$ The mouthing component was sometimes hard to identify, as the hand occasionally obscured the mouth from view.
} 
In addition to sign by sign coding, some coding decisions, which will be relevant particularly in chapters 5 and 6, were made for the description as a whole (see table 4.4 below). Coding for the whole description was for frame of reference, viewpoint, and perspective, based on the definitions provided in chapter 3. Descriptions were coded as being in observer perspective if the signer was external to the event and all referents in the scene were mapped onto the sign space in front of the signer. The use of character perspective, on the other hand, entails the mapping of an animate referent within the scene onto the signer's body (see chapter 6 for more detail on the characteristics of perspective). In frame of reference use, the relative frame of reference locates the figure with respect to the ground from an external viewpoint. This viewpoint is the deictic "origo" and may be the signer's own viewpoint, the addressee's viewpoint, or another referent's viewpoint. Descriptions were coded in the intrinsic frame of reference if they expressed only a binary relationship between the figure and the ground, without corresponding to an external viewpoint. Finally, to exhibit the use of an absolute frame of reference, descriptions must make use of fixed bearings in the environment, independent of a particular viewpoint. Tables 4.3 and 4.4 illustrate the coding scheme for the signed description in example (1), repeated below for convenience.
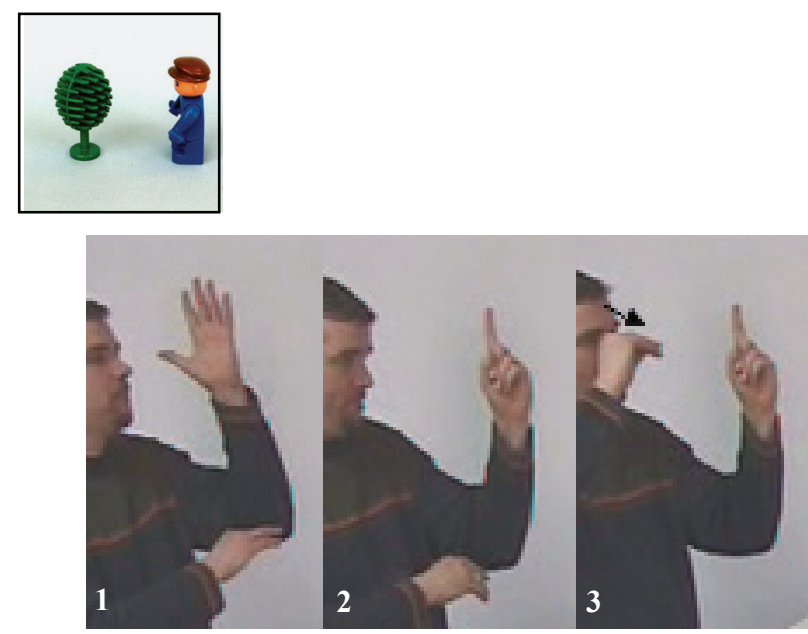

RH:

LH: TREE

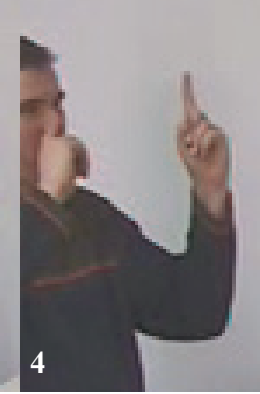

BROWN

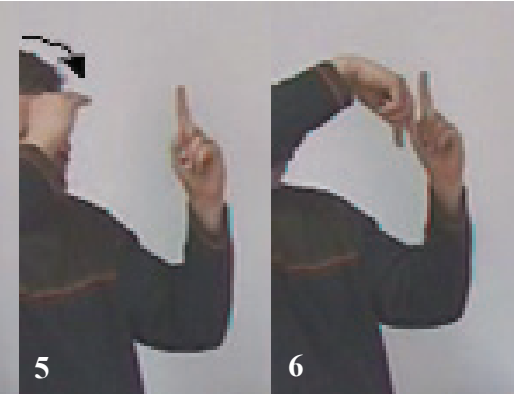

SASS(hat) $\quad \mathrm{CL}_{\mathrm{E}}(\mathrm{man})$ : locR,ornL

$\mathrm{CL}_{\mathrm{E}}($ tree): locL---hold--- 
Table 4.3. Example of sign by sign coding of the spatial scene description in example (1).

\begin{tabular}{|c|c|c|c|c|c|c|}
\hline$\#$ & $\begin{array}{l}\text { Sign } \\
\text { (meaning) }\end{array}$ & $\begin{array}{l}\text { Type sign } \\
\text { (e.g. lexical, } \\
\text { num., CL, } \\
\text { pred.) }\end{array}$ & Mouthing & Hand & $\begin{array}{l}\text { Hold of previous } \\
\text { classifier (which } \\
\text { hand? which } \\
\text { object?) }\end{array}$ & $\begin{array}{l}\text { Simultaneity of separate } \\
\text { classifiers (which } \\
\text { objects?) }\end{array}$ \\
\hline 1 & TREE & lexical & tr- & ndom (LH) & NA & NA \\
\hline 2 & loc-tree & $\mathrm{CL}$ & -ee & ndom (LH) & NA & NA \\
\hline 3 & MAN & lexical & man & dom $(\mathrm{RH})$ & yes (LH, tree) & NA \\
\hline 4 & BROWN & lexical & bro- & dom (RH) & yes (LH, tree) & NA \\
\hline 5 & shape-hat & SASS $^{54}$ & -own & dom (RH) & yes (LH, tree) & NA \\
\hline 6 & loc-man & $\mathrm{CL}$ & - & dom (RH) & yes (LH, tree) & yes (tree, man) \\
\hline
\end{tabular}

\begin{tabular}{|l|l|l|l|l|l|}
\hline$\#$ & $\begin{array}{l}\text { Sign } \\
\text { (meaning) }\end{array}$ & Classifier type & $\begin{array}{l}\text { Location of } \\
\text { classifier }\end{array}$ & $\begin{array}{l}\text { Orientation, dir of } \\
\text { facing, or movement }\end{array}$ & Figure / Ground \\
\hline 1 & TREE & NA & NA & NA & Ground (lex. ident.) \\
\hline 2 & loc-tree & entity (upright) & L, lat & non-feat. & Ground (CL) \\
\hline 3 & MAN & NA & NA & NA & Figure (lex. ident.) \\
\hline 4 & BROWN & NA & NA & NA & Figure (lex. ident.) \\
\hline 5 & shape-hat & NA & NA & NA & Figure (lex. ident.) \\
\hline 6 & loc-man & entity (2-legged) & R, lat & face L (=face tree) & Figure (CL) \\
\hline
\end{tabular}

Table 4.4. Example of whole scene coding of the spatial scene description in example (1).

\begin{tabular}{|l|l|l|l|l|}
\hline$\#$ & $\begin{array}{l}\text { Sign } \\
\text { (meaning) }\end{array}$ & Frame of reference & Viewpoint & Perspective \\
\hline 1 & TREE & & & \\
\hline 2 & loc-tree & & \\
\cline { 1 - 2 } 3 & MAN & relative & signer's & observer \\
\hline 4 & BROWN & & & \\
\hline 5 & shape-hat & & & \\
\hline 6 & loc-man & & & \\
\hline
\end{tabular}

The next section presents a detailed analysis of the DGS static scene descriptions elicited with the stimulus materials described above and coded according to the schema above. The analysis proceeds according to the five structural properties listed in section 4.1 .

\subsection{DGS locative descriptions}

The analysis of DGS locative descriptions presented in this chapter is based on the five structural properties inherent in the locative construction in example (1). The properties are

\footnotetext{
54 The sequence "BROWN SASS(hat)" is part of a complex NP in which the noun, i.e. HAT, is elided. The full NP would be "HAT BROWN SASS(hat)", where HAT is the noun, and "BROWN SASS(hat)" is the modifier that contains a color adjective as well as an adjectival size and shape specifier that specifies the actual shape of the hat.

It is important to remember that objects of a type can come in different shapes and sizes, and that the citation form of signs reflects only certain properties or certain subtypes of their referent. In DGS, the citation form of HAT depicts the action of putting on a hat. Information about the size and shape properties of specific hats, like the one worn by the man in the stimulus picture, can be conveyed through the use of size and shape specifiers like "SASS(hat)" that follow the noun.
} 
stated here as conventions, which are hypothesized to determine the canonical structure of DGS locative descriptions. The data is tested against these conventions.

1. Each referent in the scene is identified (lexically) before spatial information about it is predicated

2. Classifier predicates are used to encode all relevant spatial information about referents (i.e. location, orientation, number) ${ }^{55}$

3. The ground object is coded before the figure object

4. A simultaneous classifier construction is used to directly depict the spatial relationship between two entities

5. The spatial array is described from the signer's own (external) viewpoint as the viewer of the scene

While convention 1 reflects general discourse and information structure principles, the remaining conventions may be tied to properties of the visual-spatial modality, and exploit its iconic affordances. Conventions 2 and 4 depend on the imagistic and diagrammatic iconicity of classifiers. That is, the imagistic iconicity of the classifiers themselves (i.e. the handshapes) means that size, shape, and featural characteristics of referents can be represented. In addition, through imagistic iconicity, one hand (as the iconic linguistic form) corresponds to one entity. The placement of the classifiers in sign space (as classifier predicates) allows referent location and orientation to be encoded. The use of simultaneous classifier constructions (convention 4) to make visible the spatial relationship between referents highlights the diagrammatic iconicity inherent in classifier predicates. Another type of iconicity, namely iconicity of perception, motivates encoding the spatial array from the signer's viewpoint (convention 5), by which the representation of a scene should match its perception (cf. Emmorey 1998, who likewise suggests that signers prefer to describe scenes from their own viewpoint because it is concordant with their visual perception of the scene). Representing the scene from a different viewpoint (e.g. that of the addressee) would require an intermediate operation, making the representation itself less iconic. Finally, though not based on iconicity, encoding the ground before the figure (convention 3), particularly, encoding large objects before small objects, may be typical to the visual modality (cf.

\footnotetext{
55 This includes SASSes that are spatialized (see Glück and Pfau 1997, 1998; Zwitserlood 2003 on the morphosyntactic differences between entity and handling classifiers, on the one hand, and SASSes, on the other hand).
} 
Emmorey 1996). As defined by these conventions, the canonical structure is maximally iconic, as well as maximally transparent and economic, in its structure.

In the analysis that follows, I focus primarily on the simple scene descriptions. As shown in section 4.2.2, the simple scenes all contained two referents, and were comprised of either two animates, two inanimates, or one animate/one inanimate. As I outlined in chapter 3 (in sections 3.2 and 3.5), the imposition of a figure-ground structure is important in determining the linguistic structure of spatial expressions. Since the simple scenes contain exactly two referents, they can be analyzed as single figure-ground expressions. In contrast, the descriptions of complex scenes, containing up to nine individual referents, are complex constructions that involve multiple figure-ground structures, and multiple simultaneous classifier constructions. Thus, distinguishing individual figure-ground structures is a much more complicated matter. For this reason, the complex scene descriptions were not analyzed in detail with respect to figure-ground structures. In all other respects, however, they were coded in the same way as the simple scene descriptions. Throughout the analysis, the complex scene descriptions are brought in to supplement and corroborate the findings arising from the simple scene descriptions.

To give a general impression before launching into the analysis itself, less than onefifth of the 88 simple scene descriptions analyzed fully conform to the five canonical structure conventions (14 out of $88,16 \%$ ). The data shows a substantial amount of variation in the forms used to represent referents, in the use of simultaneous classifier constructions, and in the way that referents are identified and spatialized. However, nearly all the descriptions exhibit structure that supports the validity of at least two of the conventions (83 out of $88,94 \%$ ), slightly over half the descriptions support at least three conventions (48 out of $88,55 \%$ ), and one quarter conform to at least four conventions (22 out of $88,25 \%)$. This information is represented graphically in table 4.5 below.

Table 4.5. Percentage of simple scene descriptions that conform to at least one, two, three, four, or all five conventions of the canonical locative construction hypothesis.

\begin{tabular}{|l|c|}
\hline$\#$ conventions supported & $\%$ descriptions \\
\hline one convention & $100 \%$ \\
\hline two conventions & $94 \%$ \\
\hline three conventions & $55 \%$ \\
\hline four conventions & $25 \%$ \\
\hline five conventions & $16 \%$ \\
\hline
\end{tabular}


In addition, and more specifically for the individual conventions, table 4.6 below shows an overview of the number of descriptions that exhibited each structure.

Table 4.6. Overview of number and percentage of simple scene descriptions that exhibit or do not exhibit the structure specified by each of the five conventions hypothesized to define the canonical structure.

\begin{tabular}{|c|l|c|c|c|}
\hline$\#$ & Convention & obtains & does not obtain & total \\
\hline 1 & Lexical identification precedes spatialization & $39(44 \%)$ & $49(56 \%)$ & 88 \\
\hline 2 & Classifiers encode all spatial information & $30(34 \%)$ & $58(66 \%)$ & 88 \\
\hline 3 & Encode ground before figure & $31(84 \%)$ & $6(16 \%)$ & $37^{56}$ \\
\hline 4 & Simultaneous construction depicts spatial relation & $65(74 \%)$ & $23(26 \%)$ & 88 \\
\hline 5 & Description is from signer's viewpoint & $87(99 \%)$ & $1(1 \%)$ & 88 \\
\hline
\end{tabular}

In the following sections, I present the analysis of DGS simple scene spatial descriptions by looking at "exceptions" to the canonical structure, sometimes on a case by case basis and by giving a quantitative overview of the patterns in the data. Each of the five conventions that are hypothesized to define the canonical structure are addressed in turn, defining the convention in more detail and specifying how the signed descriptions can "fail" to conform to it. Any of the five conventions can obtain or not obtain for any description, and for each convention there are different ways in which not obtaining can manifest itself.

\subsubsection{Convention 1: Identification precedes spatialization}

There are various ways in which descriptions can exhibit a syntactic structure in which individual referents are not lexically identified before spatial information is predicated about them. To validate the convention, under the strict conditions of this analysis, a simple scene description must explicitly exhibit the following structure: $\left[\mathrm{NP}_{1}\right]\left[\mathrm{CL}_{1}\right]\left[\mathrm{NP}_{2}\right]\left[\mathrm{CL}_{2}\right]$. Note that with respect to this convention, the order of figure and ground is irrelevant. "Referent 1" and "referent 2" refer simply to the first and second referent mentioned. The "identification followed by spatialization" convention does not obtain if the following structures appear. They are discussed in turn below.

i. Identification holds for both referents (single identification)

ii. Referents are spatialized simultaneously (simultaneous spatialization)

\footnotetext{
56 The denominator that appears here for the number of descriptions that encoded ground before figure (convention 3) is the number of man/tree stimulus pictures. Only these simple scenes exhibited a natural segregation into figure and ground objects. The figure-ground structure of descriptions of scenes that contained two objects of the same kind (i.e. the remaining 51 simple scene descriptions) is discussed in section 4.3 .3 according to linguistic criteria.
} 
iii. A referent is identified and spatialized in a single constituent (collapsed identification and spatialization)

iv. A referent is spatialized before it is identified (inverted identification and spatialization)

v. The relevant spatial information for a referent is distributed across constituents (distributed spatialization)

Table 4.7 below shows the number of descriptions that exhibit each of these structures for the three different types of simple scene stimulus picture descriptions. The total number of simple scene descriptions is 88 , comprised of $37 \mathrm{man} /$ tree descriptions, $34 \mathrm{man} / \mathrm{man}$ descriptions, and 17 cone/cone descriptions.

Table 4.7. Distribution of simple scene descriptions according to different structures for which convention 1 does not obtain, across the different types of simple scene stimulus pictures.

\begin{tabular}{|l|c|c|c|c|}
\hline $\begin{array}{c}\text { Structure for which } \\
\text { conv. 1 does not obtain }\end{array}$ & $\begin{array}{c}\text { \# man/tree } \\
\text { descriptions }\end{array}$ & $\begin{array}{c}\text { \# man/man } \\
\text { descriptions }\end{array}$ & $\begin{array}{c}\text { \# cone/cone } \\
\text { descriptions }\end{array}$ & $\begin{array}{c}\text { total \# descriptions for } \\
\text { which conv. 1 does not } \\
\text { obtain (out of 49) }\end{array}$ \\
\hline single identification & - & 26 & 7 & $33(67 \%)$ \\
\hline simult. spatialization & - & 13 & - & $16(33 \%)$ \\
\hline collapsed ident./spat. & 11 & - & 2 & $11(22 \%)$ \\
\hline inverted ident./spat. & 4 & - & - & $6(12 \%)$ \\
\hline distributed ident./spat. & 1 & 4 & $-10 \%)$ \\
\hline
\end{tabular}

Exception (i) to convention 1: Single identification. Single identification occurs only in descriptions of stimulus pictures that depict two entities of the same type (i.e. man/man or cone/cone pictures). This is illustrated in example (2), where the noun phrase MAN BROWN SASS(hat) (frames 1-3) serves to identify both referents. There is no further identifying material (e.g. lexical nouns or modifiers) between the two classifier forms that are used to spatialize (i.e. provide location and orientation information about) the referents (frames 4-5).

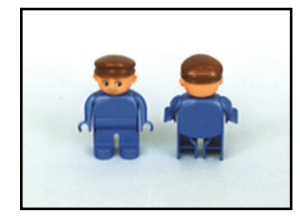

${ }^{57}$ Note that the numbers in this column add up to more than 49 (as shown in table 4.6), as the different structures are not mutually exclusive. A single description can "fail" to conform to convention 1 in more than 


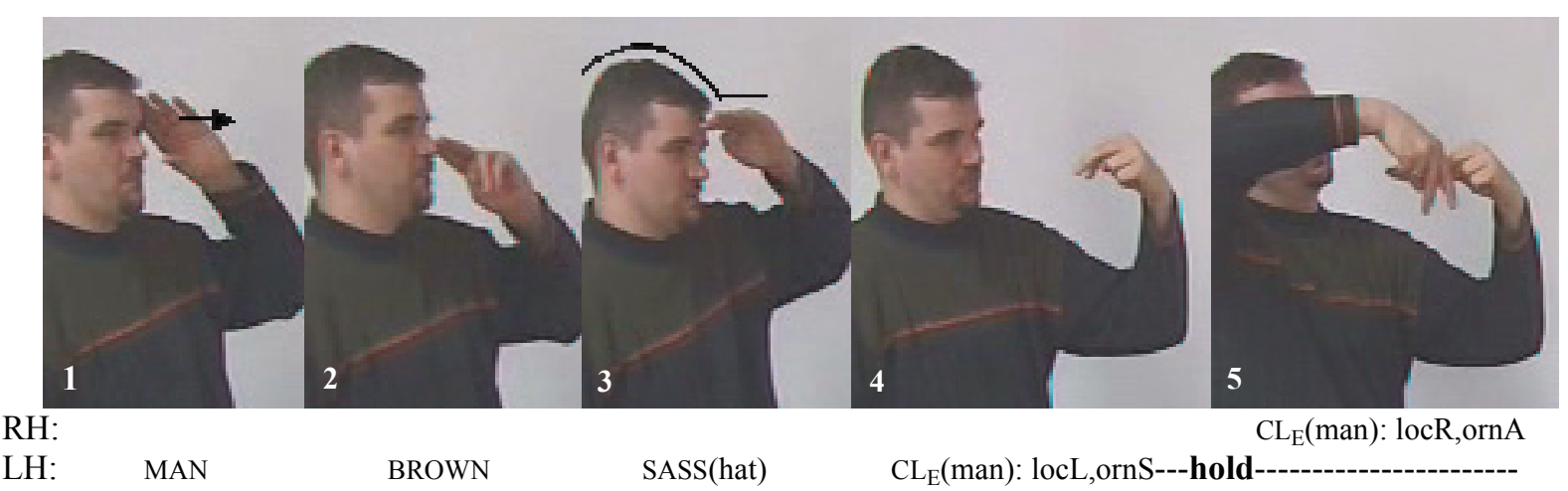

As the next example shows, single identification of referents also applies to descriptions in which more than one noun phrase is used for identification. The crucial point is that all signs that are used to identify the referents precede, and never follow, the signs that provide location and orientation information about the referents. This means that the noun phrase used for referent identification must hold for both entities. In the description shown in (3), the signer uses a total of three separate noun phrases in his identification of the referents: TWO CONE, CONE, and TWO RED CONE. ${ }^{58}$ All of them occur before there is any spatial information given. In total, 33 descriptions were characterized by having a single identification component. This amounts to $67 \%$ of the total number of descriptions of pictures with referents of the same type (34 man/man and 17 cone/cone descriptions) - a prerequisite for the possibility of single identification.

(3)
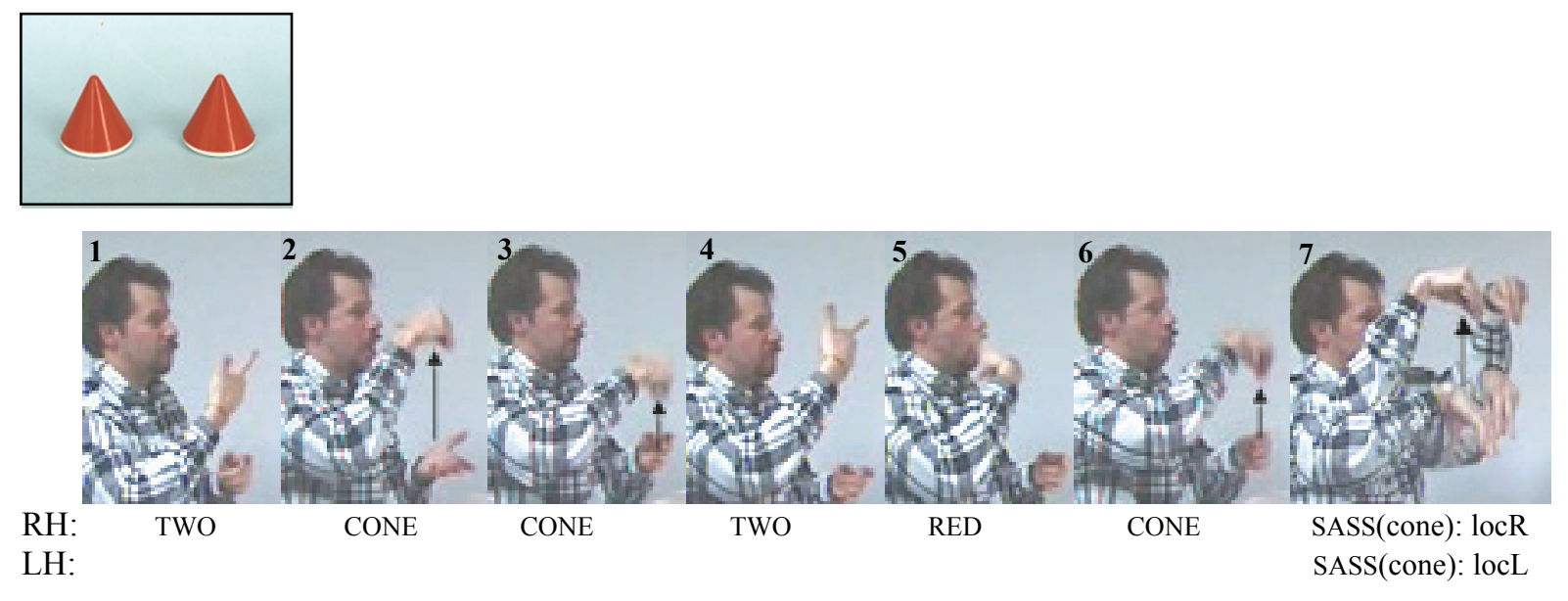

one way. For example, as we will see, the total number of descriptions that showed "simultaneous spatialization" are a subset of those with "single identification".

${ }^{58}$ In subsequent examples of descriptions of cone/cone stimulus pictures, the reader will notice that the signs glossed as CONE do not all look the same, as signers use different forms to sign cone. These signs are all glossed in the same way, as they function as nouns (i.e. they are accompanied by mouthing and are used for referent identification). Similarly, the reader will notice that more than one lexical form for MAN appears in the data. 
Half of these descriptions were additionally characterized by "simultaneous spatialization", that is, simultaneous placement of the forms used to encode spatial information (as can be seen in example (3), frame 7). Simultaneous spatialization is the focus of the next subsection. Furthermore, note that the third noun phrase in example (3), TWO RED CONE (frames 4-6), contains the use of a numeral. 27 of the 33 single identification descriptions (82\%) exhibited the use of numerals. Four of the six descriptions that did not contain a numeral were produced by the same signer (LL). The use of numerals to specify the number of referents is a different type of "violation" of the hypothesized canonical structure that will be discussed with respect to convention 2 in section 4.3.2. In general, as we shall see, there is a significant amount of overlap and co-occurrence of structures that do not conform to one of more of the conventions taken to define the canonical structure.

Exception (ii) to convention 1: Simultaneous spatialization. As has already been exemplified in example (3), simultaneous spatialization means that the forms used to encode spatial information (specifically, classifier predicates) are placed into sign space at the same time. (Note that the terms "simultaneous spatialization" and "simultaneous construction" are not synonymous. However, descriptions that exhibit simultaneous spatialization are simultaneous constructions. In fact, they demonstrate a simultaneous classifier construction par excellence, as the construction is not the result of the sequential placement of classifiers with a hold of the first classifier predicate, but rather the immediately simultaneous presentation of classifiers.) Descriptions with simultaneous spatialization are a subset of those characterized by single identification, and thus also occurred only for stimulus pictures that depict two entities of the same type (i.e. man/man or cone/cone pictures).

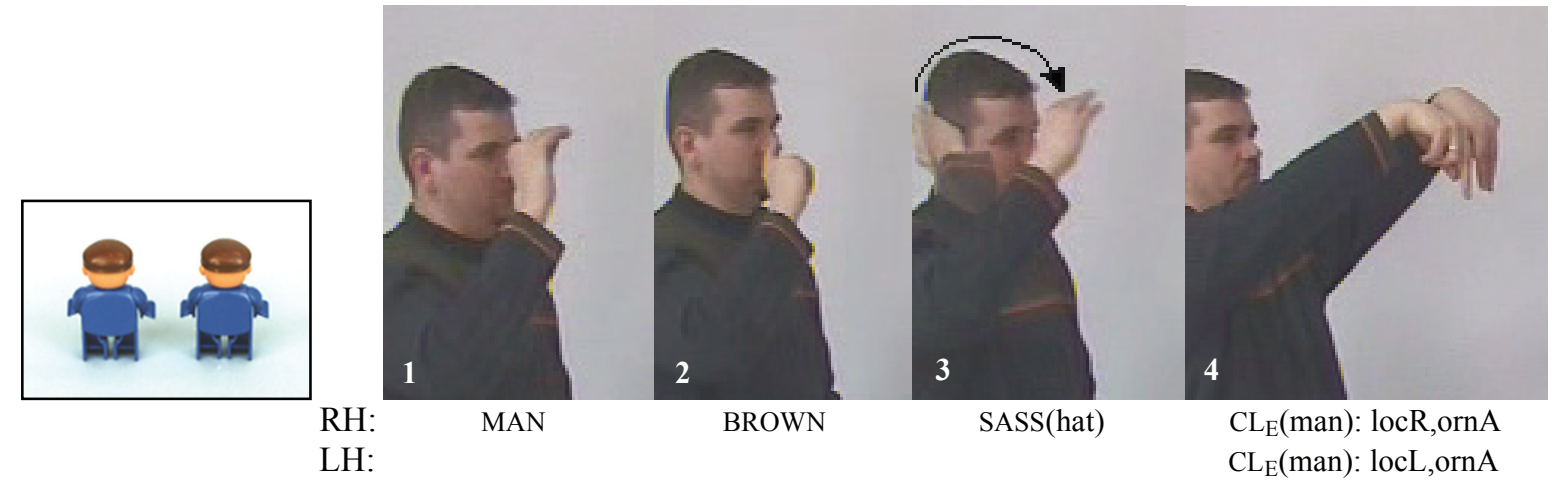

Example (4) above shows the simultaneous placement of classifier predicates in the description of a man/man stimulus picture. Again, this signer identifies the men by including 
the physical attribute "with the brown hat" in the NP (frames 1-3), and then simultaneously places two 2-legged entity classifiers into sign space. The spatial relationship between the two men (left and right on the lateral axis, and facing away from the signer) is simultaneously encoded (frame 4). Example (5) shows another description of the stimulus picture that we have already seen in example (3) (two red cones, left and right on the lateral axis). This signer uses the noun phrase TWO RED CONE for referent identification (frames 1-3) and then uses upright entity classifiers to simultaneously encode the cones' left and right locations. The upright entity classifiers are used here as generic predicates of location that do not give entity-specific size and shape information.
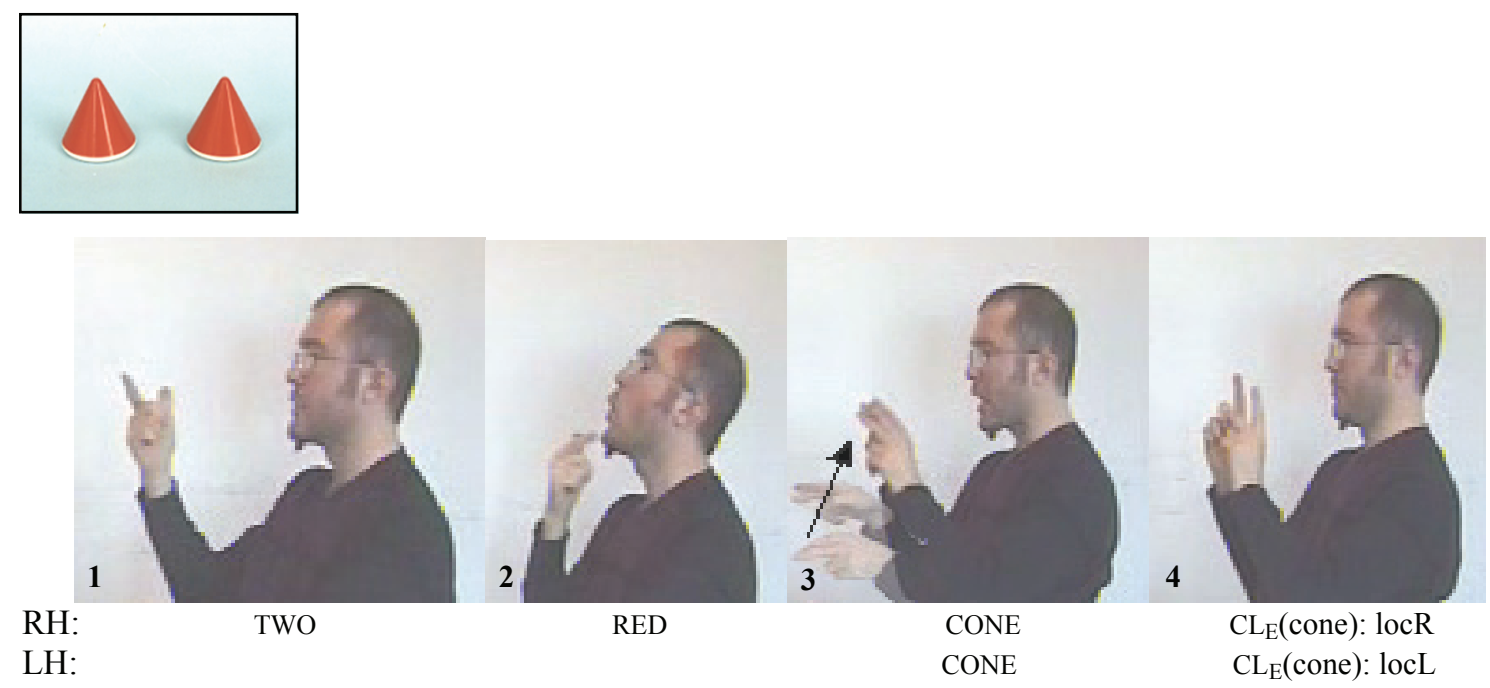

In all, 16 descriptions - $13 \mathrm{man} / \mathrm{man}$ and 3 cone/cone descriptions - were characterized by the simultaneous spatialization of referents. This is half of the 33 descriptions that exhibited single identification of referents. Note that while simultaneous spatialization is presented here as a structure for which convention 1 does not obtain, it conforms wholly to the convention regarding the use of simultaneous classifier constructions (convention 4).

Besides occurring only in descriptions of stimulus pictures with referents of the same type (man/man or cone/cone), there was a further constraint on simultaneous spatialization in the data. The constraint concerns the orientational relationship between the two men in the man/man stimulus pictures. Simultaneous spatialization did not occur in man/man pictures in which the men are facing in directions that are at a right angle to each other (e.g. one man is facing the signer, and one man is facing right). It occurred only when both men are facing in the same direction (as in example (4) above) or when the men are facing in opposite 
directions (i.e., literally, directions that differ by $180^{\circ}$ ) (as in example (6) below, where the men are facing each other).

(6)
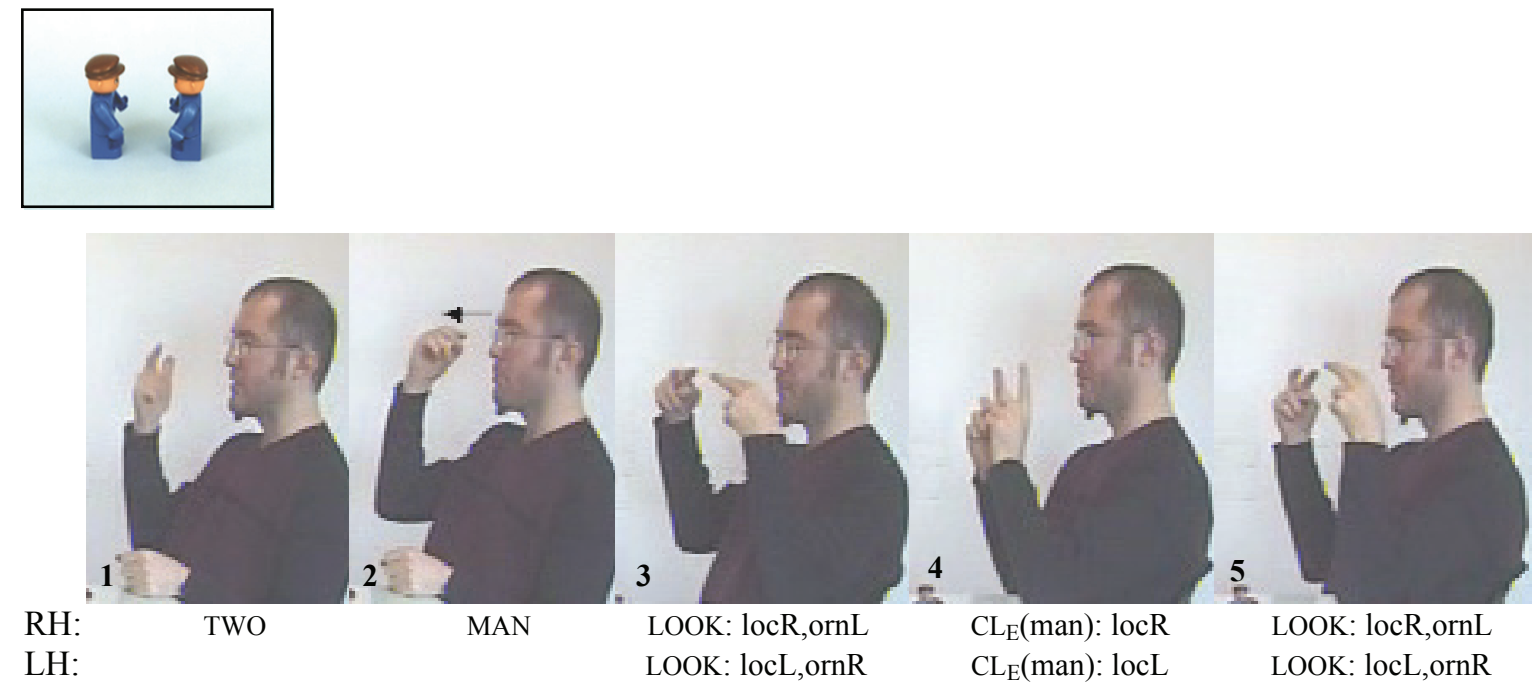

The reason for this constraint, I suggest, is because simultaneous spatialization entails a single predication, which is allowed in DGS only for dual or reciprocal relationships. Thus, dual relationships like both facing left and both facing away (from the signer) and reciprocal relationships like facing one another or facing away from one another (with their backs to one another), including standing next to one another, facing in opposite directions are possible single predications in DGS. In contrast, there is no simultaneous spatialization in descriptions of stimulus pictures that show the men facing in directions that differ by $90^{\circ}$. This type of orientational relationship cannot be expressed as a single predication in DGS. Rather, the descriptions of such spatial relationships require a figure-ground structure, in which one referent is located with respect to the other referent, and where classifier placement must thus be sequential. (The validity of this constraint on single predications in DGS must be tested with more data, as there were only a small number of descriptions of this type of picture in the data set used here. ${ }^{59}$ )

However, additional support is lent to the claim that single predications in DGS are possible only for dual and reciprocal relationships by the fact that simultaneous spatialization did not occur for man/tree stimulus pictures. It is conceivable that a signer could lexically identify both referents, one after the other, and then depict the spatial relationship between them by simultaneously placing two classifier predicates (one for the man and one for the

\footnotetext{
${ }^{59}$ Only two of the $12 \mathrm{man} / \mathrm{man}$ stimulus pictures showed the men facing in directions that differed by $90^{\circ}$ (see stimulus pictures 2 (group 2) and 8 (group 3) in appendix A).
} 
tree) in sign space. Since the two referents in question are appropriately represented with classifiers that are easily distinguishable and associated with their intended referent (i.e. an upright entity classifier for the tree and a 2-legged entity classifier for the man), there would be no ambiguity about which classifier belonged to which NP. However, a construction like this, a hypothetical example of which is given in glosses in example (7) for the stimulus picture shown, is not attested in the DGS data. ${ }^{60}$

(7)

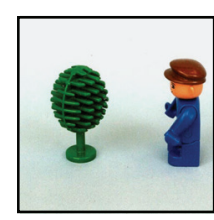

$$
\begin{aligned}
& \text { * RH: } \quad \text { MAN } \quad \mathrm{CL}_{\mathrm{E}}(\mathrm{man}): \text { locR,ornL } \\
& \text { LH: TREE } \text { CL }_{\mathrm{E}} \text { (tree): locL }
\end{aligned}
$$

It is furthermore interesting to note a parallel between simultaneous spatialization and the form of balanced two-handed signs, which meet the symmetry condition (Battison 1978). This condition expresses a form constraint on two-handed signs in which both hands move. It specifies that the hands have the same handshape and that the hands move either symmetrically or in alternation (see chapter 2, section 2.2). The simultaneous placement of 2legged entity classifiers that face in the same or in opposite directions is reminiscent of the types of movement that occur in balanced two-handed signs. Symmetrical and parallel directionality is somehow favored, and the avoidance of simultaneous placement of classifiers whose orientation is not symmetrical in this way may be the result of linguistic constraints, as well as visual-perceptive and motoric constraints on form complexity (cf. Battison (1978) on restrictions in ASL on "complex combinations of manual-visual components" (p. 56)).

Finally, a brief remark on simultaneous spatialization in the complex scene descriptions. Like in the simple scenes, signers simultaneously located two referents of the same kind in space. Two trees (located left and right in the scene) appeared in eight of the twelve complex scene stimulus pictures, corresponding to 23 descriptions. The trees were simultaneously spatialized in $16(70 \%)$ of these descriptions (as can be seen e.g. in example (25) in section 4.3.3). ${ }^{61}$ Six descriptions indicated the trees' locations sequentially, and one

\footnotetext{
${ }^{60}$ In the example, I have assigned the NPs for the two referents to different hands (corresponding to their location in sign space). It would also have been possible to assign them to the same hand.

${ }^{61}$ This includes one description given in character perspective, in which the signer mapped the man onto her body (see section 4.3.5 and section 5.4 in chapter 5). The signer simultaneously located the trees behind her shoulders, using open C-hands to represent the large round shape of the tree trunk (corresponding to the lifesized event space of character perspective).
} 
description used a spatially modifiable sign with prepositional meaning to indicate the location of the trees relative to the other referents (see section 4.3.2 on the use of prepositions). In descriptions of pictures that contained multiple pigs (five or six), signers also occasionally placed two animal classifiers into space simultaneously. This occurred at the beginning of the sequence of animal classifiers used to represent the configuration of pigs, frequently with a subsequent hold of the non-dominant hand.

Following this discussion of both "single identification" and "simultaneous spatialization" in the descriptions, it is worthwhile looking at the relationship between them in terms of a grammatical analysis. "Single identification" occurs with two different types of constructions. On the one hand, it occurs with single predications (as evidenced by simultaneous spatialization, i.e. simultaneous placement of classifiers), and, on the other hand, it occurs with expressions that have a figure-ground structure (as evidenced by a sequential placement of classifiers). Based on this, I suggest that the NP that serves as the "single identification" corresponds to two different types of subject NP, depending on the type of construction it occurs with. In constructions with "simultaneous spatialization", the noun phrase must be interpreted as referring to a plural subject (as in the English reciprocal predication The men are facing each other). ${ }^{62}$
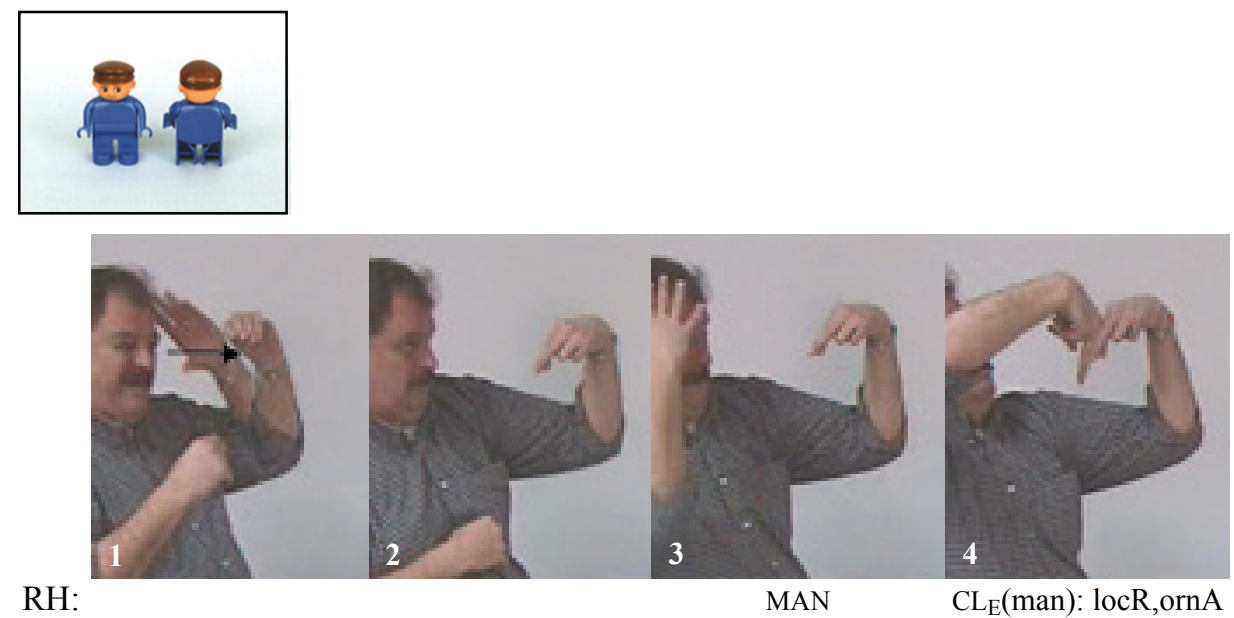

RH:

LH: $\quad$ MAN

$\mathrm{CL}_{\mathrm{E}}(\mathrm{man})$ : locL,ornS--hold

On the other hand, in the case of sequential placement of classifier predicates, there are actually two separate clauses, each (underlyingly) containing an NP, i.e. to identify the referent, and a predicate, i.e. to provide spatial information about the referent. This is

\footnotetext{
${ }^{62}$ Nouns in DGS are inherently transnumeral. That is, the signs themselves exhibit no inherent number marking (Biermann 1982; Krifka 1989), and can be interpreted as singular or plural depending on the context (Perniss 2001).
} 
exemplified in example (8) above ${ }^{63}$, and corresponds to an English paraphrase like (One) man, on the left, is facing forward, and (another) man, next to him on the right, is facing away.

However, in the case of "single identification", there is only one NP that is valid for both predicates. Structurally, this is reminiscent of subject elision in conjoined clauses (e.g.

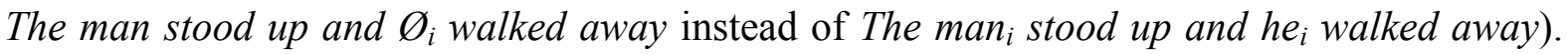
The subject of the second clause can be elided because it is coreferent with the subject of the first clause. However, in the signed examples that display "single identification", we are not dealing with two clauses that share the same subject (i.e. the $\operatorname{man}_{i} / \varnothing_{i}$ or $\operatorname{man}_{i} / h e_{i}$ ). Instead, we have two clauses with different subjects, but subjects which identify referents of the same type (i.e. the $\operatorname{man}_{i} /$ the $\operatorname{man}_{j}$ ). Thus in DGS, it is possible through subject elision to conjoin two clauses predicating spatial location information of two different referents if the two referents are of the same type. As with elision in general, "single identification" elides redundant material from the signed construction. However, in contrast to spoken languages, the elided element is not redundant in the sense of being coreferent. Instead, in the DGS examples that exhibit "single identification" (i.e. subject elision), what is redundant is the relationship between the lexical item identifying the referent and the classifier form used to represent the referent. Thus, crucially, the elision of the second subject NP seems to require that the two referents be representable by means of the same classifier form.

In terms of discourse constraints, the subject elision in these contexts seems to result from various properties of the modality. This includes the imagistic iconicity principles by which one hand represents one referent and by which sign language classifiers encode figural information. The number of hands can indicate the number of entities, and the hand configuration indicates the type of referent. The necessary information about the reference of the subject NP is thus available from the number and configuration of the hands. ${ }^{64}$ The structural variation that results from "single identification" and "simultaneous spatialization", in particular, thus depends on the interaction of spatially iconic properties of classifier predicates (special to the visual-spatial modality) and general discourse principles (e.g. the elision of redundant material).

\footnotetext{
${ }^{63}$ Note that the description in (8) is an example of a description that conforms to all five conventions, thus exhibiting the hypothesized canonical structure of locative descriptions (see section 4.3.3 on figure and ground encoding in $\mathrm{man} / \mathrm{man}$ descriptions).
} 
Exception (iii) to convention 1: Collapsed identification and spatialization. Sometimes signers did not use separate signs for referent identification and spatialization, but rather collapsed them into a single form. In these cases, signers spatialized the entity using an entity classifier or a SASS and insured identification through simultaneous mouthing of the German word for the entity. In example (9), the signer collapses spatialization and identification for both entities in the stimulus picture (i.e. for both the tree and the man). In frame 1, the signer places an upright entity classifier into sign space and simultaneously mouths Baum, the German word for "tree". ${ }^{65}$ The same thing happens in frame 2 for the man. That is, referent location is indicated with a classifier predicate and referent identification is achieved through simultaneous mouthing of Mann (the German word for "man"). The man's orientation is encoded separately in frame 3 with a direction of looking predicate. In total, 11 out of the 88 simple scene descriptions (12\%) exhibited collapsed identification and spatialization for at least one of the two referents in the spatial scene. These occurrences were primarily due to two signers (one a native signer (FB), the other an early signer (HT)), each of whom collapsed referent identification and spatialization in five different descriptions.
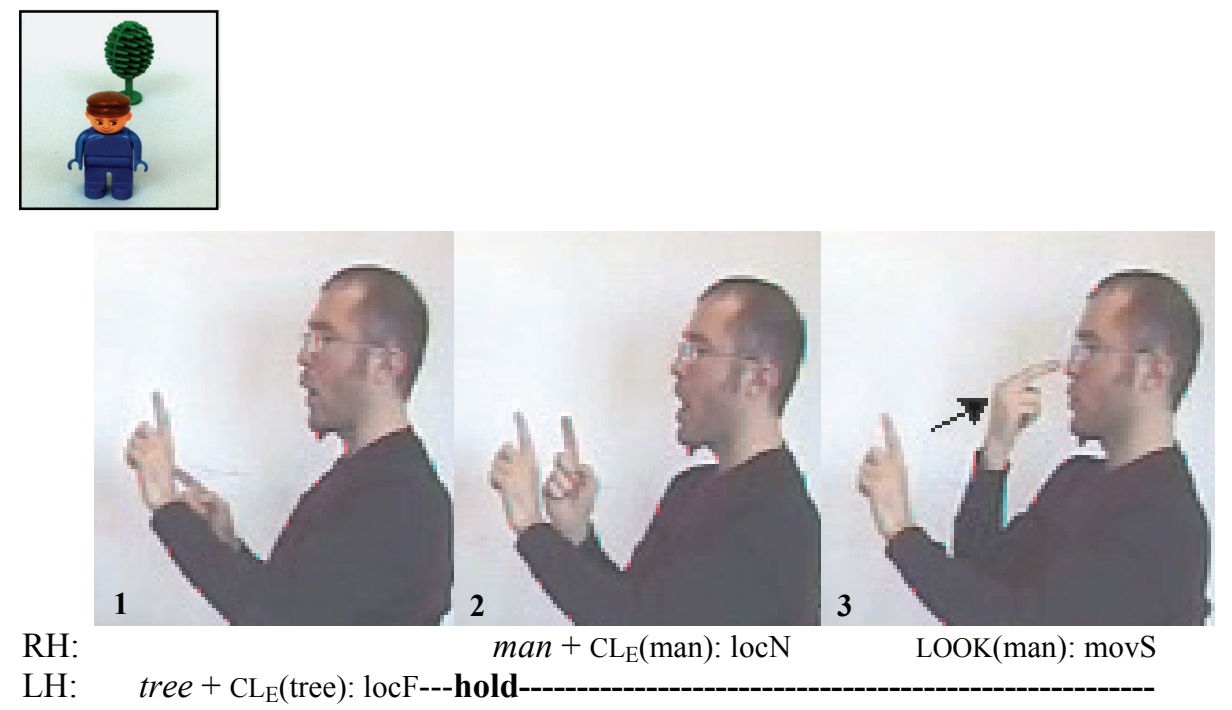

Exception (iv) to convention 1: Inverted identification and spatialization. In some descriptions, a classifier predicate was used to indicate referent location before the referent was identified, thus exhibiting an inversion of identification and spatialization. In example

\footnotetext{
${ }^{64}$ Recall from above that a high percentage of descriptions with "single identification" contained a numeral in the NP (e.g. TWO MAN). In these cases, the use of two classifier predicates, each predicating information about "one man", may also be expected from the NP itself.
} 
(10), the signer locates the second referent (the red cone) in space (frame 4) before identifying the cone's color (frame 5). Since the two referents in the picture can be distinguished by color only, the specification of the color is necessary for proper identification of the referent, and is thus necessary for a complete description. However, since both referents are cones, it is not necessary to repeat the noun CONE for full referent identification.
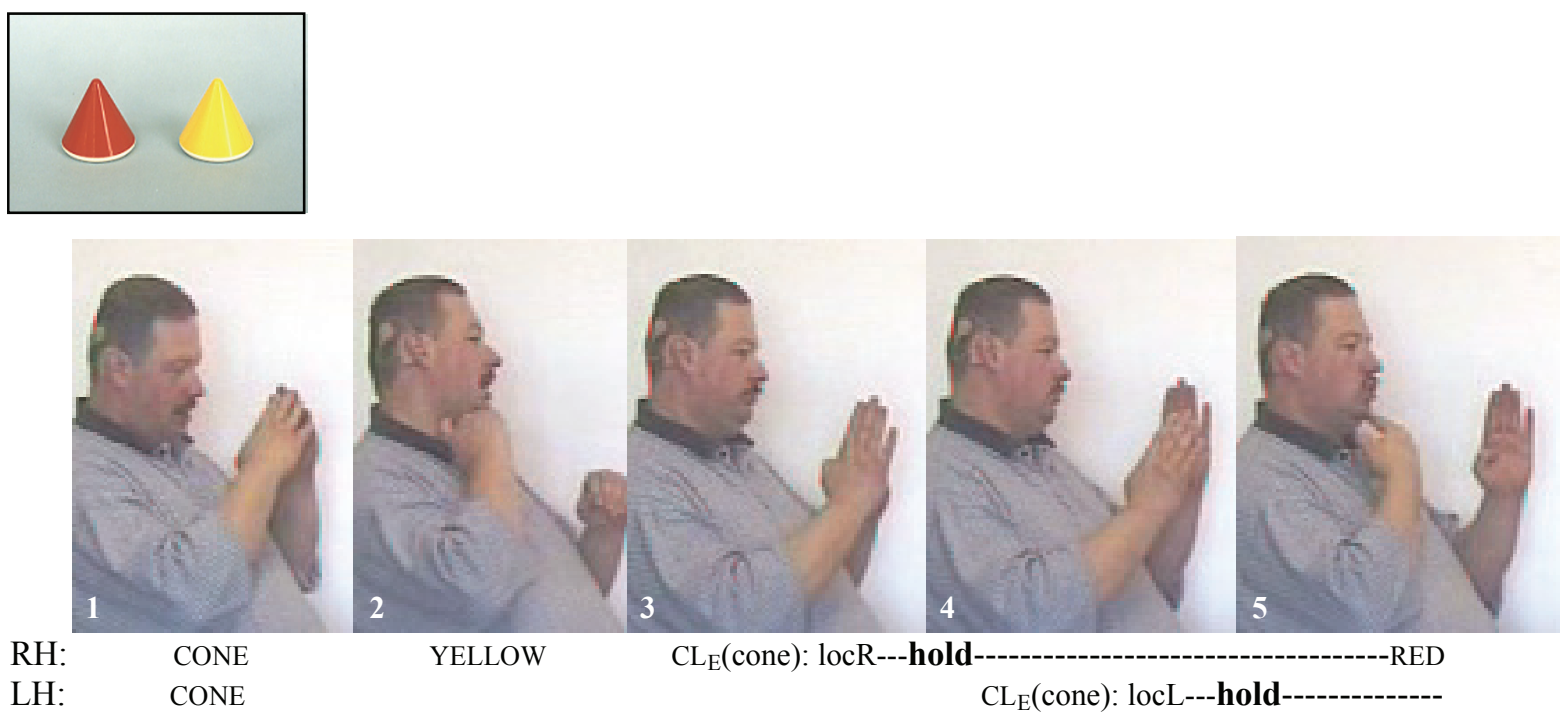

There were six descriptions in which the order of the lexical sign and the classifier predicate was reversed for one or both referents. So, overall, the inversion of identification and spatialization was very infrequent, happening in only $7 \%$ of the descriptions. Moreover, all six descriptions that exhibited this inversion were signed by two signers, HT and SB (three descriptions each), who happened to be partners for all groups of pictures. The example in (10) shows an inversion of identification and spatialization for a cone, but inversion, in fact, occurred for all three types of referents (man, tree, and cone).

Exception (v) to convention 1: Distributed identification and spatialization. Finally, in five descriptions, spatial information about the second entity is encoded before all relevant spatial information about the first entity has been expressed. One of these is discussed further below (in example (16) in section 4.3.2). In these descriptions, the repeated sequence of referent identification and spatialization is interrupted, as the full sequence for the first referent is not completed before proceeding to the second entity. In example (16), the signer first encodes

\footnotetext{
${ }^{65}$ Though it is not included in the gloss, notice that the signer uses his right hand to index the classifier form produced on his left hand. This underscores the reference of the mouthed identification.
} 
the locations of the two men (in frames 4 and 5) and then encodes their respective directions of facing (in frames 10 and 14). In another example, the signer points to two locations in space to index the men's locations before showing their orientations.

As a final note about structures that do not conform to convention 1 of the canonical locative construction hypothesis, the occurrence of "collapsed", "inverted", and "distributed" identification/spatialization of referents was sporadic in the data. In the DGS descriptions analyzed here, they appeared to be idiosyncratic, and attributable to a few individual signers. Nevertheless, they occurred in one-quarter of all simple scene descriptions (22 out of 88). In contrast, the occurrence of "single identification" and "simultaneous spatialization" in the DGS descriptions was systematic, and could be generalized, thereby constituting motivated sub-patterns to the structure of locative descriptions. However, the types of structures that appear to be idiosyncratic in DGS may occur in a more systematic manner in other sign languages. For example, collapsing the identification and spatialization of a referent into a single sign by placing lexical signs in non-neutral space may be more common in other sign languages. The spatialization of lexical signs as a means of expressing location information has been noted in the literature e.g. by Emmorey 1996 for ASL and by Zeshan 2000 for IPSL.

\subsubsection{Convention 2: Classifiers encode all spatial information}

The second convention hypothesized to define the canonical structure of locative constructions states that the affordances of classifier predicates for clear and efficient expression of spatial information will be maximally exploited. The spatial information that is relevant to the descriptions at hand is referent location, referent orientation, and referent number. Due to the structure-preserving possibilities of the modality, all of this information can be encoded with classifier predicates. ${ }^{66}$ The location/orientation of the hands in sign space corresponds to the location/orientation of entities in the event space, and the number of predicates/hands corresponds to the number of entities. ${ }^{67}$

The DGS static scene descriptions show that signers, in fact, do not rely solely on the properties of classifier predicates in conveying information about location, orientation, and

\footnotetext{
${ }^{66}$ As noted already, though they have arguably different properties than real classifier predicates (e.g. the ability to use them for reference tracking), convention 2 was taken to obtain for the use of spatialized SASSes (e.g. to encode the tree).

${ }^{67}$ Because there are two manual articulators, there can be two referents visually depicted in sign space at a time. However, referents can exist conceptually at unique locations in sign space. For these conceptual locations, as well, it holds that the number of predicates corresponds to the number of referents (that are presented as different entities).
} 
number. The convention of "classifiers encode all spatial information" does not obtain for the following structures:

i. Referent location and/or orientation is represented with forms other than classifier predicates (non-classifier-predicate referent representation)

ii. Referent number is numerically quantified (numerals specify referent number)

iii. Space is demarcated with forms other than classifier predicates (prepositions mark location)

Exception (i) to convention 2: Non-classifier-predicate referent representation. The "classifiers encode all spatial information" convention stipulates that signers shall use the form that is most efficient, and that maximally exploits the iconic potential of the modality. The focus of analysis in this subsection is on the representation of the human entities, i.e. the men, in the stimulus pictures. For these featured entities, this means that classifiers should encode both location and orientation information. Thus, to conform to this convention, it is predicted that the intrinsically featured 2-legged entity classifier form should be used to encode the man's location and orientation, as its imagistic and diagrammatic iconic properties afford maximally transparent and economic expression.

Table 4.8 shows the distribution of different forms that were used to encode the location and/or orientation of the man in the man/tree (37) and man/man (34) stimulus pictures. ${ }^{68}$ For each form and combination of forms used (labeled (A) through (F)), the table indicates whether location or orientation, or both, were encoded. The data reveals that, in contrast to what is predicted by convention 2, signers did not primarily use the 2-legged classifier form to encode the man's location and orientation. The 2-legged classifier appeared alone in only $30 \%$ of the descriptions (21 out of 71 ) (see (A) in the table). Instead of a conventional classifier, the form that appeared most commonly across all descriptions was the directional predicate LOOK. (We consider below whether it might in fact be considered a type of classifier predicate.) Alone or in combination with a classifier predicate, it occurred in 47 out of 71 descriptions ( $66 \%$ in total) (in (B), (C), (D), and (E)). The different types of forms and combinations of forms in table 4.8 are discussed in more detail below.

\footnotetext{
${ }^{68}$ In the man/man stimulus picture descriptions, signers always used the same form (or combination of forms) to encode the location and orientation information of the two men. Thus, the men are not counted individually, but per picture.
} 
Table 4.8. Distribution of different forms used to encode the location $(+/-10 \mathrm{c})$ and orientation $(+/-$ ornt) of the man in the man/tree (37) and man/man (34) stimulus picture descriptions.

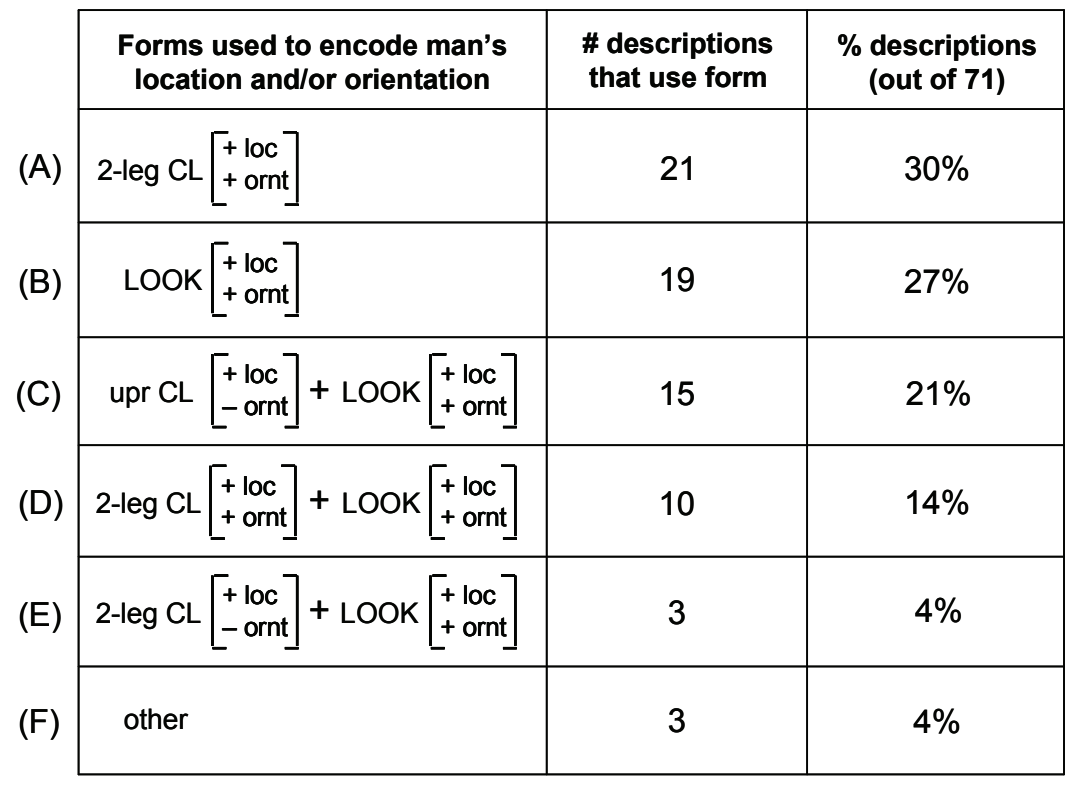

As the table shows, the three most common ways of encoding the man's location and orientation were (A) the 2-legged entity classifier alone (2-leg CL; 21 descriptions), (B) the use of LOOK alone (LOOK; 19 descriptions), and (C) the use of LOOK together with an unfeatured, upright entity classifier (upr CL + LOOK; 15 descriptions).
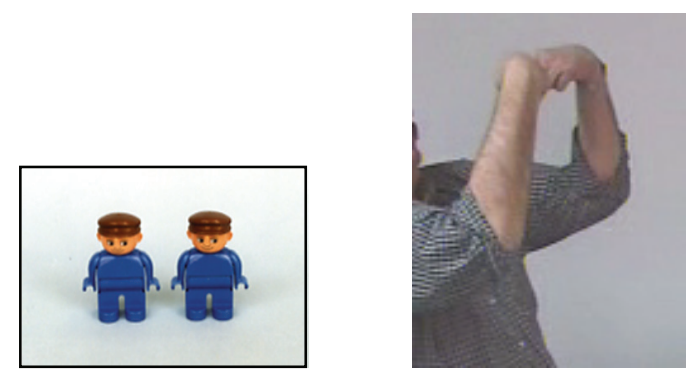

RH: $\quad \mathrm{CL}_{\mathrm{E}}(\mathrm{man})$ : locR,ornS

LH: $\quad \mathrm{CL}_{\mathrm{E}}(\mathrm{man})$ : locL,ornS

(12)
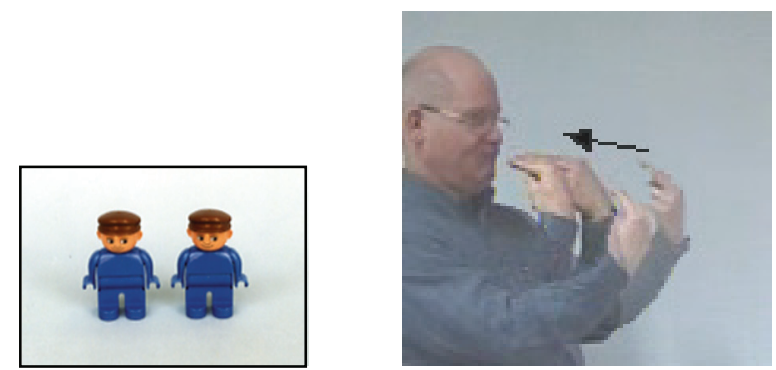

RH: $\quad$ LOOK: locR,movS

LH: LOOK: locL,movS 


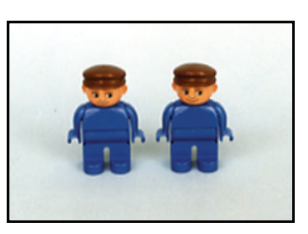

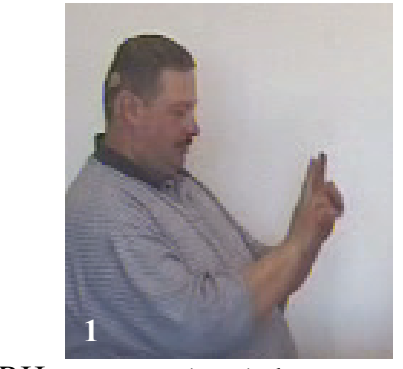

RH:
$\mathrm{CL}_{\mathrm{E}}(\mathrm{man}):$ locR $\mathrm{CL}_{\mathrm{E}}(\mathrm{man}):$ locL

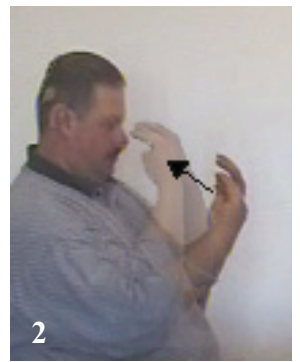

LOOK: locR,movS LOOK: locL, movS

Examples of their use, from three descriptions of the same stimulus picture, are shown in (11), (12), and (13) above, respectively. The examples show only the spatialization part of the descriptions. As it happened, all three signers placed classifier forms into space simultaneously, but each signer chose a different means of encoding the men's location and orientation.

In examples (11) and (12), a single form encodes both location and orientation. In (11), this is achieved through the use of a 2-legged entity classifier predicate ((A) in table 4.8). This use conforms to what is presumed by convention 2 of the canonical structure. In (12), in contrast, the signer uses what is not a classifier predicate, but actually a directional predicate, i.e. LOOK, whose movement through space indicates the direction of looking ((B) in table 4.8). As it is used in these descriptions, the starting location marks the location of the entity whose orientation or direction of looking is being encoded. The direction of the fingers, and the direction of movement indicates the direction of looking. That is, the initial location of the hands in space in (12) indicates the locations of the men, and the movement of the hands to the face signifies that the men are facing in the direction of the signer. (As indicated in table 4.8, all occurrences of LOOK were taken to encode both location and orientation.) The use of the LOOK predicate, which takes on the function of a classifier predicate in these descriptions, is discussed in more detail at the end of this section. Moreover, the LOOK predicate will be relevant in the analysis of the addressees' interpretations of spatial descriptions in section 5.5 of chapter 5 .

The third most common means of encoding the man's location and orientation is shown in example (13) (upr CL + LOOK; see (C) in table 4.8). The signer first simultaneously places upright entity classifiers into locations right and left in space (frame 1) to mark the entity locations. The upright entity classifier is unfeatured, and thus encodes only location, and not orientation. The placement of the entity classifiers is followed by the simultaneous use of the 
directional predicate LOOK (frame 2) to indicate the direction of facing of the entities. As in example (12), LOOK is directed to the face (specifically, the eyes), to indicate that the men are facing the signer. In all cases of "upr CL + LOOK" coding, location was expressed before orientation.

Finally, signers combined the 2-legged entity classifier with LOOK (see (D) in table 4.8). In 10 descriptions (labeled "2-leg CL + LOOK"), the 2-legged classifier was oriented in space such that both location and orientation were encoded (see frame 7 of example (14)), and was followed by LOOK to additionally encode the man's direction of facing (frame 8). As with the "upr CL + LOOK" coding described above, the 2-legged entity classifier always preceded the LOOK predicate.

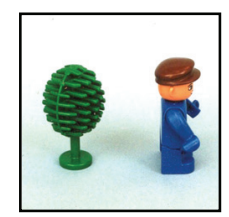

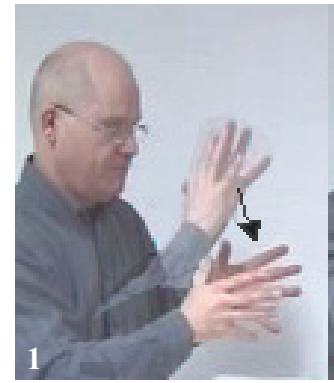

RH: tree + SASS(tree): locL $\mathrm{LH}$ tree $+\mathrm{SASS}($ tree): locL

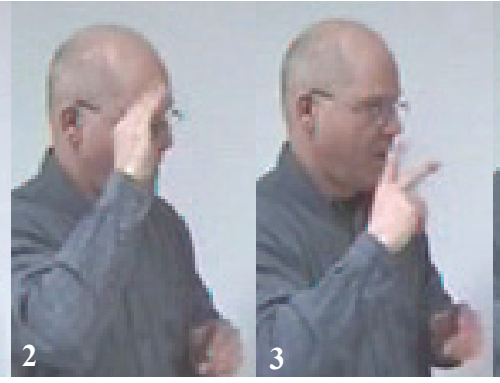

BLUE

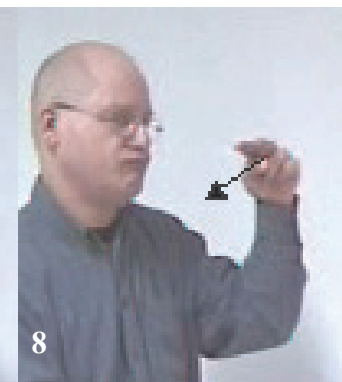

RH:

LH $\quad \mathrm{CL}_{\mathrm{E}}(\mathrm{man})$ : locR,ornR

LOOK: locR,movR

Interestingly, three further descriptions exhibited a combination of the 2-legged entity classifier and LOOK, but were unique in that they did not contain redundant orientation information. These descriptions are labeled "2-leg CL + LOOK", with the specification [+ loc / - ornt] for the classifier (see (E) in table 4.8). The orientation of the 2-legged entity classifier in these descriptions did not corresponded to the orientation of the man in the stimulus 
picture. Thus, these descriptions are functionally similar to those coded as "upr CL + LOOK" in that the necessary information about the man's direction of facing was provided only by the LOOK predicate that followed the entity classifier, and not by the classifier itself.

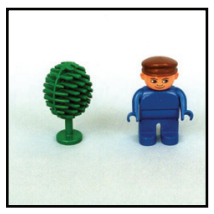

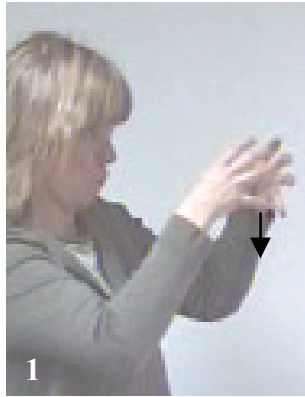

RH: tree + SASS(tree): locL

LH: $\quad$ tree + SASS(tree): locL

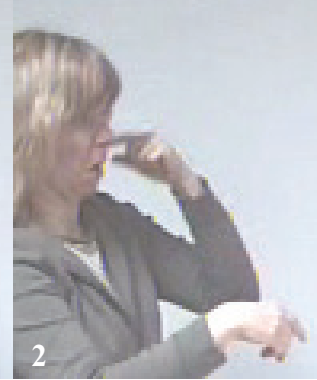

MAN

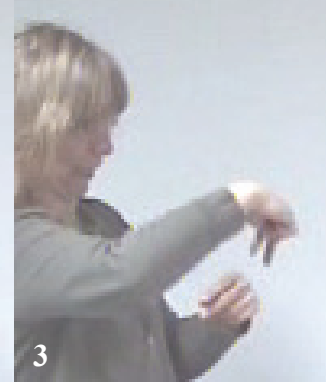

$\mathrm{CL}_{\mathrm{E}}(\mathrm{man}):$ locR

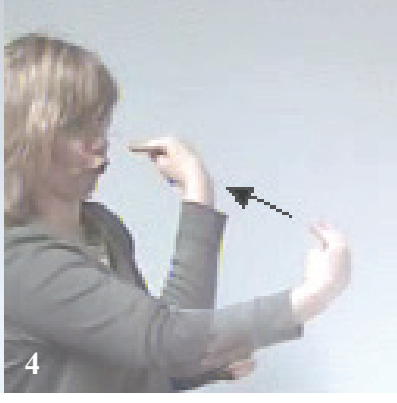

LOOK: locR,movS

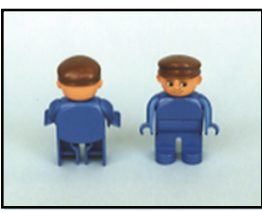

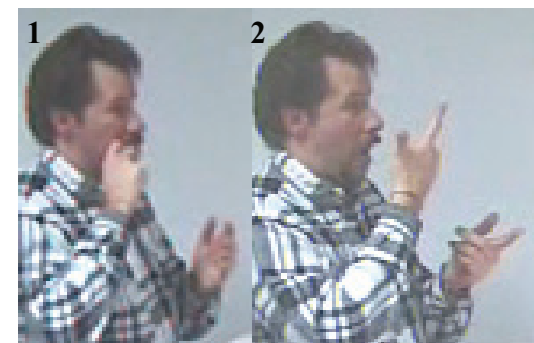

RH:

LH:

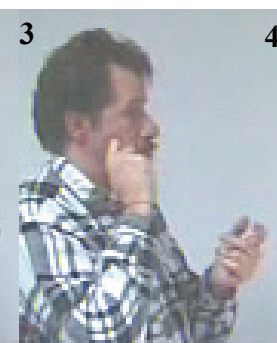

MAN

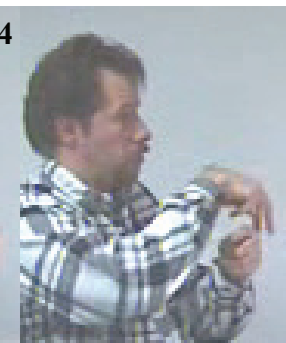

$\mathrm{CL}_{\mathrm{E}}(\mathrm{man}):$ locL

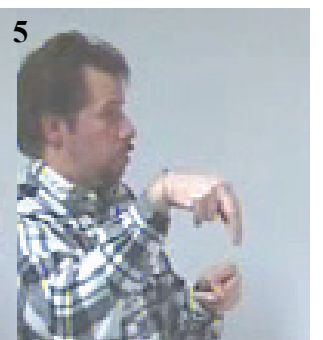

$\mathrm{CL}_{\mathrm{E}}$ (man): locR

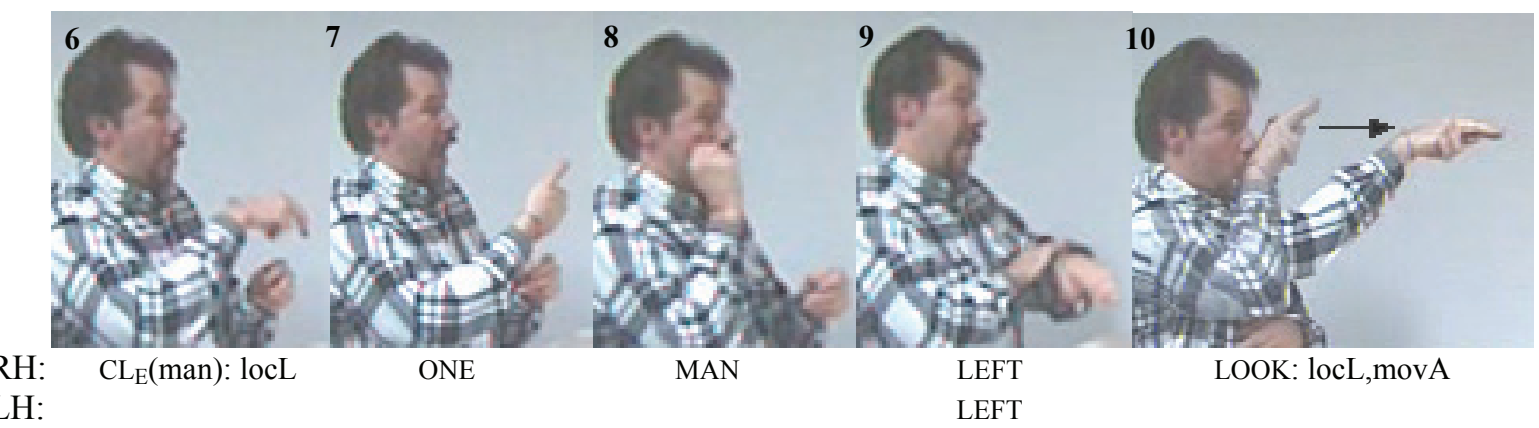




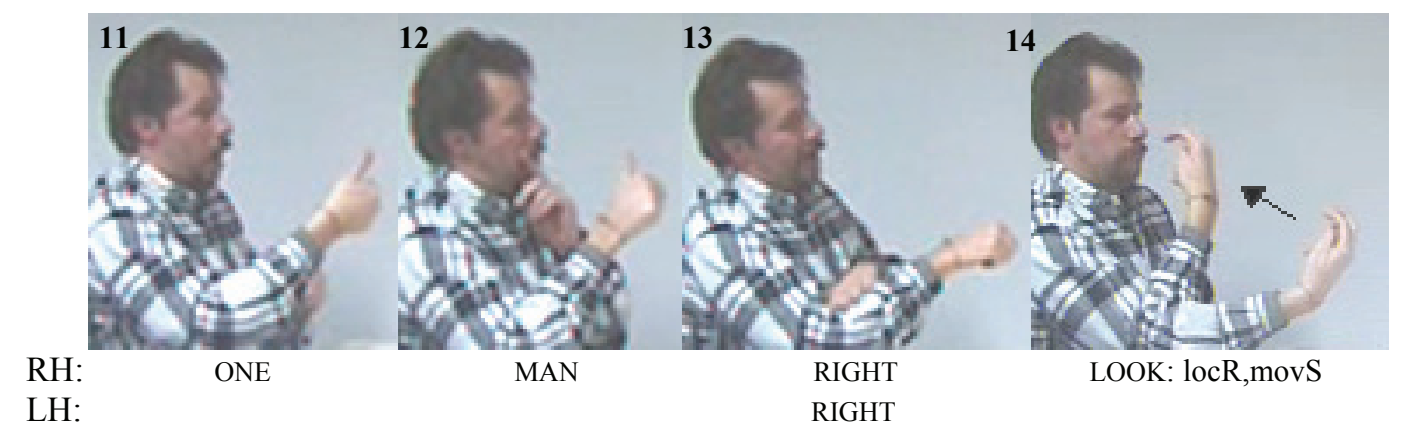

In these three descriptions (two of which are shown in examples (15) and (16) above), the entity classifier is oriented in space in the articulatorily easiest way, that is, with the inside of the hand held toward the body (see frame 3 in example (15) and frames 4 and 5 in example (16)). Crucially, the orientation of the entity classifier does not correspond to the orientation of the men in the stimulus pictures. ${ }^{69}$ The correct orientation information is conveyed only through the direction of the LOOK predicates used after the entity classifiers (see frame 4 in example (15) and frames 10 and 14 in example (16)). (There was one further occurrence of this type in the complex scene descriptions; it occurs in frame 12 of example (24) below.) Note that the signer in example (16) uses the lexical prepositions LEFT and RIGHT to make explicit to which entity each LOOK predicate corresponds. The use of prepositions is discussed later in this section.

The 2-legged entity classifiers that occur in the DGS corpus analyzed here encode orientation in the majority of cases. However, the existence of cases like we see in examples (15) and (16) suggests that the use of the 2-legged entity classifier does not necessarily entail orientation, but may rather only implicate it. This means that if no other information regarding orientation is provided, then the orientation indicated by the 2-legged classifier form is implicated. If other orientation information is provided, however, the orientation represented by the 2-legged classifier may conflict with it, as the classifier does not entail orientation. In the task at hand, it was obvious that both location and orientation needed to be conveyed. Signers depicted location and orientation with the 2-legged entity classifier even in the presence of other forms encoding orientation, i.e. LOOK. In other types of descriptions and discourse situations, we may expect more occurrences of the type illustrated in examples (15) and (16).

We can observe in the data that the 2-legged entity classifier was not the most commonly-used form for encoding location and orientation information across signers. In the

\footnotetext{
${ }^{69}$ The orientation of the 2-legged entity classifier in frame 4 of example (16) happens to coincide with the man's direction of facing.
} 
data, 19 of the 21 descriptions that used only the 2-legged entity classifier to encode location and orientation were signed by GL and LL, both of whom are DGS instructors, and who were partners for two groups of stimulus pictures. The remaining two descriptions were signed by LL's other partner, JP, and occurred in JP's descriptions directly after LL had been the director. For the first two descriptions in which a man appeared, JP used the 2-legged classifier form only, but then transitioned to using only LOOK (via a description containing the 2-legged classifier form together with LOOK). This suggests that JP may personally prefer to use LOOK to encode orientation, but that his signing style was initially influenced by his partner's (LL's) preference for the 2-legged entity classifier. Similarly, HT and SB, who were paired for all four groups of pictures, were together responsible for 13 out of the 15 "upr CL + LOOK" descriptions. The use of the other forms - LOOK only and "2-leg CL + LOOK" - were distributed across multiple signers. This distribution lends further support to the notion that DGS does not rely only on the use of a featured entity classifier, a maximally iconic form, to encode location and orientation information.

Finally, I return to the use of LOOK in the data. The prominent use of LOOK in the data overall (66\%) suggests that it is the directional predicate and not the featured entity classifier ( $30 \%$ alone, $14 \%$ with LOOK) that is favored for encoding orientation information. In the DGS descriptions in which it was used, the directional predicate LOOK took on the properties of a classifier predicate in encoding spatial information. The handshape encoded salient properties of the referent type (i.e. the fingertips correspond to the eyes, particularly, the direction of viewing, of an animate entity), and the location and orientation of the hand in sign space encoded information about the location and orientation of the referent in the event space.

We have seen that LOOK is used in conjunction with both non-featured and featured entity classifiers. That is, in some $25 \%$ of cases it bore the sole burden of encoding orientation, while in others (about 40\%) it encoded orientation redundantly. We have also seen cases in which LOOK alone carried the function of encoding orientation, even occurring in combination with the featured 2-legged entity classifier. In these descriptions, the orientation of the entity classifier did not correspond to the referent's orientation in the scene. Thus, as was stated already, though infrequent in the data, these occurrences suggest that only location may be truly entailed by the 2-legged entity classifier in DGS, while orientation is implicated in the absence of other information. Further research will help determine whether it is true that the 2-legged classifier may not entail orientation in DGS in all contexts. If it is true, it means that signers can create a spatial scene in sign space without committing to all 
types of spatial information encodable by classifier predicates. Thus, like the example of the cat on the fence from ASL (in section 4.1), DGS may have spatially "neutral" spatial forms.

Exception (ii) to convention 2: The use of numerals. In addition to the representation of location and orientation information, classifier predicates also represent the number of entities. In terms of iconic mappings, one classifier corresponds to one entity, and the number of entities can be appropriately and sufficiently communicated through the number of individual (entity) classifiers that the signer uses. Thus, the use of numerals to quantify the number of entities, even in the presence of classifiers, does not support convention 2 because signers are not relying fully, and thus solely, on the iconic potential of classifier predicates.

As before, we are looking only at the simple scenes, which contained only two entities. The use of numerals is thus restricted to the use of either ONE or TwO. The numeral TWO occurred in descriptions of man/man and cone/cone pictures, which contained two of the same type of entity. ONE occurred in descriptions of man/tree and man/man pictures. ${ }^{70}$ Table 4.9 below shows the distribution of numeral use across the different types of simple scene stimulus pictures.

Table 4.9. Number of descriptions that exhibited the use of numerals per type of simple scene stimulus picture.

\begin{tabular}{|c|c|c|}
\hline & $\begin{array}{c}\text { \# descriptions } \\
\text { with numeral }\end{array}$ & $\begin{array}{c}\text { total \# descriptions } \\
\text { per picture type }\end{array}$ \\
\hline Man/man pictures & 25 & $34(71 \%)$ \\
\hline Man/tree pictures & 5 & $37(14 \%)$ \\
\hline Cone/cone pictures & 6 & $17(35 \%)$ \\
\hline total descriptions & 36 & $88(41 \%)$ \\
\hline
\end{tabular}

Table 4.9 shows that TWO occurred in almost three-quarters of man/man descriptions (see example (6)) and in one-third of the cone/cone descriptions (see example (5)). Indeed, the numeral TwO was used at least once by all but one signer. Moreover, as examples (17) (frame 1) and (18) (frame 3) below show, TwO occurred in descriptions with simultaneous (example (17), frame 5) as well as sequential placement (example (18), frames 4 and 5) of entity classifiers. (See section 4.3.4 for a discussion of simultaneous and sequential classifier placement.) Only five descriptions of man/tree pictures contained a numeral (i.e. ONE), four 
of which were produced by SB (a late signer). The use of ONE may mark a contrast with the duality of entities in the other stimulus pictures. It may also be due to an influence from spoken German (where the use of an indefinite/definite article is obligatory; see BoyesBraem 1985 and Heßmann 2001 on register variation).
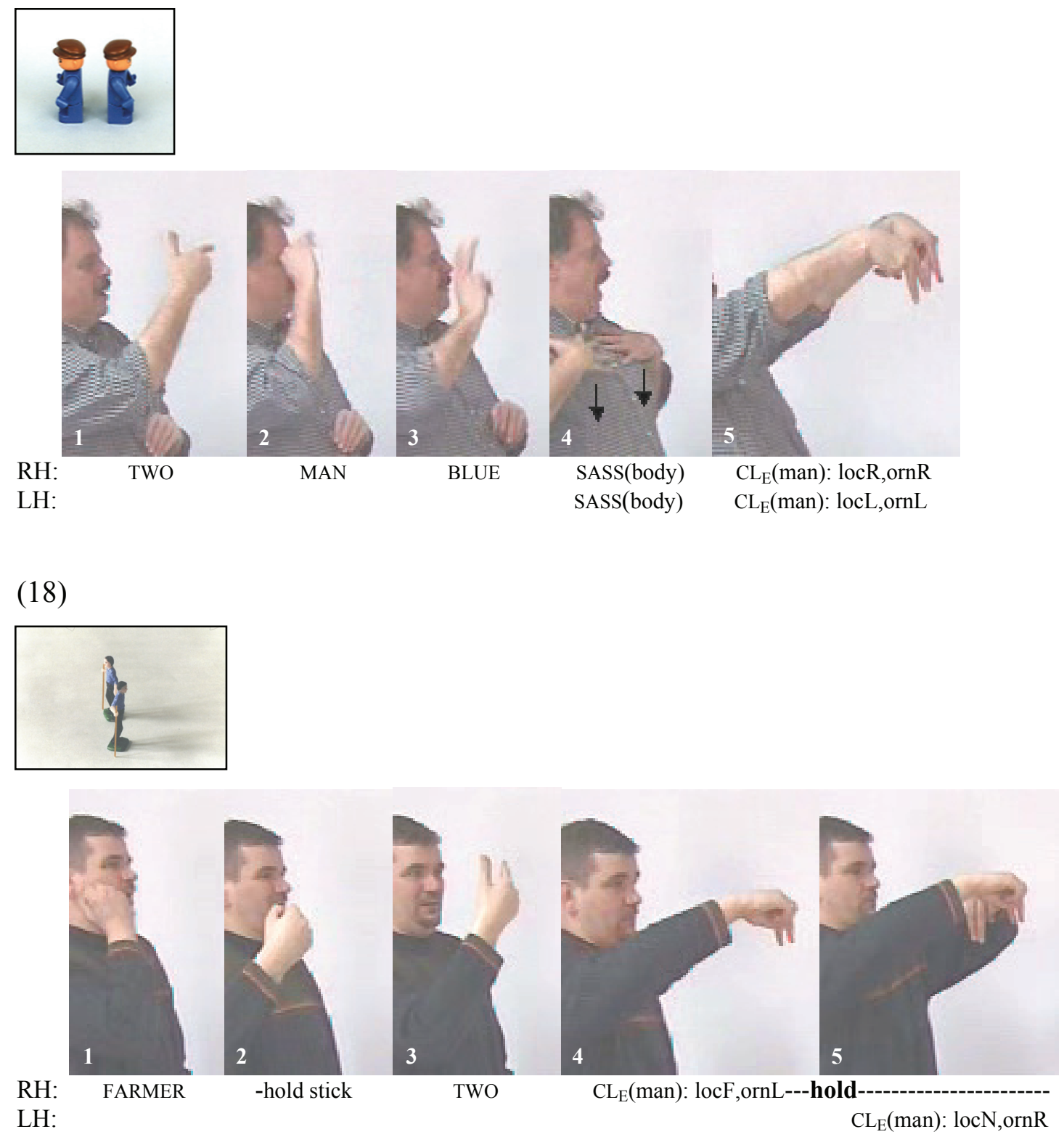

With regard to the co-occurrence of different exceptions to the conventions, TWO appeared together with "single identification" and "simultaneous spatialization" in almost all cases, but was not so frequent in descriptions that did not exhibit "single identification". This suggests that the use of numerals in DGS spatial descriptions is used as an explicit plural quantifier for

${ }^{70}$ When ONE occurred in man/man descriptions, it followed a previous use of TWO (see examples (16) and (30)). 
the inherently transnumeral noun phrase. This is supported by the use of numerals in the complex scene descriptions. Here, numerals occurred with "single identification" and "simultaneous spatialization" to explicitly encode the number of referents of the same specific type (e.g. FIVE pigs). However, numerals were not used to quantify different referents of the same ontological type (e.g. three different kinds of animals). (See further below for more on the use of numerals in complex scene descriptions.)

The results of a comparative study on the expression of number and quantification in DGS and TİD by Perniss and Özyürek (2004a) are also interesting in this respect. In this study, signers were asked to describe pictures that contained different amounts of the same type of referents (e.g. one, two, three, four, or many chairs). The overall results of the study showed that Turkish signers consistently used numerals more frequently and used classifier predicates less frequently than did German signers in their spatial descriptions. That is, German signers relied more on the use of classifier predicates to depict the spatial configuration as well as the number of referents in a scene, and additionally used numerals for explicit quantification in some cases. Interestingly, the difference between frequency of numeral use between German and Turkish signers was least pronounced where there were two referents of the same type. German signers used the numeral TwO comparatively more frequently than other numerals (like ONE, THREE, or FOUR). The use of TWO may underscore the special property of signed language of being able to represent two entities individually, yet at the same time through the use of the two manual articulators.

In general, the use of numerals in DGS may be motivated by the particular context, and may thus be constrained by particular discourse properties. For example, the presence of contrastive contexts may promote the use of numerals. Numerals may also be more likely to occur in static scene descriptions (where the focus is on object type, number, and configuration) than in other types of discourse. In addition, as mentioned above, the transnumerality of nouns in DGS may also motivate the use of an explicit quantifier to prefigure a plural interpretation of the noun phrase. The discussion of numeral use illustrates the interdependence of different discourse constraints.

Exception (iii) to convention 2: The use of prepositions. As has become clear, the diagrammatic iconicity of the visual-spatial modality makes it possible to create visual representations of spatial relationships in sign space, such that the relative placement of the hands depicts the relative locations of entities. As is assumed in convention 2, the arrangement of classifier predicates in space can sufficiently and efficiently mark the relevant 
locations in sign space. Thus, in this analysis, the use of other signs, for example, prepositions, to mark locations constitutes an exception to the "classifiers encode all spatial information" convention.

In the DGS data, a total of 12 simple scene descriptions (14\% of 88$)$ contained spatial prepositions. The prepositions that occurred were of two different types: (1) lexical prepositions (so-called because they have a fixed form), specifically LEFT and RIGHT, and (2) signs that can be glossed as NEXT-TO, which are spatially modifiable depending on the direction of proximity. ${ }^{71}$ The distribution of preposition use across the different types of simple scene stimulus pictures is shown in table 4.10 below. As the table shows, the spatially modifiable type of preposition occurred more frequently, and was used by five different signers. In contrast, the lexical prepositions LEFT and RIGHT appeared in only three descriptions, and were all produced by the same signer $(\mathrm{KO}){ }^{72}$ This same signer was also responsible for four of the nine descriptions that contained NEXT-TO signs (the man/man and man/tree descriptions, as well as one of the cone/cone descriptions).

Table 4.10. Number of descriptions that exhibited the use of prepositions per type of stimulus picture.

\begin{tabular}{|l|c|c|c|}
\hline & $\begin{array}{c}\text { \# descriptions } \\
\text { with LEFT/RIGHT }\end{array}$ & $\begin{array}{c}\text { \# descriptions } \\
\text { with NEXT-TO }\end{array}$ & $\begin{array}{c}\text { total \# } \\
\text { descriptions }\end{array}$ \\
\hline man/man pictures & 1 & 1 & 34 \\
\hline man/tree pictures & 1 & 2 & 37 \\
\hline cone/cone pictures & 1 & 6 & 17 \\
\hline total descriptions & $3(3 \%)$ & $9(10 \%)$ & 88 \\
\hline
\end{tabular}

Example (19) below shows a cone/cone description that contains a NEXT-TO preposition. NEXT-TO is generally bimanual, whereby both hands mark a location (e.g. the spatial location of one entity) and, from this location, one hand moves to another location (e.g. the location of a second entity). The sign is produced with a B-handshape, held vertically, and the movement is an upward arc from the start location to the finish location. Depending on the direction of movement, this form can be translated by English prepositional phrases like in front of, to the left of, to the right of, or in back of. In example (19), the NEXT-TO sign (frame 3) moves to the

\footnotetext{
${ }^{71}$ There was one occurrence of a lexical preposition expressing a topological relationship. The sign TOGETHER was used in a cone/cone description. The signer had set up the cones on the tabletop so close to each other that they were touching, and depicted the scene accordingly in his subsequent description.

${ }^{72}$ One description - shown in example (20) below - contained both kinds of preposition. Thus, there were 12 individual occurrences of prepositions, but in only 11 different descriptions.
} 
right, starting from the left hand hold of the bimanual size and shape specifier used for the first cone (frame 2). The signer continues to hold his left hand in place while he signs RED (frame 4), and then drops it for the production of the bimanual form that depicts the second cone (frame 5). The use of NEXT-TO here fulfills a function basically equivalent to that of a simultaneous classifier construction. At the end point of the sign (frame 3), both hands are present in sign space, simultaneously marking the locations of the two cones. (Compare example (19) to example (28) below. The two descriptions differ only in the presence of a NEXT-TO sign before the identification of the second referent. In (28) there is no instance within the description in which both referent locations are simultaneously represented in sign space.)

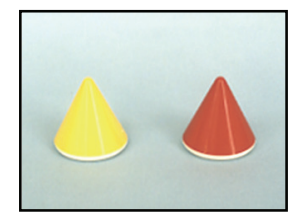

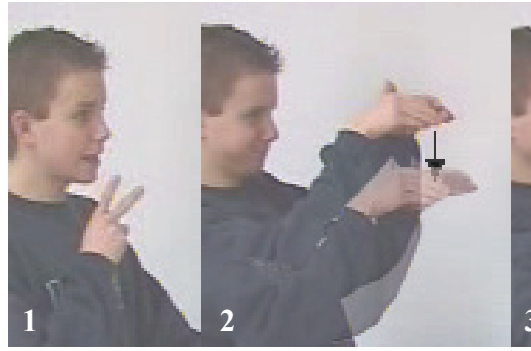

$\mathrm{RH}$ : LH:

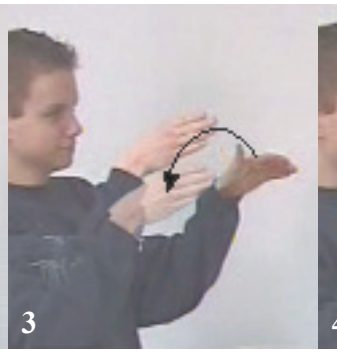

NEXT-TO: movR ---hold

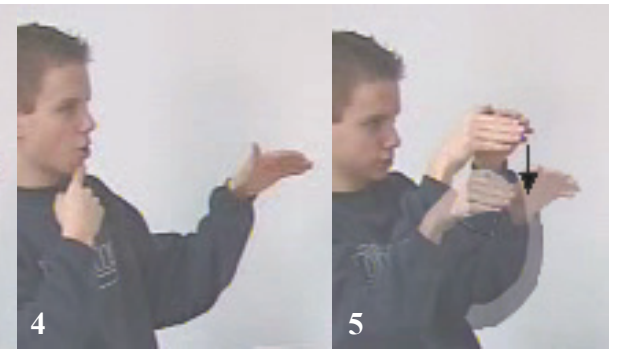

RED cone + SASS(cone): locR -cone + SASS(cone): locR

The next example, (20), contains both kinds of prepositions: a spatial NEXT-TO sign (again to the right) and the form-invariant, non-spatialized preposition LEFT. It is interesting to note the signer's choice of sign for expressing the man's orientation in this description. The signer moves his hand, in a B-handshape, away from the body as a general directional sign/gesture (frame 3). The direction of the sign's movement, and the direction in which the fingertips point, indicate the man's direction of facing. Before this, in frame 2, the signer uses the preposition LEFT, and not a classifier predicate, to indicate the man's location. Thus, neither location nor orientation is encoded with a classifier (or classifier-like form (e.g. LOOK)). The signer then signs NEXT-TO to the right (frame 4) to mark the tree's location. The left hand holds at the start location, which coincides with the man's location (but which is not a hold of the directional sign). Through the hold, the description ends with both referent locations visually depicted (frame 5). 


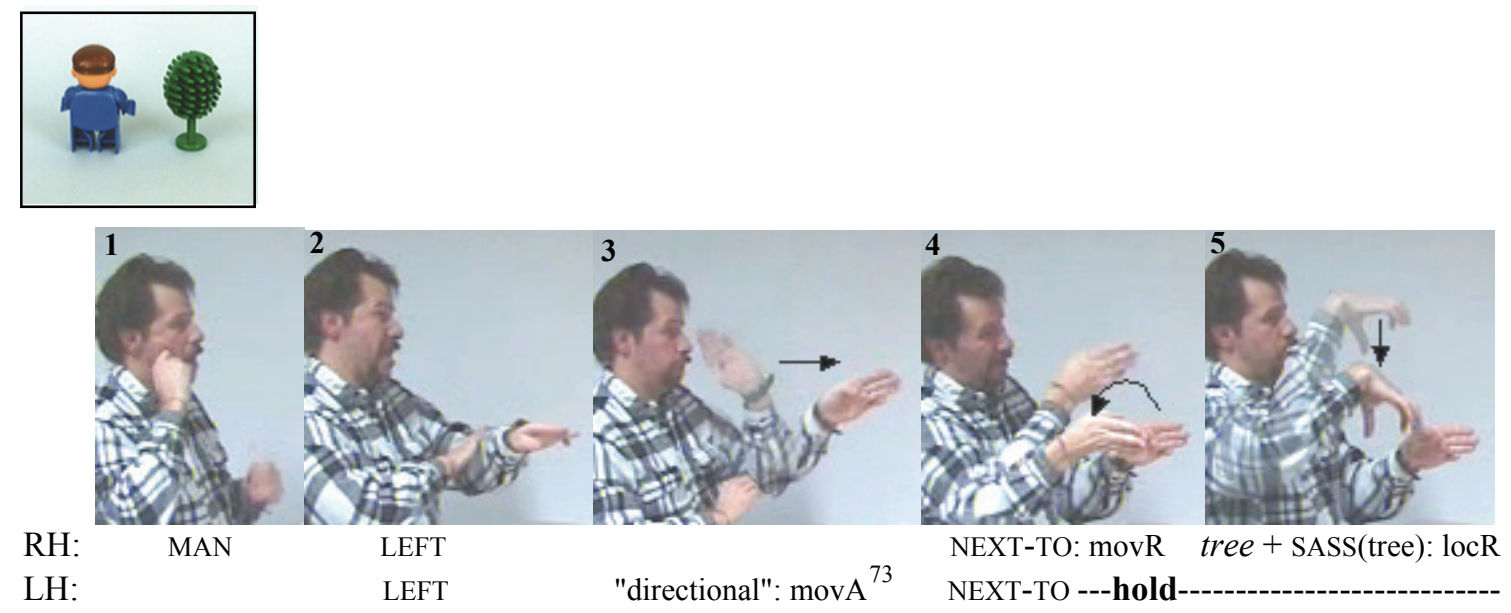

Though the data set produces too few instances of relevant cases to allow more than speculation, it is worth asking why prepositions occur comparatively more in the cone/cone descriptions than in the other descriptions. One reason may be related to the use of bimanual forms to represent the cones. In these cases, the use of a NEXT-TO preposition can insure that there will be a simultaneous representation of referent locations in the description. Through the hold of one hand at the starting location (i.e. the location of one entity) and the movement of the other hand to the end location (i.e. the location of the other entity), both entity locations are visually marked at the same time.

The use of a NEXT-TO preposition also emphasizes the figure-ground structure of the descriptions in which it appears by making explicit that with respect to the first entity at location $\mathrm{X}$, there is a second entity at location $\mathrm{Y}$. As with the numerals, the information provided by the preposition is redundant in that it is also encoded by the classifier predicates themselves. As suggested above, their occurrence may be more frequent under certain conditions (e.g. the presence of articulatory constraints), and possibly in certain discourse types, to make spatial relationships more explicit spatially.

The next section digresses from the simple scene descriptions to the complex scene descriptions, as a way of checking whether the observations so far are generalizable. The use of numerals and prepositions was quite prevalent in the complex scene descriptions. In addition, motion predicates and tracing signs were used to indicate referent configuration and the imposed motion of referents within the scene. The complex scene descriptions show considerably more variation than the simple scene descriptions in the forms used to encode spatial information.

\footnotetext{
${ }^{73}$ In table 4.8 above, this form is in the "other" category.
} 
Exceptions to convention 2 in the complex scene descriptions. This section gives an overview of the use of forms other than classifier predicates to encode spatial information in the complex scene descriptions. Four complex scene stimulus pictures contained a group of five or six pigs in some configuration around a man, with two trees in the back (see appendix A). Of the 12 descriptions of these pictures, only four individually depicted the location of every pig. The other eight descriptions used forms like the ones shown in examples (21) and (22) (the full descriptions in which these forms occurred are shown in examples (25) and (26) below). In example (21), the signer uses a general motion predicate to convey the random movement of the group of pigs - a sort of "milling about" - around the man. Similarly, example (22) shows a circle traced around the classifier predicate depicting the man to represent the configuration of the pigs encircling the man. In addition, the direction in which the circle is traced represents the pigs' direction of facing (counter-clockwise).
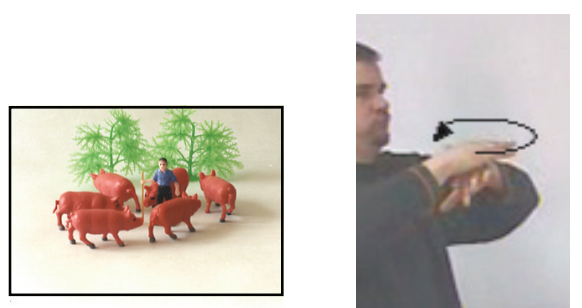

RH: MOT PRED: plural entity, circle

LH: MOT PRED: plural entity, circle

(22)

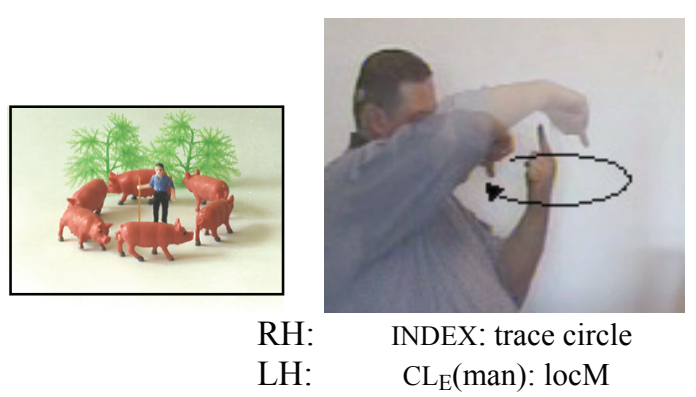

Both numerals and prepositions were used more frequently in the complex scene descriptions than in the simple scene descriptions. Prepositions occurred in 18 of the 34 (53\%) complex scene descriptions. Spatially modifiable NEXT-TO signs were common, and lexical prepositions like CENTER and INSIDE also occurred, but the lexical prepositions LEFT and RIGHT did not occur in the complex scene descriptions. This may be because the complex scenes contained more spatial contrasts than left/right only. The DGS prepositions LEFT and RIGHT may be used more readily to mark a binary contrast on the lateral axis. 
Examples (23) and (24) below show two descriptions of the same stimulus picture. Both descriptions use three NEXT-TO signs to mark entity locations in relation to a referent whose location is marked throughout the description by the hold of the non-dominant hand, thereby providing a spatial anchor for the scene.

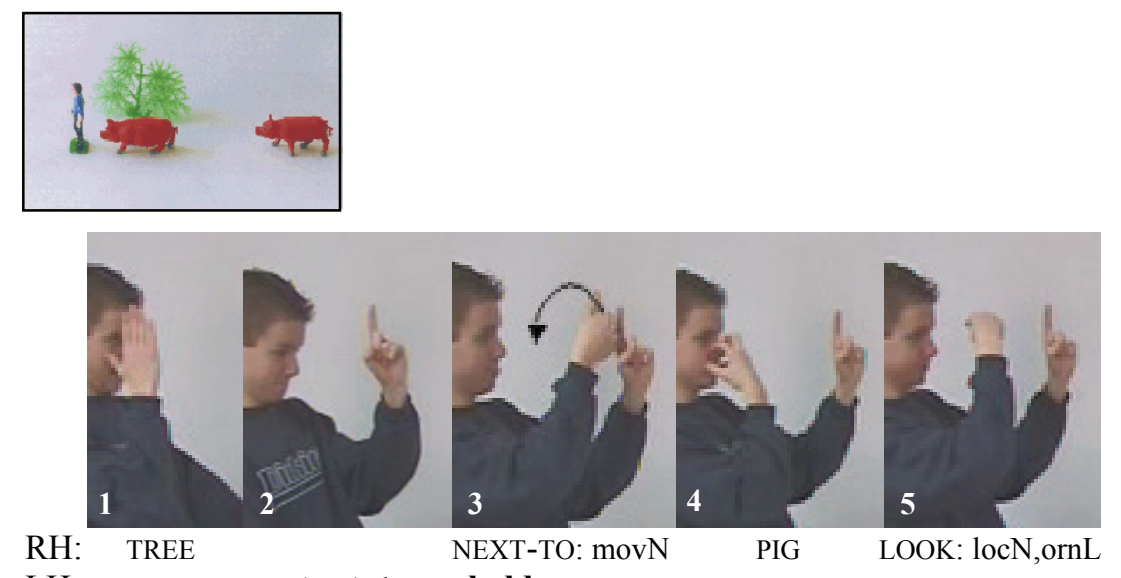

LH:

$\mathrm{CL}_{\mathrm{E}}$ (tree): locM--hold-
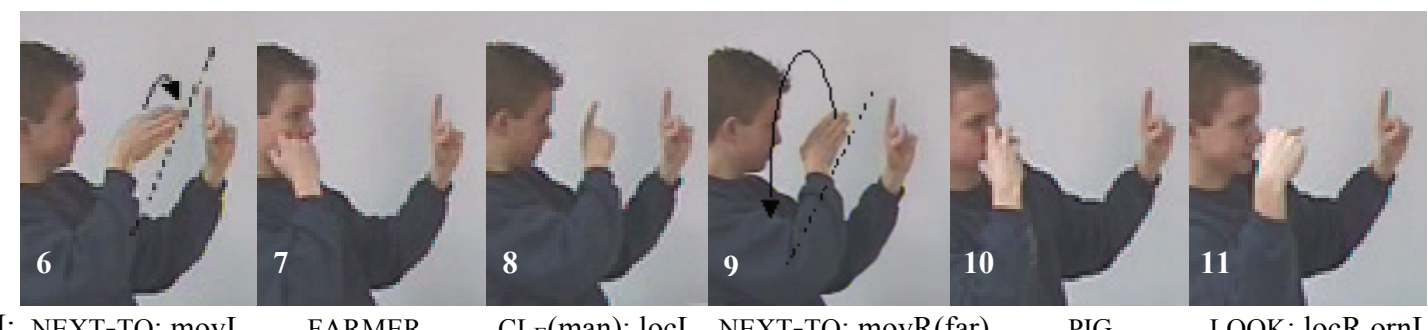

RH: NEXT-TO: movL

FARMER

$\mathrm{CL}_{\mathrm{E}}(\mathrm{man})$ : locL NEXT-TO: movR(far)

PIG

LOOK: locR,ornL

LH: ---hold-
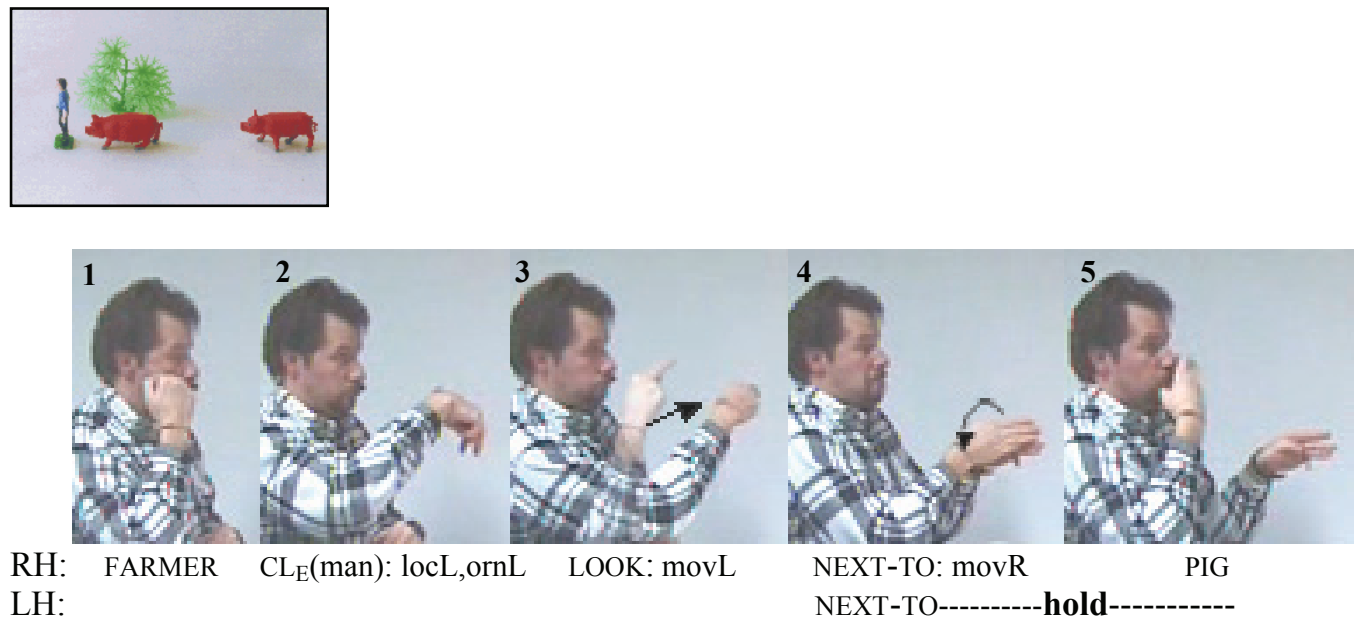


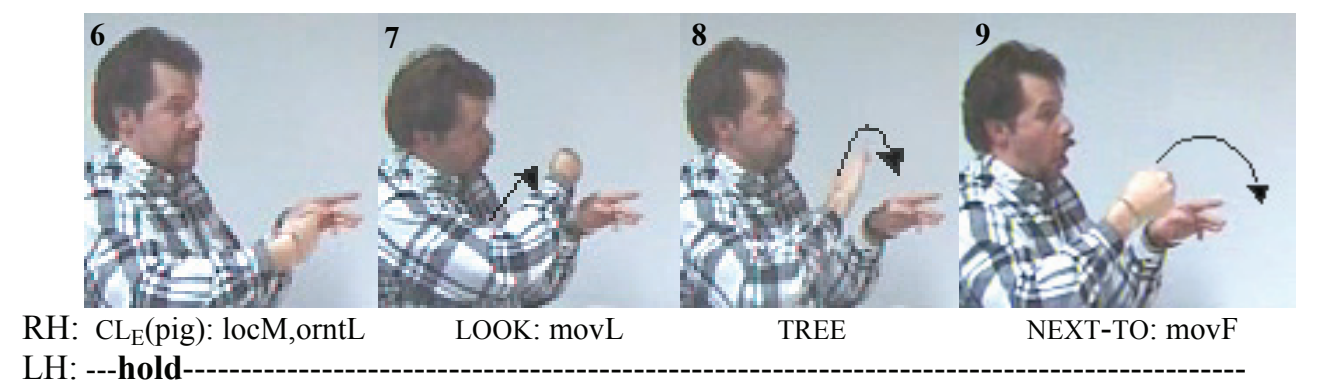

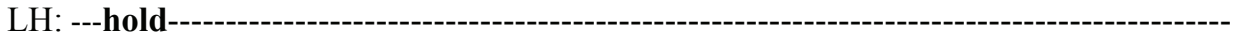

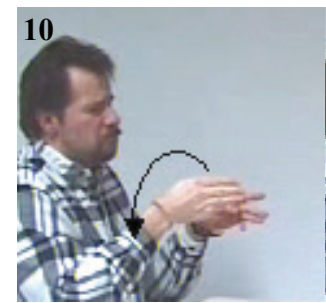

RH: NEXT-TO: movR(far) LH: ---hold-

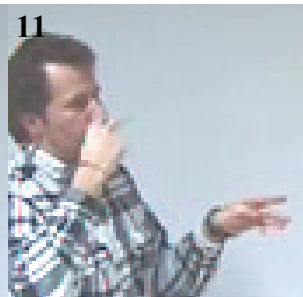

PIG

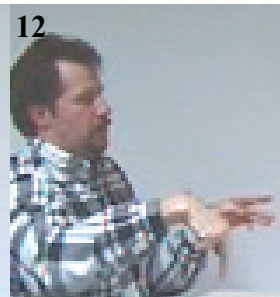

$\mathrm{CL}_{\mathrm{E}}$ (pig): locR

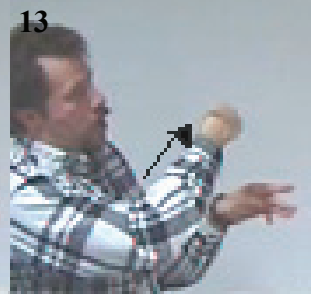

LOOK: movL

In (23), the description proceeds from the tree's location, marked with an upright classifier form. In contrast, in (24), it is the man's location from which the other referent locations are indicated, and the non-dominant hand of the first NEXT-TO sign (frame 4) replaces the initial entity classifier for the man (frame 2) to maintain a spatial anchor. The examples also show that the spatial modification of NEXT-TO can indicate not only direction, but also distance from a certain location, as shown in the contrast between frames 6 and 9 in example (23) and between frames 4 and 10 in example (24). The use of the non-dominant hand to hold backgrounded information in place is addressed again in section 4.3 .3 below.

Turning now to numerals, 24 of the 34 (71\%) complex scenes descriptions exhibited the use of numerals to indicate referent number. With multiple pictures that contained two or more entities of a type, the complex scenes provided opportunity for the use of numerals other than ONE and TWO. In the descriptions of pictures containing five or six pigs, all signers used numerals to identify their specific number. This was done both when the signer used classifiers to locate each individual pig and when the pigs' configuration was depicted with, for example, a general motion predicate (as shown in example (21) above) or a trace of the shape (as in example (22) above).

In addition, the overall count of the use of numerals was boosted through what appears to be a task-specific inventarization strategy developed between the partners HT and SB. These two signers began many of their descriptions by first giving an inventory of the objects in the scene and then starting the actual description, often repeating numerals within the description. Since the task required the addressee (i.e. the matcher) to construct the described 
scene from a set of plastic figures, the initial inventarization of entities could allow the addressee to reach for the appropriate figures before the actual description started, thereby minimizing the time between the description and the completion of the scene construction. Signers did sometimes spend some seconds picking figures from the pile after having seen the description, which means that the spatial information seen had to be held in memory longer before it could be reproduced.

The more frequent use of numerals in complex scene descriptions may also be explained in terms of memory load. As there are only two manual articulators, it is possible to directly encode the spatial relationship between maximally two referents at the same time. With just the location and orientation of individual classifier predicates to go on, addressees would have to keep in mind the locations of referents in sign space (including the number of locations), even in the absence of visual information, over lengthy stretches of discourse. In scenes that contain multiple referents, the use of numerals to encode the number of a particular type of referent may aid in the mental construction of the spatial scene from the signed description.

\subsubsection{Convention 3: Encode ground before figure}

The third convention that defines the canonical structure of locative constructions states that the ground object is encoded before the figure object in signed descriptions. To understand this, we must first clearly define what is meant by figure and ground. As a perceptual phenomenon, figure and ground assignment refers to the process by which a scene is organized into foregrounded (i.e. figure) and backgrounded (i.e. ground) regions. This segregation follows configural, image-based Gestalt principles like those of area, symmetry, convexity, and border closure (e.g. Rubin 1915/1958), but also cues like memory of object structure (Peterson et al. 2000), and familiarity (Vecera and O'Reilly 1998). The terms figure and ground were introduced into linguistics by Talmy $(1975,1978,1983)$. For Talmy, the figure is the entity that attracts the focal attention and whose situation or properties are characterized with respect to the ground, a reference entity in the periphery of attention.

Thus, Talmy draws a parallel between the focus on figures in visual perception and in language. Just as figural regions are visually discerned from backgrounded regions, so do the spatial schemas of languages allow speakers to specify the location or motion of a figure with respect to a larger ground (see also Talmy 2003). Importantly, the linguistically-determined definition of figure and ground has parallels with, but is not dependent on perceptual distinctions between figure and ground. That is, while Talmy takes the figure to be generally 
smaller, more salient, and more moveable than the bigger, more permanent and fixed ground, a linguistic figure-ground structure is also possible when the two objects are of the same type. Crucially, in utterances that exhibit a figure-ground structure, the figure is located with respect to the ground object.

The DGS descriptions that exhibit a figure-ground structure are tested according to two claims, both of which are found in the sign language literature referring to figure-ground constructions:

1. the ground object is encoded before the figure object

2. the non-dominant hand encodes the ground object

The analysis is divided into two parts, depending on whether the objects in the scene can be perceptually segregated into a figure and a ground object. This is the case for the man/tree pictures, where the tree is the larger, more permanent ground object, and the figure is the smaller, more moveable figure object. Thus, in order to support convention 3, the man/tree descriptions should encode the tree before the man (claim 1 above) and should use the nondominant hand to encode the tree (claim 2 above). ${ }^{74}$

The man/man and cone/cone pictures cannot be perceptually segregated into a figure and a ground, because the objects are of the same type. For these descriptions, the two claims above cannot be independently tested, because there is no independent (i.e. perceptuallydetermined) evidence for claim 1. The conformity of the man/man and cone/cone descriptions to the "ground before figure" convention of the hypothesized canonical locative construction must be tested by combining claims 1 and 2 . The first entity (i.e. the first man or the first cone encoded in each description, respectively), as the ground, should be encoded with the non-dominant hand. By testing the man/man and cone/cone descriptions in this way, it is possible to determine whether DGS has a truly linguistically-determined figure-ground structure that holds regardless of whether figure and ground status can be assigned on a perceptual basis.

\footnotetext{
${ }^{74}$ In the analysis that follows, hand dominance is defined in terms of dexterity. That is, the non-dominant hand refers to the left hand of right-handed signers and to the right hand of left-handed signers (see Friedman 1975; Krifka 2006; Nespor and Sandler 1999). Most people show a clear preference for the use of one hand (the more dexterous hand) over the other (the less dexterous hand). In signing, then, the more dexterous hand tends also to be the active or dominant hand, and the less dexterous hand is passive or non-dominant (cf. also Padden and Perlmutter (1987) on the distinction between the strong and weak hand). While dexterity and dominance generally go together, the "non-dominant hand" does not necessarily refer to the less dexterous hand (i.e. the left hand of right-handed signers and the right hand of left-handed signers) (see Frishberg's 1985 discussion of "dominance reversal"). Instead, the "non-dominant" hand can be more generally seen as the passive hand in a given construction. However, a test of the claim that the non-dominant hand has a function qua non-dominant hand requires a clear definition of what the non-dominant hand is. The strong correlation between dexterity and dominance provides the definition of the non-dominant hand which is used here to test this claim.
} 
Also note that convention 3 - "encode ground before figure" - can only be tested on descriptions that exhibit a sequential placement of classifier forms, thus locating one entity with respect to another. This excludes the $13 \mathrm{man} / \mathrm{man}$ and 3 cone/cone picture descriptions which were characterized by simultaneous spatialization (see table 4.7 in section 4.3.1). These descriptions were analyzed as single predications (as in the reciprocal relationship The two men are facing each other), and not as figure-ground expressions.

In addition to these descriptions, one man/tree description signed in character perspective is excluded from the analysis of figure-ground structure. In this description, the figure and ground entities are not both located in the sign space in front of the signer's body. This means that the convention that the non-dominant hand encodes the ground (while, by implication, the dominant hand encodes the figure) cannot be tested. The analysis thus includes a total of 71 descriptions, comprised of $36 \mathrm{man} /$ tree descriptions (excluding the character perspective description), $21 \mathrm{man} / \mathrm{man}$ descriptions (excluding 13 descriptions with simultaneous spatialization), and 14 cone/cone descriptions (excluding 3 descriptions with simultaneous spatialization). ${ }^{75}$

Testing convention 3 in the man/tree pictures, where there is perceptual figure-ground segregation. In the sign language literature, most examples of locative constructions involve referents that can be assigned figure and ground status based on perceptual features. In claiming that the ground object is encoded before the figure object, it is the bigger, more permanent object that is located in space before the smaller, more moveable object. In terms of referent type, the examples in the literature are comparable to the man/tree picture descriptions analyzed here, where the tree corresponds to the bigger, more permanent ground object, and the man to the smaller, more moveable figure object. In order to support the first claim above, namely that the ground is encoded before the figure, the DGS man/tree descriptions should encode the tree before the man. In addition, to support the second claim, the tree, as the ground, should be encoded by the non-dominant hand. (More precisely, the claim is that the non-dominant hand should provide spatial information about the ground entity. This does not have to be the same hand that is used to identify the entity.)

Table 4.11 below shows the distribution of man/tree descriptions as tested against the two claims. For both claims, each description can either pass or fail. Descriptions that pass on

${ }^{75}$ In table 4.6 in section 4.3 , the "encode ground before figure" convention is applied only to the total of 37
man/tree descriptions, based on scenes that could be naturally divided into figure and ground entities, as it 
claim 1 exhibit a tree-man order of encoding, while those that fail encode the man before the tree. For claim 2, those that pass encode the ground (i.e. the tree) with the non-dominant hand. Descriptions that fail on claim 2 use the dominant hand to encode the tree. In some descriptions, the tree is coded using a bimanual form. For these descriptions, claim 2 is taken to be non-applicable (NA), and they are coded separately.

Table 4.11. Distribution of man/tree descriptions tested against claim 1 (encode ground, i.e. tree, before figure, i.e. man) and claim 2 (use non-dominant hand to encode ground, i.e. tree).

\begin{tabular}{|c|c|c|c|}
\hline \multirow{2}{*}{ Conv. 2: ndom. hand encodes ground } & \multicolumn{2}{|c|}{ Conv. 1: encode ground before figure } & \multirow[b]{2}{*}{ total \# descriptions } \\
\hline & $\begin{array}{c}\text { pass } \\
\text { (tree-man order) }\end{array}$ & $\begin{array}{c}\text { fail } \\
\text { (man-tree order) }\end{array}$ & \\
\hline pass $($ ground $=$ ndom. hand $)$ & 16 & 3 & $19(53 \%)$ \\
\hline fail (ground = dom. hand) & 7 & 2 & $9(25 \%)$ \\
\hline NA (ground = biman.) & 7 & 1 & $8(22 \%)$ \\
\hline total \# descriptions & $30(83 \%)$ & $6(17 \%)$ & $36(100 \%)$ \\
\hline
\end{tabular}

As table 4.11 shows, the tsree is encoded before the man in the majority of descriptions (30 out of $36 ; 83 \%$ ), thus passing on claim 1 . Slightly over half of these descriptions also pass on claim 2 , in that the non-dominant hand is used to encode the tree (16 out of $30 ; 53 \%)$. About a quarter of the tree-man descriptions fail on claim 2, because the dominant hand encodes the tree $(7$ out of $30 ; 23 \%)$. The final quarter cannot be subsumed under convention 2 , because the tree is encoded bimanually, with no unimanual hold of the tree's location in space during the remainder of the description.

Let us return to the seven descriptions in which the tree is encoded before the man (pass claim 1), but in which the dominant hand encodes the tree (fail claim 2). Recall that the stimuli were comprised of pictures that showed the entities arranged both on the lateral (left/right) and sagittal (near/far) axes. Thus, for pictures that depict the tree on the right and the man on the left, there is a conflict between claims 1 and 2 that derives from the iconic mapping between the right and left sides of the event space, on the one hand, and of the sign space, on the other hand. If signers represent the ground object, i.e. the tree, first (passing on claim 1), the description fails on claim 2 because the tree is on their dominant-hand side. ${ }^{76}$

preceded the introduction of the non-dominant hand convention being tested here for scenes with entities of the same type.

${ }^{76}$ As mentioned, one of the signers who participated in the task is left-handed. Thus, the situation is reversed for this signer, since the right side is her non-dominant side. As it happens, however, this signer described only 
Table 4.12 below shows the distribution of man/tree descriptions, and their conformity to claims 1 and 2, based on the axial configuration of the two entities. As the schematic diagrams in the table show (under the heading "loc. of entities on axis"), an entity can appear in one of four locations, coded from the signer's viewpoint as on the signer's left, on the signer's right, near to the signer, or far from the signer.

Table 4.12. Distribution of man/tree descriptions according to order of encoding (claim 1) and hand used to encode ground (claim 2), and divided by entity location on lateral or sagittal axes. In the column labeled "loc. of entities on axis", the bigger shaded circle represents the tree and the smaller unshaded circle represents the man.

\begin{tabular}{|c|c|c|c|c|c|c|c|c|}
\hline $\begin{array}{c}\text { entities on } \\
\text { which axis? }\end{array}$ & \# descr. & $\begin{array}{c}\text { loc. of entities } \\
\text { on axis }\end{array}$ & \# descr. & $\begin{array}{r}\text { order of } \\
\text { encoding }\end{array}$ & $\begin{array}{l}\text { tree: non- } \\
\text { dom. hand }\end{array}$ & $\begin{array}{l}\text { tree: dom. } \\
\text { hand }\end{array}$ & $\begin{array}{c}\text { tree: } \\
\text { biman. }\end{array}$ & $\begin{array}{l}\text { total \# } \\
\text { descr. }\end{array}$ \\
\hline \multirow{4}{*}{$\begin{array}{l}\text { lateral axis } \\
\text { (left/right) }\end{array}$} & \multirow{4}{*}{20} & \multirow[b]{2}{*}{$\infty$} & \multirow{2}{*}{12} & tree-man & 8 & $\mathbf{0}$ & 3 & 11 \\
\hline & & & & man-tree & 1 & 0 & 0 & 1 \\
\hline & & & \multirow{2}{*}{8} & tree-man & $\mathbf{0}$ & 4 & 2 & 6 \\
\hline & & $\theta$ & & man-tree & 0 & 2 & 0 & 2 \\
\hline \multirow{4}{*}{$\begin{array}{c}\text { sagittal axis } \\
\text { (near/far) }\end{array}$} & \multirow{4}{*}{16} & & \multirow{2}{*}{8} & tree-man & 5 & 1 & 1 & 7 \\
\hline & & $\infty$ & & man-tree & 0 & 0 & 1 & 1 \\
\hline & & & \multirow{2}{*}{8} & tree-man & 3 & 2 & 1 & 6 \\
\hline & & $\infty$ & & man-tree & 1 & 1 & 0 & 2 \\
\hline tot. \# descr. & 36 & & 36 & & 19 & 9 & 8 & 36 \\
\hline
\end{tabular}

The distribution in table 4.12 shows that when the entities are located on the lateral axis, it is the left vs. right location of the ground entity that determines which hand is used to encode it (unless the tree is coded bimanually). Regardless of the order of mention, it is always the non-dominant (i.e. left) hand that encodes the tree for pictures in which the tree is on the left side of the picture as the signer views it (9 descriptions). ${ }^{77}$ When the tree is on the right side of the picture as seen by the signer, it is the dominant (i.e. right) hand that encodes the tree (6 descriptions). Thus, claim 2, which states that the non-dominant hand encodes the ground object (i.e. the tree) is overridden by iconic mapping principles.

three man/tree pictures, of which one was a sagittal axis and two were lateral axis pictures. In both lateral axis descriptions, the signer used a bimanual form to encode the tree.

${ }^{77}$ Note that the uneven distribution of lateral axis pictures with the tree on the left (12) vs. the tree on the right (8) introduces a positive bias with respect to the use of the non-dominant hand to encode the tree (claim 2). 
In the sagittal axis pictures, where the man and the tree are in near/far locations with respect to the viewer, there is no direct connection between the entity location and the hand that is used to encode it. In both types of sagittal axis pictures, the non-dominant hand could easily and equally be used to encode the tree. The analysis shows that the non-dominant hand is indeed used slightly more often than the dominant hand or a bimanual form for both the near and the far location of the tree.

For the man/tree picture descriptions analyzed in this section, perceptual features of the entities served as criteria for identifying a figure and a ground object. As the analysis has shown, the DGS descriptions support claim 1 (i.e. encode the ground object before the figure object) when a figure-ground distinction can be assigned on perceptual grounds. Moreover, we saw some evidence that the descriptions also support claim 2 (i.e. use the non-dominant hand to encode the ground). However, the use of the non-dominant hand to encode the ground is influenced by iconic principles, in that the location of the tree in the scene motivates the choice of hand to represent it. In the next section, we look more closely at the use of the non-dominant hand, and at its putative function of encoding the ground object, by ruling out the influence of visually-iconic mappings.

Testing convention 3 in the man/man and cone/cone pictures, where there is no perceptual figure-ground segregation. The man/man and cone/cone pictures feature two entities of the same kind, and thus provide no perceptual cues to identify figure and ground. This means that there is no independent criterion, outside of the description itself, for distinguishing between a figure and a ground. In contrast to the man/tree pictures, we cannot a priori identify the ground object based on its features (as we were able to call the tree the ground in the previous section).

This type of situation, or rather the difference between these two types of scenes, has not been systematically addressed in the literature. As I have said, the examples of locative constructions found in the literature generally contain entities that can be perceptually segregated into figure and ground. These examples have motivated the claim that sign languages encode the ground object before the figure object. What about the structure of locative constructions that describe the location of one object with respect to the location of another object of the same type? Another specification that appears in the literature with regard to the encoding of figure and ground is independent of perceptual features, and has been stated above as claim 2 . It specifies that the non-dominant hand functions to spatialize 
the ground object, and, more generally, to represent backgrounded information (cf. EngbergPedersen 1994; Miller 1994).

As mentioned above, to test the DGS man/man and cone/cone descriptions in terms of these claims about figure and ground, we must proceed from the assumption that the first entity (i.e. the first man or the first cone encoded in each description, respectively) is the ground and that this entity should be encoded with the non-dominant hand. If the first entity is systematically represented with the non-dominant hand when referents are of the same type (or cannot be perceptually segregated into figure and ground), this would provide evidence that DGS makes a purely linguistic distinction between figure and ground.

There were 35 descriptions of man/man and cone/cone pictures in which the two referents were sequentially (as opposed to simultaneously) located in sign space. These 35 descriptions (21 man/man and 14 cone/cone) can thus be tested against the "non-dominant hand encodes the ground" claim, where the ground is taken to be the first entity. Table 4.13 below shows the distribution of man/man and cone/cone picture descriptions according to the hand used to encode the first entity.

Table 4.13. Distribution of man/man and cone/cone descriptions tested against claims 1 and 2 (encode the first entity, as the ground, with the non-dominant hand).

\begin{tabular}{|l|c|c|}
\hline $\begin{array}{l}\text { first entity (i.e. ground) } \\
\text { encoded by: }\end{array}$ & $\begin{array}{c}\text { \# descriptions } \\
\text { man/man pictures }\end{array}$ & $\begin{array}{c}\text { \# descriptions } \\
\text { cone/cone pictures }\end{array}$ \\
\hline non-dominant hand & $6(29 \%)$ & $4(28.5 \%)$ \\
\hline dominant hand & $15(71 \%)$ & $4(28.5 \%)$ \\
\hline bimanually & $0(0 \%)$ & $6(43 \%)$ \\
\hline total \# descriptions & 21 & 14 \\
\hline
\end{tabular}

The data in the table shows that the non-dominant hand is not systematically, and not even predominantly, used in DGS static scene descriptions of this type. This suggests that the nondominant hand, in DGS, does not fulfill the function of encoding the ground qua nondominant hand. Furthermore, it suggests that there is no established linguistic figure-ground structure in DGS for scenes that contain entities that cannot be assigned figure and ground status based on perceptual features. The convention is established only for spatial scenes in which there is a clear perceptual distinction between a figure and a ground object (as in the man/tree scenes discussed in the previous section).

Because nearly all examples of simple locative constructions that occur in the literature (e.g. Emmorey 1996; Liddell 1980, 2003a for ASL, and Keller 1998; Leuninger and Happ 1997 for DGS) involve entities of different types that can be clearly segregated into a figure 
and a ground, it is not clear how locative descriptions in ASL, for example, would look for objects of the same type. In contrast, in Engberg-Pedersen's $(1993,1994)$ discussion of simultaneous constructions to express location and motion events in DSL, the holdmorpheme that represents the ground referent is typically associated with the non-dominant hand. This analysis includes representations of motion events in which both hands represent the same type of referent. For example, she describes a signed narrative of a basketball game, where the hands represent two basketball players. The non-dominant hand holds the backgrounded player visually present in space while the relative motion of the foregrounded player is represented on the dominant hand (1993: 283). Though this example comes from a motion event narrative, and not a simple locative description, it may be that DSL displays a systematic figure-ground structure across all spatial descriptions, including location and motion.

Testing convention 3 in the complex scene pictures. The complex scene descriptions are also relevant with regard to the question of figure-ground encoding, and thus some examples are given in this section. All of the complex scenes contained entities of different types that could be assigned figure-ground status based on perceptual features (e.g. the trees, the fences). However, at the same time, many of the complex scenes contained entities of the same, or essentially the same, type (i.e. the cows, horses, and pigs). Thus, the complex scenes can be analyzed in terms of the two claims that we have been testing — that is "encode the ground before the figure" (claim 1) and "use the non-dominant hand to encode the ground" (claim 2) — both for entities that can be perceptually segregated into a figure and a ground, and those that cannot. As previously mentioned, the coding of the complex scene descriptions has not included a systematic figure-ground analysis. Here, we can only give a general impression of the figure-ground structures found in the complex scene descriptions. On the one hand, it focuses on the order of referent encoding for referents that can be assigned figure-ground status by perceptual features. On the other hand, it focuses on the use of the non-dominant hand to encode the ground object, and to keep referents visually accessible as (back-)ground information.

Three-quarters of the complex scene descriptions (25 out of $34 ; 74 \%$ ) exhibited a ground-figure ordering in that they encoded the perceptually ground-like elements (i.e. the trees, the fence, the trough) before encoding the animate, figure referents. Of the eight signers that participated in the task, five consistently encoded the ground objects before the figure objects. The other three signers varied in whether they encoded the figure or the ground 
objects first. (Incidentally, these same three signers were responsible for the figure before ground encodings in the simple scene man/tree descriptions, showing that individual signers exhibit different usage patterns.)

In addition, the complex scene descriptions that included a human figure hint at the influence of an animacy hierarchy (cf. Comrie 1981; Yamamoto 1999) in determining the order of referent encoding, albeit in variable directions. In total, 20 descriptions included a human figure, in addition to trees and pigs. Of these, 6 descriptions exhibit an inanimate animate - human (tree - pig - man) ordering and 5 descriptions exhibit a human - animate inanimate (man - pig - tree) ordering. Thus, half of these complex scene descriptions (11 of $20 ; 55 \%$ ) assigned salience to the human figure. Either the man was placed in sign space last, as the foregrounded object within an already established background of trees and pigs (as in example (25) below), or the man was located first and the rest of the scene was built up around him (as in example (26) below).
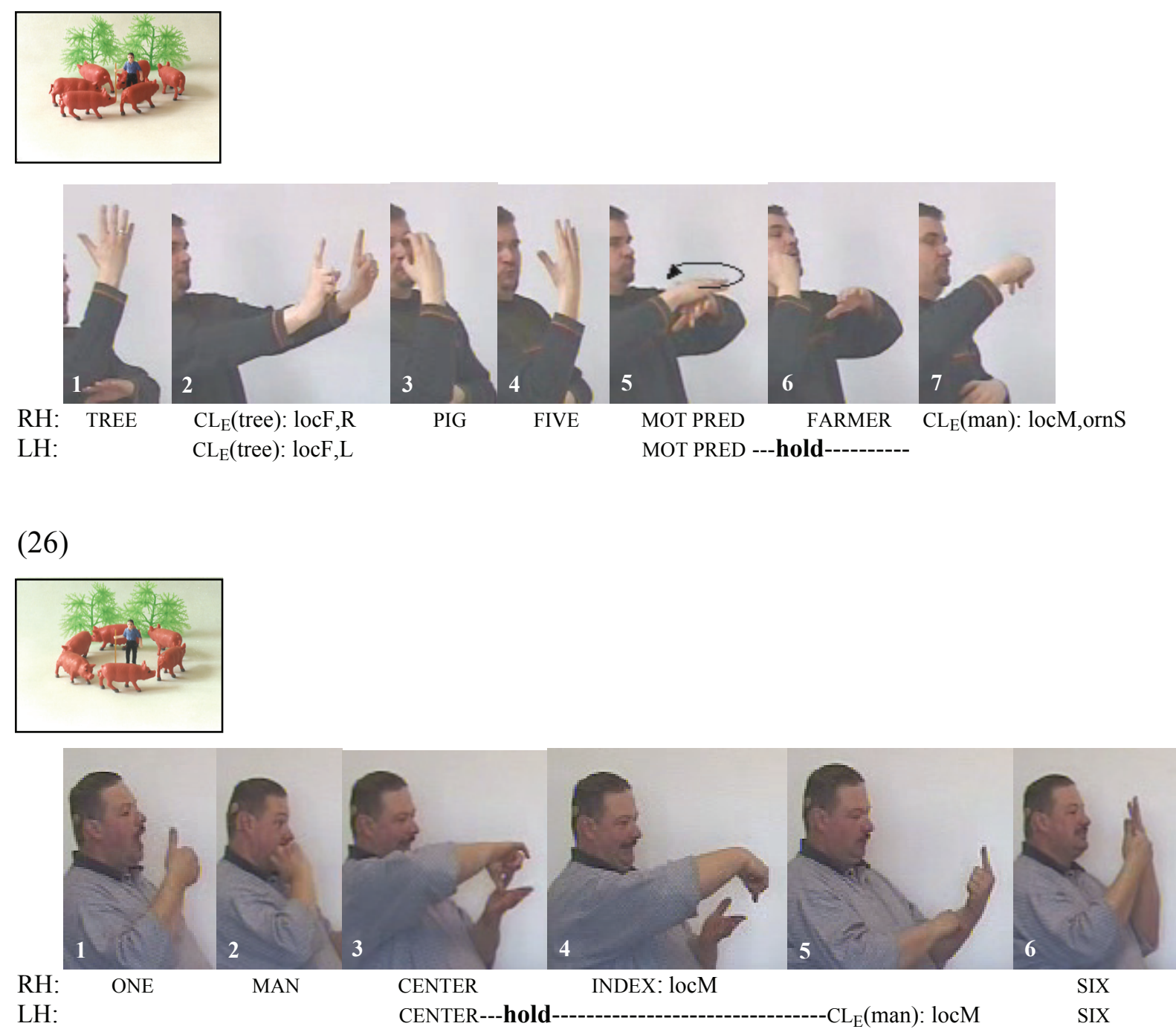

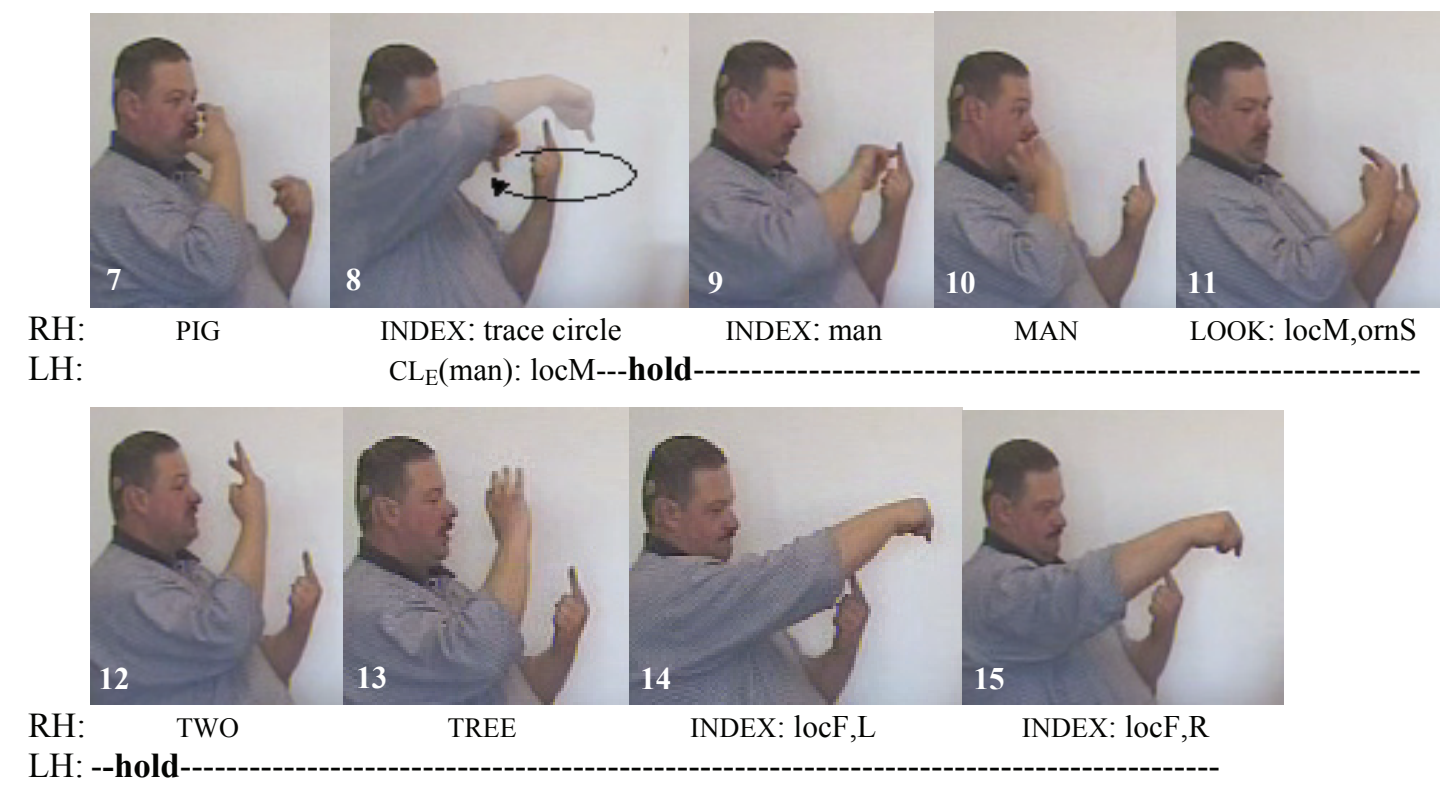

LH: --hold--

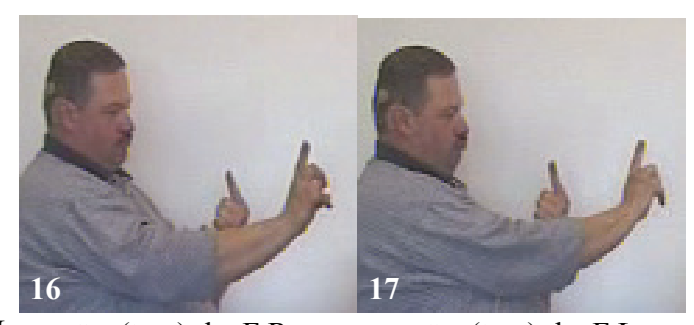

RH: $\quad \mathrm{CL}_{\mathrm{E}}$ (tree): locF, R $\quad \mathrm{CL}_{\mathrm{E}}$ (tree): locF,L

LH: --hold-

In some cases, the configuration of the entities in the scene seems to have influenced the order of encoding of referents. In the stimulus picture in example (27) below, the three animate entities are in a line on the sagittal axis. The pig - man - pig order of the entities is mirrored in the signer's description. Thus, the visual/perceptual order imposed onto the scene is reflected in the signed description.
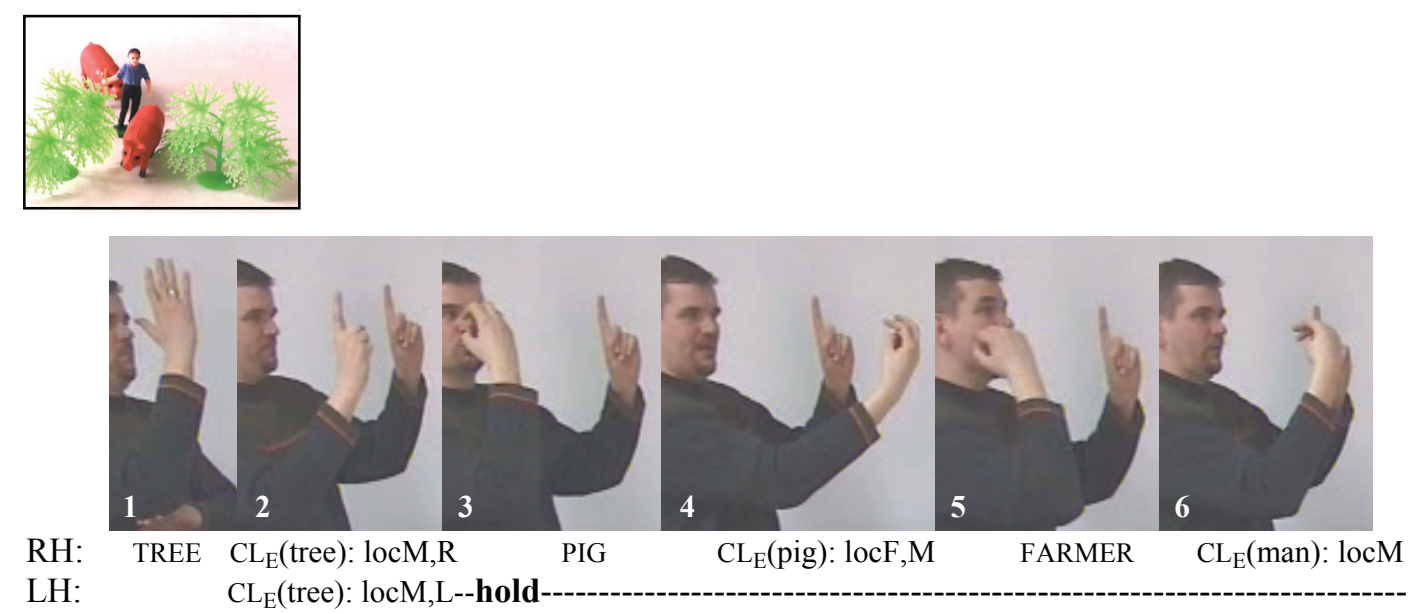


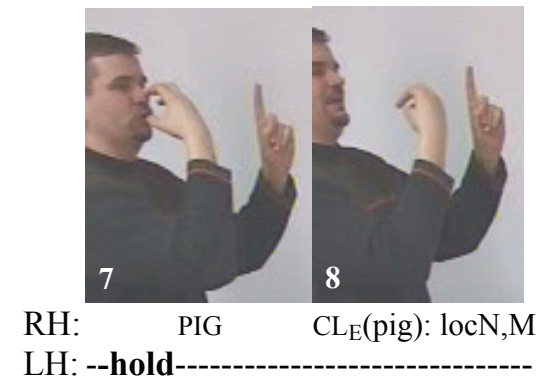

We now focus on the use of the non-dominant hand in complex scene descriptions. Examples (26) and (27) above both exhibit a prolonged hold of the non-dominant. In (26), the signer first locates the man in sign space with his non-dominant hand, and then holds the classifier predicate in place for the remainder of the description (continuously for frames 8 - 17). Before the placement of the upright entity classifier for the man (frame 5, resumed in frame 8), the signer uses a preposition (CENTER) to explicitly establish the man's location in the center of the scene (frame 3). All other information about the spatial array is given with respect to the man's central location in the scene. In (27), the signer holds the tree on the left in place during the depiction of the other entities (frames 3 - 8). Semantically, this makes explicit that the man and the pigs are walking through the trees. Here again, the nondominant hand holds the classifier in place, and the other referents are located in sign space with respect to it.

Thus, in both examples, the non-dominant hand plays an important role in that it provides a spatial anchor for the scene created, keeping a single referent (i.e. the man in example (26) and the tree in example (27)) visible in sign space throughout the description. In terms of information structure and figure-ground structure, regardless of perceptual features, the referent held in place by the non-dominant hand is the (back)ground(ed) object, with respect to which more focal referents are located.

Overall, the complex scene descriptions contained multiple examples of the use of the non-dominant hand to hold classifiers in place over significant portions of descriptions. However, as with the simple scene descriptions, the non-dominant hand was not systematically used to hold referents in place during the production of other signs. Sometimes there was no hold, and at other times the dominant hand was used. Thus, these results also suggest that the convention of using the non-dominant hand to encode the ground does not systematically hold in DGS. Yet, the non-dominant hand was used to maintain a referent in space across a stretch of discourse more often than the other alternatives. This suggests that the non-dominant hand does serve a discourse function in maintaining backgrounded 
information in DGS descriptions. Although I do not commit to its morphemic status, the nondominant hand here functions in a way similar to Engberg-Pedersen's hold-morpheme (as shown e.g. in examples (26) and (27)). In Engberg-Pedersen's (1993: 285) terms, when two classifier predicates (or polymorphemic verbs, as she calls them) occur simultaneously, "the one that takes the hold-morpheme at any given moment serves the Ground function". In Engberg-Pedersen (1994), this function is explicitly brought into connection with the passive, non-dominant hand (cf. also Krifka 2006). In using the non-dominant hand to provide a visible spatial anchor for the descriptions, the dominant hand - generally the more active and possibly dexterous hand - is free to perform the bulk of the signing. Thus, there may also be an articulatory component to the backgrounding discourse function of the nondominant hand.

\subsubsection{Convention 4: Use simultaneous classifier constructions}

As has become clear, simultaneity is an important component of sign language structure. It is a unique property of the visual modality that spatially and temporally co-occurring objects or events can be directly represented as such. Convention 4 of the hypothesis of the canonical structure of DGS locative descriptions being tested here states that the spatial relationship between two entities will be visually represented through a simultaneous classifier construction. Assuming a figure-ground structure (i.e. locating one referent with respect to another), the classifiers used to represent the entities should be placed into space sequentially, whereby the first hand remains in place through the identification and spatialization of the second referent. However, we have already seen that an utterance-final simultaneous classifier construction can also be achieved through the simultaneous placement of classifiers ("simultaneous spatialization" as I have called it in section 4.3.1). As noted in section 4.3.1, simultaneous spatialization occurs under special circumstances, namely when the two referents are of the same type and the spatial relationship between them can be expressed by means of a single predication.

The "simultaneous classifier construction" convention does not obtain only when there is sequential placement of two classifier predicates with no hold of the first classifier. Figure 4.3 below schematically depicts the three possibilities of visual and temporal coordination of classifier placement. The spatial relationship between the two entities is directly visually perceptible in sign space in (1) and (2), but not in (3). In (3), the relevant information about the first referent must be held in memory in order to understand the spatial relationship between the two referents. 


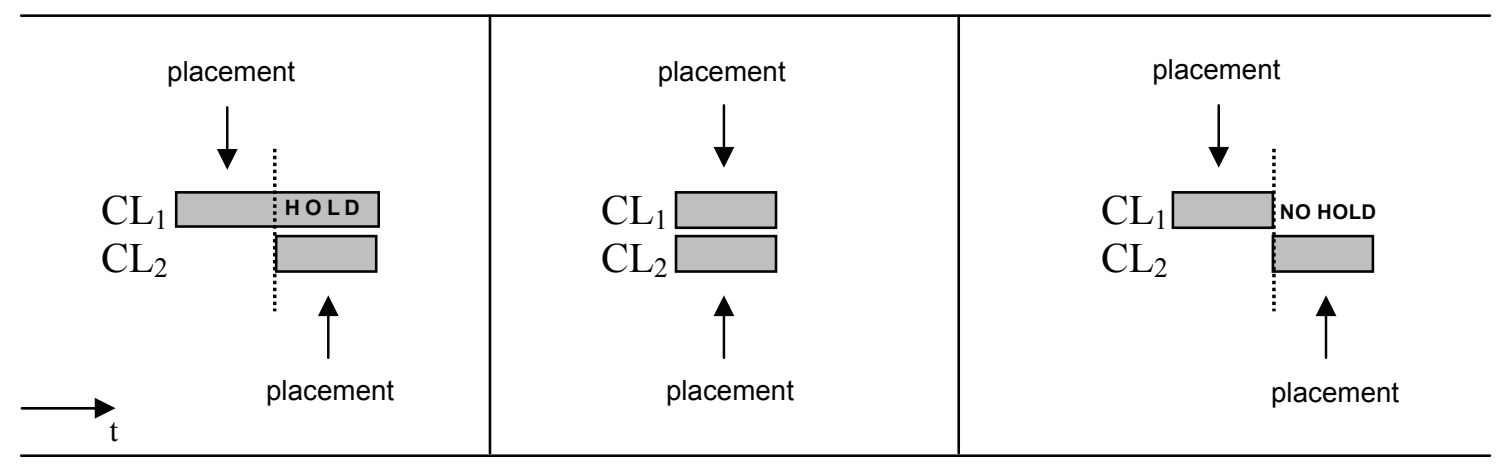
(1) Sequential with hold
(2) Simultaneous spatialization
(3) Sequential without hold (conv. 4 does not obtain)

Figure 4.3. Different possibilities of temporal and visual coordination of classifiers for representing the two entities $\left(\mathrm{CL}_{1}\right.$ and $\left.\mathrm{CL}_{2}\right)$ in the simple scene stimulus pictures. (Time (t) increases to the right.)

Table 4.14 below shows how these different possibilities of classifier placement were distributed across the three different types of simple scene stimulus pictures (man/tree, man/man, cone/cone). Note that the table also includes a row labeled "Multiple (all three)", in which one description is listed. In this cone/cone description, the signer coded the spatial relationship between the cones multiple times, using all three possibilities of classifier placement in the course of the description. As the table shows, three-quarters of all descriptions end with a simultaneous classifier construction $(73 \%)$, either through a hold of the first form or through immediate simultaneous placement of both forms. Only 23 descriptions (26\%) "violate" the "simultaneous classifier construction" convention by displaying no hold of the first classifier through the production of the second classifier.

Table 4.14. Distribution of the different types of classifier predicate placement across the three types of stimulus pictures.

\begin{tabular}{|l|c|c|c|c|}
\hline Type of classifier placement & man/tree & man/man & cone/cone & total \\
\hline 1. Sequential with hold & 27 & 18 & 3 & $48(55 \%)$ \\
\hline 2. Simultaneous & 0 & 13 & 3 & $16(18 \%)$ \\
\hline 3. Sequential without hold ("violation") & 10 & 3 & 10 & $23(26 \%)$ \\
\hline Multiple (all three types) & 0 & 0 & 1 & $1(1 \%)$ \\
\hline Total & 37 & 34 & 17 & 88 \\
\hline
\end{tabular}

More than half of the cone/cone descriptions have no hold of the first cone in space during the depiction of the second cone, exhibiting a "violation" of convention 4. One reason for this 
is the use of a bimanual classifier form to depict the cone, in which case a final simultaneous classifier construction is articulatorily blocked (see example (28) below). Note that the signer does hold one hand of the first cone during the production of the sign RED (frame 3 ), to identify the second cone. The hold is terminated, however, when it is needed for the bimanual trace of the second cone (frame 4).
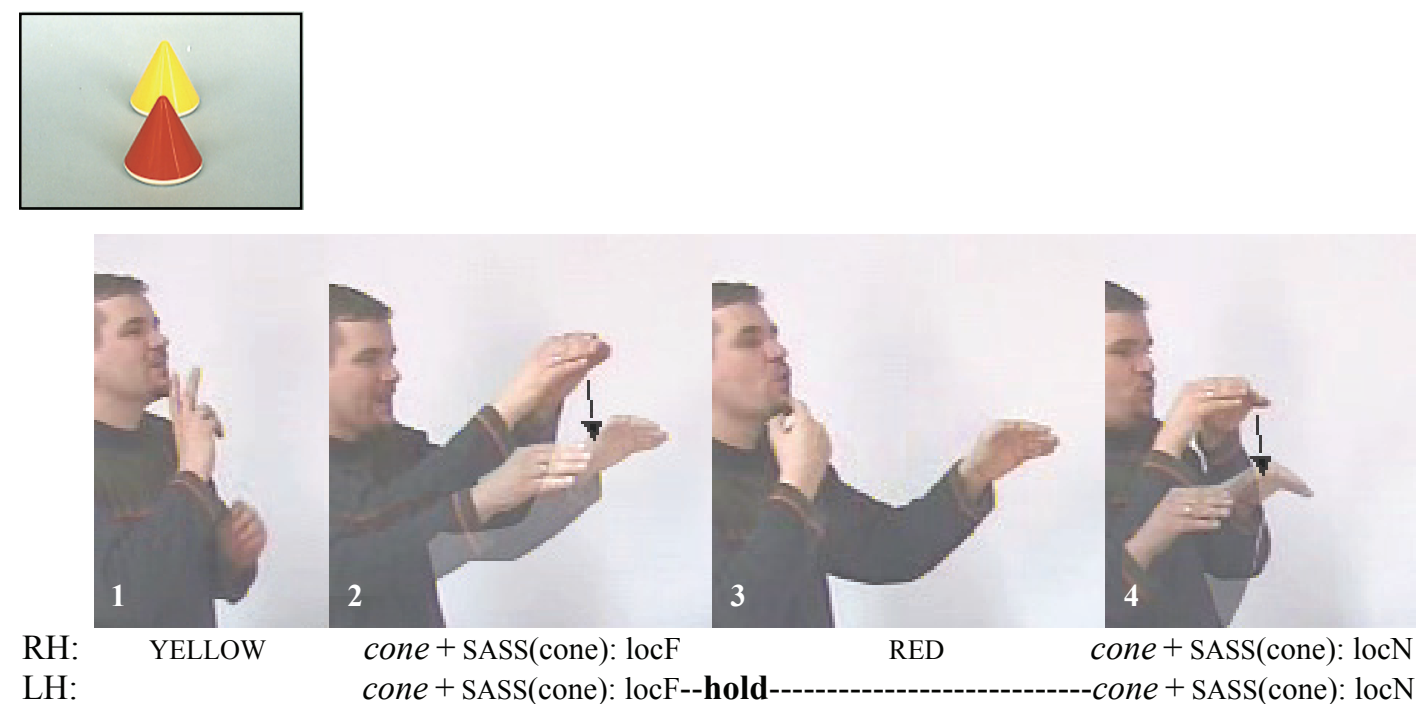

Not all ten of the no-hold cone/cone descriptions were due to such articulatory constraints, however. Bimanual forms were used for the cones in seven of the ten descriptions (by four different signers). ${ }^{78}$ The other three descriptions contained unimanual classifier forms for the cones, but did not display a hold of the first classifier. In these three descriptions, the same hand was used to encode the location of both entities.

Articulatory constraints also played a role in the man/tree descriptions that did not exhibit a simultaneous classifier construction. In eight of the ten no-hold man/tree descriptions, the tree was signed with a bimanual form. As can be seen in example (29) below, the signer first signs the tree, collapsing identification and spatialization of the entity into a single bimanual form (frame 1). He then introduces the man by describing the figure's salient physical attributes (blue body, brown hat). During the first of these signs (BLUE) (frame 2), the non-dominant left hand is held in place, marking the tree's location, but it is dropped for the subsequent bimanual sign (SASS(body)) (frame 3). The hold does not resume afterwards (which would be possible), and there is no final simultaneous classifier construction to indicate both locations in space. This particular signer (one of the native 
signers) is responsible for five of the eight man/tree descriptions that use a bimanual form for the tree. Thus, here again, we see that individual signer preference is strongly responsible for the variation in the data. In the two remaining man/tree descriptions that did not have a hold of the first classifier, the same hand was used to represent both entities.
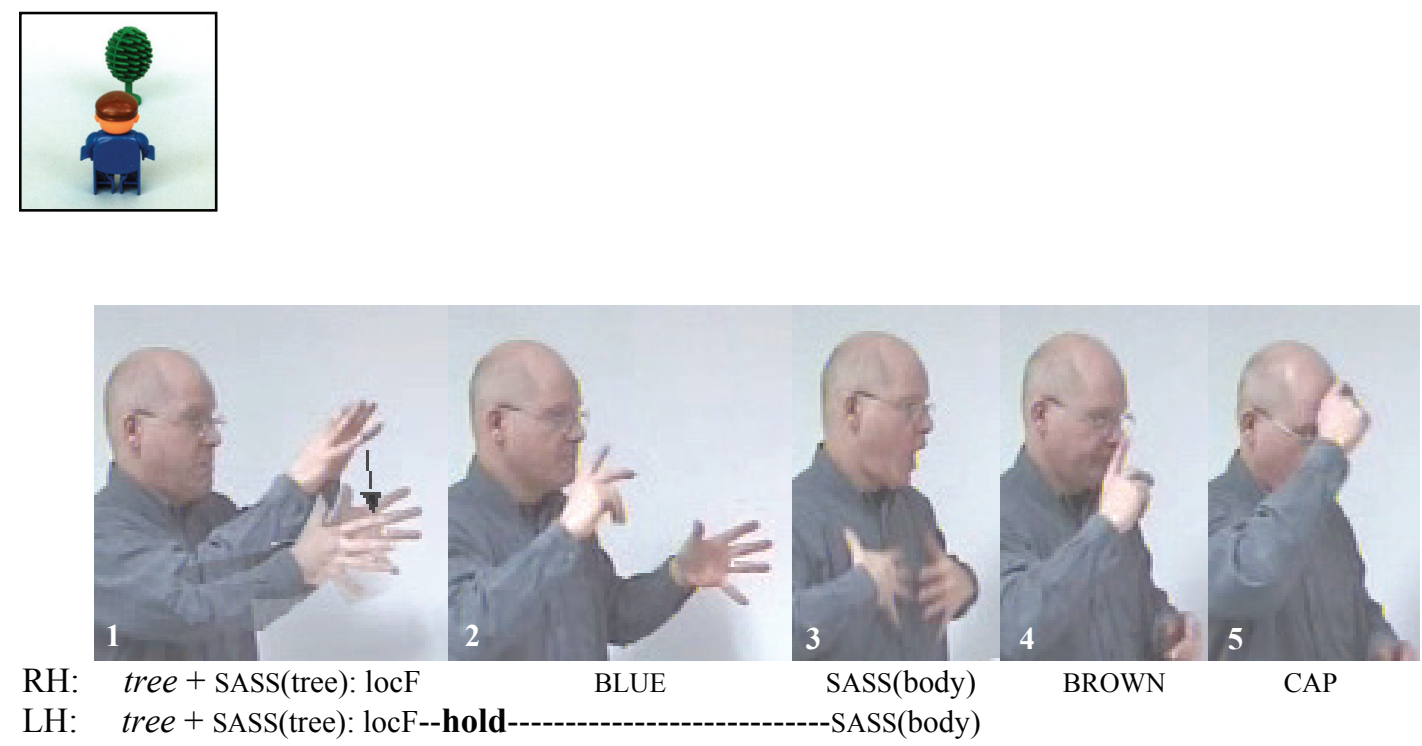

LH: $\quad$ tree + SASS(tree): locF--hold-----------------------SASS(body)

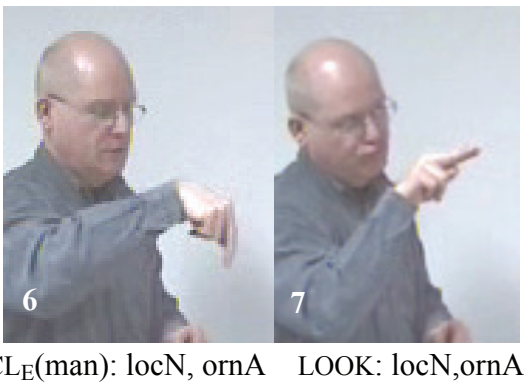

RH: $\mathrm{CL}_{\mathrm{E}}(\mathrm{man})$ : locN, ornA LOOK: $\operatorname{locN}$, ornA LH:

The use of the same hand for both entities also characterized the three man/man descriptions in which there was no hold of the first classifier predicate (as shown in example (30) below). The signer represents both entities with her dominant left hand, using the featured 2-legged entity classifier form in conjunction with LOOK. There was no example of a no-hold description in which one hand was used for the first entity, then dropped, and the other hand then depicted the second entity. This suggests that a sequential classifier placement with no hold of the first classifier may also be related to articulatory constraints. In using the same hand to encode both referents, the signer may avoid an articulatorily difficult hand orientation

\footnotetext{
${ }^{78}$ In six of these seven descriptions, the bimanual form was like the one shown in frames 2 and 4 of example (28). In the remaining description, the signer used his non-dominant hand as a base hand for both entities, tracing the shape of the cone with an upward movement of the dominant hand.
} 
(as would be encountered e.g. if frames 11 and 13 in example (30) were executed with the right hand).
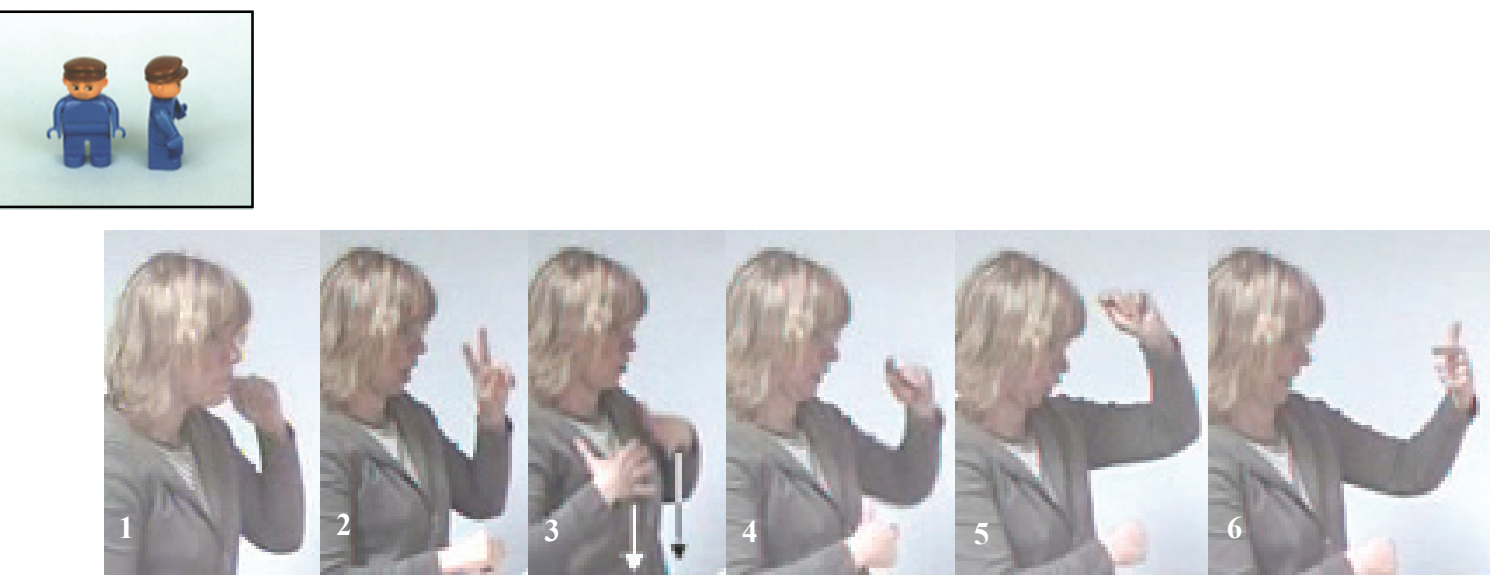

RH:

LH:

MAN

BLUE

SASS(body)

WITH

HAT

TWO

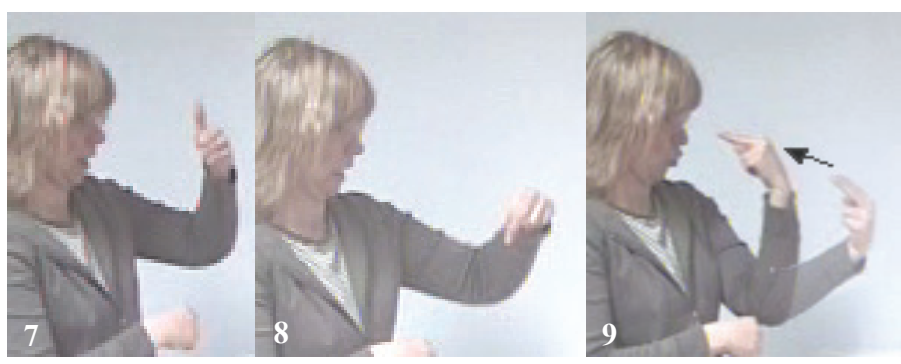

RH:

LH:

ONE $\quad \mathrm{CL}_{\mathrm{E}}(\mathrm{man})$ : locL,ornS

LOOK: locL,movS
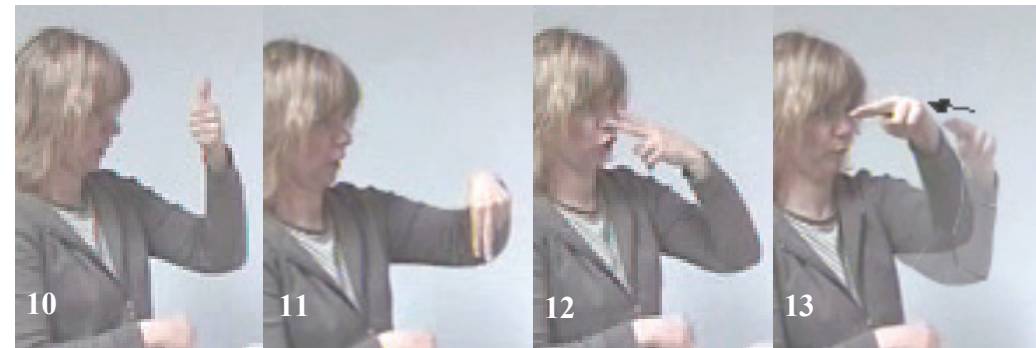

$\mathrm{RH}$ :

LH:

ONE

$\mathrm{CL}_{\mathrm{E}}$ (man): locR,ornR

LOOK

LOOK: locR,movR

To sum up, no-hold utterances have been analyzed here as exhibiting a "violation" of convention 4 , because they do not fully exploit the imagistic and diagrammatic iconicity afforded through the use of two independent manual articulators to represent two independent referents in a visually accessible spatial representation. However, no-hold descriptions do exploit the visual-spatial affordances of the modality in that they use distinct locations in space for the entities. That is, even though these descriptions do not simultaneously represent both entities in space, the separate locations of the entities in space are uniquely marked. Though the location of the first entity does not remain physically occupied by a classifier 
form, it remains conceptually occupied in the visual spatial array that the signer creates in sign space.

Testing convention 4 in the complex scene descriptions. These three types of classifier placement - that is, sequential placement with hold, sequential placement with no hold, and simultaneous placement - also characterize the complex scene descriptions. Take, for example, the three descriptions of the complex scene in which one pig is inside and a second pig is outside of a square fenced enclosure (see picture 3 in group 1 of the stimulus pictures in appendix A). In all three descriptions, the order of referents represented was the same: (1) the fence, (2) the pig inside the fence, (3) the pig outside the fence. In one of these three descriptions, the signer used a simultaneous classifier construction to depict both the spatial relationship between the first and second referents (i.e. the fence and the pig inside) and between the second and third referents (i.e. the pig inside and the pig outside). Thus, this description was characterized by sequential placement of classifiers with hold for both figureground pairs of referents. The signer held one hand of the fence in place while depicting the pig inside the fence, and then held this pig in place while depicting the pig outside the fence. The other two descriptions of this picture, however, contained a hold for only the second figure-ground pair of referents (i.e. the two pigs). That is, there was no hold of the fence during the representation of the pig's location inside the fence. These two descriptions display sequential placement of classifiers with no hold for the first pair of referents (i.e. the fence and the pig inside), and sequential placement of classifiers with hold for the second pair (i.e. the pig inside and the pig outside).

Finally, as noted already at the end of section 4.3.1, signers used simultaneous spatialization in complex scene descriptions to depict the locations of two trees or two pigs. Overall, the use of these three types of classifier placement in the complex scene descriptions is surely influenced by clause structure and prosody (Fischer 2005; van der Kooij et al. 2004; Sandler 1999). These issues are not investigated here, leaving a more detailed analysis of the structure of the complex scene descriptions to future research.

\subsubsection{Convention 5: Sign from the signer's (external) viewpoint}

The fifth and final convention states that signers should give spatial descriptions from their own vantage point, as they are viewing the scene. Stated as a principle of iconicity of perception, the most straightforward solution to the problem of spatial mapping onto sign space is for the signer to represent the scene as he/she has seen or experienced it (cf. 
Emmorey et al. 2002). There are two ways in which this convention does not obtain. On the one hand, signers can give descriptions from a different viewpoint. For example, descriptions can be given from the addressee's point of view (as in The man is to the left of the tree from your point of view) (see Emmorey 1996, 2002 for ASL; Schober 1993 for English). In sign space, descriptions from the addressee's point of view effectively involve a mental rotation of the scene by $180^{\circ}$ (see chapter 5 on addressee interpretation). On the other hand, instead of signing from the external vantage point from which they are viewing the scene, signers could place themselves within the scene, taking a character's perspective of the scene. This requires mapping an animate referent onto the body and describing the scene from that referent's scene-internal point of view. In this case, as discussed in section 3.5 of chapter 3 , the signer's body is the ground object with respect to which other objects are located.

Of all the static scene descriptions, simple and complex scenes combined, only two descriptions were not signed from the signer's own external viewpoint as the viewer of the scene. This conforms to the iconicity of perception principle and to the traditional notion of the topographic use of sign space (Klima and Bellugi 1979), whereby referent locations are schematically mapped from real-world space onto sign space, and a mapping from the signer's own point of view is implicitly assumed. However, one simple scene (of 88) and one complex scene (of 34) were signed not from the signer's external viewpoint of the scene, but rather from a scene-internal character's perspective. In these descriptions, the signer mapped the human referent in the scene onto his/her body. The locations of the other referents were represented with respect to the signer's location as a character in the scene. (These descriptions are discussed again in chapter 5 , in connection with the use of perspective and frames of reference in static scene spatial description.)

The choice of different manners of spatial mapping (e.g. the use of character perspective) may require some reorganization of the correspondence between the real-world event space and sign space. For example, in the case of the complex scene description from character perspective, the man in the scene that the signer mapped onto her body was not facing in the same direction as the signer. Thus, the signer had to, in effect, rotate the scene in order to make the representation in sign space correspond to the character's internal point of view.

The use of viewpoint and perspective may be influenced by different discourse types (see chapter 6 on the encoding of location and motion in event narratives). In addition to discourse constraints, linguistic and cognitive constraints may play a role in the choice of mapping between event space and sign space. Further research is needed to determine the 
influence of these different factors on viewpoint and perspective in signed spatial descriptions.

\subsection{Summary and discussion}

This chapter provided a detailed analysis of DGS static scene spatial descriptions. The analysis proceeded by testing the DGS data against five conventions that were hypothesized to represent the canonical structure of locative descriptions in DGS, and which are generally valid for most Western sign languages (as the literature review in section 4.1 showed). The analysis has discussed, on a descriptive-linguistic case-by-case basis, where the descriptions of simple and complex static scenes did not conform to the hypothesis put forward. We have seen that the order of referent identification followed by spatialization (convention 1) is influenced by linguistic and discourse constraints with regard to predication type and the interpretation of the subject NPs of the utterances. Furthermore, signers do not rely on classifier predicates alone to encode location, orientation, and number information about referents (convention 2). Faced with discourse as well as articulatory constraints, signers emphasize entity quantity and the spatial relationships between entities through the use of numerals and prepositional forms, respectively. Moreover, the data show that DGS signers rely heavily on the directional predicate LOOK to encode location, and especially orientation. LOOK carried the sole burden of encoding orientation when it appeared together with an upright entity classifier form, and sometimes also when it occurred with a 2-legged entity classifier. This suggests that the 2-legged entity classifier, in particular, may not entail orientation information in DGS, even though it is a featured classifier form. This suggests that DGS has spatial forms that do not necessarily commit to the information they can potentially encode. With respect to convention 3, concerning the order of figure-ground encoding, we saw that there is a systematic figure-ground order in DGS only for entities that can be perceptually segregated into a figure and a ground object, confirming the object-based claim mentioned with regard to DGS in the literature review in section 4.1. In contrast, there was no systematic use of the non-dominant hand to encode the ground objects in scenes that contained objects of the same kind, with no natural figure-ground. This indicates that there is no convention in DGS governing the linguistic structure of figure-ground constructions that is independent of referent type.

Finally, iconicity assumptions proved to play a strong role with respect to conventions 4 and 5. The majority of descriptions visually represented the spatial relationship between entities in sign space through the use of a simultaneous classifier construction, and signers 
almost invariably represented the spatial scene from their own vantage point as the viewer. Representation from the signer's viewpoint was predicted by the iconicity of perception principle, but may not be favored across sign languages to the same degree. For example, ASL signers showed a higher proportion of use of the addressee's viewpoint in static scene spatial descriptions (Emmorey 1996). In addition, in data from TID, signers represent referents on the sagittal axis in sign space (e.g. two cars, one behind the other), though the scene presented to them depicts the objects on the lateral axis (Arik 2006).

Overall, the analysis has shown that iconicity, as the general principle underlying the conventions taken to define the canonical structure of locative constructions, is a strong factor in determining the structure of locative constructions in DGS. Yet, it cannot account for all the variation found in the DGS static scene spatial descriptions, which rather suggests the influence of other constraints in shaping the descriptions in the data. For each convention, the role of different types of constraints has been discussed, including linguistic, discourse, and articulatory constraints. The analysis makes clear that the explanation of the structure of DGS locative constructions cannot rely on the assumption of iconicity alone, and that such an analysis must therefore form a part of any grammatical description of the language.

An analysis of the kind presented in this chapter, that is, of a corpus of locative constructions in an individual sign language, is necessary to delimit the effects of iconicity on spatial encoding in the visual-spatial modality, and to further our understanding of the different ways in which the structure of spatial descriptions may be constrained.

The next chapter analyzes the static scene spatial descriptions in terms of perspective and frame of reference use. This includes the encoding of figure and ground and the issue of viewpoint, which have already been addressed in sections 4.3.3 and 4.3.5 above, respectively. 


\section{Perspective and frames of reference in DGS static scene spatial description}

Chapter 5

In this chapter, I analyze the use of perspective and frames of reference in DGS static scene spatial descriptions. I remind the reader of the two systems here. Signing perspective refers to the way in which sign space is structured for spatial representation. Specifically, it defines the way in which an event space is projected onto sign space. When signers sign in observer perspective, they are external to the event space. Referents are represented in the area of space in front of the body, on which the entire event space, in a reduced size, is projected. In contrast, in character perspective, the signer is conceptually within the event space in the role of an animate referent. The event space is projected as life-sized, encompassing the signer, and other referents are represented as correspondingly large within the space.

Frames of reference are needed in order to specify the location of a figure object with respect to a spatially non-coincident ground object. In a relative frame of reference expression, the location of a figure is specified with respect to a ground from an external viewpoint that is different from both the figure and ground entities. The intrinsic frame of reference encodes a binary relationship between the figure and ground only, based on the inherent features of the ground object. Finally, the use of an absolute frame of reference depends on the availability of fixed bearings (e.g. a system of cardinal directions or landscape features) with which to specify the location of the figure entity with respect to the ground.

As argued in chapter 3 (section 3.5), perspective and frames of reference are independent systems. In representing a spatial scene, perspective is in fact a precondition for the use of a frame of reference for location specification. However, based on certain shared properties between perspective and frames of reference (shown in table 3.3 in section 3.5.1 of chapter 3), I have argued that prototypical alignments exist between them. On the one hand, character perspective aligns or prototypically co-occurs with the intrinsic frame of reference. In character perspective, referent location is represented in relation to the character mapped onto the signer's body. Thus, expressed in intrinsic frame of reference terms, the character is the ground object with respect to which another, figure, object is located. On the other hand, observer perspective aligns with the relative frame of reference. The signer's external vantage 
point as the observer of the scene corresponds to the deictic origo whose viewpoint defines the relationship between figure and ground in the relative frame.

Due to the visual properties of the modality, it was furthermore argued in chapter 3 that the relative and intrinsic frames of reference can simultaneously occur within a perspective. Thus, in character perspective, in the life-sized event space projection, the signer can indicate the relationship of two referents from the character's point of view. The character is the deictic origo for the relative frame of reference expression, but simultaneously remains the ground with respect to which both referents are located. In observer perspective, a featured ground object allows an intrinsic orientational relationship between the figure and the ground to be simultaneously encoded with the relative relationship between them, as seen e.g. from the signer's point of view.

This chapter presents an analysis of perspective and frame of reference use in static scene spatial descriptions in DGS. If we see correspondences between the use of observer perspective and the relative frame of reference, on the one hand, and between the use of character perspective and the intrinsic frame of reference, on the other hand, we will have evidence for the alignments presented above (and in chapter 3). If the data also provides evidence for the simultaneous use of frames of reference within a particular perspective, we will have evidence that the two systems are in fact independent.

In general, there is a considerable body of research that investigates the use of frames of reference in spoken languages (see the overview in chapter 3). In comparison, much less is known about how frames of reference are used in sign languages, and about their relationship to signing perspective. This section looks first at the use of perspective (section 5.2) and then at the use of frames of reference (section 5.3) in DGS static scene spatial descriptions. Section 5.4 discusses the relationship between perspective and frames of reference. In addition, an analysis of addressee comprehension of location and orientation information investigates the conventionalization of frame of reference use in DGS (section 5.5).

\subsection{Data}

The analysis in this chapter is based on the same DGS static scene description data as in the previous chapter. The analysis of perspective use is based on both the simple ( 88 in total) and complex (34 in total) scene descriptions. The frame of reference analysis is based only on the simple scene descriptions, for ease of comparison of figure-ground structures. The analysis excludes the 16 descriptions in which referents were simultaneously spatialized, since these descriptions do not exhibit a figure-ground structure (cf. section 4.3.1 in chapter 4) Thus, the 
frame of reference analysis comprises a total of 72 descriptions. The descriptions are divided into three groups depending on the type of stimulus picture: $37 \mathrm{man} / \mathrm{tree}, 21 \mathrm{man} / \mathrm{man}$, and 14 cone/cone descriptions. Details about data coding are given in the relevant sections.

\subsection{Perspective in DGS static scene descriptions}

This section presents an analysis of the use of perspective in the DGS simple and complex static scene spatial descriptions. As figure 5.1 below shows, signers described the static scene stimulus pictures almost exclusively in observer perspective. Specifically, 87 out of 88 simple scene descriptions and 33 out of 34 complex scene descriptions were given in observer perspective. Character perspective was thus used only twice, once for a simple scene and once for a complex scene description.

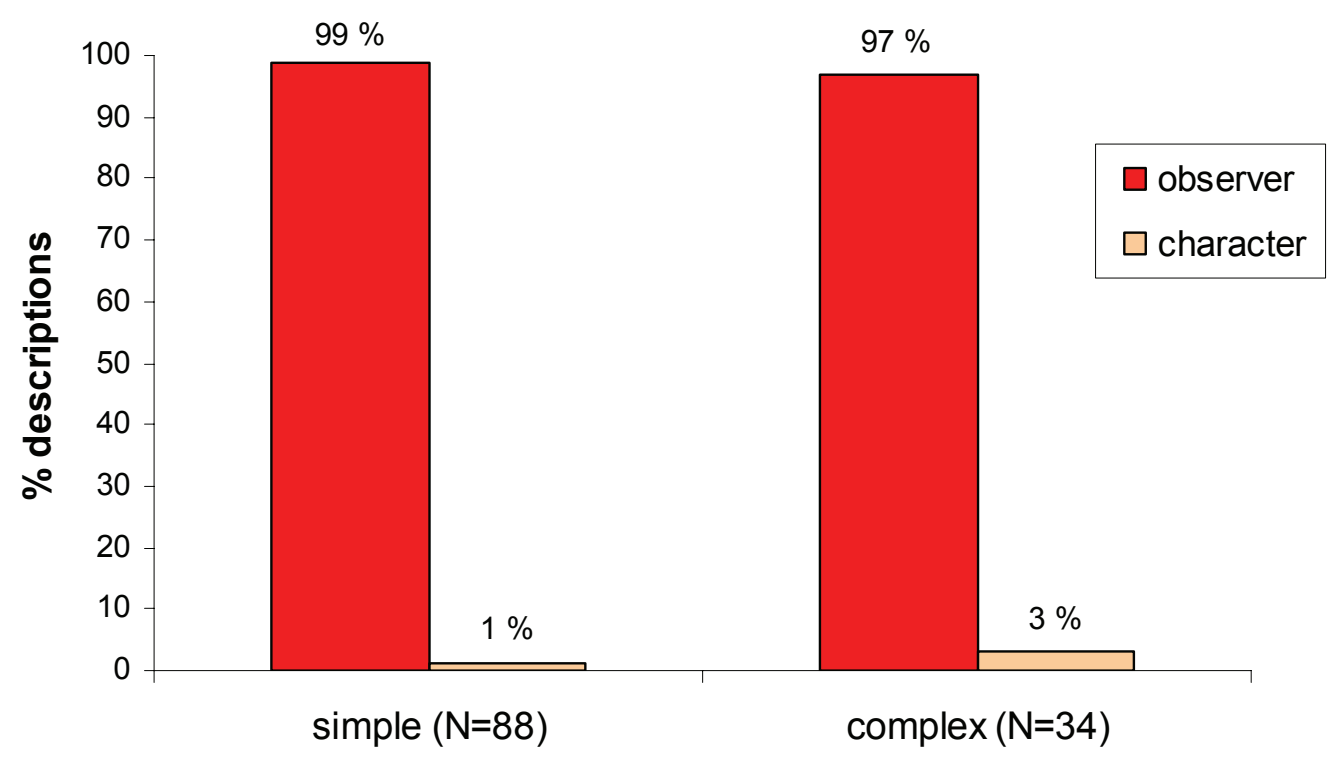

Figure 5.1. Distribution of use of observer and character perspective signing across simple and complex scene descriptions.

The results indicate that, in DGS, observer perspective is the preferred perspective for spatial descriptions of static scenes containing both animate and inanimate entities. The signer is external to the event space and the referents in the spatial array are mapped onto the sign space in front of the body. In addition, referents are represented using entity classifiers, which correspond in scale to the reduced size of the observer perspective event space projection. These characteristics are apparent in example (1) below. ${ }^{79}$

\footnotetext{
${ }^{79}$ Emmorey and Falgier (1999) identify a "relatively low horizontal signing space" as a property characterizing the use of "diagrammatic space", i.e. observer perspective, whereas a "relatively high horizontal signing space"
} 
(1)
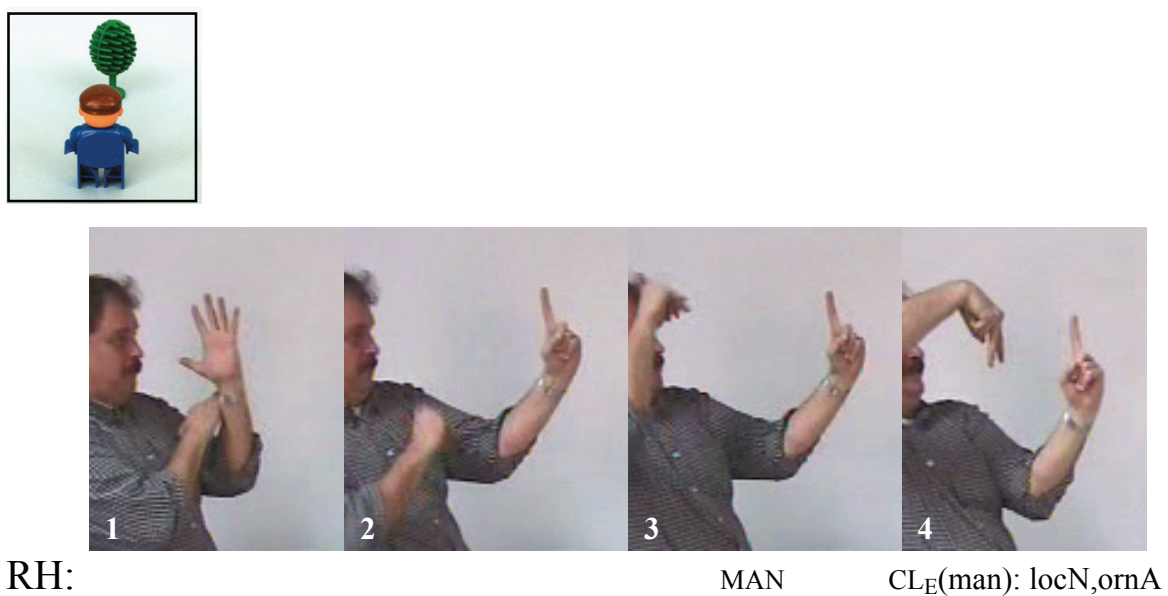

RH:

TREE

$\mathrm{CL}_{\mathrm{E}}$ (tree): locF---hold-

In the two character perspective descriptions, in contrast, the human figure in the scene was mapped onto the signer's body and the other referents were located either in front of or behind the signer, depending on the spatial relations in the scene. Example (2) below shows the simple scene description given in character perspective. The signer takes the role of the man and locates the tree behind his body by pointing with his thumb over his shoulder (frame 5). The sagittal configuration of the man and the tree in the stimulus picture, as well as the congruence between the man's and the signer's orientation may have facilitated the use of character perspective here. However, this observation does not apply to the complex scene description in character perspective. In the complex scene picture described in character perspective, the man faces the signer (see picture 10 (group 3) in appendix A). Thus, in the description, the signer turned the scene around - locating the pigs in a semicircle in front of her body and reaching over her shoulders to represent the trees. ${ }^{80}$

(2)

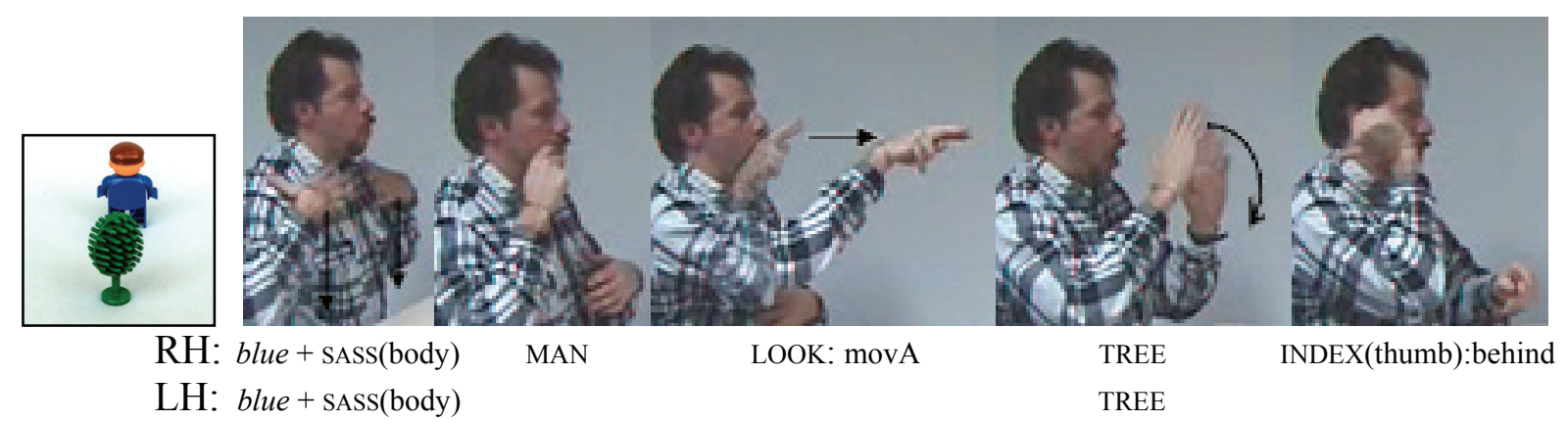

is associated with the use of "viewer space", i.e. character perspective (see also Emmorey et al. 2000). As noted in footnote 51 in chapter 4, the high horizontal plane apparent in the examples of DGS static scene descriptions shown here is the result of the divider between the signer and addressee, which shields the tabletop space in front of each individual from the other's view (see figure 4.2 in section 4.2.4 of chapter 4). 


\subsection{Frames of reference in DGS static scene descriptions}

As stated above, the choice of signing perspective is a prerequisite for creating a spatial representation in sign space, and thus a frame of reference must be instantiated within a particular perspective. As shown in the previous section, DGS signers chose an observer perspective event space projection almost invariably for their static scene spatial descriptions, although the use of character perspective also occurred. Given a particular event space projection, we now look at how these descriptions can be analyzed in terms of frame of reference use.

As stated in section 5.1 above, this analysis includes only descriptions that display a figure-ground structure, that is, in which one referent is located with respect to the other referent. A total of 72 descriptions - comprised of man/tree (37), man/man (21), and cone/cone (14) descriptions - displayed a sequential placement of classifier forms, with or without a hold of the first classifier (cf. section 4.3.4 in chapter 4).

Each of the 72 descriptions was categorized according to whether it displayed features consistent with the use of a relative, intrinsic, or absolute frame of reference, as described above. As I have argued, the linguistic structure of individual propositions is important in determining the figure-ground structure, and thus, the instantiation of a particular frame of reference.

We know that a frame of reference expression requires the location of a figure object to be specified in relation to the location of a ground object. In the relative frame of reference, an external viewpoint, or deictic origo, is needed in order to specify the spatial relationship. The origo's own intrinsic (body) coordinates are used for the angular specification of location by mapping them onto the ground object. The ground object's own intrinsic features, if present, do not enter into a relative frame of reference expression. In contrast, in an intrinsic frame of reference expression, the ground object's intrinsic features are crucial. The figure object is located with respect to the intrinsic features of the ground object. The analysis of DGS static scene descriptions in terms of frame of reference use follows these strict definitions. In addition, across the different types of stimulus pictures, I take the ground object to be the first referent that is located in sign space (see section 4.3.3 in chapter 4). It is the reference object with respect to which the second referent (i.e. the figure) is located.

Figure 5.2 below shows the proportion of use of frames of reference in the DGS static scene descriptions, divided by type of stimulus picture (man/tree, man/man, cone/cone).

\footnotetext{
${ }^{80}$ The signer did not, however, rotate the orientation of the pigs by $180^{\circ}$.
} 
Descriptions exhibited the use of the relative and intrinsic frames, either alone or simultaneously (cf. chapter 3, section 3.5.1). There were no descriptions that exhibited an absolute frame of reference. The results presented in the figure are discussed in more detail below.

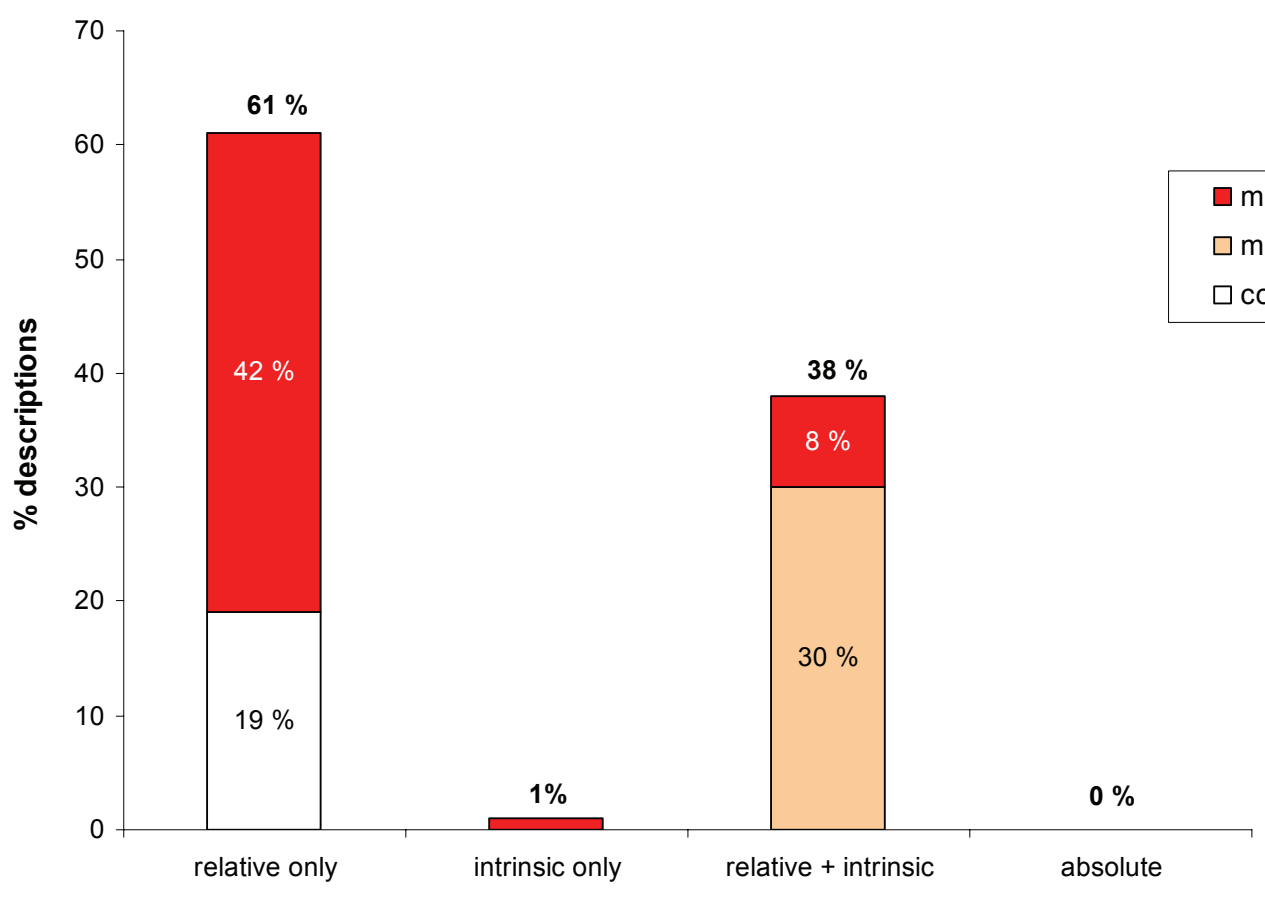

Type of frame of reference

Figure 5.2. Percentage use of relative only, intrinsic only, relative+intrinsic, and absolute frame of reference in man/tree, man/man, and cone/cone simple scene descriptions.

In all but one of the 72 descriptions, the signer encoded the spatial relationship between the two entities in the spatial scene from his own external viewpoint. Thus, in these descriptions, the signer is the deictic origo, which fulfills one criterion for the use of a relative frame of reference. These 71 descriptions appear in figure 5.2 in the "relative only" (61\%; 44 out of $71)$ and "relative+intrinsic" $(38 \% ; 27$ out of 71$)$ columns. What other characteristics do they exhibit that ultimately categorize them as exhibiting only the relative frame, or both the relative and intrinsic frames of reference?

In the descriptions characterized by the use of the relative frame of reference only, the ground object is unfeatured. This is the case for all of the cone/cone descriptions included in this analysis (14 descriptions; 19\%). The ground object is the first referent, i.e. an unfeatured cone, located in sign space. The figure object, i.e. the second cone, is located with respect to the signer's external viewpoint only. An example of a relative frame cone/cone description is given in example (3) below (repeated from example (19) in chapter 4). 
(3)

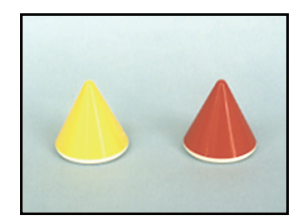

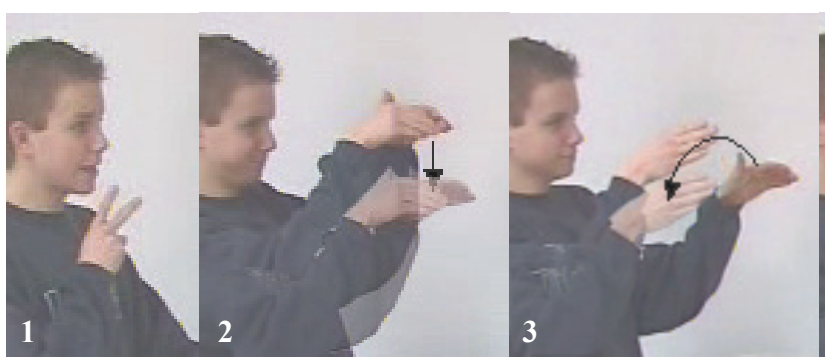

RH: LH:
YELLOW cone + SASS(cone): locL cone + SASS(cone): locL---hold

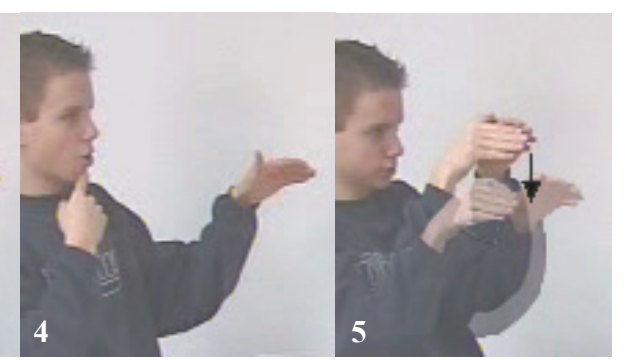

RED cone + SASS(cone): locR cone + SASS(cone): locR

In addition, the use of the relative frame of reference only is exhibited in the $30 \mathrm{man} /$ tree descriptions in which the tree is encoded before the man (42\%). As shown in example (4) below (repeated from example (1) above), the unfeatured ground object (i.e. the tree) is placed into sign space first. The figure object (i.e. the man) is located with respect to the tree from the signer's external viewpoint of the scene.
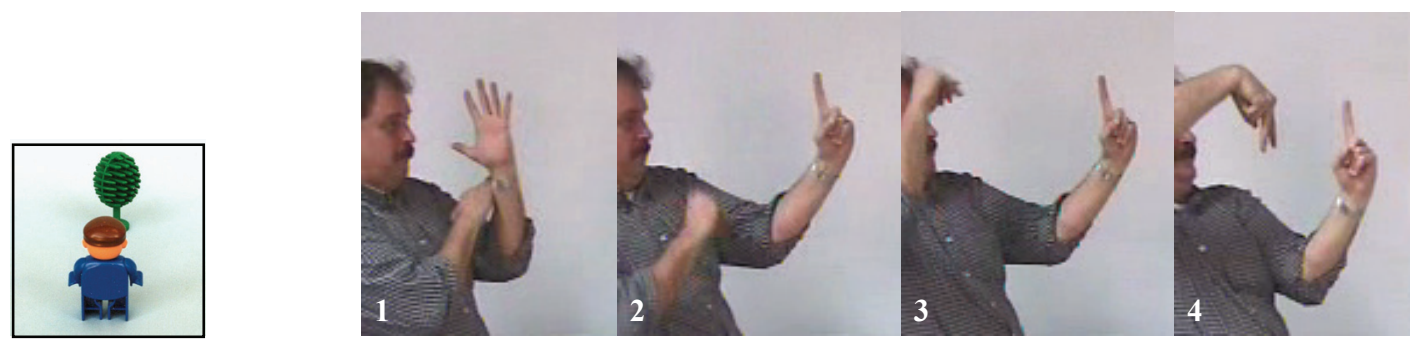

RH:

MAN $\quad \mathrm{CL}_{\mathrm{E}}(\mathrm{man}):$ locN,ornA

LH: TREE

$\mathrm{CL}_{\mathrm{E}}($ tree $)$ : locF---hold-

In contrast, the descriptions that fall into the "relative+intrinsic" category are all characterized by a featured ground object. This group of descriptions is comprised of the $21 \mathrm{man} / \mathrm{man}$ descriptions and the $6 \mathrm{man} /$ tree descriptions in which the man was located before the tree. In all of these 27 (38\%) descriptions, the ground object is a man, and the figure object (i.e. another man or the tree) is located with respect to the man's intrinsic features. This fulfills the requirements for the use of an intrinsic frame of reference. In addition, the figure's location is specified with respect to the signer as deictic origo, as required in the relative frame of reference. An example of a man/man description that exhibits a sequential classifier placement, and thus exhibits the simultaneous use of relative and intrinsic frames, is given in 
example (5) below (repeated from example (2) in chapter 4). An example of a man/tree description which simultaneously uses the intrinsic and relative frames of reference is shown in example (6) below. The man is encoded first, as the featured ground object. Since the tree (as the figure object, here) is located in relation to the man's intrinsic features, the description exhibits the use of an intrinsic frame of reference. At the same time, the tree is located as seen from the signer's viewpoint in a relative frame of reference.

(5)

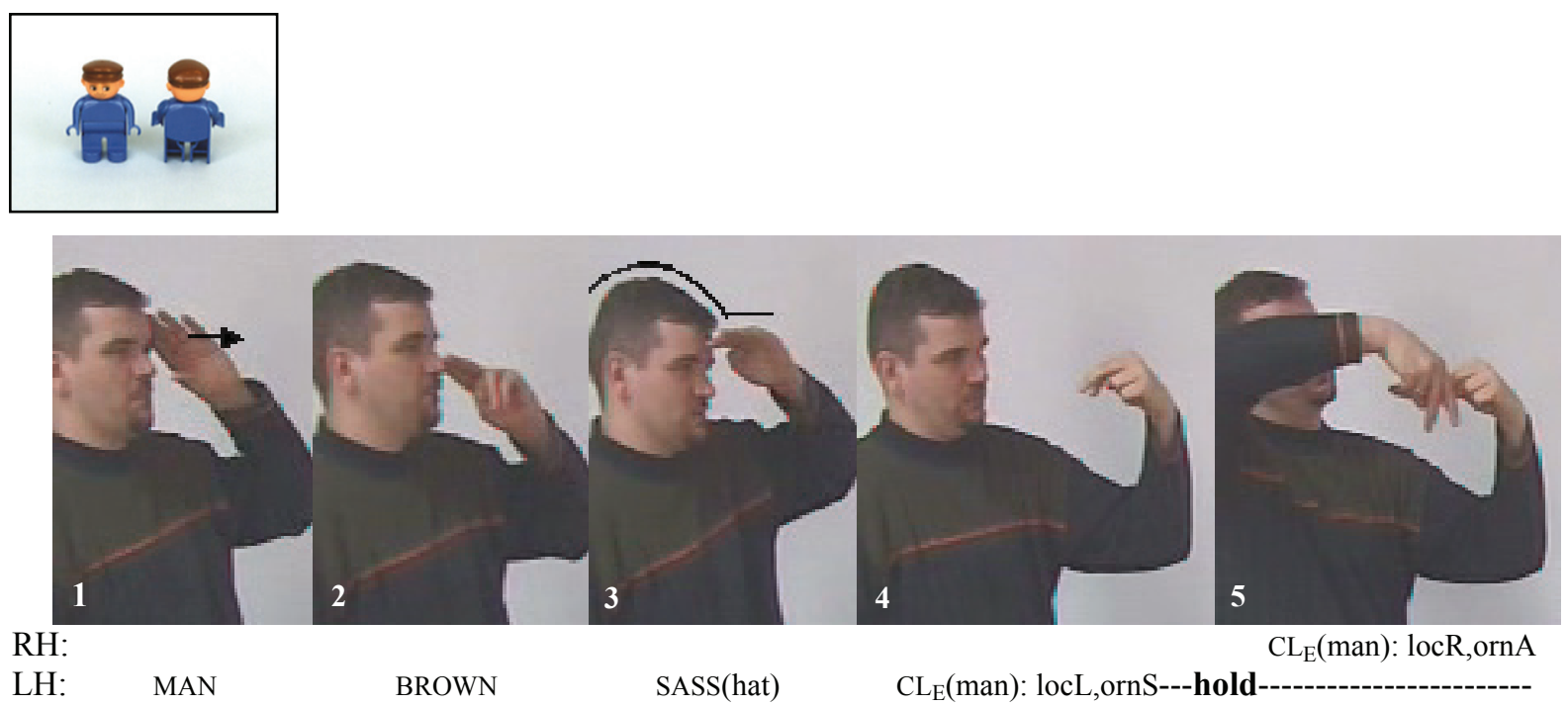

(6)

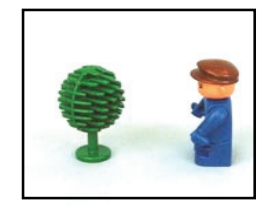

RH: LH:
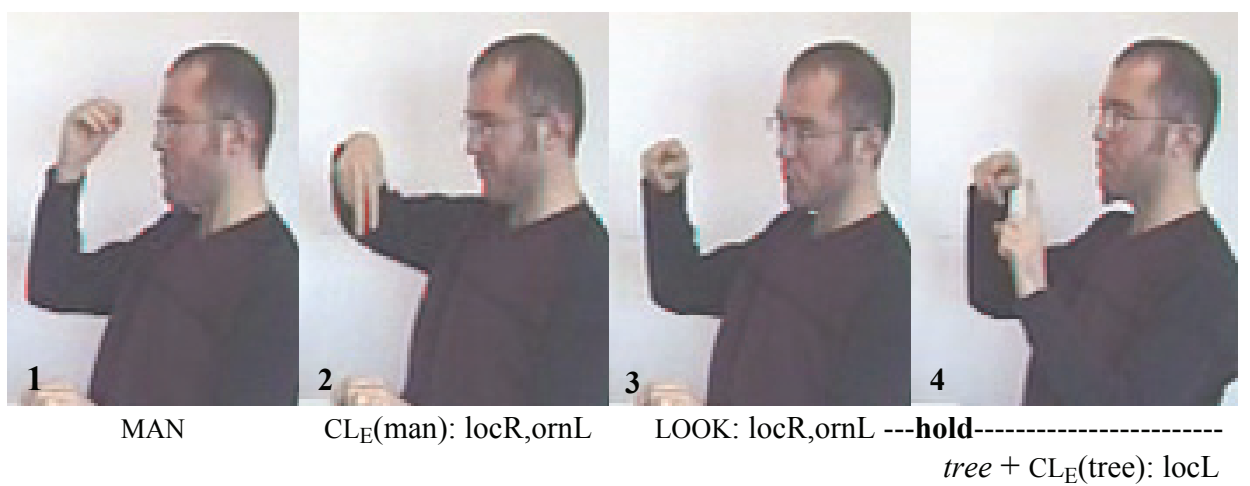

Finally, the single simple scene description that is not given from the signer's external viewpoint exhibits the use of an intrinsic frame of reference only (1\%). In this description, shown in example (7) below (repeated from example (2) above), the signer maps the man onto his body and then locates the tree with respect to his body. Thus, the man, represented by the signer's own body, is the ground object, and the figure (i.e. the tree) is located with respect to the man's intrinsic features. 


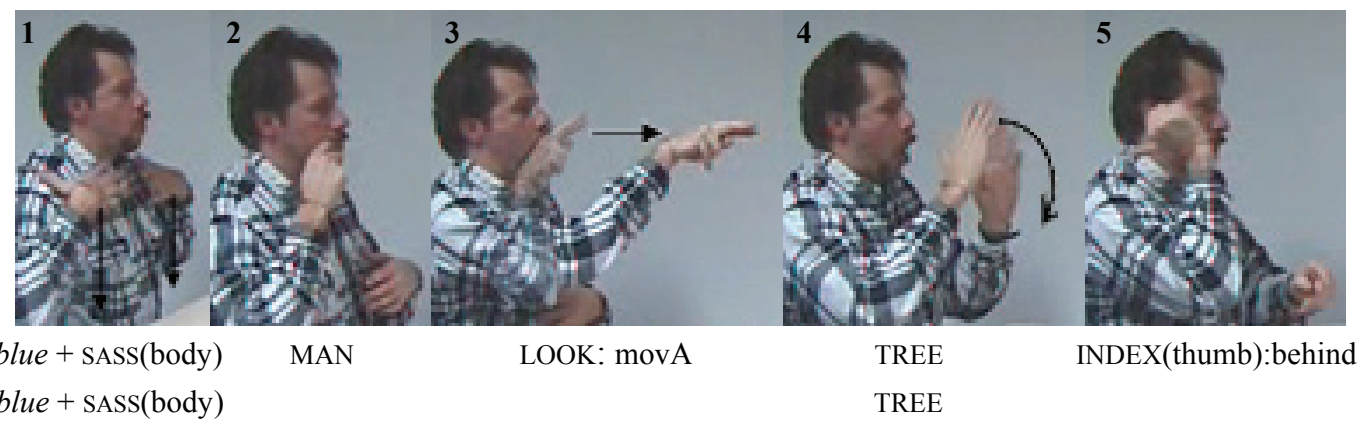

RH: blue + SASS(body)
LH: blue + SASS(body)

TREE

\subsection{Perspective and frame of reference alignment}

In this section, the relationship between perspective and frames of reference is investigated by comparing the results of the analyses presented in the previous two sections. The perspective analysis in section 5.2 has shown that signers made almost exclusive use of observer perspective in the simple scene descriptions. Only one description was signed in character perspective. The frame of reference analysis in section 5.3 has shown that all but one description exhibited the use of a relative frame of reference, either alone or simultaneously with an intrinsic frame. A single description used an intrinsic frame of reference alone.

These correspondences provide evidence for the alignments between perspective and frames of reference which were presented in chapter 3 (section 3.5.1). On the one hand, there is an alignment between observer perspective and the relative frame of reference. Both are characterized by an external viewpoint on the scene that is separate from the figure and ground entities. On the other hand, we see an alignment between character perspective and the intrinsic frame of reference. In descriptions that use only the intrinsic frame of reference, the signer's body cannot represent a third element (i.e. the viewpoint). Instead, the signer's body must represent the ground, and the figure must be located with respect to the signer's intrinsic features. In addition, the frame of reference analysis in section 5.3 provides evidence for the independence of signing perspective and frames of reference. This is apparent in the descriptions in which two frames of reference (i.e. the relative and intrinsic frames) occurred simultaneously in observer perspective (as in examples (5) and (6) above). 
The simultaneous encoding of two frames of reference within a single form is afforded by the visual properties of the modality, specifically, by the fact that classifier predicates used to represent referents can simultaneously provide location and orientation information. Indeed, the possibility of simultaneously-occurring frames of reference seems to be a unique property of the visual-spatial modality. In spoken language, it is not possible to encode two frames simultaneously within a single form, or often even within a single proposition. For example, in English, the relative frame of reference expression The man is to the left of the tree gives us no intrinsic information. By the same token, the intrinsic frame of reference expression The tree is in front of the man (in other words, The man is facing the tree) gives us no information about relative location.

Note, however, that the presence of orientational information (i.e. through the use of featured classifier forms) is not tantamount to the use of an intrinsic frame of reference. This is particularly relevant to the man/tree descriptions. Recall from figure 5.2, and the accompanying discussion, that we have been careful to distinguish between the man/tree descriptions that encoded the tree first and those that encoded the man first. In observer perspective, the tree-first (i.e. tree as unfeatured ground) descriptions exhibit a relative frame of reference only (see example (4)), while the man-first (i.e. man as featured ground) descriptions exhibit a simultaneous relative and intrinsic frame of reference (see example (6)). However, we have seen in chapter 4 that all 36 observer perspective man/tree descriptions encode the man's orientation. That is, the orientational relationship between the man and the tree is preserved (from the signer's viewpoint) in all of these man/tree descriptions, regardless of the order of referent placement. Here, we come upon another special property of the modality. In signed language, the orientational relationship between two referents is encoded regardless of the order of referents if featured forms are used to represent featured referents. ${ }^{81}$ In other words, the orientational information encoded in a featured form is a necessary, but not sufficient condition for the use of an intrinsic frame of reference if we take into account the properties of what is encoded as the ground. How to define the figure and ground in locative descriptions has been emphasized in this thesis as a crucial point in determining the instantiation of frames of reference in signed language.

In spoken language, in contrast, it seems that the orientational features of two referents can be simultaneously expressed only in special cases, e.g. reciprocal relationships like

\footnotetext{
${ }^{81}$ As has been suggested in chapter 4, DGS may have featured forms that do not entail the inherently present orientational information. The extent to which and the circumstances under which this is true must be further investigated.
} 
"facing each other"; otherwise, a single intrinsic frame of reference expression can encode the orientational features of only one of the two referents. In signed language, we can make a distinction between the use of an intrinsic frame of reference and the encoding of sceneinternal orientational properties. The iconic properties of the modality, and of classifier predicates, in particular, create a modality-specific difference in the instantiation of frames of reference and in the encoding of orientational information, in general.

Finally, there is another important modality-specific difference between signed and spoken language frame of reference use. In a relative frame of reference expression in spoken language, the "presence" of a deictic origo (e.g. the speaker's viewpoint) is made clear through the choice of a particular spatial term related to the viewer's body coordinates (e.g. The man is to the left of the tree.) In the visual-spatial modality, however, the situation is slightly more complicated. Unless signers use lexical prepositions like LEFT and RIGHT, which unequivocally refer to the signer's body coordinates, the only cue that the signer is "committing" to an origo is the fact that the scene which the signer creates in sign space matches the signer's view of the scene being described. The individual forms that the signer uses in the description, however, as well as their syntax, could just as well characterize an expression encoding mere referent proximity (e.g. the man is next to the tree).

The analysis of simple scene descriptions presented in this section has shown that signers do indeed encode spatial relationships from their own point of view, corresponding to the use of a relative frame of reference. However, whether the commitment to a viewpoint is truly part of the linguistic system of DGS spatial descriptions can be verified only by looking at the addressees' interpretations of the descriptions. Only by looking at the interpretations can we know whether the use of a relative frame of reference in static scene spatial descriptions is conventionalized in DGS.

\subsection{Analysis of addressees' interpretations of static scene spatial descriptions}

With the notable exception of Emmorey et al. (1998) and Emmorey et al. (2000), the interpretation (as opposed to the production) of spatial descriptions has not been investigated in any detail in the research on sign languages to date. Emmorey et al. (1998) asked participants to judge whether a signed description of a room matched a picture of the room which they had viewed just previous to seeing the description. Descriptions were given either from the point of view of the signer (on videotape), indicated by the initial sign I-ENTER, or from the point of view of the addressee (i.e. the participant, seated in front of the television screen), indicated by the initial sign YOU-ENTER and rotated by $180^{\circ}$. The results of the study 
show that ASL signers (as addressees) performed better - that is, they were more likely to notice when objects were placed in the wrong location - when viewing descriptions signed from the signer's point of view.

Emmorey et al. (2000) confirmed this finding in a study more similar to the static scene spatial descriptions task presented here for DGS spatial description. Their study involved two signers seated opposite one another, conversing about an environment (a total of eleven pairs participated in the study). Signers were asked to describe a town (from a town map) to a partner so that he or she could subsequently reproduce the map. Signers mapped the town landmarks and the layout of the streets onto sign space in an observer perspective event space projection and from their own viewpoint. When redescribing the town, all but one addressee rotated the observed locations in signing space, thus interpreting the descriptions from the signer's own viewpoint. The results of this study suggest that the use of a relative frame of reference in ASL spatial descriptions is indeed codified. The analysis presented in this section will determine whether this is also the case for DGS spatial descriptions.

The analysis includes the interpretations of both the simple and complex scene spatial descriptions by the DGS signers that acted as matchers in the task. In the elicitation task, the matchers (seated across from and facing the directors who gave the description) were asked to construct the spatial scene described to them using a set of plastic figurines (as described in section 4.2.1 in the previous chapter). This task required an externalization of the addressees' mental interpretations of scenes, thereby giving us explicit information about how a scene was construed. As the analyses above have shown, the directors consistently described the scenes from their own external viewpoint as viewers of the scene (with the exception of two descriptions from scene-internal, i.e. character perspective, viewpoints). The analysis presented in this section concerns the viewpoint that is implicit in the addressees' (i.e. matchers') interpretations of the spatial relationships they see mapped onto sign space by the signers (i.e. directors). In particular, the analysis addresses the question of whether DGS has an established viewpoint convention for representing spatial relationships and maintaining constancy across production and interpretation. As discussed in chapter 3, section 3.2.2, one must caution against assuming that the visual-spatial modality presents a unique solution to the issue of viewpoint in spatial description. Conventions in description and interpretation must be established in a language in order to ensure successful communication about spatial relationships. 


\subsubsection{Coding and stimulus categorization}

To investigate the question posed above and to assess how signers of DGS interpret the spatial information they see in sign space, matchers' responses (i.e. their object set-ups) were coded for the following aspects:

(1) From what point of view was object location interpreted - from the director's point of view or from the matcher's point of view?

(2) From what point of view was object orientation interpreted - from the director's point of view or from the matcher's point of view?

Location and orientation information was not equally relevant across the different kinds of simple scene stimulus pictures, and for this analysis, it was necessary to divide the simple scenes into two main groups: viewpoint-dependent and viewpoint-independent pictures. Viewpoint-independent pictures look the same from the director's and the matcher's points of view (opposite each other). The viewpoint-independent stimulus pictures comprised two types of pictures: two cones of the same color (as in figure 5.3(a)) and two men facing in opposite directions (that is, directions differing by $180^{\circ}$ ) (as in figures 5.3(b) and (c)). In (a), there is a red cone on the left and on the right, regardless of whether the picture is viewed from the director's or the matcher's vantage point. Similarly, in (b), both the director and the matcher see that the men have their backs to each other and that they are in right and left locations on the lateral axis. Finally, in (c), from both viewpoints, the man on the right is facing the viewer and the man on the left is facing away from the viewer.

Matcher
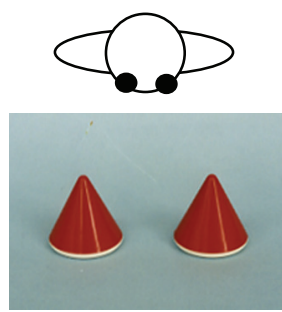

(a)

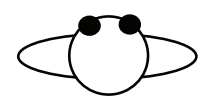

(b)
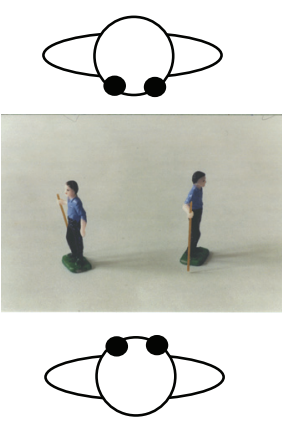

(c)
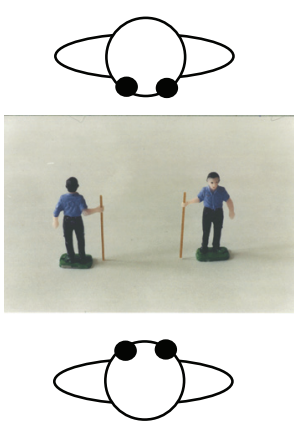

Figure 5.3. Examples of viewpoint-independent stimulus pictures.

For these stimulus pictures, it is not possible to discriminate between an interpretation of location and orientation information from the director's or the matcher's point of view. Both interpretations result in the same object set-up. There were eight viewpoint-independent 
stimulus pictures in all, two cone/cone and six man/man pictures, corresponding to 24 descriptions.

The remaining 23 simple scene stimulus pictures were viewpoint-dependent, and did not look the same from different viewpoints. These were the cone/cone pictures with cones of different colors (as in figure 5.4(a); 4 pictures in all), the man/man pictures with the men facing in the same direction (as in figure 5.4(b); 4 pictures) or different (but not opposite) directions (as in figure 5.4(c); 2 pictures), and all of the man/tree pictures (as in figure 5.4(d); 13 pictures).

Matcher

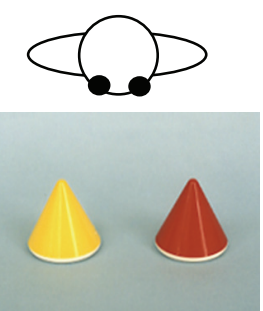

Director

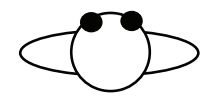

(b)

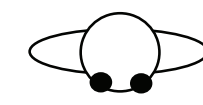

1
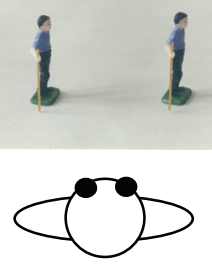

(c)
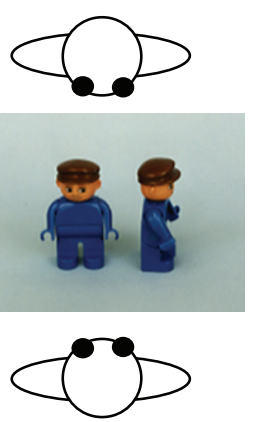

(d)
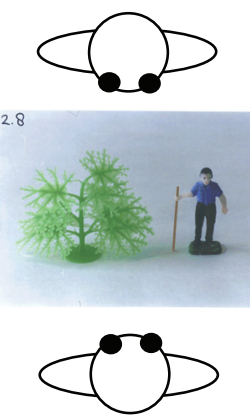

Figure 5.4. Examples of stimulus pictures that differ with respect to location and/or orientation information depending on the viewpoint.

In these pictures, viewpoint is crucial to the interpretation of location and orientation information. However, the different types of pictures differ with respect to the type of information that is viewpoint-dependent. Since cones are non-featured objects, with no orientation specification, the different color cone/cone pictures (as in figure 5.4(a)) are viewpoint-dependent with respect to location only. Viewing the cones from the director's vantage point, the red cone is on the right and the yellow cone is on the left. From the matcher's vantage point, however, the yellow cone is on the right and the red cone is on the left. Similarly, in the man/man pictures that have both men facing in the same direction (as in $5.4(b))$, it is only orientation information that is viewpoint-dependent. The men are both facing right as the director sees them, while they are both facing left as the matcher sees them. Finally, in the man/man pictures in which the men face directions that differ by $90^{\circ}$ (as in 5.4(c)) and in the man/tree pictures (as in 5.4(d)), both location and orientation are viewpoint-dependent.

In total, there were 64 descriptions of viewpoint-dependent stimulus pictures. In 11 of these, only location was relevant; in 10, only orientation was relevant; and in the remaining 43 descriptions, both location and orientation mattered (see the overview in table 5.1 below). 
In the data, there were no differences in interpretation that could be attributable to location vs. orientation viewpoint-dependence. Thus, the response types for all 64 viewpointdependent descriptions are discussed together.

Table 5.1. Overview of the number of each category of stimulus picture and the number of descriptions for each category.

\begin{tabular}{|c|c|c|c|c|c|c|}
\hline & \multicolumn{4}{|c|}{ SIMPLE } & COMPLEX & TOTAL \\
\cline { 2 - 6 } & \multicolumn{2}{|c|}{ Viewpoint-dependent } & Viewpoint-independent & Viewpoint-dependent & \\
& LOC+ORNT & ORNT & LOC & & & \\
\hline stim. pic. & 15 & 4 & 4 & 8 & 34 & 12 \\
\hline descr. & 43 & 10 & 11 & 24 & 34 \\
\hline
\end{tabular}

\subsubsection{Rotation and translation in interpretation}

For the matcher seated opposite the director, there are two possible interpretations of location and orientation information: (1) from the director's point of view, or (2) from the matcher's own point of view. An interpretation by the matcher from the director's point of view involves what is essentially a $180^{\circ}$ mental rotation of the representation that the director "inscribes" into sign space (Emmorey 1996). An interpretation by the matcher from the matcher's own point of view involves what I will call a mental translation of the representation from the director's (tabletop or sign) space to the matcher's (tabletop or sign) space.

The two types of interpretation, involving rotation in one case and translation in the other, are illustrated using a man/tree stimulus picture in figures 5.5 and 5.6 below, respectively. If matchers rotate location and orientation information, the object configuration looks identical from both the director's and the matcher's points of view. In figure 5.5, the tree is on the left and the man is on the right facing the viewer from both vantage points. In figure 5.6 , on the other hand, the object configuration in front of each signer differs in referent location and orientation from the two points of view. For the director, the tree is on the left and the man is on the right facing him/her. But, after translation, as the matcher sees the object configuration, the tree is on the right and the man is on the left facing away from him/her. ${ }^{82}$ The translation interpretation bears a resemblance to what Emmorey (2002)

\footnotetext{
${ }^{82}$ As discussed in section 3.4.1 of chapter 3, Emmorey (1996) has discussed the difference between signer and addressee viewpoint, but with a focus on production, not interpretation (but see Emmorey et al. 1998).
} 
describes as the use of shared space, where two signers (located opposite each other) jointly view an environment (see figure 3.14 in chapter 3, section 3.4.2).

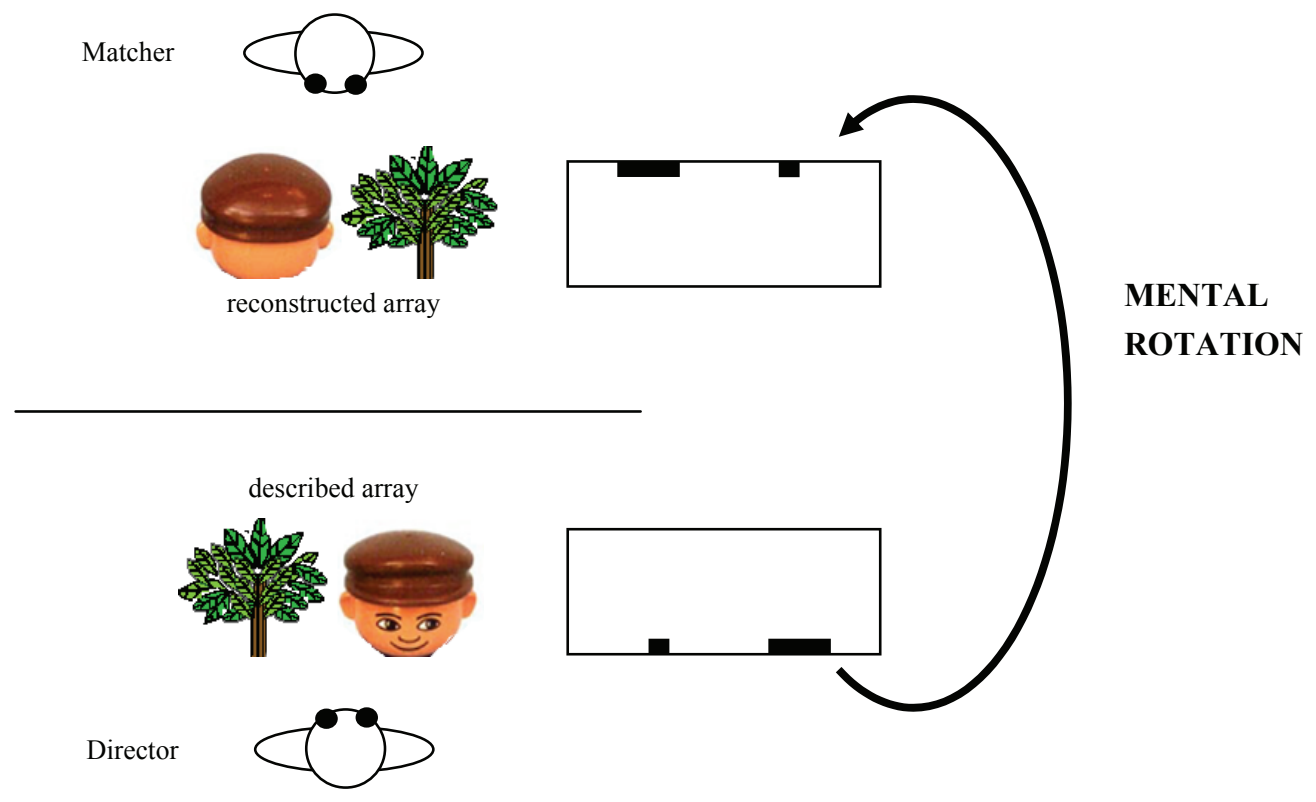

Figure 5.5. The matcher interprets location and orientation information from the director's point of view, rotating the spatial description.

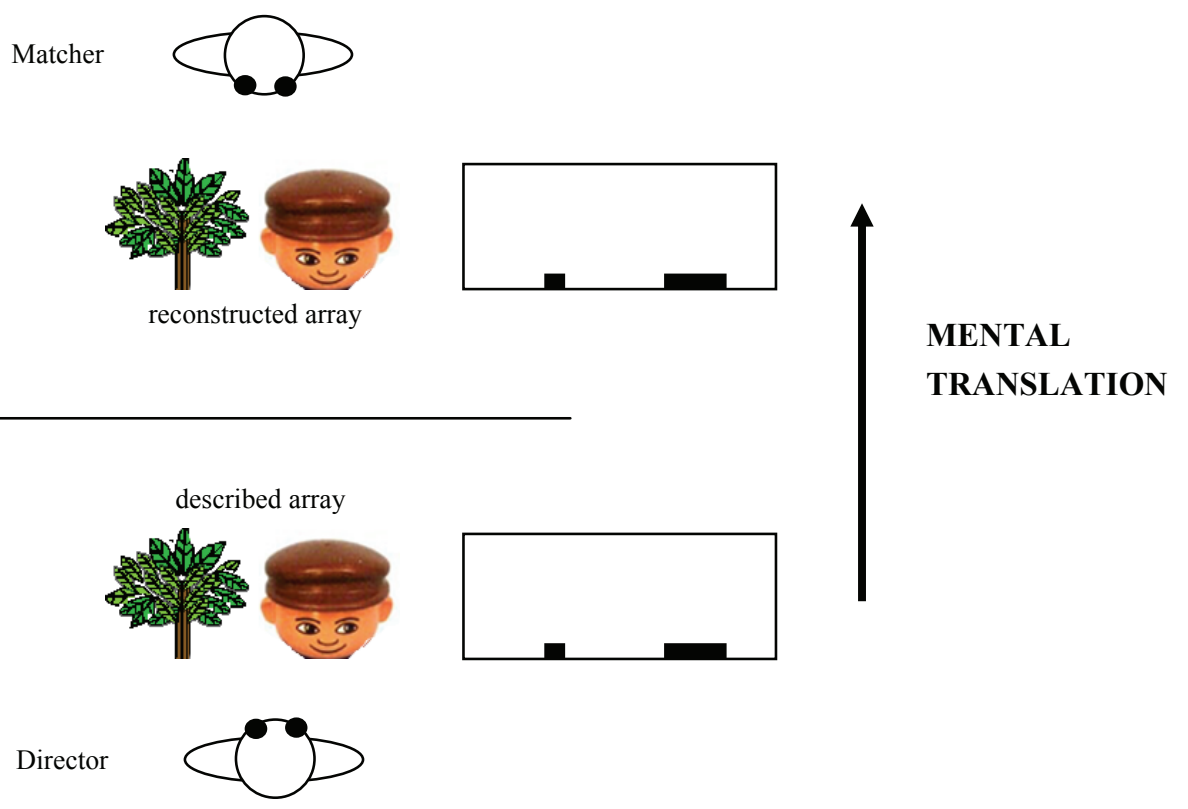

Figure 5.6. The matcher interprets location and orientation information from his/her own point of view, translating the spatial description.

\subsubsection{Rotation and translation interpretations in the data}

This section looks at the occurrence of rotation and translation interpretations in the data. The analysis focuses on the 64 descriptions of viewpoint-dependent pictures. As shown in table 
5.1 above, the viewpoint-dependent stimulus pictures are of different types, depending on which information is dependent on viewpoint: both location and orientation information (LOC+ORNT), only orientation (ORNT), or only location information (LOC).

\subsubsection{Simple scenes}

In the majority of cases, matchers interpreted both location and orientation information from the director's point of view, thus mentally rotating the scene in sign space. In other cases, matchers translated both location and orientation information to their own space. And in still other instances, matchers partially rotated and partially translated the spatial information in the director's signed description. Table 5.2 summarizes the distribution of these different response types in the data.

Table 5.2. Number and percentage of rotation and translation (full and partial) response types for all 64 viewpoint-dependent simple scene spatial descriptions, by type of viewpoint-dependence (location and orientation, orientation only, location only).

\begin{tabular}{|c|c|c|c|c|c|c|}
\hline & LOC+ORNT & ORNT & LOC & $\begin{array}{c}\text { ORNT trans.; } \\
\text { LOC rot. }\end{array}$ & $\begin{array}{c}\text { LOC trans.; } \\
\text { ORNT rot. }\end{array}$ & Total \\
\hline Rotation & 30 & 9 & 8 & 0 & 0 & $47(73 \%)$ \\
\hline $\begin{array}{c}\text { Translation } \\
\text { (full) }\end{array}$ & 5 & 1 & 3 & 0 & 0 & $9(14 \%)$ \\
\hline $\begin{array}{c}\text { Translation } \\
\text { (partial) }\end{array}$ & 0 & 0 & 0 & 4 & 4 & $8(13 \%)$ \\
\hline
\end{tabular}

Of the 64 descriptions of viewpoint-dependent pictures, 47 (73\%) were interpreted from the director's point of view. Thus, in these cases, the matchers rotated the encoded location and orientation information (as diagrammed in figure 5.5 above) and both the director's and the matcher's object set-ups looked like the object configuration in the picture. In $17(27 \%)$ instances, spatial information was translated, either fully or partially, from the director's space to the matcher's space. In about half of these translation responses, comprising all types of viewpoint-dependent stimulus pictures, the configuration was fully translated (as in figure 5.6 above). In the other half, comprising only LOC+ORNT viewpoint-dependent pictures, there was a partial translation. That is, either location information was translated, while orientation information was rotated, or vice versa. Two examples of partial translation- one of each kind - are given in figures 5.7 and 5.8. 

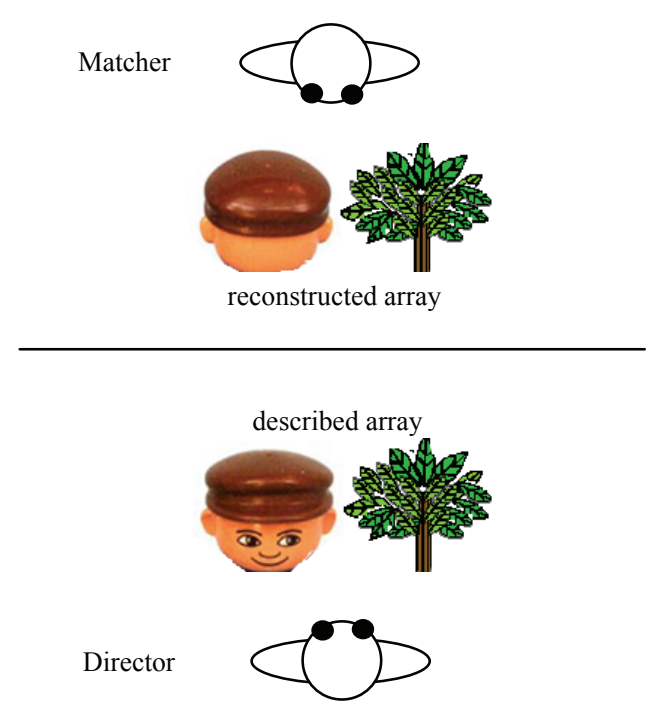

Figure 5.7. The matcher translates the locations of the man and tree, and rotates the man's orientation.
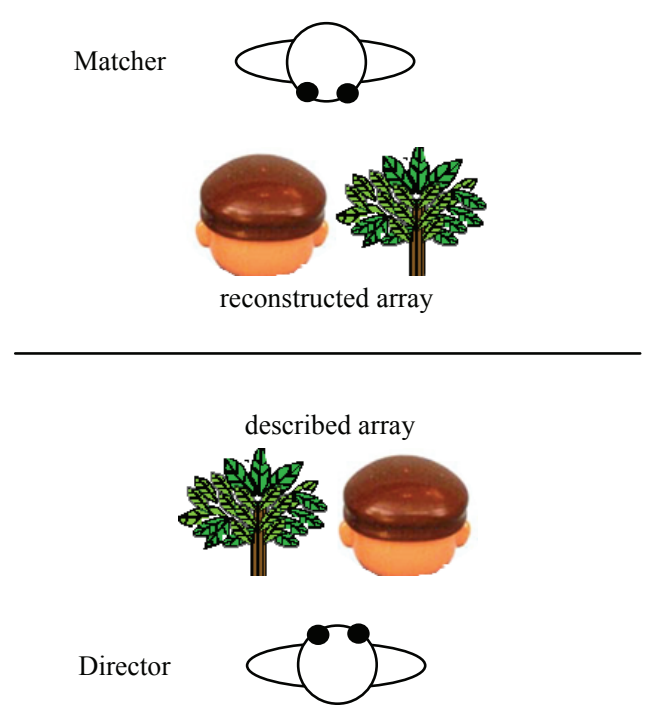

Figure 5.8. The matcher rotates the locations of the man and tree, and translates the man's orientation.

In the interpretations, individual signers show consistent patterns. For example, with respect to partial translation of descriptions, JP tended to rotate orientation information and translate location information (as in figure 5.7), while SR exhibited the opposite pattern, rotating location and translating orientation (as in figure 5.8). In addition, at the beginning of the task, HT translated both location and orientation information, in particular, in his set-ups of man/tree scenes. This was the case, however, only in the first group of pictures, which suggests that HT adopted a full rotation strategy (as evidenced by subsequent interpretations) in the course of the task. The presence of possible training effects are discussed in section 5.5.5 at the end of this chapter.

There is another factor that may have contributed to the translation interpretation of facing information. Recall from chapter 4 that the directional predicate LOOK, functioning as a classifier predicate, frequently appeared in descriptions to indicate direction of facing. Signers who used this sign executed it in different ways. In some cases, it was placed in a location in sign space and held. In other cases, however, the hand was moved through space in the direction of looking. While the mode of execution in the former case resembles that of a classifier predicate of location, that of the latter retains the form of a directional verb. Grammatically, the locations between which directional verbs (also called agreement or indicating verbs) move in space indicate the subject and object argument of the verb. Thus, in discourse, if the signer moves a directional verb in the direction of the addressee, the addressee interprets herself as the verb's object. Thus, the movement of the directional 
predicate LOOK (or LOOK-AT, as it might be glossed, cf. Liddell 1998) toward the addressee means "look at you". Similarly, if the signer moves a directional verb toward her own body, the signer herself (or the referent mapped onto her body) is the object of the verb ("look at me").

The use of LOOK to fulfill the function of a classifier predicate (that is, to mark location and/or orientation) instead of the function of a directional predicate (that is, to indicate the subject and object arguments of the verb) introduces a source of ambiguity that may have been a confounding factor in the interpretation of the man's direction of facing. In a description containing "LOOK: movA" to encode the man facing away from the signer, the movement of LOOK toward the addressee (in the manner of a directional predicate) may have induced an interpretation by the addressee consistent with the meaning of a directional predicate. Thus, instead of interpreting the form as meaning "face away from the signer", the addressee may have interpreted the form as "looks at me", which is coded as a translation interpretation of facing information.

\subsubsection{Complex scenes}

Slightly over half the interpretations of descriptions of complex scene stimulus pictures, as evidenced by the matchers' object set-ups, depicted the scene as it was in the stimulus picture, thus exhibiting full rotation (18 out of $34 ; 53 \%$ ). There were no interpretations that exhibited full translation from the director's to the matcher's space. Rather, the remaining interpretations all exhibit partial translation - some elements were rotated while others were translated. Within these partial translations, there was greater variation in how different aspects of the description were interpreted compared to the simple scenes. One reason for this may be the difference in quantity of location and orientation information that signers must keep track of. In the complex scene descriptions, not all information could be represented simultaneously in space, because there were more than two referents. In addition, information like the geometric shape of entities (e.g. the shape of the fenced enclosures) was present in the complex scenes. Another reason for the greater variation in interpretation may be that the complex scene descriptions exhibited a greater variety of different types of signs to encode spatial information, e.g. the use of motion predicates (as in example (9) below) and tracing signs (as in figure 5.13 below).

Different types of partial translation/rotation were evident, for example, in the interpretations of the complex scenes shown in figures 5.9 and 5.10 below. In both scenes, the horse is facing the viewer, and the cow drinking from the trough is to the left of the horse. 
The scenes differ only in the orientation of the enclosure surrounding the horse. All six descriptions of these scenes start with the shape of the fence, then locate the horse inside the fence, and then depict the trough and the cow to the left. In the interpretations, i.e. in the reconstructed arrays, all six matchers (three per picture) rotated the shape of the triangular fence. However, in three cases, other location and orientation information in the scene was translated from the director's to the matcher's space.

Matcher
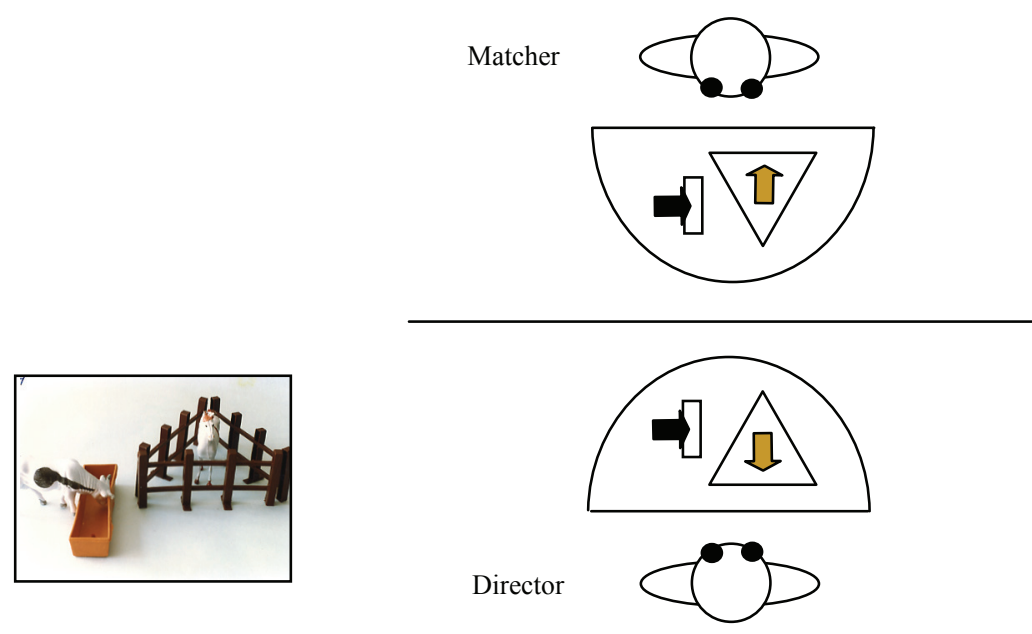

Figure 5.9. The matcher rotates orientation information and translates location information.

Matcher
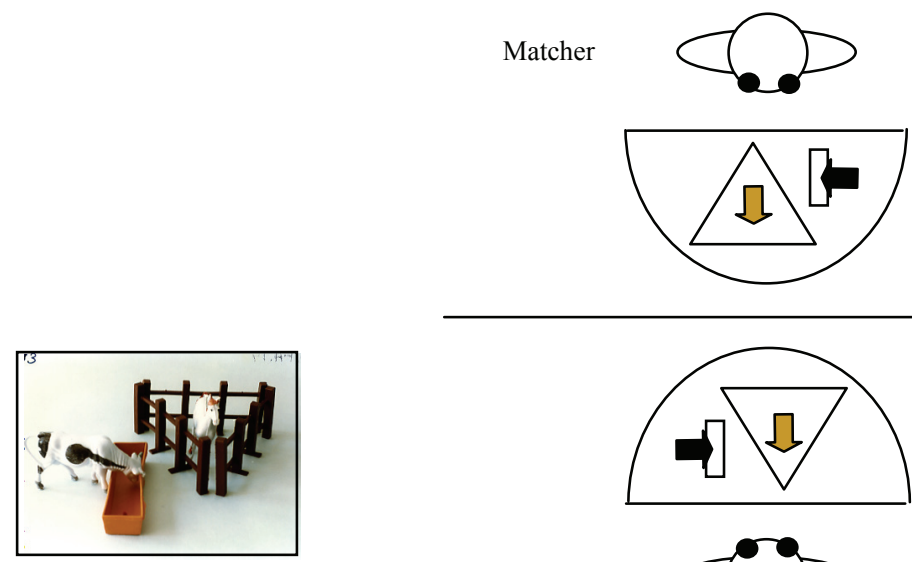

Director

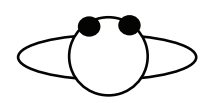

Figure 5.10. The matcher rotates location information and partially translates orientation information

Figures 5.9 and 5.10 above give a schematic representation of two examples. In figure 5.9, the matcher rotates the orientations of both the fence and the horse, but translates the locations of the two main elements (the horse/fence and cow/trough). That is, the matcher places the cow and trough to the right side of the horse and fence, which does not correspond to the relative locations depicted in the stimulus picture. In figure 5.10, on the other hand, the 
relative locations are rotated, as is the orientation of the fence, but the horse's orientation is translated. This preserves the orientation information from the director's sign space in an absolute sense (i.e. the horse is facing the director in both set-ups), but it does not preserve the scene-internal intrinsic information (i.e. in the matcher's set-up, the horse is not facing a corner of the fence and the cow is not on its right side).

The patterns of interpretation represented in figures 5.9 and 5.10 parallel those in figures 5.7 and 5.8, respectively, and indeed the examples come from the same two signers, JP and SR (both early signers) As shown in figure 5.9, JP rotates orientation information and translates location information. ${ }^{83}$ SR exhibits the opposite pattern, in figure 5.10. She rotates location and translates orientation information.

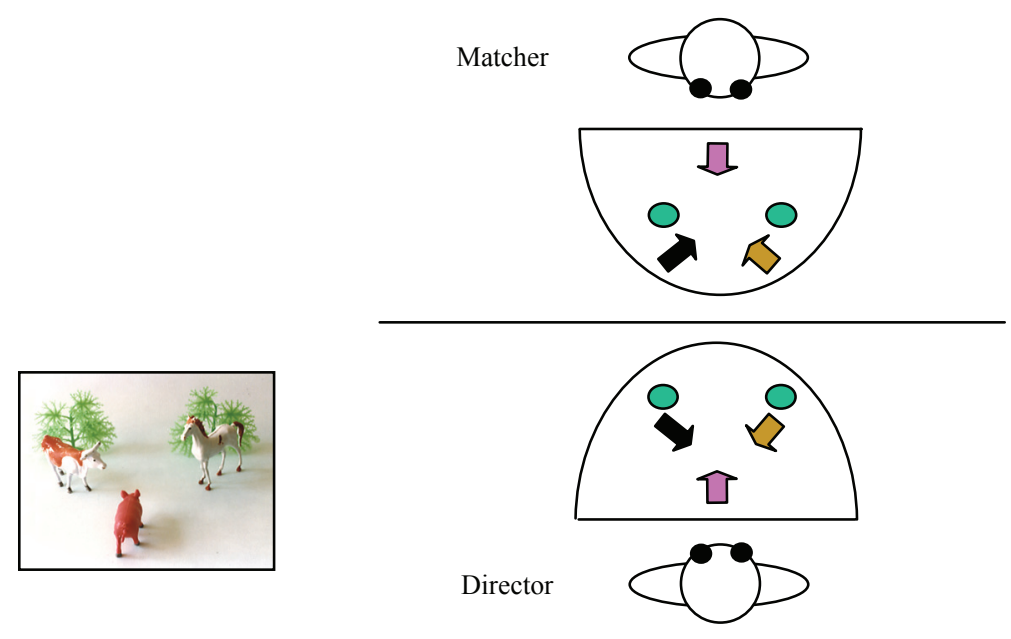

Figure 5.11. The matcher rotates orientation information and partially translates location information.

Figure 5.11 represents another interpretation by JP, in which orientation information is rotated and location information is translated. Here, JP rotates the orientations of all three animals in the scene (the pig, cow, and horse) - they are all facing toward the middle. The location information, however, except for the location of the pig, is translated. This causes the matcher's set-up to look quite different from the stimulus picture. In terms of relative distance from the body, the trees are medial and not distal, and the left and right locations of the cow and horse are reversed.

\footnotetext{
${ }^{83} \mathrm{JP}$ was 16 years old at the time of data collection. Rotation in interpretation may be something learned quite late in the acquisition process, maybe even as late as the mid-teens (Mathilde de Geus, personal communication; cf. also Haun 2007).
} 


\subsubsection{When iconicity is not enough: miscommunications of spatial relationships}

For the complex scenes, it was not always the case that differences between the matcher's setup and the stimulus picture scene were due to full or partial translation interpretations. In their descriptions, the directors sometimes relied on sign space alone to indicate the relative locations of referents. That is, they relied fully on the iconic potential of the modality in creating a schematic mapping between real-world referent locations and locations in sign space associated with referents, as the examples below will make clear.

For example, in (8) below, the signer uses a simultaneous classifier construction to represent the location and orientation of the two pigs with respect to each other, but does not use a simultaneous construction (or other sign) to make explicit the locations of the pigs with respect to the fence. Thus, only the distance between the animal classifiers in sign space (and the distance between the classifiers and the signer's body) indicates the relative locations of the pigs and the fence. The matcher's interpretation of the pigs' locations suggests that a full reliance on iconicity in the sense of location-to-location correspondences may not be sufficient when more than two referent locations are involved. In his interpretation, the matcher does not place one pig inside the fence and the other in front of the fence, but rather places both pigs inside the fence.
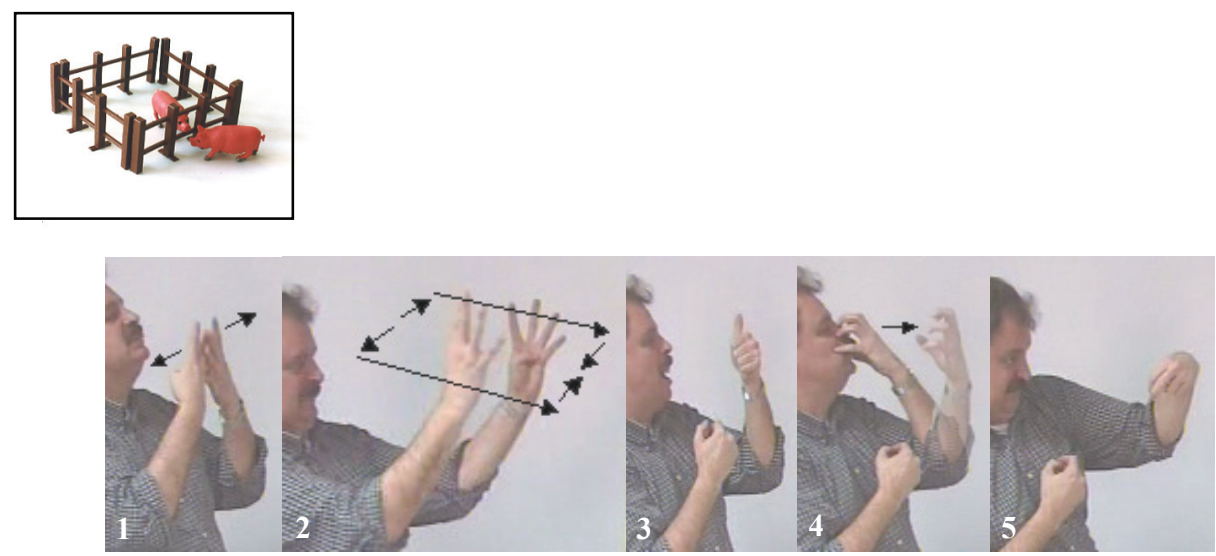

RH: FENCE SASS(fence): trace square

LH: FENCE SASS(fence): trace square ONE PIG $\quad \mathrm{CL}_{\mathrm{E}}$ (pig): locF,orntS--hold---

RH:

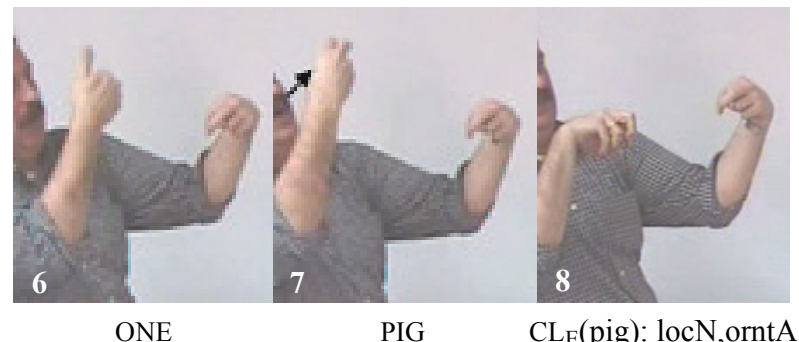

LH: 
In his description, the director places his hands quite far apart in sign space to represent the locations of the pigs, inscribing unique locations for the pigs that are different from the location of the fence. In giving the description, he seems to have considered this distance to be sufficient as an indication that the fence separated the two pigs. Had the pigs actually both been inside the fence, he might have placed his hands much closer together. For the matcher, however, the distance between the hands does not cue the intended meaning. In his interpretation, the matcher may be relying instead on what he may consider to be the most typical configuration in this situation - namely that both pigs are inside the fenced enclosure. Whatever the case may be, it is clear that iconicity alone is not sufficient to unambiguously convey the intended meaning.
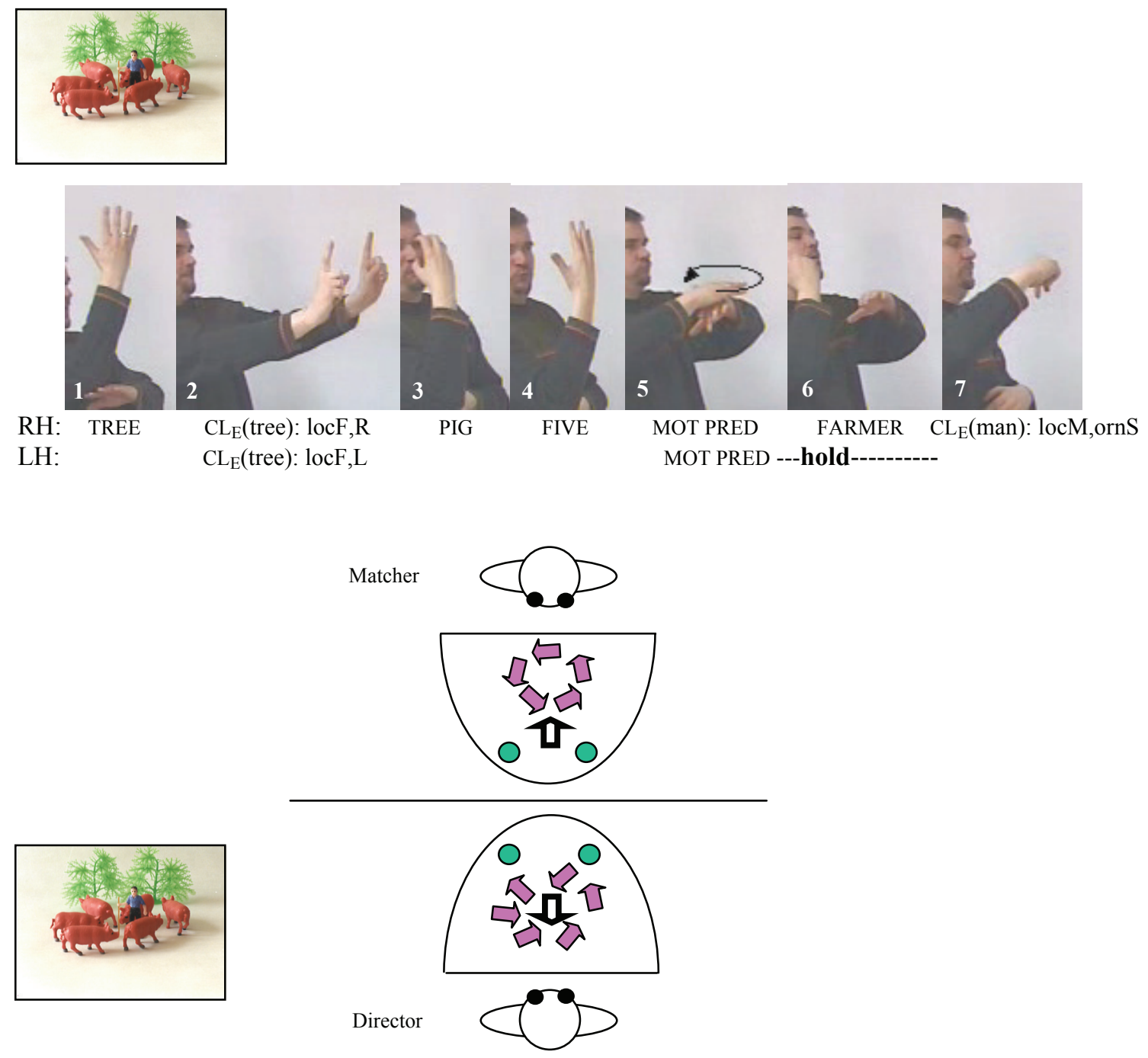

Figure 5.12. Matcher's interpretation of man's location (behind the pigs) and pigs' configuration (in a circle) based on the director's classifier predicate placement and use of motion predicate. 
Similarly, in the description with the general motion predicate that we have seen already in example (25) in chapter 4 (repeated in example (9) above), the signer indicates the location and orientation of the man with respect to the pigs without the use of a simultaneous classifier construction or other sign (e.g. a preposition). In the interpretation, the matcher does not place the man in the middle of the pigs, as in the stimulus picture, but rather behind the pigs (i.e. between the pigs and the trees) (see the schematic representation in figure 5.12 above).

For both examples (8) and (9), subsequent negotiation between the two signers cleared up the misunderstanding. In both cases, the director uses a preposition to clarify the nature of the spatial relationship between the referents. For the picture in example (8), the signer resigned the description, adding the lexical preposition INSIDE and a NEXT-TO preposition moving toward the body to make explicit the location of the pigs with respect to the fence. Thus, he uses prepositional forms to add explicit spatial information about the pigs' locations. As in his original description, he uses a simultaneous classifier construction to represent the two pigs. He does not, however, use an additional simultaneous construction involving the representation of the fence together with one of the pigs.

As an additional point, the matcher interprets the circular movement of the motion predicate in example (9) as encoding specific orientation, while the director uses it to convey the general meaning of "milling about" (i.e. in a random configuration). In the matcher's setup (as shown in the schematic representation in figure 5.12), the pigs are in a circle, all facing in a counter-clockwise direction. This shows that signs have multiple (potential) semantic components, and that those components which are intended to be conveyed and those which are understood may not overlap completely in the first instance.

The interpretation of the tracing sign shown in figure 5.13 below (see example (26) in chapter 4 for the full description) also illustrates this point. The director traces a circle around the man (held in the middle with an upright entity classifier) to convey semantic information both about the pigs' configuration and their direction of facing (counter-clockwise). The matcher, however, interprets the circle as indicating configuration only, and not as additionally indicating the pigs' direction of facing. As a result, the matcher sets up all the pigs so that they are facing the center (i.e. facing the man), which does not correspond to the stimulus picture (see the schematic representation in figure 5.13). It may be that the matcher, in the absence of what he considers to be explicit marking of orientation, chooses an orientation which he finds most plausible given the situation. 


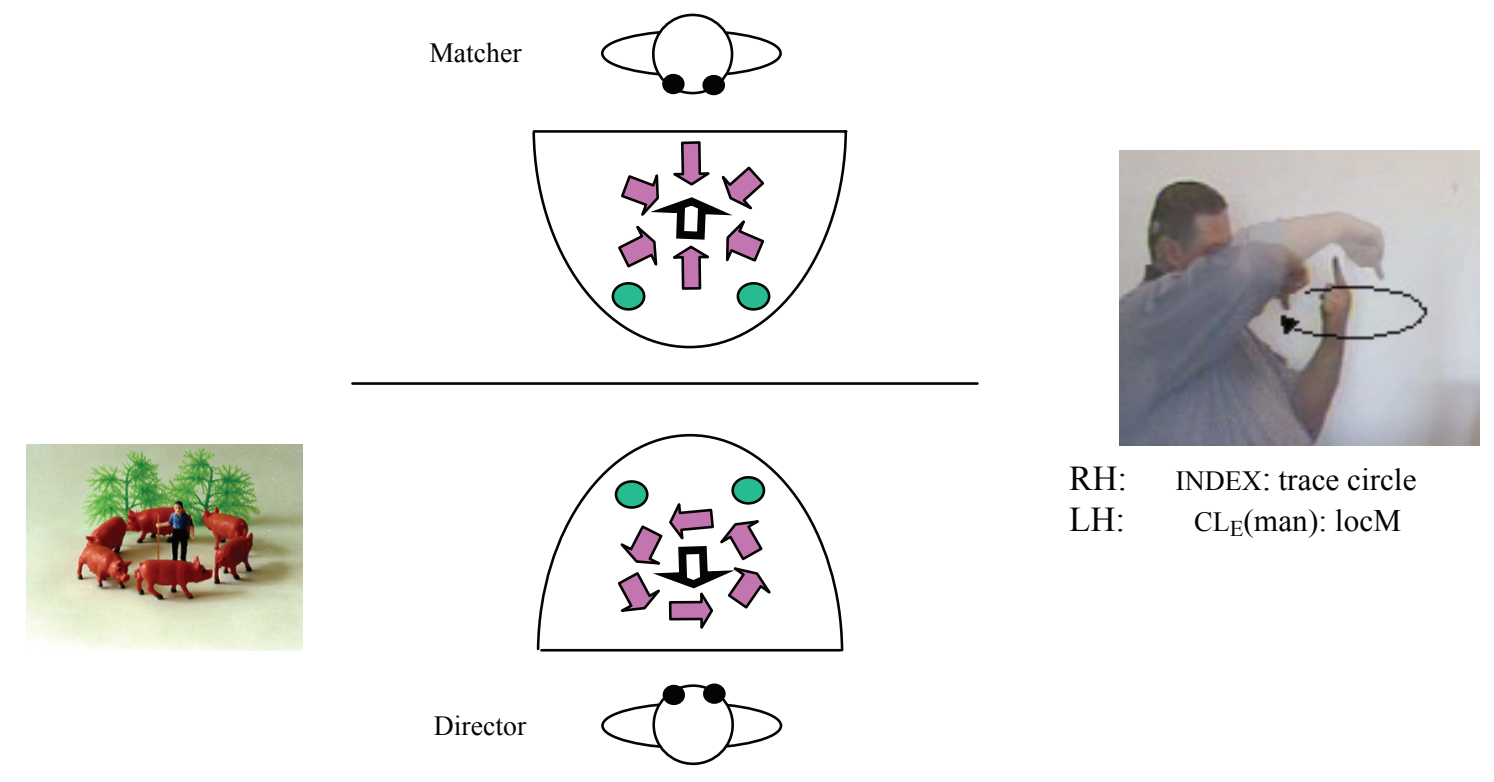

Figure 5.13. Matcher's interpretation of pigs' orientation (facing the center) based on the director's use of a circular trace to indicate configuration and orientation.

\subsubsection{General discussion of interpretation results}

Overall, the results suggest that interpretation from the director's point of view (requiring rotation by the addressee) is conventionalized - or at least the dominant strategy - for the interpretation of spatial relationships of location and orientation in DGS. This echoes Emmorey's findings for ASL, which showed that addressees rotate the spatial information they observe in sign space when re-describing a map (Emmorey et al. 2000), and that addressees are better able to assess the match between an actual room and a signed description of the room when the description is signed from the signer's point of view than when it is signed from the addressee's point of view (Emmorey et al. 1998).

As we have seen, in the DGS data analyzed here, all descriptions were signed from the director's point of view, with no explicit specification of viewpoint. (Viewpoint was mentioned explicitly only a few times in subsequent negotiations between the director and matcher, when there were differences between the director's and matcher's respective object configurations.) Three-quarters of the matchers' reconstructed arrays revealed an interpretation from the director's point of view, thus exhibiting mental rotation of the spatial scene inscribed in sign space. This provides quite robust evidence for the conventionalization of a relative frame of reference, with the signer as viewpoint, in DGS static scene spatial descriptions. 
Nevertheless, we have also seen that the interpretation of location and orientation information is not entirely straightforward. The data reveals that different interpretations arise from the same (or virtually the same) signed description, suggesting that some of the forms used in the spatial descriptions may be, at least partially, semantically general. For example, certain spatial predicates can be unspecified in the sense that they potentially convey multiple semantic aspects, not all of which will apply in a given situation. The selection of intended semantic aspects and interpreted semantic aspects may not overlap. In addition, we saw that a reliance on the iconicity of location-to-location mappings between the event space and sign space can lead to ambiguities. In the complex scenes, descriptions that lacked explicit spatial marking (e.g. through the use of a preposition) were misinterpreted due to difficulties in discriminating relative locations in sign space. The use of LOOK to encode the man's orientation and the potential ambiguity between interpreting it in the function of a classifier predicate vs. a directional predicate was discussed in section 5.5.3.1.

Though not part of the analysis, it is interesting to note the order of referents in the signed description by the director compared to the order of object set-up by the matcher. In the majority of cases, for both simple and complex scenes, matchers set up their object configurations in the same order in which the directors located entities in sign space in their descriptions. ${ }^{84}$ Though I have used the term rotation to describe the end state of interpretation, this does not entail that signers actually perform a mental rotation of the locations/orientations they see in their interlocutor's sign space. Indeed, when asked, native signers of ASL did not report experiencing a sensation of rotating a mental image (Emmorey et al. 1998). Emmorey suggests that addressees may comprehend spatial descriptions as if they were producing the description themselves (by performing a type of self-rotation into the signer's position) or that they may somehow transform the perceived articulations into their own signed productions (involving a "motor theory of sign perception") (1998: 241). In the data presented here, the fact that the order of object set-up by the matcher was the same as the order of articulation by the director suggests that the transformation that takes place in interpretation is not a rotation of the representation itself (as a whole), but indeed a type of self-rotation or "comprehension by imagined doing".

As a final remark concerning the interpretation of spatial information in DGS, recall from the methodology section in chapter 4 (in section 4.2.1) that I signaled to the director

\footnotetext{
${ }^{84}$ The order differed, for example, when matchers set up objects simultaneously (in the simple scenes) or left a question about the location/orientation of a certain entity to the very end (in the complex scenes).
} 
whether or not the matcher's set-up looked the same as the scene depicted in the stimulus picture during the task. If the two configurations were not the same, the director re-signed the description, sometimes in precisely the same way, and sometimes making spatial information more explicit (for example, through the additional use of simultaneous classifier constructions, prepositions, or by explicitly marking spatial contrasts). It is possible that these re-signings and negotiations produced a type of training effect, such that the signers were trained to interpret location and orientation from the director's viewpoint. However, while one or two individual signers who showed a propensity for translation of location and orientation information may have been "instructed" by the director to rotate this information in the course of a particular negotiation, there was no true learning curve observable in the data.

\subsection{Summary and discussion}

The overall results of this chapter have shown that DGS signers use an observer perspective event space projection for static scene descriptions. In addition, the use of a relative frame of reference, either alone or in combination with an intrinsic frame, is predominant for specifying the spatial relationship between a figure and a ground object. The correspondence between the use of observer perspective and the relative frame provides evidence for their alignment or prototypical co-occurrence, while the simultaneous use of the relative and intrinsic frames within observer perspective shows that perspective and frames of reference are indeed independent systems. Furthermore, the simultaneous use of two frames of reference reveals the unique affordances of the visual-spatial modality. Finally, the analysis of addressee interpretation of static scene descriptions has shown that the use of the relative frame of reference, with the signer as deictic origo, is conventionalized in DGS.

Because of the very low frequency of use of character perspective in DGS static scene descriptions, however, we have seen only little evidence for the alignment between character perspective and the intrinsic frame of reference, and no evidence for the simultaneous use of the relative and intrinsic frames of reference within character perspective. Moreover, this chapter provides evidence for the relationship between perspective and frames of reference for only one type of discourse (i.e. static scene description). To investigate these issues, the next chapter turns to the use of perspective and frames of reference in a different discourse type in DGS, namely event narratives. 


\section{Perspective and frames of reference in DGS event narratives}

Chapter 6

The present chapter looks at the use of perspective and frames of reference in the expression of location and motion events in event narratives. In comparison to chapter 5, a different discourse type is being examined and the expression of motion, in addition to static location is investigated. The overall structure of event narratives may be a closer approximation of naturally-occurring language, and discourse type, in general, may have an effect on the way in which spatial information is expressed. We first analyze the encoding of static scenes (i.e. location scenes) in narratives and compare it to the static scene descriptions in chapter 5. Secondly, within event narratives, we compare the expression of location and motion. Thus, we are able to investigate within event narratives whether the use of perspective and frame of reference is influenced by static vs. motion event type.

This chapter further addresses the relationship between perspective and frames of reference. In the previous chapter, we saw evidence, on the one hand, for the alignment between observer perspective and the relative frame of reference. On the other hand, we also saw the simultaneous use of relative and intrinsic frames within observer perspective, providing support for the independence of the two systems. However, the evidence from the previous chapter was essentially restricted to observer perspective representations, since the use of character perspective was very rare. The event narratives analyzed in this chapter are characterized by the activity and interaction of animate referents within an event space. Thus, there is a higher expectation for character perspective representations. This chapter seeks further evidence for the alignments between perspective and frames of reference, as well as for the independence of the two systems in both observer and character perspective event space projections.

The next section details the methodology and data collection for the event narratives (section 6.1). Section 6.2 then investigates the use of perspective and frames of reference in the expression of location in the event narratives. Section 6.3 investigates the use of perspective and frames of reference to express location and motion in event narratives, and the relationship between the two systems is discussed in section 6.4. Finally, I address again the conventionalization of the relative frame of reference by looking at the retellings of 
narratives by addressees (section 6.5). In contrast to chapter 5, where the addressee was asked to construct a model of the signer's description, this analysis looks at the signed production of the relative frame of reference by addressees who have seen the events narrated. If retellers exhibit mental rotation of the sign space in representing referent motion or location, this provides further support for the conventionalization of viewpoint in the relative frame of reference in DGS.

\subsection{Methodology}

\subsubsection{Stimulus materials and task description}

Short cartoon films were used as stimulus materials to elicit event narratives. The films were taken from a German children's television series called Die Sendung mit der Maus, and feature a large personified mouse and a smaller personified elephant as the main protagonists. The films have a fixed "camera" perspective, which defines a constant vantage point on the event. Narratives of six mouse/elephant stimulus films form the basis of the perspective and frame of reference analysis presented here. ${ }^{85}$

In the task, pairs of DGS signers were seated in front of a camera as shown in figure 6.1 below. For each film, one signer (whom I call the teller), was asked to watch the cartoon (presented on a Sony DV-Watchman), and to then relate the event to the other signer.

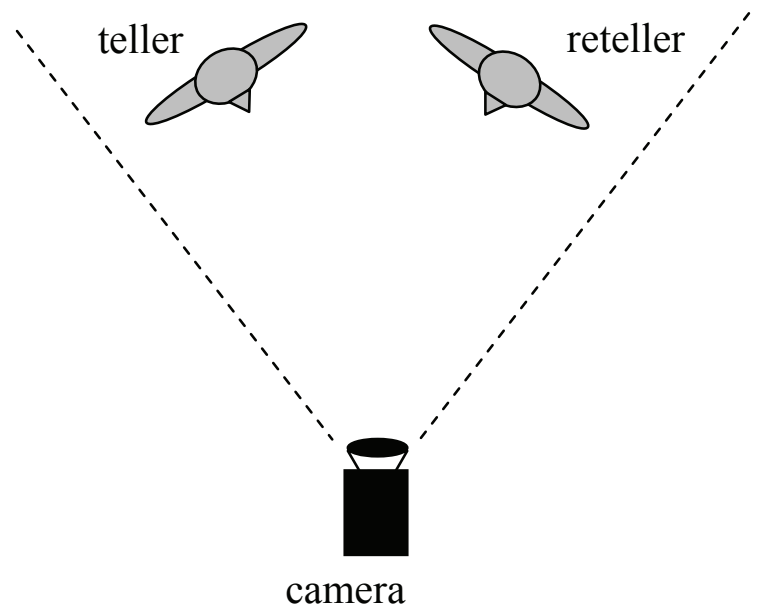

Figure 6.1. Camera set-up in the event narratives elicitation task.

This second signer (whom I call the reteller) was asked to retell the story to the camera (while still seated with the teller), such that another signer watching the film would in turn be

\footnotetext{
${ }^{85}$ In total, narratives were collected for eleven mouse/elephant stimulus films. Of these, six films were chosen for the final analysis, based on the spatial setting, the number of referents, and the nature of referent locations and movements.
} 
able to retell the story. This was meant to insure maximum information reproduction about the content of the story on the part of the reteller.

Signers were told that they could watch the films as often as necessary in order to retain the information they deemed necessary for good narration. Nevertheless, in nearly all cases, signers chose to watch the film only once. Although it was not stated explicitly in the instructions, signers were told upon inquiry that interruptions of the narratives were allowed. Many, though not all, pairs of signers interacted to some extent during the event narrations. For example, addressees (i.e. retellers) sometimes asked clarification questions during the tellings. In some cases, corrections or amendments were made by the teller during the retellings.

\subsubsection{Data collection}

A total of 14 individual signers participated in the task. Data was collected from seven pairs of signers for five stimulus films, and from six pairs for one stimulus film. Signers ranged in age between 16 and 45, with a mean age of 34. Three signers (GL, FB, DM) were native signers (i.e. with deaf parents or older deaf siblings), one signer (SB) was a late signer (i.e. sign language acquisition after the age of six), and the remaining signers were early signers (i.e. sign language acquisition by the age of six) (GP, JP, KF, SP, SR, KO, CS, SS, LL, HT).

The signers in each pair alternated roles with respect to who was the teller and who was the reteller, usually every two films. With some exceptions, the pairings of signers stayed the same for all six stimulus films (GL and GP, JP and LL (or FB), KF and DM, HT and SB, KO and SR (or FB), CS and SS, SP and HT). The signer called HT was a participant in two different pairs. After having been paired with SB, he was paired with SP some months later, in the role of the reteller for all films. FB participated in the narrations of only two individual films, once with JP and once with KO. (The pairings of signers for each stimulus film are shown in appendix B.) Signers told between two and four narratives as the teller in a pair, depending on the pairing and role alternations. ${ }^{86}$

\subsubsection{Data coding}

For each stimulus film, a list was made of all scenes that involved spatial relationships of location and motion that could be expressed in frame of reference terms. In total, the

${ }^{86}$ The exception is FB, who told only one narrative. 
encoding of 74 scenes was analyzed, corresponding to 477 descriptions. ${ }^{87}$ Table 6.1 below shows the number of location and motion scenes and number of descriptions for each type of scene.

Table 6.1. Total number of location and motion scenes in the stimulus films and total number of coded descriptions for each type of scene in the narratives.

\begin{tabular}{|c|c|c|}
\hline & scenes & descriptions \\
\hline Location & 11 & 79 \\
\hline Motion & 63 & 398 \\
\hline
\end{tabular}

The location and motion scenes for one of the six stimulus film events (the "pillow case event") are shown in figure 6.2 below.

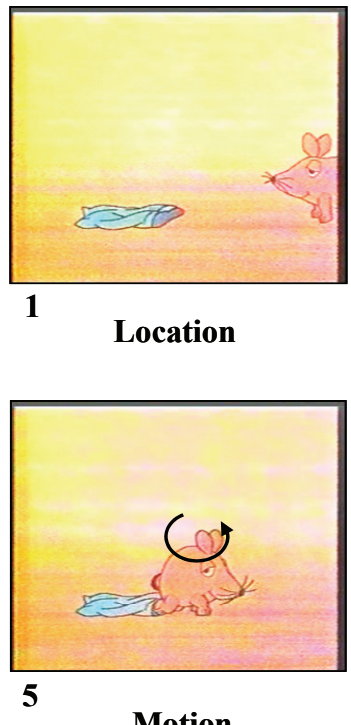

Motion

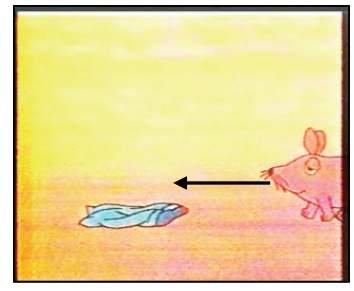

2

Motion

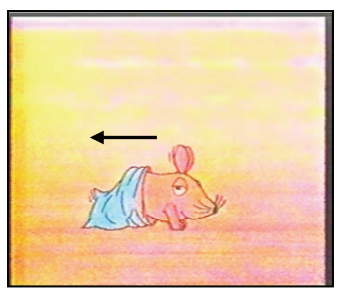

Motion

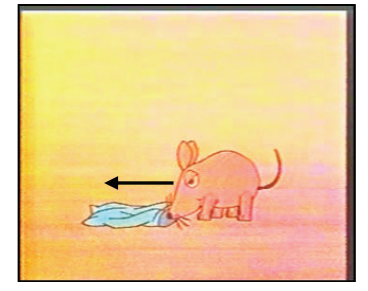

3

Motion

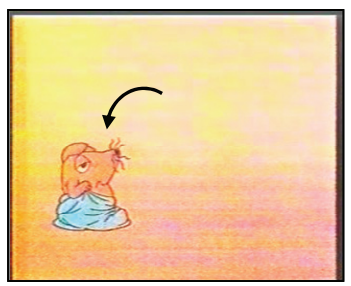

Motion

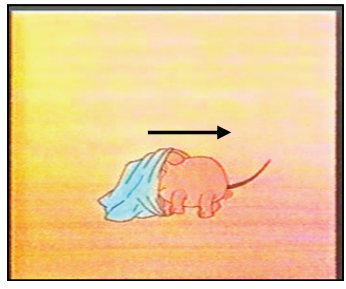

Motion

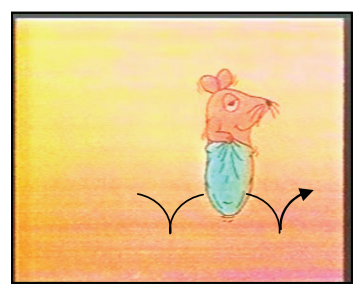

Motion

Figure 6.2. Still images illustrating the location and motion scenes that were coded in the "pillow case event"

The first scene (in still image (1) in the figure) is a location scene that shows the mouse on the right and the pillow case on the left of the screen. The remaining scenes are motion scenes. In (2), the mouse is walking toward the pillow case. Having reached it, the mouse crawls inside the pillow case in (3), and then exits it crawling backwards in (4), after realizing that there is no opening on the other side. The mouse then turns around (5) and crawls backwards into the pillow case (6). It stands up, clutching the pillow case to its waist, and

\footnotetext{
${ }^{87}$ Appendix C, which includes all location and motion scenes for all stimulus events, contains a total of 83 scenes that were identified in the six stimulus films. Ultimately, nine scenes were excluded from the final analysis, because their descriptions could not be clearly distinguished in frame of reference terms. The excluded scenes were: scene 15 from the bar event, scenes 11 and 14 from the chestnut event, and scenes $4-9$ from the apple tree event.
} 
falls backward, landing on its bottom (7). The mouse gets up and starts jumping in place, and then jumps forward, moving laterally to the right off the screen (8). (Appendix C contains similar descriptions and stills for all six films).

As the distribution in table 6.1 and the event description in figure 6.2 show, the events contained many more motion scenes than location scenes. The motion scenes included the agentive motion of an animate referent (i.e. the mouse, elephant, or another character) and the caused motion of animate and inanimate referents. With respect to location, I distinguish between two different types of location scenes, depending on where they occur in the stimulus films. The first scene of each stimulus film reveals the event's spatial setting and configuration of referents (as in still 1 figure 6.2). In total, there are 6 event-initial location scenes. The remaining 5 location scenes are event-medial, and occur when new referents enter the scene and thus become part of the spatial setting, occupying a location within it.

The expression of each location and motion scene required the representation of referents in sign space (i.e. the association of referents with particular locations in sign space). The analysis of how these scenes were encoded can inform us about the use of perspective and frames of reference in event narratives.

Perspective. As in chapter 5, the nature of the event space projection for each scene determined whether it exhibited the use of an observer or a character perspective. Scenes were coded as signed in observer perspective if the whole event space was projected onto the area of space in front of the signer's body. In this projection, referents are represented on a correspondingly small reduced-size scale, and the signers themselves remain external to the event. In contrast, if the event space projection was life-sized, surrounding and encompassing the signer, the scene was coded as represented in character perspective. Referents in character perspective are represented on a life-sized scale, and the signer is in the role of a character within the event. In addition, the axis of representation (i.e. lateral or sagittal, with respect to the body) was a criterion in deciding whether an event space was projected from character or from observer perspective. In the stimulus films, the referents are predominantly located on the left and right sides of the screen as seen by the viewer, and their movements are laterally directed. Representation of location and/or motion on the lateral (i.e. left/right) axis is thus a cue to an event space projection from the perspective of an external observer. On the other hand, as seen from the perspective of the referents in the stimulus films themselves, motion is directed toward or away from the body, and other referents are located in front of or behind the body. Thus, representation on the sagittal axis is associated with an event space projection 
from a character's perspective. ${ }^{88}$ An overview of the criteria that define signing perspective is shown in table 6.2 below.

Table 6.2. Characteristics of observer and character perspective in terms of event space projection, including the axis of representation of referent location/motion.

\begin{tabular}{|l|c|c|}
\hline $\begin{array}{l}\text { Projection of } \\
\text { event space } \\
\text { onto sign space }\end{array}$ & $\begin{array}{c}\text { Character perspective } \\
\text { vantage point } \\
\text { Encompasses signer } \\
\text { Life-sized }\end{array}$ & $\begin{array}{c}\text { Observer perspective } \\
\text { vantage point } \\
\text { In front of signer } \\
\text { Reduced size }\end{array}$ \\
\hline $\begin{array}{l}\text { Representation } \\
\text { of referent } \\
\text { location/motion }\end{array}$ & On the sagittal axis & On the lateral axis \\
\hline
\end{tabular}

Frame of reference. In addition to signing perspective, each scene was coded for frame of reference use. As I have stressed in chapter 5, the instantiation of a frame of reference depends crucially on the construal of figure and ground. In the event narratives analyzed here, the figure entity was the animate entity located or moving with respect to a reference or ground object (another animate or inanimate entity present in the spatial setting of the event space). For a scene to exhibit coding in the relative frame of reference, the figure and ground objects had to be located in sign space from an external viewpoint, e.g. that of the viewer watching the stimulus film. In contrast, use of the intrinsic frame of reference does not involve an external viewpoint. The binary relationship expressed by the intrinsic frame simply locates the figure object with respect to the ground object.

Specifically, for the location scenes, the figure is the (more) active animate referent (or in two cases, the smaller inanimate referent) that is located with respect to an inanimate referent or the (more) passive animate referent. Table 6.3 below identifies the figure and ground objects for all event-initial and event-medial location scenes in the six stimulus films. Descriptions from an external viewpoint in which an unfeatured ground object (e.g. the tree or bar) was encoded first exhibit the use of the relative frame of reference only. Conversely, descriptions from an external viewpoint that encoded a featured ground referent first (i.e. the mouse or elephant) exhibit the use of the relative and intrinsic frames simultaneously (cf. the discussion of figure and ground encoding in chapter 4, section 4.3.3). The use of an intrinsic frame of reference only is exhibited in descriptions that locate the figure object with respect to the referent mapped onto the signer's body (i.e. with respect to the body's intrinsic

\footnotetext{
${ }^{88}$ The axis of representation is a cue for the use of perspective based on the nature of the particular stimuli I have used. I do not claim that axis will be an important factor in determining choice of perspective in all signed narratives.
} 
features). Simultaneous spatialization of two referents was excluded from the analysis, as in chapter 4.

Table 6.3. Overview of the figure and ground elements in the event-initial and event-medial location scenes in each stimulus film.

\begin{tabular}{|l|l|l|l|}
\hline Location scene & Stimulus film & Figure objects & Ground objects \\
\hline Event-initial & Pillow case & mouse & pillow case \\
& Pancake & mouse & stove \\
& Ball & mouse & elephant \\
& Bar & mouse & bar \\
& Chestnut & mouse & tree \\
& Apple tree & mouse & fence/tree \\
\hline Event-medial & Pillow case & - & - \\
& Pancake & elephant & mouse \\
& Ball & - & - \\
& Bar & mouse or elephant & bar \\
& Chestnut & chestnut & mouse/tree \\
& & plank $(2 \mathrm{x})$ & mouse $(2 \mathrm{x})$ \\
& Apple tree & - & - \\
\hline
\end{tabular}

For the motion scenes, the figure object is the moving referent. It can either be animate and featured (e.g. the mouse or elephant), or inanimate and unfeatured (e.g. the pancake or ball). The ground is the source or the goal of the figure's motion, depending on the order of mention of referents. The motion can be construed as, for example, "moving forward" or "moving left" from a particular source, or as moving toward an animate goal (i.e. movement with respect to the intrinsic features of an animate goal). Table 6.4 below gives an overview of the figure-ground combinations that occur in the motion scenes in the six stimulus films.

Table 6.4. Overview of the figure and ground elements in the motion scenes in each stimulus film.

\begin{tabular}{|l|l|l|}
\hline Stimulus film & Figure objects & Ground objects (source or goal) \\
\hline Pillow case & mouse & pillow \\
\hline Pancake & $\begin{array}{l}\text { mouse } \\
\text { pancake } \\
\text { elephant }\end{array}$ & $\begin{array}{l}\text { stove } \\
\text { mouse/elephant } \\
\text { mouse }\end{array}$ \\
\hline Ball & $\begin{array}{l}\text { mouse } \\
\text { ball }\end{array}$ & $\begin{array}{l}\text { elephant } \\
\text { mouse/elephant }\end{array}$ \\
\hline Bar & $\begin{array}{l}\text { mouse } \\
\text { elephant } \\
\text { man }\end{array}$ & $\begin{array}{l}\text { bar } \\
\text { bar } \\
\text { bar }\end{array}$ \\
\hline Chestnut & mouse & tree/chestnut \\
\hline Apple tree & mouse & tree/fence \\
\hline
\end{tabular}


As a final point regarding the assignment of figure and ground status, the presence of both referents within single motion event utterances was not taken to be a strict determining factor of figure-ground structure in the event narratives. In the analysis, previously established referent-location associations, e.g. for a ground entity, were valid across stretches of discourse. In general, the influence of discourse and narrative structure principles on the order of encoding is a complex matter that is not analyzed in any detail here.

Table 6.5 below gives an impression of how each location and motion scene in the pillow case event (cf. figure 6.2 above) would need to be signed in order to exhibit a relative (only) or intrinsic (only) frame of reference encoding. (Appendix $\mathrm{C}$ contains similar tables for all six stimulus films.) The elements in bold face in the table represent the spatial encoding in sign space that corresponds to the use of a relative frame and an intrinsic frame of reference, respectively.

Table 6.5. Possible spatial relationships encoded in signed descriptions of the pillow case stimulus film, corresponding to expression in relative and intrinsic frame of reference terms.

\begin{tabular}{|l|l|l|l|}
\hline$\#$ & Type & RELATIVE frame of reference coding & INTRINSIC frame of reference coding \\
\hline 1 & Loc & Mouse on right; pillow case on left & Pillow case in front of mouse \\
\hline 2 & Mot & $\begin{array}{l}\text { Mouse enters screen on right, moves left } \\
\text { toward pillow case }\end{array}$ & Mouse moves forward, toward pillow case \\
\hline 3 & Mot & Mouse moves left into pillow case, facing left & Mouse moves forward, into pillow case \\
\hline 4 & Mot & $\begin{array}{l}\text { Mouse moves right out of pillow case, facing } \\
\text { left }\end{array}$ & Mouse moves backward, out of pillow case \\
\hline 5 & Mot & $\begin{array}{l}\text { Mouse turns around so that it is facing right } \\
\text { (of screen) away from pillow case }\end{array}$ & $\begin{array}{l}\text { Mouse turns to its left } \text { so that it is facing } \\
\text { away from pillow case }\end{array}$ \\
\hline 6 & Mot & Mouse moves left into pillow case, facing right & Mouse moves backward into pillow case \\
\hline 7 & Mot & Mouse falls to left & Mouse falls backward \\
\hline 8 & Mot & Mouse jumps across screen, moves right & Mouse jumps forward \\
\hline
\end{tabular}

As I have argued, the instantiation of a frame of reference requires the projection of an event space onto sign space from a particular perspective. However, signers did not always use perspective to encode scenes. In these cases, signers used lexical predicates (e.g. the lexical predicate JUMP, as shown in example (1) below), signed in neutral space, to express a motion event. ${ }^{89}$ The movement of this predicate does not express a directed path motion from a source to a goal (e.g. moving from left to right, as in an observer-perspective representation of the event space). The action of jumping is simply stated as occurring, without being encoded within a particular event space projection.

\footnotetext{
${ }^{89}$ Predicates like JUMP have been called "frozen" (Klima and Bellugi 1979; Schick 1990; Supalla 1986). They are evolved from predicates of location and motion involving classifier forms. In contrast to polymorphemic classifier predicates, they are argued to be monomorphemic.
} 
(1)
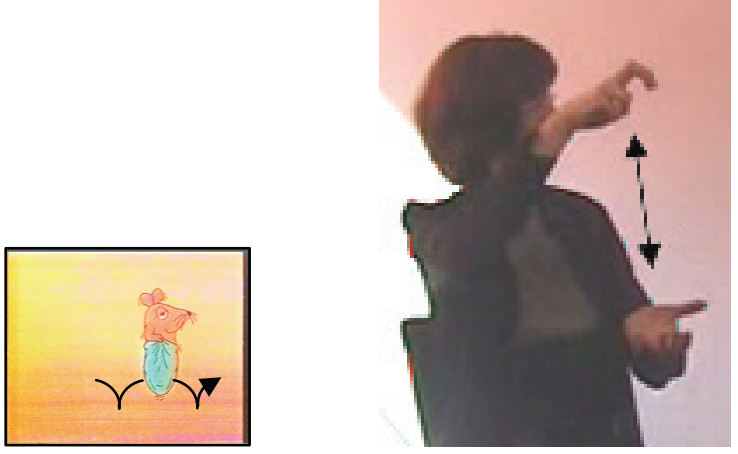

RH: $\quad$ LEX(jump)

LH: $\quad$ LEX(jump)

persp: none

Finally, there was no one-to-one correspondence between the number of scenes that were coded and the number of descriptions that were analyzed. Signers sometimes encoded a scene in more than one way. Specifically, signers used both observer and character perspectives sequentially (with variable order) for the same scene. In these cases, the descriptions were counted individually, one for each type of perspective.

\subsection{The encoding of location in event narratives}

This section presents the analysis of the tellers' encoding of location scenes in the event narratives. The use of perspective is presented first, followed by the use of frames of reference. The results are compared to the results of the analysis of perspective and frame of reference use in static scene descriptions presented in chapter 5 .

\subsubsection{The use of signing perspective to encode location}

Figure 6.3 below shows the percentage of location scenes encoded in observer perspective, character perspective, or without perspective (i.e. with a lexical predicate in neutral space). The total number of location scene descriptions is 79 . The graph shows that all location scenes were encoded in a particular perspective. Furthermore, the graph shows that location was encoded predominantly in character perspective ( $72 \% ; 57$ out of 79 scene descriptions). This is more than twice as high as the use of observer perspective $(28 \% ; 22$ out of 79 descriptions). 


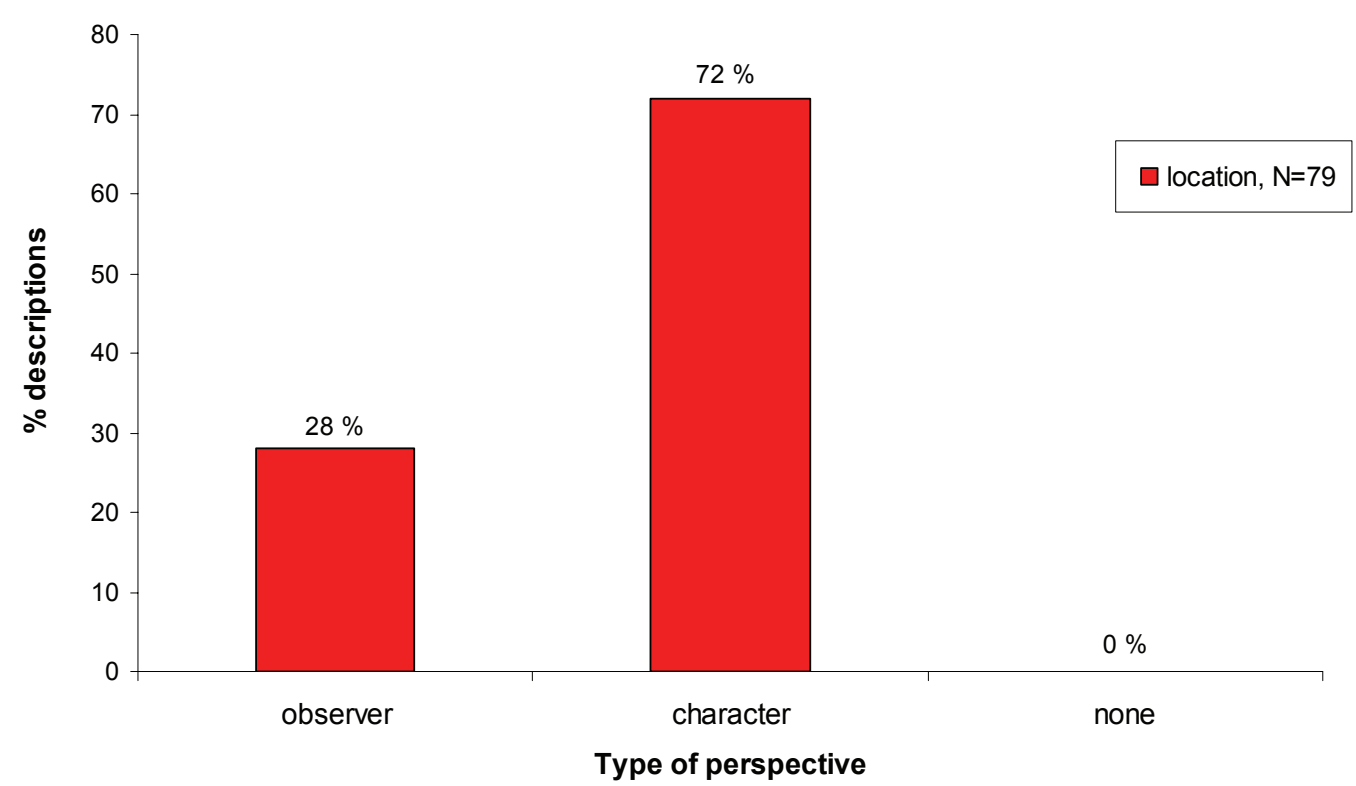

Figure 6.3. Percentage of location scene descriptions that use observer, character, or no perspective.

The finding that location scenes in event narratives are coded predominantly in character perspective stands in marked contrast to the findings of the previous chapter. Recall from chapter 5 that in static scene descriptions, location was coded almost exclusively in observer perspective. The results suggest that discourse type plays an important role in the use of perspective in DGS. (See section 7.5 in chapter 7 for further discussion of the motivation to use character perspective in event narratives.)

The results found here also contrast with findings on the use of perspective in event narratives in other sign languages. The use of observer perspective at the beginning of event narratives has been reported for scene-setting purposes and the establishment of referentlocation associations (Morgan 1999, 2002, 2005; Poulin and Miller 1995; Pyers and Senghas 2007). In his analysis of event narratives in BSL, Morgan $(2002,2005)$ attributes the use of observer perspective (fixed referential space, in his terms) to establishing the spatial layout (or, in his words, the "set" elements of the event). Moreover, Morgan (1999) explicitly argues that signers use observer perspective (i.e. the fixed referential space) at the beginning of an event narrative in order to establish the spatial setting as a foundation, or anchor, through which subsequent shifts in perspective are facilitated and understood.

Similar claims have been made by Pyers and Senghas (2007), comparing referential shift in ASL and NicaSL, as well as by Poulin and Miller (1995) for LSQ. Pyers and Senghas note about the use of observer perspective (which they call diagrammatic space, following 
Emmorey and Falgier 1999) that it "provides a 'big picture' view of how characters and objects are spatially related to one another" and, importantly, that "it is typically expressed early in a narrative, when the narrator sets the spatial stage on which the narrative is to take place" (p. 283). Having established the relevant referent-location associations in sign space, the signer may then use character perspective (viewer space), in which spatial relationships are represented from the perspective of a character within the event, in a way that is consistent with the observer perspective representation. Poulin and Miller (1995) establish this relationship explicitly by distinguishing between a "main frame of reference" and "dependent frames of reference". In the main frame, the narrator associates referents with particular locations in space. The representation of spatial relationships from the perspective of a character is dependent on the spatial indices that are established in the main frame.

These studies provide evidence from research on other sign languages that observer perspective is used in narratives to establish referent locations. The analysis presented here on scene-setting in DGS event narratives (through the encoding of both event-initial and eventmedial location scenes) has not found similar evidence to support the explicit use of observer perspective for this purpose.

Example (2) below shows the encoding of an event-medial location scene sequentially in both a character and an observer perspective event space projection. The represented scene is from the ball event (see the still image above the example): The elephant (on the left, facing right) and the mouse (on the right, facing left) are standing across from each other, looking at each other. In the example, the signer first represents this relationship in a character perspective representation, taking the role of both characters. That is, first, the signer is in the role of the mouse, holding the ball, looking at the elephant (still 1). Then, the signer is in the role of the elephant, standing, looking at the mouse (still 2). ${ }^{90}$ Finally, the signer represents the relationship between the mouse and the elephant in an observer perspective event space projection (still 3). The mouse and the elephant are depicted with entity classifiers on the right and left sides of sign space, respectively, and the orientation of the hands depicts the entities facing each other.

\footnotetext{
${ }^{90}$ Note that the orientation of the signer's head and shoulders in the role of each character corresponds to that character's direction of facing. When the signer is in the role of the mouse, she is oriented toward the left, as the mouse is facing left in the stimulus film. When the signer is in the role of the elephant, she is oriented to the right, corresponding to the elephant's orientation in the stimulus film. The use of a shoulder shift to represent different characters is a common device in DGS narratives for marking switches in subject reference (Perniss and Özyürek 2004b).
} 
(2)
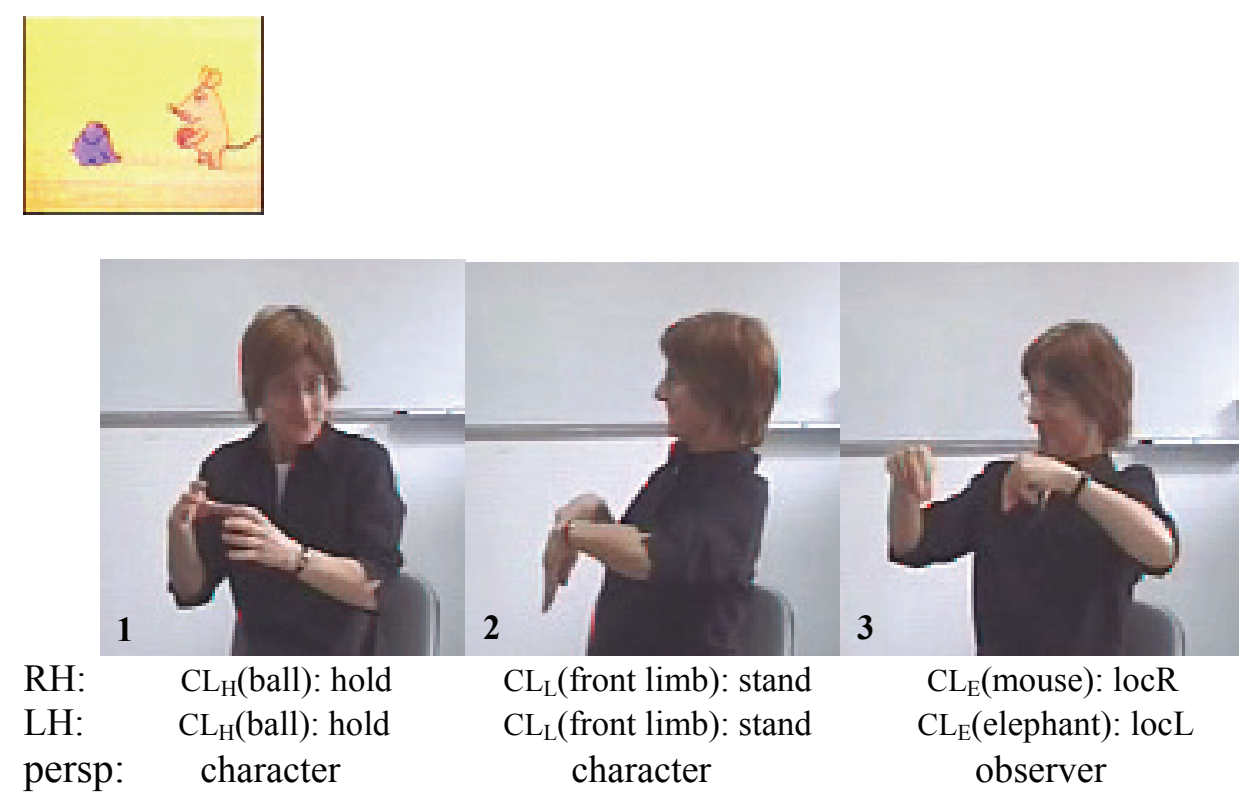

This is not to say that scene-setting is not an important component in DGS event narratives. Instead of observer perspective, however, the data shows that DGS signers prefer to use character perspective for scene-setting. The importance of scene-setting is emphasized, e.g., by the fact that signers never omitted event-initial scenes in the narratives; only event-medial location scenes were omitted. This suggests that initial scene-setting, i.e. introducing the referents present in the event space, is indeed important in providing an anchor from which further spatial information is interpreted.

In this regard, it is interesting to note an observation made in Slobin's (1997) discussion of scene-setting in spoken narratives and literary texts. Slobin (1997) suggests that speakers of verb-framed languages (compared to speakers of satellite-framed languages) devote more attention to scene setting, that is, to describing the spatial setting or physical context in which a motion event takes place. He suggests that speakers of verb-framed languages may choose to organize discourse in a way that gives path information indirectly by focusing on the layout of the spatial setting in which the event takes place (instead of by adjoining multiple path segments to a single verb, as is possible in satellite-framed languages). In other work, Slobin has suggested that sign languages should be analyzed as "complex verb-framed languages" (see Hoiting and Slobin 2001; Slobin and Hoiting 1994). Given these assumptions, Slobin would presumably predict that signers devote time to establishing the spatial setting and to making spatial relationships between referents explicit early on in the narrative, as indeed they do. 


\subsubsection{The use of frames of reference to encode location}

An analysis of frame of reference use depends on the identification of figure and ground entities, and on the way in which the relationship between these entities is expressed. The identification of figure and ground for both location and motion scenes was outlined in section 6.1.3 above. The frame of reference analysis in this section is based on a total of 78 descriptions (after the exclusion of one description in which both entities were simultaneously located in space (shown in still 3 of example (2) above)).

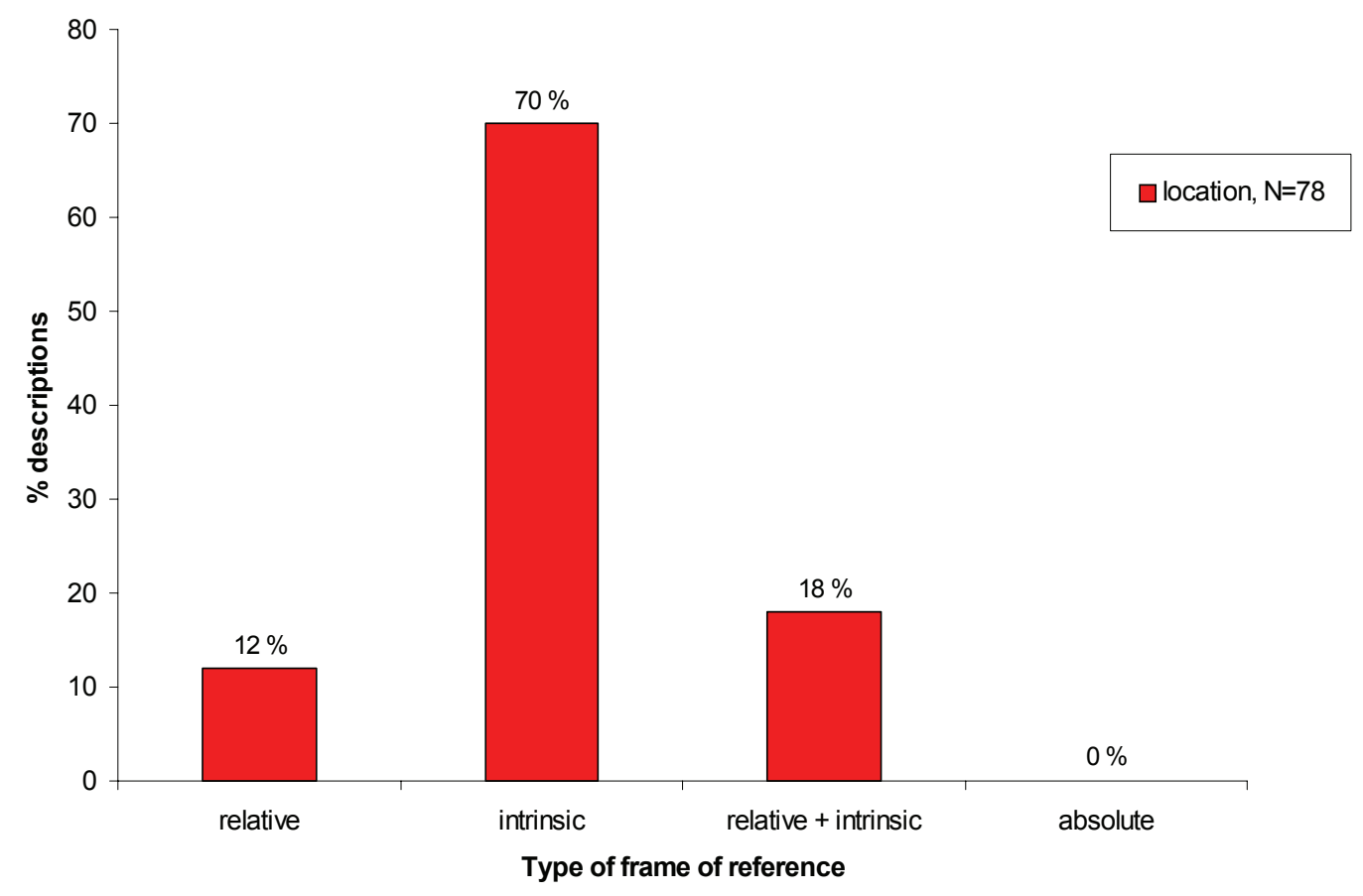

Figure 6.4. Percentage of descriptions that exhibited the use of a relative only, intrinsic only, relative+intrinsic, and absolute frame of reference for the location scenes.

The graph in figure 6.4 above shows that location was encoded in terms of the intrinsic features of the ground object in the vast majority of descriptions, making the intrinsic frame of reference by far the most common type (70\%; 55 out of 78$)$. In comparison, the relative frame of reference was used very infrequently. Scenes that were coded in the relative frame of reference only $(12 \% ; 9$ out of 78$)$ were characterized by an inanimate ground object whose location in the event space setting was encoded before that of the animate figure. Slightly more common was the occurrence of relative and intrinsic coding simultaneously $(18 \% ; 14$ out of 78). In these descriptions, the figure and ground objects are located in sign space from an external (relative) viewpoint, and the figure object is simultaneously located with respect to the intrinsic features of the ground object. 
The use of frames of reference to encode referent location in event narratives again contrasts sharply with the findings in chapter 5. Overall, the results of the analyses in this and the previous chapter indicate that discourse type (i.e. static scene descriptions vs. event narratives) influences the expression of location scenes. In the static scene descriptions analyzed in chapter 5, location/orientation was expressed almost exclusively in observer perspective and in a relative frame of reference, either alone or simultaneously with the intrinsic frame. In contrast, in the event narratives analyzed in this chapter, referent locations were predominantly indicated in a character perspective event space projection. Moreover, the use of the intrinsic frame of reference far exceeded the use of the relative frame alone or "relative+intrinsic".

What could account for the strong influence of discourse type that we see in the encoding of location in event narratives compared to static scene descriptions? One reason may lie in the overall nature of the events, especially the fact that they are characterized by the activity of animate referents. Another reason may be the more direct involvement in the narrative that the identification with a protagonist allows. This may make it more likely for signers to encode the event from a character perspective. In addition, signers may use character perspective for encoding location in anticipation of an upcoming transitive motion event involving manual activity (cf. Engberg-Pedersen 1993; Zwitserlood 2003; also see chapter 7, section 7.5).

Narrative discourse typically involves not only location, but also the motion and action of referents. This is examined in the following section, where the encoding of location and motion is contrasted with respect to perspective and frame of reference use.

\subsection{The encoding of location and motion in event narratives}

\subsubsection{The use of signing perspective to encode location and motion}

The use of perspective to encode location and motion scenes in the event narratives is shown in figure 6.5 below. The figure shows the proportion of descriptions that used an observer, character, or no event space projection, for all location and motion scenes across all signers and all narratives. The percentages for the encoding of location scenes are repeated from figure 6.3 (in section 6.2.1) above. 


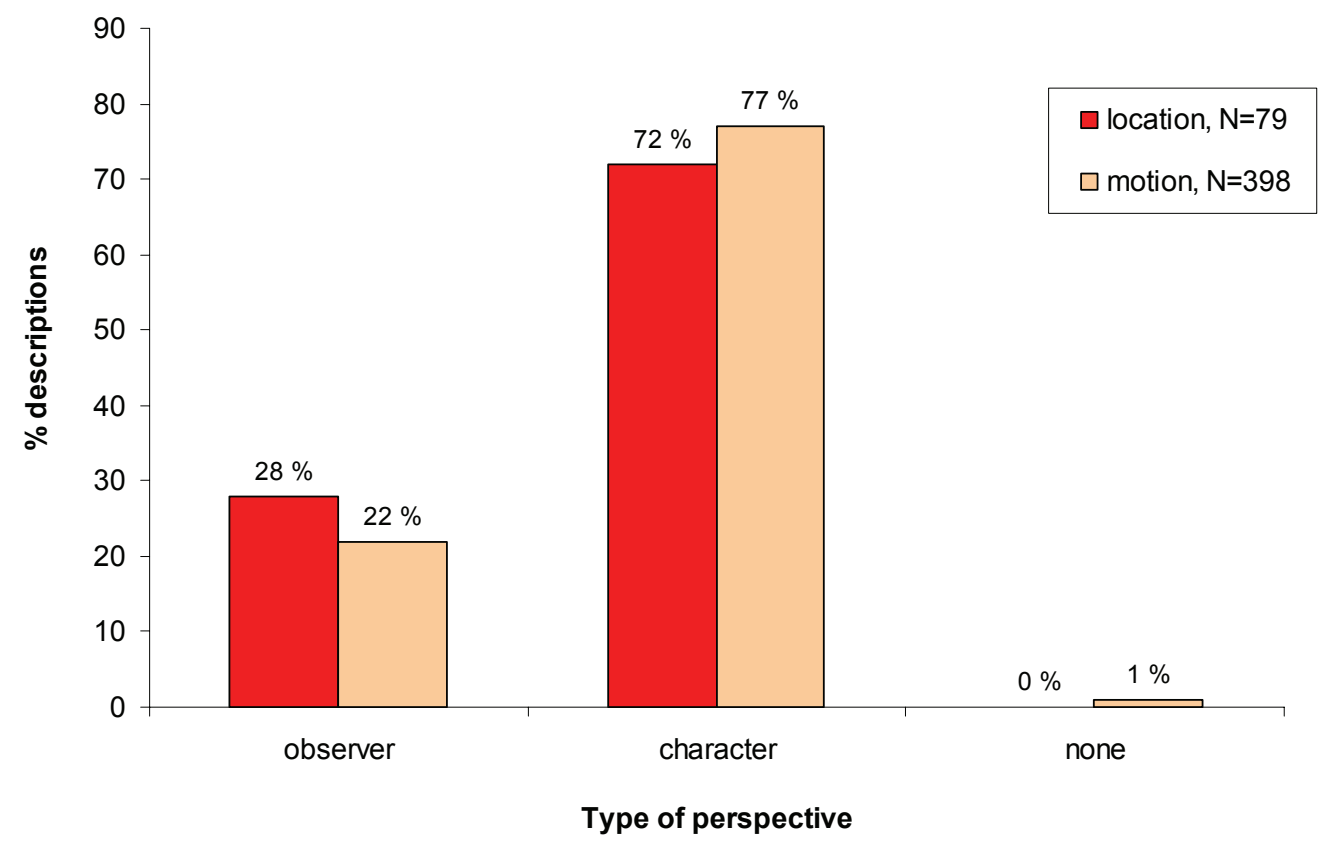

Figure 6.5. Percentage of perspective use in DGS event narratives for scenes depicting static location vs. motion.

The graph shows that the distribution of use of observer and character perspective does not differ between location and motion event encoding. In these event narratives, DGS signers prefer character perspective for both types of spatial scene.

As already noted at the end of the previous section, the preference for character perspective signing in event narratives may be related to the expression of certain semantic components of the stimulus events. In particular, the relevant motion events in the stimulus films are characterized by different manners of motion, including manner of locomotion by an agent (e.g. walking, jumping, turning) and caused motion of inanimate objects by an agent (e.g. by throwing, hitting, flipping). Moreover, many of the agentive motion events are accompanied by an additional manner element, namely, the manner of handling or conveying an object while moving, e.g. holding a pan (as in the pancake event) or dribbling a ball (as in the ball event). Thus, many of the manner of motion elements that we encounter here are necessarily represented with handling classifiers, and felicitously represented in character perspective. In addition, affect and emotion demand the use of character perspective, as they are necessarily represented on the character's (i.e. the signer's) face. (The relationship between signing perspective and classifier predicates, as well as the role of different semantic components of motion events are discussed in more detail in the next chapter.) 
The use of character perspective has been discussed elsewhere as a pragmaticallydetermined preference to focus on the interaction between characters (Leeson and Saeed 2002) and to represent events from an "egocentric" (i.e. protagonist) point of view (EngbergPedersen 1993). In addition, a preference for signing from character perspective seems to be supported by certain factors inherent in the event structure. For example, if there is only one animate referent, or a referent that is identified as the primary protagonist, signers tend to adopt this referent's perspective as the dominant point of view from which to narrate an event (cf. Engberg-Pedersen's 1993 empathy convention for referent mapping). This is strengthened further if the protagonist engages in manual activity (as is the case in the stimulus events used here), which the signer must necessarily represent in character perspective, where the signer's hands represent the character's hands and the use of handling classifiers represents the character's manipulation of objects. These observations are congruent with the DGS data.

\subsubsection{The use of frames of reference to encode location and motion}

In this section, I look at the use of frames of reference to encode location and motion scenes in event narratives. The analysis is based on a total of 392 motion scene descriptions, excluding six scenes that were represented with lexical predicates without an event space projection.

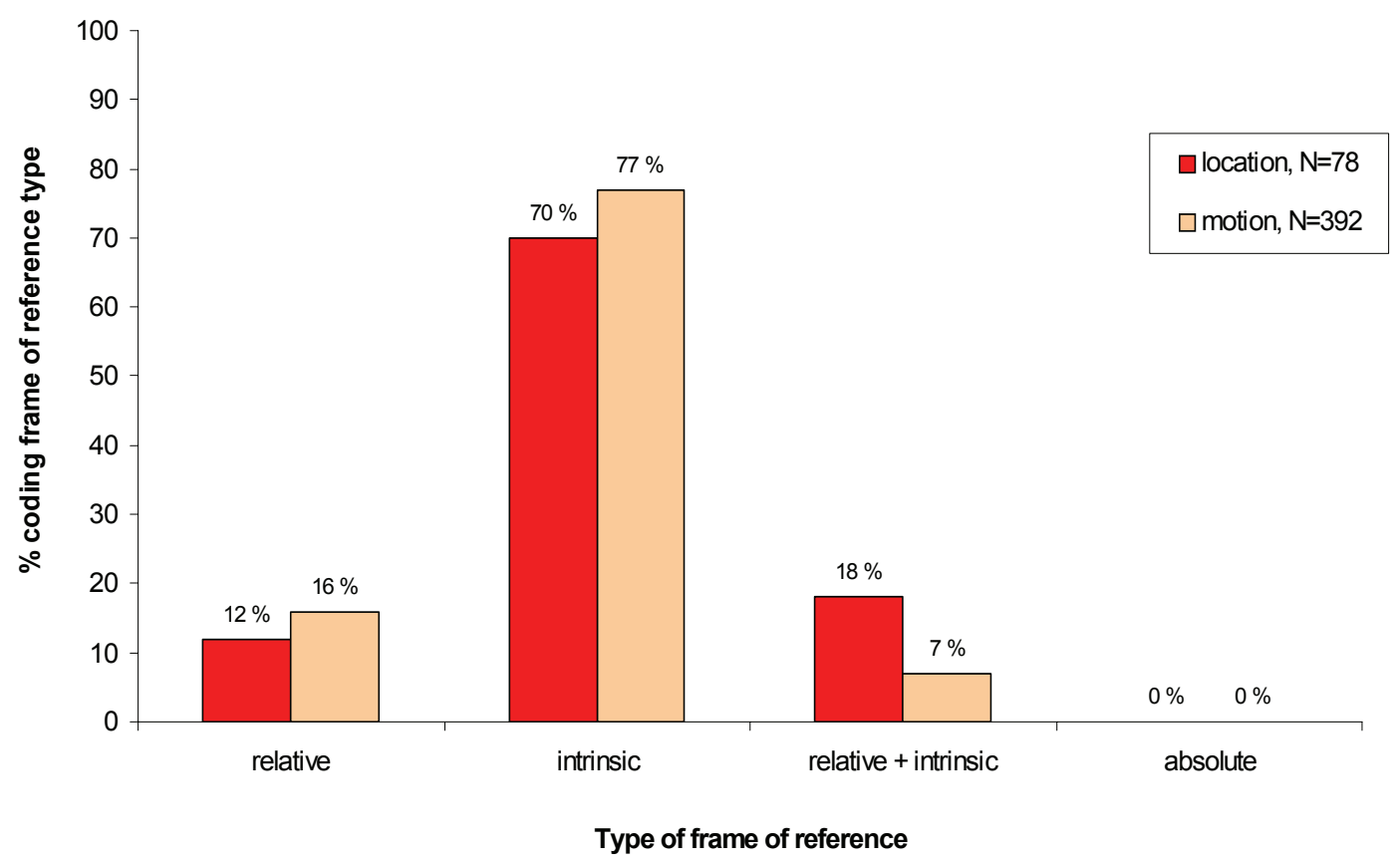

Figure 6.6. Percentage of frame of reference use in DGS event narratives for scenes depicting static location vs. motion 
Figure 6.6 above shows the distribution of use of frames of reference in location and motion events across all signers and all event narratives. The percentages for the location events are repeated from figure 6.4 (in section 6.2.2) above.

As the graph shows, the use of the relative and intrinsic frames is distributed very similarly between location and motion scenes. Both location and motion scenes are encoded predominantly in an intrinsic frame of reference (about three-quarters of the time), and the relative frame is seldom used in comparison (only about one-sixth of the time). There are no occurrences of the absolute frame of reference for either type of scene. The graph also shows the proportions of location and motion descriptions that involved the simultaneous use of a relative and an intrinsic frame of reference. In total, there were 14 location scene descriptions (18\%) and 28 motion scene descriptions (7\%) that were characterized by the use of both the relative and intrinsic frames. Here, the difference between the percentages of use for location and motion scenes is comparatively pronounced.

The reason for the greater difference seems to lie in the fact that signers often started their narratives by indicating the location and/or motion of the mouse. It was the mouse, then, that functioned as the featured ground referent relative to whose features another referent was located. This difference may thus be related to the signers' preference to identify with the mouse as the primary protagonist (cf. the discussion at the end of the previous section). The simultaneous use of relative and intrinsic frames is discussed in more detail in the next section.

\subsection{Alignment between perspective and frames of reference in location and motion scenes}

Figure 6.5 in section 6.3.1 above shows that signers used character perspective about three times as frequently as they used observer perspective for both location and motion scenes. In figure 6.6 in section 6.3.2, the graph shows that the relationship between figure and ground referents was encoded in an intrinsic frame of reference about five times more often than in a relative frame of reference for both location and motion scenes. Thus, we see a predominance of use of character perspective and the intrinsic frame over observer perspective and the relative frame of reference. This corresponds to the alignments between perspective and frames of reference that I have argued for in chapters 3 and 5.

Indeed, all of the location and motion scenes that used a relative frame of reference were encoded in an event space projected from observer perspective. The signer's body is external to the event space, and defines a viewpoint on the event space that determines the 
relative locations of figure and ground entities. In the event descriptions, referent locations expressed in observer perspective appeared on the left and right sides of sign space (i.e. on the lateral axis). Similarly, all the scenes that used an intrinsic frame of reference were encoded in an event space projected from character perspective. When signers map an animate referent onto the body, as required by character perspective, locations of other referents are mapped onto sign space with respect to the signer's body (i.e. opposite the signer's body on the sagittal axis). The signer's body, in the role of a character within the event, functions as the ground object in an intrinsic frame of reference expression.

These alignments, however, do not equate perspectives and frames of reference. In the event narratives analyzed in this chapter, we see the simultaneous use of relative and intrinsic frames in both observer and character perspectives. This is further evidence for the independence of the two systems. Table 6.6 below shows the distribution of "relative+intrinsic" codings in both perspectives for location and motion scenes.

Table 6.6. Distribution of use of relative and intrinsic frames of reference simultaneously in observer and character perspective event space projections.

\begin{tabular}{|l|c|c|c|}
\hline & $\begin{array}{c}\text { OBS persp. } \\
\text { "rel.+intr." }\end{array}$ & $\begin{array}{c}\text { CHAR persp. } \\
\text { "rel.+intr." }\end{array}$ & Total \\
\hline Location & 12 & 2 & 14 \\
\hline Motion & 13 & 15 & 28 \\
\hline
\end{tabular}

Examples of the simultaneous use of the relative and intrinsic frames are given below. Example (3) shows the depiction of a motion scene from a narrative of the ball event (see the image from the stimulus film above the example) which exhibits "relative+intrinsic" in observer perspective. The signer first establishes the elephant's location in an observer perspective event space projection by depicting the floor as a horizontal plane in the sign space in front of his body ( $\left(\right.$ till 2) and locating the elephant on it (still 3). ${ }^{91}$

\footnotetext{
${ }^{91}$ Although the signer does not use an entity classifier here to represent the whole elephant, the elephant's location is nevertheless represented in an event space projection that corresponds to observer perspective. This is evidenced by the location of the signer's hands in sign space on the horizontal plane and at some distance from the signer's body. In the stimulus film, the elephant is facing away from the viewer (i.e. facing in the same direction as the viewer), and the signer uses his own body to represent the elephant's orientation. The signer's hands represent the elephant's front limbs, giving information about the elephant's posture. In a fully character perspective representation, the signer's hands (as limb classifiers) would be held under his chin. The representation is coded here as being in observer perspective because we are interested in the perspective in which referent location is expressed.
} 
(3)
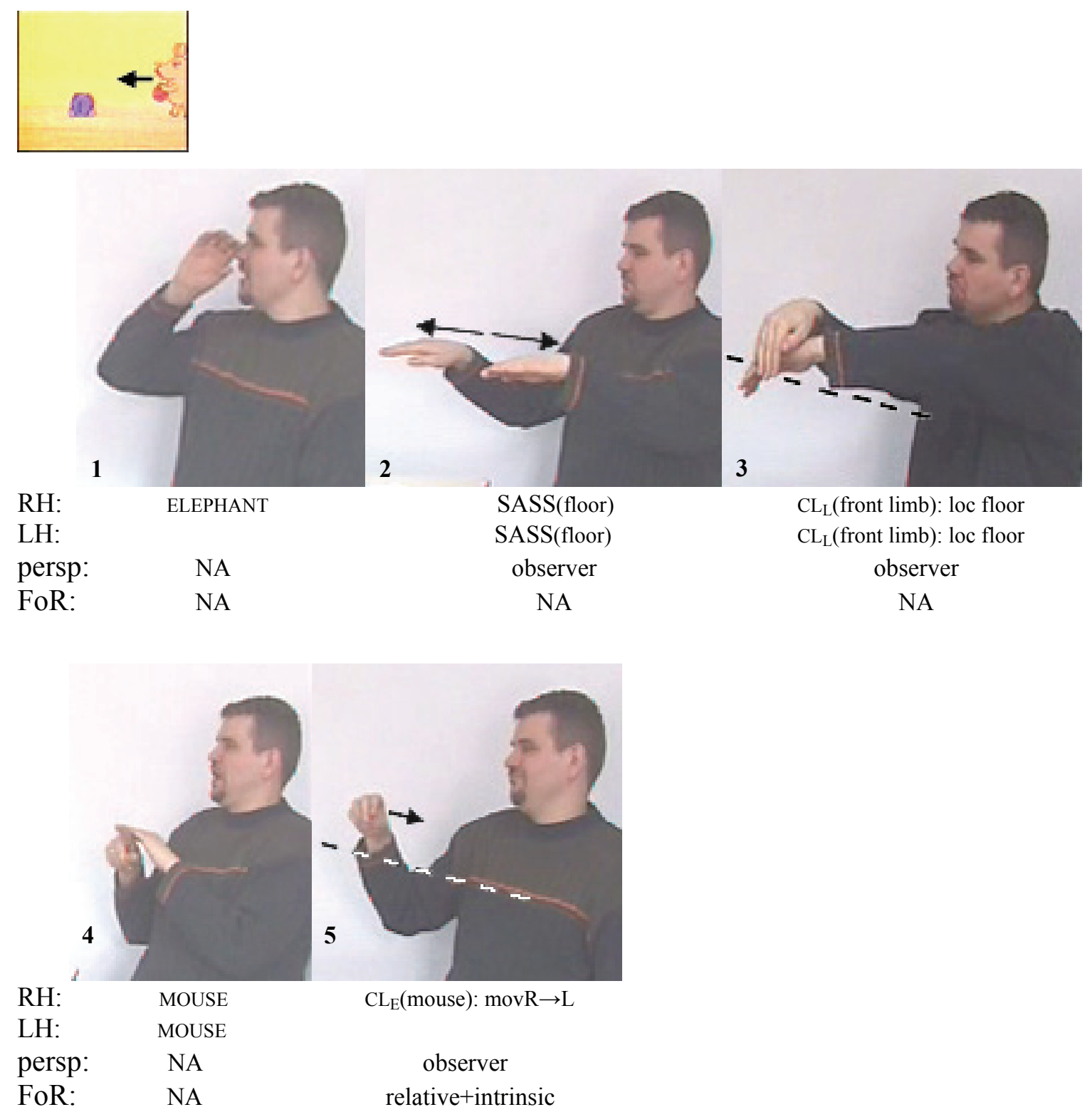

After identifying the mouse (still 4), the signer then represents its motion in the observerperspective event space projection (still 5), moving from right to left, on the lateral axis. This corresponds to the signer's external view of the stimulus event, and thus corresponds to a relative frame of reference expression with the signer as deictic origo. Simultaneously, the mouse's direction of motion is construed with respect to the elephant's orientation (indicated in still 3 as facing forward). The mouse is to the elephant's right, and the mouse is moving toward it, as a featured goal.

Example (4) below shows the simultaneous use of relative and intrinsic frames to depict referent locations in character perspective. The signer uses size and shape specifiers (SASS) to represent the locations of the stove and the basket of wood in the kitchen scene of the 
pancake event (see the still image above the example). The SASS forms are large in scale, corresponding to the life-sized, character perspective event space projection, in which the signer is conceptually in the role of the mouse (cf. figure 7.1 in section 7.2.3 of chapter 7).

(4)

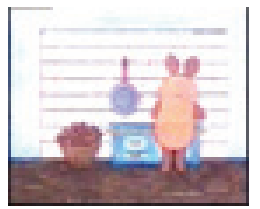

RH:

LH:

persp: (a)

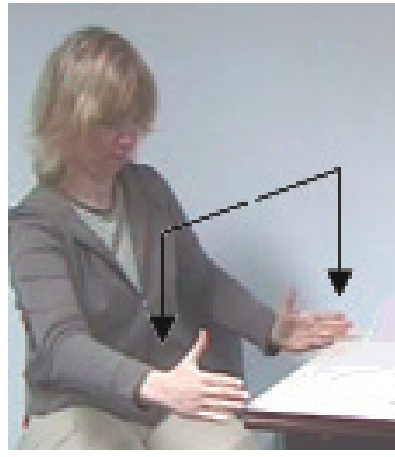

SASS(stove): loc

SASS(stove): loc

character (b)

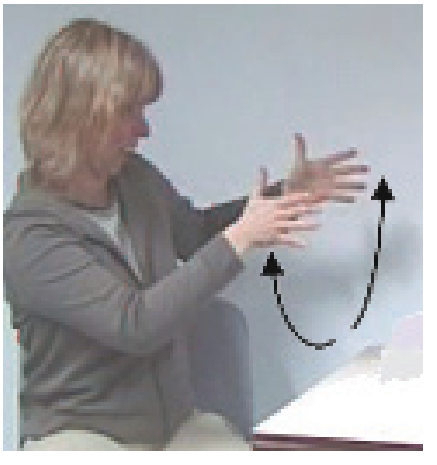

SASS(basket): loc

SASS(basket): loc

character

In (4a), the signer locates the stove directly in front of her body (i.e. in front of the mouse's body). The basket of wood (in (4b)) is located to the left of the stove, and thus also to the left of the signer's/mouse's body. Thus, both entities are located with respect to the inherent features of the mouse as the ground object, thus displaying the use of the intrinsic frame of reference. At the same time, the relative locations of the stove and the basket are expressed from a third location, that is, from the mouse's viewpoint as deictic origo.

(a)

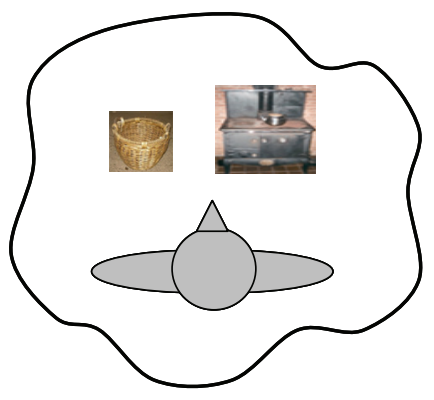

(b)

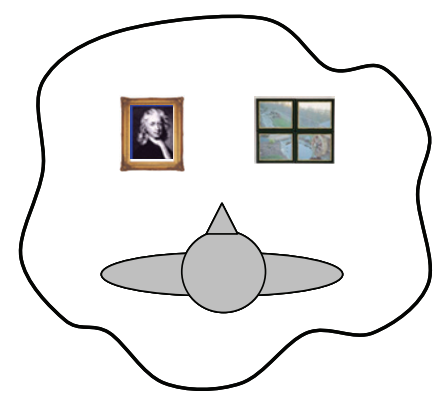

Figure 6.7. Schematic illustrations, in (a), of the representation in example (4), which I analyze as exhibiting the simultaneous use of relative and intrinsic frames of reference in character perspective, and, in (b), of Emmorey's (2002) example for the use of the relative frame of reference in character perspective.

This example is like Emmorey's (2002) example of the use of the relative frame of reference in character perspective. In figure 6.7 above, Emmorey's example (repeated from figure 3.9(b) in section 3.2.2 in chapter 3 ) is compared schematically to example (4). In contrast to 
Emmorey, I analyze this construction as exhibiting both the relative and intrinsic frames simultaneously.

The simultaneous use of relative and intrinsic coding in a character perspective event space projection was due almost exclusively to the signer shown in example (4), and occurred predominantly in the pancake event narrative. Overall, the low incidence of "relative+intrinsic" in character perspective for location scenes may be due to the lack of scenes containing two spatially non-coincident referents in addition to the (animate) referent that can be mapped onto the signer's body (as with the mouse, stove, and basket in the pancake event).

\subsection{Encoding of viewpoint in the relative frame of reference in teller and reteller narratives}

In chapter 5, we saw that essentially all descriptions were given from the signer's external viewpoint, thus in a relative frame of reference with the signer as deictic origo. The addressee responses were analyzed to verify the conventionalization of viewpoint in the relative frame, and the results confirmed a predominance of rotation responses. Here, for the event narratives, though the use of the relative frame proved to be a minor strategy overall, we look to see if it is deployed in the same way.

This section therefore focuses on the location and motion scenes that were encoded in observer perspective using a relative frame of reference by both tellers (having watched the stimulus film) and retellers (having seen the teller's narrative). As with the static scene descriptions in chapter 5 , the retellers (facing the tellers) might produce either a rotation or translation response in their own encoding of referent location and motion on the lateral axis. A rotation response indicates that the addressee is interpreting location and motion information from the signer's point of view, providing evidence for the conventionalization of the signer as deictic origo in the relative frame of reference. In contrast, a translation response indicates that the addressee is interpreting the information he sees in the interlocutor's sign space from his own point of view.

In total, 39 scenes were encoded by both the teller and the reteller from an external viewpoint. The analysis reveals that retellers rotated location and/or motion information $80 \%$ of the time (i.e. for 31 out of 39 scenes). This again provides evidence (from another discourse type, in addition to static scene description in the previous chapter) that the use of the relative frame of reference with the signer as deictic origo is indeed conventionalized in DGS. 
The teller-reteller codings of two scenes from the ball event in which the reteller exhibited rotation of motion information are shown in examples (5) and (6) below. In (5a), the teller depicts the mouse entering the scene from the right, on the lateral axis (corresponding to the direction of motion she has observed on the screen while watching the stimulus film). In (5b), the reteller depicts the scene in the same way, representing the mouse's motion from right to left. The reteller rotates the direction of motion information, interpreting it from the signer's point of view. Similarly in example (6), both the teller (in example (6a)) and the reteller (in example (6b)) represent the mouse moving to the right on the lateral axis, after being hit in the stomach with the ball. Here again, the direction of motion in sign space corresponds to the mouse's direction of motion in the stimulus film as seen by the viewer.

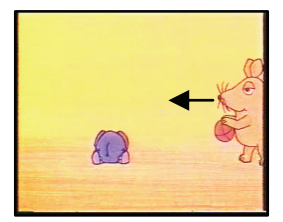

$\mathrm{RH}:$

LH:

persp: $\quad$ observer FoR: $\quad$ relative

(6)

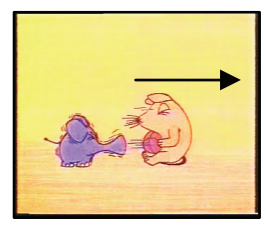

$\mathrm{RH}$ :

(a) Teller

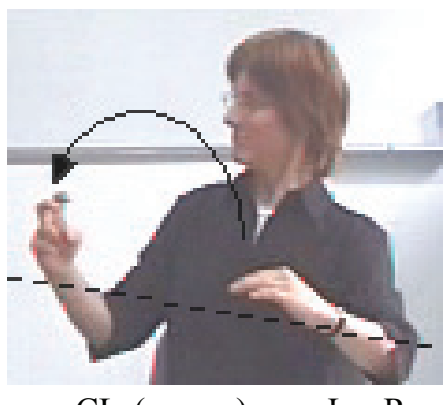

LH:

persp:

FoR: (a) Teller

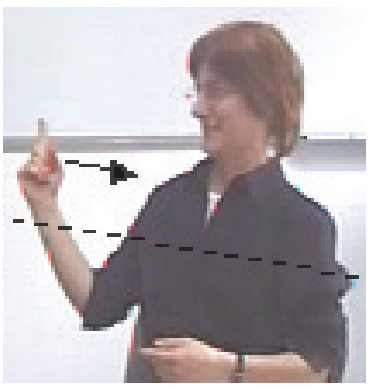

$\mathrm{CL}_{\mathrm{E}}$ (mouse): movR $\rightarrow \mathrm{L}$
RH:

LH:

persp:

FoR: (b) Reteller

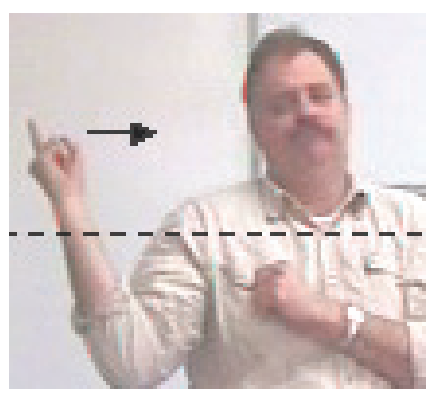

$\mathrm{CL}_{\mathrm{E}}$ (mouse): movR $\rightarrow \mathrm{L}$

observer relative (b) Reteller

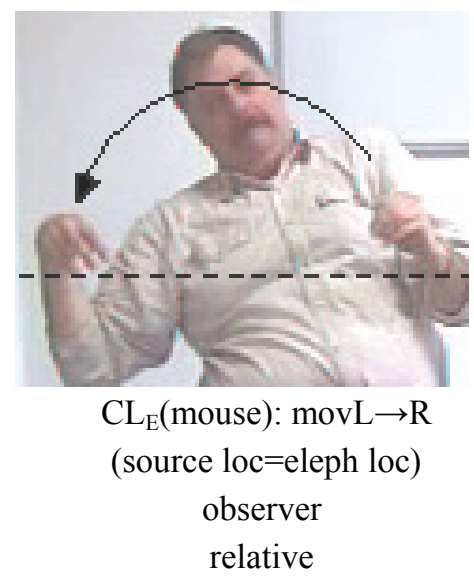

A closer look at the scenes for which retellers represented the event by translation (not rotation) of location and/or motion information suggests that hand dominance is a significant 
factor. For example, in the chestnut event, the mouse exits the event space on the left and shortly thereafter re-enters on the left (see the image from the stimulus film shown with example (7) below). These scenes in the chestnut event accounted for five of the eight nonrotated encodings. In all the teller narratives, the mouse's motion was represented to and from the left, respectively, as it appeared on the screen. The mouse's motion was represented to the left even when the teller used his dominant, right hand to depict the movement, resulting in an articulatorily more marked contralateral movement across the body (as in (7a) below). In the translation responses in the reteller narratives, the retellers represented the motion to and from the right, using their dominant, right hands, thereby depicting the movement ipsilaterally (in an articulatorily less marked way).

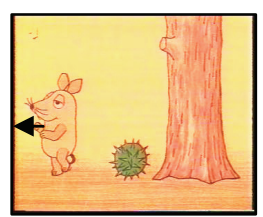

RH:

LH:

persp:

FoR: (a) Teller

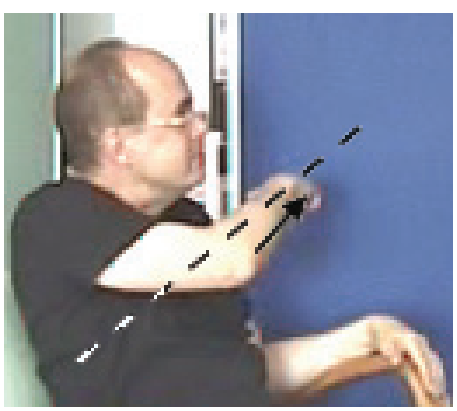

$\mathrm{CL}_{\mathrm{E}}$ (mouse):movR $\rightarrow \mathrm{L}$

observer

relative (b) Reteller

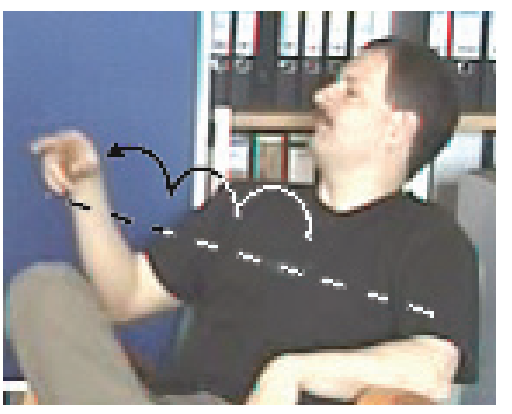

RH:

LH:

persp:

FoR:
$\mathrm{CL}_{\mathrm{E}}$ (mouse):movL $\rightarrow \mathrm{R}$

observer relative

The proportion of rotation vs. translation interpretations in this chapter are very similar to what was found in chapter 5 . In the static scene descriptions in chapter $5,73 \%$ of the matchers' object set-ups (which represent externalizations of their mental interpretations of the signed descriptions) exhibited a rotation of location and orientation information. In this chapter, rotation was exhibited by the addressee in $80 \%$ of the scenes for which both the teller and reteller used observer perspective/relative frame of reference to encode location or motion. As was discussed above, hand dominance seems to have influenced the representation of location/motion on the lateral axis in the retellers' narratives, arguably lowering the proportion of rotation responses. Had signers in the narrative task not been asked to sign, but rather to externalize their interpretations in a non-linguistic way (as with the object set-ups in the static scene tasks), the proportion of rotation may have been closer to $100 \%$. 
This suggests that addressees maintain a high degree of spatial coherence in comprehension, integrating information about referent location and motion into a coherent mental representation of the event space. The construction of an event space within which activity and interaction between referents takes place, as in event narratives, may also affect the interpretation of certain forms that appear in the discourse. For example, in section 5.5.3.1 of chapter 5, the interpretation of LOOK as a directional predicate (i.e. as "look at") was cited as a possible reason for translation responses of orientation. That is, in the static scene descriptions, addressees may have interpreted the goal location of the LOOK predicate within the context of the speech event (e.g. as looks at me), and not within the context of the narrated event (e.g. as looks away from the tree). In event narratives, however, signers may be less likely to interpret directional predicates outside of the context and space of the narrated event.

\subsection{Summary and discussion}

The comparison of analyses presented in this and the previous chapter shows that there is an effect of discourse type in perspective and frame of reference use to encode location in DGS. Whereas signers predominantly use observer perspective representations (and the relative or "relative+intrinsic" frame of reference) to encode location in static scene descriptions (chapter 5), they predominantly use character perspective (and the intrinsic frame) for location scenes in event narratives (this chapter). In addition, this chapter showed that within the event narrative discourse type, there was no difference in the encoding of location and motion scenes, and that character perspective/intrinsic frame was predominantly used to encode both types. This may, in part, be attributed to the type of discourse, which may favor, for example, an identification with the main protagonist. However, the results contrast with previous findings for other sign languages (BSL, ASL, and LSQ in particular), where observer-perspective representations are preferred for scene setting. This suggests that the distribution of perspective/frame of reference use for the encoding of location (for scene setting) and motion may differ between sign languages. In addition, it suggests that location and motion events in DGS are not treated fundamentally differently, and that discourse type affects their specific expression.

The distribution of perspective/frame of reference use in event narratives may also be influenced by language-specific constraints, in particular, by the availability of a role shift mechanism (cf. Liddell 1998; Padden 1986). DGS signers can distinctly mark switches in subject reference by shifting their shoulders slightly to the right or the left. This allows 
signers to represent different protagonists in character perspective based on the orientation of their shoulders. The availability of this type of role shift mechanism may motivate a continuous use of character perspective. In particular, it may allow an activity-based representation of the source and goal locations of transitive events (e.g. animate referent at source throws, animate referent at goal catches). Such a shifting mechanism was not found to be used in TID event narratives similar to the DGS narratives analyzed here (Perniss and Özyürek 2004b). ${ }^{92}$ In contrast, TİD narratives exhibited a higher proportion of use of observer perspective to convey source/goal referent locations in transfer events. (See section 7.6 in chapter 7 for further discussion of this difference between the use of perspective in DGS and TIDD narratives.)

In relation to perspective and frames of reference, the findings from this chapter, taken together with those from chapter 5, show that the two systems are indeed independent, but that they exhibit prototypical co-occurrences or alignments. In addition, the conventionalization of the signer's viewpoint in the relative frame of reference was found to hold in both discourse types (event narratives and static scene descriptions). Moreover, the representation of location and motion information by the tellers (and then by the retellers) consistently matched their view of the events in the stimulus films, which suggests that signers (in both production and comprehension) pay attention to relative and intrinsic spatial information within an event space, and maintain a high degree of spatial coherence in discourse.

\footnotetext{
92 This is not to say that TiD never explicitly marks role shift. Our ongoing research with different types of narratives (e.g. reported dialogue) will provide more insight into this.
} 


\section{The relationship between signing perspective and spatial/activity predicates}

Chapter 7

The analysis presented in this chapter focuses on the use of spatial/activity predicates to depict referents within a particular event space projection. Spatial/activity predicates refer to all signs that indicate the location, orientation, motion, or action/activity of referents in the event. The most studied of these types of signs are classifier predicates, which represent whole entities or the manipulation of entities. Signers may also use lexical predicates and index signs to encode information pertaining to referent location, motion, and action. Lexical predicates can identify a referent's manner of motion or type of activity within an event space, and index signs can indicate referent location within an event space. ${ }^{93}$

This chapter seeks to answer the following questions: What is the relationship between signing perspective and different types of spatial/activity predicates (in particular, classifier predicates, lexical predicates, and index signs) that can be used to depict referents in an event space? What are the different construction types that arise from the combination of these predicates with signing perspectives? What is the frequency of occurrence of different construction types? What motivates the use of different construction types in event narratives?

\subsection{The relationship between the use of perspective and different spatial/activity predicates in the sign language literature}

This section discusses the use of classifier predicates, lexical predicates, and index signs as different spatial devices that can depict referent location, motion, and action in relationship to an observer or character perspective event space projection. I begin with the use of classifier predicates, as they have received the most attention in the literature.

The use of a particular perspective to encode referent location, motion, and action typically involves the use of classifier predicates. While the use of classifier predicates, in general, has been widely studied in the field of sign language linguistics, the relationship

\footnotetext{
${ }^{93}$ It is this function of index signs with which I am concerned here, and it is in this sense that I refer to them as predicates. This type of index signs excludes those that have a purely referential function, in that the association made between the referent and a location in sign space is not "arbitrary".
} 
between classifier predicates and signing perspective in discourse has not been explicitly investigated in either qualitative or quantitative analyses.

Where the relationship between classifiers and perspective is addressed (or at least implicitly present) in the literature, it is generally couched in terms of argument structure and verb semantics (cf. Engberg-Pedersen 1993; McDonald 1982; Zwitserlood 2003). In these analyses, there is a systematic correspondence, on the one hand, between entity classifiers and intransitive verbs, and, on the other hand, between handling classifiers and transitive verbs. ${ }^{94}$ Zwitserlood (2003) describes the difference between the use of entity classifiers (with intransitive verbs) and handling classifiers (with transitive verbs) in terms of independent and dependent motion representation. In each case, the handshape of the classifier predicate encodes the theme argument of the verb. With entity classifiers, the whole entity is represented, and moves independently. Handling classifiers, in contrast, are used to depict how an entity is manipulated or handled. The entity which is handled, and thereby in motion (in dependence of the manipulation), is not represented directly, but rather indirectly.

Though Zwitserlood does not make the association explicit, the correspondence between the use of classifiers and signing perspective is apparent. With handling classifiers, the entity in motion is represented on the hands through a depiction of its manipulation by the character mapped onto the body. The representation of motion and action correspond to the character's own experience, and are depicted from the character's perspective. On the other hand, when entity classifiers are used, the entity in motion is represented directly, through a depiction of the whole entity on the hand. The location/motion of the entity is represented through the movement/position of the hand. This corresponds to the signer's external vantage point in observer perspective.

Morgan (1999, 2005) makes a slightly more explicit statement of the relationship between classifier predicates and perspective in BSL. He attributes the use of entity classifiers in what he calls the fixed referential space (FRS) (which corresponds to observer perspective signing) to establishing referent locations and marking spatial relationships. In contrast, the shifted referential space (SRS) (which corresponds to character perspective signing) is used for direct discourse and for representing characters' actions.

In addition, although not widely characterized as such in the literature, articulatory and semantic constraints can also be argued to influence the relationship between classifier

\footnotetext{
${ }^{94}$ McNeill (1992:119) makes a similar claim with regard to the distribution of occurrence of observer and character viewpoint gestures; character viewpoint gestures appear more with transitive verbs, while observer viewpoint gestures appear more with intransitive verbs.
} 
predicates and signing perspective. That is, the type of information that certain forms are able to felicitously represent plays a role in determining this relationship. As we have seen, entity classifiers are well-suited to the representation of referent location and motion within a reduced-size event space, as the whole entity is mapped onto the hand, and the hand is free to move within the area of sign space in front of the signer's body. In addition, the featural properties of entity classifiers, in particular the 2-legged entity classifier, allow signers to represent postural information (e.g. lying vs. standing), direction of movement (e.g. forward vs. backward), and even manner of locomotion (e.g. walking vs. jumping). The orientational features of the 2-legged entity classifier (i.e. fingers=legs; back of the fingers=front of the body), however, do not include parts that correspond to the human figure's arms or head. As a result, the 2-legged entity classifier is not suited for the expression of manual activity. To depict the handling of an entity, the signer's hands have to function as the (handling) referent's hands. As we have seen, this implies a character perspective representation, in which the referent is mapped onto the body. Similarly, this mapping of a character onto the body also restricts the type of information that can be felicitously expressed. While the expression of manual activity is afforded, the depiction of path motion, for example, is impeded. It is infelicitous in DGS for a signer to actually move her body to indicate a referent's change of location. Thus, the depiction of path motion requires the use of entity classifiers. $^{95}$

We turn now to the use of lexical predicates as spatially modifiable signs. As we have seen already in the previous chapter, signers need not always represent the referents whose actions they are describing with classifier predicates. In addition to classifier predicates, signers can use lexical predicates (e.g. PLAY, LIE, or COOK). The handshape neither reflects the manipulation of a referent nor the referent itself, as it does with handling or entity classifier predicates, but is part of the phonological form of the lexical predicate. In citation form, lexical predicates or verb signs are produced in neutral space. That is, the location(s) at which they are produced carry no meaning. However, in most sign languages, verbs can be spatially modified to perform various grammatical functions, including the marking of argument relations, person, number, and location. Thus, for example, signers can modify a verb like GIVE (a typical "agreement verb" (see Padden 1990) or "indicating verb" (Liddell 2003a)) to mean I give (to) you by moving the hand from the body toward the addressee, or to

\footnotetext{
${ }^{95}$ In AdaSL, where entity classifiers are not used for referent depiction, the depiction of path motion on the body is possible. For example, to represent a referent walking back and forth, signers use an intransitive motion marker and then move their torso laterally from side to side (Nyst 2007; Nyst and Perniss 2004).
} 
mean you give (to) me by moving the hand from the addressee toward the body. The production of lexical predicates in "non-neutral" space has been noted in the literature (e.g. Emmorey 1996 for ASL; Senghas and Coppola 2001 for NicaSL; Vermeerbergen 1996 for VGT (see also Johnston et al. 2007); Zeshan 2000 for IPSL), but has not been specifically discussed in relationship to event space projection.

Moreover, there has been little systematic investigation of the relationship between the use of perspective and lexical predicates in extended discourse. Exceptions are Liddell (1998, 2003a) and Dudis (2004b, 2006) who discuss the occurrence of different types of signs, including lexical predicates, in different types of spatial representation. For example, Liddell (1998) discusses the narration of a cartoon, in which the signer, in the role of a character within the event, directs the sign LOOK-AT toward the location of another character. The direction of the sign corresponds to the characters' locations in a life-sized projection of the event space. It is not clear, however, whether LOOK-AT, or similar predicates, may also appear in other event space projections, nor what the relative frequency of different types of constructions is.

Finally, as context-dependent, deictically-motivated signs, index signs are prime examples of spatially modifiable signs. Their use to refer to both present and non-present referents has been amply researched. Like in co-speech gesture, physically present referents can be directly pointed to for reference. Index signs used to refer to non-present referents have been analyzed along two main lines in the literature. On the one hand, their use is explained in terms of a "syntactic" use of space, where locations in space are arbitrarily chosen for referent association (e.g. Klima and Bellugi 1979; Lillo-Martin and Klima 1990). On the other hand, their use is taken to parallel the indication of physically present referents and is explained in terms of pointing to the conceptual locations of non-present referents (e.g. Liddell 1998, 2003a; Vogt-Svendsen and Bergman 2007). However, the studies do not explicitly specify the use of pointing signs in relation to the use of different perspectives.

\subsection{Methodology}

This section presents the methodology used in this analysis, and describes how the use of these three types of predicates in different perspectives was coded. The treatment of perspective and different types of spatial/activity predicates (classifier predicates, in particular) as independent spatial devices allows a systematic analysis of the relationship between perspective and the use of signs to depict referents within the projected event space. 


\subsubsection{Stimulus materials and data collection}

The analysis in this chapter is based on the same six stimulus events that were analyzed in chapter 6. Short cartoon films taken from the German children's television series Die Sendung mit der Maus were used to elicit signed event narratives. The films feature a large personified mouse and a smaller personified elephant as the main protagonists, and are characterized by a fixed "camera" perspective, which defines a constant vantage point on the event.

In total, 45 narratives produced by 14 signers were analyzed. The signers ranged in age between 16 and 45 (see section 6.1.2 in chapter 6). The number of narratives told by each signer varied because of the different constellations of pairs of signers that took turns being the teller and reteller in the pair (see appendix B).

\subsubsection{Coding of spatial/activity predicates and perspective}

The signed narratives elicited from the stimulus films were transcribed sign by sign. Overall, $55 \%$ of all signs were categorized as spatial/activity predicates that indicated the location, orientation, motion, or action of referents in the event. ${ }^{96}$ The remaining signs were nouns (used to identify the predicates' arguments), adjectives, adverbs, and signs that were part of the signer's meta-narrative commentary. The meta-narrative commentary included segments that could be translated e.g. as The mouse wants to flip the pancake over in the pan. Although it expresses spatial information, the predicate in this utterance was not included in the spatial/activity predicate count since it does not function to depict the mouse's activity directly, but rather to provide a narrative comment about the mouse's intentions.

To recapitulate, there were three categories of spatial/activity predicates: (1) classifier predicates, in which the hands represent salient or relevant size and shape properties of referents (e.g. the use of a handling classifier in throw the ball to represent the size of the ball and the activity of throwing); (2) lexical predicates, in which the hands execute the citation form of the sign, and do not provide information about geometric properties of referents (e.g. the use of the lexical predicate PLAY in play ball to identify the activity engaged in); (3) index signs, in which the index finger points to a location in sign space to indicate the location of a referent within the event space.

\footnotetext{
${ }^{96}$ There were a total of 2529 individual signs in the 45 narratives that were coded. 1392 of these signs (55\%) were categorized as spatial/activity predicates.
} 


\subsubsection{The relationship between perspective and classifier predicates}

Section 7.1 above described a correspondence between the use of entity classifiers and observer perspective, on the one hand, and handling classifiers and character perspective, on the other hand. These correspondences - implied or stated in the literature, and derivable from the interplay of articulatory and semantic constraints - can also be motivated by a principle of scale iconicity, whereby different parts of a representation should have the same size, insuring an internal consistency in scale within the event space projection. Based on the notion of scale iconicity, specifically the match between size of referent projection and size of event space projection, I take these correspondences to exhibit what I call alignments between classifier type and perspective (see table 7.1 below and section 3.5.2 in chapter 3).

Table 7.1. Characteristics of alignments between event space projection and referent projection.

\begin{tabular}{|c|c|c|}
\hline & Observer perspective & Character perspective \\
\hline $\begin{array}{l}\text { Projection of } \\
\text { Event Space }\end{array}$ & $\begin{array}{ll}\text { - } & \text { Event-external vantage } \\
\text { point } \\
\text { - In front of signer } \\
\text { - } \quad \text { Reduced size }\end{array}$ & $\begin{array}{ll}\text { - } & \text { Event-internal vantage } \\
& \text { point } \\
\text { - } & \text { Encompasses signer } \\
\text { - } & \text { Life-sized }\end{array}$ \\
\hline $\begin{array}{l}\text { Representation } \\
\text { of ref. loc/mot }\end{array}$ & - On the lateral axis & - On the sagittal axis \\
\hline Classifiers & $\begin{array}{ll}\text { - } & \text { Entity } \\
\text { - } & \text { Reduced-size SASS }\end{array}$ & $\begin{array}{ll}\text { - } & \text { Handling } \\
\text { - } & \text { Life-sized SASS }\end{array}$ \\
\hline
\end{tabular}

I briefly summarize the characteristics of these alignments again. ${ }^{97}$ In character perspective, the event space is life-sized. The character mapped onto the signer's body is by definition lifesized, and all other referents within the event space are equally represented on a life-sized scale. Handling classifiers are aligned in scale because they represent the manipulation of life-sized entities by the character. In observer perspective, the event space is reduced in size, and is represented in the area of space in front of the signer's body. The scale of referent representation within the event space is correspondingly small, and thus aligned with entity classifiers.

In addition to the use of entity classifiers, the use of SASSes (size and shape specifiers) that correspond in reduced size to the scale of an observer perspective event space projection are taken to be aligned with observer perspective. Similarly, the use of SASSes that represent referents as life-sized are taken to be aligned with character perspective. Figure 7.1 below shows the difference in size between SASS forms used to represent the stove in the pancake 
event. In (a), the signer represents the stove by means of a life-sized SASS. The size of the stove corresponds to a life-sized event space in which the signer, as the mouse, is present. In (b), the stove is represented by a SASS on a much smaller scale. The scale corresponds to a reduced-size observer perspective event space.

(a)

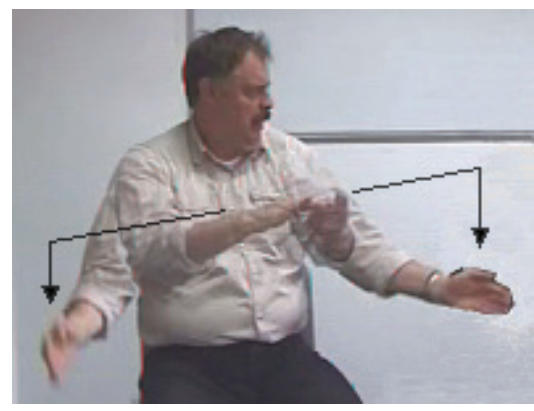

(b)

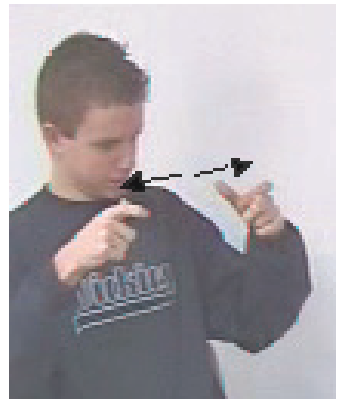

Figure 7.1. In (a), the stove is represented with a life-sized SASS, corresponding to a character perspective event space, while in (b), the stove is represented with a reduced-size SASS, on a scale corresponding to an observer perspective event space.

In terms of iconic mappings, the constancy of scale in the alignments between classifiers and perspective is taken to be more preserving of structural relationships within the event space than referent representation on differing scales. In extended discourse, however, referent representation on different scales is quite common. For example, a person approaching the signer can be indicated by moving an upright entity classifier toward the body. The entity classifier corresponds in scale to a reduced-sized observer perspective representation, while the signer's body (representing either the signer himself or another animate referent) corresponds in scale to a life-sized character perspective representation. This is an example of what I call a non-alignment between the scale of the event space projection and the classifier predicate. Table 7.2 summarizes the characteristics of perspective with non-aligned classifiers. They are the converse of the alignments shown in table 7.1 above. $^{98}$

\footnotetext{
${ }^{97}$ As in chapter 6, table 7.1 includes the axis of referent representation as a criterion for identifying perspective, based on the nature of referent location/motion in the stimulus films.

${ }^{98}$ SASSes are included in table 7.2 for the sake of completeness, though when they occurred, generally at the beginning of a narrative for scene-setting, it was the size of the SASS itself that was used to determine the size of the event space projection.
} 
Table 7.2. Characteristics of non-alignments between event space projection and referent projection.

\begin{tabular}{|c|c|c|}
\hline & Observer perspective & Character perspective \\
\hline $\begin{array}{l}\text { Projection of } \\
\text { Event Space }\end{array}$ & $\begin{array}{ll}\text { - } & \text { Event-external vantage } \\
\text { point } \\
\text { - } \quad \text { In front of signer } \\
\text { - } \text { Reduced size }\end{array}$ & $\begin{array}{ll}\text { - } & \text { Event-internal vantage } \\
& \text { point } \\
\text { - } & \text { Encompasses signer } \\
\text { - } & \text { Life-sized }\end{array}$ \\
\hline $\begin{array}{l}\text { Representation } \\
\text { of ref. loc/mot }\end{array}$ & - On the lateral axis & - On the sagittal axis \\
\hline
\end{tabular}

\begin{tabular}{|l|ll|ll|}
\hline Classifiers & $\bullet$ & Handling & $\bullet$ & Entity \\
& $\bullet$ & Life-sized SASS & $\bullet$ & Reduced-size SASS \\
\hline
\end{tabular}

\subsubsection{The relationship between signing perspective and lexical predicates}

As noted in section 7.2.2 above, lexical predicates can occur in neutral or non-neutral space. When signers produce lexical spatial/activity predicates in neutral space (e.g. PLAY), referents' actions are semantically identified, but no spatial information about the referents or the event space is conveyed. When signers produce lexical predicates in non-neutral space, the location is associated with some meaning. In event narratives, it is possible to modify the execution of lexical predicates in sign space to conform to meaningful locations within a particular event space projection. That is, the predicates can be directed (e.g. GIVE) or located (e.g. LIE) in space in a way that betrays a perspective from either within the event (a character's perspective) or outside of the event (an observer's perspective). In this data, lexical predicates that moved or were located along the lateral axis were coded as occurring within an observer perspective event space representation. The use of lexical predicates on the sagittal axis was taken as a cue for representation in a character perspective event space projection.

\subsubsection{The relationship between signing perspective and index signs}

Typically, index signs are analyzed as referential devices that function to establish and maintain the association between discourse referents and locations in sign space. The locations at which referents are established can be meaningful within a particular event space projection. Index signs directed at observer perspective locations occur on the plane of sign space in front of the signer's body, onto which the event space is projected on a reduced-size scale. In character perspective, on the other hand, index signs can be directed to any location around the signer's body, corresponding to possible referent locations in a life-sized 
projection of the event space. The use of index signs in the event narratives was coded for their occurrence within a particular event space projection based on these criteria. ${ }^{99}$

\subsection{Combinations of perspective and spatial/activity predicates in the data: Qualitative}

\section{analysis}

As argued in the sections above, a systematic analysis of the possible combinations of spatial/activity predicates and perspective requires that these different spatial devices be regarded as independent systems. This section presents a qualitative analysis of the DGS event narrative data, giving examples of the form and structure of the different construction types found.

All the different possible combinations of perspective with the three types of spatial/activity predicates discussed above were found to occur in the DGS narratives. That is, the spatial and activity predicates in the DGS narratives could reveal any of the nine combinations represented in figure 7.2.

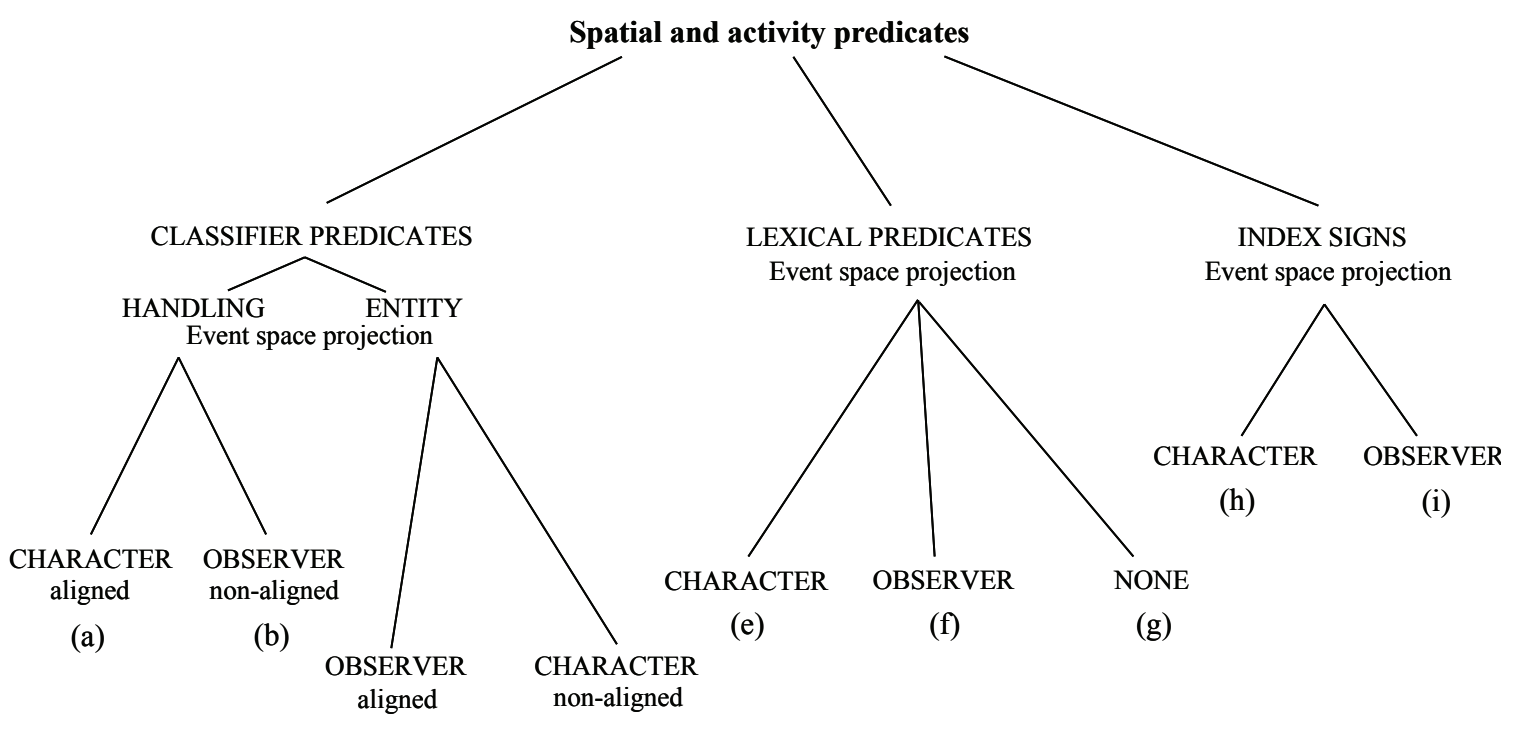

(c)

(d)

Figure 7.2. Different possible construction types of spatial and activity predicates and event space projection.

Examples of occurrences of all nine constructions types in the data are described and illustrated in (a) - (i) below.

\footnotetext{
${ }^{99}$ Of course, index signs may also occur without an event space projection, and indeed, their referential function is generally discussed in terms of "arbitrary" referent-location associations (cf. Klima and Bellugi 1979; LilloMartin and Klima 1990). However, in this data, the event space was predefined by the stimuli, and index signs occurred only to refer to referent locations within an event space projection.
} 
a. Handling classifier in character perspective (aligned): In aligned character perspective signing, the signer assumes the role of a character within the event. The character's head, torso, and hands are mapped onto the signer. The signer's movements can be attributed to the character and interpreted as occurring within a life-sized event space that encompasses the signer. Handling classifiers are used to depict the way in which a referent would carry out a particular action (i.e. through the manipulation or handling of an object). ${ }^{100}$ In example (1), the signer represents the mouse holding the ball in the ball event (see the image from the stimulus film shown with the example). The signer is in the role of the mouse, and his hands represent the mouse holding the ball (with handling classifiers). The signer holds the ball directly in front of the body, as does the mouse in the event.
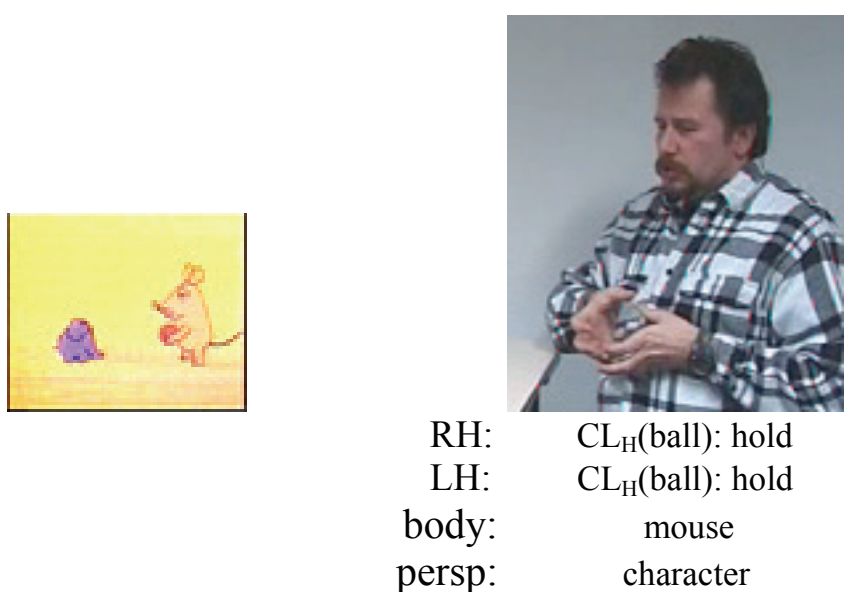

b. Handling classifier in observer perspective (non-aligned): In non-aligned observer perspective constructions, the event space is projected onto sign space from an external, observer's perspective. The signer's head and body are external to the event space, and the placement of the hands in space corresponds to referent locations as seen from an observer perspective. The hands do not, however, represent the referents themselves (cf. entity classifiers). Rather, they represent the manner of object manipulation by the referent, namely with handling classifiers. In example (2) below, the signer represents the elephant holding the pan in the pancake event (see the image from the stimulus film). The location of the signer's left hand (on the left side of sign space) depicts the elephant's location (on the left side of the

\footnotetext{
${ }^{100}$ It is also possible for the signer's hands to represent another character's hands, as e.g. to depict being hit in the eye by directing the fist to the eye (see Morgan and Woll 2003; Supalla 1986). This type of construction did not occur in the data analyzed here.
} 
event space) from an observer's perspective. The handshape depicts the elephant grasping the pan.

(2)
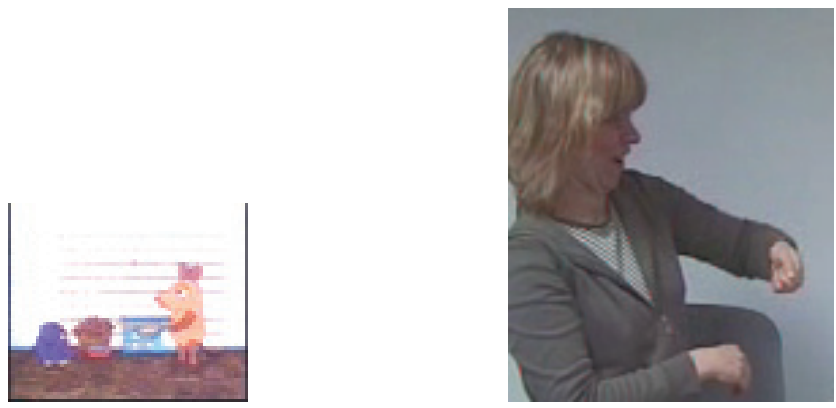

RH:

LH: $\mathrm{CL}_{\mathrm{H}}($ pan): hold at locL

body: signer

persp: observer

c. Entity classifier in observer perspective (aligned): In aligned observer perspective representations, the event space is projected from an observer's external perspective and is represented on a reduced scale in the area of space in front of the signer's body. Entity classifiers are used to represent referents within the event space. In example (3) below, the signer uses entity classifiers to represent the mouse and the elephant standing across from each other, facing each other, in the ball event (see the image from the stimulus film). The classifiers are located on the left (i.e. the elephant) and right (i.e. the mouse) sides of sign space (on the lateral axis), and are oriented toward one another. The signer's head and body are external to the event space projection.

(3)
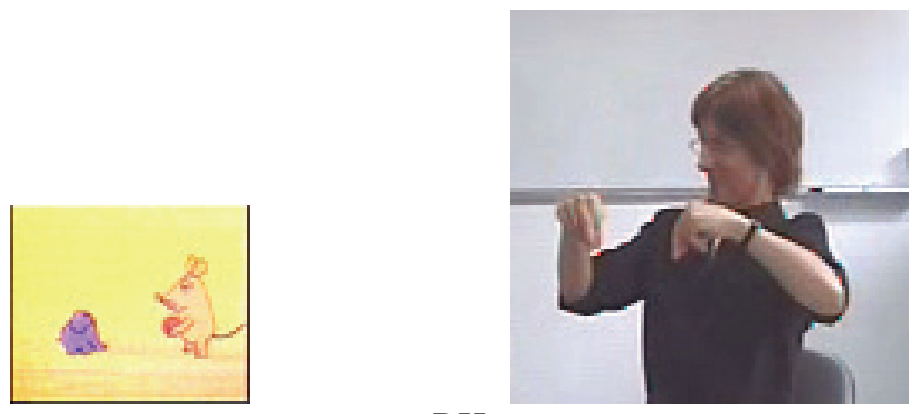

RH: $\quad \mathrm{CL}_{\mathrm{E}}$ (mouse): locR

LH: $\quad \mathrm{CL}_{\mathrm{E}}($ elephant): locL

body:

persp:

signer

observer 
d. Entity classifier in character perspective (non-aligned): In this non-aligned type, the event space is life-sized and projected from the vantage point of an event protagonist. Thus, referent location and motion is depicted in a life-sized character perspective event space. However, the character from whose perspective the event is projected is only partially mapped onto the signer's body. At least one of the signer's hands does not represent the hand of the character, but rather, is used to represent a different referent within the event by means of an entity classifier. In example (4) below, from a narrative of the pancake event, the signer uses an entity classifier (on his left hand) to represent the pancake on the floor, while his head, body, and right hand (holding the pan) represent the mouse. The location of the entity classifier in sign space corresponds to the pancake's location in the event space from the mouse's perspective (i.e. in front of the body and low). ${ }^{101}$

(4)
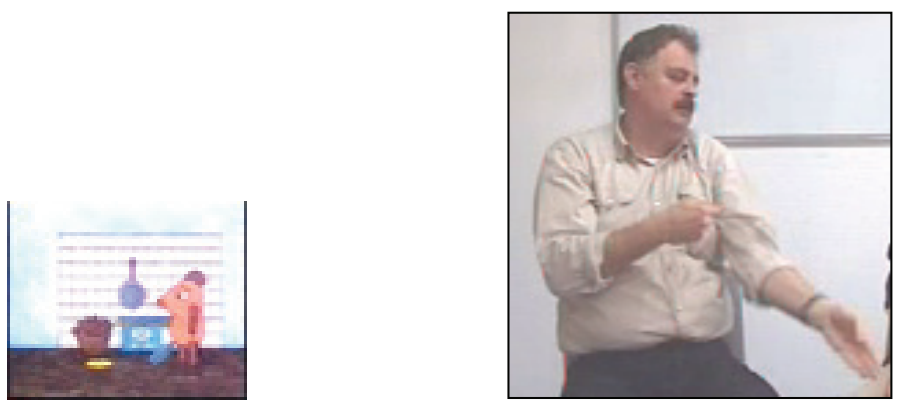

RH: $\quad \mathrm{CL}_{\mathrm{H}}($ pan): hold

LH: $\quad \mathrm{CL}_{\mathrm{E}}$ (pancake): loc floor

body: mouse

persp: $\quad$ character

e. Lexical predicate in character perspective: In this construction type, signers identify the actions of characters through the use of lexical predicates that are executed in a character perspective event space projection. The handshape does not depict spatial properties of an entity or reflect its handling, but rather encodes the lexical meaning of the predicate. However, its placement or directionality in space gives information about the location of referents in a life-sized character perspective event space. In example (5) below, the signer uses the lexical predicate "to see, to spot at a location" to indicate the location of the pillow case ahead of the mouse (see the image from the stimulus film). Through the direction of the predicate, the location of the pillow case is indicated as somewhere opposite signer's body, corresponding to a projection of the event space from the mouse's perspective.

${ }^{101}$ The signer's body here is turned slightly to the left, corresponding to the mouse's orientation facing away 

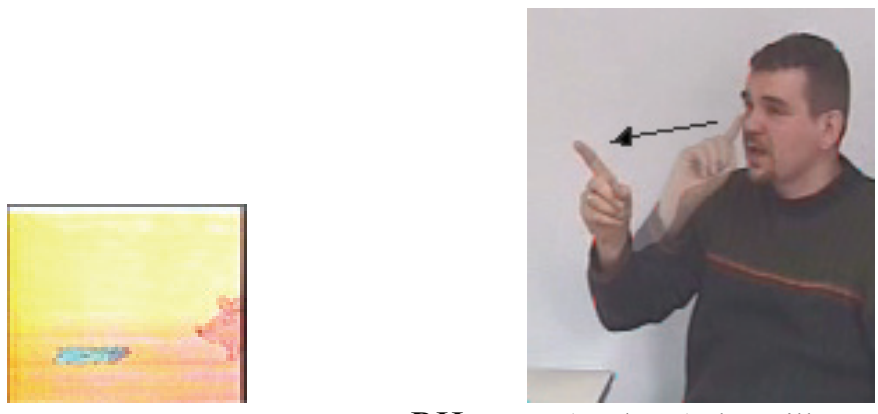

RH: LEX(see/spot): loc pillow case

LH:

body:

persp:

mouse

character

f. Lexical predicate in observer perspective: In this construction type, lexical predicates are likewise associated with meaningful locations in space. Here, the locations are determined by an observer perspective event space projection. In example (6) below, the signer starts the lexical predicate "to take/get (sth.)" on the left and moves the hand back to the body (to the right) along the lateral axis. This representation encodes the two images from the chestnut stimulus film shown next to the example. The mouse exits the screen, moving to the left (see the first still from the stimulus film), and returns, moving to the right (see the second still from the stimulus film), carrying a plank of wood and a stone. Through the direction of the lexical predicate, the signer encodes the direction of the mouse's movement in an observer perspective event space projection, corresponding to the signer's view of the event.

(6)
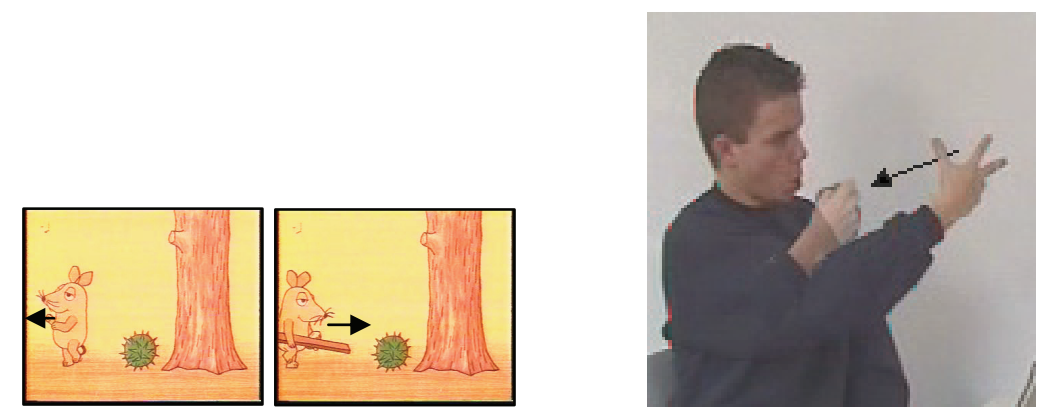

RH: LEX(take/get): from locL

LH:

body: signer

persp: observer

from the stove (see chapter 8 , section 8.5.1). 
g. Lexical predicate only (no event space projection): Referent actions that occur in the stimulus films can be semantically expressed by signers using lexical predicates that are executed in neutral space. This means that there is no event space projection. In example (7) below, the signer represents the mouse turning around in the pillow case event (from facing the pillow case to facing away from the pillow case) with a lexical predicate that is not spatially modified within an event space projection.
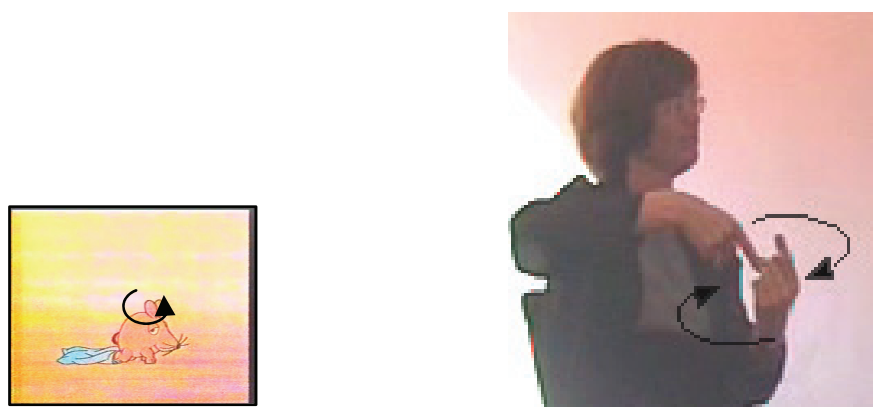

RH: LEX("um"-turn around)

LH: LEX("um"-turn around)

body: $\quad$ signer

persp: none

h. Index sign in character perspective: In this construction type, an index sign is used to refer to the location of a referent within a character perspective event space projection. In example (8) below, the signer points to the elephant's location from the perspective of the mouse in the pancake event. The signer points to a location opposite his body, and slightly downward, representing the mouse seeing the elephant enter the kitchen.

(8)
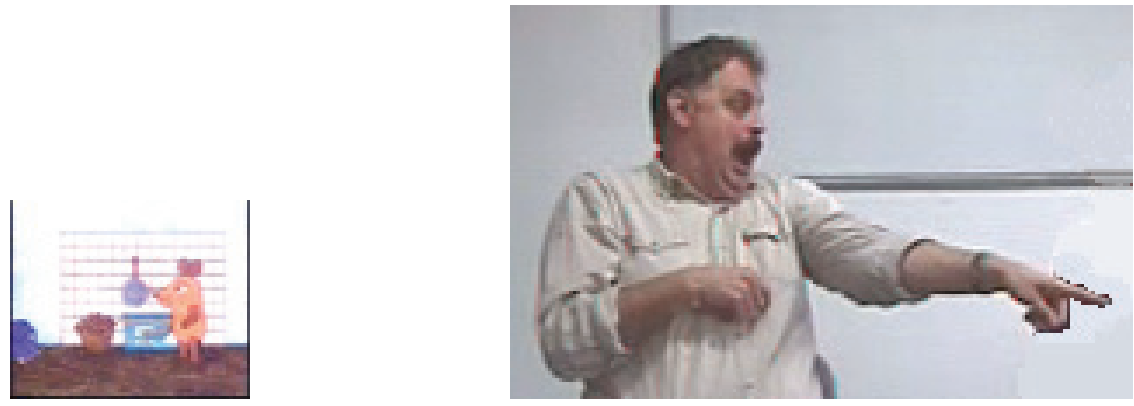

RH:

LH:

body:

INDEX: loc eleph

persp:

mouse

character 
i. Index sign in observer perspective: An index sign can also refer to a referent location within an observer perspective event space projection. In example (9) below, the signer is not a character pointing to another referent within the life-sized event (as above). Rather, the signer points to the elephant at a location that corresponds to the reduced-size scale of the event space in observer perspective. The elephant's location corresponds to the signer's external vantage point as the viewer of the ball event stimulus film.

(9)
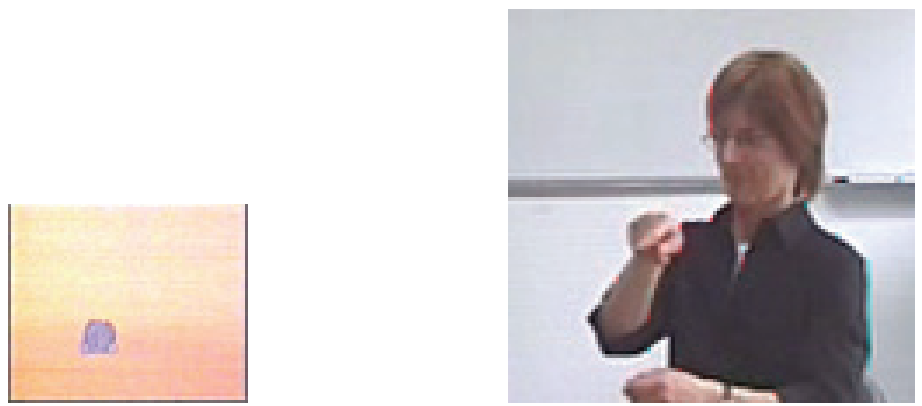

RH: $\quad$ INDEX: loc eleph

LH:

body: $\quad$ signer

persp: $\quad$ observer

The next section presents a quantitative analysis of the distribution of these different construction types in the DGS event narratives. Based on the assumption of scale iconicity, as well as assumptions about the relationship between classifier predicates and perspective implicit in much of the literature, one might expect the predominant, or even exclusive, use of aligned classifier-perspective construction types. In addition, the general emphasis on the use of classifier predicates to encode spatial information does not predict the strong use of other types of spatial/activity predicates in event space representations. The quantitative analysis tests these hypotheses.

\subsection{Distribution of perspective and spatial/activity predicates in the data: Quantitative analysis}

If we collect all the spatial/activity predicates coded, we have a large sample over which to generalize. Figure 7.3 below presents the proportion of use of the different predicate types in the different types of event space projection. In addition, the graph includes an "other" category, which contains constructions that are not discussed here, but which will appear in the analysis presented in the next chapter. 


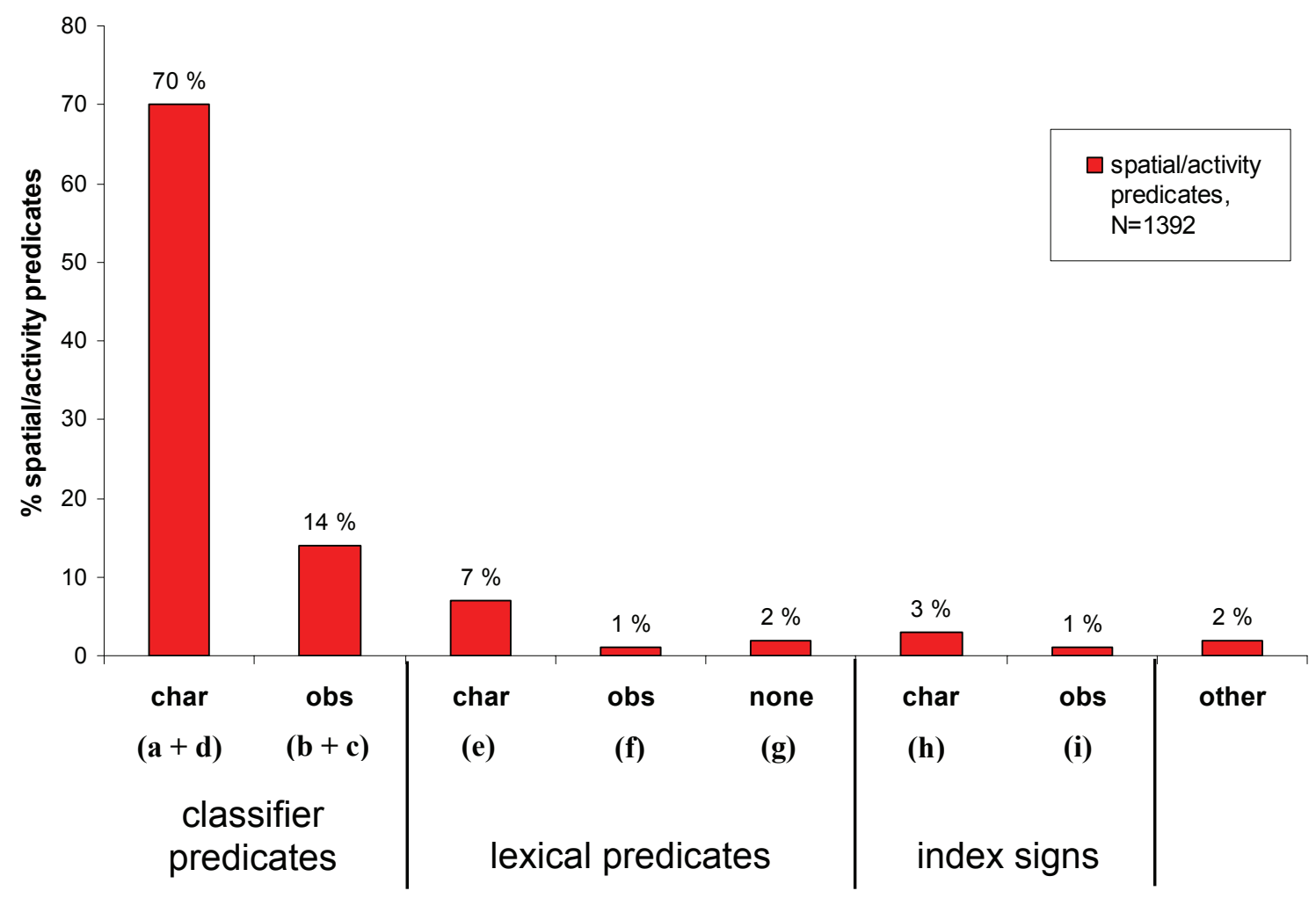

Figure 7.3. Percentage of use of different predicate types (classifier, lexical, index) with different event space projections (character, observer, none). The letters correspond to those in figure 7.2.

The graph in figure 7.3 shows that the majority of spatial/activity predicates was encoded with classifier predicates. The use of lexical predicates and index signs was rare in comparison. As we already saw in the previous chapter, the use of character perspective dominates. This is most striking with the use of classifier predicates, but is also significantly greater with the use of lexical predicates and index signs. ${ }^{102}$

It is important to note that none of the lexical spatial/activity predicates that occurred were body-anchored verbs or "plain" verbs (cf. Padden 1986), which cannot be spatially modified. Thus, all lexical predicates that were used without perspective (that appear in $(\mathrm{g})$ in figure 7.3) could potentially have been spatially modified (i.e. produced in non-neutral space).

\footnotetext{
${ }^{102}$ The more frequent use of character perspective was found to be significant for all types of predicates using a Chi square test. In the use of classifier predicates, the likelihood of choosing character perspective was significantly greater than that of choosing observer perspective (Chi-square $=1031.123 ; \mathrm{df}=1 ; \mathrm{p}<0.001$ ). In the use of lexical predicates, the likelihood of choosing character perspective was significantly greater than that of choosing observer perspective (Chi-square $=118.271 ; \mathrm{df}=1 ; \mathrm{p}<0.001)$ and than that of choosing no perspective (Chi-square=82.66; $\mathrm{df}=1 ; \mathrm{p}<0.001$ ). Finally, in the use of index signs, the likelihood of choosing character perspective was significantly greater than that of choosing observer perspective (Chi-square $=11.57 ; \mathrm{df}=1$; $\mathrm{p}<0.001)$. However, the results of these tests must be interpreted with caution, since the two perspectives are not completely exclusive independent variables, as is necessary for the use of a Chi square test.
} 
In the rest of the chapter, we will be concerned only with the codings that used classifier predicates. We go one step further and look at the distribution of different types of classifier predicates between the different perspective types. The graph in figure 7.4 below shows the use of aligned and non-aligned classifier predicates in character and observer perspective.

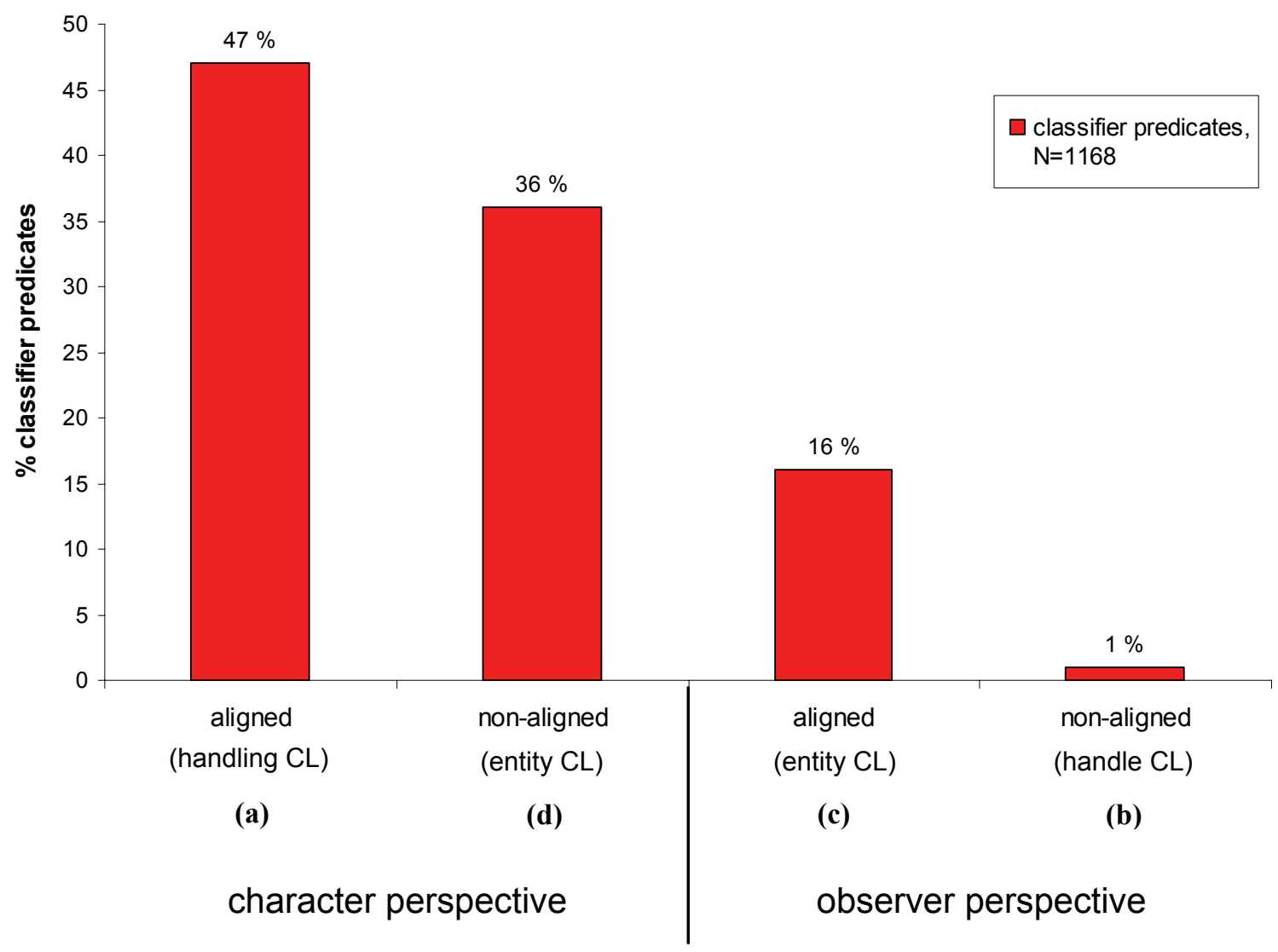

Figure 7.4. Percentage of aligned and non-aligned classifier-perspective construction types. The letters correspond to those in figure 7.2.

The graph shows that DGS signers prefer aligned constructions in both event space representations. The difference is especially pronounced in observer perspective, but is significantly different in both perspectives. ${ }^{103}$ While signers exhibit a high proportion of both aligned (handling classifiers) and non-aligned (entity classifiers) character perspective

\footnotetext{
${ }^{103}$ The more frequent use of aligned constructions was found to be significant in both character and observer perspectives using a Chi square test. Within character perspective uses, the likelihood of using an aligned construction was significantly greater than that of using a non-aligned construction (Chi-square $=37.44 ; \mathrm{df}=1$; $\mathrm{p}<0.001$ ). Within observer perspective uses, the likelihood of using an aligned construction was significantly greater than that of using a non-aligned construction (Chi-square $=72.46 ; \mathrm{df}=1 ; \mathrm{p}<0.001$ ). Again, the results of these tests must be interpreted with caution, since the two construction types are not completely exclusive independent variables, as is necessary for the use of a Chi square test.
} 
representations, the use of non-aligned (handling classifiers) observer perspective representations was quite rare.

\subsection{The relationship between semantic event components and classifier-perspective constructions}

This section discusses the motivations for using aligned and non-aligned construction types in DGS narratives. The description of complex events entails encoding information about the spatial setting in which the event takes place (e.g. location, orientation, and motion information) and information about the actions of animate referents within that setting. As we have seen, different semantic components are associated with different signing perspectives and represented with different predicate types. How do the different semantic components get distributed across construction types in event narratives and what motivates signers' choice of classifier predicates and signing perspectives?

I look first at aligned and non-aligned character perspective representations (section 7.5.1). Section 7.5.2 then discusses motivations for the use of aligned observer perspective representations. (The use of non-aligned observer perspective representations is not discussed separately, as they appeared very infrequently in the data.)

\subsubsection{The motivation for character perspective coding}

The stimulus films used to elicit DGS event narratives are characterized by the action and interaction of animate and inanimate referents in a particular spatial setting. There are numerous components in the stimulus events themselves that may induce signing in character perspective. Firstly, the mouse is the predominant protagonist in most of the events - and indeed is the only protagonist in half of the events. Even in the events in which the elephant is present with the mouse throughout the event (the ball and bar events), the type of interaction between the two characters betrays the mouse's primary role. This may be a factor that motivates signers to focus on the mouse and encode the events from the mouse's perspective. Secondly, the events are characterized by the presence of affect and emotion on the mouse's and elephant's faces. In character perspective, the signer can represent the characters' emotions on her own face. Thirdly, there is an abundance of transitive manner of motion in the stimulus films. This includes manner of handling and manner of conveyance, both of which involve manual activity that is most felicitously represented with handling classifiers in character perspective. Finally, in relating events of this type, identifying with the actors in the event (by taking their perspective) shifts the focus into the event. This 
involvement strategy may help to highlight how the protagonists contribute to the event's unfolding.

The presence of manual activity, manner of activity, and affect/identification in the events are all factors that motivate the use of aligned character perspective representations. However, in the DGS narratives, we also find a high frequency of use of non-aligned character perspective representations, that is, where signers used entity classifiers in an event space projected from character perspective to depict referent location and motion. What motivates the use of non-aligned character perspective constructions? The analysis here focuses on a certain type of event for which non-aligned character constructions were very frequent, namely where an intransitive path component occurs together with a transitive manner component. (See chapter 8 for further analysis of the discourse functions of this type of construction, and for a review of similar types of construction in the literature.)

Consider the scene in which the mouse enters the event space in the ball event (see the image from the stimulus film shown with example (10) below). The two main components that characterize this motion event are an intransitive path component (the mouse is moving along a straight path) and a transitive manner component (the mouse is holding/dribbling a ball). ${ }^{104}$ These event components occur simultaneously: that is, the mouse is moving forward (walking) while holding/dribbling the ball. Example (10) shows a representation of this motion event with the use of a non-aligned character perspective construction.

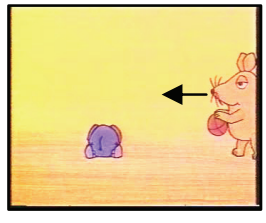

1

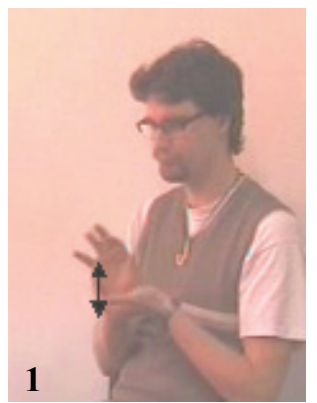

RH: $\mathrm{CL}_{\mathrm{H}}$ (ball): hold/dribble

LH: $\mathrm{CL}_{\mathrm{H}}($ ball): hold/dribble body:

persp:

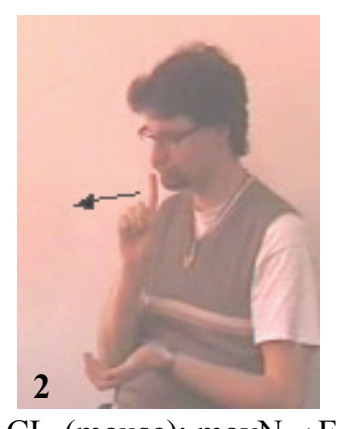

$\mathrm{CL}_{\mathrm{E}}$ (mouse): $\operatorname{movN} \rightarrow \mathrm{F}$

$\mathrm{CL}_{\mathrm{H}}$ (ball): hold mouse character

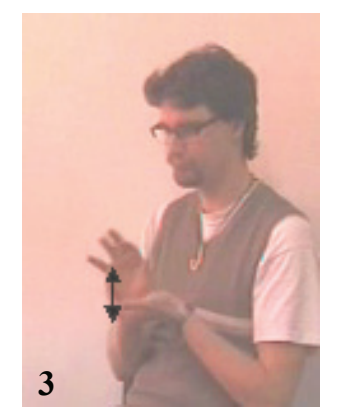

$\mathrm{CL}_{\mathrm{H}}$ (ball): hold/dribble $\mathrm{CL}_{\mathrm{H}}$ (ball): hold/dribble mouse character

Stills 1 and 3 in example (10) show the signer in an aligned character perspective representation depicting only the transitive manner component (holding/dribbling the ball). In still 2, however, the signer adds an entity classifier to represent the intransitive path

${ }^{104}$ The intransitive manner of locomotion (i.e. walking) is not considered here. 
component (i.e. the mouse moving forward). During the representation of this path motion, the non-dominant hand stays in place, in the configuration of the "holding/dribbling" manner component. Thus, in the non-aligned character perspective construction in still 2 , both the intransitive path and the transitive manner components are simultaneously represented. ${ }^{105}$ Note also that the signer uses a "sandwich" construction, in which the path component (in still 2) is sandwiched between two representations of the manner component (stills 1 and 3). This structural device links the components together, additionally indicating their simultaneity (cf. Senghas et al. 2004).

Compare the representation in example (10) with that in example (11) below, which shows the encoding of the same motion event by a different signer. The signer in (11) does not use a non-aligned construction type, but rather uses two aligned constructions to encode the intransitive path and transitive manner components completely separately. In still 1, the signer represents the mouse's path motion along the lateral axis (in an aligned observer perspective representation). The mouse's manner of activity (i.e. dribbling the ball) is subsequently represented in an aligned character perspective representation (still 2).
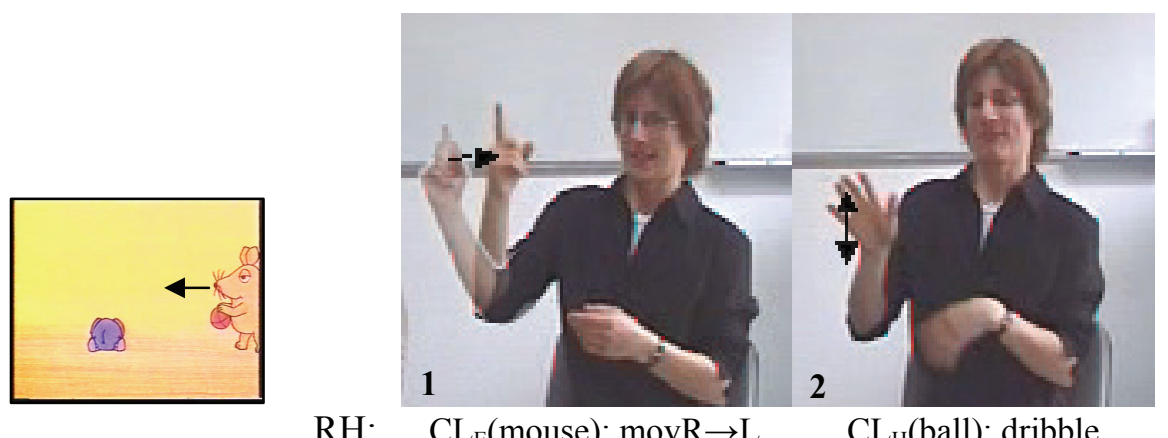

RH:

LH:

body:

persp:

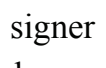

mouse

observer

character

I have argued for the alignments between perspective and classifiers on the basis of an iconicity of scale. The signer in example (11) achieves scale iconicity by using an aligned observer perspective construction followed by an aligned character perspective construction. It is clear, however, that in doing so, a different type of iconicity, namely temporal iconicity is lost. In using aligned constructions, it is not possible to simultaneously represent simultaneously occurring event components that are associated with or felicitously

\footnotetext{
${ }^{105}$ This differs from the simultaneous representation of intransitive path motion and manner of motion (i.e. the manner of locomotion), which can be simultaneously represented on a single articulator. For example, a 2-
} 
represented in different perspectives (i.e. intransitive path motion (observer) and transitive manner motion (character)). In the non-aligned character perspective construction in example (10), we see the opposite situation. The signer can preserve the temporal iconicity of the event, simultaneously representing simultaneously occurring aspects of the event, but loses the iconicity of scale in doing so. In addition, the non-aligned construction does not compromise the spatial coherence of the representation by associating the same referent with different locations in space (as happens with the aligned constructions). On the other hand, the use of the aligned representations in example (11) allows the signer to encode additional spatial information lacking from the representation in example (10), namely the encoding of relative frame of reference information. These observations show that the choice of construction type is influenced by different types of iconicity and different types of spatial information. Chapter 9 of this dissertation further explores the relationships between different iconicity principles.

\subsubsection{The motivation for observer perspective coding}

We now turn to an analysis of the use of observer perspective coding in the DGS event narratives. As figure 7.3 above shows, observer perspective was used only one-fifth as often as character perspective. Moreover, it was used almost exclusively in the aligned construction type (i.e. with entity classifiers to represent location and motion) (see figure 7.4). Below, I present three categories of path-salient event types that taken together account for $86 \%$ of the aligned observer perspective codings: (1) Agentive path motion with no manual activity; (2) Entering or exiting the event space; (3) Path of caused motion.

Event type 1: Agentive path motion with no manual activity. This category includes elements where change of location (directed path motion) is the primary salient event component. The whole pillow case event (except for the initial static location scene) can be characterized in this way (see the stills in appendix C). The pillow case event consists in the mouse's maneuvering toward and away from the pillow case, and, in contrast to the other stimulus events, the mouse walks on all four limbs (instead of standing upright) and there is no manual activity, not is there interaction with other animate referents. The incidence of observer perspective representation for this event type 30\% (56 out of 188 described scenes). Example (12) below shows the location of the pillow case on the left (still 1), and the mouse's 
subsequent movement toward (still 2) and into the pillow case (still 3) along the lateral axis from right to left. ${ }^{106}$
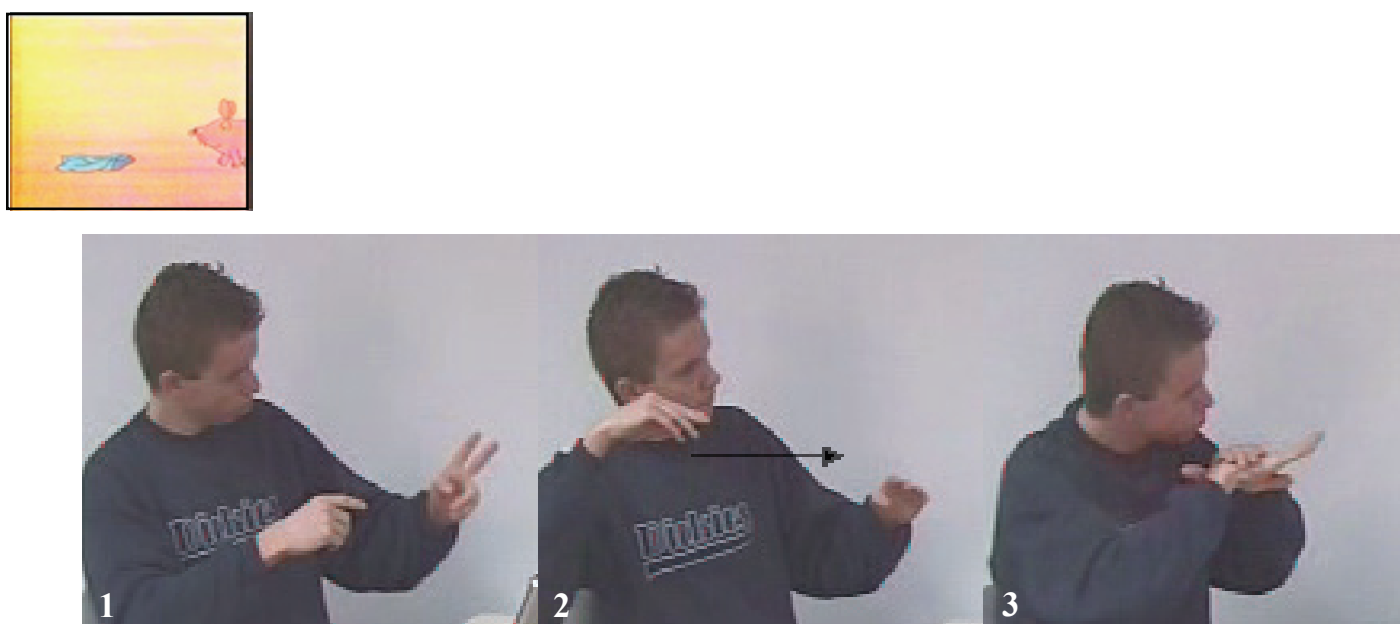

$\mathrm{RH}:$

$\mathrm{CL}_{\mathrm{E}}$ (mouse): movR $\rightarrow \mathrm{L}$

LH: LEX(lie): pillow case locL

body: $\quad$ signer

persp: observer
$\mathrm{CL}_{\mathrm{E}}$ (pillow case): locL signer observer
$\mathrm{CL}_{\mathrm{E}}$ (mouse): locL (under pillow case) $\mathrm{CL}_{\mathrm{E}}$ (pillow case): locL

signer

observer

Event type 2: Entering or exiting the event space. All of the stimulus films contain an entering and/or exiting event. The characters - that is, the mouse, the elephant, and the man (in the chestnut event) - enter or exit the scene from either the left or the right, moving laterally across the screen from the viewer's point of view. Conceptually, entering and exiting can be defined as a figure entity moving into or out of a "containment" relationship with another (ground) entity (Kita 1999). In the enter/exit scenes in the stimulus events, the event space itself (bounded for the viewer by the screen) may be thought of as the container into or out of which the figure moves. In the visual-spatial modality, this "event space container" can be projected as a whole directly onto sign space from an external, observer perspective.

Movement in and out of the "event space container" - i.e. entering and exiting the bounded space - can be represented through the depiction of referent path motion in observer perspective. Thus, one might expect, and does indeed find, an increased use of observer perspective to encode entering and exiting. Here again, about a third of the total number of encoded enter/exit scene scenes were expressed in observer perspective $(34 \%$; 64

\footnotetext{
${ }^{106}$ The reader will notice that there is some character perspective representation of the mouse's movements on the body (e.g. the head bends down in still 3 where the mouse is shown crawling into the pillow case). These blended character perspective elements lie outside the focus of the current study, which is on the representation of location, motion, and action within an event space projection.
} 
out of 188 described scenes). Noteworthy characteristics of the enter/exit scenes themselves seem to additionally motivate the use of observer vs. character perspective across signers.

For example, the enter and exit scenes in the chestnut event were unanimously represented in observer perspective. In the chestnut event, the mouse, as the only animate referent in the event, temporarily disappears from the event space (the relevant still images from the stimulus film are shown in example (6) above). Consider a representation of this scene in character perspective: The mouse must be mapped onto the body, and must thus necessarily be within the event space. In addition to the fact that moving the body is not felicitous to represent path motion in character perspective, the boundaries of the event space in character perspective are not as clearly defined as in observer perspective. The observer perspective lateral path representation ensures that the meaning of entering and exiting the bounded event space can be truly conveyed.

The scene in which the man enters the event space in the bar event was also unanimously coded in observer perspective. In the event, the mouse and elephant are busy trying to straighten the bar, when the man unexpectedly comes along. There is no direct connection between his arrival and what is otherwise happening in the event, which may decrease the motivation to depict his movement from an event-internal perspective (e.g. from the mouse's character perspective). The use of the observer perspective may reflect this distance.

Event type 3: Path of caused motion. The third category of path-salient event types is the path component of transitive caused motion. Overall, $22 \%$ of scenes encoded in observer perspective (41 out of 188) corresponded to this category. The caused motion was of both inanimate (i.e. the pancake and ball) and animate (i.e. the mouse) referents, and consisted of repeated back and forth motion (the inanimates) as well as a single path (the mouse) (see the stills in appendix C). The events in this category (as those described in section 7.5.1) are characterized by the presence of a transitive manner component (i.e. that causes the motion) and an intransitive path components (i.e. the caused motion itself). However, here, the two components do not occur simultaneously, but rather sequentially (in a causal relationship). Signers who used observer perspective to encode the caused path motion used character perspective to encode the preceding manner component. Switching between perspectives for the representation of the different event components may highlight the causal relationship between them. 


\subsection{Summary and discussion}

This chapter investigated the relationship between signing perspective and different types of spatial/activity predicates that can be used to express referent location, motion, and action. It was found that character perspective was used significantly more often than observer perspective for all predicate types, of which classifier predicates were by far the most commonly-used type. This supports the idea that signers, exploiting the affordances of the visual-spatial modality, prefer the use of more iconic forms over less iconic forms. Furthermore, it was found that aligned constructions were used significantly more often than non-aligned constructions in both perspectives, though there was frequent use of non-aligned character perspective constructions.

I have suggested that different types of iconicity (which I call scale iconicity and temporal iconicity) influence the choice of aligned and non-aligned character perspective constructions. The use of aligned representations allows signers to preserve scale iconicity, but not temporal iconicity. Conversely, the use of non-aligned character representations allows signers to simultaneously encode simultaneously occurring intransitive path and transitive manner components, thereby preserving temporal iconicity. This suggests that different iconicity principles are valued by different signers under different circumstances. An Optimality Theory type of analysis, a suggestion of which is given in chapter 9, but the elaboration of which must be left to future research, may be helpful in ranking different iconicity principles to predict the choice of construction at a given time.

In addition, an analysis of the use of aligned observer perspective constructions showed that this construction was primarily used to encode agentive path motion with no manual activity, entering/exiting the event space, and the path of caused motion. The use of nonaligned observer perspective representations occurred only very infrequently in the data. By comparison, this construction was used frequently by Turkish signers narrating the pancake and ball events in TID. In an analysis that used a subset of the DGS data analyzed here, Turkish signers used non-aligned observer perspective $11 \%$ of the time compared to not even $1 \%$ by the German signers (Perniss and Özyürek 2004b, in press). ${ }^{107}$ As noted in chapter 6, Turkish and German signers in the cited study also differed significantly in the way subject reference switches were marked. In DGS, signers shifted their shoulders to distinguish two different animate referents mapped onto the body, a spatial device which did not appear systematically in the TID narratives. That is, DGS signers could mark the roles of the mouse

\footnotetext{
${ }^{107}$ The analysis compared the use of perspective and classifier type in 182 TiD and 176 DGS spatial/activity
} predicates. 
and the elephant flipping the pancake back and forth to each other by shifting the shoulders between two distinct orientations. In contrast, TID signers achieved expression of the mouse and elephant's reciprocal manual activity through the use of handling classifiers on each hand, in the locations of the mouse and elephant in an observer perspective event space projection (i.e. with a non-aligned observer perspective construction).

Finally, by identifying the illustrated construction types, this chapter has provided the tools with which further analyses and cross-linguistic comparisons can be carried out (see also Perniss and Özyürek, in press). To date, there is a lack of comparative research across sign languages on the use of perspective, and on the relationship between perspective and different types of spatial/activity predicates in encoding semantic components of an event. Systematic investigations of the occurrence of different construction types across different sign languages and across different discourse types will offer a wealth of knowledge on the possible typological variation between sign languages in the spatial domain. 


\section{Form and function of simultaneity in DGS event narratives}

Chapter 8

The use of space for linguistic expression affords the notion of simultaneity a special status in signed language. The availability of multiple independent articulators makes possible the simultaneous representation of independent meaningful elements. This chapter looks at the use of multiple articulators, both manual and non-manual (e.g. the body, the head, the eyes), in constructions that simultaneously encode elements associated with different perspectives. Two main types of construction are focused on: (1) non-aligned character perspective constructions and (2) what I call "double-perspective constructions". The focus of analysis in this chapter is on the function of these construction types in maintaining spatial coherence in discourse, especially in relationship to principles of temporal and scale iconicity.

In non-aligned character perspective constructions, already discussed in the previous chapter, manual and non-manual articulators associated with different perspectives are used simultaneously within a character perspective event space projection. As shown in the previous chapter, entity classifiers (represented manually, and prototypically aligned with observer perspective) are used in an otherwise character perspective representation (where the head and body, as non-manual articulators, represent the character itself, and the hands can represent the character's activity). In chapter 7, the use of non-aligned character perspective constructions was shown to achieve temporal iconicity through the simultaneous representation of simultaneously occurring aspects of the event. In double-perspective constructions, different articulators, manual and non-manual, represent spatial information simultaneously in two different event space projections, that is, simultaneously in observer and character perspectives. This construction type has not been previously described for other sign languages (see Perniss 2007a for DGS).

Two main functions related to the maintenance of spatial coherence in discourse are attributed to the use of non-aligned character and double-perspective constructions. These functions are explained in terms of iconicity principles and, more generally, in terms of achieving efficiency and informativeness in the expression of spatial information within discourse. I show that through the use of non-aligned character perspective representations, signers can achieve full semantic specification of a spatial event. In exhibiting temporal 
iconicity with the event, the highly efficient construction also insures a high degree of informativeness, encoding multiple event components, and contributing to overall spatial coherence. With double-perspective constructions, signers maintain spatial coherence between perspectives by creating an explicit mapping between them. I demonstrate how coreferent locations in observer and character perspective event space projections are simultaneously indexed, and how this allows signers to be maximally informative and efficient in the expression of spatial relationships.

Before presenting the analysis of the form and function of such constructions in the DGS narratives, I give an overview of previous literature (section 8.1) and provide background on efficiency and informativeness principles as discourse structure constraints (section 8.2).

\subsection{Previous research on simultaneous constructions related to perspective}

As the overview in chapter 2 (section 2.4) showed, there has been substantial research on the use of simultaneous constructions that use the two manual articulators. Here, I discuss previous research on constructions that exhibit the use of different articulators (manual and non-manual) that are associated with different perspectives. The majority of this research corresponds to what I have called non-aligned character perspective constructions (Aarons and Morgan 2003; Dudis 2002, 2004a; Engberg-Pedersen 1993; Fridman-Mintz and Liddell 1998; Liddell 1998, 2000, 2003a; Liddell and Metzger 1998; Morgan 2002; Risler 2007; Sallandre 2007).

Liddell (1998, 2003a) and Dudis (2002, 2004a) describe the production of what they call simultaneous blends, where elements corresponding in scale to character and observer perspective representations are mapped onto different articulators and/or locations in space. The simultaneous representation of elements with different scale properties is made possible through the availability of different partitionable zones of the body, including the hands, face, mouth, and torso (Dudis 2004a). For example, a signer can simultaneously represent a vehicle on one hand using an entity classifier and the driver of the vehicle on the body (Dudis 2004a; Liddell 2000 for ASL). A "zoomed out", or observer perspective view of the scene, is exhibited in the use of an entity classifier to depict, in Liddell's example, a car stopped at an intersection, and in Dudis' example, a motorcycle going up a hill. By mapping the drivers of the vehicles onto the body, the signer can simultaneously depict their facial expressions and behaviors (e.g. the driver of the car looking both ways before crossing the intersection) through a "zoomed in" or character perspective view of the scene. Aarons and Morgan (2003) 
describe similar constructions from SASL in what they call the creation of multiple perspectives. For example, a signer maps a parachutist floating through the air simultaneously onto his hand and onto his body.

In addition, Aarons and Morgan (2003) for SASL and Engberg-Pedersen (1993) for DSL describe the encoding of spatial relationships between two referents through the simultaneous representation of one referent on the body and the other referent on the hand. For Engberg-Pedersen, the backgrounded referent is represented on the body as the patient or spectator of the event (cf. the mapping of the backgrounded player onto the non-dominant hand with a hold-morpheme discussed in section 2.4.1 of chapter 2). The motion and location of the agent is represented with an entity classifier from the patient's vantage point. For example, the interaction between two basketball players can be depicted by mapping one player onto the body and representing the motion of the other player relative to the body with an entity classifier on the hand. Finally, Morgan (2002) discusses similar examples to encode temporal simultaneity of events in BSL narratives. For example, to encode the meaning "the boy gazed on while the dog sat waiting nearby", the signer can represent the boy on the body and the dog with an entity classifier next to the body (p. 144).

\subsection{Discourse structure constraints of efficiency and informativeness}

The explication of efficiency and informativeness principles in discourse dates back to Grice's (1975) conversational maxims. Grice's two principles of quantity are formulated as "make your contribution as informative as required" (Q1) and "do not make your contribution more informative than is required" (Q2). In later research on pragmatic theory, the essence of these maxims was reformulated as the Q-principle (Principle of Quantity) and the I-principle (Principle of Informativeness), respectively (Atlas and Levinson 1981; Horn 1984; Levinson 2000). ${ }^{108}$ In a discourse context, the two principles pull in opposite directions, minimizing the speaker's effort on the one hand (i.e. maximizing the speaker's efficiency via the I-principle), and minimizing the addressee's effort on the other hand (i.e. maximizing the hearer's input via the Q-principle).

This chapter examines efficiency and informativeness in the expression of spatial information in narratives. On the one hand, there is pressure to be as informative and precise as possible in encoding spatial information. On the other hand, there is pressure to be as efficient and concise as possible. The measure of efficiency is determined by the number of

${ }^{108}$ Horn uses the label R-principle for what Levinson calls the I-principle. 
simultaneously-occurring event components that signers encode within a single expression. That is, when two event components (e.g. path and manner) are encoded simultaneously (temporally iconic with the event), this is taken to be more efficient than when they are encoded sequentially. The measure of informativeness is the amount of spatial information made explicit (either through simultaneous or sequential expression) for every spatial event encoded. How efficiency and informativeness of expression are specifically revealed through the use of multiple articulators associated with different perspectives will become clear in the discussions of individual constructions below.

\subsection{Data}

As in the previous two chapters, the DGS event narratives form the basis of the analysis. The analysis focuses on two construction types. Of the total of 1392 spatial/activity predicates that occurred in the event narratives, $551(40 \%)$ were expressed in non-aligned character perspective. The double-perspective constructions analyzed in this chapter appeared in the category "other" in figure 7.3 in section 7.4 of chapter 7 . There were a total of $21(2 \%)$ constructions of this type in the DGS event narratives.

\subsection{Non-aligned character perspective constructions: the use of multiple articulators to achieve full semantic specification}

The semantic specification of an event can be expressed in terms of the individual semantic components of the event that are encoded. Here we are concerned with the expression of path and manner components (including path and manner of motion, manner of handling, and manner of transfer). For the purposes of this analysis, an event is fully specified semantically when the path and manner components that characterize it are explicitly encoded. This section discusses the use of non-aligned character perspective constructions that simultaneously depict different aspects of the same event on different articulators (discussed in chapter 7 as a way of achieving temporal iconicity with the event). Here, the focus of analysis is on the encoding of simultaneously-occurring components to achieve a full specification of the event through semantic supplementation of a component prototypically aligned with observer perspective. Two examples are presented below.

The first example is from the pancake event. In the stimulus film, the mouse moves forward in an exaggerated lunge, attempting to catch the pancake in the pan it is holding in its hand (see the image from the stimulus film above the example below). In example (1), the signer depicts the mouse moving the pan upward to flip the pancake (still 1), then moving the 
hand and body forward to catch the pancake (still 2). In the third still, the signer represents the pancake landing on the mouse's head.

(1)

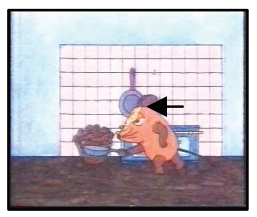

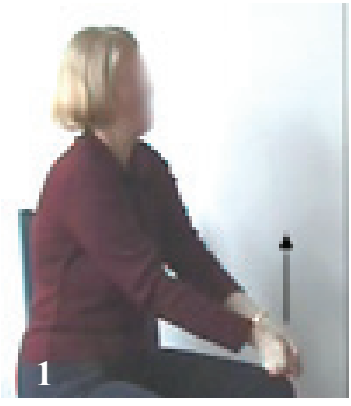

RH: $\quad \mathrm{CL}_{\mathrm{H}}(\mathrm{pan})$ : hold LH: $\quad \mathrm{CL}_{\mathrm{H}}($ pan): hold body: mouse: loc post persp: character

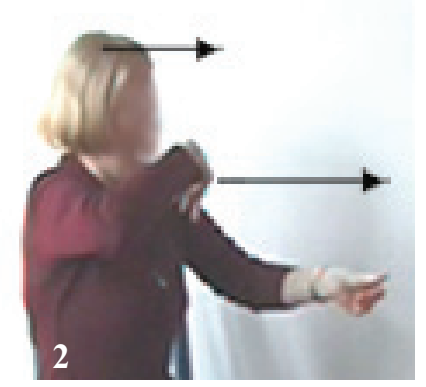

$\mathrm{CL}_{\mathrm{E}}$ (mouse): mov $\operatorname{loc}_{1} \rightarrow \mathrm{loc}_{2}$ $\mathrm{CL}_{\mathrm{H}}$ (pan): hold

mouse: mov post $_{1} \rightarrow$ post $_{2}$ character

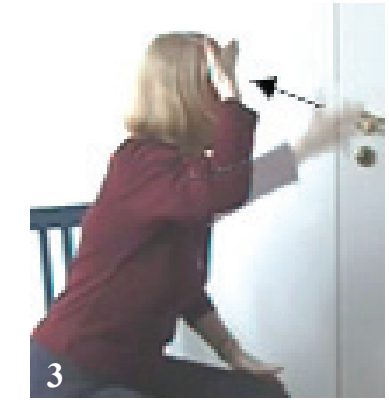

$\mathrm{CL}_{\mathrm{E}}($ pancake): mov pan $\rightarrow$ head

mouse: loc post ${ }_{2}$

character

The analysis focuses on the representation in the second still of example (1). The mouse's movement consists of two separate event components: (1) a change of location, i.e. the mouse moves forward (moving from location 1 to location $_{2}$ ), and (2) a change of posture, i.e. the mouse leans forward (moving from posture ${ }_{1}$ to posture 2 ). In addition, the mouse is holding the pan in its hand during this movement.

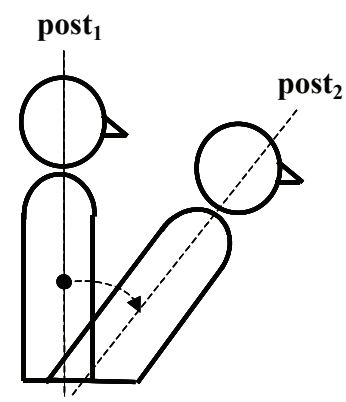

Figure 8.1. Representation of the mouse's change of posture encoded by moving the torso from posture ${ }_{1}$ to posture .

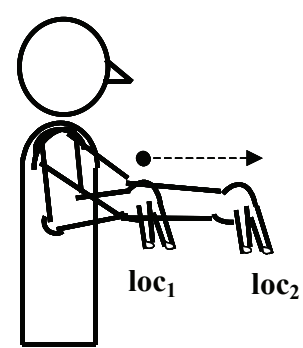

Figure 8.2. Representation of the mouse's change of location encoded by moving the hand from location 1 to location $_{2}$.

Of the two motion components, the change of posture can be represented with a form prototypically aligned with character perspective, i.e. by leaning the torso forward (see the schematic representation in figure 8.1 above). However, the mouse's change of location cannot be felicitously represented with an aligned character perspective form, as it would entail a movement of the whole body. The movement must be encoded instead with an entity 
classifier, a form prototypically aligned with observer perspective (see the schematic representation in figure 8.2 above). ${ }^{109}$

In example (1), the signer combines the two motion components in a single non-aligned construction type, leaning her torso forward to encode the mouse's change of posture while simultaneously representing the mouse's path motion by moving an entity classifier on the right hand along the sagittal axis. In addition, manner of handling information is simultaneously represented by means of a handling classifier on the left hand, depicting the mouse holding the pan. Taken together, the different articulators involved in the construction encode distinct pieces of information that are necessary to achieve a full semantic specification of the event. The meaning contributed by the non-aligned entity classifier supplements the otherwise character perspective-determined representation.

The use of the non-aligned character perspective construction contributes to spatial coherence in different ways. Firstly, it preserves temporal iconicity by simultaneously encoding simultaneously occurring event components. Secondly, it is both very efficient and informative in encoding locative information. The construction is maximally informative for the addressee because it fully specifies the mouse's movement, encoding both the path and manner of motion components, in addition to encoding manner of handling. Finally, the expression of all three components within a single expression makes the construction very efficient, additionally boosting spatial coherence.

The second narrative analyzed in this section is from the ball event. In the stimulus film, the mouse and the elephant pass the ball back and forth between them using different body parts. The elephant uses its trunk to hit the ball, and the mouse uses its hands, feet, and snout to transfer the ball to the elephant. In example (2) below, the signer uses limb classifiers to encode the mouse's action of kicking (still 1), and then uses an entity classifier to represent the ball's path along the sagittal axis (still 2). Similarly, in example (3), the signer uses an entity classifier, on his dominant right hand, to depict the path motion of the ball along the sagittal axis (approaching the body in still 1; moving away from the body in still 2), and represents the elephant's trunk on his left hand with a limb classifier. ${ }^{110}$

\footnotetext{
109 In figure 8.2, the path motion is represented along the sagittal axis, corresponding to the signer's representation in example (1). An aligned observer perspective representation of the mouse's change of location would be directed along the lateral axis to the left.

${ }^{110}$ Note that in example (3), the entity classifier (representing the ball) and the limb classifier (representing the elephant's trunk) have the same handshape.
} 


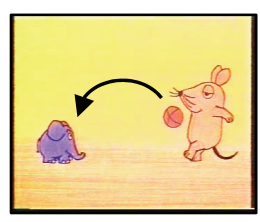

(3)

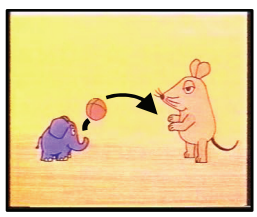

$\mathrm{RH}:$

LH:

body:

persp:

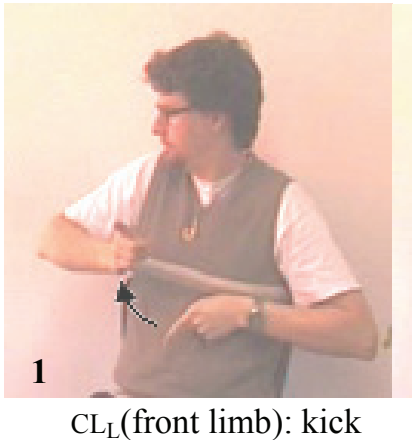

LH: body: persp:

$$
\mathrm{CL}_{\mathrm{L}} \text { (front limb) }
$$$$
\text { character }
$$

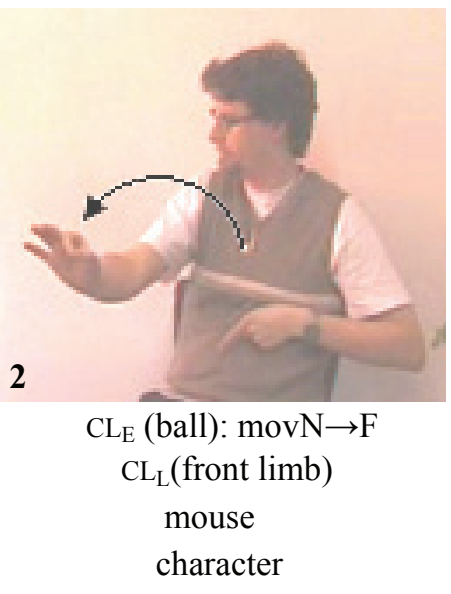

character

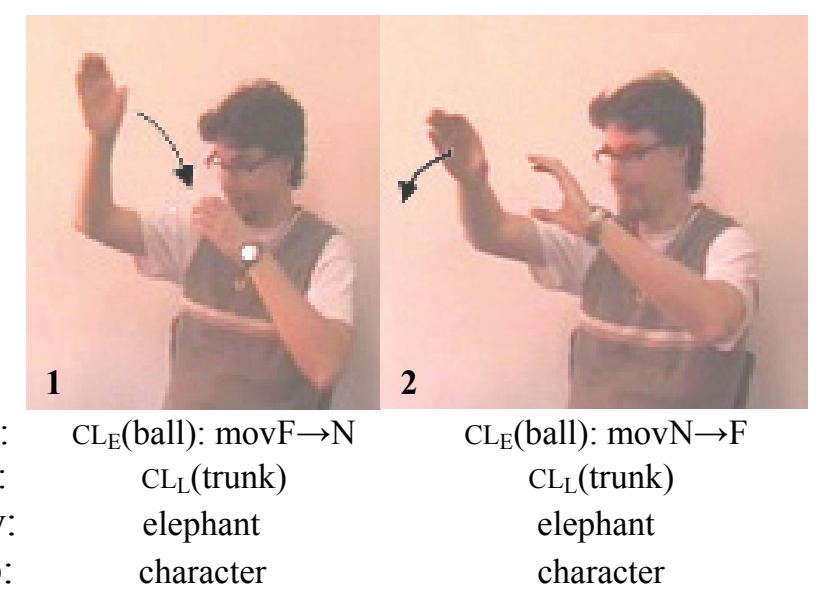

In the representations in examples (2) and (3), the entity classifiers used to depict the ball's path motion are forms associated with observer perspective that occur in otherwise character perspective representations. Through the use of these non-aligned character perspective representations, the signer is able to depict both the path motion of the ball between the mouse and elephant, as well as its manner of transfer. To make the contribution to spatial coherence made by the non-aligned constructions especially clear, their use is compared to that of aligned character perspective constructions to encode the same scenes in the corresponding reteller's narrative.

Examples (4) and (5) below show how the same scenes (i.e. the mouse kicking the ball and the elephant hitting the ball with its trunk) were encoded by the reteller after seeing the teller's narrative. Throughout her narrative, the reteller uses only aligned character perspective constructions, or lexical predicates without an event space projection, to encode the animate referents' interactions with the ball. In the character perspective representations, 
all movements of the signers hands, head, and body are attributable to the animate referent mapped onto the body. The signer only represents body-part movements that are isomorphic with felicitously-used parts of her own body. To represent the movement and use of other body parts (for example, the mouse's foot/leg or the elephant's trunk), she does not use manual limb classifiers, like the teller, but rather uses a comparable human body part or a lexical predicate. Thus, to represent the kicking event, the signer first points to her leg (still 1 in example (4)) and then signs the lexical predicate KICK (still 2). Here, the relevant body part (i.e. the foot/leg) does have an isomorphic counterpart on the human body, but it is not felicitously used in character perspective representations. To depict the elephant hitting the ball with its trunk in example (5), the signer moves her own head up. In keeping with an aligned character perspective depiction of the event, where the hands represent only the hands of the animate referent mapped onto the signer's body, the signer does not specify the shape of the trunk using a limb classifier form.

(4)

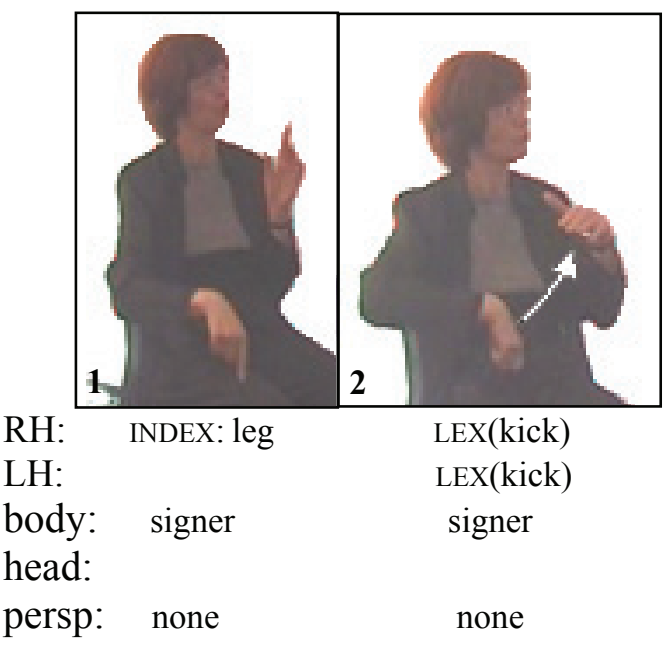

(5)

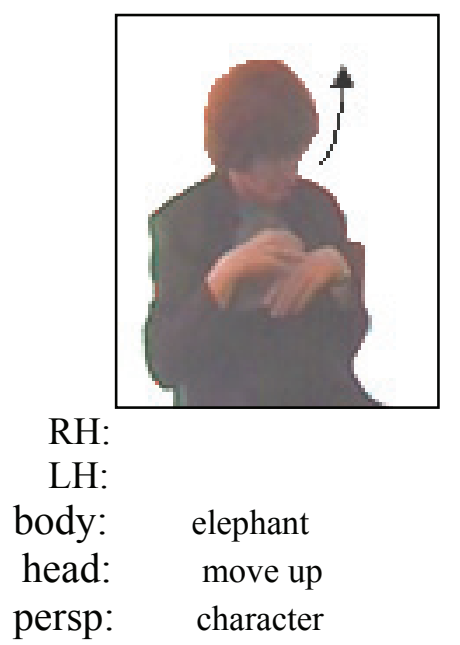

Though the use of aligned character constructions preserves scale iconicity, the reteller's narrative is less informative in expressing different event components than the teller's narrative. Information that is explicitly encoded through the use of non-aligned forms in the teller's narrative (in examples (2) and (3)), specifically information about shape, trajectory (including source and goal), and instrument, must here be inferred. Moreover, the teller's use of different entity classifiers to represent the ball allows him to introduce scale iconicity into his narrative, despite the non-aligned constructions. In example (2), the mouse's legs are represented with G-hand limb classifiers (still 1). Correspondingly, the ball is represented with an F-hand (in still 2). In example (3), both the elephant's trunk and the ball are 
represented with a C-hand. The "scale adjustment" of classifier forms allows the representation of the body part that makes contact with the ball and the ball itself to correspond in size. ${ }^{111}$

\subsection{Double-perspective constructions: The use of multiple articulators to achieve a mapping between different event space projections}

This section describes the use of what I call "double-perspective constructions", a new kind of construction in which different perspectives are blended that has not been previously described in the literature. These constructions are characterized by the simultaneous coindexing of referent location in two perspectives.

When signers inscribe sign space in both observer and character perspective event space projections in the course of a narrative, single referents may get associated with more than one location in sign space. This creates potential problems for spatial coherence, and thus the relationship between the referent-location associations in the two perspectives has to be clear. One way of making this relationship clear is through the use of a double-perspective construction. The simultaneous representation of an event from both observer and character perspectives can explicitly establish a mapping between corresponding meaningful locations in the two event spaces. The next two sections illustrate examples of double-perspective constructions in event narratives of the pancake event.

\subsubsection{Mapping between perspectives for spatial coherence: efficiency of expression}

The double-perspective construction discussed in this section achieves an explicit mapping between referent-location associations in observer and character perspective event space projections. Used early in the narrative, it allows the signer maximum narrative efficiency, while maintaining a high degree of spatial coherence regarding the relevant spatial relationships. Here, the relevant spatial relationships are the two orientations of the mouse with respect to the stove (i.e. the mouse faces the stove or is turned $90^{\circ}$ to the left of the stove), as shown in the still images from the stimulus film above example (6a) below.

Before using the double-perspective construction, the signer's narrative exhibits a linear sequence of aligned observer and character perspective representations (seen in stills $1-4$ in example (6a)). The signer first uses entity classifier forms in an observer perspective event

${ }^{111}$ In this regard, Slobin et al. (2003: 19) note that when a classifier predicate "depicts a figure moving or located with respect to a ground, it is critical that the scale of the figure and ground 'classifiers' correspond with 
space projection to depict the spatial relationship between the mouse and the stove (still 1). The mouse's manual activity in this orientation (i.e. facing the stove) is then represented in a character perspective representation (still 2). ${ }^{112}$ Following this, the signer returns to an entity classifier representation in observer perspective to depict the mouse's change of orientation (still 3). The signer turns the 2-legged entity classifier from an orientation facing outward (away from the signer) to the left. In still 4, the signer depicts the mouse's activity in this new orientation (i.e. holding a pan, now visible to the viewer) in an aligned character perspective representation. Example (6a) can be divided into two blocks: the first block (represented by stills 1 and 2) encodes the mouse's activity in an orientation facing the stove, while the second block (represented by stills 3 and 4) encodes the mouse's activity turned away from the stove. In the event itself, the two components in each block (i.e. orientation and manual activity) are simultaneous.

(6a)
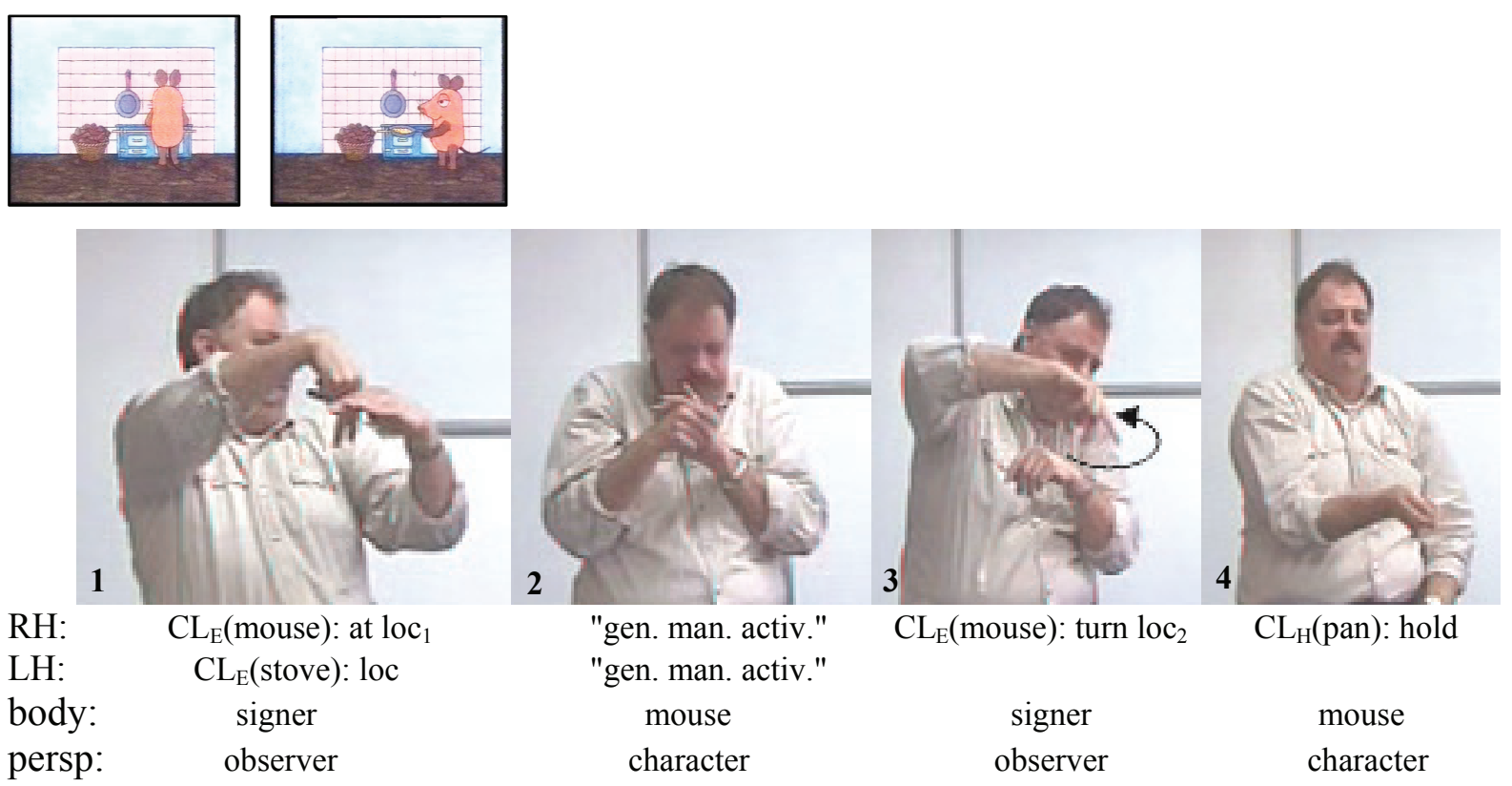

Having explicitly established the mouse's orientations with respect to the stove in observer perspective, the signer then employs the special construction in focus here, a doubleperspective construction (shown as still 5 in example (6b) below) to map the observer

each other...(T)he same referent can be represented by different handshapes, depending on how their relative sizes are conceptualized in the projection from mental space into signing space."

${ }^{112}$ At this point in the stimulus film, the mouse's precise activity is not yet apparent to the viewer. The mouse is visible only from the back, and the viewer sees only the movement of its shoulders. The signer therefore represents "generic manual activity" here, indicating that the mouse is busy "doing something", the exact nature of which is not yet clear. 
perspective orientations onto two distinct orientations of the torso in character perspective. ${ }^{113}$ In this construction, multiple independent articulators encode the same spatial information (i.e. the mouse turning to face the stove) simultaneously in character and observer perspective. It consists of a lateral turn of the hands, shoulders, and torso from left to right. The beginning location of the construction, in both perspectives, indexes the mouse in the orientation turned away from the stove; the end location, in both perspectives, indexes the mouse facing the stove.

RH:

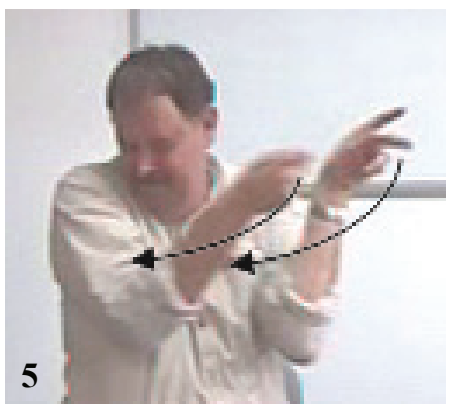

LH: LEX(back/return-to): $\operatorname{mov} \operatorname{loc}_{2} \rightarrow \operatorname{loc}_{1}$

body: mouse/signer

persp: $\quad$ observer + character

Character perspective is manifested through the turn of the torso and shoulders (representing the mouse turning), as well as the handling classifier on the signer's right hand (representing the mouse holding the pan). At the same time, the spatial orientations specified earlier with 2legged entity classifiers in observer perspective (in stills 1 and 3 in example (6a)) are accessed by a spatially modifiable lexical predicate meaning BACK/RETURN-TO, which is executed by the left hand. The spatial modification of the lexical predicate is understood within an observer perspective event space projection, that is, with conceptual recourse to the spatial information previously specified with the 2-legged entity classifiers.

Through the double-perspective construction, the leftward orientation of the torso and shoulders becomes distinctively linked to the orientation of the mouse turned $90^{\circ}$ from the stove, and the rightward orientation is linked to the orientation of the mouse facing the stove. Following the special construction, the signer shifts between the two orientations of the body in character perspective to explicitly and unambiguously encode the mouse's movement between the two orientations with respect to the stove, as illustrated in the sequence in

\footnotetext{
${ }^{113}$ The double-perspective construction did not immediately follow the representation shown in still 4 in example 6(a). In between, the signer depicted the mouse flipping the pancake into the air, the pancake landing on the floor, and the mouse picking it up (see the still images from the pancake stimulus film in Appendix C).
} 
example (6c) below. In still 6, the signer represents the mouse facing the stove; in still 7, the body orientation indicates that the mouse is turned away from the stove. The doubleperspective construction links the two (character perspective) orientations of the torso to the two (observer perspective) orientations specified by the entity classifiers by simultaneously co-indexing locations in both event space projections. The orientations of the torso are thereby explicitly and unambiguously associated with meaning they would not otherwise have (see Perniss 2007a on the ambiguity of an unmarked turn of the torso).

$(6 c)$

RH:

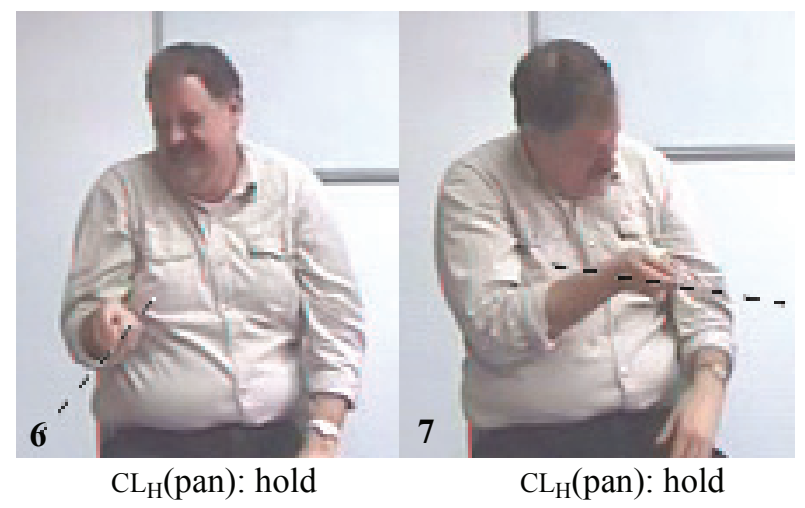

LH:

body: $\quad$ mouse(at $\left.\operatorname{loc}_{1}\right)$

persp: character

mouse $\left(\right.$ at $\left.\operatorname{loc}_{2}\right)$

character

The mapping achieved by the double-perspective construction allows the signer to simultaneously encode, in aligned character perspective, different event components (i.e. orientation and manual activity) in a single expression throughout the remainder of the narrative. The construction thereby affords the signer a temporal iconicity with the event that would otherwise be possible only with a non-aligned construction and an additional articulator. By staying in aligned character perspective, the signer additionally achieves scale iconicity, representing different components of the event on the same scale.

Figure 8.3 below shows a schematic representation of the signer's narrative. Blocks 1 and 2 correspond to example (6a), stills $1-4$, and show the signer's initial uses of aligned observer and character perspective event space projections. The double-perspective construction in block 3 (corresponding to example (6b), still 5) creates a mapping between both event space projections by simultaneously co-indexing locations in them. The narrative is subsequently continued in character perspective (blocks 4 and 5; corresponding to example (6c), stills 6 and 7).

In allowing the signer to simultaneously encode different simultaneous event components (i.e. orientation and manual activity), this double-perspective construction is 
similar to the non-aligned constructions discussed in section 8.4. However, here, the different event components are not encoded by supplementing the character perspective representation with observer perspective information expressed in a non-aligned form. Instead, the character perspective representation occurs together with an observer perspective representation, thereby creating an explicit mapping of spatial information between the two event space projections. Crucially, the double-perspective construction uses two event space projections simultaneously, where the non-aligned character perspective constructions uses only one.

time
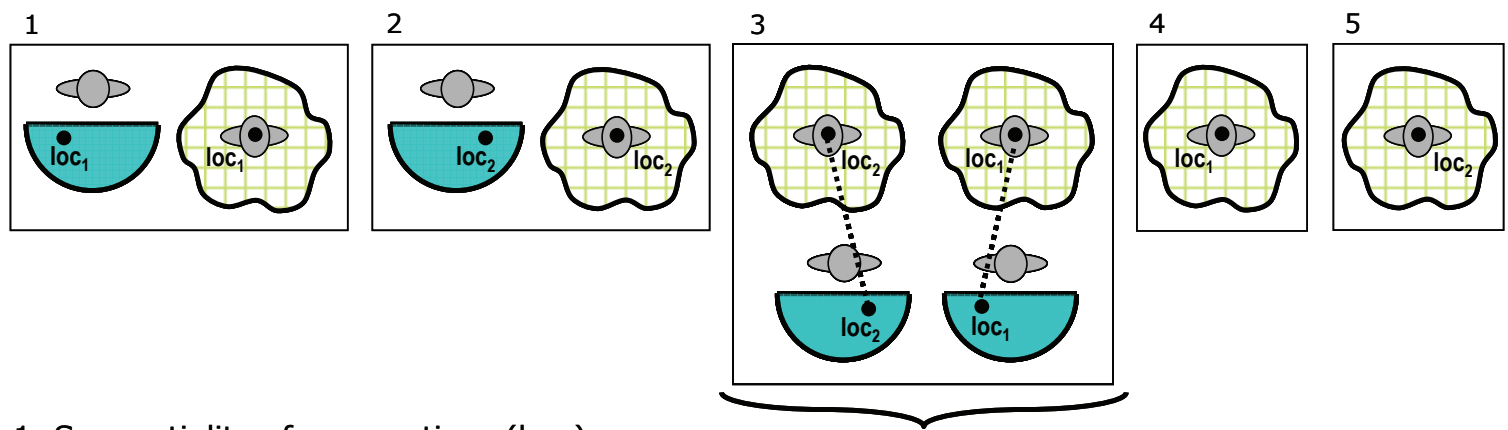

1. Sequentiality of perspectives ( $\left(\operatorname{loc}_{1}\right)$

2. Sequentiality of perspectives ( $\left.\operatorname{loc}_{2}\right)$

Double-perspective construction

3. Simultaneity of perspectives ( $\operatorname{loc}_{1}$ and $\operatorname{loc}_{2}$ )

4. Character perspective $\left(\operatorname{loc}_{1}\right)$

5. Character perspective $\left(\mathrm{Ioc}_{2}\right)$

observer perspective event space

$\square$ character perspective event space

Loc $_{1}$ : referent orientation $=$ facing stove

$\mathbf{L o c}_{\mathbf{2}}$ : referent orientation $=$ turned from stove

Figure 8.3. Schematization of the use of sequentiality and simultaneity of perspectives in the teller's narrative.

This narrative is compared to the corresponding reteller's narrative to make the function of the simultaneous perspective construction especially clear. The signer who retold the event based on seeing this narrative also used both character and observer perspective event space projections, but used a different strategy to express the relationship between the representations in the two projections. Instead of a double-perspective construction, the reteller uses aligned character and observer perspective constructions throughout the entire narrative. Example (7a) below shows the beginning of the reteller's narrative. In terms of event space projection, it is the same as the beginning of the teller's narrative (cf. example (6a)). The signer represents the mouse standing at the stove in observer perspective with a 2- 
legged entity classifier (stills 1 and 2). The mouse's turn away from the stove to the left is also represented with a 2-legged entity classifier (still 4). In each orientation, the mouse's manual activity is represented in what corresponds to a character perspective representation (stills 3 and 5). ${ }^{114}$

(7a)

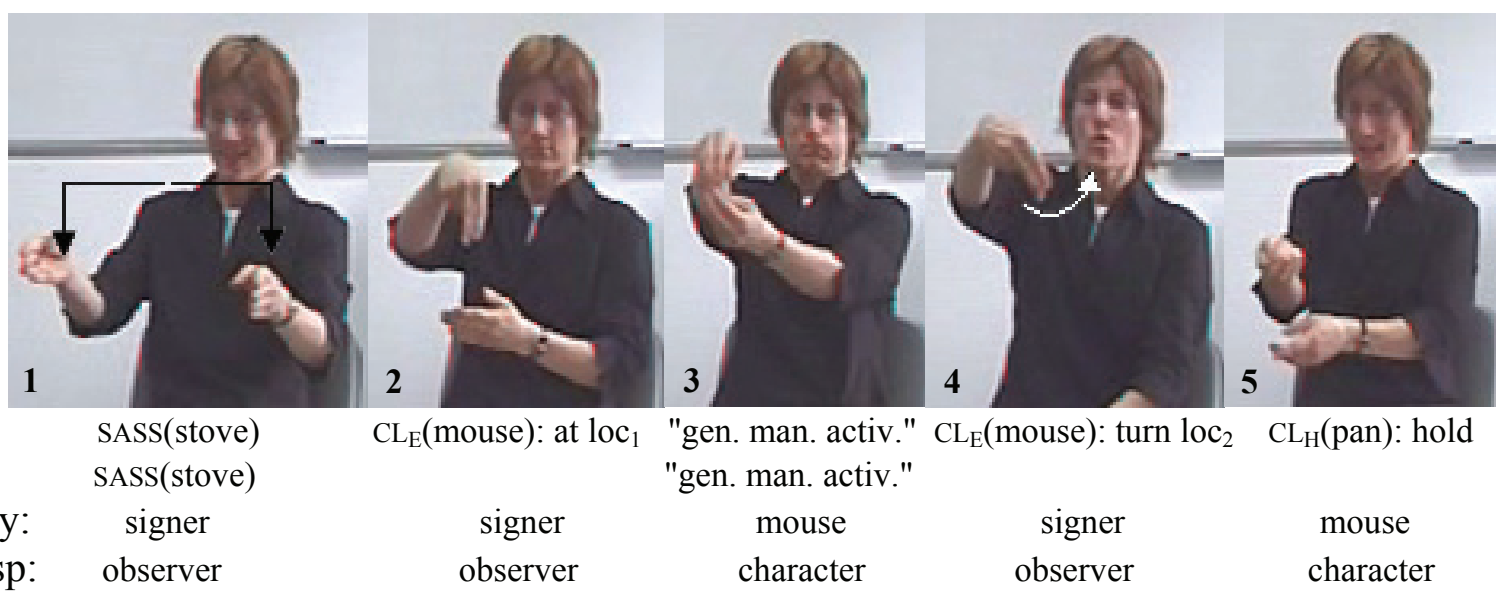

From this point in the narrative, however, the reteller adopts a different strategy than the teller. The reteller continues her narrative with aligned observer and character perspective representations, and does not explicitly co-index locations through the simultaneous representation of the two event space projections. Every change of orientation is thus indicated with a 2-legged entity classifier (see stills 6 and 8 in example (7b) below). The manual activity performed by the mouse in each orientation is represented in character perspective (see stills 7 and 9 in example (7b)). This affords the signer scale iconicity in her representation, but it is at the loss of temporal iconicity. The reteller's narrative is represented schematically in figure 8.4 below.

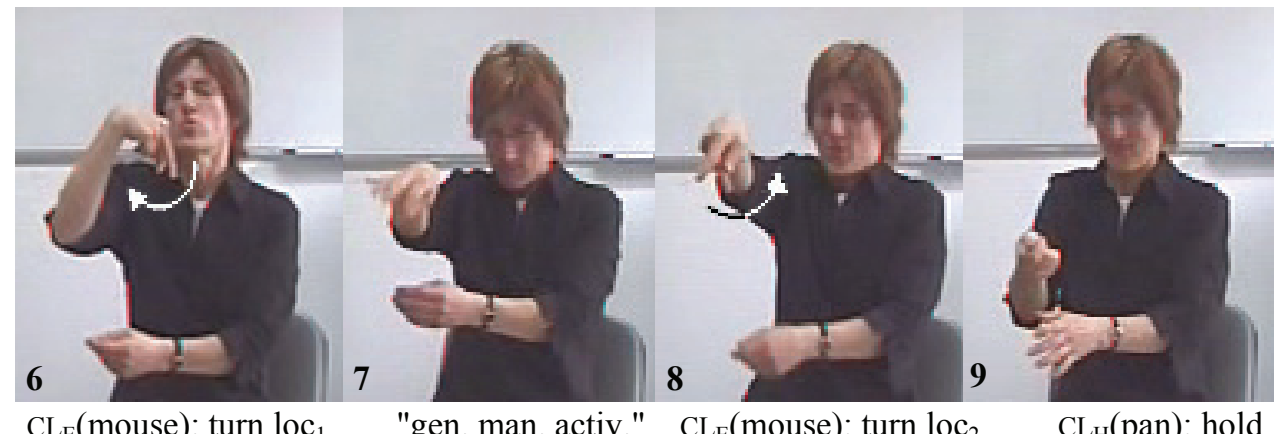

LH

body:

persp

$$
\text { signer }
$$

observer mouse
character

\section{signer}

observer mouse

character

\footnotetext{
114 The representation in still 3 again depicts "generic manual activity", as the mouse's real activity is not visible to the viewer at this point of the stimulus film.
} 

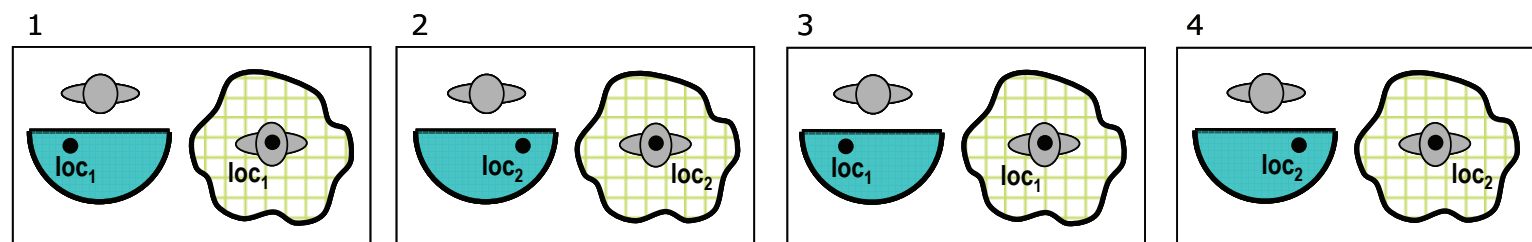

1. Sequentiality of perspectives $\left(\operatorname{loc}_{1}\right)$

2. Sequentiality of perspectives $\left(\operatorname{loc}_{2}\right)$

3. Sequentiality of perspectives (loc1)

4. Sequentiality of perspectives (loc2)

observer perspective event space

character perspective event space

Loc $_{1}$ : referent location $=$ facing stove

Loc $_{\mathbf{2}}$ : referent location $=$ turned from stove

Figure 8.4. Schematization of the use of sequentiality of perspectives in the reteller's narrative.

The comparison of the teller and reteller narratives demonstrates how the use of the doubleperspective construction increases the teller's efficiency in explicitly encoding spatial information about referent location/orientation. For equally precise and explicit encoding of spatial relationships throughout the narratives, the teller (who employs a double-perspective construction) needs only about half as many utterances (7 vs. 12 utterances) as the reteller (who uses a fully sequential strategy). ${ }^{115}$

\subsubsection{Mapping between perspectives for spatial coherence: informativeness of expression}

This section exemplifies the use of a double-perspective construction to co-index referent locations in two event space projections by shifting from character to observer perspective within the representation of a single transitive event. Through the shift, the signer explicitly identifies the goal location of the transitive event in both event space representations.

In the five stills in example (8) below, the signer represents the mouse flipping the pancake to the elephant after the elephant has entered the scene (see the image from the pancake stimulus film above the example). The flipping of the pancake is a transitive event that involves the caused motion of an inanimate referent through the manual activity of an

\footnotetext{
115 The utterances here are counted from the point in the narrative where the teller uses the double-perspective construction to the elephant's entrance on the scene (see Appendix C), and are broadly defined as the propositions used to encode the mouse's location/orientation and activity for each change in location/orientation.
} 
animate referent. The signer represents this manual activity in an aligned character perspective representation (still 1). The signer is in the role of the mouse, and moves the pan upward to flip the pancake to the elephant. The handling classifier (holding the pan) is held in front of the body, and the path of the pancake is projected along the sagittal axis. This is supported by the signer's eye gaze, which follow the path of the pancake upward out of the pan. However, the signer's gaze does not follow the pancake back down to the conceptual location of the elephant opposite the signer's body, as would be expected in an aligned character perspective representation of this event. Instead, the eyes follow the path of the pancake to a goal location located to the left of the signer's body (still 2). That is, at the apex of the pancake's trajectory, the eyes separate out as an independent articulator from the character perspective representation to complete the pancake's trajectory at a goal location determined in an observer perspective event space representation.

The double-perspective construction shown in stills 1 and 2 of example (8) involves the body, head, eyes, and dominant hand as articulators in character perspective and the eyes (and head) as articulators in observer perspective. The eye gaze following the pancake's conceptual path marks the transition from one event space projection to the other. Through this transition, the signer explicitly co-indexes the goal location of the transitive event simultaneously on the sagittal and lateral axes. The handling classifier on the dominant hand remains in place, keeping the character perspective space active until the transitive event is completely encoded (i.e. until the pancake reaches the goal location, the elephant).
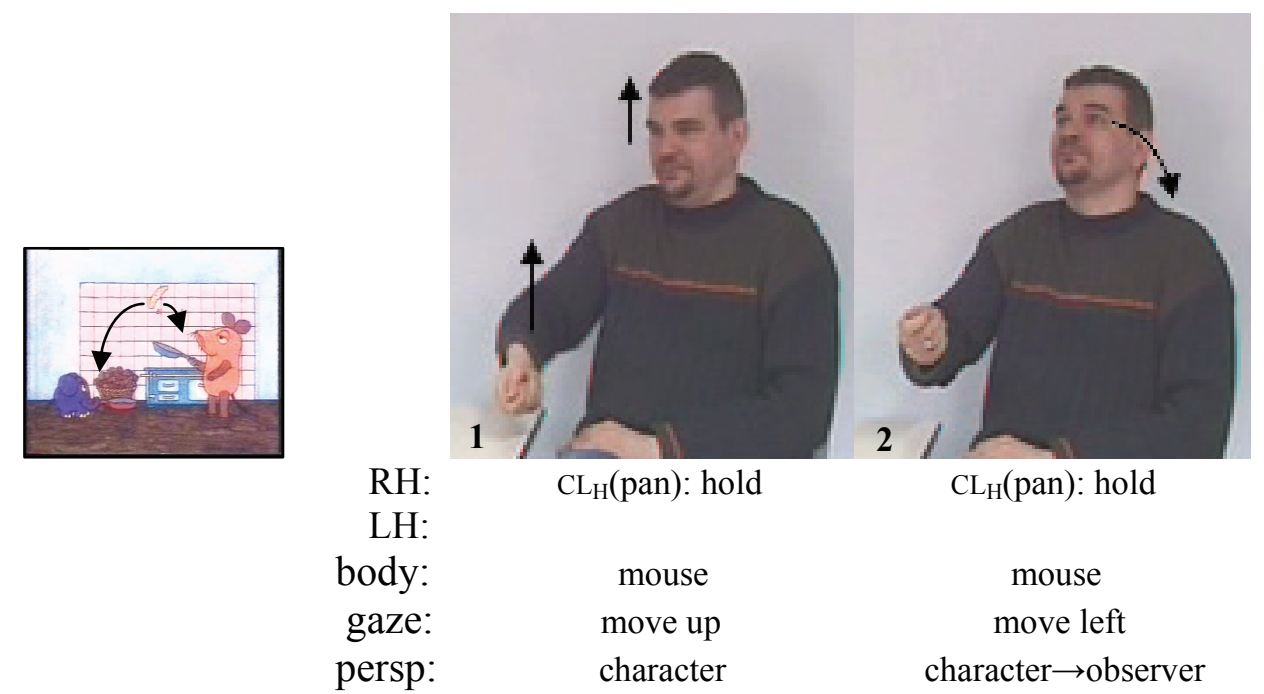


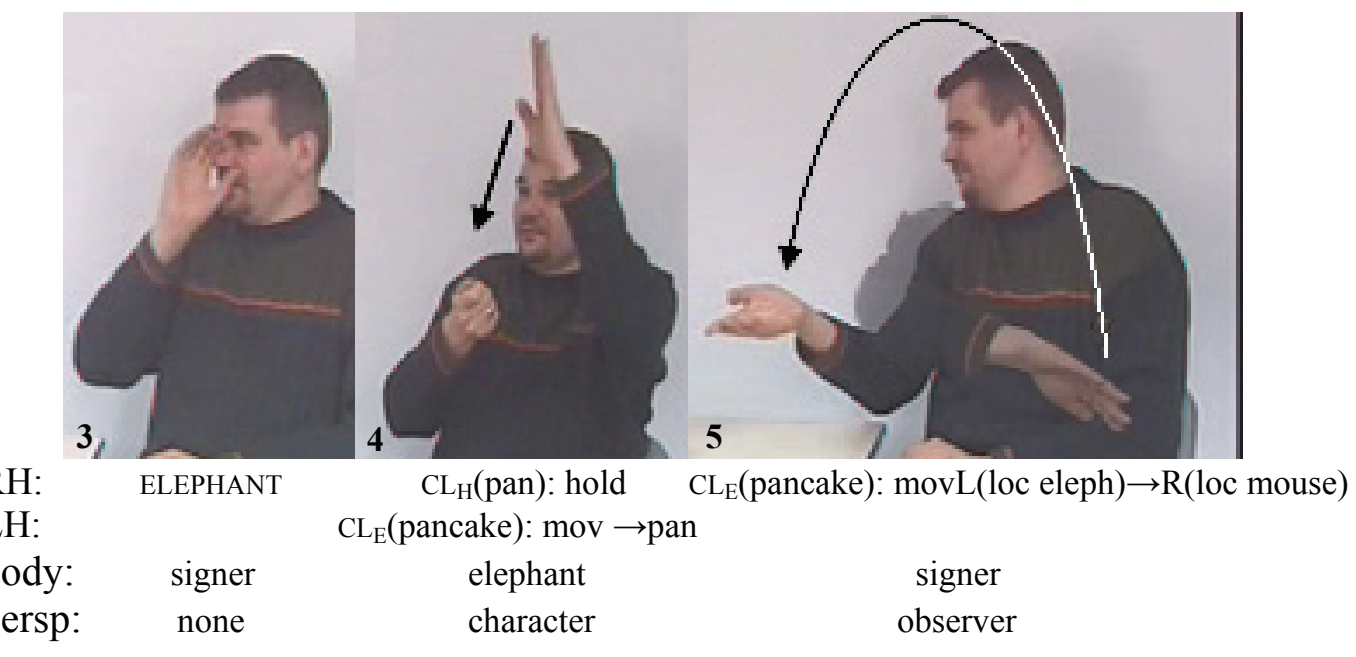

Immediately after the simultaneous construction, the signer lexically identifies the elephant (still 3) and then takes on the elephant's role to represent the pancake landing in the pan (still 4). Following this, the signer changes to observer perspective to represent the path of the pancake between the two animate referents on the lateral axis (still 5). The path starts on the left side, that is, on the side explicitly associated with the elephant through the doubleperspective construction.

Figures 8.5 and 8.6 below show the conceptual locations of the mouse and the elephant in character and observer perspective event space projections, respectively. The figures show that the relative spatial relationship between the referents is the same in each projection, but that the specific referent-location associations in sign space are different.

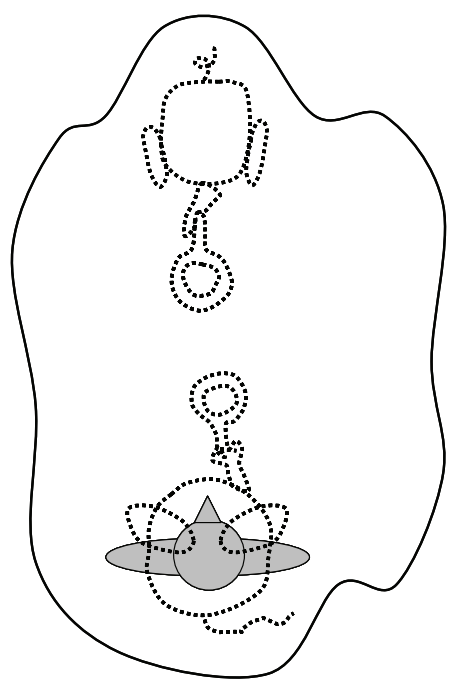

Figure 8.5. Locations of mouse and elephant in a character perspective event space projection.

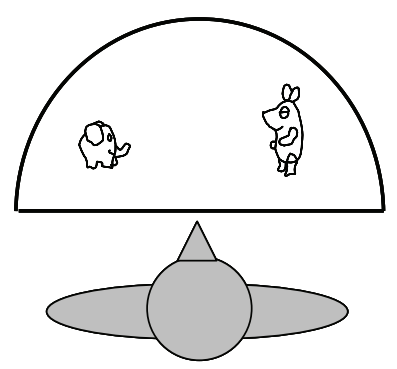

Figure 8.6. Locations of mouse and elephant in an observer perspective event space projection. 
As mentioned earlier, when signers use both character and observer event space projections in the course of a narrative, referents get associated with different locations in sign space, and the correspondence between co-referential locations must be inferred by the addressee if it is not made explicit by the signer. The use of the double-perspective construction shown in example (8) makes the correspondence between referent locations in character and observer perspective event space projections maximally explicit, and thus maximally informative in terms of spatial coherence. Moreover, the construction lets the signer be fully explicit very efficiently in terms of the signer's own effort and in terms of the amount of information integration that the addressee is faced with.

\subsection{Summary and discussion}

This chapter presented an analysis of the use of non-aligned character and double-perspective constructions in DGS narratives. These two construction types are characterized by the use of different manual and non-manual articulators to simultaneously encode spatial information associated with different perspectives. The use of these constructions was discussed in terms of maintaining spatial coherence across perspectives and in terms of achieving temporal and scale iconicity in the event representation. In addition, these functions were discussed in relation to efficiency and informativeness, taken to be discourse-structure constraints that interact with iconicity principles. In the literature, the simultaneous representation of elements from different perspectives (i.e. both observer and character perspective) and on multiple independent articulators (i.e. both manual and non-manual articulators) has not been extensively investigated with regard to specific functions related to discourse and iconicity.

Specifically, this chapter discussed the use of non-aligned character perspective constructions as a way to achieve full semantic specification of an event. The non-aligned constructions allow the signer to supplement a character perspective representation with information prototypically aligned with observer perspective. In this way, multiple event components can be expressed within a single expression. The signer achieves a high degree of efficiency through temporal iconicity, by which simultaneity within the event can be encoded as such. At the same time, the supplemented event components increase spatial coherence through a high degree of informativeness about spatial relationships.

To emphasize the function of supplementing information for a full semantic specification, I elaborate on the example given in Dudis (2004a), in which a signer depicts a motorcyclist ascending a hill (cf. section 8.1). Dudis notes that the representation of the motorcyclist alone (i.e. in a character perspective representation with the motorcyclist 
mapped onto the body) would suffice to convey the information that the top of the hill had been reached, without showing any path movement of the motorcycle using an entity classifier. For example, Dudis lists the following cues on the face and body from which the completed ascent of the hill could be inferred: the eye gaze changes from being directed upward to horizontal; the facial expression changes from tense to relaxed; the torso and hands (gripping the handlebars) move slightly forward. Thus, while the addition of the explicit path component with an entity classifier certainly encodes more information about the event (giving a richer, more detailed representation), Dudis argues that it does not encode additional semantic information that would otherwise be lacking. In contrast to this, in the non-aligned character perspective construction that I have presented in example (1) in this chapter, in particular, the information encoded in one perspective cannot be inferred from what is encoded in the other perspective. The full semantic specification of the mouse's movement in example (1) (in section 8.4; see also figures 8.1 and 8.2) necessarily involves the encoding of both the mouse's change of location and its change of posture. Thus, the semantic totality of the event cannot be encoded without both the representation associated with observer perspective (i.e. to encode the change of location with an entity classifier) and the representation associated with character perspective (i.e. to encode the change of posture with the torso and holding the pan).

The second type of construction discussed in this chapter were double-perspective constructions, whose description is a novel contribution to the discussion of simultaneity of perspectives in the literature. With double-perspective constructions, signers can create a mapping between the referent-location associations in observer and character perspective event space projections by simultaneously co-indexing them. The mapping between perspectives allows signers to be maximally informative and explicit about the relationship between two perspectives and achieves a high degree of spatial coherence. In addition, the double-perspective constructions may demonstrate a unique property of signed language (DGS, in particular) in that they allow two different perspectives to be simultaneously held and represented. This exhibits a simultaneity of perspectives that is generally not taken to be possible, as evidenced e.g. by the impossibility of simultaneously seeing both perspectives in a Neckar-cube (i.e. one sees the cube as either popping out of the page or receding into the page). Determining the exact nature of the perspectival simultaneity in double-perspective constructions remains for future research.

Finally, while non-aligned character perspective constructions occurred frequently in the data (cf. figure 7.4 in section 7.4 of chapter 7), the use of double-perspective 
constructions, as they have been presented here, was very infrequent (cf. the "other" category in figure 7.3 in chapter 7). This suggests that there are constraints on the way that the affordances of the visual-spatial modality can be exploited. The nature of the modality allows different meaning elements to be simultaneously encoded using independent articulators. This makes possible the simultaneous representation of two event space projections, as we have seen in this chapter. However, the overall low occurrence of such constructions indicates that this type of simultaneous representation is disfavored. This may be due to their greater complexity, which may be cognitively more demanding. More research with more data is needed to learn about how signers exploit the affordances of simultaneity in discourse. 


\section{Conclusion}

Chapter 9

The main aim of this dissertation has been to provide an analysis of spatial language and investigate the role of iconicity in spatial representation in DGS. Specifically, the dissertation has examined the use of important spatial devices, including frames of reference, perspective, and classifier predicates, in two different types of discourse. The results presented in the foregoing chapters have important implications for our understanding of the role of iconicity in the visual-spatial modality, the possible language-specific variation within the spatial domain in the visual-spatial modality, the structure of spatial language in both natural language modalities, and the relationship between spatial language and cognition.

In addition to the empirical analyses, the dissertation has provided an overview of the relevant linguistic and theoretical domains. Chapter 2 discussed the notion of iconicity in language systems, in general, and in the visual-spatial modality, in particular. Moreover, it introduced the reader to the phonology and relevant morphosyntactic structures of DGS (and sign languages, generally), including the system of classifier predicates and simultaneous constructions. Chapter 3 presented the investigation of the spatial domain, especially the two major subdomains of location and motion, as an important undertaking in linguistics and cognitive science. The literature on the use of frames of reference in both spoken and sign languages was reviewed, and the concept of signing perspective as a projection of the event space onto sign space was introduced and defined. Frames of reference and signing perspective were presented as independent systems, whose relationship is determined by certain prototypical alignments (discussed again in section 9.3 below). The next section summarizes the main findings of the analyses of the expression of spatial relationships in DGS. Section 9.2 discusses the practical implications of the methodologies developed in this dissertation, and section 9.3 highlights important theoretical implications of the findings.

\subsection{Summary of main findings}

To date, research on the spatial domain in sign languages has concentrated primarily on the use of classifier predicates, and on the ability to create mappings between real space and sign space based on their structure-preserving properties. In general, the use of space in sign 
language expression has been widely assumed to be guided by iconic principles, which are furthermore assumed to hold in the same way across sign languages. Thus, there has been little expectation of variation between sign languages in the spatial domain, particularly in the use of classifier predicates, perspective, and viewpoint. Moreover, the notion of iconicity itself is invoked broadly, without differentiation of different types of iconicity. Consequently, perhaps, there has been little systematic investigation of linguistic expression in the spatial domain in individual sign languages, and less investigation of spatial language in extended signed discourse. Most of the research to date that has addressed the use of space in the visual-spatial modality has been based on ASL, and comparatively little is known about encoding in the spatial domain in other sign languages. As a result, we know little also about the possible variation in this domain in the visual-spatial modality.

This dissertation has provided such an investigation of DGS in the expression of spatial relationships. The study has focused on spatial encoding in two different discourse types, static scene spatial description and event narratives, and on the expression of both location and motion events. Its primary theoretical objectives have been to characterize the structure of locative descriptions in DGS; to explain the use of frames of reference and perspective in the expression of location and motion; to clarify the interrelationship between the systems of frames of reference, signing perspective, and classifier predicates; and to characterize the interplay between iconicity principles, on the one hand, and grammatical and discourse constraints, on the other hand, in the use of these spatial devices.

Chapter 4 provided an analysis of the structure, and structural variation, of locative constructions in DGS, based on descriptions of static object configurations. The results showed that DGS signers do rely to a large extent on the iconic properties of classifier predicates to encode location, orientation, and number of referents, and on the properties of sign space to create "isomorphic" representations of real-space scenes in sign space. In this, DGS descriptions conform to general assumptions about how iconicity shapes spatial language in the visual-spatial modality. However, the results also reveal structural properties that may be special to DGS. For example, they suggest a constraint in DGS on simultaneous spatialization of referents, such that single predication is possible only for dual or reciprocal relationships. In addition, the non-dominant hand was not found to be used systematically to encode the ground object (contrary to what has been mentioned in literature on DGS and other sign languages). This suggests that DGS does not have a strictly linguistic figureground structure. A systematic ordering of figure and ground was found only when entities could be perceptually segregated into a figure and ground (e.g. in the man/tree pictures), 
supporting the notion that the encoding of bigger, more permanent objects before smaller, more moveable objects may be a property of the modality (Cogill-Koez 2000; Emmorey 1996, 2003). Furthermore, as predicted by the iconicity of perception principle, almost 100\% of static scene descriptions were coded from the signer's viewpoint. However, as suggested by the relatively higher occurrence of spatial descriptions from the addressee's viewpoint in ASL (Emmorey 1996), different sign languages may exhibit different preferences in the use of viewpoint.

Finally, the analysis showed that the 2-legged entity classifier in DGS may not entail orientation information, although it is a featured form. This indicates that classifiers in DGS may appear in unmarked vs. marked configurations, as determined, for example, by articulatory effort (cf. Liddell 1980 on ASL, and the example of the cat on the fence cited in chapter 3). Marked configurations (i.e. marked locations/orientations of the hand) may always be intended to commit the signer to all the represented spatial information, while unmarked configurations may not be so intended. This is theoretically interesting in that it shows that sign languages can be semantically general over spatial configurations in a way in which, for examples, pictures or diagrams cannot. More research with different types of spatial scenarios and on different types of discourse situations is needed to determine the influence of these different factors.

Chapter 5 investigated the use of perspective and frames of reference. It was found that DGS static scene descriptions are characterized by the use of an observer perspective event space projection and a relative frame of reference. The use of both relative and intrinsic frames simultaneously, as a special property of the visual-spatial modality, was also found to occur in observer perspective. Developing criteria for the differentiation between the use of relative and intrinsic frames of reference is an important contribution of this chapter. The analysis of addressee interpretation is a novel contribution to the investigation of frame of reference use in signed language. By asking the addressees to reconstruct the spatial scene, as they interpreted the signed description, it was possible to test whether the use of viewpoint within the relative frame of reference in DGS is conventionalized within the language system across both production and comprehension. The results of the analysis indicate that interpretation of location and orientation information from the signer's point of view (i.e. with the signer as deictic origo) is indeed preferred.

The use of perspective and frames of reference in event narratives was the topic of chapter 6. It was found that the use of character perspective and the intrinsic frame of reference is preferred in DGS event narratives to encode both location and motion events. 
The difference in perspective and frame of reference use between static scene descriptions and event narratives shows a clear effect of discourse type in the encoding of location. The use of character perspective in DGS for location (i.e. for scene setting) contrasts with findings for other sign languages (e.g. ASL and BSL), in which scene setting in narratives is argued to take place in observer perspective. Overall, the predominant use of character perspective and the intrinsic frame in DGS narratives may be related to the type of events contained in the stimulus films (e.g. manual manipulation of objects and events of object transfer by animate referents). However, the influence of discourse type on the use of perspective and frames of reference may differ between sign languages, depending on the availability of languagespecific mechanisms for spatial encoding. For example, the use of a body-shift mechanism in DGS to explicitly mark switches in subject reference between two animate referents may facilitate the continuous use of character perspective. The possible lack of body shift as a systematic mechanism in TID, in contrast, may motivate a higher frequency of use of observer perspective in narratives (cf. Perniss and Özyürek, in press).

Chapter 7 investigated how referent location, motion, and action is encoded in event narratives, and proposed nine different construction types based on the use of perspective with classifier predicates, lexical predicates, or index signs. Furthermore, the analysis proposed a definition of aligned and non-aligned combinations of classifier predicates and perspective. The analysis of distribution of use of the different construction types showed that DGS signers use character perspective abundantly in both aligned and non-aligned construction types. In observer perspective, however, only aligned representations were used, specifically for path-salient event types. The use of aligned and non-aligned construction types was discussed in terms of iconicity principles, showing that scale iconicity and temporal iconicity may be competing types of iconic representation (see section 9.3.2 below). Other data (e.g. from TID narratives) suggest that the distribution of use of aligned and nonaligned construction types reflects language-specific constraints on the simultaneous expression of different aspects of the event (cf. Özyürek and Perniss, in press). In addition, other sign languages may use completely different constructions. For example, the expression of motion events in AdaSL looks radically different from that in DGS. AdaSL does not use entity classifiers at all to encode location and motion, and instead uses generic directional signs and character perspective-like representations (cf. Nyst and Perniss 2004).

In addition, the chapter 7 finding of lexical predicates used without an event space projection is interesting in relation to the general question of spatial representation in a spatial medium. Together with the finding from chapter 4 that 2-legged entity classifiers, though 
featured, may not entail orientation information (discussed above), the use of lexical predicates in neutral space shows that spatial information can be represented in the visualspatial modality without committing to iconic spatial representation. That is, it is possible to be semantically general or non-committal over details of spatial representation in a spatial language, despite iconicity principles (like imagistic and diagrammatic iconicity) which might make it seem impossible.

Finally, chapter 8 focused on the special affordance of simultaneity in the visual-spatial modality. The chapter presented occurrences of non-aligned character perspective and double-perspective constructions in the DGS event narratives, and analyzed them in terms of the interaction between iconicity principles and discourse principles of efficiency and informativeness in the expression of spatial information. Their functions were discussed, respectively, as: (1) encoding different aspects of the same event simultaneously to achieve full semantic specification of the event and to preserve temporal iconicity, and (2) encoding the same aspect of an event in two different ways simultaneously to achieve a mapping between two perspectives to maintain spatial coherence. It seems incidentally to be the first time that these double-perspective constructions have been identified.

\subsection{Methodological implications}

This section addresses the methodologies employed in this dissertation for investigating the spatial domain in a signed language. Methodologies have been provided for analyzing the structure of locative descriptions, the use of perspective, frames of reference, and different types of predicates across both production and comprehension. In addition, the dissertation has been careful to provide real signed examples, with still images cut from the signed descriptions themselves.

Chapter 4 provides a detailed format for investigating the structure of locative descriptions in a signed language. The system of coding involves a sign-by-sign transcription of not only the type of sign, but the type of spatial information it encodes, its location and orientation in sign space, the hand with which it is signed, and the type of classifier form. The analysis is at a level of granularity that allows structural regularities (or idiosyncrasies) to be picked up. As such, it offers a format for cross-linguistic comparison of locative constructions across sign languages. Such analyses will help to uncover even very finegrained language-specific differences between sign languages in the expression of spatial relationships of location and orientation. 
In addition, chapters 4-6 have offered precise characterizations of the distinction between figure and ground in location and motion events, in different discourse types and with different types of entities. Such a specification of figure and ground is important for identifying the instantiation of frames of reference in sign languages, and has not been previously worked out in detail. The frame of reference analysis is based on the syntax of individual propositions, and on the semantic contribution of its parts. The definitions and format offered here also allow careful comparisons across sign languages of the use of frames of reference. Such comparisons might uncover differences in the preference for particular frames of reference and in the conventionalization of linguistic figure-ground structures.

Chapter 7 presented a descriptive format for investigating the relationship between signing perspective and different predicate types used to encode referent location, motion, and action in event narratives. Different construction types were defined based on the possible combinations of perspective with classifier predicates, lexical predicates, and index signs. This again provides a format for future cross-linguistic comparisons across different sign languages, and can help uncover variation in the use of aligned and non-aligned forms, and in the different types of uses of simultaneity of perspectives and predicate types.

Finally, the analysis of addressee comprehension, in conjunction with the analysis of the original descriptions themselves, offers important insights into the conventionalization of the spatial systems investigated in this dissertation. The matchers' scene reconstructions in the static scene description task and the addressee retellings in the narrative task allowed an assessment of how location and orientation information was interpreted.

\subsection{Theoretical implications}

\subsubsection{Perspective and frames of reference analysis}

One major theoretical contribution of this dissertation is its analysis of perspective and frames of reference in the visual-spatial modality. It has provided principled definitions for the use of perspective and for the identification of frames of reference in sign, and has investigated the relationship between the two systems. With regard to the investigation of frames of reference, in particular, this dissertation has gone beyond what has been previously available on frames of reference in the visual-spatial modality. The analyses and definitions set forth are important for an understanding of the influence of the modality on the way that frames of reference can appear in signed language. 
I have provided evidence for the independence of perspective and frames of reference as spatial systems in the visual-spatial modality. There are two main pieces of evidence for this. Firstly, the specification of the locative relationship between two objects (i.e. a figure and a ground object) as encoded in a frame of reference utterance must take place within a particular event space projection (i.e. from observer or character perspective). Secondly, relative and intrinsic frames of reference can simultaneously co-occur within both observer and character event space projections. The simultaneity of frames of reference within a single proposition is a special property of the visual-spatial modality.

The independence of the two systems notwithstanding, this dissertation has argued for the existence of prototypical alignments between perspective and frames of reference, based on shared properties. On the one hand, the use of observer perspective aligns with the use of a relative frame of reference. Both observer perspective signing and the relative frame presuppose an external viewpoint from which spatial relationships are represented. In the relative frame, the figure and ground objects are different from the signer's body, which represents the viewpoint. Similarly, in observer perspective, the signer's body is external to the represented event space. The intrinsic frame of reference can be simultaneously present if the ground object (i.e. the first object located in sign space) is featured. On the other hand, the use of character perspective aligns with the use of an intrinsic frame of reference. The binary relationship between the figure and ground objects encoded by the intrinsic frame corresponds to character perspective signing, where an animate referent is mapped onto the signer's body. The signer's body represents the ground object and the figure object is located with respect to the body. The simultaneous use of a relative frame of reference is possible when two referents are located within the life-sized event space projection from the character's point of view.

In relation to the representation of entities in space in the visual-spatial modality, one could speculate about the hierarchical relationship between the use of these systems. To represent an event space, it seems signers must first decide on a perspective (i.e. on a way of projecting the event space onto sign space). Then, if the scene contains elements that are noncoincident in space, signers must choose a frame of reference in which to represent the spatial relationship(s) between the elements. Finally, in the relative frame of reference, signers must choose a viewpoint from which to represent the scene. (Similarly, having decided on a character perspective, or the use of an intrinsic frame of reference, signers must choose the character from whose perspective they wish to represent the scene.) The logical ordering 
between these systems is suggestive of a processing order, the investigation of which requires further research.

A further alignment that has been motivated in this dissertation is between the use of signing perspective and classifier predicates. These alignments are based on the scale of event space projection and on the felicitousness of representation of different types of information within each perspective (e.g. transitive vs. intransitive motion events). In observer perspective, the event space is reduced in size and is projected onto the area of sign space in front of the signer's body. Entity classifiers represent the location and motion of entities within the event on a correspondingly small, reduced-size scale. In character perspective, the event space is life-sized, and the signer is in the role of a character within the event. The signer's hands thus represent the character's hands, and the manipulation of referents by the character is represented through the use of handling classifiers.

Bringing together perspective, frames of reference, and classifier predicates, we can thus motivate a three-way alignment. The use of observer perspective prototypically aligns with the relative frame of reference and entity classifiers. The use of character perspective prototypically aligns with the intrinsic frame of reference and handling classifiers. Importantly, the analyses of the event narratives in this dissertation have shown that what appears in spatial descriptions are not always aligned forms. The use of non-aligned forms can fulfill different functions related to the expression of simultaneous aspects of an event and, generally, to the efficiency and informativeness of spatial encoding. In addition to exploring the affordance of simultaneity of frames of reference within one perspective, the simultaneity of perspectives and the simultaneous use of manual and non-manual articulators within different perspectives has been investigated. This furthers our understanding of simultaneous mapping in the visual-spatial modality.

The investigation of perspective and frames of reference in the visual-spatial modality is especially important for our understanding of these two systems in language, in general, that is, across language modalities. First of all, the visual features of classifier forms make it possible for location and orientation information to be simultaneously expressed, and thus for the relative and intrinsic frames to be simultaneously encoded in a single form. This type of simultaneity is not possible in spoken languages, where the information within a single form is restricted to a single frame of reference.

In addition, the nature of the visual-spatial modality defines a relationship between the systems of perspective and frames of reference that is qualitatively different from that in spoken languages. In spoken languages, the use of observer vs. character perspective has 
counterparts in spatial descriptions of different types of environments (e.g. living space, building space) in the use of "survey" vs. "route" descriptions (Emmorey et al. 2000; Taylor and Tversky 1992, 1996) and "gaze" vs. "body" tours (Ehrich and Koster 1983; Linde and Labov 1975). In addition, the use of observer vs. character perspective in discourse, e.g. narratives, has counterparts in "diegesis (description)" vs. "mimesis (depiction)" (Clark and Gerrig 1990), and the use of "constructed action" in character perspective signing (Metzger 1995) bears similarity to "constructed dialogue" in spoken discourse (Tannen 1989). ${ }^{116}$

The use of different "perspectives" in spoken language, however, is not directly related to the use of frames of reference, as we have seen for DGS. The use of space to talk about space in the visual-spatial modality means that frame of reference use is dependent on perspective. This creates the correspondences, or prototypical alignments, between the two systems that we have seen. Moreover, the obligatory nature of perspective in the visualspatial modality - that is, the fact that perspective is a precondition for most spatial representation - seems to lend it a different status than in spoken languages. In the visualspatial modality, language involving the expression of spatial relationships hinges on the use of perspective in creating a space in which to project the event space being talked about. Thus, the use of perspective is inextricably bound to the use of all other types of spatial devices and systems. In this way, perspective in signed language may constrain language in the spatial domain in a much more thorough way than in spoken language.

\subsubsection{Space, iconicity, and discourse}

In the sign language literature, the term iconicity is typically used very broadly, covering all types of form-meaning resemblance and visual motivation (cf. the discussion in chapter 2). This dissertation has made clear the need to differentiate between different types of iconicity, showing that different iconicity principles affect the structure of signing and interact with grammatical and discourse functions. As a close to the dissertation, this section offers a

\footnotetext{
${ }^{116}$ Furthermore, though not directly related to spatial language, the use of character perspective in narratives bears similarity to what Labov (1972) calls internal evaluation. In telling stories, speakers use strategies like direct quotation and expressive phonology to create a sense of identification and involvement with characters. It is also reminiscent of what Tannen (1980) refers to as maintaining a direct perspective in story telling, which is characterized by a direct relating of events/actions. Labov opposes internal evaluation to external evaluation, where the point of a story is communicated not through the manner of telling and the involvement in the story, but rather through explicit verbal statements. Tannen opposes direct perspective to film perspective, where a stimulus film's events are recounted from the perspective of an external viewer and evaluator, and explicit reference to the fact of film viewing is made throughout the narrative. It is also interesting in this respect that Kay (1977) and Tannen (1982) link interpersonal involvement strategies in storytelling to oral tradition.
} 
synthesis of the types of iconicity we have encountered in the previous chapters and attempts to make clear the relationships between them.

One major type of iconicity is scale iconicity, which demands that different parts of the representation should have the same size. In particular, the size of event space projection (i.e. perspective) and the size of referent projection should match. Another major type of iconicity is temporal iconicity, which requires that simultaneously occurring things should be represented as such. Finally, iconicity of perception holds that the perception of an event space should match its representation.

These iconicity principles have different implications for how spatial information can be represented, and they cannot all be satisfied at the same time. In chapters 7 and 8 , especially, different iconicity principles were invoked to motivate the choice of construction type in event narratives. In each situation, the choice of a type of iconicity seems to be modulated by different constraints, the interaction of which may be explained by means of an Optimality Theory-type ranking of principles. The choice of one type of iconicity buys you one thing at the cost of another, and grammatical, articulatory, and discourse constraints may adjudicate between using one over another. This approach is outlined below, in an elaboration of the analyses given in chapters 7 and 8. Sharpening and expanding such an account further is left to future research.

The principle of scale iconicity motivates the alignments between classifier predicates and signing perspective. Event representation in one scale (e.g. in observer perspective with entity classifiers) also insures that referent-location associations within sign space are unique, which satisfies one aspect of the iconicity of perception principle. However, due to articulatory and grammatical constraints, as we have seen, not all information can be represented in aligned observer perspective representations. Thus, to represent certain types of information (e.g. manual activity in transitive manner events), signers must switch into character perspective. If signers choose to preserve scale iconicity by using aligned observer and character perspective representations, it is, however, at the cost of temporal iconicity. That is, certain simultaneously occurring events (e.g. intransitive path and transitive manner events) cannot be simultaneously represented. Conversely, the use of non-aligned constructions (in particular, non-aligned character perspective) allows signers to gain the temporal iconicity, but at the cost of scale iconicity.

Switching between two event space projections or perspectives may also cost signers the spatial coherence of a single scale (inherent in the iconicity of perception principle). But through the affordance of simultaneity and the special nature of the double-perspective 
construction (described in chapter 8), the spatial coherence can be won back. With a doubleperspective construction, different locations, in different perspectives, that are associated with a single referent are simultaneously co-indexed and thereby mapped onto each other. As chapter 8 showed, the different types of iconicity interact with discourse function. For example, we saw that the use of non-aligned character constructions preserves temporal iconicity, while affording a high degree of informativeness and efficiency.

Finally, I return to the Peircean types of iconicity introduced in chapter 2, namely imagistic and diagrammatic iconicity, and address their presence in the spatial representations studied in this dissertation. In chapter 4 , the imagistic properties of classifier handshapes (encoding size, shape, and intrinsic features of referents), and the diagrammatic properties of classifier predicates (encoding location and orientation of referents, especially in simultaneous classifier constructions) were invoked to motivate the canonical structure of DGS locative descriptions. Furthermore, in keeping with the alignments between different systems for which I have argued throughout the dissertation, diagrammatic iconicity may be prototypically associated with observer perspective representations, while imagistic iconicity may be prototypically associated with character perspective representations. The external viewpoint and the use of entity classifiers in a reduced-size event space allows signers to diagrammatically represent the relationships between individual referents in space. In character perspective, on the other hand, the signer's body and hands correspond imagistically to the body and hands of the character whose role the signer assumes.

\subsection{General conclusions}

Overall, the analyses and findings presented in this dissertation are important for our understanding of visual vs. grammatical/discourse constraints on the representation of imagistic content in the visual-spatial modality. Moreover, the results show that the use of space to represent space in signed language is a more complex and varied matter than it superficially appears to be. The differentiation of iconicity into different principles, acting on different levels, has helped to demonstrate the interaction between effects of iconicity and effects of grammatical and discourse constraints. Through the discussion of differences between DGS and other sign languages in the use of space (most notably TID and AdaSL), this dissertation has also demonstrated evidence of language-specific constraints and strategies in the expression of spatial relationships in DGS. In general, the results suggest that different sign languages may use a variety of different mechanisms and strategies in the expression of spatial relationships. 
By providing an analysis of spatial description in DGS, this dissertation has contributed to our general understanding of the possible variation in the visual-spatial modality in the spatial domain. Cross-linguistic comparisons between sign languages are necessary to uncover the whole range of variation and the specific forms that can appear. Further research on DGS and other sign languages will continue to delineate the modality-specific effects of iconicity and the effects of language-specific grammatical and discourse constraints on the shape of spatial language. 
Appendix A

The static scene stimulus pictures were compiled from various sets of stimuli in the Max Planck Institute for Psycholinguistics stimulus archive. The original labels of the stimulus materials are indicated after the picture. Pictures labeled with an "M", "R", or "C" are from the Annie Senghas Man and Tree object-object matching task; pictures labeled with "FA" are from the Farm Animals task; pictures labeled as "set 2" and "set 4" are from the Original Man and Tree picture-picture matching task.

\section{Group 1}
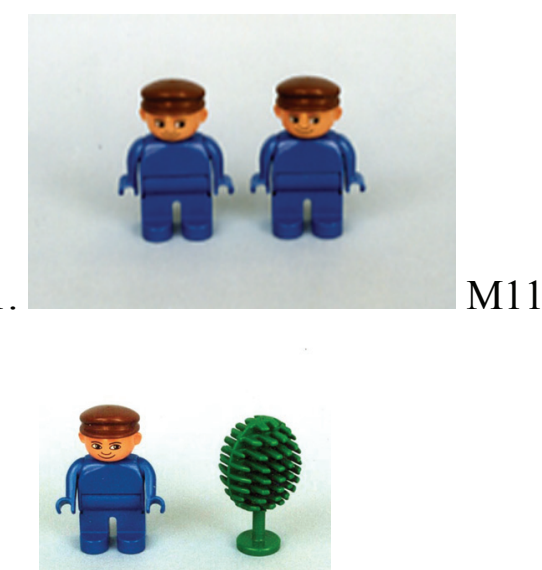

2.

R14

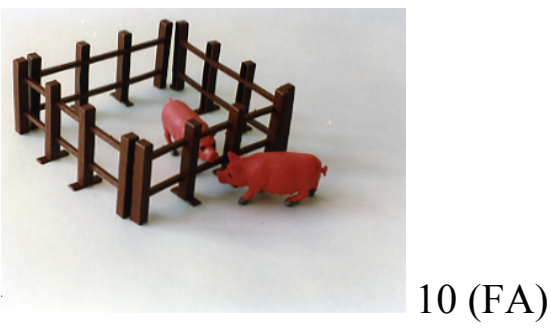

4.

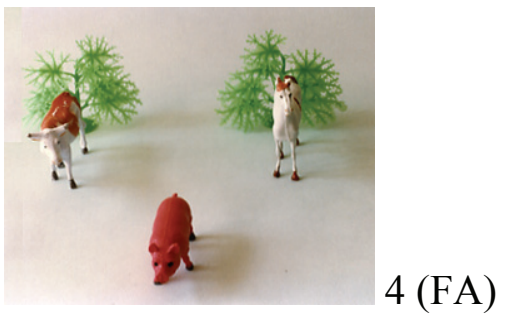

5.

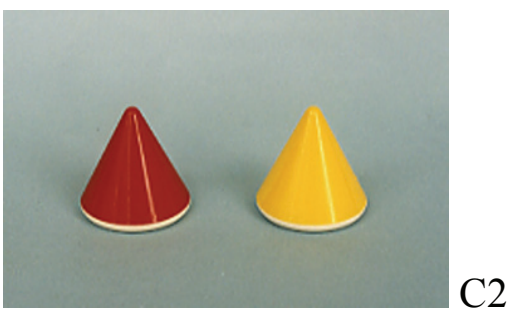


6.

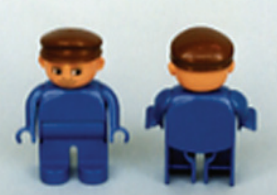

6.

M13

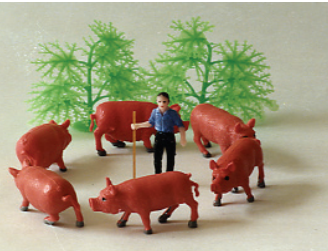

7. 4 (set 4)

8.
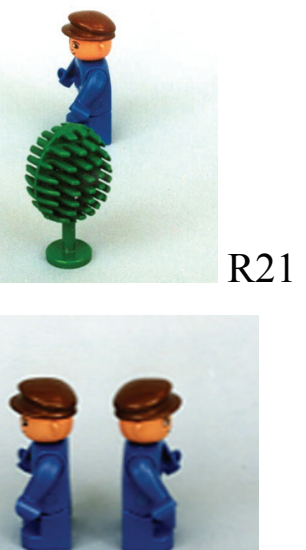

9.

M24

10.

R33

\section{Group 2}

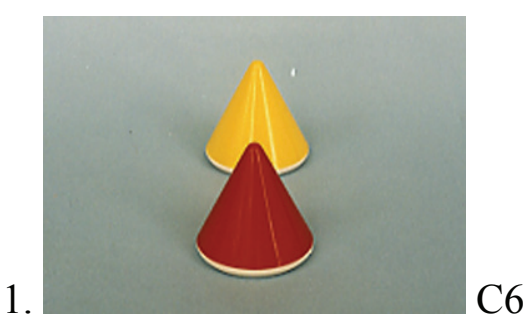


2.

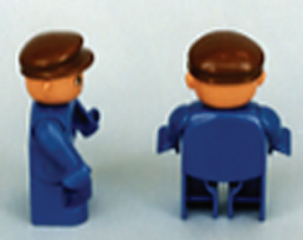

M43

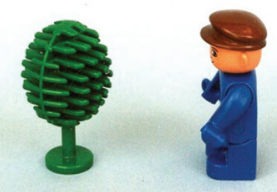

3.

R22

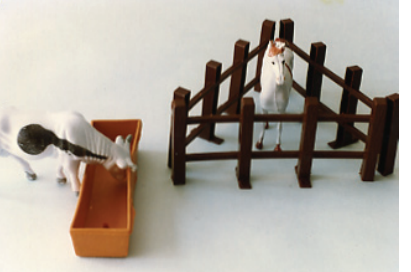

4.

14 (FA)

5.

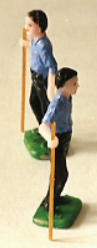

10 (set 4)

6.
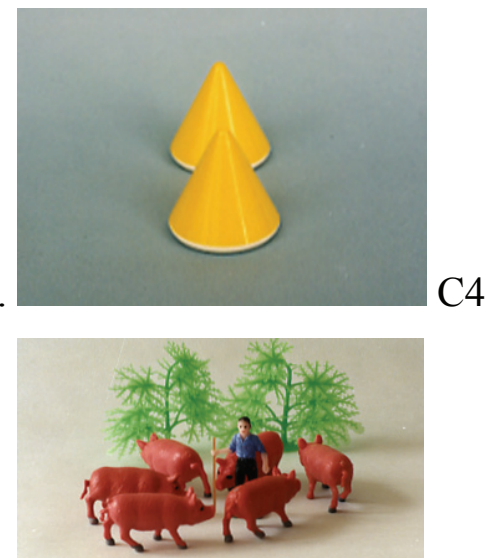

7. $2(\operatorname{set} 4)$

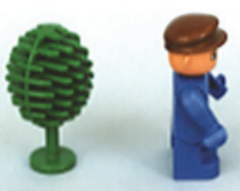

8.

R42 
9.

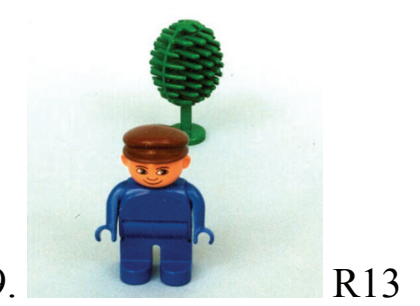

10.

M22

11.

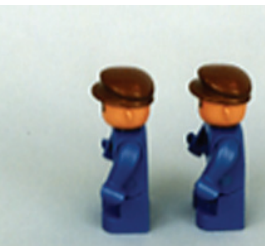

0.
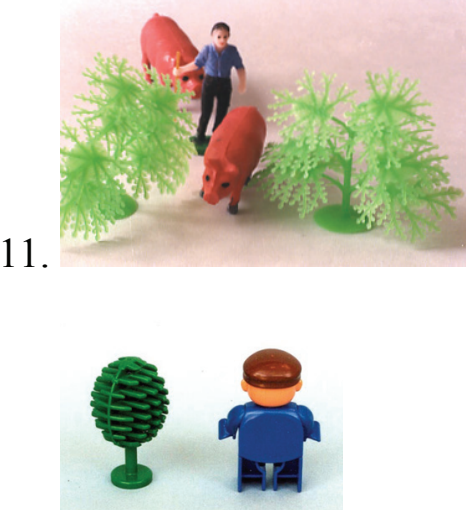

12. R31

\section{Group 3}

1.

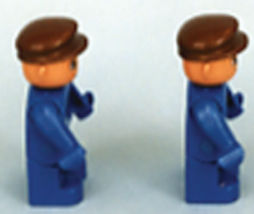

2.

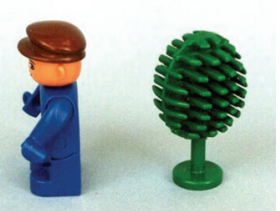

R24

3.

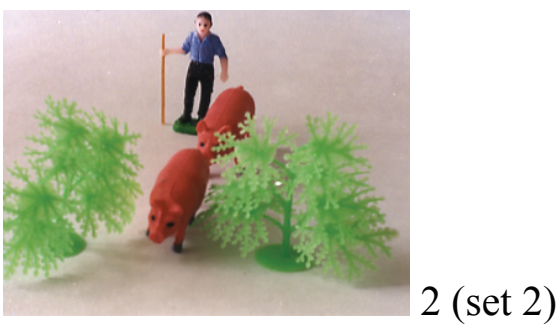


4.

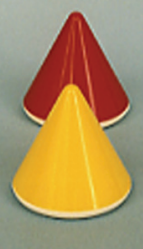

C5

5.

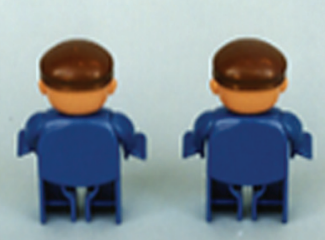

5.

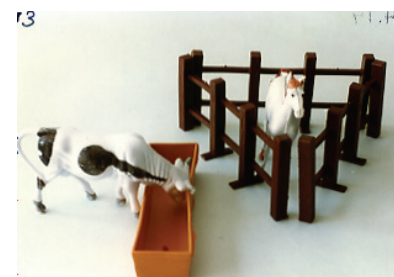

6.

13 (FA)

7.

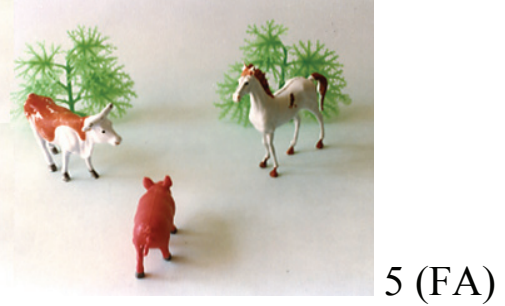

8. M14

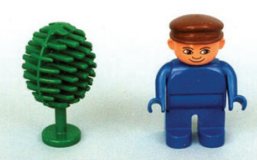

9.

R12

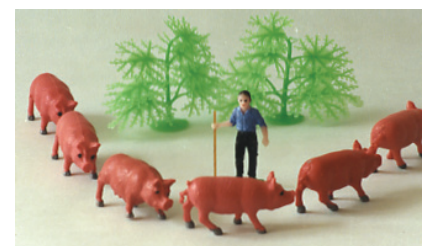

10.

1 (set 4) 
11.

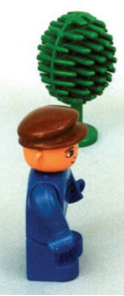

R43

Group 4

1.

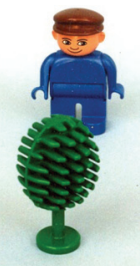

R11

2.
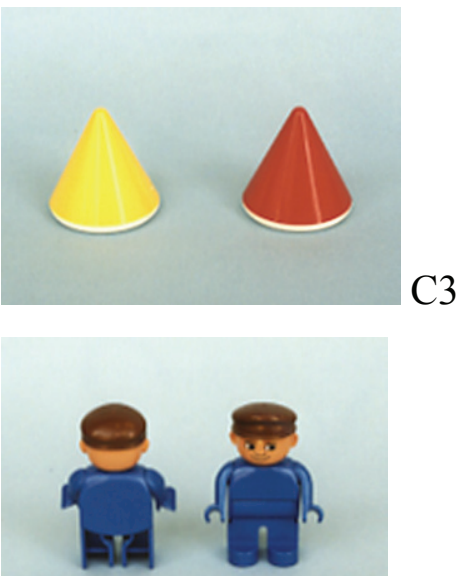

3.

4.

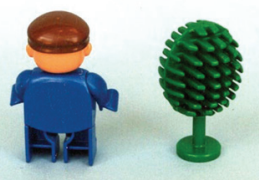

R34

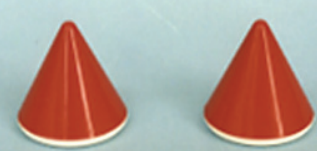

5.

C1

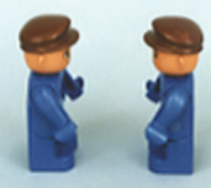

6. 


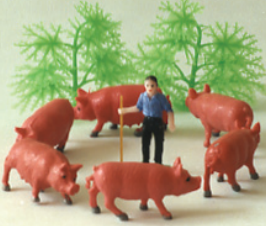

7.

3 (set 4)

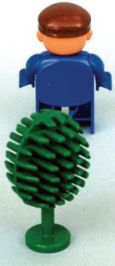

8.

R31

9.
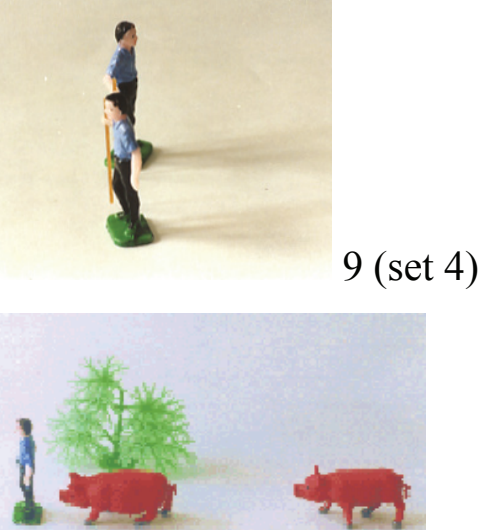

10.

8 (FA) 
Appendix B

\begin{tabular}{|c|c|c|c|}
\hline Signer pair & Teller & Reteller & stimulus films: \\
\hline \multirow[t]{2}{*}{ GL and GP } & GL & GP & $\begin{array}{l}\text { Pillow case } \\
\text { Pancake } \\
\text { Chestnut } \\
\text { Apple tree }\end{array}$ \\
\hline & GP & GL & $\begin{array}{l}\text { Ball } \\
\text { Bar }\end{array}$ \\
\hline \multirow[t]{2}{*}{ JP and LL } & JP & LL & $\begin{array}{l}\text { Pillow case } \\
\text { Pancake } \\
\text { Chestnut }\end{array}$ \\
\hline & LL & JP & $\begin{array}{l}\text { Ball } \\
\text { Bar }\end{array}$ \\
\hline \multirow[t]{2}{*}{$\mathrm{KF}$ and $\mathrm{DM}$} & $\mathrm{KF}$ & $\mathrm{DM}$ & $\begin{array}{l}\text { Pillow case } \\
\text { Pancake } \\
\text { Chestnut } \\
\text { Apple tree }\end{array}$ \\
\hline & DM & $\mathrm{KF}$ & $\begin{array}{l}\text { Ball } \\
\text { Bar }\end{array}$ \\
\hline \multirow{2}{*}{$\mathrm{HT}$ and SB } & HT & SB & $\begin{array}{l}\text { Pillow case } \\
\text { Ball } \\
\text { Apple tree }\end{array}$ \\
\hline & $\mathrm{SB}$ & HT & $\begin{array}{l}\text { Pancake } \\
\text { Bar } \\
\text { Chestnut }\end{array}$ \\
\hline \multirow{2}{*}{$\mathrm{SR}$ and $\mathrm{KO}$} & $\mathrm{SR}$ & $\mathrm{KO}$ & $\begin{array}{l}\text { Pillow case } \\
\text { Pancake }\end{array}$ \\
\hline & $\mathrm{KO}$ & SR & $\begin{array}{l}\text { Ball } \\
\text { Bar }\end{array}$ \\
\hline \multirow[t]{2}{*}{ CS and SS } & $\mathrm{CS}$ & SS & $\begin{array}{l}\text { Pillow case } \\
\text { Pancake } \\
\text { Chestnut } \\
\text { Apple tree }\end{array}$ \\
\hline & SS & $\mathrm{CS}$ & $\begin{array}{l}\text { Ball } \\
\text { Bar }\end{array}$ \\
\hline $\mathrm{SP}$ and HT & SP & HT & $\begin{array}{l}\text { Pillow case } \\
\text { Pancake } \\
\text { Ball } \\
\text { Bar } \\
\text { Chestnut } \\
\text { Apple tree }\end{array}$ \\
\hline FB and JP & FB & $\mathrm{JP}$ & Apple tree \\
\hline $\mathrm{KO}$ and $\mathrm{FB}$ & $\mathrm{KO}$ & FB & Chestnut \\
\hline
\end{tabular}


Appendix C

A description of each stimulus film used to elicit DGS narratives is given, together with still images from the films themselves. The numbers in the descriptions and under the images correspond to those given in the tables that list the possible spatial relationships encoded in signed descriptions of the stimulus films, corresponding to expression in relative and intrinsic frame of reference terms.

\section{(1) The pillow case event:}

The stimulus film begins with a pillow case lying on the ground and the mouse (1). The mouse walks toward the pillow case (2). Having reached it, the mouse crawls inside it (3), but then crawls out of it again (4) after finding that it can't get out the other side. The mouse then turns around (5) and crawls into the pillow case again, this time backward (6). It stands up, clutching the pillow case to its waist, and falls backward, landing on its bottom (7). The mouse gets up and starts jumping in place, and then jumps forward, moving laterally off the screen (8).

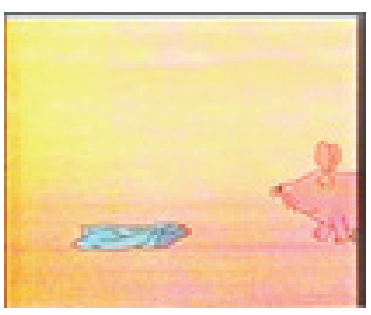

1

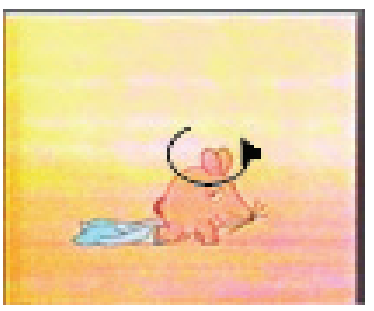

5

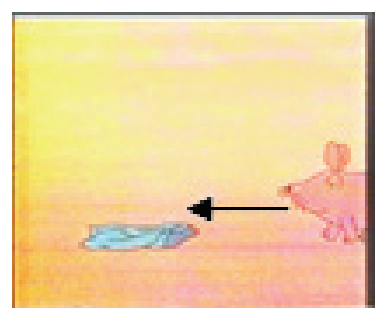

2

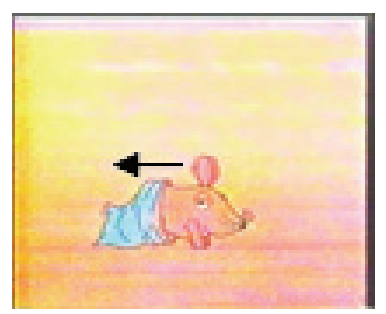

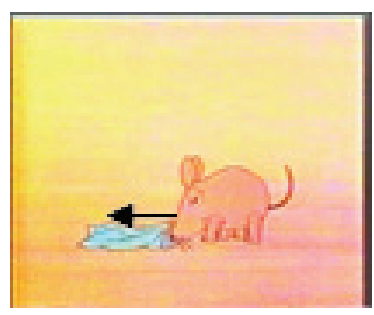

3

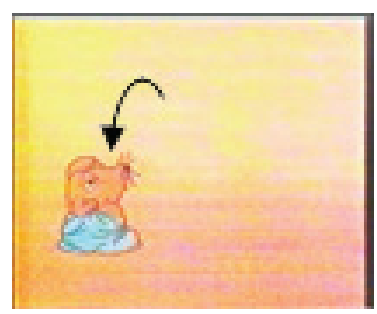

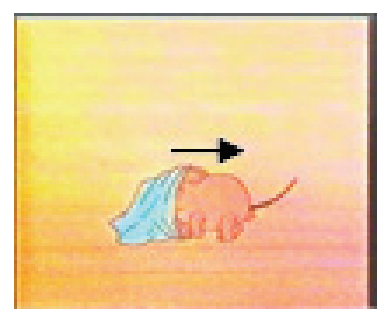

4

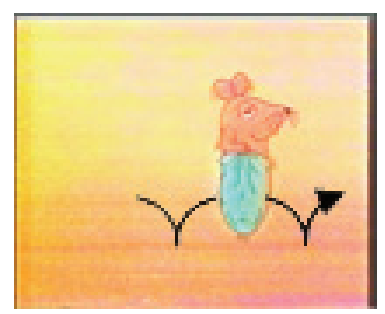

\begin{tabular}{|l|l|l|l|}
\hline$\#$ & Type & RELATIVE frame of reference coding & INTRINSIC frame of reference coding \\
\hline 1 & Loc & $\begin{array}{l}\text { Mouse is on } \text { right of screen. } \\
\text { Pillow case is on left of screen }\end{array}$ & Pillow case is in front of mouse \\
\hline 2 & Mot & Mouse moves left toward pillow case & Mouse moves forward, toward pillow case \\
\hline 3 & Mot & Mouse moves left into pillow case, facing left & Mouse moves forward toward pillow case \\
\hline 4 & Mot & Mouse moves right out of pillow case, facing left & Mouse moves backward, out of pillow case \\
\hline 5 & Mot & $\begin{array}{l}\text { Mouse } \text { turns } \text { around so that it is facing right (of } \\
\text { screen) }\end{array}$ & $\begin{array}{l}\text { Mouse } \text { turns to } \text { its left so that it is } \text { facing } \\
\text { away from pillow case }\end{array}$ \\
\hline 6 & Mot & Mouse moves left into pillow case, facing right & Mouse moves backward into pillow case \\
\hline 7 & Mot & Mouse falls to left & Mouse falls backward \\
\hline 8 & Mot & Mouse jumps across screen, moves right & Mouse jumps forward \\
\hline
\end{tabular}




\section{(2) The pancake event:}

In the pancake stimulus film, the mouse is standing at a kitchen stove with its back to the viewer, its arms and shoulders moving (1). After some time, the mouse turns away from the stove to the left (2) and the viewer sees that it is holding a pan with a pancake in it. The mouse flips the pancake, but instead of landing in the pan, the pancake arcs through the air and lands on the floor (3). The mouse moves forward and bends down to pick the pancake up off the floor (4) and turns back to the stove (5) to prepare a new pancake. This sequence repeats two more times (see the additional numbers under the still images and the numbers in the table). The fourth time the mouse turns away from the stove to try to flip the pancake (14), it runs forward (15) to catch the pancake in the location in which it has been landing. This time the pancake lands on the mouse's forehead. Needing a new strategy, the mouse calls the elephant for assistance, who enters the screen from the left (16). The mouse motions for the elephant to stand at a distance, and they are standing across from each other, facing each other (17). The mouse then moves toward the elephant to hand it the other pan hanging on the wall (18). The mouse flips the pancake, and it arcs through the air and lands in the elephant's pan (19). The elephant flips the pancake, and it arcs back through the air into the mouse's pan (20). The pancake moves back and forth between the mouse and the elephant three times, until the screen fades (21 (in the table only)).

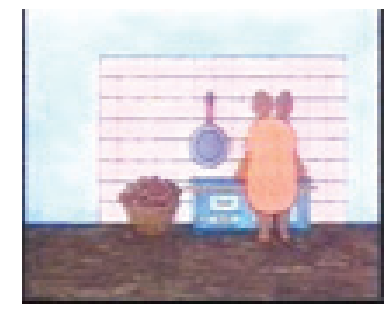

1

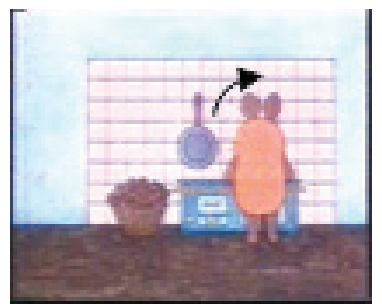

5 (also 9, 13)

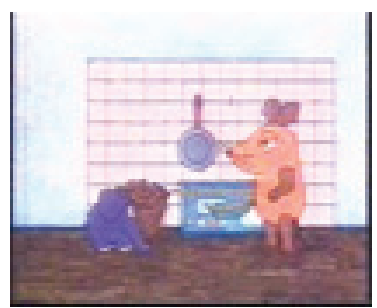

17

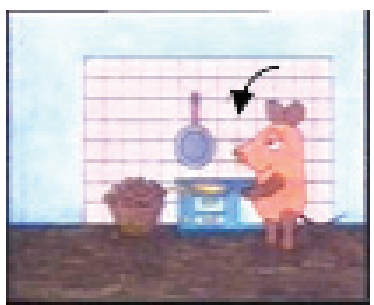

2 (also 6, 10)

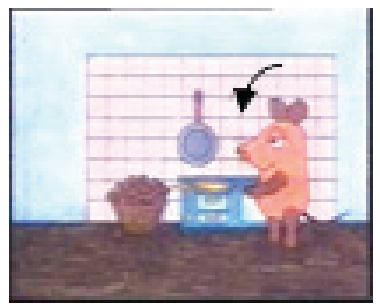

14

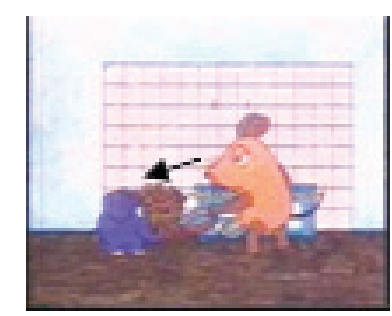

18

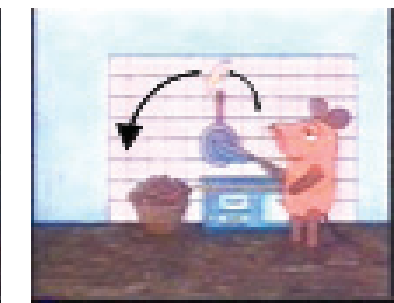

3 (also 7, 11)

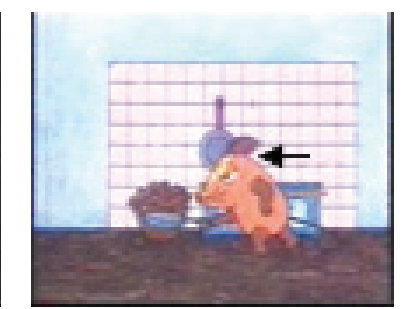

15

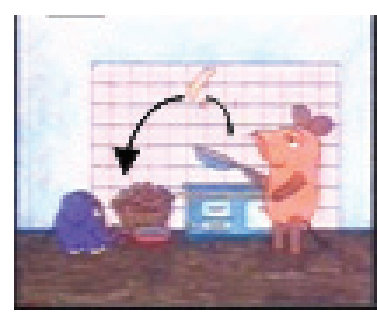

19

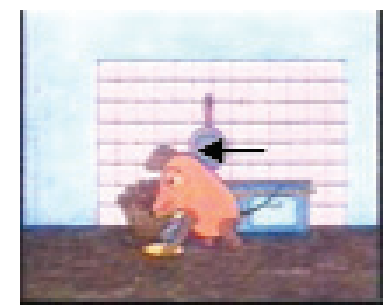

4 (also 8, 12)

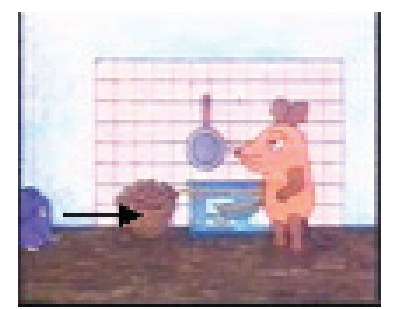

16

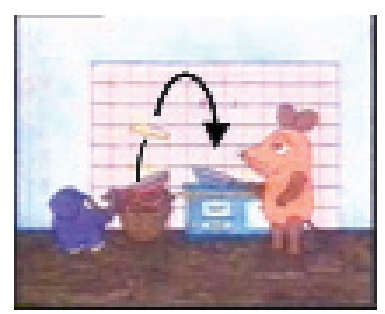

20 


\begin{tabular}{|c|c|c|c|}
\hline$\#$ & Type & RELATIVE frame of reference coding & INTRINSIC frame of reference coding \\
\hline 1 & Loc & Mouse is between stove and viewer & Mouse is facing the stove \\
\hline 2 & Mot & Mouse turns left, away from stove & Mouse turns to its left, away from stove \\
\hline 3 & Mot & Pancake arcs left (onto floor) & Pancake moves away from mouse \\
\hline 4 & Mot & Mouse moves left (picks up pancake) & Mouse moves forward (picks up pancake) \\
\hline 5 & Mot & Mouse turns right, to stove & Mouse turns to its right, to stove \\
\hline 6 & Mot & Mouse turns left, away from stove & Mouse turns to its left, away from stove \\
\hline 7 & Mot & Pancake arcs left (onto floor) & Pancake moves away from mouse \\
\hline 8 & Mot & Mouse moves left (picks up pancake) & Mouse moves forward (picks up pancake) \\
\hline 9 & Mot & Mouse turns right, to stove & Mouse turns to its right, to stove \\
\hline 10 & Mot & Mouse turns left, away from stove & Mouse turns to its left, away from stove \\
\hline 11 & Mot & Pancake arcs left (onto floor) & Pancake moves away from mouse \\
\hline 12 & Mot & Mouse moves left (picks up pancake) & Mouse moves forward (picks up pancake) \\
\hline 13 & Mot & Mouse turns right, to stove & Mouse turns to its right, to stove \\
\hline 14 & Mot & Mouse turns left, away from stove & Mouse turns to its left, away from stove \\
\hline 15 & Mot & Mouse moves left (to catch pancake) & Mouse moves forward (to catch pancake) \\
\hline 16 & Mot & Elephant enters left, moves right & Elephant moves toward mouse \\
\hline 17 & Loc & Elephant stands left, mouse stands right & $\begin{array}{l}\text { Elephant and mouse stand opposite each } \\
\text { other, facing each other }\end{array}$ \\
\hline 18 & Mot & Mouse moves left (gives pan to elephant) & $\begin{array}{l}\text { Mouse moves forward (gives pan to } \\
\text { elephant) }\end{array}$ \\
\hline 19 & Mot & Pancake arcs left (from mouse to elephant) & $\begin{array}{l}\text { Pancake moves away from mouse, toward } \\
\text { elephant }\end{array}$ \\
\hline 20 & Mot & Pancake arcs right (from elephant to mouse) & $\begin{array}{l}\text { Pancake moves away from elephant, toward } \\
\text { mouse }\end{array}$ \\
\hline 21 & Mot & ( 3 times back and forth in total) & (3 times back and forth in total) \\
\hline
\end{tabular}

\section{(3) The ball event:}

At the beginning of the ball event, the viewer sees the elephant lying on the ground on its stomach, facing away from the viewer. The mouse is on the right side of the screen, facing the elephant (1). The mouse moves toward the elephant, dribbling a ball (2), and gets the elephant's attention. The elephant turns to the right, toward the mouse (3), and then gets up and stands across from the mouse, facing the mouse (4), in order to play ball. The mouse starts the game by throwing the ball to the elephant (5), and the elephant uses its trunk to hit the ball back to the mouse (6). The ball is passed back and forth between the mouse and the elephant in this manner five times (7). The mouse then kicks the ball to the elephant (8). The elephant hits the ball back with its trunk (9), kicking its legs out as it does so. The ball passes back and forth between them in this way two times (10). The mouse then uses its nose to hit the ball (11), and the elephant, in turn, stretches its trunk high into the air to hit the ball (12). The ball passes back and forth between them in this way two times (13), when suddenly, the elephant swallows the ball instead of hitting it back. Worried, the mouse walks over to the elephant (14). The mouse tickles the elephant's trunk, causing the elephant to sneeze. The ball flies out of the elephant's trunk and hits the mouse in the stomach (15). From the impact of the ball, the mouse flies backward through the air (16). 


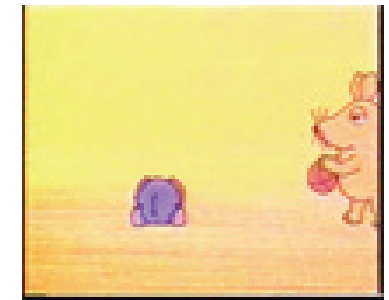

1

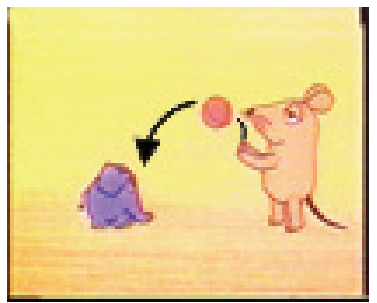

5

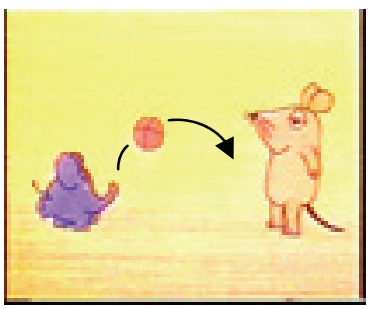

9

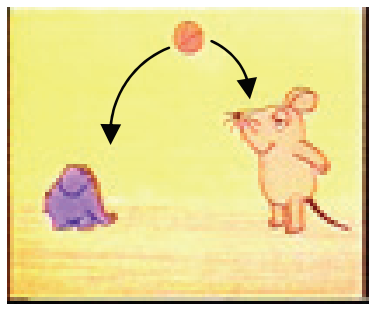

13

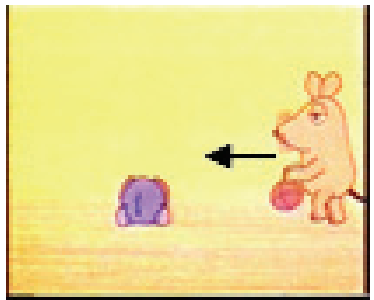

2

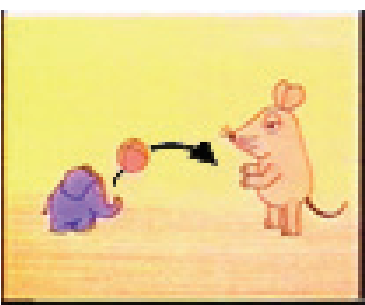

6

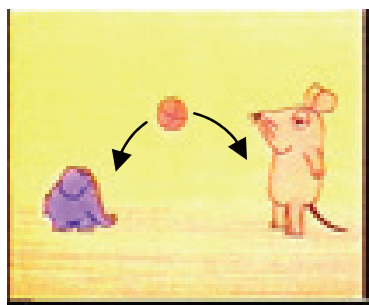

10

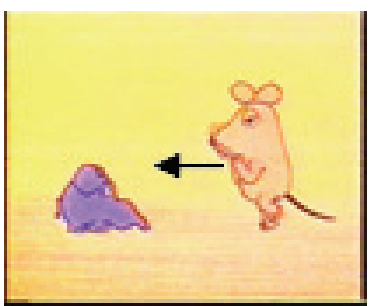

14

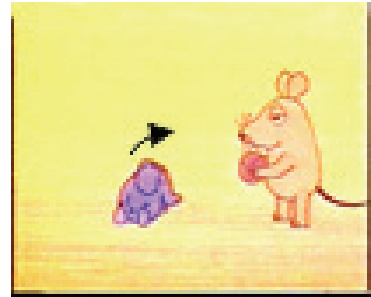

3

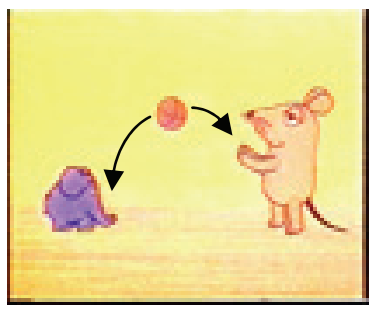

7

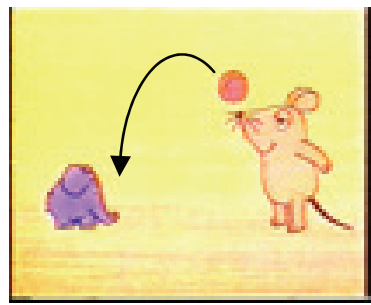

11

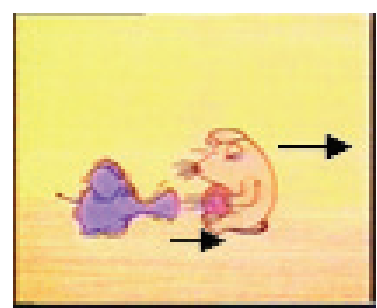

$15 \& 16$

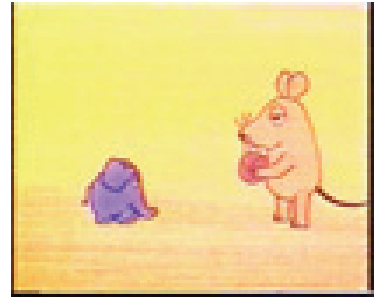

4

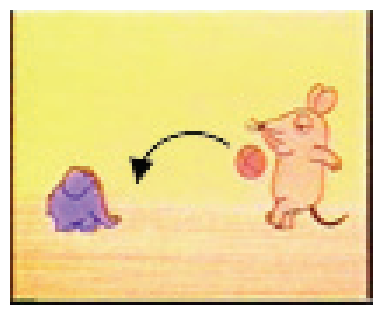

8

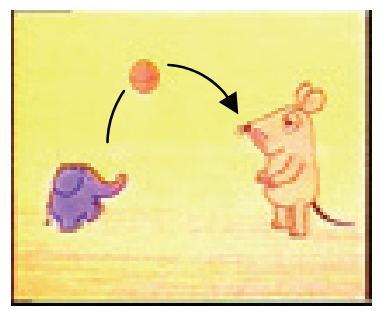

12

\begin{tabular}{|c|c|c|c|}
\hline \# & Type & RELATIVE frame of reference coding & INTRINSIC frame of reference coding \\
\hline 1 & Loc & $\begin{array}{l}\text { Elephant is on left of screen. } \\
\text { Mouse is on right of screen }\end{array}$ & $\begin{array}{l}\text { Elephant is in front of mouse. } \\
\text { Mouse is on elephant's right }\end{array}$ \\
\hline 2 & Mot & Mouse moves left toward elephant & Mouse moves forward, toward elephant \\
\hline 3 & Mot & Elephant turns right to mouse & Elephant turns right to mouse \\
\hline 4 & Loc & Elephant stands left, mouse stands right & Elephant and mouse stand facing each other \\
\hline 5 & Mot & Ball arcs left, from mouse to elephant & $\begin{array}{l}\text { Ball moves away from mouse, toward elephant } \\
\text { (mouse throws ball) }\end{array}$ \\
\hline 6 & Mot & Ball arcs right, from elephant to mouse & $\begin{array}{l}\text { Ball moves away from elephant, toward mouse } \\
\text { (elephant hits ball with trunk) }\end{array}$ \\
\hline 7 & Mot & (5 times back and forth in total) & ( 5 times back and forth in total) \\
\hline 8 & Mot & Ball arcs left, from mouse to elephant & $\begin{array}{l}\text { Ball moves away from mouse, toward elephant } \\
\text { (mouse kicks ball) }\end{array}$ \\
\hline 9 & Mot & Ball arcs right, from elephant to mouse & $\begin{array}{l}\text { Ball moves away from elephant, toward mouse } \\
\text { (elephant hits ball with trunk) }\end{array}$ \\
\hline 10 & Mot & (2 times back and forth in total) & (2 times back and forth in total) \\
\hline 11 & Mot & Ball arcs left, from mouse to elephant & $\begin{array}{l}\text { Ball moves away from mouse, toward elephant } \\
\text { (mouse hits ball with nose) }\end{array}$ \\
\hline 12 & Mot & Ball arcs right, from elephant to mouse & $\begin{array}{l}\text { Ball moves away from elephant, toward mouse } \\
\text { (elephant hits ball with trunk) }\end{array}$ \\
\hline
\end{tabular}




\begin{tabular}{|l|l|l|l|}
\hline 13 & Mot & (2 times back and forth in total) & (2 times back and forth in total) \\
\hline 14 & Mot & Mouse moves left, toward elephant & Mouse moves forward, toward elephant \\
\hline 15 & Mot & Ball moves right, hits mouse & Ball moves away from elephant, hits mouse \\
\hline 16 & Mot & Mouse moves right, from impact & Mouse moves backward, from impact \\
\hline
\end{tabular}

\section{(4) The bar event:}

At the beginning of this stimulus film, the mouse is standing in front of a gymnast's bar (1). The mouse moves toward the bar, until it is standing under it (2), jumps up to grab hold of the bar, and starts swinging back and forth on it (3). At this point, the elephant enters the screen from the right (4) and watches the mouse as it starts to swing around the bar (5) and then as it dismounts from the bar (6). The mouse turns around to look at the elephant and moves away from the bar (7), such that the mouse and elephant are on either side of the bar (8). The elephant also wants to swing on the bar and moves toward it (9). The elephant reaches the bar with its trunk, but the bar bends under the elephant's weight. The elephant lets go of the bar, swinging backward onto the ground (10). Furious, the mouse moves toward the bar to try to straighten it (11), but is unable to do so and moves away from the bar again (12). Suddenly, a man dressed in a green suit and top hat enters the screen from the left and walks under the bar, straightening the bar as he does so (13). Amazed, the mouse and elephant look at each other (14) and then at the viewer, standing on either side of the bar (15). (Scene 15 was excluded from the final analysis.)

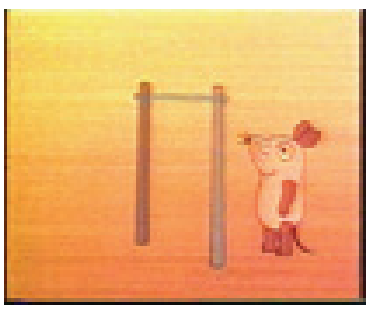

1

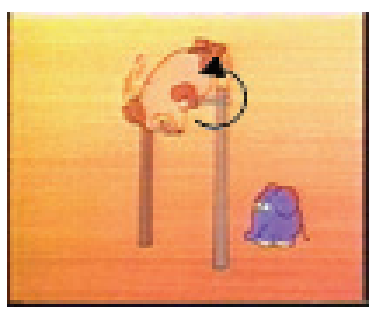

5

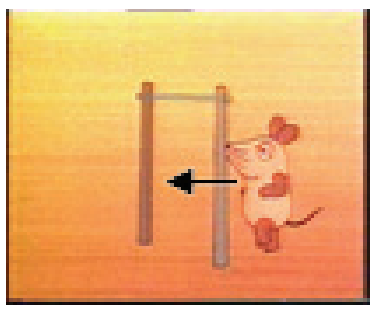

2

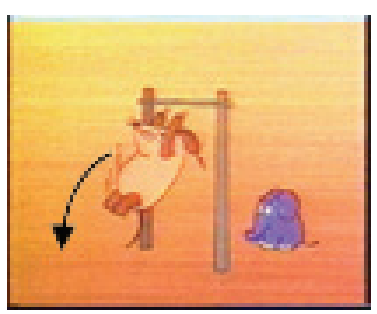

6

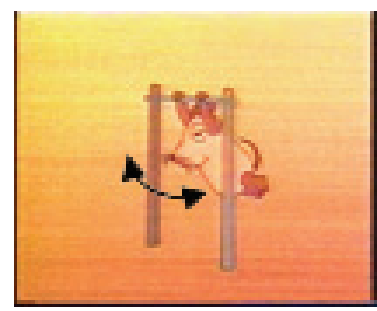

3

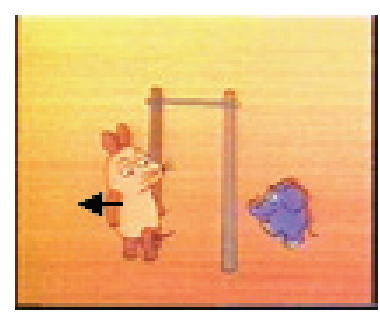

7

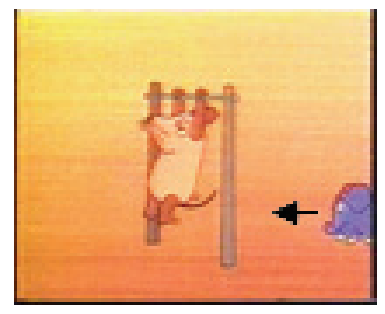

4

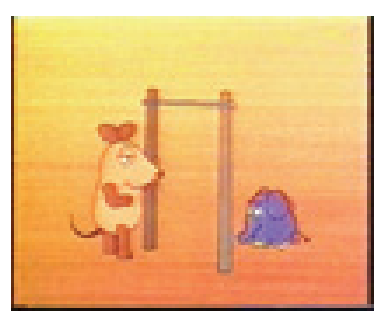

8 


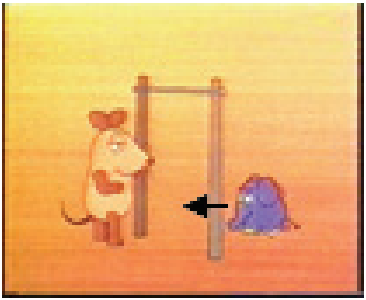

9

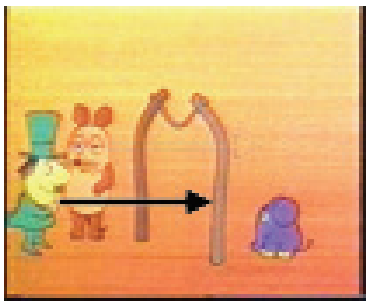

13

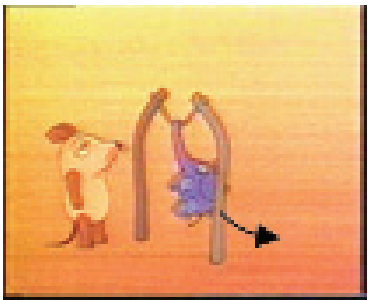

10

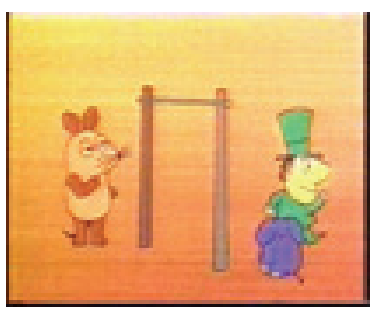

14

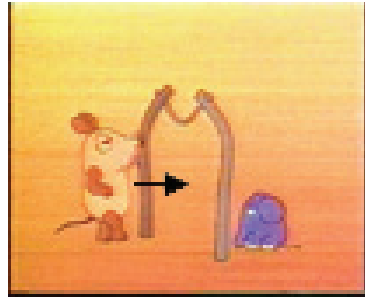

11

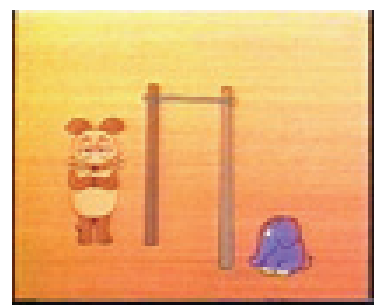

15

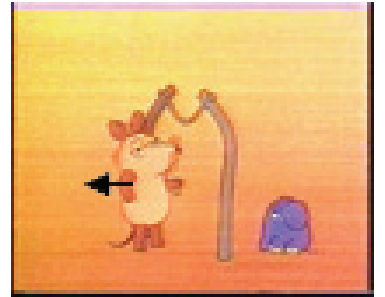

12

\begin{tabular}{|c|c|c|c|}
\hline \# & Type & RELATIVE frame of reference coding & INTRINSIC frame of reference coding \\
\hline 1 & Loc & $\begin{array}{l}\text { Bar is in middle of screen. } \\
\text { Mouse is on right of screen. }\end{array}$ & $\begin{array}{l}\text { Bar is in front of mouse. } \\
\text { Mouse is facing bar. }\end{array}$ \\
\hline 2 & Mot & Mouse moves left, to bar. & Mouse moves forward, to bar. \\
\hline 3 & Mot & $\begin{array}{l}\text { Mouse swings left and right, hanging from } \\
\text { bar. }\end{array}$ & $\begin{array}{l}\text { Mouse swings forward and backward, hanging } \\
\text { from bar. }\end{array}$ \\
\hline 4 & Mot & Elephant enters right, moves left, to bar. & Elephant moves forward, to bar. \\
\hline 5 & Mot & $\begin{array}{l}\text { Mouse swings around bar, lateral } \\
\text { movement (left-right) }\end{array}$ & $\begin{array}{l}\text { Mouse swings around bar, sagittal movement } \\
\text { (forward) }\end{array}$ \\
\hline 6 & Mot & Mouse dismounts to left of bar. & Mouse dismounts in front of bar. \\
\hline 7 & Mot & Mouse moves left & Mouse moves backward \\
\hline 8 & Loc & $\begin{array}{l}\text { Mouse is on left of bar. } \\
\text { Elephant is on right of bar. }\end{array}$ & $\begin{array}{l}\text { Mouse and elephant facing each other, with bar } \\
\text { between them. }\end{array}$ \\
\hline 9 & Mot & Elephant moves left, to bar. & Elephant moves forward, to bar. \\
\hline 10 & Mot & Elephant moves right, away from bar. & Elephant moves backward, away from bar. \\
\hline 11 & Mot & Mouse moves right, to bar. & Mouse moves forward, to bar. \\
\hline 12 & Mot & Mouse moves left, away from bar. & Mouse moves backward, away from bar. \\
\hline 13 & Mot & $\begin{array}{l}\text { Man enters left, moves right across screen } \\
\text { (under bar). }\end{array}$ & Man moves forward, to and under bar. \\
\hline 14 & Loc & $\begin{array}{l}\text { Mouse facing right (of screen) to elephant, } \\
\text { then facing viewer. } \\
\text { Elephant facing left (of screen). }\end{array}$ & $\begin{array}{l}\text { Mouse looks to its left to the elephant, then faces } \\
\text { forward. } \\
\text { Elephant facing forward. }\end{array}$ \\
\hline 15 & Loc & $\begin{array}{l}\text { Mouse is left of bar, elephant is right of } \\
\text { bar. }\end{array}$ & Bar is between mouse and elephant. \\
\hline
\end{tabular}

\section{(5) The chestnut event:}

This stimulus film starts with the mouse asleep against a tree (1). The mouse is awoken by a chestnut falling next to it onto the ground (2). The mouse looks at the chestnut (3), gets up to walk around and inspect it (4), and then moves toward it to touch it (5). The chestnut's thorns prick the mouse's paws and it jumps back, away from the chestnut, in pain (6). The mouse then looks off the screen to the left (7) and exits the screen in that direction (8). When it returns, it is carrying a plank of wood and a stone (9), which it uses to make a seesaw, placing 
the chestnut on the far side of the plank (10). The mouse jumps onto the elevated side of the plank (11), sending the chestnut flying upward. The mouse moves the plank out of the way (12), and when the chestnut lands on the ground, it breaks open, so that the mouse can move toward it and take the nut inside (13). The mouse eats the nut and looks at the viewer, pleased with itself (14). (Scenes 11 and 14 were excluded from the final analysis.)

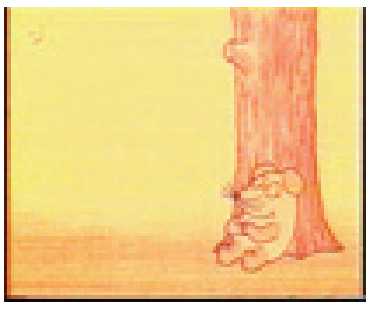

1

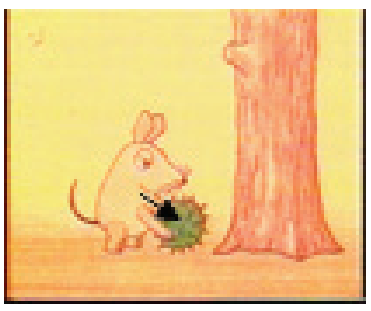

5

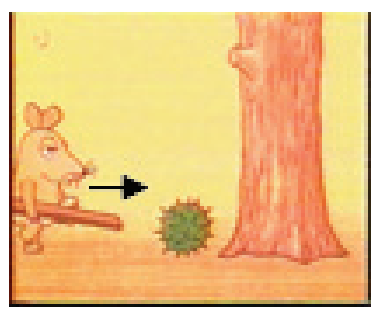

9

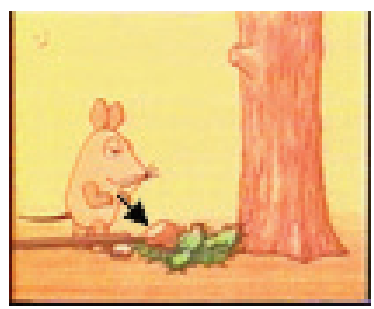

13

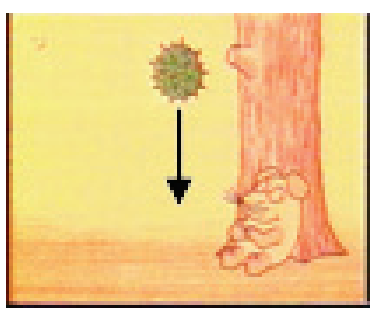

2

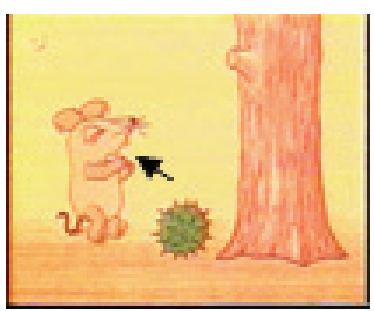

6

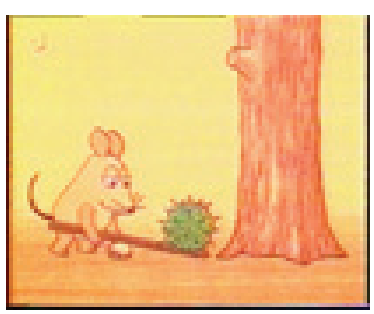

10

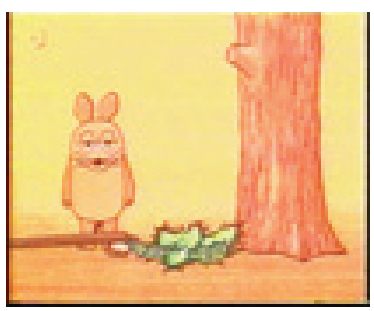

14

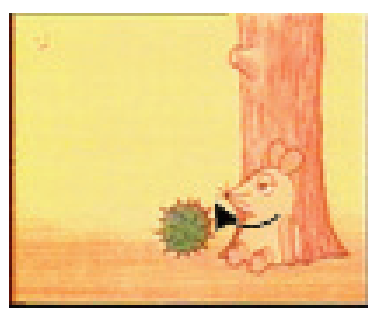

3

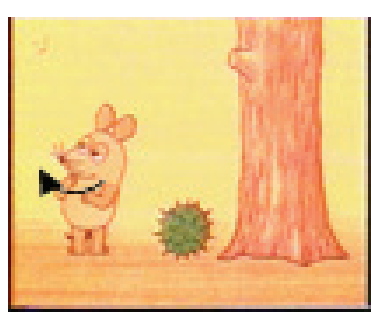

7

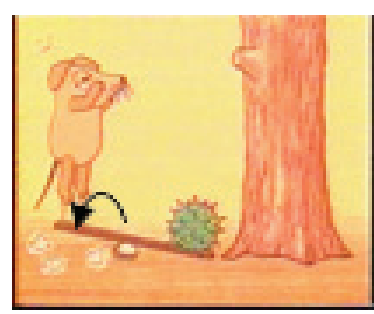

11

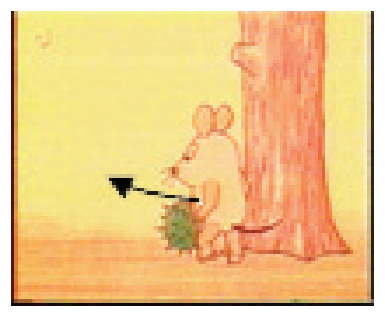

4

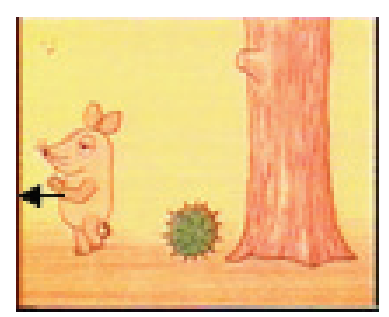

8

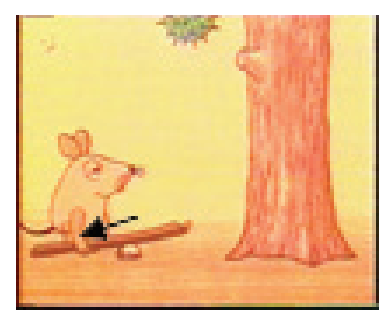

12

\begin{tabular}{|c|c|c|c|}
\hline \# & Type & RELATIVE frame of reference coding & INTRINSIC frame of reference coding \\
\hline 1 & Loc & Mouse and tree on right side of screen. & Mouse is leaning back against tree. \\
\hline 2 & Loc & Chestnut falls left of tree. & Chestnut falls to mouse's right. \\
\hline 3 & Mot & Mouse looks left (on screen) at chestnut. & Mouse looks to its right at chestnut. \\
\hline 4 & Mot & Mouse moves left in front of chestnut. & Mouse walks sideways to its left, facing chestnut. \\
\hline 5 & Mot & Mouse reaches right. & Mouse reaches forward. \\
\hline 6 & Mot & Mouse moves left. & Mouse moves backward. \\
\hline 7 & Mot & Mouse looks left. & Mouse looks to its right. \\
\hline 8 & Mot & Mouse exits left, moves left. & Mouse walks forward, away from chestnut. \\
\hline 9 & Mot & Mouse enters left, moves right. & Mouse walks forward, toward chestnut. \\
\hline 10 & Loc & Plank lies left-right across screen; chestnut & Plank extends away from mouse; chestnut is on \\
\hline
\end{tabular}




\begin{tabular}{|l|l|l|l|}
\hline & & is on right side. [moved right] & far side. [moved forward] \\
\hline 11 & Loc & $\begin{array}{l}\text { Mouse facing right (of screen), jumps } \\
\text { toward viewer onto plank. }\end{array}$ & $\begin{array}{l}\text { Mouse facing chestnut/tree; jumps to its right onto } \\
\text { plank. }\end{array}$ \\
\hline 12 & Mot & Plank is moved left. & Plank is moved backward. \\
\hline 13 & Mot & Mouse reaches right (to pick up chestnut). & Mouse reaches forward (to pick up chestnut). \\
\hline 14 & Mot & Mouse turns to face viewer (eats chestnut). & Mouse turns to its right (eats chestnut). \\
\hline
\end{tabular}

\section{(6) The apple tree event:}

The viewer sees the tree in the middle of the screen, with a fence in front of it, and the mouse on the left (1). The mouse walks along the fence (2), and stops in front of the tree, looking up at it and turning toward it (3). The mouse tries to reach the apples in the tree in various ways, first by trying to squeeze through the fence (4). Then it tries to climb the fence and stretch toward the apples (5). Finally, the mouse pulls at the fence (6), and when a piece breaks off, the mouse turns it to the right so that it is vertical (7), and leans it against the tree to use as a ladder ( 8 and 9). The mouse climbs the ladder, picks and apple, and happily eats it. (Scenes 4-9 were excluded from the final analysis.)

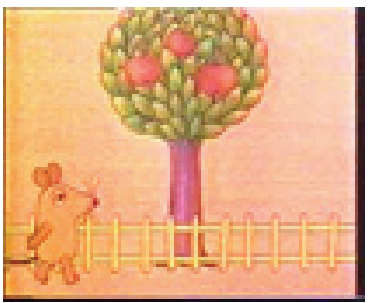

1

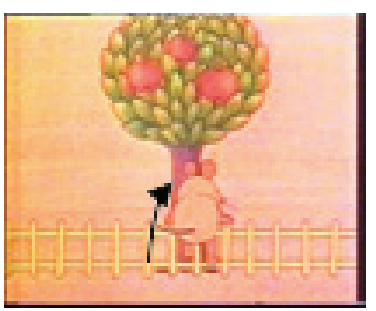

5

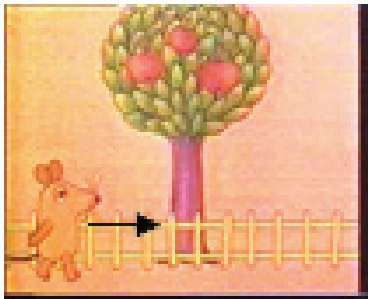

2

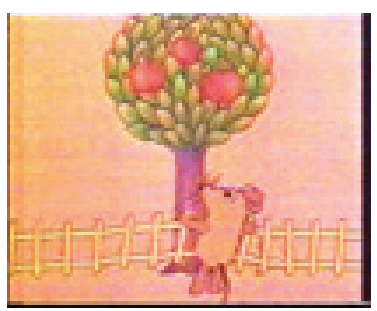

6

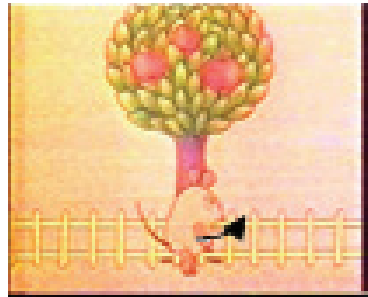

3

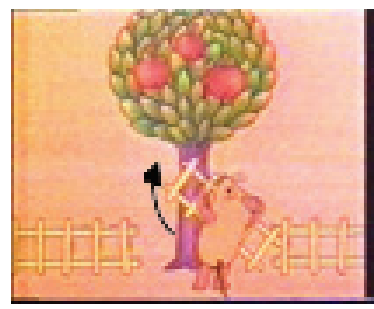

7

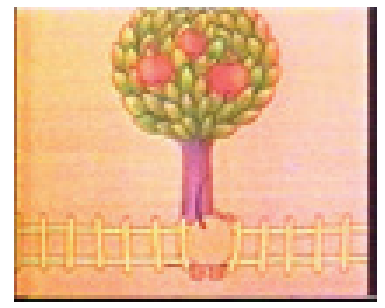

4

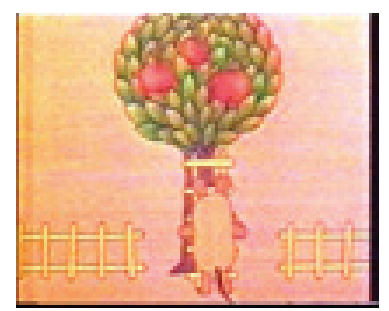

$8 \& 9$

\begin{tabular}{|l|l|l|l|}
\hline$\#$ & Type & RELATIVE frame of reference coding & INTRINSIC frame of reference coding \\
\hline 1 & Loc & Tree behind fence; mouse in front of fence. & Fence and tree (behind fence) on mouse's left. \\
\hline 2 & Mot & Mouse moves right, along fence & Mouse moves forward along fence. \\
\hline 3 & Mot & Mouse turns away from viewer. & Mouse turns left. \\
\hline 4 & Mot & Mouse pushes through fence. & Mouse pushes through fence. \\
\hline 5 & Mot & Mouse climbs fence. & Mouse climbs fence. \\
\hline 6 & Mot & Mouse pulls at fence (breaks off piece). & Mouse pulls at fence (breaks off piece). \\
\hline 7 & Mot & Mouse turns "ladder" rightward. & Mouse turns "ladder" rightward. \\
\hline 8 & Mot & Mouse leans ladder against tree. & Mouse leans ladder against tree. \\
\hline 9 & Loc & Ladder behind mouse; tree behind ladder. & Ladder and tree in front of mouse. \\
\hline
\end{tabular}




\section{References}

Aarons, D. (1996). Topics and topicalization in ASL. Stellenbosch Papers in Linguistics 30: 65-106.

Aarons, D. and R. Morgan (2003). Classifier predicates and the creation of multiple perspectives in South African Sign Language. Sign Language Studies 3:2, 125-156.

Aikhenvald, A.Y. (2003). Classifiers in Spoken and Signed Languages: How to Know More. In: Emmorey, K. (Ed.) Perspectives on Classifier Constructions in Sign Languages. Mahwah, NJ: Lawrence Erlbaum Associates, pp. 87-90.

Allen, S., Özyürek, A., Kita, S., Brown, A., Furman, R., Ishizuka, T., and M. Fujii (2006). Language-specific and universal influences in children's syntactic packaging of Manner and Path: A comparison of English, Japanese, and Turkish. Cognition 102: 16-48.

Arik, E. (2006). Locative Constructions in Turkish Sign Language. Poster presented at TISLR 9 (Theoretical Issues in Sign Language Research), Florianópolis, Brazil, December 2006.

Arik, E. and M. Nadolske (2006). Conceptualization of Space in Turkish Sign Language (TID). Paper presented at CSDL 2006 (Conceptual Structure, Discourse \& Language), , San Diego, California, November 2006.

Aronoff, M., Meir, I., Padden, C.A., and W. Sandler (2003). Classifier Constructions and Morphology in Two Sign Languages. In: Emmorey, K. (Ed.), Perspectives on Classifier Constructions in Sign Languages. Mahwah, NJ: Lawrence Erlbaum Associates, pp. 53-84.

Aronoff, M., Meir, I., and W. Sandler (2005). The paradox of sign language morphology. Language 81(2): 301-344.

Atlas, J.D. and S.C. Levinson (1981). IT-clefts, informativeness, and logical form. In: Cole, P. (Ed.), Radical Pragmatics. Academic Press, New York, pp. 1-62.

Battison, R. (1978). Lexical Borrowing in American Sign Language. Silver Spring, MD: Linstok Press.

Benedicto, E. and D. Brentari (2004). Where did all the arguments go?: Argument-changing properties of classifiers in ASL. Natural Language and Linguistic Theory 22(4): 743-810.

Berlin, B. (1992). Ethnobiological Classification: Principles of Categorization of Plants and Animals in Traditional Societies. Princeton: Princeton University Press.

Berlin B. (1994). Evidence for pervasive synesthetic sound symbolism in ethnozoological nomenclature. In: Hinton, L., Nichols, J., and J.J. Ohala (Eds.), Sound Symbolism. Cambridge: Cambridge University Press, pp. 76-103.

Berman, R.A. and D.I. Slobin (Eds.) (1994). Relating events in narrative: A cross-linguistic developmental study. Hillsdale, NJ: Lawrence Erlbaum Associates. 
Biermann, A. (1982). Die grammatische Kategorie Numerus. In: Seiler, H.-J. and C. Lehmann (Eds.), Apprehension. Die sprachliche Erfassung von Gegenständen. Tübingen: Gunter Narr, pp. 229-243.

Bloom, P., Peterson, M.A., Nadel, L., and M. Garrett (Eds.) (1996). Language and Space. Cambridge, MA: MIT Press.

Bohnemeyer, J. and P. Brown (in press). Standing divided: Dispositional verbs and locative predications in two Mayan Languages. To appear in special issue of Linguistics, edited by F. Ameka and S.C. Levinson.

Bowerman, M. (1996). The origins of children's spatial semantic categories: Cognitive vs. linguistic determinants. In: Gumperz, J.J. and S.C. Levinson (Eds.), Rethinking Linguistic Relativity. Cambridge: Cambridge University Press, pp. 145-176.

Boyes-Braem, P. (1985). Studying sign language dialects. In: Stokoe, W. and V. Volterra (Eds.), SLR 1983: Proceedings of the III International Symposium on Sign Language Research, Rome, 22-26 June 1983. Silver Spring, MD: Linstok Press, pp. 247-253.

Boyes-Braem, P. (1995). Einführung in die Gebärdensprache und ihre Erforschung. (Internationale Arbeiten zur Gebärdensprache und Kommunikation Gehörloser, Band 11) Hamburg: Signum Verlag.

Boyes-Braem, P. and R. Sutton-Spence (Eds.) (2001). The Hands are the Head of the Mouth: The Mouth as Articulator in Sign Languages. (International Studies on Sign Language and Communication of the Deaf 39). Hamburg: Signum Press.

Brennan, M. (1990). Word Formation in British Sign Language. Stockholm: University of Stockholm.

Brennan, M. (1992). The Visual World of British Sign Language: An Introduction. In: Brien, D. (Ed.), Dictionary of British Sign Language/English. London: Faber and Faber.

Brentari, D. (1995). Sign language phonology. In: Goldsmith, J. (Ed.), The handbook of phonological theory. Cambridge, MA: Blackwell, pp. 615-639.

Brentari, D. (1998). A prosodic model of sign language phonology. Cambridge, MA: MIT Press.

Brown, P. (2006). A sketch of the grammar of space in Tzeltal. In: Levinson, S.C. and D.P. Wilkins (Eds.) (2006), Grammars of Space. Cambridge: Cambridge University Press, pp. $230-272$.

Brown, P. and S.C. Levinson (1993). Linguistic and non-linguistic coding of spatial arrays: Explorations in Mayan cognition. Working Paper No. 24 of the Cognitive Anthropology Research Group. Max Planck Institute for Psycholinguistics, Nijmegen.

Burgess, N., Jeffrey, K.J., and J. O'Keefe (1999). Integrating hippocampal and parietal functions. In: Burgess, N., Jeffrey, K.J., and J. O'Keefe (Eds.), The hippocampal and parietal foundations of spatial cognition. Oxford: Oxford University Press, pp. 3-32. 
Bybee, J. (1985). Diagrammatic iconicity in stem-inflection relations. In: Haiman, J. (Ed.), Iconicity in syntax. Amsterdam: John Benjamins, pp. 11-48.

Byun, K.-S. (2004). Gender marking in Korean Sign Language. Talk given at the MPI for Psycholinguistics, Nijmegen.

Clark, H.H. (1973). Space, time, semantics, and the child. In: Moore, T.E. (Ed.), Cognitive development and the acquisition of language. New York: Academic Press, pp. 27-63.

Clark, H.H. and R.J. Gerrig (1990). Quotations as demonstrations. Language 66: 764-805.

Cogill-Koez, D. (2000). Signed Language Classifier Predicates: Linguistic structures or schematic visual representation? Sign Language and Linguistics 3(2): 153-207.

Comrie, B. (1981). Language universals and linguistic typology: Syntax and morphology.

Chicago: University of Chicago Press.

Cormier, K. (2007). Do all pronouns point? Indexicality of first person plural pronouns in BSL and ASL. In: Perniss, P.M., Pfau, R., and M. Steinbach (Eds.), Visible variation: Crosslinguistic studies $n$ sign language structure. Berlin: Mouton, pp.63-101.

Coulson, S. (2001). Semantic Leaps: Frame-shifting and Conceptual Blending in Meaning Construction. Cambridge: Cambridge University Press.

Coulson, S. and G. Fauconnier (1999). Fake Guns and Stone Lions: Conceptual Blending and Privative Adjectives. In: Fox, B., Jurafsky, D., and L. Michaelis (Eds.), Cognition and Function in Language. Palo Alto, CA: CSLI, pp. 143-158.

Croft, W. (1990). Typology and universals. (Cambridge Textbooks in Linguistics) Cambridge: Cambridge University Press.

Croft, W. (2003). Typology. In: Nadel, L. (Ed.), Encyclopedia of Cognitive Science. London: Nature Publishing Group.

Cuxac, C. (1996). Fonctions et structures de l'iconicité des langues des signes. Doctoral dissertation, Université René Descartes, Paris V.

Cuxac, C. (1999). French sign language: proposition of a structural explanation by iconicity. In: Braffort, A., Gherbi, R., Gibet, S., Richardson, J., and D. Teil (Eds.), Lecture Notes in Artificial Intelligence. Proceedings of the 3rd Gesture Workshop '99 on Gesture and Sign Language in Human-Computer Interaction. Berlin: Springer, pp. 165-184.

DeMatteo, A. (1977). Visual Imagery and Visual Analogues in American Sign Language. In: Friedman, L.A. (Ed.), On the Other Hand. London: Academic Press, pp. 109-136.

Dudis, P. (2002). Grounded blend maintenance as a discourse strategy. In: Lucas, C. (Ed.), Turn-taking, Fingerspelling, and Contact in Signed Languages (Sociolinguistics in Deaf Communities 8). Washington, D.C.: Gallaudet University Press, pp. 53-72.

Dudis, P. (2004a). Body partitioning and real-space blends. Cognitive Linguistics 15-2: 223238. 
Dudis, P. (2004b). Depiction of events in ASL: Conceptual integration of temporal components. Doctoral dissertation, University of California, Berkeley.

Dudis, P. (2006). ASL Constructions and Blending. Paper presented at TISLR 9 (Theoretical Issues in Sign Language Research), Florianópolis, Brazil, December 2006.

Ebbinghaus, H. (1998). Warum deutsche Wörter wesentliche Bestandteile der Deutschen Gebärdensprache sind. Teil I. Das Zeichen 45(12): 443-451; Teil II. Das Zeichen 46(12): 594-611.

Ebbinghaus, H. and J. Heßmann (1990). Deutsche Wörter in der Deutschen Gebärdensprache - Theoretische Überlegungen zu einem empirischen Tatbestand. Das Zeichen 11: 60-71.

Ebbinghaus, H. and J. Heßmann (1996). Signs and Words - Accounting for Spoken Language Elements in German Sign Language. In: Edmondson, W. and R. Wilbur (Eds.), International Review of Sign Linguistics 1. Mahwah, NJ: Lawrence Erlbaum Associates, pp. 23-56.

Edmondson, W. (1990). A Non-Concatenative Account of Classifier Morphology in Signed and Spoken Languages. In: Prillwitz, S and T. Vollhaber (Eds.), Current Trends in European Sign Language Research. Hamburg: Signum Press, pp. 187-203.

Ehrich, V. and C. Koster (1983). Discourse organization and sentence form: The structure of room descriptions in Dutch. Discourse Processes 6, 169-195.

Emmorey, K. (1996). The confluence of space and language in signed language. In: Bloom, P., Peterson, M.A., Nadel, L., and M. Garrett (Eds.), Language and Space. Cambridge, MA: MIT Press, pp. 171-210.

Emmorey, K. (1998). The impact of sign language use on visual-spatial cognition. In: Marschark, M. and M.D. Clark (Eds.), Psychological perspectives on deafness: Vol. 1. Hillsdale, NJ: Lawrence Erlbaum Associates, pp. 19-52.

Emmorey, K. (2001). Space on hand. In: Gattis, M. (Ed.), Spatial schemas and abstract thought. Cambridge, MA: MIT Press, pp. 147-174.

Emmorey, K. (2002). Language, cognition and the brain. Mahwah, NJ: Lawrence Erlbaum Associates.

Emmorey, K. (Ed.) (2003). Perspectives on Classifier Constructions in Sign Languages. Mahwah, NJ: Lawrence Erlbaum Associates.

Emmorey, K., Corina, D., and U. Bellugi (1995). Differential processing of topographic and referential functions of space. In: Emmorey, K. and J. Reilly (Eds.), Language, Gesture, and Space. Hillsdale, NJ: Lawrence Erlbaum Associates, pp. 43-62.

Emmorey, K., Damasio, H., McCullough, S., Grabowski, T., Ponto, L., Hichwa, R., and U. Bellugi (2002). Neural systems underlying spatial language in American Sign Language. Neuroimage 17, 812-824. 
Emmorey, K. and B. Falgier (1999). Talking about space with space. In: Winston, E.A. (Ed.), Story Telling and Conversation: Discourse in Deaf Communities. Washington, DC: Gallaudet University Press, pp. 3-26.

Emmorey, K., Grabowski, T., McCullough, S., Ponto, L.L., Hichwa, R.D., and H. Damasio (2005). The neural correlates of spatial language in English and American Sign Language: a PET study with hearing bilinguals. Neuroimage 24(3): 832-840.

Emmorey, K. and M. Herzig (2003). Categorical Versus Gradient Properties of Classifier Constructions in ASL. In: Emmorey, K. (Ed.), Perspectives on Classifier Constructions in Sign Languages. Mahwah, NJ. Lawrence Erlbaum Associates, pp. 221-246.

Emmorey, K., Klima, E.S., and G. Hickok (1998). Mental rotation within linguistic and nonlinguistic domains in users of American Sign Language. Cognition 68: 221-246.

Emmorey, K., Kosslyn, S.M., and U. Bellugi (1993). Visual imagery and visual-spatial language: Enhanced imagery abilities in deaf and hearing ASL signers. Cognition 46: 139181.

Emmorey, K. and J. Reilly (Eds.) (1995). Language, Gesture, and Space. Hillsdale, NJ: Lawrence Erlbaum Associates.

Emmorey, K. and B. Tversky (2002). Spatial perspective choice in ASL. Sign Language and Linguistics 5(1): 3-25.

Emmorey, K., Tversky, B., and H.A. Taylor (2000). Using space to describe space: Perspective in speech, sign, and gesture. Spatial Cognition and Computation 2: 157-180.

Engberg-Pedersen, E. (1993). Space in Danish Sign Language: The semantics and morphosyntax of the use of space in a visual language. Hamburg: Signum Press.

Engberg-Pedersen, E. (1994). Some simultaneous constructions in Danish Sign Language. In: Brennan, M. and G.H. Turner (Eds.), Word-order issues in sign language. Working papers presented at a workshop held in Durham, 18 - 22 September, 1991. Durham: Isla, pp. 73-87.

Erlenkamp, S. (2002). Syntaktische Kategorien und lexikalische Klassen: Typologische Aspekte der Deutschen Gebärdensprache. Doctoral dissertation. Munich: Lincom.

Fauconnier, G. (1994). Mental spaces. Aspects of meaning construction in natural language. Cambridge: Cambridge University Press.

Fauconnier, G. (1997). Mappings in Thought and Language. Cambridge: Cambridge University Press.

Fauconnier, G. and M. Turner (1996). Blending as a Central Process of Grammar. In: Goldberg, A.E. (Ed.), Conceptual Structure, Discourse and Language. Stanford, CA: CSLI, pp. 113-130.

Fehrmann, G. (2001). Dikursive Organisationsstrukturen in "strukturell mündlichen" Erzähltexten der Deutschen Gebärdensprache (DGS). Sprache und Literatur 32(2): 53-68. 
Fehrmann, G., Huber, W., Jäger, L., and H. Sieprath (1991). Tätigkeitsbeschreibung des Aachener Projekts zur Deutschen Gebärdensprache. Zeichen 5(18): 486-488.

Fillmore, C.J. (1982). Frame semantics. In: Linguistics in the Morning Calm, ed. by The Linguistic Society of Korea. Seoul: Hanshin, pp. 111-137.

Fischer, S.D. (1975). Influences on Word Order Change in American Sign Language. In: Li, C. (Ed.), Word Order and Word Order Change. Austin, TX: University of Texas Press, pp. 125.

Fischer, S.D. (2005). Prosody and operator scope marking in Western and Asian sign languages. Talk given at the MPI for Psycholinguistics, Nijmegen, November 2005.

Fridman-Mintz, B. and S.K. Liddell (1998). Sequencing mental spaces in an ASL narrative. In: Koenig, J.P. (Ed.), Discourse and Cognition: Bridging the Gap. Cambridge: Cambridge University Press, pp. 255-268.

Friedman, L.A. (1975). Space, time, and person reference in American Sign Language. Language 51, 940-961.

Frishberg, N. (1975). Arbitrariness and Iconicity: Historical Change in American Sign Language. Language 51: 696-719.

Frishberg, N. (1985). Dominance relations and discourse structure. In: Stokoe, W. and V. Volterra (Eds.), SLR '83. Proceedings of the Third International Symposium on Sign Language Research. Silver Spring, MD: Linstok Press, pp. 79-90.

Gee, J.P. and J. Kegl (1983). Narrative/story structure, pausing, and American Sign Language. Discourse Processes 6:3, 243-258.

Gennari, S.P., Sloman, S.A., Malt, B.C., and T. Fitch (2002). Motion events in language and cognition. Cognition 83: 49-79.

Givón, T. (1985). Iconicity, isomorphism, and non-arbitrary coding in syntax. In: Haiman, J. (Ed.), Iconicity in Syntax. Amsterdam: John Benjamins, pp. 187-220.

Givón, T. (1991). Isomorphism in the Grammatical Code: Cognitive and Biological Considerations. Studies in Language 1, no. 15: 85-114.

Glück, S. (2001). Morphosyntaktische Eigenschaften der Klassifikatoren in Deutscher Gebärdensprache. In: Leuninger, H. and K. Wempe (Eds.), Gebärdensprachlinguistik 2000 Theorie und Anwendung. Vorträge vom Symposium "Gebärdensprachforschung im deutschsprachigen Raum", held 11-13 June 1999, Frankfurt. Hamburg: Signum Verlag, pp. 127-145.

Glück, S. and R. Pfau (1997). Einige Aspekte der Morphologie und Morphosyntax in Deutscher Gebärdensprache. Frankfurter Linguistische Forschungen 20, pp. 30-48.

Glück, S. and R. Pfau (1998). On Classifying Classification as a Class of Inflection in German Sign Language. In: Cambier-Langeveld, T., Lipták, A., and M. Redford (Eds.), "Console VI" proceedings. Sixth Annual Conference of the Student Organization of Linguistics in Europe, pp. 59-74. 
Goldin-Meadow, S., McNeill, D., and J. Singleton (1996). Silence is liberating: Removing the handcuffs on grammatical expression in the manual modality. Psychological Review 103, 34-55.

Greenberg, J.H. (1963). Some universals of grammar with particular reference to the order of meaningful elements. In: Greenberg, J.H. (Ed.), Universals of language. Cambridge, MA: MIT Press, pp. 73-113.

Grice, H.P. (1975). Logic and conversation. In: Cole, P. and J.L. Morgan (Eds.), Speech Acts. New York: Academic Press, pp. 41-58.

Grinevald, C. (2003). Classifier Systems in the Context of a Typology of Nominal Classification. In: Emmorey, K. (Ed.) Perspectives on Classifier Constructions in Sign Languages, pp. 91-110.

Grote, K. and E. Linz (2003). The Influence of Sign Language Iconicity on Semantic Conceptualization. In: Nanny, M. and O. Fischer (Eds.) From sign to signing: Iconicity in Language and Literature, vol. 3. Amsterdam: John Benjamins, pp. 23-40.

Grote, K. and K. Willmes (2003). Do organizational principles in the mental lexicon influence the internal architecture of visual-semantic categories? In: Baker, A., Bogaerde, B. van der, and O. Crasborn (Eds.), Cross-linguistic perspectives in sign language research. Selected papers from TISLR 2000. Hamburg: Signum Press, pp. 283-306.

Haiman, J. (1980). The iconicity of grammar: isomorphism and motivation. Language 56(3): 515-540.

Haiman, J. (Ed.) (1985). Iconicity in syntax. Amsterdam: John Benjamins.

Hanke, T. (2002). HamNoSys in a sign language generation context. In: Schulmeister, R. and H. Reinitzer (Eds.), Progress in sign language research. In honor of Siegmund Prillwitz. Hamburg: Signum, pp. 249-264.

Hanke, T., Konrad, R., and A. Schwarz (2001). GlossLexer: A multimedia lexical database for sign language dictionary compilation. Sign Language \& Linguistics 4(1/2): 171-189.

Happ, D. and H. Leuninger (1998). DGS zum Kennenlernen. Frankfurter Linguistische Forschungen, Sonderheft RELEX.

Haug, T. and W. Mann (2005). Projekt Gebärdensprachtest: Entwicklung von Testverfahren zur Deutschen Gebärdensprache für gehörlose Kinder und Jugendliche. Das Zeichen 19(71): 370-380.

Haun, D.B.M. (2007). Cognitive Cladistics and the Relativity of Spatial Cognition. Doctoral dissertation, MPI Series in Psycholinguistics 41, University of Nijmegen.

Heßmann, J. (2001). GEHÖRLOS SO! Materialien zur Gebärdensprache. Band 1: Grundlagen und Gebärdenverzeichnis. Internationale Arbeiten zur Gebärdensprache und Kommunikation Gehörloser, 32. Hamburg: Signum Verlag.

Hilzensauer, M. and A. Skant (2001). Klassifikation in Gebärdensprachen. In: Leuninger, H. and K. Wempe (Eds.), Gebärdensprachlinguistik 2000 - Theorie und Anwendung: Vorträge 
vom Symposium "Gebärdensprachforschung im deutschsprachigen Raum". Hamburg: Signum Verlag, pp. 91-111.

Hinton, L., Nichols, J., and J. Ohala (1994a). Introduction: Sound symbolic processes. In: Hinton, L., Nichols, J., and J. Ohala (Eds.), Sound symbolism. Cambridge: Cambridge University Press, pp. 1-14.

Hinton, L., Nichols, J., and J. Ohala (Eds.) (1994b). Sound symbolism. Cambridge: Cambridge University Press.

Hockett, C.F. (1960). The origin of speech. Scientific American 203: 88-96.

Hohenberger, A., Happ, D., and H. Leuninger (2002). Modality-dependent aspects of sign language production: Evidence from slips of the hands and their repairs in German Sign Language. In: Meier, R.P., Cormier, K., and D. Quinto-Pozos (Eds.), Modality and Structure in Signed and Spoken Languages. Cambridge: Cambridge University Press, pp. 112-142.

Hoiting, N. and D.I. Slobin (2001). Typological and Modality Constraints on Borrowing. Examples from the Sign Language of the Netherlands. In: Brentari, D. (Ed.), Foreign Vocabulary in Sign Languages. Mahwah, NJ: Lawrence Erlbaum Associates, pp. 121-37.

Horn, L.R. (1984). Toward a new taxonomy for pragmatic inference. In: Schiffrin, D. (Ed.), Meaning, form, and use in context. Washington, D.C.: Georgetown University Press, pp. 1142.

Huber, W., Sieprath, H., and I. Werth (2001). Sprachdiagnostik in der Deutschen Gebärdensprache. In: Leuninger, H. and K. Wempe (Eds.), Gebärdensprachlinguistik 2000 Theorie und Anwendung: Vorträge vom Symposium "Gebärdensprachforschung im deutschsprachigen Raum". Hamburg: Signum Verlag, pp. 255-276.

Iversen, W., Nuerck, H.C., and K. Willmes (2004). Do signers think differently? The processing of number parity in deaf participants. Cortex 40: 176-178.

Jackendoff, R. (1990). Semantic Structures. Cambridge, MA: MIT Press.

Janzen, T. (2004). Space rotation, perspective shift, and verb morphology in ASL. Cognitive Linguistics 15-2: 149-174.

Johnston, T. (1996). Function and medium in the forms of linguistic expression found in a sign language. In: Edmondson, W.H. and R.B. Wilbur (Eds.), International Review of Sign Linguistics, Vol. 1. Mahwah, NJ: Lawrence Erlbaum Associates, pp. 57-94.

Johnston, T., Vermeerbergen, M., Schembri, A., and L. Leeson (2007). "Real data are messy": Considering cross-linguistic analysis of constituent ordering in Auslan, VGT, and ISL. In: Perniss, P.M., Pfau, R., and M. Steinbach (Eds.), Visible variation: Cross-linguistic studies $n$ sign language structure. Berlin: Mouton, pp. 163-205.

Kant, I. (1768). Von dem ersten Grunde des Unterschieds der Gegenden im Raume [On the first ground of the distinction of regions in space]. In: Cleve, J. van and R.E. Frederick (Eds.), The philosophy of right and left: Incongruent counterparts and the nature of space. Dordrecht: Kluwer, pp. 27-34. 
Kay, P. (1977). Language evolution and speech style. In: Blount, B. and M. Sanches (Eds.), Sociocultural dimensions of language change. New York: Academic Press, pp. 21-34.

Keller, J. (1998). Aspekte der Raumnutzung in der Deutschen Gebärdensprache. Hamburg: Signum Verlag.

Keller, J. (1999). Mundbilder in europäischen Gebärdensprachen. Das Zeichen 13(47): 136143.

Kita, S. (1999). Japanese Enter/Exit verbs without motion semantics. Studies in Language 23, 307-330.

Kita, S. and A. Özyürek (2003). What does cross-linguistic variation in semantic coordination of speech and gesture reveal? Evidence for an interface representation of spatial thinking and speaking. Journal of Memory and Language, 48, 16-32.

Klann, J. and W. Huber (2005). Die zerebrale Repräsentation der Gebärdensprache. In: Leuninger, H. and D. Happ (Eds.), Gebärdensprachen: Struktur, Erwerb, Verwendung. (Linguistische Berichte Sonderheft, 13). Hamburg: Buske, pp. 359-380.

Klima, E.S. and U. Bellugi (1979). The signs of language. Cambridge, MA: Harvard University Press.

Kooij, E. van der (2001). Weak drop in Sign Language of the Netherlands. In: Dively, V.L., Metzger, M., Taub, S., and A.M. Baer (Eds.), Signed languages. Discoveries from international research. Washington, DC: Gallaudet University Press, pp. 27-42.

Kooij, E. van der, Crasborn, O., and W. Emmerik (2004). Prosodic features of focus in NGT. Paper presented at TISLR 8 (Theoretical Issues in Sign Language Research), Barcelona, September 2004.

Krifka, M. (1989). Nominal reference, temporal constitution and quantification in event semantics. In: Bartsch, R., Benthem, J. van, and P. van Emde Boas (Eds.), Semantics and contextual expression. Dordrecht: Foris Publications, pp. 75-115.

Krifka, M. (2006). The Origin of Topic/Comment Structure, of Predication, and of Focusation in Asymmetric Bimanual Coordination. Talk given at the Nascent Language Conference, Rockefeller Foundation, Bellagio, Italy, October, 2006.

Landau, B. and R. Jackendoff (1993). "What" and "Where" in Spatial Language and Spatial Cognition. Behavioral and Brain Sciences 16: 217-265.

Labov, W. (1972). Sociolinguistic patterns. Philadelphia, PA: University of Philadelphia Press.

Langacker, R.W. (1987). Foundations of cognitive grammar: Theoretical prerequisites. Stanford, CA: Stanford University Press.

Langacker, R.W. (1999). Grammar and Conceptualization. Berlin: Mouton de Gruyter. 
Leeson, L. and J. Saeed (2002). Windowing of attention in simultaneous constructions in Irish Sign Language (ISL). Paper presented at HDSL 5, University of New Mexico, Albuquerque, November, 2002.

Leuninger, H. and D. Happ (1997). Lena. Gebärdenspracherwerb trotz Input. Frankfurter Linguistische Forschungen 20, 83-96.

Leuninger, H., Happ, D., and A. Hohenberger (2000). Assessing modality-neutral and modality-dependent aspects of language production: Slips of the tongue and slips of the hand and their repairs in spoken German and German sign language. Paper presented at the DFGcolloquium at Dagstuhl, September 2000.

Levelt, W.J.M. (1984). Some Perceptual Limitations on Talking About Space. In: Doorn, A.J., Grind, W.A. van der, and J.J. Koenderink (Eds.), Limits on Perception. Utrecht: VNU Science Press, pp. 323-358.

Levelt, W.J.M. (1996). Perspective taking and ellipsis in spatial development. In: Bloom, P., Peterson, M.A., Nadel, L., and M. Garrett (Eds.), Language and Space. Cambridge, MA: MIT Press, pp. 77-108.

Levinson, S.C. (1994). Vision, shape, and linguistic description: Tzeltal body-part terminology and object description. In: Haviland, J.B. and S.C. Levinson (Eds.), Spatial conceptualization in Mayan Languages. Special issue of Linguistics 32, 791-855.

Levinson, S.C. (1996a). Frames of reference and Molyneux's question. In: Bloom, P., Peterson, M.A., Nadel, L., and M. Garrett (Eds.), Language and Space. Cambridge, MA: MIT Press, pp. 109-170.

Levinson, S.C. (1996b). Language and Space. Annual Review of Anthropology 25: 353-382.

Levinson, S.C. (2000). Presumptive Meanings. Cambridge, MA: MIT Press.

Levinson, S.C. (2003). Space in Language and Cognition: Explorations in Cognitive Diversity. Cambridge: Cambridge University Press.

Levinson, S.C., Kita, S., Haun, D.B., and B.H. Rasch (2002). Returning the tables: Language affects spatial reasoning. Cognition 84(2): 155-188.

Levinson, S.C. and S. Meira (2003). "Natural concepts" in the spatial topological domain adpositional meanings in crosslinguistic perspective: An exercise in semantic typology. Language 79(3): 485-516.

Levinson, S.C. and D.P. Wilkins (2006a). The background to the study of the language of space. In: Levinson, S.C. and D.P. Wilkins (Eds.), Grammars of Space. Explorations in Cognitive Diversity. Cambridge: Cambridge University Press, pp. 1-23.

Levinson, S.C. and D.P. Wilkins (Eds.) (2006b). Grammars of Space. Explorations in Cognitive Diversity. Cambridge: Cambridge University Press.

Li, P. and L. Gleitman (2002). Turning the tables: language and spatial reasoning. Cognition 83(3): 265-294. 
Liddell, S.K. (1980). American Sign Language Syntax. New York: Mouton.

Liddell, S.K. (1995). Real, Surrogate and Token Space: Grammatical Consequences in ASL. In: Emmorey, K. and J.S. Reilly (Eds.), Language, Gesture, and Space. Hillsdale, NJ: Lawrence Erlbaum Associates, pp. 19-41.

Liddell, S.K. (1996). Spatial representations in discourse: comparing spoken and signed language. Lingua 98: 145-167.

Liddell, S.K. (1998). Grounded blends, gestures, and conceptual shifts. Cognitive Linguistics 9-3: 283-314.

Liddell, S.K. (2000). Blended spaces and deixis in sign language discourse. In: McNeill, D. (Ed.), Language and gesture: Window into thought and action. Cambridge: Cambridge University Press, pp. 331-357.

Liddell, S.K. (2003a). Grammar, Gesture, and Meaning in American Sign Language. Cambridge: Cambridge University Press.

Liddell, S.K. (2003b). Sources of meaning in ASL classifier predicates. In: Emmorey, K. (Ed.), Perspectives on Classifier Constructions in Sign Languages. Mahwah NJ: Lawrence Erlbaum Associates, pp. 199-220.

Liddell, S.K. and R.E. Johnson (1989). American Sign Language: The phonological base. Sign Language Studies 64: 195-277.

Liddell, S.K. and M. Metzger (1998). Gesture in sign language discourse. Journal of Pragmatics 30, 657-697.

Lillo-Martin, D. and E.S. Klima (1990). Pointing out Differences: ASL Pronouns in Syntactic Theory. In: Fischer, S.D. and P. Siple (Eds.), Theoretical Issues in Sign Language Research. Chicago: University of Chicago Press, pp. 191-210.

Linde, C. and W. Labov (1975). Spatial Networks as a Site for the Study of Language and Thought. Language, vol. 51: 924-939.

MacSweeney, M., Woll, B., Campbell, R., Calvert, G., McGuire, P., David, A., Simmons, A., and M. Brammer (2002). Neural Correlates of British Sign Language Comprehension: Spatial Processing Demands of Topographic Language. Journal of Cognitive Neuroscience 14: 10641075.

Majid, A., Bowerman, M., Kita, S., Haun, D.B., and S.C. Levinson (2004). Can language restructure cognition? The case for space. Trends in Cognitive Sciences 8(3): 108-114.

Mandel, M. (1977). Iconic Devices in American Sign Language. In: Friedman, L.A. (Ed.), On the Other Hand. London: Academic Press, pp. 57-107.

Marsaja, I G. (2003). Sociocultural, sociolinguistic, and linguistic patterns in a Deaf community: An ethnographic study of a Deaf village in North Bali. Doctoral dissertation, La Trobe University, Melbourne, Australia.

Marr, D. (1982). Vision. New York: W.H. Freeman. 
Mathur, G. (2002). On the Syntax-Phonology Interface in ASL: The Case of HOLD. Paper presented at the LSA Annual Meeting, 2002.

McDonald, B. (1982). Aspects of the American Sign Language Predicate System. Doctoral dissertation, State University of New York, Buffalo.

McNeill, D. (1992). Hand and Mind: What Gestures Reveal about the Mind. Chicago: University of Chicago Press.

Meier, R.P. (1990). Person deixis in American Sign Language. In: Fischer, S.D. and P. Siple (Eds.), Theoretical Issues in Sign Language Research, Vol. 1: Linguistics. Chicago: University of Chicago Press, pp. 175-190.

Meier, R.P. (2002). Why different, why the same? Explaining effects and non-effects of modality upon linguistic structure in sign and speech. In: Meier, R.P, Cormier, K., and D. Quinto-Pozos (Eds.), Modality and structure in signed and spoken languages. Cambridge: Cambridge University Press, pp. 1-25.

Meier, R.P, Cormier, K., and D. Quinto-Pozos (Eds.) (2002). Modality and structure in signed and spoken languages. Cambridge: Cambridge University Press.

Meir, I. (1998). Syntactic-Semantic Interaction in Israeli Sign Language verbs: The Case of Backwards Verbs. Sign Language \& Linguistics, Vol. 1: 3-33.

Meir, I. (2002). A Cross-Modality Perspective on Verb Agreement. Natural Language and Linguistic Theory 20, pp. 413-50.

Metzger, M. (1995). Constructed dialogue and constructed action in American Sign Language. In: Lucas, C. (Ed.), Sociolinguistics in Deaf Communities. Gallaudet University Press, Washington D.C., pp. 255-271.

Miles, M. (2000). Signing in Seaglio: Mutes, dwarfs, and gestures at the Ottoman Court 1500-1700. Disability and Society, Vol. 15(1), 115-134.

Miller, C. (1994). Simultaneous constructions in Quebec Sign Language. In: Brennan, M. and G.H. Turner (Eds.), Word-order issues in sign language. Working papers presented at a workshop held in Durham, 18 - 22 September, 1991. Durham: Isla, pp. 89-112.

Miller, G.A. and P.N. Johnson-Laird (1976). Language and Perception. Cambridge MA: Harvard University Press.

Morgan, G. (1999). Event packaging in British Sign Language discourse. In: Winston, E. (Ed.), Story Telling and Conversation: Discourse in Deaf Communities. Washington, DC: Gallaudet University Press, pp. 27-58.

Morgan, G. (2002). Children's Encoding of Simultaneity in BSL Narratives. Sign Language \& Linguistics 5:2, 131-165.

Morgan, G. (2005). The development of narrative skills in British Sign Language. In: Schick, B., Marschark, M., and P. Spencer (Eds.), Advances in Sign Language Development in Deaf Children. Oxford: Oxford University Press. 
Morgan, G. and B. Woll (2003). The Development of Reference Switching Encoded Through Body Classifiers in British Sign Language. In: Emmorey, K. (Ed.), Perspectives on Classifier Constructions in Sign Languages. Mahwah, NJ: Lawrence Erlbaum Associates, pp. 297-310.

Nespor, M. and W. Sandler (1999). Prosody in Israeli Sign Language. Language and Speech, 42(2-3), 143-176.

Newmeyer, F.J. (1992). Iconicity and generative grammar. Language 68:756-796.

Newport, E.L. and R.P. Meier (1985). The acquisition of American Sign Language. In: Slobin, D.I. (Ed.), The Cross-Linguistic Study of Language Acquisition. Vol. II, The Data. Hillsdale, NJ: Lawrence Erlbaum Associates, pp. 881-938.

Newport, E.L. and T.R. Supalla (1980). Clues from the Acquisition of Signed and Spoken Language. In: Bellugi, U. and M. Studdert-Kennedy (Eds.), Signed and Spoken Language: Biological Constraints on Form. Weinheim: Verlag Chemie, pp. 187-211.

Newport, E.L. and T.R. Supalla (2000). Sign language research at the millennium. In: Emmorey, K. and H. Lane (Eds.), The signs of language revisited. An anthology to honor Ursula Bellugi and Edward Klima. Mahwah, NJ: Lawrence Erlbaum Associates, pp. 103114.

Nyst, V. (2007). A Descriptive Analysis of Adamorobe Sign Language (Ghana). Doctoral dissertation, Netherlands Graduate School of Linguistics (LOT) 151, University of Amsterdam.

Nyst, V. and P.M. Perniss (2004). Classifiers or verb series: motion in German Sign Language and Adamorobe Sign Language (Ghana). Paper presented at ESF workshop "Modality Effects on the Theory of Grammar: A Cross-linguistic View from Sign Languages of Europe", Barcelona, November 2004.

Orlansky, M.D. and J.D. Bonvillian (1984). The role of iconicity in early sign language acquisition. Journal of Speech and Hearing Disorders 49: 287-292.

Özyürek, A. and P.M. Perniss (in press). Event representation in sign language: A crosslinguistic perspective. To appear in: Bohnemeyer, J. and E. Pederson (Eds.), Event Representation in Language: Encoding Events at the Language-Cognition Interface. Cambridge: Cambridge University Press.

Padden, C.A. (1983). Interaction of morphology and syntax in American Sign Language. Doctoral dissertation (published in 1988 in Outstanding Dissertations in Linguistics Series IV). New York: Garland.

Padden, C.A. (1986). Verbs and role-shifting in ASL. In: Padden, C.A. (Ed.), Proceedings of the Fourth National Symposium on Sign Language Research and Teaching. Silver Spring, MD: National Association of the Deaf, pp. 44-57.

Padden, C.A. (1990). The relation between space and grammar in ASL verb morphology. In Lucas, C. (Ed.), Sign Language Research: Theoretical Issues. Washington, DC: Gallaudet University Press, pp. 118-132. 
Padden, C.A. and D.M. Perlmutter (1987). American Sign Language and the architecture of phonological theory. Natural Language and Linguistic Theory, vol. 5, 335-375.

Papafragou, A., Masely, C., and L. Gleitman (2002). Shake, rattle, 'n' roll: the representation of motion events in language and cognition. Cognition 84: 184-219.

Papaspyrou, C. (1990). Gebärdensprache und universelle Sprachtheorie. Versuch einer vergleichenden generativ-transformationellen Interpretation von Gebärdensprache und Lautsprache sowie der Entwurf einer Gebärdenschrift. (Internationale Arbeiten zur Gebärdensprache und Kommunikation Gehörloser, Band 8). Hamburg: Signum Verlag.

Pederson, E., Danzinger, E., Wilkins, D.P., Levinson, S.C., Kita, S., and G. Senft (1998). Semantic typology and spatial conceptualization. Language 74(3): 557-589.

Peirce, C.S. (1902). Logic, Considered as Semeiotic (The Carnegie application). Manuscript L75, from the Nachlass of Charles Sanders Peirce (reconstructed by J. Ransdell).

Perniss, P.M. (2001). Numerus und Quantifikation in der Deutschen Gebärdensprache. MA thesis, University of Cologne.

Perniss, P.M. (2007a). Locative functions of simultaneous perspective constructions in German Sign Language narratives. In: Vermeerbergen, M., Leeson, L., and O. Crasborn (Eds.), Simultaneity in Signed Languages: Form and function. Amsterdam: John Benjamins, pp. 27-54.

Perniss, P.M. (2007b) Achieving spatial coherence in German Sign Language narratives: The use of classifiers and perspective. Lingua 117(7): 1315-1338.

Perniss, P.M. and A. Özyürek (2004a). How many pots on the window sill? Number and quantification in German and Turkish Sign Language. Paper presented at "Cross-linguistic perspectives in sign language research", workshop at German Linguistics Society Conference (DGfS), Mainz, February 2004.

Perniss, P.M. and A. Özyürek (2004b). Expressing spatial relationships in German Sign Language (DGS) and Turkish Sign Language (TID). Poster presented at TISLR 8 (Theoretical Issues in Sign Language), Barcelona, November, 2004.

Perniss, P.M. and A. Özyürek (in press). Constructing action and locating referents: A comparison of German and Turkish sign language narratives. To appear in: Quer, J. (Ed.). Signs of the time. Selected papers from TISLR 2004. Hamburg: Signum Press.

Perniss, P.M., Pfau, R., and M. Steinbach (Eds.) (2007). Visible variation: Cross-linguistic studies in sign language structure. Berlin: Mouton.

Perniss, P.M. and U. Zeshan (in preparation). Possessive and existential constructions in Kata Kolok (Bali). To appear in: Perniss, P.M. and U. Zeshan (Eds.), Possessive and existential constructions in sign languages. (Sign Language Typology Series No. 2). Nijmegen: Ishara Press.

Peterson, M.A., Gelder, B. de, Rapcsak, S.Z., Gerhardstein, P.C., and A. Bachoud-Levi (2000). Object memory effects on figure assignment: conscious object recognition is not necessary or sufficient. Vision Research 40(10-12): 1549-67. 
Petitto, L.A. and P.F. Marentette (1991). Babbling in the manual mode: Evidence for the ontogeny of language. Science 251: 1483-1496.

Pfau, R. (1997). Zur phonologischen Komponente der Deutschen Gebärdensprache: Segmente und Silben. Frankfurter Linguistische Forschungen 20: 1-29.

Pfau, R. (2002). Applying morphosyntactic and phonological readjustment rules in natural language negation. In: Meier, R.P., Cormier, K., and D. Quinto-Pozos (Eds.), Modality and Structure in Signed and Spoken Languages. Cambridge: Cambridge University Press, pp. 263-295.

Pfau, R. and M. Steinbach (2003). Optimal reciprocals in German Sign Language. Sign Language \& Linguistics 6(1): 3-42.

Pfau, R. and M. Steinbach (2004). An optimality-theoretical account of German Sign Language pluralization. In: Agbayani, B., Samiian, V., and B.V. Tucker (Eds.), Proceedings of the Western Conference on Linguistics (WECOL), Vol. 15. Fresno, CA: CSU, pp. 234-245.

Pickering, M.J. and S.C. Garrod (2004). Towards a mechanistic theory of dialogue. Behavioral and Brain Sciences 27, 169-226.

Pike, K.L. (1943). Phonetics: A critical analysis of phonetic theory and a technic for the practical description of sounds. Ann Arbor: University of Michigan Press.

Pizzuto, E. and V. Volterra (2000). Iconicity and transparency in sign languages: a crosslinguistic cross-cultural view. In: Emmorey, K. and H. Lane (Eds.), The Signs of Language Revisited: An Anthology in Honor of Ursula Bellugi and Edward Klima. Hillsdale, NJ: Lawrence Erlbaum, pp. 261-286.

Poizner, H., Klima, E.S., and U. Bellugi (1987). What the hands reveal about the brain. Cambridge, MA: MIT Press.

Poulin, C. and C. Miller (1995). On Narrative Discourse and Point of View in Quebec Sign Language. In: Emmorey, K. and J. Reilly (Eds.), Language, Gesture, and Space. Hillsdale, NJ: Lawrence Erlbaum Associates, pp. 117-132.

Prillwitz, S. (1985). Skizzen zu einer Grammatik der Deutschen Gebärdensprache. Hamburg: Signum Verlag.

Pyers, J. and A. Senghas (2007). Referential shift in Nicaraguan Sign Language: A comparison with American Sign Language. In: Perniss, P.M., Pfau, R. and M. Steinbach (Eds.), Visible variation: Cross-linguistic studies in sign language structure. Berlin: Mouton, pp. 279-302.

Risler, A. (2007). A cognitive linguistic view of simultaneity in process signs in French Sign Language. In: Vermeerbergen, M., Leeson, L. and O. Crasborn (Eds.), Simultaneity in Signed Languages: Form and function. Amsterdam: John Benjamins, pp. 73-102.

Rubin, E. (1915/1958). Figure and ground. In: Beardslee, D.C. and M. Wertheimer (Eds.), Readings in perception. Princeton, NJ: Van Nostrand, pp. 194-203. (Original published in 1915 as Synsoplevede Figurer. Copenhagen: Gyldendalske.) 
Russo, T. (2004). Iconicity and productivity in sign language discourse: an analysis of three LIS discourse registers. Sign Language Studies 4(2): 164-197.

Sallandre, M.-A. (2007). Simultaneity in French Sign Language discourse. In: Vermeerbergen, M., Leeson, L. and O. Crasborn (Eds.), Simultaneity in Signed Languages: Form and function. Amsterdam: John Benjamins, pp. 103-126.

Sallandre, M.-A. and C. Cuxac (2002). Iconicity in Sign Language: a theoretical and methodological point of view. In: Wachsmuth I. and T. Sowa (Eds.), Gesture-based communication in human-computer interaction. Proceedings of the International Gesture Workshop. (GW 2001) Berlin: Springer Verlag, pp. 171-180.

Sandler, W. (1989). Phonological Representation of the Sign: Linearity and Nonlinearity in American Sign Language. Dordrecht: Foris Publications.

Sandler, W. (1996). Representing Handshapes. In: Edmondson, W.H. and R.B. Wilbur (Eds.), International Review of Sign Linguistics, Vol. 1. Mahwah, NJ: Lawrence Erlbaum Associates, pp. 115-158.

Sandler, W. (1999). Prosody in two natural language modalities. Language and Speech 42: 127-142.

Sandler, W. and D. Lillo-Martin (2003). Natural sign languages. In: Aronoff, M. and J. ReesMiller (Eds.), The Handbook of Linguistics. Oxford: Blackwell, pp. 533-562.

Sauer, A., Wotschke, M., Glück, S., Happ, D., and H. Leuninger (1997). DGS-Syntax: Raumnutzung und Satztypen. Frankfurter Linguistische Forschungen 20, 49-82.

Saussure, F. de (1916). Cours de linguistique générale. Paris: Payot.

Schembri, A. (2002). The representation of motion events in signed language and gesture: A preliminary report. In: Schulmeister, R. and H. Reinitzer (Eds.), Progress in sign language research: In honor of Siegmund Prillwitz. Hamburg: Signum Verlag, pp. 99-126.

Schembri, A. (2003). Rethinking "Classifiers" in Signed Languages. In: Emmorey, K. (Ed.), Perspectives on Classifier Constructions in Sign Languages, pp. 3-34.

Schembri, A., Jones, C., and D. Burnham (2005). Comparing Action Gestures and Classifier Verbs of Motion: Evidence From Australian Sign Language, Taiwan Sign Language, and Nonsigners' Gestures Without Speech. Journal of Deaf Studies and Deaf Education 10(3): 272-290.

Schick, B. (1990). Classifier Predicates in American Sign Language. International Journal of Sign Linguistics 1: 15-40.

Schober, M.F. (1993). Spatial Perspective-taking in Conversation. Cognition 47, 1-24.

Seifart, F. (2005). The Structure and Use of Shape-based Noun Classes in Miraña (North West Amazon). Doctoral dissertation, MPI Series in Psycholinguistics 32, University of Nijmegen. 
Senghas, A. and M. Coppola (2001). Children creating language: how Nicaraguan Sign Language acquired a spatial grammar. Psychological Science 12: 323-328.

Senghas, A., Kita, S., and A. Özyürek (2004). Children Creating Core Properties of Language: Evidence from an Emerging Sign Language in Nicaragua. Science 17, 1779-1782.

Slobin, D.I. (1996). From "thought and language" to "thinking for speaking". In: Gumperz, J. and S.C. Levinson (Eds.), Rethinking Linguistic Relativity. Cambridge: Cambridge University Press, pp. 70-96.

Slobin, D.I. (1997). Mind, code, and text. In: Bybee, J., Haiman, J., and S.A. Thompson (Eds.), Essays on language function and language type: Dedicated to T. Givón. Amsterdam: John Benjamins, pp. 437-467.

Slobin, D.I. and N. Hoiting (1994). Reference to movement in spoken and signed languages: Typological considerations. Proceedings of the Twentieth Annual Meeting of the Berkeley Linguistics Society, pp. 487-505.

Slobin, D.I., Hoiting, N., Anthony, M., Biederman, Y., Kuntze, M., Lindert, R., Pyers, J., Thumann, H., and A. Weinberg (2001). Sign language transcription at the level of meaning components: The Berkeley Transcription System (BTS). Sign Language \& Linguistics 4, 6396.

Slobin, D.I., Hoiting, N., Kuntze, M., Lindert, R., Weinberg, A., Pyers, J., Anthony, M., Biederman, Y., and H. Thumann, (2003). A Cognitive/Functional Perspective on the Acquisition of "Classifiers". In: Emmorey, K. (Ed.), Perspectives on Classifier Constructions in Sign Languages. Mahwah, NJ: Lawrence Erlbaum Associates, pp. 271-298.

Steinbach, M. and R. Pfau (2007). Grammaticalization of auxiliaries in sign languages. In: Perniss, P.M., Pfau, R., and M. Steinbach (Eds.), Visible variation: Cross-linguistic studies $n$ sign language structure. Berlin: Mouton, pp. 303-339.

Stokoe, W.C. (1960). Sign Language Structure. An outline of the visual communication systems of the American Deaf. Silver Spring, MD: Linstok Press.

Supalla, T.R. (1978). Morphology of verbs of motion and location in American Sign Language. In: Caccamise, F. (Ed.), American Sign Language in a bilingual, bicultural context: Proceedings of the National Symposium on Sign Language Research and Teaching. Silver Spring, MD: National Association of the Deaf, pp. 27-45.

Supalla, T.R. (1982). Structure and Acquisition of Verbs of Motion and Location in American Sign Language. Unpublished doctoral dissertation. University of California, San Diego.

Supalla, T.R. (1986). The Classifier System in American Sign Language. In: Craig, C. (Ed.), Noun Classes and Categorization. Philadelphia: John Benjamins, pp. 181-214.

Supalla, T.R. (1990). Serial Verbs of Motion in ASL. In: Fischer, S.D. and P. Siple (Eds.), Theoretical Issues in Sign Language Research. Chicago: University of Chicago Press, pp. 127-62. 
Supalla, T.R. and E.L. Newport (1978). How many seats in a chair? The derivation of nouns and verbs in American Sign Language. In: Siple, P. (Ed.), Understanding Language through Sign Language Research. New York: Academic Press, pp. 91-132.

Supalla, T.R. and R. Webb (1995). The grammar of International Sign: A new look at pidgin languages. In: Emmorey, K. and J. Reilly (Eds.), Language, Gesture, and Space. Hillsdale, NJ: Lawrence Erlbaum Associates, pp. 333-354.

Sutton-Spence, R. and B. Woll (1999). The Linguistics of British Sign Language: An Introduction. Cambridge: Cambridge University Press.

Talmy, L. (1975). Semantics and syntax of motion. In: Kimball, J. (Ed.), Syntax and semantics (Vol. 4). New York: Academic Press, pp. 181-238.

Talmy, L. (1978). Figure and ground in complex sentences. In: Greenberg, J.H. (Ed.), Universals of human language. Stanford: Stanford University Press, pp. 625-649.

Talmy, L. (1983). How language structures space. In: Pick, H. and L. Acredolo (Eds.), Spatial orientation: Theory, research, and application. New York: Plenum Press, pp. 225282.

Talmy, L. (1985). Lexicalization Patterns: Semantic Structure in Lexical Forms. In: Shopen, T.E. (Ed.), Language Typology and Syntactic Description, Vol. 3. Cambridge: Cambridge University Press, pp. 57-149.

Talmy, L. (1991). Path to realization: A typology of event conflation. Proceedings of the Berkeley Linguistics Society, 17, 480-519.

Talmy, L. (1996). Fictive motion in language and "ception". In: Bloom, P., Peterson, M.A., Nadel, L., and M.F. Garrett (Eds.), Language and Space. Cambridge, MA: MIT Press, pp. 211-276.

Talmy, L. (2003). The Representation of Spatial Structure in Spoken and Signed Language. In: Emmorey, K. (Ed.), Perspectives on Classifier Constructions in Sign Languages. Mahwah, NJ: Lawrence Erlbaum Associates, pp. 169-196.

Tannen, D. (1980). A Comparative Analysis of Oral Narrative Strategies: Athenian Greek and American English. In: Chafe, W. (Ed.), The Pear Stories: Cognitive, Cultural, and Linguistic Aspects of Narrative Production. Norwood, NJ: Ablex, pp. 51-87.

Tannen, D. (1982). Oral and Literate Strategies in Spoken and Written Narratives. Language 58(1): $1-21$.

Tannen, D. (1989). Talking voices. Repetition, dialogue, and imagery in conversational discourse. Cambridge, MA: Cambridge University Press.

Tervoort, B.T. (1953). Structurele analyse van visueel taalgebruik binnen een groep dove kinderen. Amsterdam: Noord-Hollandsche Uitgevers Maatschappij.

Taub, S. (2001). Language from the body: Iconicity and metaphor in American Sign Language. Cambridge: Cambridge University Press. 
Taub, S. and D. Galvan (2001). Patterns of Conceptual Encoding in ASL Motion Descriptions. Sign Language Studies 1:2, 175-200.

Taylor, H.A. and B. Tversky (1992). Spatial mental models derived from survey and route descriptions. Journal of Memory and Language 31(2): 261-292.

Taylor, H.A. and B. Tversky (1996). Perspective in spatial descriptions. Journal of Memory and Language 35: 371-391.

Uyechi, L. (1993). The geometry of visual phonology. Stanford, CA: CSLI.

Valli, C. and C. Lucas (1995). Linguistics of American Sign Language: An Introduction. Washington, DC: Gallaudet University Press.

Vecera, S.P. and R.C. O'Reilly (1998). Figure-Ground Organization and Object Recognition Processes: An Interactive Account. Journal of Experimental Psychology: Human Perception and Performance 24, 441-462.

Vermeerbergen, M. (1996). ROOD KOOL TIEN PERSOON. Morfo-syntactische aspecten van gebarentaal. Doctoral dissertation, Vrije Universiteit Brussel, Brussels.

Vermeerbergen, M. (2001). Simultane constructies in de Vlaamse Gebarentaal. In: Beyers, R. (Ed.), Handelingen (Koninklijke Zuid-Nederlandse Maatschappij voor Taal- en Letterkunde en Geschiedenis), LIV 2000. Brussels: LIV, pp. 69-81.

Vermeerbergen, M., Leeson, L., and O. Crasborn (Eds.) (2007). Simultaneity in Signed Languages: Form and function. Amsterdam: John Benjamins.

Vigliocco, G., Vinson, D., Woolfe, T., Dye, M., and B. Woll (2005). Language and imagery: effects of language modality. Proceedings of the Royal Society: Biological Sciences 272(1574): 1859-1863.

Vogel, H. (1999). Geschichte der Gehörlosenbildung. In: Beecken, A., Keller, J., Prillwitz, S., and H. Zienert (Eds.), Grundkurs Deutsche Gebärdensprache, Stufe I, Arbeitsbuch. Hamburg: Signum Verlag, pp. 46-49.

Vogt-Svendsen, M. and B. Bergman (2007). Point buoys: The weak hand as a point of reference for time and space. In: Vermeerbergen, M., Leeson, L., and O. Crasborn (Eds.), Simultaneity in Signed Languages: Form and function. Amsterdam: John Benjamins, pp. 217-236.

Wassmann, J. and P. Dasen (1998). Balinese Spatial Orientation. Journal of Royal Anthropological Institute 4(4): 689-713.

Wilcox, S. (2004). Cognitive iconicity: Conceptual spaces, meaning, and gesture in signed languages. Cognitive Linguistics 15(2): 119-147.

Wilcox, S. (2006). Iconicity, Sign Language. In: Brown, K., Anderson, A.M., Bauer, L., Berns, M., Hirst, G., and J. Miller (Eds.), Encyclopedia of Language and Linguistics (Second Edition), Vol. 5. Amsterdam: Elsevier, pp. 472-475. 
Wilcox, S. and P. Wilcox (1995). The Gestural Expression of Modality in ASL. In: Bybee, J. and S. Fleishman (Eds.), Modality in Grammar and Discourse. Amsterdam: John Benjamins, pp. 135-162.

Wilkinson, E., Rossini, P., Sallandre, M.-A., and E. Pizzuto (2006). Deixis, Anaphora, and Highly Iconic Structures: Cross-linguistic Evidence on American (ASL), French (LSF), and Italian (LIS) Signed Languages. Paper presented at TISLR 9 (Theoretical Issues in Sign Language Research), Florianópolis, Brazil, December 2006.

Woll, B. (2001). The sign that dares to speak its name: Echo phonology in British Sign Language (BSL). In: Boyes-Braem, P. and R. Sutton-Spence (Eds.), The Hands are the Head of the Mouth: The Mouth as Articulator in Sign Languages. (International Studies on Sign Language and Communication of the Deaf 39). Hamburg: Signum, pp. 87-98.

Woll, B. (2003). Modality, universality, and the similarities among sign languages: An historical perspective. In: Baker, A., Bogaerde, B. van der, and O. Crasborn (Eds.), Crosslinguistic perspectives in sign language research. Hamburg: Signum Press, pp. 119-131.

Woll, B., Capek, C., Waters, D., Campbell, R., MacSweeney, M., Brammer, M., McGuire, P., and T. David (2006). Do mouths sign? Paper presented at TISLR 9 (Theoretical Issues in Sign Language Research), Florianópolis, Brazil, December 2006.

Yamamoto, M. (1999). Animacy and Reference: a cognitive approach to corpus linguistics. Amsterdam: John Benjamins.

Yang, J.-H., (2004). From Character to Sign: Processes of Lexical Borrowing in Chinese Sign Language. Talk given at the MPI for Psycholinguistics, Nijmegen.

Zeshan, U. (2000). Sign Language in Indo-Pakistan. Amsterdam: John Benjamins.

Zeshan, U. (2003). "Classificatory" Constructions in Indo-Pakistani Sign Language: Grammaticalization and Lexicalization Processes. In: Emmorey, K. (Ed.), Perspectives on Classifier Constructions in Signed Languages. Mahwah, NJ: Lawrence Erlbaum Associates, pp 113-141.

Zwitserlood, I. (2003). Classifying hand configurations in Nederlandse Gebarentaal (Sign Language of the Netherlands). Doctoral dissertation, Netherlands Graduate School of Linguistics (LOT) 78, University of Utrecht. 
This dissertation investigates the expression of spatial relationships in German Sign Language (Deutsche Gebärdensprache, DGS). The analysis focuses on linguistic expression in the spatial domain in two types of discourse: static scene description (location) and event narratives (location and motion). In more general terms, the dissertation provides a usagebased account of iconic mapping in the visual-spatial modality. Through a systematic investigation of spatial expressions in DGS, it goes beyond previous research in the spatial domain in sign languages, and in DGS, in particular, by investigating the impact of different constraints - e.g. linguistic, discourse, and articulatory — on iconicity in sign languages.

Chapters 1-3 of the dissertation introduce sign languages as the natural languages of the deaf, explicate the structure of language in the visual-spatial modality, define the main theoretical questions underlying the research presented in the dissertation, and outline the relevant theoretical domains. Specifically, chapter 2 discusses iconicity as a subject of semiotic and linguistic theory, and describes the system of classifier predicates with which signers can create iconic mappings between real-world or conceptual space and sign space. In these morphologically complex predicates, the classifier (i.e. the handshape) expresses information about the size and shape of a referent, and the position and movement of the hand(s) in sign space encode information about the location and motion of the referent(s). The investigation focuses on two main types of classifiers: entity classifiers, in which the handshape represents a whole entity, and handling classifiers, in which the handshape represents the manipulation of an entity by an agent.

Chapter 3 gives an overview of the spatial domain (that is, the linguistic encoding of location and motion) in spoken and signed language and defines the use of frames of reference in spatial description. Frame of reference systems are employed in spoken and signed language to express the spatial relationship between two objects (a figure and a ground object) that are located at some remove from one another in space. There are three major types of frame of reference that have been identified in natural languages. The relative frame of reference expresses a ternary relationship by specifying the figure's location with respect to the ground from a particular viewpoint (or deictic origo). In contrast, the intrinsic frame of reference utilizes the inherent features of the ground object to express a binary relationship between the figure and ground. Finally, the absolute frame of reference makes use of fixed, 
external bearings (e.g. cardinal directions) to specify the location of the figure with respect to the ground.

In addition, chapter 3 introduces signing perspective as an important spatial device. Signing perspective refers to the way in which an event space is projected onto sign space. It determines how sign space is structured for spatial representation, and therefore plays a significant role in the depiction of spatial relationships. There are two ways in which this projection can take place, depending on the signer's conceptual location in relation to the event space. On the one hand, signers can have an external vantage point. The signer's role is that of an observer who can oversee the whole event space, which is mapped in a reduced size onto the space in front of the body. This corresponds to an observer perspective event space projection. On the other hand, the signer's vantage point can be within the event. Such an event-internal perspective is achieved by assuming the role of a character within the event. In this way, the character's location coincides with that of the signer, and entities in the event space are mapped onto sign space as they are seen by that character. This corresponds to a character perspective event space projection.

Chapters 4-8 form the empirical investigation of the dissertation. Deaf signers of DGS were asked to participate in two tasks. In the static scene description task, signers described simple and complex static scenes to (deaf) addressees, who reconstructed the scene using plastic figures. In the event narratives task, signers narrated cartoon stimulus films to an addressee, who then retold the event. The main findings related to the expression of location and motion events in static scene descriptions and event narratives, and to the interplay between the relevant spatial systems (classifier predicates, perspective, and frames of reference) are summarized below for each of these chapters.

Chapter 4 presents a qualitative and quantitative investigation of the conventions put forth in the sign linguistics literature for the expression of spatial information in simple locative descriptions. DGS descriptions of simple and complex static scenes were analyzed on a descriptive-linguistic case-by-case basis. Importantly, the analysis shows that signers do not rely fully on the iconic properties of classifier predicates and space in giving spatial descriptions, but rather communicate locative information additionally through the use of directional predicates, numerals, and prepositional forms, as well as through predication and subject NP type. In addition, the chapter shows that the figure-ground structure of DGS locative descriptions is dependent on the perceptual features of the objects.

Chapter 5 investigates the use of perspective and frames of reference in DGS static scene description and shows that DGS signers consistently map location and orientation 
information onto sign space in an observer perspective event space projection. Moreover, spatial information is represented in a relative frame of reference or in both relative and intrinsic frames simultaneously, as a unique property of the visual-spatial modality. The results support an alignment, or prototypical co-occurrence, between observer perspective and the relative frame of reference, based on shared semantic properties, but also show that perspective and frames of reference are independent systems. A comprehension analysis shows that addressees (sitting across from the signers) predominantly demonstrate mental rotation in interpreting the location and orientation information they see in the signer's sign space. The addressee responses provide further evidence that the use of the relative frame of reference, with the signer as deictic origo, is conventionalized in DGS for static scene spatial descriptions.

Chapter 6 turns to event narratives, as another discourse type, to investigate the use of perspective and frames of reference to encode location and motion. The results of the analysis show that both location and motion in event narratives are predominantly expressed in a character perspective event space projection and in an intrinsic frame of reference. These results contrast with those of chapter 5 and indicate that discourse type strongly influences the choice of perspective and frame of reference in the coding of spatial information in DGS. The results also indicate that location and motion are not treated fundamentally differently from each other in DGS. In addition, the chapter argues for an alignment, or prototypical cooccurrence, between character perspective and the intrinsic frame, but again provides evidence for the independence of the two systems by showing that the relative and intrinsic frames also occur simultaneously within character perspective.

Chapter 7 discusses the use of different types of spatial/activity predicates (i.e. classifier predicates, lexical predicates, and index signs) that can depict referent location, motion, and action in relationship to their use in observer or character perspective event space projections. The chapter presents a qualitative analysis, identifying and showing examples of different predicate-perspective construction types, as well as a quantitative analysis, showing the distribution of the different construction types in the data. In addition, the analysis motivates an alignment between entity classifiers and observer perspective, on the one hand, and between handling classifiers and character perspective, on the other hand, based on iconicity principles of the scale of representation (between the size of referent representation and the size of the event space projection) and on the type of information felicitously encoded by different classifier forms. The use of "aligned" vs. "non-aligned" constructions is discussed in relation to event simultaneity and iconicity of expression. 
Chapter 8 focuses on the affordance of simultaneity in the visual-spatial modality and looks at constructions that use multiple articulators, both manual and non-manual (e.g. the body, the head, the eyes), to simultaneously encode elements associated with different perspectives. The use of these constructions is discussed in terms of maintaining spatial coherence across perspectives and in terms of achieving temporal and scale iconicity in the event representation. Their occurrence and function in discourse is discussed in relation to efficiency and informativeness, taken to be discourse-structure constraints that interact with iconicity principles.

The concluding chapter (chapter 9) highlights the methodological and theoretical contributions of the dissertation. It emphasizes the importance and necessity of careful, systematic investigation of sign language use, in different types of discourse, within the spatial domain. Furthermore, it synthesizes the outcomes of the previous chapters in relation to perspective, frames of reference, and classifier predicates, underscoring again the alignments between them, but also the importance of distinguishing them as independent systems. In addition, it discusses the influence of different iconicity principles (including scale iconicity, temporal iconicity, and iconicity of perception) on the structure of spatial representations and their interaction with linguistic and discourse-structure principles. 


\section{Samenvatting}

Gebarentaal wordt voornamelijk uitgedrukt met de handen, waarbij situaties en gebeurtenissen worden uitgebeeld in de ruimte voor het lichaam van de gebaarder; de gebarenruimte. Net als bij gesproken talen kunnen daarbij complexe scènes worden geschetst, waarbij duidelijkheid wordt gegeven over bijvoorbeeld ruimte, tijd en causaliteit. In dit proefschrift wordt onderzocht hoe ruimtelijke relaties worden uitgedrukt in de Duitse Gebarentaal (Deutsche Gebärdensprache, DGS). Het onderzoek concentreert zich op het linguïstisch uitdrukken van ruimtelijke verbanden in twee soorten van discours: de beschrijving van statische scènes (locatie) en het navertellen van gebeurtenissen (locatie en beweging). Meer in het algemeen gaat het in dit proefschrift over de mogelijkheden om iconische afbeeldingen in de visueel-ruimtelijke modaliteit te gebruiken, door studie van het gebruik ervan in de praktijk. Door systematisch onderzoek naar ruimtelijke uitdrukkingsvormen in DGS voegt deze dissertatie nieuwe kennis toe aan het onderzoeksgebied van gebarentalen, met name de DGS, waarbij belang en invloed van verschillende beperkingen op iconiciteit in gebarentalen worden bestudeerd (bijvoorbeeld linguïstische, discoursgerelateerde en articulatorische beperkingen).

In de hoofdstukken 1-3 van dit proefschrift worden gebarentalen geïntroduceerd als de natuurlijke talen van dove mensen en wordt de structuur van taal in de visueel-ruimtelijke modaliteit uitgelegd. Hier worden ook de belangrijkste theoretische vraagstellingen van dit proefschrift beschreven en de relevante theoretische onderzoeksgebieden gedefinieerd. In hoofdstuk 2 wordt specifiek ingegaan op iconiciteit (het bestaan van gelijkenissen tussen vorm en betekenis) als linguïstisch en semiotisch onderwerp. Verder wordt het systeem van classifierpredicaten (classifier predicates) beschreven, waarmee men in de gebarenruimte iconische afbeeldingen van een echte of conceptuele ruimte kan creëren. Zulke morfologisch complexe predicaten worden samengesteld uit een handvorm (de classifier), die informatie geeft over de vorm of de semantische klasse van een object, en de positie en beweging van de handen in de gebarenruimte, waardoor informatie over de locatie en beweging van de objecten wordt weergegeven. Het onderzoek concentreert zich op twee typen classifiers: entiteitsclassifiers (entity classifiers), waar de handvorm een geheel object (of wezen) weergeeft (bv. een auto of een boom) en hanteerclassifiers (handling classifiers), waar de 
handvorm de manipulatie van een object door een Agens uitbeeldt (bv. het gooien van een bal of het vasthouden van een pan).

Hoofdstuk 3 geeft een overzicht van beschrijvingen van ruimte in gesproken en gebarentaal (met name het linguïstisch uitdrukken van locatie en beweging) en bespreekt het gebruik van relatiekaders (frames of reference) in zulke beschrijvingen. Relatiekadersystemen worden zowel in gesproken talen als in gebarentalen gebruikt om de ruimtelijke relatie uit te drukken tussen twee objecten (een gestaltobject (figure object) en een basisobject (ground object)) die in de ruimte gescheiden zijn. De onderlinge relatie tussen de objecten kan daarbij op drie verschillende manieren worden beschreven. Een relatief relatiekader drukt een driehoeksrelatie uit door de locatie van het gestaltobject in verhouding tot het basisobject te beschrijven vanuit een extern standpunt of origo (bv. de man staat links van de boom). Dit in tegenstelling tot een intrinsiek relatiekader dat de onderlinge ruimtelijke relatie tussen gestalt- en basisobject uitdrukt, gezien vanuit de inherente kenmerken van het basisobject (bv. de man staat voor de auto). Tenslotte maakt het absolute relatiekader gebruik van een algemeen vastgesteld oriëntatiesysteem (bv. kompasrichting) om de locatie van het gestaltobject in verhouding tot het basisobject te specificeren (bv. de rivier ligt ten noorden van de stad).

In hoofdstuk 3 wordt verder het gebaarperspectief (signing perspective) geïntroduceerd als een belangrijk middel om over ruimte te praten. Het gebaarperspectief bepaalt de manier waarop een ruimtelijke scène op de gebarenruimte wordt geprojecteerd. Het bepaalt hoe de gebarenruimte voor het representeren van ruimte word gestructureerd, dus speelt deze een belangrijke rol in het afbeelden van ruimtelijke relaties. Er zijn twee manieren waarop deze projectie kan plaatsvinden Deze zijn afhankelijk van de locatie van de verteller in relatie tot de afgebeelde ruimte. Aan de ene kant kan de verteller een extern observatiepunt innemen, waarbij hij de rol heeft van een observeerder die de gehele ruimte kan overzien. Wanneer deze ruimte in verkleinde vorm op de gebarenruimte van de observerende verteller wordt afgebeeld, noemen we dit een een projectie van de beschreven situatie vanuit het perspectief van een externe toeschouwer (observer perspective event space projection). Aan de andere kant kan het observatiepunt van de verteller binnen de afgebeelde ruimte liggen. Dit interne perspectief kan worden bereikt door het aannemen van de rol van een karakter in de beschreven scène. Hierdoor valt de locatie van dit karakter samen met dat van de verteller en worden objecten in de gebarenruimte afgebeeld zoals die worden gezien vanuit de locatie van het aangenomen karakter. Dit noemen we een projectie van de gebeurtenissenruimte vanuit het perspectief van één van de karakters (character perspective event space projection). 
De hoofdstukken 4-8 tonen de resultaten van het empirisch onderzoek van het proefschrift. Dove proefpersonen waren gevraagd om eenvoudige en complexe statische ruimtelijke scènes en dynamische gebeurtenissen (met name tekenfilmpjes) te beschrijven aan een, eveneens dove, toeschouwer. Dit deden zij in de DGS, op de manier die zij in hun dagelijks leven gewend zijn. Toeschouwers moesten vervolgens de ruimtelijke scène construeren (door middel van speelgoedpoppetjes) of het verhaal aan de verteller navertellen, zoals ze hem hadden begrepen. Hieronder volgt een samenvatting van de belangrijkste uitkomsten van het onderzoek, waarbij de nadruk ligt op het uitdrukken van locatie en beweging bij het beschrijven van statische scènes en complexere gebeurtenissen aan de ene kant, en op de wisselwerking tussen de verschillende manieren om ruimte te beschrijven aan de andere kant (classifierpredicaten, gebaarperspectief en ruimtelijke relatiekaders).

Hoofdstuk 4 presenteert een kwalitatief en kwantitatief onderzoek naar de conventies die in de wetenschappelijke literatuur over gebarentaal belangrijk zijn voor het uitdrukken van locatie en oriëntatie in beschrijvingen van ruimtelijke scènes. Onderzocht is wat de geldigheid van deze conventies is voor de DGS. Het resultaat laat zien dat vertellers in de DGS inderdaad gebruik maken van de iconische eigenschappen van classifierpredicaten en van de gebarenruimte in hun beschrijvingen van ruimtelijke scènes maar daarnaast ook ruimtelijke informatie overbrengen door gebruik te maken van directionele werkwoorden (directional predicates; predicaten waarvan de bewegingsrichting in de gebarenruimte bepaald wordt door de (echte of conceptuele) locatie van de objecten/mensen waarover wordt gesproken), getallen en voorzetselvormen, en verder door het type van predicatie en subject NP. Verder toont hoofdstuk 4 dat bij DGS-beschrijvingen van locatie de gestaltbasisstructuur afhangt van de perceptuele kenmerken van de objecten zelf.

In hoofdstuk 5 wordt met behulp van dezelfde opzet gekeken hoe perspectief en relatiekaders worden gebruikt in beschrijvingen van statische scènes in de DGS. Aangetoond wordt dat vertellers in de DGS op consistente wijze informatie over locatie en oriëntatie op hun gebarenruimte projecteren vanuit het perspectief van een (externe) observeerder (in observerperspectief). Bovendien worden ruimtelijke relaties beschreven ofwel door middel van een relatief relatiekader ofwel door een simultane combinatie van een relatief en een intrinsiek relatiekader. Dit laatste is onmogelijk in gesproken talen en daardoor uniek voor gebarentalen. De resultaten van deze studie wijzen op een prototypisch samenvallen (alignment) van observerperspectief en een relatief relatiekader in de DGS, op grond van kenmerken die door beide systemen worden gedeeld. Toch laten de uitkomsten ook zien dat 
de verschillende vormen van perspectief en van relatiekader onafhankelijke middelen zijn voor het uitdrukken van ruimtelijke relaties.

In hoofdstuk 5 is ook vastgesteld op welke wijze de toeschouwers (die recht tegenover de verteller zitten) de informatie over locatie en oriëntatie die zij in de gebarenruimte zien interpreteren. De resultaten laten zien dat zij de ruimtelijke configuratie mentaal roteren, waardoor zij de beschreven scène in dezelfde oriëntatie plaatsen als deze voor de verteller was. Dit is een verder bewijs dat het relatieve relatiekader, met het standpunt van de verteller als origo, de conventie is in de DGS voor het beschrijven van statische ruimtelijke scènes.

In hoofdstuk 6 worden vertellingen van complexe gebeurtenissen (event narratives) onderzocht, om het gebruik te bestuderen van perspectief en relatiekader bij het beschrijven van ruimtelijke relaties (locatie en beweging) in een andere type discours. Deze studie toont aan dat zowel locatie als beweging in complexe gebeurtenissen voornamelijk worden uitgebeeld in characterperspectief en met behulp van een intrinsiek relatiekader. Dit resultaat is niet in overeenstemming met de uitkomsten van hoofdstuk 5 en wijst erop dat het type discours een sterke invloed heeft op de keuze van perspectief en relatiekader in het overbrengen van ruimtelijke informatie in de DGS. Ook wordt duidelijk dat locatie en beweging niet op fundamenteel verschillende wijzen worden uitgedrukt door gebruikers van de DGS. Hoofdstuk 6 pleit voor een prototypisch samenvallen van het characterperspectief en het intrinsieke relatiekader, maar toont ook aan dat deze twee systemen onafhankelijk zijn, aangezien de resultaten laten zien dat het relatieve en het intrinsieke relatiekader gelijktijdig kunnen voorkomen in het characterperspectief.

Hoofdstuk 7 bespreekt het gebruik van verschillende manieren waarop de locatie, beweging en/of actie van een object kunnen worden weergeven (classifierpredicaten, lexicale predicaten en wijsgebaren), in relatie tot hun gebruik in observer- of characterperspectief. Dit hoofdstuk bevat een kwalitatieve analyse, waarbij voorbeelden worden getoond van verschillende soorten combinaties van predicaat en perspectief (predicate-perspective construction types) en een kwantitatieve analyse, die de verdeling van de verschillende constructietypen aantoont in de DGS event narratives. Deze analyse suggereert een samenvallen van entiteitsclassifiers en observerperspectief aan de ene kant en van hanteerclassifiers en characterperspectief aan de andere kant. Dit wordt gebaseerd op een iconiciteit op de schaal van representatie (tussen de grootte van de gerepresenteerde objecten en de gerepresenteerde ruimte) en op het soort informatie die effectief kan worden uitgedrukt door middel van verschillende classifiervormen. Het gebruik van constructies waarin 
classifiertype en perspectief samenvallen (aligned) versus constructies met niet-samenvallend classifiertype en perspectief (non-aligned) wordt besproken in relatie tot iconiciteit.

Hoofdstuk 8 ligt de nadruk op simultaneïteit als speciale toepassing van de visueelruimtelijke modaliteit. Constructies worden onderzocht waarin verscheidene articulatoren, zowel manueel (de handen) als niet-manueel (het hoofd, de ogen, de schouders), tegelijkertijd worden ingezet om elementen uit te drukken die met verschillende gebaarperspectieven geassocieerd zijn. Het gebruik van zulke constructies wordt beschreven als een middel om ruimtelijke coherentie (spatial coherence) tussen perspectieven te bevestigen (dat wil zeggen dat ruimtelijke relaties tussen objecten blijven kloppen, zelfs als van perspectief wordt gewisseld). Ook kunnen zulke constructies worden ingezet om iconiciteit van tijd (temporal iconicity) en/of schaal (scale iconicity) in de representatie van de gebeurtenis te bewaren. Hun voorkomen en functie in een verhaal in de DGS wordt verder besproken in verband met efficiency (een spreker streeft naar economie en doelmatigheid) en informativeness (een spreker streeft naar het duidelijk overbrengen van informatie). Deze zijn mede bepalend voor het verloop van een discours en werken in samenspel met iconiciteitsprincipes.

Het afsluitende hoofdstuk (hoofdstuk 9) draagt de methodologische en theoretische bijdrage van het proefschrift opnieuw tevoorschijn. Het benadrukt dat zorgvuldige en systematische analyse van gebarentaal in communicatief gebruik, en in verschillende typen van discours, belangrijk is voor een goed begrip van de manier waarop ruimtelijke relaties kunnen worden uitgedrukt. De uitkomsten van de voorafgaande hoofdstukken worden samengevat, in het bijzonder wat gebaarperspectief (signing perspective), ruimtelijke relatiekaders (frames of reference), en classifierpredicaten (classifier predicates) betreft. Ook wordt benadrukt dat deze systemen prototypische samenvallingen (alignments) vertonen, maar toch als onafhankelijke systemen moeten worden gezien. Verder wordt de invloed van verschillende iconiciteitsprincipes (bv. temporal iconicity en scale iconicity) op de structuur van representaties van ruimte en hun samenspel met linguïstische en discoursstructurele principes besproken. 


\section{Zusammenfassung}

Gebärdensprache wird vorwiegend mit den Händen produziert und mit den Augen erfaßt. Sprachliche Ausdrücke, z.B. Sachverhalte und Ereignisse wiedegebend, werden im sogenannten Gebärdenraum, d.h. in dem drei-dimensionalen Raum vor dem Körper des Gebärdenden, artikuliert. Wie in gesprochenen Sprachen auch müssen dabei z.B. räumliche, zeitliche und kausale Zusammenhänge deutlich gemacht werden. Diese Dissertation untersucht den Ausdruck räumlicher Information in der Deutschen Gebärdensprache (DGS). Die Untersuchung richtet sich auf den sprachlichen Ausdruck räumlicher Verhältnisse zwischen Ojbekten in zwei verschiedenen Diskurstypen: zum einen die Beschreibung statisch-räumlicher Szenen (Spezifizierung von Lokation) und zum anderen die Beschreibung komplexer Ereignisse (Spezifizierung von Lokation und Bewegung). Durch die systematische Erkundung der Struktur von sprachlichen Konstruktionen über Raum im kontext-gebundenen Sprachgebrauch erfaßt diese Dissertation die Möglichkeiten des ikonischen Abbildens in der visuell-räumlichen Modalität, und geht damit über bisherige Untersuchungen im Bereich des räumlichen Ausdrucks hinaus, sowohl für Gebärdensprachen im Allgemeinen als auch für DGS im Einzelnen. Die Erforschung verschiedener Parameter, z.B. linguistisch, diskursiv und artikulatorisch, die die ikonische Repräsentation in Gebärdensprache beeinflußen und ihr Beschränkungen auferlegen können, trägt zu unserem allgemeinen Verständnis von Ikonizität in der visuell-räumlichen Modalität bei.

Kapitel 1-3 der Dissertation stellen Gebärdensprache vor als die natürliche Sprachmodalität gehörloser Menschen, erläutern strukturelle Merkmale von Sprache in der visuell-räumlichen Modalität, definieren die den Untersuchungen zugrundeliegenden theoretischen Fragestellungen und skizzieren die relevanten theoretischen Bereiche. Speziell wird in Kapitel 2 eingegangen auf Ikonizität als Gegenstand linguistischer und semiotischer Theorie. Desweiteren beschreibt Kapitel 2 das gebärdensprachliche System van Klassifikatorprädikaten (classifier predicates), womit Gebärdende ikonische Abbildungen eines echt oder konzeptuell existierenden Raums im Gebärdenraum schaffen können. In diesen morphologisch komplexen Prädikaten drückt der Klassifikator (d.h. die Handform) Information aus über die Größe und Form eines Referenten, und die Lokation und Bewegung der Hände im Gebärdenraum geben Information über die Lokation und Bewegung der Referenten wieder. Zwei Typen von Klassifikatorprädikaten sind hierbei besonders wichtig: 
Entitäts-Klassifikatoren (entity classifiers), bei welchen die Handform einen ganzen Referenten (d.h. eine ganze Entität, z.B. ein Auto oder einen Baum) repräsentiert, und Handlungs-Klassifikatoren (handling classifiers), bei welchen die Handform die Handhabung eines Referenten durch ein Agens repräsentiert.

Kapitel 3 gibt einen Überblick über Raum als linguistisches Untersuchungsobjekt (d.h. über den sprachlichen Ausdruck von v.a. Lokation und Bewegung) in geprochenen und Gebärdensprachen und definiert den Gebrauch von Referenzrahmen (frames of reference) in solchen Ausdrücken. Referenzrahmen werden in gesprochenen und Gebärdensprachen verwendet, um die räumliche Beziehung zwischen zwei Objekten (einem Figur- und GrundObjekt) zu kennzeichnen, wenn diese Objekte voneinander räumlich getrennt sind. In natürlichen Sprachen kann diese Beziehung auf drei verschiedene Weisen ausgedrückt werden. Der relative Referenzrahmen drückt eine ternäre Relation aus, indem die Lokation des Figur-Objekts bezüglich des Grund-Objekts von einem bestimmten Blickwinkel (einem origo) aus spezifiziert wird (z.B. der Mann ist links vom Baum). Im Gegensatz hierzu dienen beim intrinsischen Referenzrahmen inhärente Merkmale des Grund-Objektes dazu, eine binäre Relation zwischen Figur- und Grund-Objekt zu spezifizieren (z.B. der Ball ist vor dem Auto). Schließlich werden beim absoluten Referenzrahmen feste, externe Bezugspunkte (z.B. die Himmelsrichtungen) verwendet, um die Lokation des Figur-Objekts bezüglich des Grund-Objekts auszudrücken (z.B. der Fluß liegt nördlich der Stadt).

In Kapitel 3 wird zudem die Gebärdenperspektive (signing perspective) eingeführt als wichtiges Mittel, um über Raum zu reden. Die Gebärdenperspektive bezeichnet die Art der Projektion des Ereignisraumes (event space) auf den Gebärdenraum. Somit bestimmt die Perspektive wie der Gebärdenraum für räumliche Repräsentation, d.h. für den Ausdruck räumlicher Beziehungen, strukturiert wird. Der Ereignisraum kann auf zwei Weisen auf den Gebärdenraum projiziert werden, in Abhängigkeit davon, wo sich der Gebärdende bezüglich des Ereignisraumes konzeptuell befindet. Auf der einen Seite kann der Gebärdende konzeptuell außerhalb des Ereignisraumes sein und einen externen Blickwinkel einnehmen. Der Gebärdende nimmt hier die Rolle eines Beobachters ein, welcher den gesamten Ereignisraum übersieht und dieser vor sich im Gebärdenraum verkleinert abbildet. Dieses entspricht einer Projektion des Ereignisraumes von einer Beobachterperspektive aus (observer perspective event space projection). Auf der anderen Seite kann sich der Gebärdende konzeptuell innherhalb des Ereignisraumes befinden und einen ereignis-internen Blickwinkel haben. Eine solche interne Perspektive wird ermöglicht durch die Übernahme der Perspektive oder Rolle eines Ereignisteilnehmers. Hierbei fällt die Lokation des 
Teilnehmers (im Ereignisraum) zusammen mit der Lokation des Gebärdenden. Die übrigen Referenten im Ereignisraum werden aus der Perspektive des Teilnehmers auf den Gebärdenraum abgebildet, was einer Projektion des Ereignisraumes von einer Teilnehmerperspektive aus entspricht (character perspective event space projection).

Kapitel 4-8 bilden den empirischen Teil der Dissertation. Zur Datenerhebung wurden gehörlose DGS-Gebärdende gefragt, an zwei verschiedenen Aufgaben teilzunehmen. Zum einen sollten Gebärdende statisch-räumliche Szenen an einen ihnen gegenübersitzenden, ebenfalls gehörlosen Adressaten beschreiben. Mithilfe von Spielfiguren mußte der Adressat die beschriebene Szene konstruieren. Zum anderen haben Gebärdende komplexe Ereignisse (in der Form von Zeichentrickfilmen) einem Adressaten erzählt; dieser mußte wiederum die Geschichte nacherzählen. Im Folgenden werden für die einzelnen empirischen Kapitel die Hauptergebnisse zusammengefaßt. Der Schwerpunkt liegt hierbei auf der Charakterisierung des Ausdrucks von Lokation und Bewegung in Beschreibungen von statischen Szenen und Ereignisnarrativen, mit besonderem Hinblick auf das Zusammenspiel der relevanten räumlichen Systeme (d.h. Klassifikatorprädikaten, Perspektive und Referenzrahmen).

Kapitel 4 untersucht DGS-Beschreibungen statisch-räumlicher Szenen und bietet eine qualitative und quantitative Analyse der Konventionen, die in der sprachwissenschaftlichen Gebärdensprachliteratur als den Ausdruck von räumlicher Information bestimmend angeführt werden, um ihre Gültigkeit für die DGS zu bewerten. Die Analyse zeigt, daß sich die DGS für den Ausdruck räumlicher Information der ikonischen Merkmale von Klassifikatorprädikaten und des Gebärdenraumes stark bedient, aber daß auch direktionale Prädikate (directional predicates; d.h. Prädikate, deren Bewegungsrichtung im Gebärdenraum durch die (echte oder konzeptuelle) Lokation der Referenten bestimmt wird), Zahlgebärden und Präpositionalformen, sowohl als auch der Prädikations- und Subjekt-NPTyp eine wichtige Rolle spielen. Zudem wird in diesem Kapitel gezeigt, daß die FigurGrund-Struktur in DGS-Beschreibungen räumlicher Beziehungen von den perzeptuellen Merkmalen der beschriebenen Objekte abhängt.

Anhand derselben Daten wird in Kapitel 5 der Gebrauch von Gebärdenperspektive und Referenzrahmen in Beschreibungen statisch-räumlicher Szenen untersucht. Gezeigt wird, daß DGS-Gebärdende Lokation und Orientation konsistent von einer Beobachterperspektive aus auf den Gebärdenraum projizieren. Zudem wird räumliche Information entweder in einem relativen Referenzrahmen oder in einer simultanen Kombination eines relativen und intrinsischen Referenzrahmens repräsentiert. Letzteres ist in gesprochener Sprache nicht möglich, und stellt daher ein besonderes Merkmal der visuell-räumlichen Modalität dar. Die 
Ergebnisse unterstützen ein prototypisches Zusammenfallen (alignment) der Beobachterperspektive und des relativen Referenzrahmens, aufgrund ähnlicher semantischer Charakteristika, deuten jedoch auch auf die Unabhängigkeit der beiden Systeme.

In Kapitel 5 wird auch eine Interpretationsanalyse dargeboten, die anhand der von den Adressaten konstruierten Szenen untersucht wie Perspektive und Referenzrahmen in Beschreibungen statisch-räumlicher Szenen interpretiert werden. Es wird gezeigt, daß Adressaten (den Gebärdenden gegenübersitzend) Lokations- und Orientierungsinformation vorwiegend mental rotieren. Das heißt, die räumliche Information, die sie im Gebärdenraum sehen, geben sie so wieder, daß es der Beobachterperspektive des Gebärdenden entspricht. Die Interpretationsanalyse liefert einen weiteren Beweis für die Konventionalisierung des relativen Referenzrahmens, mit dem Gebärdenden als origo, in der DGS.

In Kapitel 6 werden Ereignisnarrative (event narratives) analysiert, um den Gebrauch von Perspektive und Referenzrahmen im Ausdruck räumlicher Beziehungen in einem anderen Diskurstyp zu untersuchen. Die Ergebnisse zeigen, daß sowohl Lokation als auch Bewegung in Ereignisnarrativen hauptsächlich von einer Teilnehmerperspektive aus und mittels eines intrinsischen Referenzrahmens ausgedrückt werden. Der Kontrast zwischen den Ergebnissen dieses und des vorigen Kapitels zeugt von einem starken Einfluß von Diskurstyp auf die Wahl von Perspektive und Referenzrahmen im Enkodieren räumlicher Information. Die Ergebnisse zeigen auch, daß sich der linguistische Ausdruck von Lokation und Bewegung in der DGS nicht grundlegend verschiedener Konstruktionen bedient. Desweiteren wird in diesem Kapitel für das prototypische Zusammenfallen (alignment) der Teilnehmerperspektive und des intrinsischen Referenzrahmens argumentiert. Doch wird auch gezeigt, daß die Teilnehmerperspektive, ebenso als die Beobachterperspektive, die simultane Verwendung der relativen und intrinsischen Referenzrahmen zuläßt, was auf die Unabhängigkeit der beiden Systeme - Perspektive und Referenzrahmen - hinweist.

Kapitel 7 diskutiert verschiedene Prädikatstypen (Klassifikatorprädikate, lexikalische Prädikate und Zeigegebärden), die Information über Lokation, Bewegung und Handlung im Raum ausdrücken können, im Zusammenhang mit ihrer Verwendung innerhalb einer teilnehmer- oder beobachterperspektivischen Projektion des Ereignisraumes. Das Kapitel liefert sowohl eine qualitative Analyse, welche Beispiele von verschiedenen PrädikatPerspektive-Konstruktionstypen identifiziert und illustriert, als auch eine quantitative Analyse, welche die Distribution der verschiedenen Konstruktionstypen in den DGS-Daten diskutiert. Zudem wird ein prototypisches Zusammenfallen (alignment) von 
Entitätsklassifikatoren und der Beobachterperspektive, auf der einen Seite, und zwischen Handlungsklassifikatoren und der Teilnehmerperspektive, auf der anderen Seite, motiviert. Dieses Zusammenfallen basiert auf einem Ikonizitätsprinzip des Repräsentationsmaßstabs (zwischen der Größe der Referentenrepräsentation und der Größe des projizierten Ereignisraumes) und auf einer Übereinstimmung zwischen Klassifikatortyp und der durch ihn effektiv ausdrückbaren Information. Der Gebrauch von prototypisch zusammenfallenden (aligned) und nicht-zusammenfallenden (non-aligned) Konstruktionstypen wird in Bezug auf Ikonizitätsprinzipien diskutiert.

In Kapitel 8 liegt der Schwerpunkt auf Simultaneität als besondere Eigenschaft der visuell-räumlichen Modalität. Untersucht werden Konstruktionen, in denen mehrfache Artikulatoren, sowohl manuelle (die Hände) als auch nicht-manuelle (z.B. der Kopf, die Augen, die Schultern), gleichzeitig gebraucht werden um Ereignisstrukturkomponenten auszudrücken, die mit verschiedenen Perspektiven assoziiert sind. Die Verwendung solcher Konstruktionen dient der Aufrechterhaltung der räumlichen Kohärenz zwischen Perspektiven (so daß die räumlichen Verhältnisse zwischen Referenten auch bei Perspektivenwechsel bestehen bleiben) und wird im Zusammenhang mit dem Erzielen von Ikonizität der temporalen Struktur (temporal iconicity) und des Maßstabs (scale iconicity) in der Ereignisrepräsentation diskutiert. Das Vorkommen dieser Konstruktionen in der DGS wird zusätzlich in Bezug auf efficiency (ein Sprecher strebt nach Effizienz) und informativeness (ein Sprecher strebt die deutliche Kommunikation von Information an) besprochen, welche die Diskursstruktur beeinflußen und mit verschiedenen Ikonizitätsprinzipien interagieren.

Das abschließende Kapitel (Kapitel 9) hebt die methodologischen und theoretischen Leistungen und Beiträge dieser Dissertation erneut hervor. Das Kapitel betont die Wichtigkeit und Notwendigkeit der vorsichtigen, systematischen Erforschung des kontext-gebundenen Sprachgebrauchs, in verschiedenen Diskurstypen, um die linguistischen Möglichkeiten des Ausdrucks räumlicher Information zu erfassen. Desweiteren faßt das Kapitel die Ergebnisse der vorangegangen Kapitel bezüglich der Verwendung von Perspektive, Referenzrahmen und Klassifikatorprädikaten zusammen. Es unterstreicht die strukturellen Ähnlichkeiten zwischen den Systemen, welche ihr prototypisches Zusammenfallen motivieren, aber auch ihr Bestehen als voneinander unabhängig zu betrachtende und behandelnde Systeme. Schließlich wird der Einfluß verschiedener Ikonizitätsprinzipien (z.B. Ikonizität des Repräsentationsmaßstabs und temporale Ikonizität) auf die Struktur räumlicher Repräsentationen, und ihre Interaktion mit linguistischen und diskursstrukturellen Prinzipien, diskutiert. 


\section{Curriculum vitae}

Pamela Perniss (1974) studied General Linguistics (major), Comparative Literature, and Philosophy (minors) at the Eberhard Karls Universität Tübingen and the Universität zu Köln, and obtained her MA in Linguistics at the Universität zu Köln. She was granted a PhD stipend from the Max-Planck-Gesellschaft and pursued her dissertation research within the Language and Cognition group at the Max Planck Institute for Psycholinguistics, Nijmegen, The Netherlands. She is currently a postdoctoral researcher at the Radboud University, Nijmegen and the MPI for Psycholinguistics, Nijmegen in the NWO-funded VIDI project "Relations between modality and language structure: Insights from comparisons of sign languages and gestures" awarded to Dr. Asli Özyürek. She has also been granted a Marie Curie stipend as a postdoctoral fellow for a research project on "The linguistic and cognitive processing of space in signed and spoken language" at the Deafness, Cognition, and Language Research Centre (DCAL) at University College London with Professor Gabriella Vigliocco. 
1. The electrophysiology of speaking: Investigations on the time course of semantic, syntactic, and phonological processing. Miranda van Turennout

2. The role of the syllable in speech production: Evidence from lexical statistics, metalinguistics, masked priming, and electromagnetic midsaggital articulography. Niels O. Schiller

3. Lexical access in the production of ellipsis and pronouns. Bernadette M. Schmitt

4. The open-/closed-class distinction in spoken-word recognition. Alette Haveman

5. The acquisition of phonetic categories in young infants: A self-organising artificial neural network approach. Kay Behnke

6. Gesture and speech production. Jan-Peter de Ruiter

7. Comparative intonational phonology: English and German. Esther Grabe

8. Finiteness in adult and child German. Ingeborg Lasser

9. Language input for word discovery. Joost van de Weijer

10. Inherent complement verbs revisited: Towards an understanding of argument structure in Ewe. James Essegbey

11. Producing past and plural inflections. Dirk Janssen

12. Valence and transitivity in Saliba: An Oceanic language of Papua New Guinea. Anna Margetts

13. From speech to words. Arie van der Lugt

14. Simple and complex verbs in Jaminjung: A study of event categorisation in an Australian language. Eva Schultze-Berndt

15. Interpreting indefinites: An experimental study of children's language comprehension. Irene Krämer

16. Language-specific listening: The case of phonetic sequences. Andrea Weber

17. Moving eyes and naming objects. Femke van der Meulen

18. Analogy in morphology: The selection of linking elements in Dutch compounds. Andrea Krott

19. Morphology in speech comprehension. Kerstin Mauth 
20. Morphological families in the mental lexicon. Nivja H. de Jong

21. Fixed expressions and the production of idioms. Simone A. Sprenger

22. The grammatical coding of postural semantics in Goemai (a West Chadic language of Nigeria). Birgit Hellwig

23. Paradigmatic structures in morphological processing: Computational and crosslinguistic experimental studies. Fermín Moscoso del Prado Martín

24. Contextual influences on spoken-word processing: An electrophysiological approach. Daniëlle van den Brink

25. Perceptual relevance of prevoicing in Dutch. Petra M. van Alphen

26. Syllables in speech production: Effects of syllable preparation and syllable frequency. Joana Cholin

27. Producing complex spoken numerals for time and space. Marjolein Meeuwissen

28. Morphology in auditory lexical processing: Sensitivity to fine phonetic detail and insensitivity to suffix reduction. Rachèl J. J. K. Kemps

29. At the same time...: The expression of simultaneity in learner varieties. Barbara Schmiedtová

30. A grammar of Jalonke argument structure. Friederike Lüpke

31. Agrammatic comprehension: An electrophysiological approach. Marlies Wassenaar

32. The structure and use of shape-based noun classes in Miraña (North West Amazon). Frank Seifart

33. Prosodically-conditioned detail in the recognition of spoken words. Anne Pier Salverda

34. Phonetic and lexical processing in a second language. Mirjam Broersma

35. Retrieving semantic and syntactic word properties. Oliver Müller

36. Lexically-guided perceptual learning in speech processing. Frank Eisner

37. Sensitivity to detailed acoustic information in word recognition. Keren B. Shatzman

38. The relationship between spoken word production and comprehension. Rebecca Özdemir

39. Disfluency: Interrupting speech and gesture. Mandana Seyfeddinipur

40. The acquisition of phonological structure: Distinguishing contrastive from noncontrastive variation. Christiane Dietrich

41. Cognitive cladistics and the relativity of spatial cognition. Daniel B.M. Haun 
42. The acquisition of auditory categories. Martijn Goudbeek

43. Affix reduction in spoken Dutch. Mark Pluymaekers

44. Continuous-speechsegmentation at the beginning of language acquisition: electrophysiological evidence. Valesca Kooijman

45. Space and Iconicity in German Sign Language (DGS). Pamela M. Perniss 